\title{
EXPERIMENTAL INVESTIGATION AND CONCEPTUAL MODEL DEVELOPMENT FOR MULTIPHASE FLOW IN A WATER-WET POROUS MEDIUM WITH NAPL-WET SOIL LENSES
}

\author{
A thesis submitted to \\ the Faculty of Graduate Studies and Research \\ in partial fulfillment of the requirements for the degree of \\ Doctor of Philosophy \\ in Environmental Engineering \\ by \\ Mustafa Elkhedr Ahmed \\ B.Sc., Cairo University, Egypt \\ M.Sc., Cairo University, Egypt \\ Department of Civil and Environmental Engineering \\ Carleton University \\ Ottawa, Ontario \\ Ottawa-Carleton Institute for Civil and Environmental Engineering \\ April 2009
}




\section{Library and Archives}

Canada

Published Heritage

Branch

395 Wellington Street

Ottawa ON K1A ON4

Canada
Bibliotheque et

Archives Canada

Direction du

Patrimoine de l'édition

395 , rue Wellington

Ottawa ON K1A ON4

Canada
Your file Votre référence
ISBN: 978-0-494-60097-9
Our file Notre référence
ISBN: $978-0-494-60097-9$
NOTICE:

The author has granted a nonexclusive license allowing Library and Archives Canada to reproduce, publish, archive, preserve, conserve, communicate to the public by telecommunication or on the Internet, loan, distribute and sell theses worldwide, for commercial or noncommercial purposes, in microform, paper, electronic and/or any other formats.

The author retains copyright ownership and moral rights in this thesis. Neither the thesis nor substantial extracts from it may be printed or otherwise reproduced without the author's permission.
AVIS:

L'auteur a accordé une licence non exclusive permettant à la Bibliothèque et Archives Canada de reproduire, publier, archiver, sauvegarder, conserver, transmettre au public par télécommunication ou par l'Internet, prêter, distribuer et vendre des thèses partout dans le monde, à des fins commerciales ou autres, sur support microforme, papier, électronique et/ou autres formats.

L'auteur conserve la propriété du droit d'auteur et des droits moraux qui protège cette thèse. $\mathrm{Ni}$ la thèse ni des extraits substantiels de celle-ci ne doivent être imprimés ou autrement reproduits sans son autorisation.
In compliance with the Canadian Privacy Act some supporting forms may have been removed from this thesis.

While these forms may be included in the document page count, their removal does not represent any loss of content from the thesis.
Conformément à la loi canadienne sur la protection de la vie privée, quelques formulaires secondaires ont été enlevés de cette thèse.

Bien que ces formulaires aient inclus dans la pagination, il n'y aura aucun contenu manquant.

\section{Canadä}




\begin{abstract}
The remediation of Brownfield sites impacted by non-aqueous phase liquids (NAPLs) remains a challenge. Many of the multiphase flow simulators and remediation technologies developed have assumed that the soils are water-wet. Yet several studies have suggested that the assumption of water-wet conditions may not be valid for a number of field sites. In addition, the processes that impact the magnitude and distribution of the residual NAPL saturation term are not well defined in water-wet porous media and in water-wet porous media with NAPL-wet soil lenses. Therefore, this research focuses on performing experimental work at the pore scale and the representative elementary volume (REV) scale to gain a better understanding of the NAPL distribution in a water-wet porous medium with NAPL-wet soil lenses. The experimental study included measuring capillary pressure-saturation $\left(P_{c}-S\right)$ relationships in two and three-phase systems, and residual NAPL saturations measured under a large range of experimental conditions.
\end{abstract}

The experimental study was followed by development and validation of conceptual models for $P_{c}-S$ relationships for air/NAPL, air/water, and NAPL/water twophase systems as well as for an air/NAPL/water three-phase system for a water-wet porous medium with NAPL-wet soil lenses. The approach taken in this research was to consider the water-wet porous medium with NAPL-wet soil lenses as the field scale REV and to derive constitutive relationships for this REV scale based on our knowledge of the constitutive relationships of each soil fraction (i.e., water-wet and NAPL-wet). The experimental results indicated that the $P_{c} \neg S$ relationship for an REV with NAPL-wet soil lenses could be constructed based on a volume-weighted summation of the $P_{c}-S$ 
relationship for each soil fraction. Special attention is needed to address the isolation of NAPL within the NAPL-wet soil lenses as water imbibes into the system as this may substantially increase the entrapped NAPL saturation.

This research also includes development and validation of a new conceptual model for the residual NAPL saturation term in a water-wet porous medium. The new model assumes that the effective residual NAPL saturation depends on eight parameters; porous medium specific surface area, NAPL entry pressure head in NAPL/water twophase system, porous medium grain size distribution, porous medium porosity, NAPL film thickness, apparent water saturation, historic maximum apparent NAPL saturation, and the absolute maximum effective residual NAPL saturation in the porous medium. The research also includes development and validation of new conceptual models for the residual NAPL saturation term in a water-wet porous medium with isolated or connected NAPL-wet soil lenses. The model predications were compared to the effective residual NAPL saturations measured under a large range of experimental conditions. In most cases, the models were capable of predicting the measured effective residual NAPL saturations. 


\section{ACKNOWLEDGEMENTS}

The author would like to express his deepest and sincerest appreciation to his thesis supervisor Dr. Paul Van Geel. Dr. Van Geel has provided valuable support and guidance throughout the completion of this research. His time and efforts are highly appreciated.

The author would also like to express his deepest gratitude to Dr. Robert Burke for his time, constructive discussions, and permission to carry out some experiments in Chemistry Department laboratories. In addition, the support provided by Dr. John Gaydos is highly appreciated. Dr. Gaydos did not hesitate to provide his time and help in setting up the pore scale experiements.

The author would also like to especially thank Dr. Marie Tudoret, Fekade Fetahi, Stanley Conley, Pierre Trudel, Alex Proctor, Keith Bourque, and Tony O'Neil for their immense support in setting up some experiments. The author would also like to express his sincere appreciation to his friend Dr. Salem Al-Kaabi who provided him with help and support.

In addition, the scholarships awarded by Carleton University, the Natural Sciences and Engineering Research Council (NSERC), and the Canadian Water Resources Association, as well as the financial support provided by Imperial Oil are gratefully acknowledged.

The author would like to thank his dearest parents whose guidance, support, and prayers have helped him throughout his journey in research. In addition, the love and support of his brothers and sisters have encouraged him greatly.

Finally, to who stood by me with love, patience, and support, I dedicate this thesis to my wife, Dalia Said, and my beloved children, Muhammad and Farida. 


\section{TABLE OF CONTENTS}

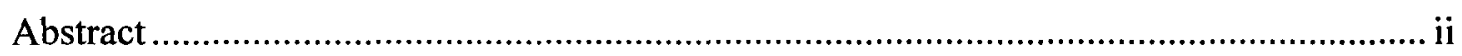

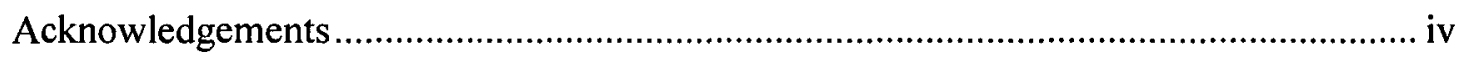

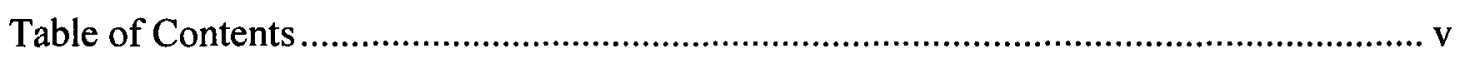

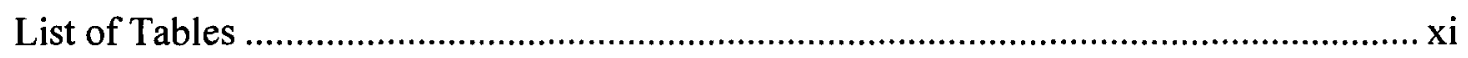

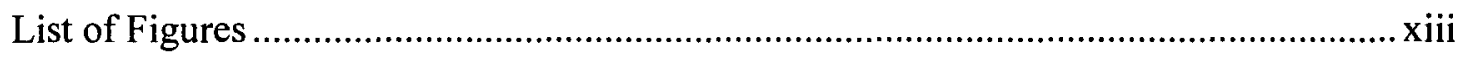

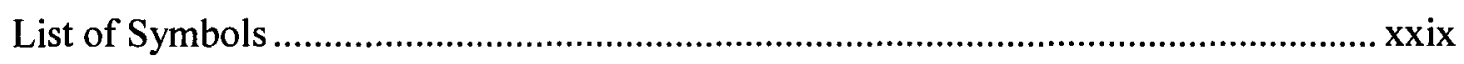

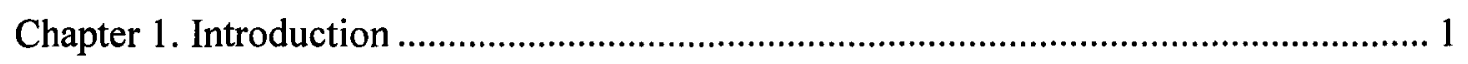

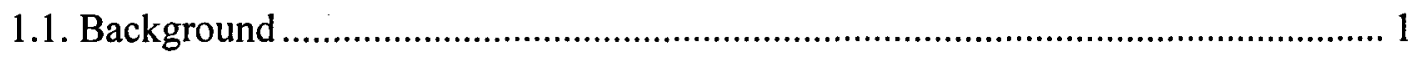

1.2. Problem Definition......................................................................................... 7

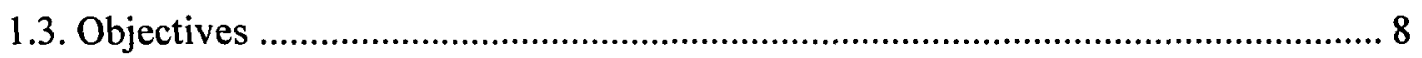

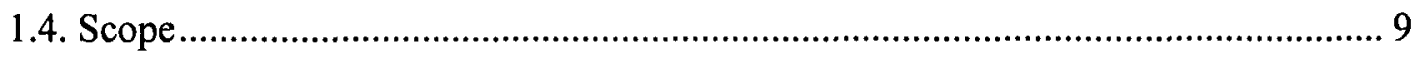

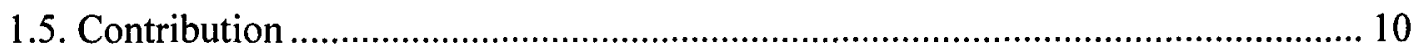

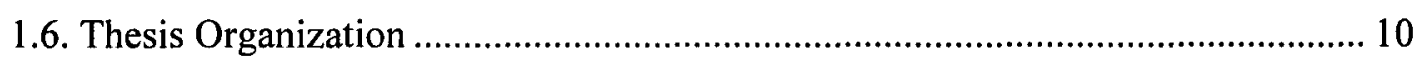

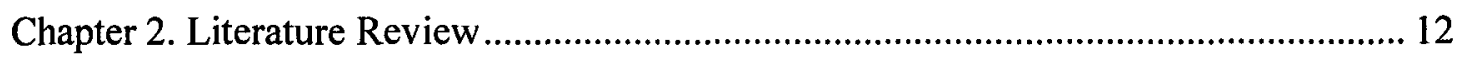

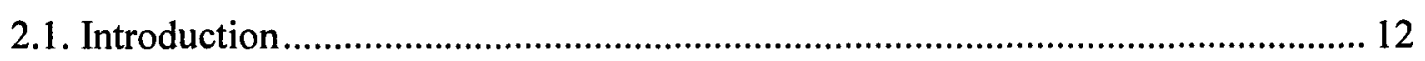

2.2. Properties of Porous Media and Immiscible Fluids ..................................... 12

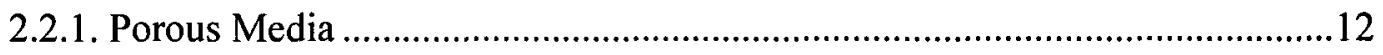

2.2.2. Characterization of Porous Media .......................................................... 13

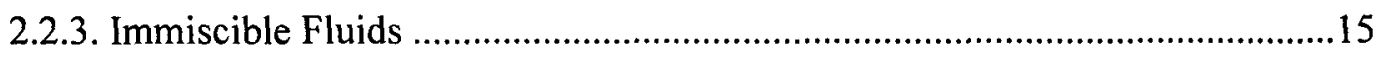

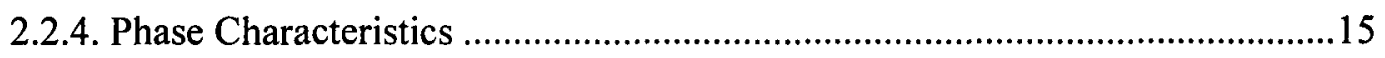

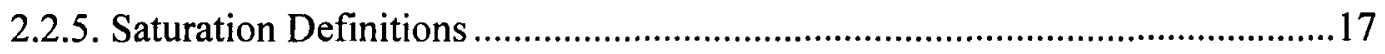

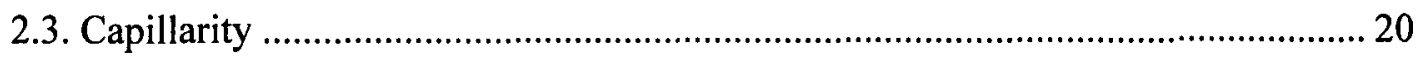


2.3.1. Surface Tension and Surface Free Energy ....................................................20

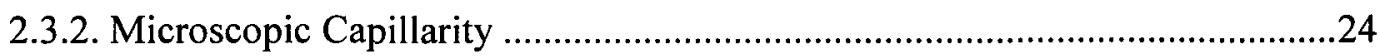

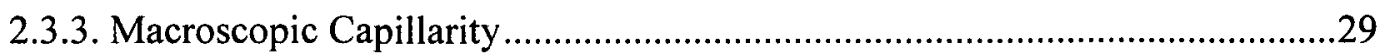

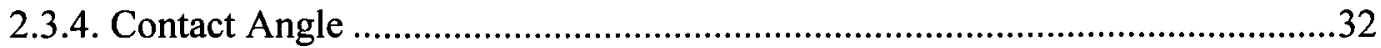

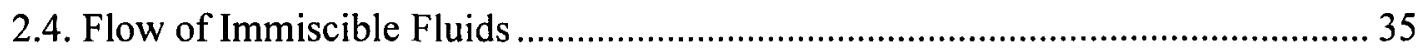

2.4.1. Mass Balance Equation ...................................................................................35

2.4.2. Constitutive Relationships .......................................................................40

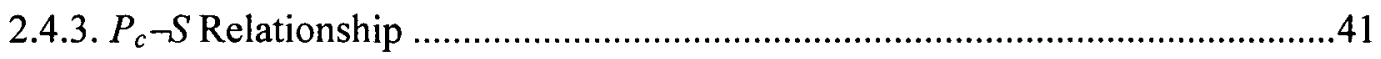

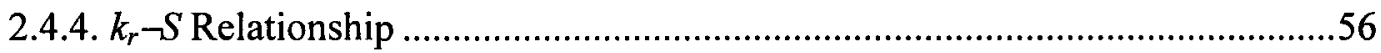

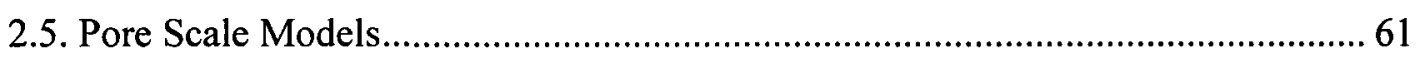

2.5.1. Describing $P_{c}-S-k_{r}$ Relationships ........................................................61

2.5.2. Describing $P c-S$ Hysteresis .......................................................................64

2.5.3. Describing NAPL Entrapment Mechanisms....................................................70

2.6. Porous Medium Wettability ............................................................................ 73

2.6.1. Evidence of Spatial Wettability Variations.........................................................76

2.6.2. Importance of Wettability on Governing Equations of Multiphase Flow ........76

2.6.3. Multiphase Flow Equations with Variable Wettability .....................................77

2.7. Residual Non-Wetting Phase Saturation in Porous Media ……………………....... 81

2.7.1. Factors Contributing to the Residual NAPL Saturation in Porous Media ........82

2.7.2. Conceptual Models Describing Residual NAPL Saturation..............................83

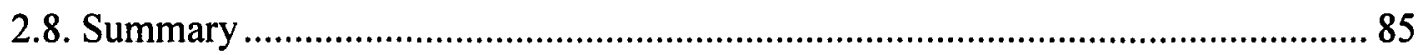

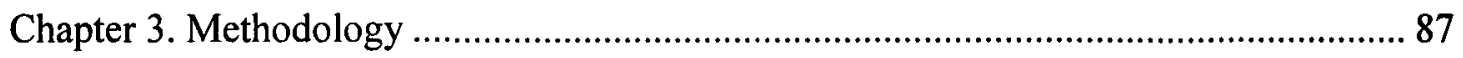

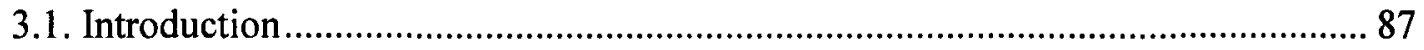




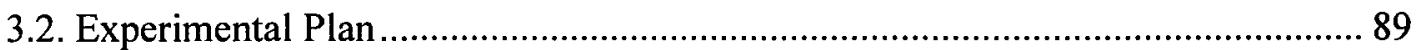

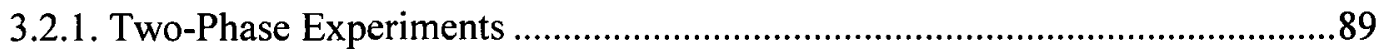

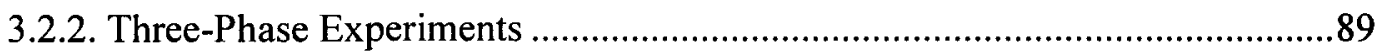

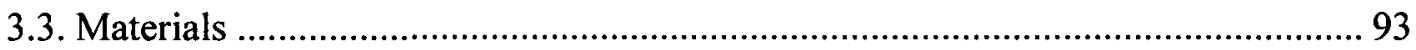

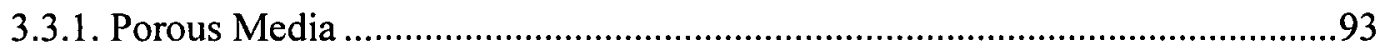

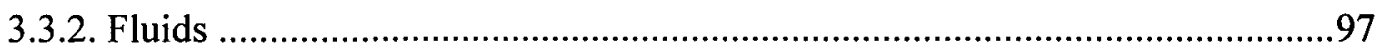

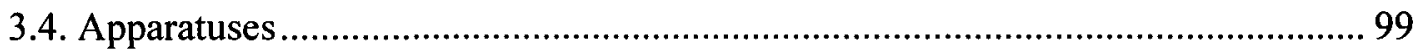

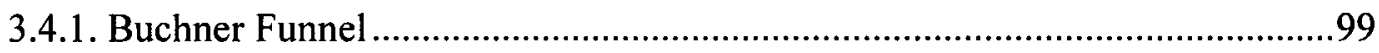

3.4.2. Modified Tempe Cell .................................................................................... 101

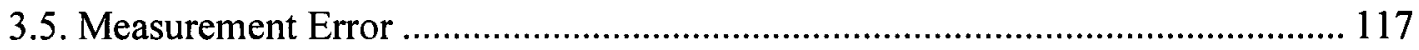

Chapter 4. Concerns Related to Using Octadecyltrichlorosilane in Rendering Soils and Porous Ceramics Hydrophobic ……......................................................................... 122

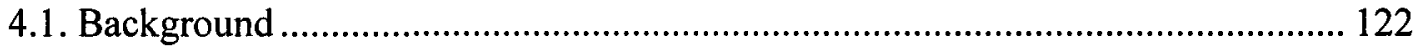

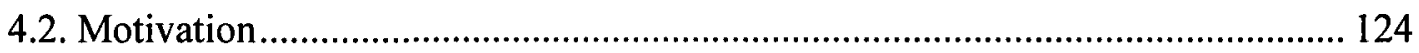

4.3. Conceptual Description of Rendering Hydrophilic Solids Hydrophobic and the

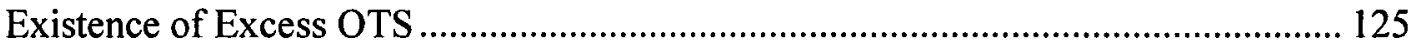

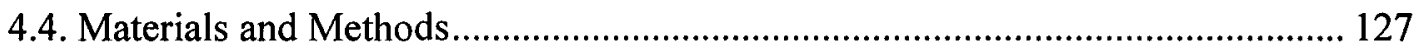

4.4.1. Basic Soil Treatment ...................................................................................127

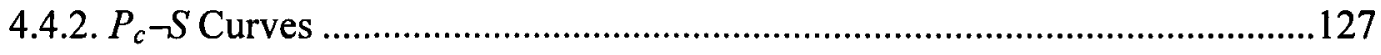

4.4.3. Enhanced Soil Washing Procedure ................................................................129

4.4.4. Verification of Excess OTS Dissolution in a Solvent...................................131

4.4.5. Identifying the Excess OTS Using Nuclear Magnetic Resonance and Infra Red

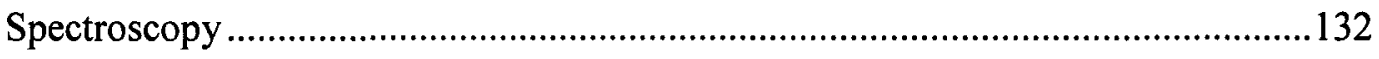


4.4.6. Determination of Soil Wettability.

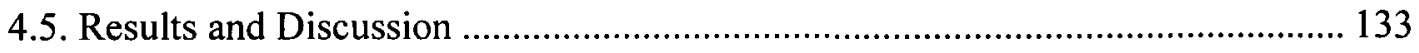

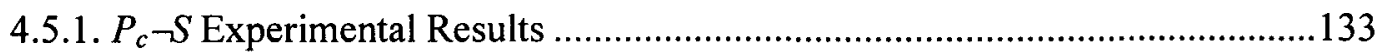

4.5.2. Assessment of OTS Treatment Procedure .............................................. 136

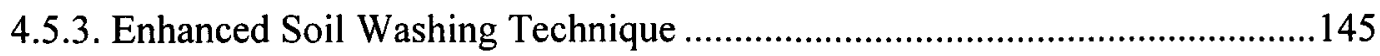

4.5.4. Porous Ceramic Plates Treatment and Washing .......................................151

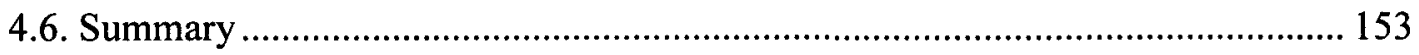

Chapter 5. Capillary Pressure-Saturation Relationships in Two-Phase Systems under

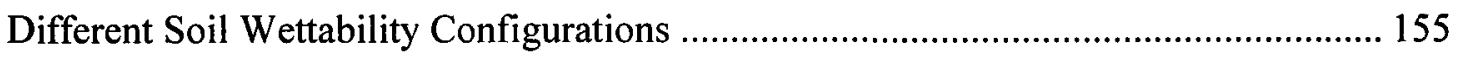

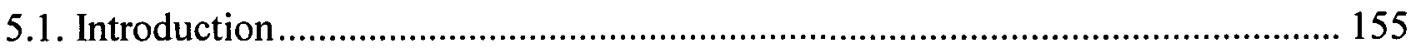

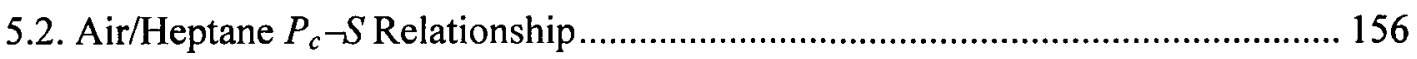

5.2.1. Capillary Pressure Head-Actual Heptane Saturation Data in Different

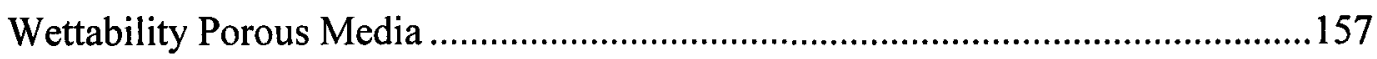

5.2.2. Conceptual Model for $P_{c} S$ Relationship under Different Wettability Configurations 159

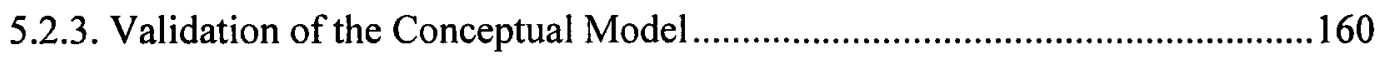

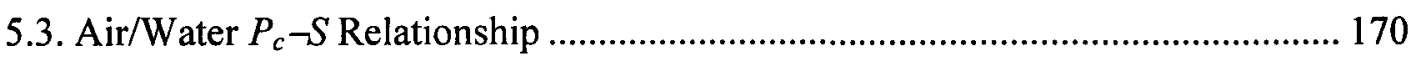

5.3.1. Capillary Pressure Head-Actual Water Saturation Data .............................170

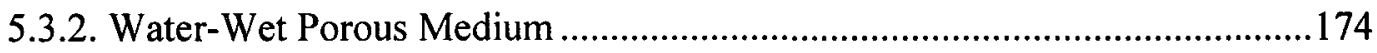

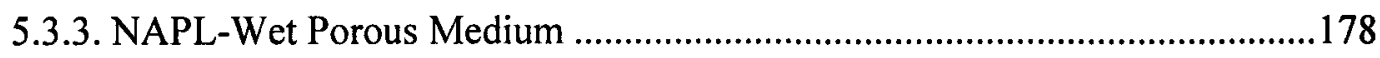

5.3.4. Water-Wet Porous Medium with a NAPL-Wet Soil Lens ...........................182

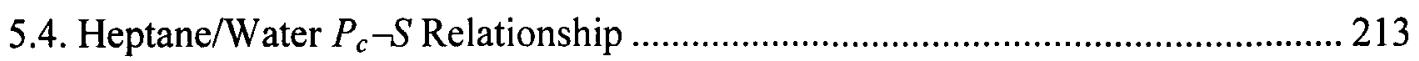

5.4.1. Capillary Pressure Head-Actual Water Saturation Data .............................213 
5.4.2. Water-Wet Porous Medium

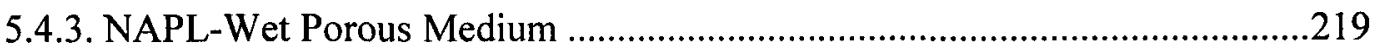

5.4.4. Water-Wet Porous Medium with a NAPL-Wet Soil Lens ...........................223

5.5. Summary 246

Chapter 6. Capillary Pressure-Saturation Relationships in Three-Phase Systems under Different Soil Wettability Configurations 247

6.1. Introduction 247

6.2. Conceptual Model for Air/NAPL Capillary Pressure-Total Liquid Saturation Relationship in a Water-Wet Porous Medium with a NAPL-Wet Soil Lens 250

6.2.1. Scenario (1): Isolated NAPL-Wet Soil Lens of a Grain Size Distribution Different than the Water-Wet Soil Fraction. 253

6.2.2. Scenario (2): Connected NAPL-Wet Soil Lens of a Grain Size Distribution Different than the Water-Wet Soil Fraction. 255

6.2.3. Conceptual Model Validation for a Water-Wet Porous Medium with a Connected NAPL-Wet Soil Lens of Similar Grain Size Distribution 260

6.3. Capillary Pressure Head-Water Saturation 282

6.4. Summary 282

Chapter 7. Residual NAPL Saturation under Different Soil Wettability Configurations 284

7.1. Introduction 284

7.2. Residual NAPL Saturation in a Water-Wet Porous Medium 287

7.2.1. Residual Heptane Saturation Data in Uniform Sand and Well Graded Sand 287

7.2.2. Conceptual Model for Residual NAPL Saturation 291

7.2.3. Validation of Residual NAPL Saturation Conceptual Model 311 
7.3. Residual NAPL Saturation in a Water-Wet Porous Medium with a NAPL-Wet Soil Lens of Different Grain Size Distribution 329

7.3.1. Conceptual Model for Residual NAPL Saturation in a Water-Wet Porous Medium with an Isolated NAPL-Wet Soil Lens 330

7.3.2. Conceptual Model for Residual NAPL Saturation in a Water-Wet Porous Medium with a Connected NAPL-Wet Soil Lens 333

7.3.3. Residual NAPL Saturation in a Water-Wet Porous Medium with a NAPL-Wet Soil Lens of Similar Grain Size Distribution 334

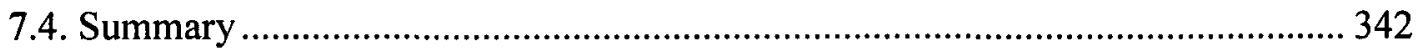

Chapter 8. Summary, Contributions, and Recommendations ................................... 344

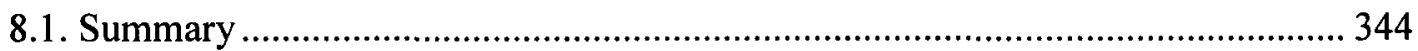

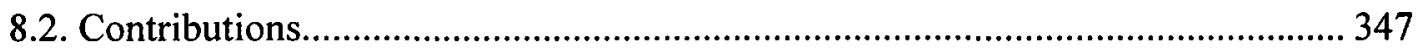

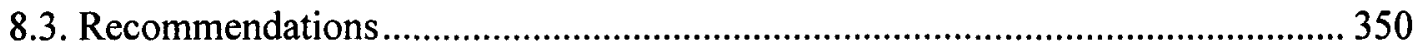

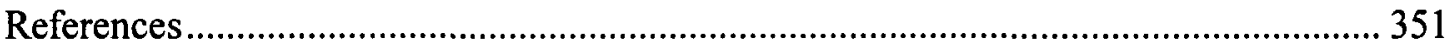




\section{LIST OF TABLES}

Table 3.1: Grain Size Distribution of Well Graded and Uniform Soils.......................... 95

Table 3.2: Coefficients of Grading and Uniformity for Well Graded Sand. .................. 95

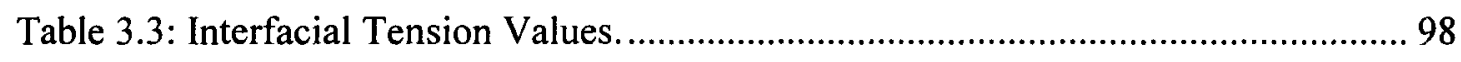

Table 3.4: Transducers Calibration Equations, Maximum Absolute Error, and Standard Error. 111

Table 4.1: Chemical Shifts from Decoupling ${ }^{13} \mathrm{C}$ NMR Spectra $\left(\mathrm{CDCl}_{3}\right) \ldots \ldots \ldots \ldots \ldots \ldots . . . . . .144$

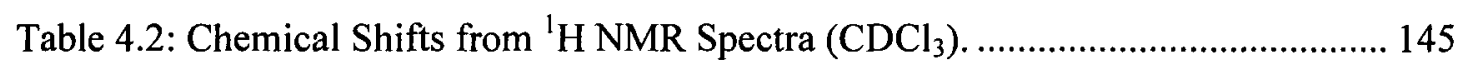

Table 4.3: FT-IR Spectra for the Excess OTS and the Derivative of OTS.................... 145

Table 5.1: Irreducible Heptane and Entrapped Air Saturations in Uniform Soil........... 159

Table 5.2: Irreducible Heptane and Entrapped Air Saturations in Well Graded Soil..... 159

Table 5.3: Van Genuchten Parameters for Uniform Sand..................................... 162

Table 5.4: Van Genuchten Parameters for Well Graded Sand. .................................. 162

Table 5.5: Irreducible Water and Entrapped Air Saturations in Uniform Soil.............. 173

Table 5.6: Irreducible Water and Entrapped Air Saturations in Well Graded Soil. ....... 173

Table 5.7: Irreducible Water Saturations in Uniform Sand. ...................................... 209

Table 5.8: Irreducible Water Saturations in Well Graded Sand. ................................. 209

Table 5.9: Irreducible and Entrapped Saturations in Uniform Sand........................... 216

Table 5.10: Irreducible and Entrapped Saturations in Well Graded Sand..................... 217

Table 5.11: Irreducible and Entrapped Water Saturations in Uniform Sand................ 232

Table 5.12: Irreducible and Entrapped Water Saturations in Well Graded Sand.......... 233

Table 6.1: Residual Heptane and Initial Water Saturations, and TLSLL..................... 264

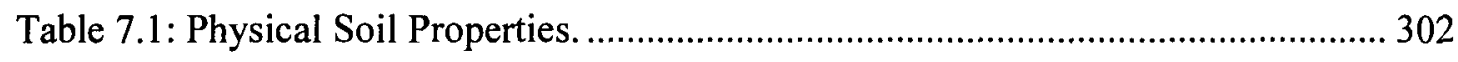


Table 7.2: Measured Versus Predicted Effective Residual Heptane Saturations in Uniform Sand

Table 7.3: Measured Versus Predicted Effective Residual Heptane Saturations in Well Graded Sand. 325

Table 7.4: Physical Properties of the Porous Medium. 326

Table 7.5: Measured Versus Predicted Effective Residual Heptane Saturations for Van Geel and Roy (2002) Experimental Data Set. 328

Table 7.6:Measured Versus Predicted Effective Residual Heptane Saturations in 90\% Water-Wet-10\% NAPL-Wet Uniform Sand. 338

Table 7.7: Measured Versus Predicted Effective Residual Heptane Saturations in 90\% Water-Wet-10\% NAPL-Wet Well Graded Sand. 339

Table 7.8: Measured Versus Predicted Effective Residual Heptane Saturations in $80 \%$ Water-Wet-20\% NAPL-Wet Uniform Sand. 339

Table 7.9: Measured Versus Predicted Effective Residual Heptane Saturations in 80\% Water-Wet-20\% NAPL-Wet Well Graded Sand. 340

Table 7.10: Measured Versus Predicted Effective Residual Heptane Saturations in 70\% Water-Wet-30\% NAPL-Wet Uniform Sand. 340

Table 7.11: Measured Versus Predicted Effective Residual Heptane Saturations in 70\% Water-Wet-30\% NAPL-Wet Well Graded Sand.

Table 7.12: Measured Versus Predicted Effective Residual Heptane Saturations in 60\% Water-Wet-40\% NAPL-Wet Uniform Sand. 341

Table 7.13: Measured Versus Predicted Effective Residual Heptane Saturations in 60\% Water-Wet-40\% NAPL-Wet Well Graded Sand. 342 


\section{LIST OF FIGURES}

Figure 1.1: Schematic of LNAPL Spill and Migration in Soil (Schwille 1988)............... 3

Figure 1.2: Schematic of DNAPL Spill and Migration in Soil (Schwille 1988)............... 3

Figure 1.3: Micro Scale Versus REV (Helmig 1997)............................................. 6

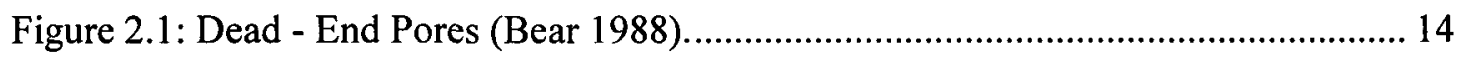

Figure 2.2: Relationship between (a) Actual, (b) Effective, and (c) Apparent Saturations

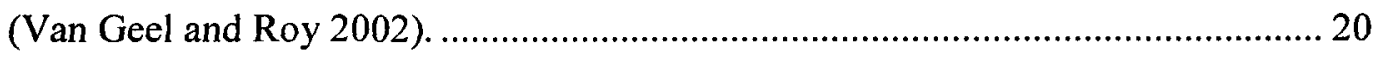

Figure 2.3 Conceptual Model of Potential Energy of Broken Bonds at Liquid Surface (Selker et al. 1999). 22

Figure 2.4: The Temperature Dependence of Air/Water Interfacial Tension (Source: CRC Handbook of Chemistry and Physics cited in Selker et al. 1999)........................... 24

Figure 2.5: Force Balance for Water Rise in Capillary Tube. .................................... 25

Figure 2.6: Definition Sketch for a Doubly Curved Liquid Surface............................. 26

Figure 2.7: Forces Acting on a Fluid Particle. ........................................................... 29

Figure 2.8: Pore Cross Section Containing Water and NAPL with Varying Water Saturations (Corey 1977). 31

Figure 2.9: (a) Cross Section of a Line Contact between a Solid Surface with a Line of Liquid/ air Contact at Equilibrium; (b) the Force Balance at the Point of Contact (Selker et al. 1999). 33

Figure 2.10: The Raindrop Effect of Contact Angle Hysteresis between Advancing and Receding Contact Angles (Selker et al. 1999). 34

Figure 2.11: Illustration of the Effect of a Microscopic Defect on the Apparent Contact Angle for (a) Advancing and (b) Receding Fluid (Selker et al. 1999). 34 
Figure 2.12: A Soil Element of Dimensions $\Delta x, \Delta y$, and $\Delta z$ in $x, y$, and $z$ Directions..... 37

Figure 2.13: Conceptual Relationship between (a) Particle Size Distribution; (b) Pore Size Distribution; (c) Pore Volume Occupied Versus Pore Filling Pressure; and (d) the Characteristic Wetting Curve (Reproduced from Selker et al. 1999). 42

Figure 2.14: The Main Characteristic Curves for Wetting, Draining, and One Set of Scanning Curves (Reproduced from Selker et al. 1999). 44

Figure 2.15: Illustration of a Capillary Pressure Head-Saturation Cycles for a Set of Equal-Size Pores with Equal-Size Throats Connecting Them (Reproduced from Selker et al. 1999). 46

Figure 2.16: Water Retention Curves for Sand, Fine Sand, and Silt Loam (Brooks and Corey 1966). 47

Figure 2.17: Influence of Initial Water Saturation on Total Liquid Saturation (Modified from Zalidis et al. 1998). 56

Figure 2.18: Relative Permeability of Water and Air in Porous Media 60

Figure 2.19: Cubic Pore Network (Reeves and Celia 1996). 63

Figure 2.20: Typical Spherical Pore Body with its Associated Bi-Conical Pore Throats (Reeves and Celia 1996) 63

Figure 2.21: A Schematic Drawing of the Characteristic Radii for a Pore and the Characteristic Filling and Emptying Pressures of that Pore (Rose 1966)...... 64

Figure 2.22: Illustration of the Probability Density Function Water Content as a Function of Filling and Draining Pressures (Selker et al. 1999). 66

Figure 2.23: Features of the Joint Density Function Given in Figure 2.22 (Selker et al. 1999). 67 
Figure 2.24: Mapping of the Water-Filled Pores through the Sequence of Wetting and Draining Characteristic Curves Presented in Figure 2.14 (Selker et al. 1999)........ 69 Figure 2.25: Water Wetting Glass Beads Surfaces in Air/Water Two-Phase System (Schwille 1988). 72

Figure 2.26: NAPL Wetting Glass Beads Surfaces in Air/NAPL Two-Phase System (Schwille 1988). 72

Figure 2.27: Water Replacing NAPL on Glass Beads Surfaces which Were Initially Saturated by NAPL in NAPL/Water Two-Phase System (Schwille 1988). 72

Figure 2.28: Possible Fluid Saturation States under Different Wettability Conditions (Bear 1988). 74

Figure 2.29: Hypothetical Distribution of Fluids and Interfacial Tensions at a Solid (s)Wetting Fluid (w)-Non-Wetting Fluid (n) Contact Line (Modified from Bradford and Leij 1995a). 75

Figure 2.30: Maximum Heptane Saturation-Residual Heptane Saturation (Van Geel and Roy 2002). 84

Figure 3.1: Grain Size Distribution of Well Graded and Uniform Soils. ...................... 95

Figure 3.2: Buchner Funnel (Modified from Van Geel and Sykes 1994). ..................... 99

Figure 3.3: Two-Phase $P_{c} \neg$ Experimental Setup. ................................................ 100

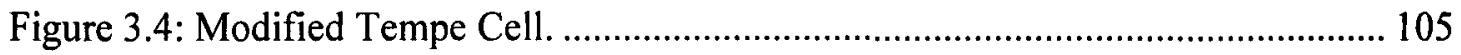

Figure 3.5: Experimental Setup for Three-Phase System....................................... 106

Figure 3.6: Evaporation Control Cells Setup...................................................... 113

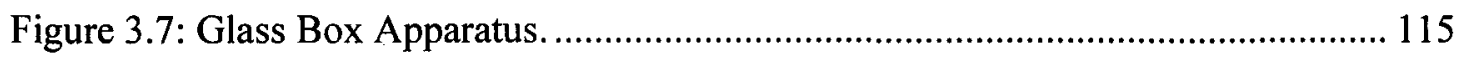

Figure 3.8: Pore Scale Experimental Setup. ........................................................ 116 
Figure 4.1: Conceptual Illustration of Bound and Unbound OTS Molecules to Silica Sand Particles.

Figure 4.2: Schematic Illustrating the Location and Extent of a Hydrophobic Sand Lens in a Hydrophilic Sand Medium. 129

Figure 4.3: Soxhlet Extractor. 130

Figure 4.4: Decane/Water Capillary Pressure Head-Actual Water Saturation Pathways. 135

Figure 4.5: Heptane/Water Capillary Pressure Head-Actual Water Saturation Pathways. 136

Figure 4.6: Decoupling ${ }^{13} \mathrm{C}$ NMR Spectrum $\left(\mathrm{CDCl}_{3}\right)$ for Excess OTS. 141

Figure 4.7: ${ }^{1} \mathrm{H}$ NMR Spectrum $\left(\mathrm{CDCl}_{3}\right)$ for Excess OTS. 142

Figure 4.8: FT-IR Spectrum for Excess OTS. 142

Figure 4.9: Decoupling ${ }^{13} \mathrm{C}$ NMR Spectrum $\left(\mathrm{CDCl}_{3}\right)$ for the Derivative of OTS........ 143

Figure 4.10: ${ }^{1} \mathrm{H}$ NMR Spectrum $\left(\mathrm{CDCl}_{3}\right)$ for the Derivative of OTS...................... 143

Figure 4.11: FT-IR Spectrum for the Derivative of OTS. ...................................... 144

Figure 4.12: Heptane/Water Capillary Pressure Head-Actual Water Saturation Pathways Using Hydrophobic Sands Free of Excess OTS. 151

Figure 4.13: Ceramic Plate Washing. 152

Figure 5.1: Capillary Pressure Head - Actual Heptane Saturation in Different Wettability Porous Media. 158

Figure 5.2: Capillary Pressure Head-Actual Heptane Saturation in Different Wettability Porous Media. 158 
Figure 5.3: Capillary Pressure Head-Apparent Heptane Saturation Main Drainage and Imbibition Pathways in a Water-Wet Porous Medium in Uniform Sand. 163

Figure 5.4: Capillary Pressure Head-Apparent Heptane Saturation Main Drainage and Imbibition Pathways in a Water-Wet Porous Medium in Well Graded Sand. 163

Figure 5.5: Capillary Pressure Head-Apparent Heptane Saturation Main Drainage and Imbibition Pathways in a $90 \%$ Water-Wet-10\% NAPL-Wet Porous Medium in Uniform Sand. 164

Figure 5.6: Capillary Pressure Head-Apparent Heptane Saturation Main Drainage and Imbibition Pathways in a $90 \%$ Water-Wet-10\% NAPL-Wet Porous Medium in Well Graded Sand. 164

Figure 5.7: Capillary Pressure Head-Apparent Heptane Saturation Main Drainage and Imbibition Pathways in an $80 \%$ Water-Wet-20\% NAPL-Wet Porous Medium in Uniform Sand. 165

Figure 5.8: Capillary Pressure Head-Apparent Heptane Saturation Main Drainage and Imbibition Pathways in an $80 \%$ Water-Wet-20\% NAPL-Wet Porous Medium in Well Graded Sand. 165

Figure 5.9: Capillary Pressure Head-Apparent Heptane Saturation Main Drainage and Imbibition Pathways in a $70 \%$ Water-Wet-30\% NAPL-Wet Porous Medium in Uniform Sand. 166

Figure 5.10: Capillary Pressure Head-Apparent Heptane Saturation Main Drainage and Imbibition Pathways in a $70 \%$ Water-Wet-30\% NAPL-Wet Porous Medium in Well Graded Sand. 166 
Figure 5.11: Capillary Pressure Head-Apparent Heptane Saturation Main Drainage and Imbibition Pathways in a $60 \%$ Water-Wet-40\% NAPL-Wet Porous Medium in Uniform Sand.

Figure 5.12: Capillary Pressure Head-Apparent Heptane Saturation Main Drainage and Imbibition Pathways in a $60 \%$ Water-Wet-40\% NAPL-Wet Porous Medium in Well Graded Sand. 167

Figure 5.13: Capillary Pressure Head-Apparent Heptane Saturation Main Drainage and Imbibition Pathways in a NAPL-Wet Porous Medium in Uniform Sand. 168

Figure 5.14: Capillary Pressure Head-Apparent Heptane Saturation Main Drainage and Imbibition Pathways in a NAPL-Wet Porous Medium in Well Graded Sand........ 168 Figure 5.15: Capillary Pressure Head-Apparent Heptane Saturation Main Drainage and Imbibition Pathways Using Pooled Data In Uniform Sand. 169

Figure 5.16: Capillary Pressure Head-Apparent Heptane Saturation Main Drainage and Imbibition Pathways Using Pooled Data in Well Graded Sand. 169

Figure 5.17: Main Drainage Pathway in Different Wettability Porous Media in Uniform Sand. 171

Figure 5.18: Main Drainage Pathway in Different Wettability Porous Media in Well Graded Sand. 171

Figure 5.19: Main Imbibition Pathway in Different Wettability Porous Media in Uniform

Sand. 172

Figure 5.20: Main Imbibition Pathway in Different Wettability Porous Media in Well Graded Sand. 172

Figure 5.21: Air and Water Distributions at Irreducible Water Saturation. 175 
Figure 5.22: Capillary Pressure Head -Saturation Main Drainage Pathway................. 176

Figure 5.23: Capillary Pressure Head-Saturation Main Imbibition Pathway................ 177

Figure 5.24: Capillary Pressure Head-Actual Water Saturation Main Imbibition and Drainage Pathways in a NAPL-Wet Porous Medium. 180

Figure 5.25: Capillary Pressure Head-Actual Water Saturation Main Imbibition and Drainage Pathways in a Uniform NAPL-Wet Porous Medium. 181

Figure 5.26: Capillary Pressure Head-Actual Water Saturation Main Imbibition and Drainage Pathways in a Well Graded NAPL-Wet Porous Medium. 182

Figure 5.27: Conceptual Model for Air/Water Capillary Pressure Head-Actual Water Saturation Imbibition and Drainage Pathways in a Water-Wet Soil Fraction and in a NAPL-Wet Soil Lens. 185

Figure 5.28: Capillary Pressure Head-Saturation Main Drainage Pathway in a Water-Wet Porous Medium with a NAPL-Wet Soil Lens. 189

Figure 5.29: Capillary Pressure Head-Actual Water Saturation Main Drainage Pathway in a Water-Wet Porous Medium in Uniform Sand. 195

Figure 5.30: Capillary Pressure Head-Actual Water Saturation Main Drainage Pathway in a Water-Wet Porous Medium in Well Graded Sand. 196

Figure 5.31: Capillary Pressure Head-Actual Water Saturation Main Drainage Pathway in a $90 \%$ Water-Wet-10\% NAPL-Wet Porous Medium in Uniform Sand. 197

Figure 5.32: Capillary Pressure Head-Actual Water Saturation Main Drainage Pathway in a 90\% Water-Wet-10\% NAPL-Wet Porous Medium in Well Graded Sand. .... 198

Figure 5.33: Capillary Pressure Head -Actual Water Saturation Main Drainage Pathway in an $80 \%$ Water-Wet-20\% NAPL-Wet Porous Medium in Uniform Sand. 198 
Figure 5.34: Capillary Pressure Head -Actual Water Saturation Main Drainage Pathway in an $80 \%$ Water-Wet-20\% NAPL-Wet Porous Medium in Well Graded Sand. .. 199

Figure 5.35: Capillary Pressure Head -Actual Water Saturation Main Drainage Pathway in a $70 \%$ Water-Wet-30\% NAPL-Wet Porous Medium in Uniform Sand. 199

Figure 5.36: Capillary Pressure Head -Actual Water Saturation Main Drainage Pathway in a 70\% Water-Wet-30\% NAPL-Wet Porous Medium in Well Graded Sand. .... 200

Figure 5.37: Capillary Pressure Head -Actual Water Saturation Main Drainage Pathway in a $60 \%$ Water-Wet $-40 \%$ NAPL-Wet Porous Medium in Uniform Sand. 200

Figure 5.38: Capillary Pressure Head -Actual Water Saturation Main Drainage Pathway in a $60 \%$ Water-Wet-40\% NAPL-Wet Porous Medium in Well Graded Sand. .... 201

Figure 5.39: Capillary Pressure Head-Saturation Main Imbibition Pathway in a WaterWet Porous Medium with a NAPL-Wet Soil Lens 203

Figure 5.40: Capillary Pressure Head-Actual Water Saturation in Different Wettability Porous Media in Uniform Sand. 206

Figure 5.41: Capillary Pressure Head-Actual Water Saturation in Different Wettability Porous Media in Well Graded Sand. 206

Figure 5.42: Capillary Pressure Head-Actual Water Saturation Data in the Water-Wet Porous Medium and the Water-Wet Soil Fractions in Uniform Sand. 210

Figure 5.43: Capillary Pressure Head-Actual Water Saturation Data in the Water-Wet Porous Medium and the Water-Wet Soil Fractions in Well Graded Sand. 210

Figure 5.44: Capillary Pressure Head-Apparent Water Saturation in the Water-Wet Porous Medium and the Water-Wet Soil Fractions in Uniform Sand. 
Figure 5.45: Capillary Pressure Head-Apparent Water Saturation in the Water-Wet Porous Medium and the Water-Wet Soil Fractions in Well Graded Sand. 212

Figure 5.46: Main Drainage Pathways in Different Wettability Porous Media in Uniform Sand. 214

Figure 5.47: Main Drainage Pathways in Different Wettability Porous Media in Well Graded Sand 214

Figure 5.48: Main Imbibition Pathways in Different Wettability Porous Media in Uniform Sand 215

Figure 5.49: Main Imbibition Pathways in Different Wettability Porous Media in Well Graded Sand 215

Figure 5.50: Heptane Located at Pore Body Centres and Surrounded by Water. 218

Figure 5.51: Water Forms at Irreducible Saturation. 218

Figure 5.52: Capillary Pressure Head-Actual Water Saturation Main Imbibition and Drainage Pathways in a NAPL-Wet Porous Medium. 221

Figure 5.53: Entrapped Water Located at the Centre of Pore Bodies and Surrounded by Heptane in a NAPL-Wet Porous Medium. 222

Figure 5.54: Conceptual Model for Heptane/Water Capillary Pressure Head-Actual Water Saturation Imbibition and Drainage Pathways in a Water-Wet Soil Fraction and in a NAPL-Wet Soil Lens. 224

Figure 5.55: Water and Gas Entrapped in Heptane in a NAPL-Wet Soil Lens. 225

Figure 5.56: $P_{c}-S$ Main Drainage Pathway in a Water-Wet Porous Medium with a NAPL-Wet Soil Lens. 227 
Figure 5.57: Capillary Pressure Head-Effective (Apparent) Water Saturation in WaterWet Porous Medium and Water-Wet Soil Fractions in Uniform Sand. 234

Figure 5.58: Capillary Pressure Head-Effective (Apparent) Water Saturation in WaterWet Porous Medium and Water-Wet Soil Fractions in Well Graded Sand. 234

Figure 5.59: $P_{c} \neg S$ Main Imbibition Pathway in a Water-Wet Porous Medium with a NAPL-Wet Soil Lens. 236

Figure 5.60: Capillary Pressure Head-Actual Water Saturation in Water-Wet Porous Medium and Water-Wet Porous Media with NAPL-Wet Soil Lenses in Uniform Sand. 240

Figure 5.61: Capillary Pressure Head-Actual Water Saturation in Water-Wet Porous Medium and Water-Wet Porous Media with NAPL-Wet Soil Lenses in Well Graded Sand. 241

Figure 5.62: Capillary Pressure Head-Actual Water Saturation Data in Water-Wet Porous Medium and Water-Wet Soil Fractions in Uniform Sand. 243

Figure 5.63: Capillary Pressure Head-Actual Water Saturation Data in Water-Wet Porous Medium and Water-Wet Soil Fractions in Well Graded Sand. 243

Figure 5.64: Capillary Pressure Head-Apparent Water Saturation in Water-Wet Porous Medium and Water-Wet Soil Fractions in Uniform Sand. 245

Figure 5.65: Capillary Pressure Head-Apparent Water Saturation in Water-Wet Porous Medium and Water-Wet Soil Fractions in Well Graded Sand. 245

Figure 6.1: Water (blue), Heptane (light red), and Air/Heptane Interface (dark line) in the Water-Wet Soil Fraction. 248 
Figure 6.2: Heptane (light red), Entrapped Water (blue), and Air/Heptane Interface (dark line) in the NAPL-Wet Soil Lens. 248

Figure 6.3: Capillary Pressure Head-Apparent Total Liquid Saturation Pathway in the Water-wet Soil Fraction. 252

Figure 6.4: Capillary Pressure Head-Apparent NAPL Saturation Pathway in an Isolated or a Connected NAPL-wet Soil Lens. 253

Figure 6.5: Capillary Pressure Head-Apparent Total Liquid Saturation Pathways in the Water-Wet Soil Fraction and in the Connected NAPL-Wet Soil Lens. 258

Figure 6.6: Air/Heptane Capillary Pressure Head-Actual Total Liquid Saturation Main Drainage Pathway at Irreducible Water Saturation. 266

Figure 6.7: Air/Heptane Capillary Pressure Head-Apparent Total Liquid Saturation Main Drainage Pathway at Irreducible Water Saturation. 266

Figure 6.8: Air/Heptane Capillary Pressure Head-Actual Total Liquid Saturation Drainage Pathway at Lowest Initial Water Saturation in the REV. 268

Figure 6.9: Air/Heptane Capillary Pressure Head-Apparent Total Liquid Saturation Drainage Pathway at Lowest Initial Water Saturation in the REV. 268

Figure 6.10: Air/Heptane Capillary Pressure Head-Actual Total Liquid Saturation Drainage Pathway at $45 \%$ Actual Water Saturation. 270

Figure 6.11: Air/Heptane Capillary Pressure Head-Apparent Total Liquid Saturation Drainage Pathway at $45 \%$ Actual Water Saturation. 271

Figure 6.12: Air/Heptane Capillary Pressure Head-Actual Total Liquid Saturation Drainage Pathway at $45 \%$ Actual Water Saturation. 272 
Figure 6.13: Air/Heptane Capillary Pressure Head - Apparent Total Liquid Saturation Drainage Pathway at $45 \%$ Actual Water Saturation.

Figure 6.14: Air/Heptane Capillary Pressure Head-Actual Total Liquid Saturation Main Imbibition Pathway at Irreducible Water Saturation. 274

Figure 6.15: Air/Heptane Capillary Pressure Head-Apparent Total Liquid Saturation Main Imbibition Pathway at Irreducible Water Saturation. 275

Figure 6.16: Air/Heptane Capillary Pressure Head-Actual Total Liquid Saturation Imbibition Pathway at Irreducible Water Saturation in the Water-Wet Soil Fraction. 276

Figure 6.17: Air/Heptane Capillary Pressure Head-Apparent Total Liquid Saturation Imbibition Pathway at Irreducible Water Saturation in the Water-Wet Soil Fraction. 276

Figure 6.18: Air/Heptane Capillary Pressure Head-Actual Total Liquid Saturation Imbibition Pathway at $45 \%$ Actual Water Saturation. 279

Figure 6.19: Air/Heptane Capillary Pressure Head-Apparent Total Liquid Saturation Imbibition Pathway at $45 \%$ Actual Water Saturation. 279

Figure 6.20: Air/Heptane Capillary Pressure Head-Actual Total Liquid Saturation Imbibition Pathway at $45 \%$ Actual Water Saturation. 280

Figure 6.21: Air/Heptane Capillary Pressure Head-Apparent Total Liquid Saturation Imbibition Pathway at $45 \%$ Actual Water Saturation. 281

Figure 7.1: Effective Residual Heptane Saturation-Apparent Heptane Saturation in Uniform Sand. 288

xxiv 
Figure 7.2: Effective Residual Heptane Saturation-Apparent Heptane Saturation in Well Graded Sand. 288

Figure 7.3: Effective Residual Heptane Saturation-Apparent Water Saturation in Uniform Sand. 290

Figure 7.4: Effective Residual Heptane Saturation-Apparent Water Saturation in Well Graded Sand. 291

Figure 7.5: Residual NAPL Forms; A. At Low Water Saturation and B. At Intermediate Water Saturation. 292

Figure 7.6: Water Wedge; A. At Low Water Saturation, B. At Intermediate Water Saturation, and C. At Higher Water Saturation. 293

Figure 7.7: Conceptual Model for Residual NAPL Saturation-Water Saturation and Residual NAPL Saturation-NAPL Saturation Relationships............................. 296

Figure 7.8: NAPL/Water Interfacial Area (SA $\mathrm{SAPL}_{\text {Water }}$--Actual Water Saturation....... 297

Figure 7.9: NAPL Film Volume-Actual Water Saturation. 298

Figure 7.10: Fitting Parameter $\lambda$-Actual Water Saturation Effect on the NAPL/Water Specific Interfacial Area. 300

Figure 7.11: Capillary Pressure Head-Actual Water Saturation in Heptane/Water TwoPhase System in Uniform Sand. 301

Figure 7.12: Capillary Pressure Head-Actual Water Saturation in Heptane/Water Two-

Phase System in Well Graded Sand. 301

Figure 7.13: Effective Saturation of Heptane Film-Apparent Water Saturation in Well Graded and Uniform Sands. 303 
Figure 7.14: Water Saturation-Porous Medium Grain Size Distribution Effect on the Effective Residual NAPL Saturation in Water Wedges. 305

Figure 7.15: NAPL Saturation Effect on the Effective Residual NAPL Saturation in Water Wedges. 307

Figure 7.16: Effective Saturation of the Heptane Residing in Water Wedges-Apparent Water Saturation in Uniform Sand. 309

Figure 7.17: Effective Saturation of the Heptane Residing in Water Wedges-Apparent Water Saturation in Well Graded Sand. 309

Figure 7.18: Effective Saturation of the Heptane Residing in Water Wedges-Apparent Heptane Saturation in Uniform Sand. 310

Figure 7.19: Effective Saturation of the Heptane Residing in Water Wedges-Apparent Heptane Saturation in Well Graded Sand. 310

Figure 7.20: Effective Residual Heptane Saturation-Apparent Total Liquid Saturation at Irreducible Water Saturation in Uniform Sand. 314

Figure 7.21: Effective Residual Heptane Saturation-Apparent Total Liquid Saturation at Irreducible Water Saturation in Well Graded Sand. 315

Figure 7.22: Effective Residual Heptane Saturation-Apparent Total Liquid Saturation at $20 \%$ Actual Water Saturation in Uniform Sand. 315

Figure 7.23: Effective Residual Heptane Saturation-Apparent Total Liquid Saturation at $20 \%$ Actual Water Saturation in Well Graded Sand. 316

Figure 7.24: Effective Residual Heptane Saturation-Apparent Total Liquid Saturation at $30 \%$ Actual Water Saturation in Uniform Sand. 316 
Figure 7.25: Effective Residual Heptane Saturation-Apparent Total Liquid Saturation at $30 \%$ Actual Water Saturation in Well Graded Sand.

Figure 7.26: Effective Residual Heptane Saturation-Apparent Total Liquid Saturation at $40 \%$ Actual Water Saturation in Uniform Sand.

Figure 7.27: Effective Residual Heptane Saturation-Apparent Total Liquid Saturation at $40 \%$ Actual Water Saturation in Well Graded Sand. 318

Figure 7.28: Effective Residual Heptane Saturation-Apparent Total Liquid Saturation at $50 \%$ Actual Water Saturation in Uniform Sand. 318

Figure 7.29: Effective Residual Heptane Saturation-Apparent Total Liquid Saturation at $50 \%$ Actual Water Saturation in Well Graded Sand.

Figure 7.30: Effective Residual Heptane Saturation-Apparent Total Liquid Saturation at $60 \%$ Actual Water Saturation in Uniform Sand.

Figure 7.31: Effective Residual Heptane Saturation-Apparent Total Liquid Saturation at $60 \%$ Actual Water Saturation in Well Graded Sand. 320

Figure 7.32: Effective Residual Heptane Saturation-Apparent Total Liquid Saturation at $70 \%$ Actual Water Saturation in Uniform Sand. 320

Figure 7.33: Effective Residual Heptane Saturation-Apparent Total Liquid Saturation at $70 \%$ Actual Water Saturation in Well Graded Sand. 321

Figure 7.34: Effective Residual Heptane Saturation-Apparent Total Liquid Saturation at $80 \%$ Actual Water Saturation in Uniform Sand. 321

Figure 7.35: Effective Residual Heptane Saturation-Apparent Total Liquid Saturation at $80 \%$ Actual Water Saturation in Well Graded Sand. 322 
Figure 7.36: Effective Residual Heptane Saturation-Apparent Total Liquid Saturation at

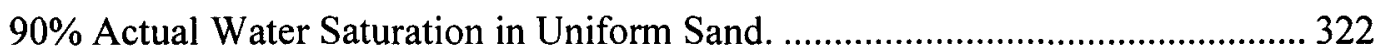

Figure 7.37: Effective Residual Heptane Saturation-Apparent Total Liquid Saturation at 90\% Actual Water Saturation in Well Graded Sand.......................................... 323 


\title{
LIST OF SYMBOLS
}

\author{
$\alpha$ \\ $\beta_{a n}$ \\ $\beta_{a w}$ \\ $\beta_{i j}$ \\ $\beta_{n w}$ \\ $\phi$ \\ $\phi_{1}$ \\ $\phi_{2}$ \\ $\phi_{a}$ \\ $\phi_{a, I}$ \\ $\phi_{r}$ \\ $\phi_{s n w}$ \\ $\Psi$ \\ $\Psi_{b}$ \\ $\sigma$ \\ $\sigma^{a b}$ \\ $\sigma^{A B}$ \\ Fitting parameter, Van Genuchten constitutive relationship \\ Capillary pressure scaling factor for air/NAPL (Parker et al. (1987)) \\ Capillary pressure scaling factor for air/water (Parker et al. (1987)) \\ Capillary pressure scaling factor for fluid pair $i, j$ ( Parker et al. \\ (1987)) \\ Capillary pressure scaling factor for NAPL/water (Parker et al. \\ (1987)) \\ Contact angle \\ Microscopic contact angle \\ Macroscopic contact angle \\ Advancing contact angle \\ Contact angle that the $a / b$ interface makes with the solid surface $(I)$ \\ measured through the wetting fluid $a$ \\ Receding contact angle \\ Angle between NAPL/water interface and the solid/water interface, \\ measured through the water \\ Matrix suction head \\ Bubbling pressure head \\ Surface or interfacial tension \\ Interfacial tension of fluid phases $a, b$ \\ Interfacial tension of fluid phases $A B$
}




\begin{tabular}{|c|c|}
\hline$\sigma_{a n}$ & Air/NAPL interfacial tension \\
\hline$\sigma_{a w}$ & Air/water interfacial tension \\
\hline$\sigma_{i}$ & Surface tension of fluid phase (i) \\
\hline$\sigma_{i j}$ & Interfacial tension of fluid phases $(i, j)$ \\
\hline$\sigma_{j}$ & Surface tension of fluid phase $(j)$ \\
\hline$\sigma_{m w}$ & NAPL/water interfacial tension \\
\hline$\sigma_{s n}$ & Interfacial tension between solid and NAPL \\
\hline$\sigma_{s w}$ & Interfacial tension between solid and water \\
\hline$\theta(h)$ & Fluid content as a function of capillary pressure head \\
\hline$\theta_{i}$ & Fluid phase $(i)$ content \\
\hline$\Delta c_{i}$ & Excess chemical concentration in surface layer \\
\hline$\Delta P$ & Pressure difference across the interface \\
\hline$\Delta x$ & Infinitesimal distance in $x$-direction \\
\hline$\Delta y$ & Infinitesimal distance in $y$-direction \\
\hline$\Delta y_{1}$ & Projection of interface (1) length in $y$-direction \\
\hline$\Delta y_{2}$ & Projection of interface (2) length in $y$-direction \\
\hline$\Delta z$ & Infinitesimal distance in $z$-direction \\
\hline$\Delta t$ & Infinitesimal time increment \\
\hline $0.6 \mathrm{WW}-0.4 \mathrm{NL}$ & $60 \%$ water-wet porous medium with $40 \%$ NAPL-wet soil lens \\
\hline $0.7 \mathrm{WW}-0.3 \mathrm{NL}$ & $70 \%$ water-wet porous medium with $30 \%$ NAPL-wet soil lens \\
\hline $0.75 \mathrm{WW}-0.25 \mathrm{NL}$ & $75 \%$ water-wet porous medium with $25 \%$ NAPL-wet soil lens \\
\hline $0.8 \mathrm{WW}-0.2 \mathrm{NL}$ & $80 \%$ water-wet porous medium with $20 \%$ NAPL-wet soil lens \\
\hline
\end{tabular}




\begin{tabular}{|c|c|}
\hline $0.9 \mathrm{WW}-0.1 \mathrm{NL}$ & $90 \%$ water-wet porous medium with $10 \%$ NAPL-wet soil lens \\
\hline${ }^{13} \mathrm{C}$ & Carbon 13 \\
\hline${ }^{1} \mathrm{H}$ & Proton \\
\hline$\Gamma$ & Dry end fitting parameter (Cary 1994) \\
\hline$\delta h_{e}$ & $\begin{array}{l}\text { Infinitesimal increase or decrease in draining capillary pressure } \\
\text { head }\end{array}$ \\
\hline$\delta h_{f}$ & Infinitesimal increase or decrease in filling capillary pressure head \\
\hline$\delta L_{1}$ & Infinitesimal length of the interface in longitudinal direction (1) \\
\hline$\delta L_{2}$ & Infinitesimal length of the interface in transverse direction (2) \\
\hline$\delta \omega_{1}$ & Infinitesimal central angle corresponding to an interface length $\delta L_{1}$ \\
\hline$\delta \omega_{2}$ & Infinitesimal central angle corresponding to an interface length $\delta L_{2}$ \\
\hline$\eta$ & Porous medium porosity \\
\hline$\eta_{e}$ & Porous medium effective porosity \\
\hline$\eta_{w w}$ & Water-wet porous medium porosity \\
\hline$\eta_{n w}$ & NAPL-wet porous medium porosity \\
\hline$\lambda$ & $\begin{array}{l}\text { Pore size distribution index, Brooks and Corey constitutive } \\
\text { relationship }\end{array}$ \\
\hline$\mu_{n}$ & Dynamic viscosity of NAPL \\
\hline$\mu_{w}$ & Dynamic viscosity of water \\
\hline$\xi$ & Capillary pressure shifting parameter (Bradford and Leij (1996)) \\
\hline$\rho$ & Fluid density \\
\hline$\rho_{i}$ & Density of fluid $(i)$ \\
\hline$\rho_{0}$ & NAPL density \\
\hline
\end{tabular}




\begin{tabular}{|c|c|}
\hline$\rho_{w}$ & Water density \\
\hline $\mathrm{Al}_{2} \mathrm{O}_{3}$ & Aluminum (III) oxide \\
\hline $\mathrm{CCP}$ & Critical capillary pressure \\
\hline $\mathrm{CDCl}_{3}$ & Deuterated chloroform \\
\hline$C_{g}$ & Grading coefficient \\
\hline$c_{i}$ & Chemical concentration in bulk liquid \\
\hline$C_{n}$ & $\begin{array}{l}\text { Residual NAPL saturation-imbibing NAPL saturation relationship } \\
\text { at fixed water saturation }\end{array}$ \\
\hline $\mathrm{CO}_{2}$ & Carbon dioxide \\
\hline$C_{u}$ & Uniformity coefficient \\
\hline$c^{w}$ & $\begin{array}{l}\text { Degree of water saturation in the NAPL-wet soil lens prior to } \\
\text { initiating the main drainage pathway }\end{array}$ \\
\hline$C_{w}$ & $\begin{array}{l}\text { Residual NAPL saturation-water saturation relationship at a fixed } \\
\text { imbibing NAPL saturation }\end{array}$ \\
\hline$d$ & Capillary tube diameter \\
\hline$d_{10}$ & Maximum particle size of the smallest $10 \%$ of the sample \\
\hline$d_{30}$ & Maximum particle size of the smallest $30 \%$ of the sample \\
\hline$d_{60}$ & Maximum particle size of the smallest $60 \%$ of the sample \\
\hline DNAPL & Dense Non Aqueous Phase Liquid \\
\hline$f$ & Fraction of the NAPL-wet soil volume in a porous medium \\
\hline$f\left(h_{a n}\right)$ & $\begin{array}{l}\text { NAPL saturation- air/NAPL capillary pressure head constitutive } \\
\text { relationship in the NAPL-wet porous medium }\end{array}$ \\
\hline$f_{I}$ & Surface area fraction of soil $I$ in a porous medium \\
\hline
\end{tabular}




\begin{tabular}{|c|c|}
\hline$F_{L g}$ & Liquid/gas surface force per unit length $=\sigma_{L g}$ \\
\hline$F_{s g}$ & Solid/gas surface force per unit length $=\sigma_{s g}$ \\
\hline$F_{s L}$ & Solid/liquid surface force per unit length $=\sigma_{s L}$ \\
\hline$f\left(\overline{\bar{S}}_{t}\right)$ & $\begin{array}{l}\text { Air/NAPL capillary pressure head-total liquid saturation } \\
\text { constitutive relationship in the water-wet porous medium }\end{array}$ \\
\hline FT-IR & Fourier transform infrared spectroscopy \\
\hline$g$ & Acceleration due to gravity \\
\hline$h$ & The elevation of a fluid particle above the datum \\
\hline$h_{a n}$ & Capillary pressure head on the air/NAPL interface \\
\hline$h_{a n}^{a n}$ & $\begin{array}{l}\text { Capillary pressure head on the air/NAPL interface in air/NAPL two- } \\
\text { phase system }\end{array}$ \\
\hline$h_{a n}^{a m w}$ & $\begin{array}{l}\text { Capillary pressure head on the air/NAPL interface in } \\
\text { air/NAPL/water three-phase system }\end{array}$ \\
\hline$h_{a w}$ & Capillary pressure head on the air/water interface \\
\hline$h_{b}$ & Bubbling capillary pressure head \\
\hline$h_{c}$ & Capillary pressure head \\
\hline$h_{e}$ & Draining capillary pressure head \\
\hline$h_{e \max }$ & Maximum draining capillary pressure head \\
\hline$h_{\text {emin }}$ & Minimum draining capillary pressure head \\
\hline$h_{f}$ & Filling capillary pressure head \\
\hline$h_{f \max }$ & Maximum filling capillary pressure head \\
\hline$h_{\text {fmin }}$ & Minimum filling capillary pressure head \\
\hline$h_{i}$ & Head of fluid $i$ \\
\hline
\end{tabular}




\begin{tabular}{|c|c|}
\hline$h_{i j}$ & Capillary pressure head on the fluid pair $i / j$ interface \\
\hline$h_{n}$ & NAPL head \\
\hline$h_{n w}$ & Capillary pressure head on the NAPL/water interface \\
\hline$h_{n w}^{a n w}$ & $\begin{array}{l}\text { Capillary pressure head on the NAPL/water interface in } \\
\text { air/NAPL/water three-phase system }\end{array}$ \\
\hline$h_{n w}^{n w}$ & $\begin{array}{l}\text { Capillary pressure head on the NAPL/water interface in } \\
\text { NAPL/water two-phase system }\end{array}$ \\
\hline$h_{w}$ & Water head \\
\hline I & Soil with a known wettability condition \\
\hline$i$ & Fluid phase \\
\hline IR & Infra Red \\
\hline$j$ & Fluid phase \\
\hline$k$ & Intrinsic permeability \\
\hline$K$ & Saturated hydraulic conductivity \\
\hline$K(\theta)$ & Unsaturated hydraulic conductivity \\
\hline$K\left(\theta_{i}\right)$ & Unsaturated hydraulic conductivity of fluid $\mathrm{i}$ \\
\hline $\mathrm{KBr}$ & Potassium bromide \\
\hline$K_{n}\left(\theta_{n}\right)$ & $\begin{array}{l}\text { Unsaturated hydraulic conductivity of NAPL as a function of NAPL } \\
\text { content }\end{array}$ \\
\hline$k_{r}$ & Relative permeability \\
\hline$k_{r}-S$ & Relative permeability - saturation \\
\hline$k_{r a}$ & Relative permeability of air \\
\hline$k_{r n}$ & Relative permeability of NAPL \\
\hline
\end{tabular}




\begin{tabular}{|c|c|}
\hline$k_{r w}$ & Relative permeability of water \\
\hline$K_{w}\left(\theta_{w}\right)$ & Unsaturated hydraulic conductivity of water as a function of water \\
\hline & content \\
\hline$K_{x}\left(\theta_{i}\right)$ & Unsaturated hydraulic conductivity of fluid $i$ in $x$-direction \\
\hline$K_{y}\left(\theta_{i}\right)$ & Unsaturated hydraulic conductivity of fluid $i$ in $y$-direction \\
\hline$K_{z}\left(\theta_{i}\right)$ & Unsaturated hydraulic conductivity of fluid $i$ in $z$-direction \\
\hline LNAPL & Light Non Aqueous Phase Liquid \\
\hline$m$ & Fitting parameter, Van Genuchten constitutive relationship \\
\hline$M_{i}$ & Mass transfer of phase $i$ \\
\hline$n$ & Fitting parameter, Van Genuchten constitutive relationship \\
\hline $\mathrm{n}$ & Non-wetting \\
\hline$N$ & Number of surfaces of different wetting properties \\
\hline $\mathrm{NaBr}$ & Sodium Bromide \\
\hline NAPL & Non Aqueous Phase Liquid \\
\hline NL & NAPL-wet soil lens \\
\hline NMR & Nuclear Magnetic Resonance \\
\hline NPT & National pipe thread \\
\hline NSLL & Lower limit of the NAPL saturation \\
\hline NW & NAPL wet porous medium \\
\hline OTS & Octadecyltrichlorosilane \\
\hline$P$ & Fluid pressure \\
\hline$P_{0}$ & Fluid pressure at water table \\
\hline$P_{a}$ & Air pressure \\
\hline
\end{tabular}




\begin{tabular}{|c|c|}
\hline$P_{a n}$ & Capillary pressure on the air/NAPL interface \\
\hline$P_{a w}$ & Capillary pressure on the air/water interface \\
\hline$P_{b}$ & Bubbling pressure \\
\hline$P_{\mathrm{c}}$ & Capillary pressure \\
\hline$P_{c 0}$ & Capillary pressure at water table \\
\hline$P_{c l}$ & Capillary pressure at water saturation $S_{w 1}$ \\
\hline$P_{c 2}$ & Capillary pressure at water saturation $S_{w 2}$ \\
\hline$P_{c}^{a b}\left(S_{a}^{a p p}\right)$ & $\begin{array}{l}\text { Predicted capillary pressure-saturation relationship in a fractionally } \\
\text { wet porous medium ( } O^{\prime} \text { Carroll et al. (2005)) }\end{array}$ \\
\hline$P_{c, I}^{A B}\left(S_{W, I}^{a p p}\right)$ & $\begin{array}{l}\text { Capillary pressure on the A/B interface as a function of the apparent } \\
\text { wetting phase saturation in the porous medium I (O'Carroll et al. } \\
(2005) \text { ) }\end{array}$ \\
\hline PCE & Perchloroethylene \\
\hline$P_{c} \neg$ & Capillary pressure-saturation \\
\hline$P_{i j}$ & Capillary pressure on the interface of fluid pair $i, j$ \\
\hline$P_{n}$ & NAPL pressure at elevation $h$, above water table \\
\hline$P_{n 0}$ & NAPL pressure at the water table \\
\hline$P_{n w}$ & Capillary pressure on the NAPL/water interface \\
\hline$P_{w}$ & Water pressure at elevation $h$, above water table \\
\hline$P_{w 0}$ & Water pressure at the water table \\
\hline$q_{x n}$ & Darcy flux of NAPL in x-direction \\
\hline$q_{x w}$ & Darcy flux of water in $\mathrm{x}$-direction \\
\hline$r$ & Pore radius or capillary tube radius \\
\hline
\end{tabular}




\begin{tabular}{|c|c|}
\hline$R_{g}$ & Gas constant \\
\hline$r_{0}$ & Pore radius that defines the NAPL entry value of the water-saturated \\
\hline & porous medium \\
\hline$r_{1}$ & Interface radius of curvature in longitudinal direction (1) \\
\hline$r_{2}$ & Interface radius of curvature in the perpendicular direction (2) \\
\hline$R^{2}$ & Square of the sample correlation coefficient \\
\hline$r_{b}$ & Radius of pore body \\
\hline$r_{b \max }$ & Radius of the largest pore body \\
\hline$r_{b \min }$ & Radius of the smallest pore body \\
\hline REV & Representative Elementary Volume \\
\hline$R_{i j}$ & Curvature of the fluid pair $i, j$ interface \\
\hline$r_{\max }$ & Maximum pore radius \\
\hline$r_{\min }$ & Minimum pore radius \\
\hline$r_{n}$ & Radius of pore throat \\
\hline$r_{n \max }$ & Radius of the largest pore throat \\
\hline$r_{n \min }$ & Radius of the smallest pore throat \\
\hline$S$ & Actual wetting phase saturation \\
\hline $\bar{S}$ & Effective wetting phase saturation \\
\hline$S_{a}$ & Actual air saturation \\
\hline $\bar{S}_{a}$ & Effective air saturation \\
\hline$S A$ & $\begin{array}{l}\text { NAPL/water specific interfacial area (interfacial area in } \mathrm{cm}^{2} \text { per } \mathrm{cm}^{3} \\
\text { of the porous medium) }\end{array}$ \\
\hline SAE/MS & Society of Automotive Engineers/Mild Steel \\
\hline
\end{tabular}




$\begin{array}{ll}\bar{S}_{a f} & \text { Free effective air saturation } \\ S_{a m} & \text { Irreducible air saturation in air/water two-phase system in a NAPL- } \\ & \text { wet porous medium } \\ \text { SAM } & \text { Self-Assembled Monolayer } \\ S_{a m^{\prime}} & \text { Irreducible air saturation in the NAPL-wet soil lens } \\ S_{\text {SAPL/Water }} & \text { NAPL/water interfacial area } \\ S_{a_{N W}} & \text { Actual air saturation in a NAPL-wet porous medium } \\ S_{a t} & \text { Actual entrapped air saturation corresponding to actual water } \\ \bar{S}_{a t} & \text { saturation } S_{w} \text { on the main imbibition pathway } \\ S_{a t_{m a x}} & \text { Trapped effective air saturation } \\ S_{a t_{\max }} & \text { Maximum actual entrapped air saturation } \\ \bar{S}_{a t_{\max }} & \text { Saturation of fluid } i \\ S_{a t_{N L}} & \text { Maximum entrapped air saturation in a water-wet soil fraction } \\ \bar{S}_{a t n} & \text { Maximum effective entrapped air saturation } \\ \bar{S}_{a t w} & \text { Entrapped air saturation in the NAPL-wet soil lens } \\ S_{a_{W W}} & \text { Effective saturation of air trapped in NAPL } \\ S_{W W-N L} & \\ & \end{array}$




\begin{tabular}{|c|c|}
\hline $\mathrm{SiO}_{2}$ & Silicon dioxide \\
\hline$S_{i r_{N W}}^{a n}$ & $\begin{array}{l}\text { Irreducible NAPL saturation in air/NAPL two-phase system in the } \\
\text { NAPL-wet porous medium }\end{array}$ \\
\hline$S_{l w}$ & $\begin{array}{l}\text { Lowest initial water saturation in the water-wet porous medium } \\
\text { with the NAPL-wet soil lens }\end{array}$ \\
\hline$S_{m}$ & Irreducible water saturation in a water wet porous medium \\
\hline$S_{m}$ & Irreducible water saturation in the water-wet soil fraction \\
\hline$S_{\text {monolayer }}$ & $\begin{array}{l}\text { Actual water saturation corresponding to a monolayer of water on } \\
\text { the porous medium grains }\end{array}$ \\
\hline$S_{n}$ & Actual NAPL saturation \\
\hline $\bar{S}_{n}$ & Effective NAPL saturation \\
\hline $\bar{S}_{n}^{a n}$ & Effective NAPL saturation in air/NAPL two-phase system \\
\hline $\begin{array}{l}\bar{S}_{n}^{h i s t}{ }_{n} \max \\
\end{array}$ & Historic maximum apparent NAPL saturation \\
\hline$S_{n_{N L}}$ & Actual NAPL saturation in the NAPL-wet soil lens \\
\hline$\overline{\bar{S}}_{n_{N L}}$ & Apparent NAPL saturation in the NAPL-wet soil lens \\
\hline$\overline{\bar{S}}_{n_{L L}}$ & Apparent NAPL saturation lower limit \\
\hline $\bar{S}_{n f}$ & Free (continuous) effective NAPL saturation \\
\hline$S_{n m}$ & $\begin{array}{l}\text { Irreducible heptane saturation in water/heptane two-phase system } \\
\text { in a NAPL-wet porous medium }\end{array}$ \\
\hline$S_{n t}$ & $\begin{array}{l}\text { Actual entrapped NAPL (heptane) saturation corresponding to } \\
\text { actual water saturation } S_{w} \text { on the main imbibition pathway }\end{array}$ \\
\hline
\end{tabular}




\begin{tabular}{|c|c|}
\hline $\bar{S}_{n t}$ & Trapped (immobile) effective NAPL saturation \\
\hline$S_{n t_{\max }}$ & $\begin{array}{l}\text { Maximum actual entrapped NAPL (heptane) saturation in a water- } \\
\text { wet porous medium }\end{array}$ \\
\hline $\bar{S}_{n t_{\max }}$ & $\begin{array}{l}\text { Maximum effective entrapped NAPL (heptane) saturation in a } \\
\text { water-wet porous medium }\end{array}$ \\
\hline$S_{n t_{\max }}$ & $\begin{array}{l}\text { Maximum actual entrapped heptane saturation in the water-wet soil } \\
\text { fraction }\end{array}$ \\
\hline$S_{n_{W W}}$ & Actual NAPL saturation in a water-wet porous medium \\
\hline$S_{n_{W W F}}$ & Actual NAPL saturation in the water-wet soil fraction \\
\hline$\overline{\bar{S}}_{n_{W W F}}$ & Apparent NAPL saturation in the water-wet soil fraction \\
\hline$S_{r}$ & Residual NAPL saturation \\
\hline $\bar{S}_{r}$ & Effective residual NAPL saturation \\
\hline$S(R)$ & Capillary pressure-saturation relationship \\
\hline$S^{*}\left(R^{*}\right)$ & Unique capillary pressure-saturation relationship \\
\hline$S_{\text {r film }}$ & Film component of the residual NAPL saturation \\
\hline $\bar{S}_{r_{-} \text {film }}$ & $\begin{array}{l}\text { Effective saturation of residual NAPL that takes the form of NAPL } \\
\text { film on water surfaces }\end{array}$ \\
\hline $\bar{S}_{r_{-} \max }$ & Maximum effective residual NAPL saturation in the porous medium \\
\hline $\bar{S}_{r_{-} \text {water wedge_n }}$ & $\begin{array}{l}\text { Effective residual NAPL saturation in water wedges due to NAPL } \\
\text { saturation effect }\end{array}$ \\
\hline wedge $_{-} w$ & Effective residual NAPL saturation in water wedges \\
\hline
\end{tabular}




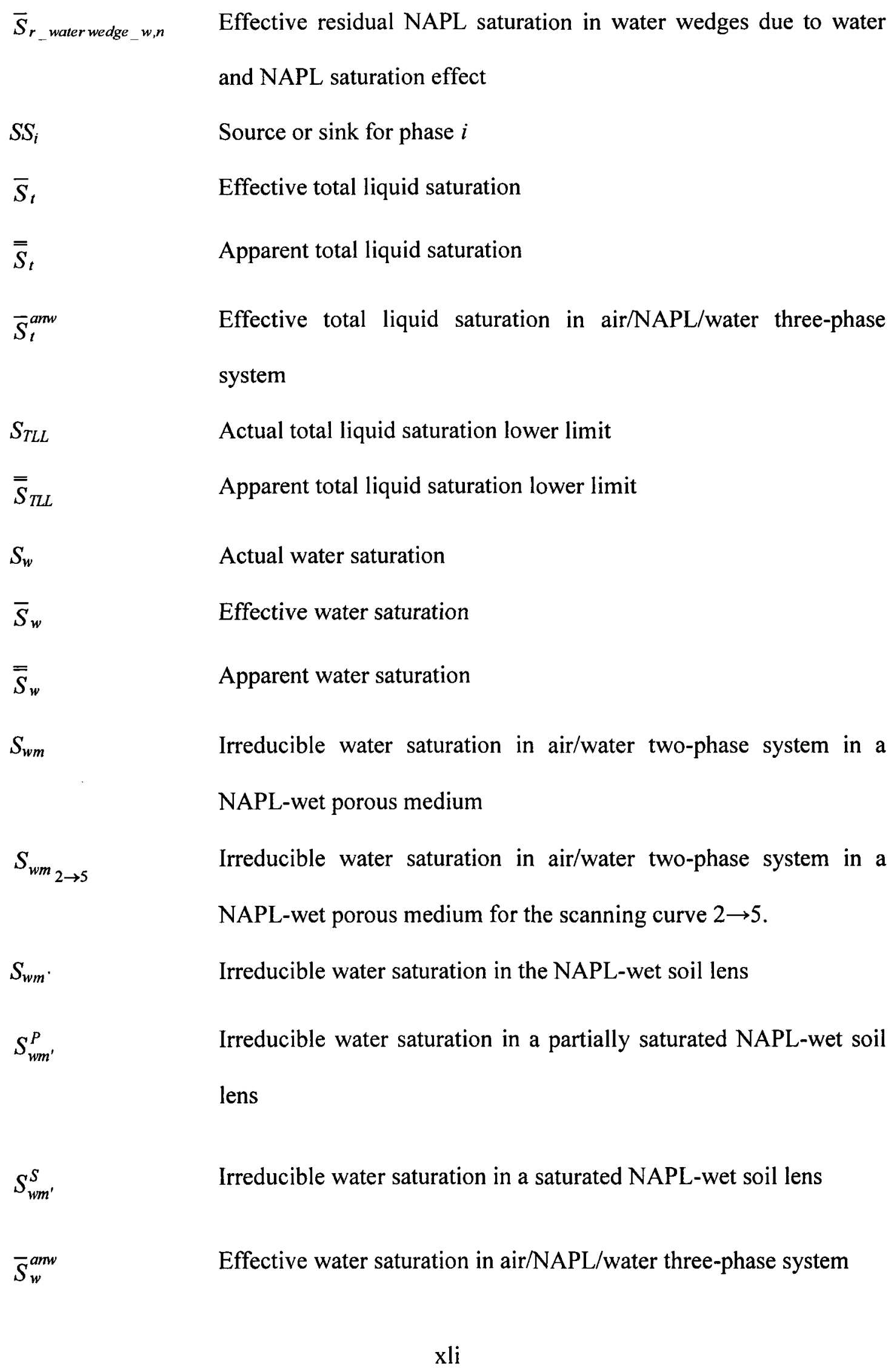




\begin{tabular}{|c|c|}
\hline $\bar{S}_{w}^{n w}$ & Effective water saturation in NAPL/water two-phase system \\
\hline$S_{w_{N L}}$ & Actual water saturation in the NAPL-wet soil lens \\
\hline$S_{W_{N W}}$ & Actual water saturation in a NAPL-wet porous medium \\
\hline$S_{w t}$ & $\begin{array}{l}\text { Actual entrapped water saturation in water/heptane two-phase } \\
\text { system in apparently saturated NAPL-wet soil lens }\end{array}$ \\
\hline$S_{w t^{\prime}}$ & $\begin{array}{l}\text { Actual entrapped water saturation in water/heptane two-phase } \\
\text { system in partially saturated NAPL-wet soil lens }\end{array}$ \\
\hline$S_{w t_{\max }}$ & $\begin{array}{l}\text { Maximum actual entrapped water saturation in water/heptane two- } \\
\text { phase system in a NAPL-wet porous medium }\end{array}$ \\
\hline$S_{w t^{\prime}}^{P}$ & $\begin{array}{l}\text { Actual entrapped water saturation in the initially partially-saturated } \\
\text { NAPL-wet soil lens. }\end{array}$ \\
\hline$S_{w t^{\prime}}^{S}$ & $\begin{array}{l}\text { Actual entrapped water saturation in the initially apparently- } \\
\text { saturated NAPL-wet soil lens. }\end{array}$ \\
\hline$S_{W_{W W-N L}}$ & $\begin{array}{l}\text { Actual water saturation in a water-wet porous medium with NAPL- } \\
\text { wet soil lenses }\end{array}$ \\
\hline$S_{W_{W W}}$ & Actual water saturation in a water-wet porous medium \\
\hline$S_{w_{W W F}}$ & Actual water saturation in the water-wet soil fraction \\
\hline$\overline{\bar{S}}_{w_{W W F}}$ & Apparent water saturation in the water-wet soil fraction \\
\hline$t$ & Time \\
\hline$T$ & Absolute temperature \\
\hline$T_{1}$ to $T_{10}$ & Transducers \\
\hline
\end{tabular}




\begin{tabular}{|c|c|}
\hline TCE & Trichloroethylene \\
\hline TLSLL & Total liquid saturation lower limit \\
\hline USDA & United States Department of Agriculture \\
\hline$V$ & Volume of a soil sample or REV \\
\hline$V_{{ }_{a t}}$ & Volume of entrapped air in the NAPL-wet soil lens \\
\hline$v_{i}$ & Darcy velocity of phase $i$ \\
\hline$V_{i}$ & Fluid phase $(i)$ volume within the REV \\
\hline$V_{n}$ & Imbibing NAPL volume \\
\hline$V_{N L}$ & NAPL-wet soil lens volume \\
\hline$V_{n t_{W W F}}$ & Volume of the entrapped heptane in the water-wet soil fraction \\
\hline$V_{n_{W W F}}$ & Volume of NAPL imbibing into the water-wet soil fraction \\
\hline$V_{r}$ & Total residual NAPL volume left in the porous medium \\
\hline$V_{r_{N L}}$ & Irreducible NAPL volume in the NAPL-wet soil lens \\
\hline$V_{r_{W W F}}$ & Residual NAPL volume in the water-wet soil fraction \\
\hline$V_{v}$ & Volume of voids within the REV \\
\hline$V_{v i}$ & Volume of the interconnected pores in a soil sample \\
\hline$V_{W W F}$ & Volume of the water-wet soil fraction \\
\hline$v_{x i}$ & Darcy flux for fluid $i$ in $x$-direction at distance $x$ \\
\hline$v_{x i+\Delta x i}$ & Darcy flux for fluid $i$ in $x$-direction at distance $x+\Delta x$ \\
\hline$v_{y i}$ & Darcy flux for fluid $i$ in $y$-direction at distance $y$ \\
\hline$v_{y i+\Delta y i}$ & Darcy flux for fluid $i$ in $y$-direction at distance $y+\Delta y$ \\
\hline
\end{tabular}


$v_{z i}$

$v_{z i+\Delta z i}$

$w$

$W_{i j}$

WW

WWF/NL
Darcy flux for fluid $i$ in $z$-direction at distance $z$

Darcy flux for fluid $i$ in $z$-direction at distance $z+\Delta z$

Wetting

Work done to separate a unit area of phase (i) from phase $(j)$

Water wet porous medium

$60 \%$ water-wet soil fraction- $40 \%$ NAPL-wet soil lens 


\section{Chapter 1. Introduction}

\subsection{Background}

Pollution can take place in different forms in an environmental system. In this research the term pollution refers to a change of physical, chemical, or biological characteristics of groundwater due to the release of another liquid substance to the soil. The soil or porous medium is composed of solid grains enclosing non-solid space. The non-solid space is called pore space. This pore space is composed of relatively large pores known as pore bodies connected with relatively small pores known as pore throats.

A soil profile in large may be divided into two zones, unsaturated zone and saturated zone. The unsaturated zone is located above the saturated zone and it is defined as the soil zone where the pore spaces normally contain water and soil gases creating a two-phase system. In case of a pollutant spill that does not dissolve in water, pore spaces might contain water, pollutant, and soil gases and hence the unsaturated zone holds three different fluid phases. The water table is not the border separating the saturated and the unsaturated zones because due to the capillary action, a tension saturated zone exists above the water table and the height of this zone is controlled by the soil type. The tension saturated zone above the water table is known as the capillary fringe. The vadose zone includes both the capillary fringe and the unsaturated zone.

Fluids, with respect to their solubility in water, may be divided into two classes. The first class is miscible fluids, which easily dissolve in water under normal pressure and temperature conditions such as ammonia and chlorine. The second class is immiscible fluids which show very low solubility levels under normal pressure and 
temperature conditions such as air, oxygen, and most petroleum hydrocarbons and solvents. The second class is the concern of the present research. The liquid phase of immiscible fluids is called Non Aqueous Phase Liquid or simply NAPL. Oil, organic liquid, and NAPL are used interchangeably in the context of this research. Many researchers such as Schwille (1988) and Dane et al. (1992) divided NAPLs into two classes depending on their density relative to water density. NAPLs of densities less than that of water are called Light Non Aqueous Phase Liquids (LNAPLs) and those with densities greater than that of water are called Dense Non Aqueous Phase Liquids (DNAPLs). Moreover, NAPLs can be classified with respect to their spreadability into two groups, spreading and non-spreading. NAPL spreadability is measured by the NAPL spreading coefficient which is a function of the interfacial tensions of the fluid phase interfaces.

After a NAPL spill, both LNAPLs and DNAPLs infiltrate in soil at a rate dependent on soil type and NAPL characteristics. Depending on the spilled volume and soil retention capacity, NAPL might reach the water table. If NAPL reaches the water table, its fate becomes largely dependent on its density. An LNAPL tends to form a pool on the water table as shown in Figure 1.1 while a DNAPL continues to migrate past the water table as shown in Figure 1.2. Upon reaching a layer which a DNAPL cannot penetrate, lateral spreading occurs. The direction of this lateral spreading normally follows the local stratigraphy regardless of groundwater flow direction. Both LNAPLs and DNAPLs leave a residual in their path (Schwille 1988, Mercer and Cohen 1990, Thomson et al. 1992). Wilson and Conrad (1984) stated that, in spite of the low solubility of NAPL in water, part of the residual NAPL dissolves in the infiltrating water. Often, the 
polluted infiltrated water exceeds drinking water standards which are set at very low concentrations for most NAPLs. By taking this fact into account, the residual NAPL acts as long term source of contamination, maybe for many decades, to local groundwater and infiltrating rainfall. In addition, Dane et al. (1992) stated that most of the current groundwater pollution problems are due to NAPL spills, which makes considerable knowledge of the area of residual NAPL very important to gain.

Figure 1.1: Schematic of LNAPL Spill and Migration in Soil (Schwille 1988).

Figure 1.2: Schematic of DNAPL Spill and Migration in Soil (Schwille 1988).

Flow and transport of miscible contaminants in water is different than the flow and transport of immiscible contaminants. For miscible contaminants, their fate can be 
described by a multi-component, single phase model where different transport mechanisms affect that single phase, such as advection and dispersion in addition to the different source and sink terms such as decay and sorption. The physical transport principles of a single phase in porous media are fairly well established (e.g., Bear 1988, Dullien 1991, and Helmig 1997).

In contrast, immiscible fluids behave differently and their modelling is much more complicated than the case of miscible fluids. In case of a NAPL spill in soil, as mentioned earlier, NAPL migrates in the downward direction through the unsaturated zone leaving a residual saturation in its path. Therefore a situation of multi-components and multiple phases is encountered. The unsaturated zone, in this case, contains three fluid phases (i.e., air, NAPL and water). Modelling the flow and transport of these fluid phases is a challenge since the mass balance equations are much more complex than in the case of multi-component, single phase flow. Part of the problem is the difficulty that arises from the fact that when NAPL migrates in the subsurface, part of this NAPL may disconnect from the infiltrating NAPL body and remain in the unsaturated zone as residual NAPL where the distribution of this residual NAPL is controlled by many factors. Among these factors are the NAPL type, soil matrix heterogeneity, and wettability, which refers to the soil's affinity to wetting by one fluid phase in the presence of other fluid phases. None of the existing multiphase models correctly describe either the amount of the residual NAPL, its distribution, or mobilization for drainage and imbibition cases under different wettability conditions.

Evidence of wettability variations with physicochemical properties of NAPL makes it possible for a porous medium to be NAPL-wet even if it was water-wet before 
exposure to certain types of NAPLs (Dwarakanath et al. 2002). As reported by Powers and Tamblin (1995), the wettability of a porous medium in the subsurface may change due to exposure to different NAPLs. That was proven using soil samples from NAPL recovery sites as well as in laboratory experiments. Powers and Tamblin (1995) classified NAPLs with respect to their strength of wetting the porous medium into three groups; namely strong, neutral or intermediate, and weak which correspond to NAPLs with high molecular weights, intermediate molecular weights, and low molecular weights respectively. Therefore, even if a porous medium is strongly water-wet, it may become NAPL-wet when exposed to high molecular weight NAPLs. Dwarakanath et al. (2002) stated that, it is highly important to recognize that the porous medium might be waterwet, mixed-wet, or NAPL-wet. It is also important to take into account that hydrocarbons are often not pure phases and contain additives which may change the wettability conditions of a porous medium. Wettability and its variations should be accounted for in flow and transport models. Also, relative permeability-saturation-capillary pressure relationships may need to include a porous medium wettability term. In addition, field materials should be used in laboratory experiments and then these laboratory data should be used in numerical modelling.

Current governing equations of flow and transport are based on an integrated look at the physical, chemical, and microbiological processes over a soil volume. Moreover, studying the behaviour of fluid phases in porous media is based on averaging their different parameters over a large volume compared to the volume of a single pore. This volume is known as a representative elementary volume or simply an REV (Figure 1.3). Wilson and Conrad (1984) stated that the continuum flow models may not be appropriate 
to simulate residual NAPL mobilization in soil since the residual NAPL exists as discontinuous blobs and pendular rings. This implies that a complete understanding of the different phenomena at the micro scale, pore scale, is important since considering the macro scale, REV scale, may result in important pore scale processes cancelling each other when lumped into a macroscopic constitutive relationship. Moreover, formation of residual NAPL in soil after a NAPL spill and the required soil remediation needed thereafter require further understanding of the migration, distribution and fate of residual NAPL at the pore scale.

micro seale

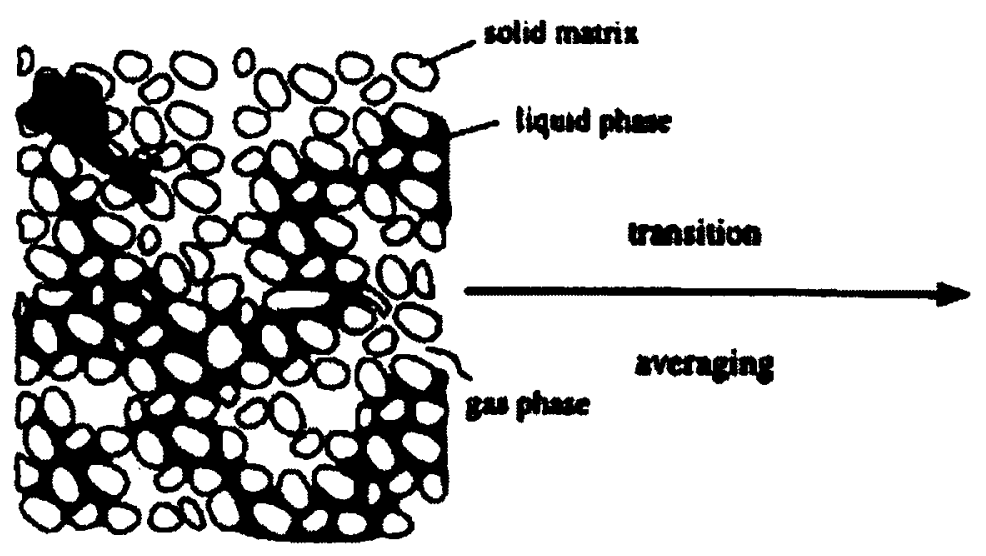

REV

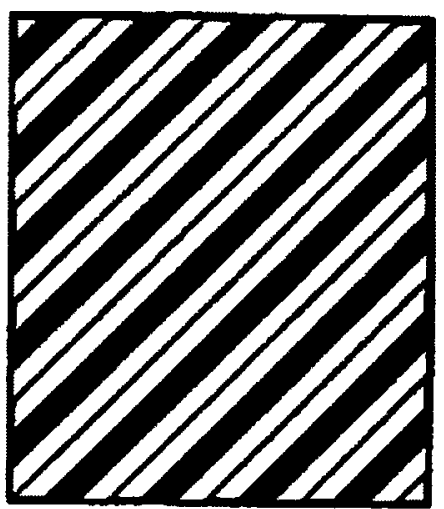

Figure 1.3: Micro Scale Versus REV (Helmig 1997).

The conceptual model used to represent multiphase flow in porous media at the REV scale is achieved by inclusion of constitutive relations into the mass balance equation for each fluid phase. These constitutive relations are originally empirical and are based on specific assumptions (e.g., water is the most wetting fluid followed by NAPL followed by air). Violating these assumptions usually happens in natural porous media. A number of attempts (e.g., Faust 1985, Kuppusamy et al. 1987) have been made to model 
one dimensional multiphase flow experiments. The main differences between the current multiphase flow models lie in the form of the mass transfer, the form of the constitutive relations for the relative permeability-saturation-capillary pressure relationships, and the method of solution. However, inclusion of medium wettability and its heterogeneity within the porous media has not been completely undertaken. Therefore introduction of heterogeneity, in terms of how wettability affects the processes at the micro scale, is an important factor to take into account. This is because understanding what is happening at the micro scale in heterogeneous porous media makes it reasonable to truly improve the existing governing equations of flow and transport and eventually develop a better understanding of the impacts on different remedial alternatives. In the context of this research, water-wet and hydrophilic are used interchangeably. In addition, NAPL-wet and hydrophobic are used interchangeably.

\subsection{Problem Definition}

Several research works have developed numerical models to simulate multiphase flow in the subsurface environment (Kuppusamy et al. 1987). However, many of these models do not account for or allow a residual NAPL to remain in the unsaturated zone after gravity drainage (Van Geel and Roy 2002). In addition, the processes that impact the magnitude and distribution of the residual NAPL are not well understood. To correctly account for a residual NAPL saturation, the constitutive relationships (i.e., relative permeability-saturation-capillary pressure relationships) for multiphase flow need to be improved to properly account for a residual NAPL saturation and the processes and properties that impact its distribution and magnitude. Only recently, conceptual models have been proposed to incorporate a residual NAPL saturation term 
into the constitutive relationships under strong water-wet porous media conditions (e.g., Wipfler and Van der Zee 2001, Van Geel and Roy 2002, Lenhard et al. 2004). Currently, none of the existing constitutive relationships includes the effects of spatial wettability variations on the residual NAPL saturation. Also, residual NAPL saturation distribution under different wettability conditions has not been clearly demonstrated.

Moreover, current constitutive relationships and scaling relations are based on Leverett's assumption which states that water is the most wetting fluid and the intermediate fluid forms a continuous layer (i.e., spreading NAPL) between the water and the gas phases. However, many natural systems are partly water-wet and some cases are NAPL-wet due to phase chemistry, spill age, and subsurface mineralogy distributions.

\subsection{Objectives}

The focus of this research comes from the growing need for a constitutive relationship, relative permeability-saturation-capillary pressure, which takes into account medium wettability and residual NAPL saturation. In order to correctly simulate multiphase systems, it is important to develop constitutive relationships that account for porous medium wettability and residual NAPL saturation. The processes by which residual NAPL can be recovered are affected by wettability conditions.

From the previous discussion, it is depicted that our understanding of the formation and distribution of residual NAPL in porous media is unclear and consequently multiphase flow models fail to properly simulate the migration and fate of NAPLs at these low saturations. Therefore, one of the research objectives is to study the NAPL behaviour at the microscopic level to better understand the physical factors which delineate the NAPL form at these low saturations. 
This research also focuses on the effects of spatial wettability variations on the constitutive relationships, the scaling relations, and the Leverett assumption because capillarity and relative permeability depend on soil texture as well as porous medium wettability characteristics. Therefore, considering wettability variations reflects the real situation in terms of NAPL distribution and migration.

In summary, the objective of this research is to perform experimental work at the pore scale and REV scale to gain a better understanding of the processes and properties that govern the formation of residual NAPL in the unsaturated zone under different wettability conditions. The goal is to develop constitutive relationships, and scaling relations that take into account porous medium wettability and residual NAPL terms. Relating the findings of this research to field applications is planned through improving the conceptual understanding of the factors that influence the movement and distribution of NAPL as well as formation and distribution of residual NAPL, under different wettability conditions, which provides needed insight and knowledge when evaluating remedial alternatives for NAPL contaminated sites.

\subsection{Scope}

This study focuses on addressing the effects of spreading NAPLs under different wettability conditions on residual NAPL magnitude and distribution in porous media both at the pore scale and REV scale. It also focuses on developing modified constitutive relations to include porous medium wettability effects and residual NAPL saturation. Two soil types were used; natural silica sand and glass beads. For the natural silica sand, two distributions were used; uniformly distributed and well graded. The soils were chemically treated to alter their wettability. The NAPL used was heptane, which is a 
spreading LNAPL. In respect to the working scale, two types of experiments were performed; Tempe cell and Buchner funnel for REV scale and a glass box apparatus for pore scale. The soil tested in the Tempe cell and Buchner funnel was silica sand, while glass beads were used in the glass box experiments. The pore scale experiments were conducted by examining the NAPL behaviour in the glass box apparatus under a microscope.

\subsection{Contribution}

In light of the previous discussion, this research addresses the following topics:

1. Gaining an understanding of the effects of changing the wettability conditions on the formation and distribution of residual NAPL at the pore scale and REV scale;

2. Development of conceptual models to include the residual NAPL term in the constitutive relationships under different wettability conditions for a spreading NAPL;

3. Validation of the scaling relations and Leverett assumption under different wettability conditions with a spreading LNAPL; and

4. Development of conceptual models to include porous medium wettability effects on the constitutive relations.

\subsection{Thesis Organization}

The previous sections of the first chapter gave an introduction of the problem in hand, objectives, scope, and contributions of this research. Following is an outline of the remaining chapters that will be covered in the thesis: 
- Chapter two is a comprehensive review of the literature focusing on porous media and immiscible fluid properties, capillarity, flow of immiscible fluids, pore scale models, wettability, and residual NAPL.

- Chapter three is devoted to the description of the research methodology and experimental setup.

- Chapter four is an experimental study that highlights some concerns related to using a traditional treatment procedure to render soil and porous ceramics NAPLwet.

- Chapter five addresses the capillary pressure-saturation $\left(P_{c}-S\right)$ relationships in twophase systems for a water-wet porous medium with NAPL-wet soil lenses.

- Chapter six addresses the $P_{c}-S$ relationships in three-phase systems for a water-wet porous medium with NAPL-wet soil lenses and validation of Leverett assumption.

- Chapter seven addresses development of conceptual models for residual NAPL saturation term in a water-wet porous medium and in a water wet porous medium with a NAPL wet soil lens.

- Chapter eight includes the thesis summary, research contributions, and recommendations for future research. 


\section{Chapter 2. Literature Review}

\subsection{Introduction}

The flow of multiple fluid phases in porous media is normally treated by considering the mass balance equations for the different fluid phases together with the closure relations (i.e., constitutive relations). The information flow in this chapter is written in a way that enables the understanding of the necessary multiphase flow topics and deficiencies leading to the research objectives. Therefore, this chapter starts by defining porous medium and immiscible fluid phase characteristics, then interactions between immiscible fluid phases and porous media through capillarity. At this stage, basic knowledge of immiscible fluids, porous media and the interaction between them is gained. Then the mass balance equations, the constitutive relations, and the deficiencies in the current multiphase flow treatment are displayed. A review of the work pertaining to incorporation of wettability and residual saturation into multiphase flow equations, which is the research focus, is then made.

\subsection{Properties of Porous Media and Immiscible Fluids}

\subsubsection{Porous Media}

Corey (1994) defined the term porous medium as a solid matrix which contains interconnected nonsolid space. The smallest dimension of the nonsolid space is large enough such that it can allow flow of fluid molecules, while its largest dimension is small enough such that when the nonsolid space contains two immiscible fluids, the orientation of the interface between these two fluid phases is largely controlled by the interfacial forces. The existence of pores in soils is either natural or is created by gas evolution 
during crystallization or by dissolution of soluble constituents in water. There are two types of soils; the first is consolidated soil, which is the case when the soil grains are cemented at their points of contact. This type of soil possesses structure and some grains form porous aggregates where an additional pore space is enclosed. The second is unconsolidated soil where its grains are not cemented at their points of contact such as sand. On the other hand, there are two types of pore spaces; the first is primary pore space which exists between the individual solid grains. The other is enclosed within the aggregates and is named secondary pore space.

\subsubsection{Characterization of Porous Media}

The pore space geometry governs the hydraulic characteristics of a porous medium. This geometry is influenced by the solid grain size distribution, solid grain shapes, and degree of consolidation (Corey 1994). Some of the parameters by which the porous medium is characterized are discussed below.

\subsection{2.a. Porosity}

There are two types of porosities; total or absolute porosity and effective porosity. The total or absolute porosity $(\eta)$ of a soil sample of volume $(V)$, according to Bear (1988), is the ratio of the pore volume $\left(V_{v}\right)$ to the soil sample volume. While the effective porosity $\left(\eta_{e}\right)$ of a soil sample of volume (V), according to Bear (1988) and Corey (1994), is defined as the ratio of interconnected pore volume $\left(V_{v i}\right)$ that contributes to flow, to the soil sample volume. The difference in use of these two porosities depends on the application. In flow problems, only the interconnected pores are considered and therefore the effective porosity is used. Even though the dead end pores and stagnant pockets are 
considered as interconnected pores, their existence does not enhance the flow as shown in Figure 2.1.

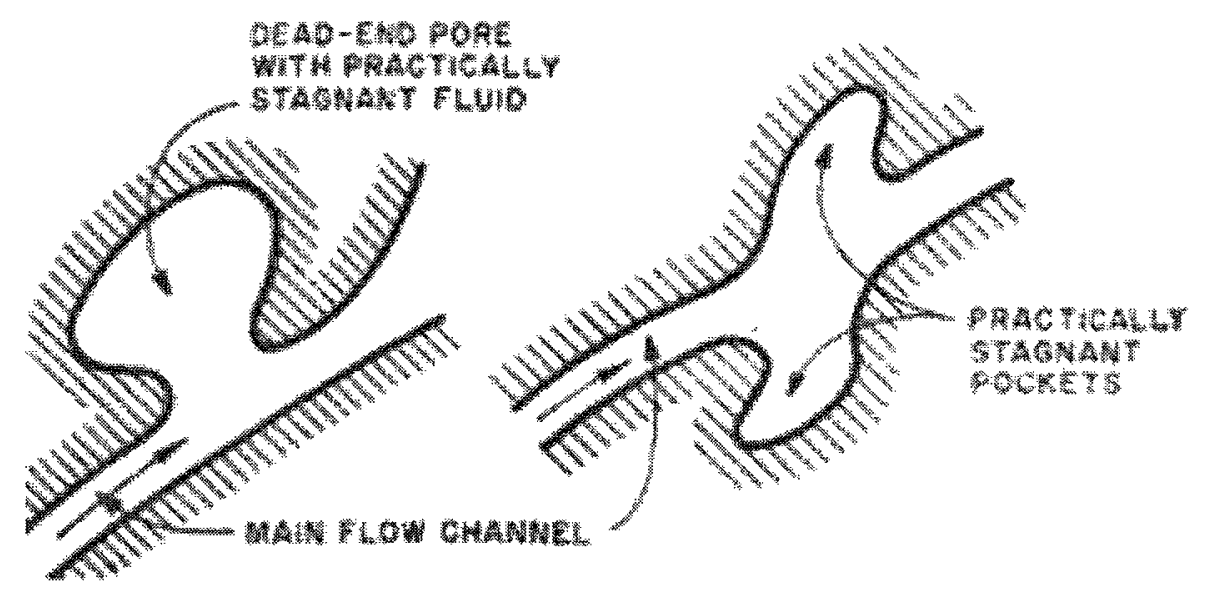

Figure 2.1: Dead - End Pores (Bear 1988).

Porosity depends on the porous medium's structure, grains shape, grain size distribution, mixing, packing, and cementation. During immiscible fluid flow experiments in a porous medium, it is permissible to ignore changes in the porous medium porosity (Corey 1994, Miller et al. 1998).

\subsection{2.b. Specific Surface}

Specific surface is the ratio of the soil grains surface area to the total volume of the soil sample. Specific surface depends on the size and shape of the individual soil grains (Corey 1994). A specific surface area of a soil sample may be measured using different experimental techniques. Among the widely used techniques are the nitrogen adsorption technique developed by Brunauer et al. (1938) and the Ethylene Glycol Monoethyl Ether adsorption technique developed by Dyal and Hendricks (1950). 


\subsection{2.c. Pore Size and Pore Size Distribution}

Average pore size of a soil sample is defined as the ratio of the sample porosity to the sample specific surface. For a soil sample that is composed of a mixture of different grain sizes, the pore sizes should differ accordingly from point to point in the soil sample. The fractions of the pore spaces represented by different sized pores are known as the pore size distribution (Corey 1994, Bear 1988).

\subsection{2.d. Stability}

Corey (1994) defined porous medium stability as the degree to which the porous medium properties, such as porosity, specific surface, pore size, and pore size distribution, remain constant under the mechanical forces caused by fluid flow. The main reason for soil instability is the amount of organic matter and clay present.

\subsubsection{Immiscible Fluids}

There are two groups of fluids. The first is the group of miscible fluids and those mix together and behave as a single fluid phase. While the second is the group of immiscible fluids and those do not mix together. Instead an interface separates every fluid pair where a discontinuity in pressure along that interface may be present. Immiscible fluids are the fluid group which will be dealt with in this research.

\subsubsection{Phase Characteristics}

\subsection{4.a. Phase State}

According to the number of fluid phases, one might have a single phase, twophases, three-phases, or more occupying the pore space. The phase state in the macro scale is determined by the phase saturation (Helmig 1997). 


\subsection{4.b. Phase Transition and Change}

Phase transition refers to a transfer of a component from one phase to another. Phase transition occurs due to vaporization, partitioning, condensation, or dissolution. While phase change means the phase state changes from a single phase system to a multiphase system. Both phase change and phase transition occur due to thermodynamic state change (Helmig 1997).

\subsection{4.c. Fluid Content and Degree of Saturation}

Within an REV, the ratio of the volume of one fluid phase $(i)$ to the total REV volume is known as the fluid phase $(i)$ content $\left(\theta_{i}\right)$. While the ratio of the volume of one fluid phase $(i)$ to the total volume of voids enclosed in an REV is known as the fluid phase $(i)$ degree of saturation $\left(S_{i}\right)$ or simply the saturation (Bear 1988, Corey 1994). $\theta_{i}=($ Volume of fluid $(i)$ in the REV) / (Total REV)

$$
\theta_{i}=\frac{V_{i}}{V} \quad\left(0<\theta_{i}<\eta\right)
$$

$S_{i}=($ Volume of fluid $(i)$ in the REV) / (Total volume of voids within the REV)

$$
\begin{aligned}
& S_{i}=\frac{V_{i}}{V_{v}} \\
& \Sigma S_{i}=1 \\
& \theta_{i}=\eta * S_{i} \\
& \Sigma \theta_{i}=\eta
\end{aligned}
$$




\subsubsection{Saturation Definitions}

\subsection{5.a. Fluids Distribution in Soil at Different Saturations}

Bear (1988) stated that the fluid distribution around soil grains, flow, and the ability of a fluid to transmit the applied pressures depend on many factors. Among these factors is the fluid saturation. At very low fluid saturation, the wetting fluid that wets the soil grains exists as a thin molecular layer around the points of contact of soil particles as pendular rings. Wetting phase does not exist as a continuous phase and no pressure can be transmitted through the wetting phase at this saturation. By increasing the wetting phase saturation, the pendular rings enlarge and become a continuous web of wetting fluid. The wetting phase saturation at this stage is known as equilibrium (or critical) saturation. As wetting phase saturation increases above the previously mentioned critical saturation, the continuous web of the wetting phase gets larger. At this stage, flow and pressure can be transmitted and the wetting phase saturation is called funicular saturation. As for the non-wetting phase, it may become discontinuous and take a shape of isolated droplets or ganglia in the soil matrix. The non-wetting phase saturation, in this case, is called insular saturation.

\subsection{5.b. Saturations}

Parker and Lenhard (1987) defined the effective saturation, in a two-phase system, as a scaled saturation that does not include other entrapped fluids in it. Therefore, it includes only one fluid form. Effective saturations were introduced to scale the actual saturations between irreducible water saturation and unity over an effective saturation between zero and unity. In a three fluid phase system, air/NAPL/water, where water is the most wetting fluid, the effective saturations of fluid phases are as follows: 
For water: $\bar{S}_{w}=\frac{S_{w}-S_{m}}{1-S_{m}}$

For NAPL: $\bar{S}_{n}=\frac{S_{n}}{1-S_{m}}$

For air: $\bar{S}_{a}=\frac{S_{a}}{1-S_{m}}$

$\bar{S}_{t}=\frac{S_{w}+S_{n}-S_{m}}{1-S_{m}}=\bar{S}_{w}+\bar{S}_{n}=1-\bar{S}_{a}$

Where:

$\bar{S}_{w}:$ effective water saturation;

$S_{w}:$ actual water saturation;

$S_{m}$ : irreducible water saturation (assumed independent of the system);

$\bar{S}_{n}:$ effective NAPL saturation;

$S_{n}:$ actual NAPL saturation;

$\bar{S}_{a}$ : effective air saturation;

$S_{a}:$ actual air saturation; and

$\bar{S}_{t}:$ effective total liquid saturation.

In addition, in a three-phase system, air/NAPL/water, where water is the most wetting fluid, Parker and Lenhard (1987) defined an apparent saturation as a scaled fluid saturation that includes the other entrapped fluids in it. Therefore, it includes multiple fluid saturations in one apparent fluid saturation term. The apparent fluid saturation reflects the location of the wetting - non-wetting fluid interface which is not impacted by the presence of the entrapped fluids. The apparent entrapped saturations are as follows: 
$\overline{\bar{S}}_{w}=\bar{S}_{w}+\bar{S}_{n t}+\bar{S}_{a t w}$

$\overline{\bar{S}}_{t}=\bar{S}_{w}+\bar{S}_{n}+\bar{S}_{a t}$

$\bar{S}_{n}=\bar{S}_{n f}+\bar{S}_{n t}$

$\bar{S}_{a}=\bar{S}_{a f}+\bar{S}_{a t}$

$\bar{S}_{a t}=\bar{S}_{a t w}+\bar{S}_{a t n}$

Where:

$\overline{\bar{S}}_{w}:$ apparent water saturation;

$\overline{\bar{S}}_{t}:$ apparent total liquid saturation;

$\bar{S}_{n t}:$ trapped (immobile) effective NAPL saturation;

$\bar{S}_{a t w}:$ effective saturation of air trapped in water;

$\bar{S}_{a t}:$ trapped effective air saturation;

$\bar{S}_{n f}:$ free (continuous) effective NAPL saturation;

$\bar{S}_{a f}:$ free effective air saturation; and

$\bar{S}_{a t n}:$ effective saturation of air trapped in NAPL.

Apparent saturations include the effective mobile and trapped saturations allowing the apparent saturation to still vary between zero and unity. Figure 2.2 shows the relationship between actual, effective, and apparent saturations. 

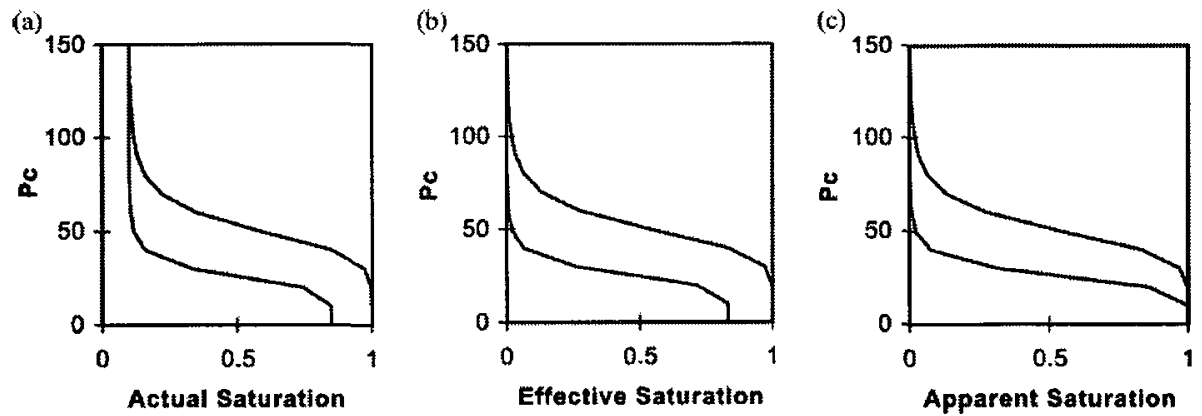

Figure 2.2: Relationship between (a) Actual, (b) Effective, and (c) Apparent Saturations (Van Geel and Roy 2002).

\subsection{Capillarity}

\subsubsection{Surface Tension and Surface Free Energy}

\subsection{1.a. Forces Contributing to the Retention of Water in Soil}

There are at least four groups of forces contributing to the retention of water in soil. These forces are Van der Waals, electrostatic dipole, osmotic, and interfacial tension forces (Selker et al. 1999). The first three forces act only at very short ranges and essentially immobilize water. In contrast, interfacial tension produces effects that affect water in bulk and is more influential in transport in the vadose zone for granular soils (e.g., sand). Therefore, a more in-depth overview is presented for interfacial tension.

\subsection{1.b. Surface Tension and Interfacial Tension}

For any two fluid phases, the work required to separate a unit area of phase $(i)$ from phase $(j)$ is known as the interfacial tension between these two-phases. Hence, the interfacial tension between two fluid phases corresponds to the interfacial free energy on the interface of these two-phases. This interfacial free energy comes from the difference between the molecules inward attraction in the interior of every phase and the molecules attraction on the interface. For the same temperature and pressure, the interfacial tension 
between two fluid phases is constant. The interfacial tension between a liquid phase and its vapour phase is known as the surface tension (Bear 1988, Corey 1994).

The Duprè formula relates the work done $\left(W_{i j}\right)$ to separate a unit area of phase $(i)$ from phase $(j)$, the interfacial tension $\left(\sigma_{i j}\right)$ between phase $(i)$ and $(j)$, and the surface tensions $\left(\sigma_{i}, \sigma_{j}\right)$ between phases $(i, j)$ and their vapour phases, respectively (Bear 1988).

$W_{i j}=\sigma_{i}+\sigma j-\sigma_{i j}$

According to Selker et al. (1999), in order to imagine how the interfacial tension develops, imagine that there is a block of water (system "a"), and this block is split into two halves (system "b") as shown in Figure 2.3. Water molecules on the new air/water surfaces are bound approximately to half of their neighbours. Energy has to be added in order to break these bonds, which is manifested as a free surface energy. Since the water surface has a constant number of molecules on its surface per unit area, the energy required to create these surfaces is directly related to the surface area created (air/water interface) and hence, the interfacial tension should have units of energy per unit area or force per unit length. 


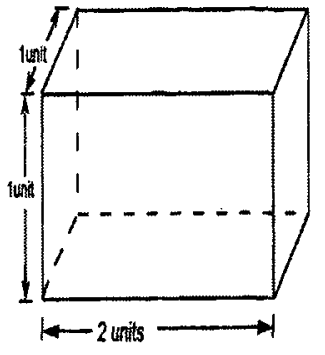

(a)

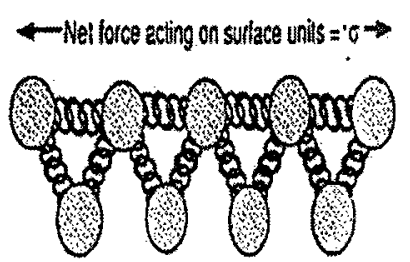

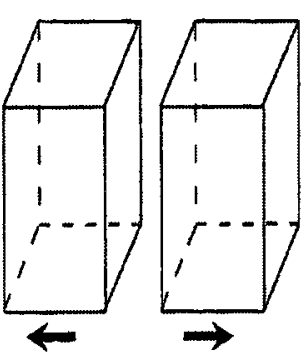

(b)

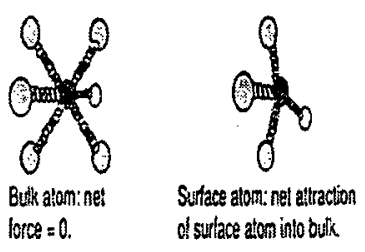

The Surface Area of System "a" is 10 Square Units, where as that of System "b" is 12 Square Units. System "b" has Greater Potential Energy due to the Broken Surface Bonds.

Figure 2.3 Conceptual Model of Potential Energy of Broken Bonds at Liquid Surface (Selker et al. 1999).

\subsection{1.c. Surface (Interfacial) Tension Dependence on the Interface Chemical Concentration}

Surface (or interfacial) tension is highly sensitive to the chemical properties of the boundary layer. Therefore, any chemical constituent that tends to accumulate in a liquid (i) boundary layer, even though the average chemical concentration in the liquid is very small, may change the surface (interfacial) tension to a large extent. This is the role surfactants (or surface active materials) play on reducing the surface (interfacial) tension of liquids. Gibbs equation calculates the change in surface (interfacial) tension with the change in concentration as follows (Corey 1977):

$\frac{d \sigma_{i}}{d c_{i}}=-R_{g} T \frac{\Delta c_{i}}{c_{i}}$ 
Where:

$\Delta c_{i}:$ excess chemical concentration in surface layer of liquid $(i)$;

$c_{i}$ : chemical concentration in bulk liquid $(i)$;

$R_{g}:$ gas constant; and

$T:$ absolute temperature.

By examining Gibbs equation, it is found that an increase in chemical concentration in the liquid surface layer with respect to its concentration in the bulk liquid tends to decrease the surface (interfacial) tension. On the other hand, when the chemical concentration in the liquid surface layer is less than its concentration in the bulk liquid, surface (interfacial) tension increases. Surface (interfacial) tension of tap water is different than soil water, where water is in contact with soil particles which contain salts.

\subsection{1.d. Temperature Dependence of Interfacial Tension}

Interfacial tension is a temperature dependent variable. The temperatureinterfacial tension relationship of air/water is well defined by a straight line. As shown in Figure 2.4 , for every $4^{\circ} \mathrm{C}$ increase in temperature, there is $1 \%$ decrease in the interfacial tension. 


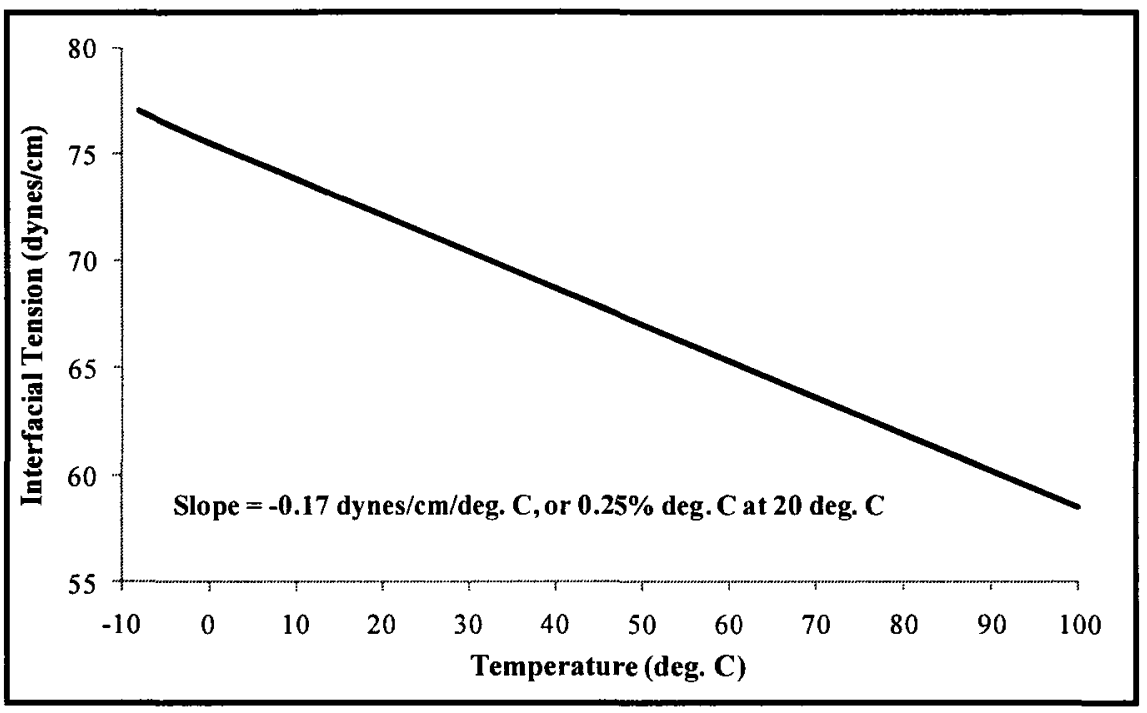

Figure 2.4: The Temperature Dependence of Air/Water Interfacial Tension (Source: CRC Handbook of Chemistry and Physics cited in Selker et al. 1999).

\subsubsection{Microscopic Capillarity}

The curvature of the interfacial boundary between two fluid phases depends on the inter-grain spaces and the proportions of fluid present. The interfacial curvature reflects the capillary pressure on that interface. The reason for this interfacial curvature is that the free energy between these fluid phases should be minimal with regards to fluid volumes and solid surfaces (Leverett 1941). The microscopic capillary pressure is the pressure difference across the wetting/non-wetting fluid interface, and it is responsible for changes occurring on the interface curvature (Reeves and Celia 1996).

A cylindrical capillary tube is taken as the simplest model for capillary attraction in soil. When a capillary tube is placed in contact with a liquid (e.g., water) surface, the static contact angle between tube material and water is established. Interfacial tension on the perimeter of the tube results in a force with a vertical component that drives water upwards. The movement continues until the vertical force due to surface tension equals to the weight of water column as shown in Figure 2.5. 
For equilibrium:

$\Sigma$ Forces in vertical direction $=0$

$h_{c} * \pi r^{2}{ }^{*}{ }^{*} \rho_{w}=\sigma_{a w} * 2 \pi r^{*} \cos \phi$

$h_{c}{ }^{*} g^{*} \rho_{w} * r=\sigma_{a w}{ }^{*}{ }^{*} \cos \phi$

$h_{c}=\frac{2 \sigma_{a w} \cos \phi}{g^{*} \rho_{w}{ }^{*} r}=\frac{2 \sigma_{a w} \cos \phi}{g^{*} \rho_{w}{ }^{*} r}$

$h_{c}=\frac{2 \sigma_{a w} \cos \phi}{g^{*} \rho_{w} * r}$

Where:

$h_{c}$ : capillary rise;

$r$ : capillary tube radius;

$g$ : acceleration due to gravity;

$\phi:$ contact angle; and

$\sigma_{a w}:$ air/water interfacial tension.

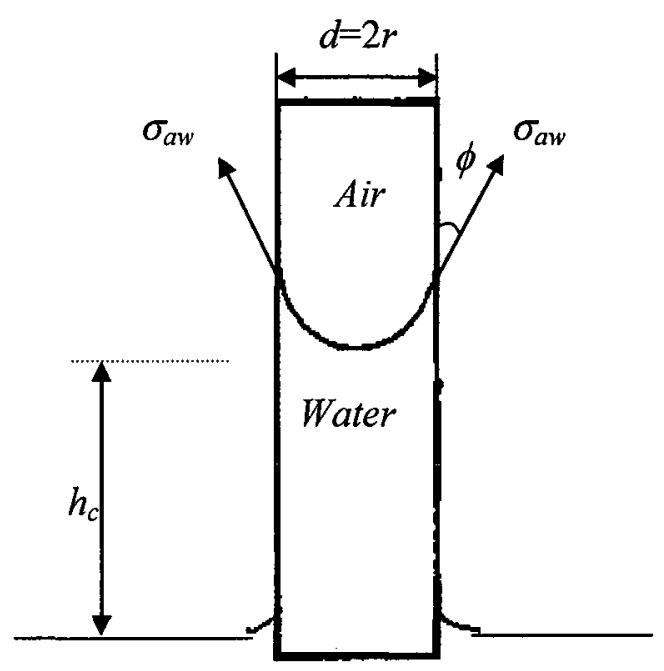

Figure 2.5: Force Balance for Water Rise in Capillary Tube. 


\subsection{2.a. Classical Derivation of Laplace Equation}

The Laplace equation is a mathematical derivation of how surface (interfacial) tension develops in porous media. This derivation relates the static fluid pressure forces which balance with the surface (interfacial) tension forces acting on the curved fluid/fluid interface within a porous medium. For this purpose, consider the infinitesimal curved fluid surface shown in Figure 2.6. The infinitesimal fluid surface has curvature in two directions of radii $r_{1}$ and $r_{2}$. Since the surface is at equilibrium, the summation of forces acting on the surface must add to zero.

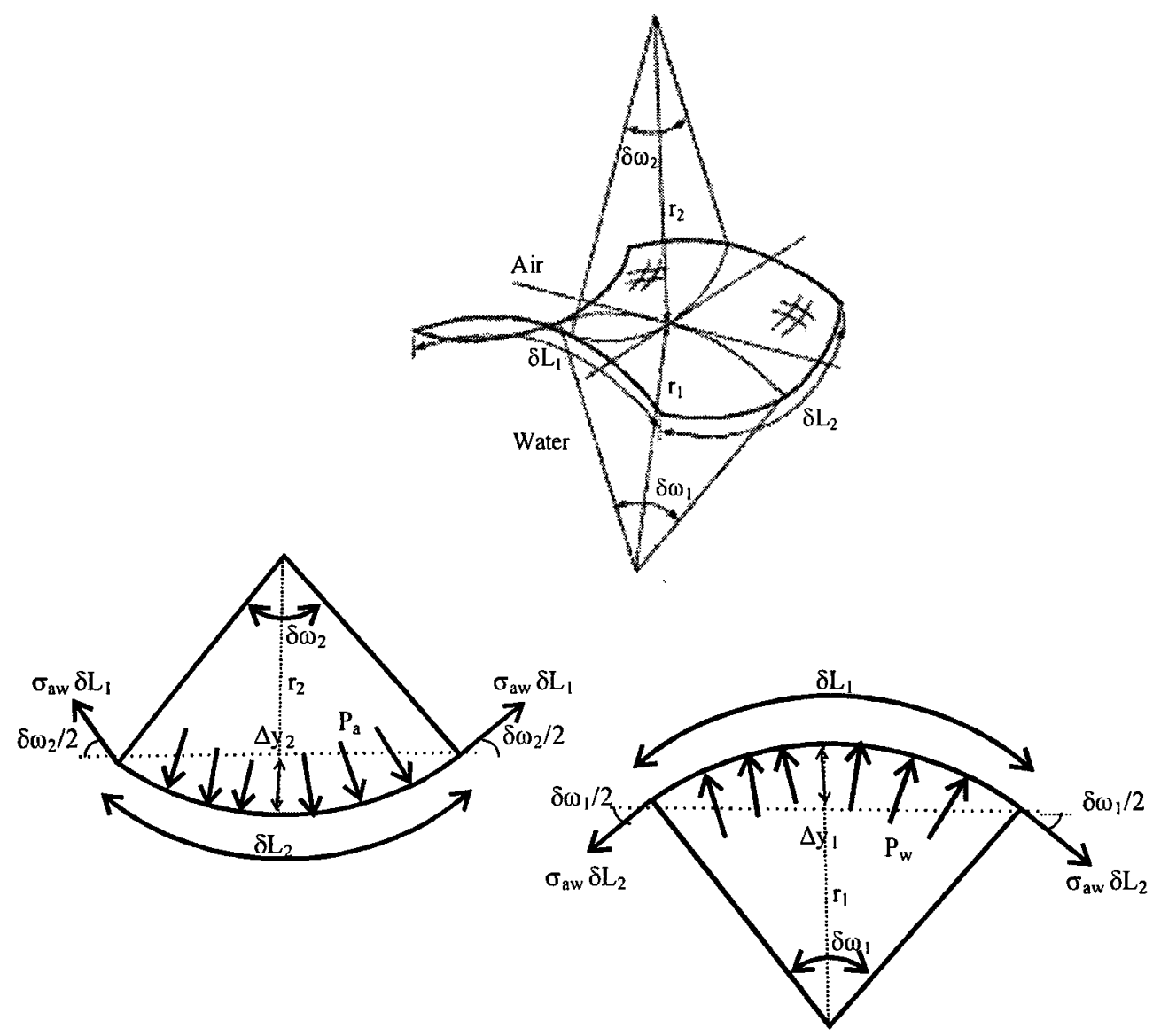

Figure 2.6: Definition Sketch for a Doubly Curved Liquid Surface (Reproduced from Selker et al. 1999). 
At equilibrium: $\Sigma$ Forces in any direction $=0$

For horizontal direction:

$$
\begin{aligned}
& {\left[\sigma_{a w} \delta L_{2} \cos \left(\frac{\delta \omega_{1}}{2}\right)-\sigma \delta L_{2} \cos \left(\frac{\delta \omega_{1}}{2}\right)\right]_{\text {along side } \delta L_{2}}+P_{w} * \Delta y_{1}-P_{w} * \Delta y_{1}+} \\
& {\left[\sigma_{a w} \delta L_{1} \cos \left(\frac{\delta \omega_{2}}{2}\right)-\sigma \delta L_{1} \cos \left(\frac{\delta \omega_{2}}{2}\right)\right]_{\text {along side } \delta L_{1}}+P_{a}^{*} \Delta y_{2}-P_{a} * \Delta y_{2}=0}
\end{aligned}
$$

Where:

$\delta L_{1}$ : infinitesimal length of the interface in longitudinal direction (1);

$\delta L_{2}$ : infinitesimal length of the interface in transverse direction (2);

$\delta \omega_{1}$ : infinitesimal central angle corresponding to an interface length $\delta L_{1}$;

$\delta \omega_{2}$ : infinitesimal central angle corresponding to an interface length $\delta L_{2}$,

$P_{w}:$ water pressure at elevation $h$, above water table;

$P_{a}:$ air pressure;

$\Delta y_{1}:$ projection of interface (1) length in $y$-direction; and

$\Delta y_{2}$ : projection of interface (2) length in $y$-direction.

For vertical direction:

$$
\begin{aligned}
& P_{w} *\left[2 r_{1} \sin \left(\frac{\delta \omega_{1}}{2}\right) * 2 r_{2} \sin \left(\frac{\delta \omega_{2}}{2}\right)\right]-2 \sigma_{a w} \delta L_{2} \sin \left(\frac{\delta \omega_{1}}{2}\right)- \\
& P_{a} *\left[2 r_{2} \sin \left(\frac{\delta \omega_{2}}{2}\right) * 2 r_{1} \sin \left(\frac{\delta \omega_{1}}{2}\right)\right]+2 \sigma_{a w} \delta L_{1} \sin \left(\frac{\delta \omega_{2}}{2}\right)=0
\end{aligned}
$$

Since $\left(\frac{\delta \omega_{2}}{2}\right)$ and $\left(\frac{\delta \omega_{1}}{2}\right)$ are very small, therefore: 


$$
\begin{aligned}
& \sin \left(\frac{\delta \omega_{1}}{2}\right)=\frac{\delta \omega_{1}}{2}=\frac{\delta L_{1}}{2 r_{1}} \\
& \sin \left(\frac{\delta \omega_{2}}{2}\right)=\frac{\delta \omega_{2}}{2}=\frac{\delta L_{2}}{2 r_{2}} \\
& P_{w} *\left[2 r_{1} * \frac{\delta L_{1}}{2 r_{1}} * 2 r_{2} * \frac{\delta L_{2}}{2 r_{2}}\right]-2 \sigma_{a w} \delta L_{2} * \frac{\delta L_{1}}{2 r_{1}}- \\
& P_{a} *\left[2 r_{2} * \frac{\delta L_{2}}{2 r_{2}} * 2 r_{1} * \frac{\delta L_{1}}{2 r_{1}}\right]+2 \sigma_{a w} \delta L_{1} * \frac{\delta L_{2}}{2 r_{2}}=0 \\
& P_{w} * \delta L_{1} * \delta L_{2}-P_{a} * \delta L_{2} * \delta L_{1}-2 \sigma_{a w} \delta L_{2} * \frac{\delta L_{1}}{2 r_{1}}+2 \sigma_{a w} \delta L_{1} * \frac{\delta L_{2}}{2 r_{2}}=0
\end{aligned}
$$

Divide by $\left(\delta L_{1} * \delta L_{2}\right)$ results:

$$
\begin{aligned}
& P_{w}-P_{a}-\frac{\sigma_{a w}}{r_{1}}+\frac{\sigma_{a w}}{r_{2}}=0 \\
& \therefore P_{w}-P_{a}=\sigma_{a w}\left(\frac{1}{r_{1}}-\frac{1}{r_{2}}\right) \\
& \Delta P=P_{c}=\sigma_{a w}\left(\frac{1}{r_{1}}-\frac{1}{r_{2}}\right)
\end{aligned}
$$

Considering a spherical air/water interface $\left(r_{l}=-r_{2}=R\right)$;

$$
\begin{aligned}
& P_{w}-P_{a}-\frac{\sigma_{a w}}{r_{1}}+\frac{\sigma_{a w}}{r_{2}}=0 \rightarrow P_{w}-P_{a}=\sigma_{a w}\left(\frac{1}{R}-\frac{1}{-R}\right)=\frac{2 \sigma_{a w}}{R} \\
& P_{c}=\frac{2 \sigma_{a w}}{R}
\end{aligned}
$$

As for NAPL/water interface, the interfacial curvature is a function of the pore dimensions where this interface is located as well as fluid proportions. If the interface centre of curvature lies in the wetting phase, then the curvature has a negative sign; otherwise it has a positive sign (Leverett 1941). 


\subsubsection{Macroscopic Capillarity}

For fluid phases, which are in capillary equilibrium and are isothermal, capillary pressure is the same on all the interfaces which are located at the same elevation. Macroscopic capillary pressure is the pressure difference between the wetting and nonwetting fluid reservoirs (e.g., Reeves and Celia 1996).

According to Corey (1977), the macroscopic capillary pressure equation can be found by initially considering the forces acting on a fluid particle in the gravitational field as shown in Figure 2.7.

$-\frac{d P}{d z}-\rho g=0$

The solution of this equation is:

$P-P_{0}=-\rho g h$

Where:

P: fluid pressure;

$P_{0}:$ fluid pressure at water table;

$\rho$ : fluid density; and

$h$ : elevation of the fluid particle above the datum at which $P=P_{0}$.

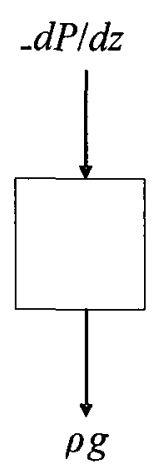

Figure 2.7: Forces Acting on a Fluid Particle. 
Equation 2.21 gives the pressure distribution in a static fluid system. This equation is also valid when applied to a mixture of immiscible fluids. In a two-phase system, water and NAPL, the pressure distribution in each fluid phase is as follows:

Water: $P_{w}-P_{w 0}=-\rho_{w} g h$

NAPL: $P_{n}-P_{n 0}=-\rho_{n} g h$

Where:

$P_{w 0}:$ water pressure at the water table;

$P_{n}:$ NAPL pressure at elevation $h$, above water table;

$P_{n 0}:$ NAPL pressure at the water table;

$\rho_{n}:$ NAPL density; and

$\rho_{w}:$ water density.

The capillary pressure distribution on the NAPL/water interface, along a vertical distance (h), assuming that each phase is interconnected along $h$, can be obtained by subtracting equations (2.22) and (2.23):

$P_{c}=\left(\rho_{w}-\rho_{n}\right) g h+P_{c 0}$

Where:

$P_{c 0}:$ capillary pressure at water table.

By neglecting the capillary pressure at water table $\left(P_{c 0}=0\right)$, the capillary pressure equation is reduced to:

$P_{c}=\left(\rho_{w}-\rho_{n}\right) g h$ 
This equation gives the capillary pressure at any elevation $(h)$ above water table in a static fluid system. In order to relate the interface mean curvature to the elevation above water table, equations (2.21) and (2.25) result in:

$P_{c}=\sigma_{n w}\left(\frac{1}{r_{1}}-\frac{1}{r_{2}}\right)=\left(\rho_{w}-\rho_{n}\right) g h$

Where:

$\sigma_{n w}:$ NAPL/water interfacial tension.

Equation (2.26) shows that there is a direct relationship between changes in interface curvature and the interface elevation. The interface mean curvature increases (i.e., radii of curvature decrease) at higher elevations.

Corey (1977) further showed that a change in interface mean curvature and capillary pressure is accompanied by a change in fluid saturations. As elevation increases, the wetting phase saturation decreases while the non-wetting phase saturation increases. This can be visualized by considering a cross section in a pore where water and NAPL are present as shown in Figure 2.8.

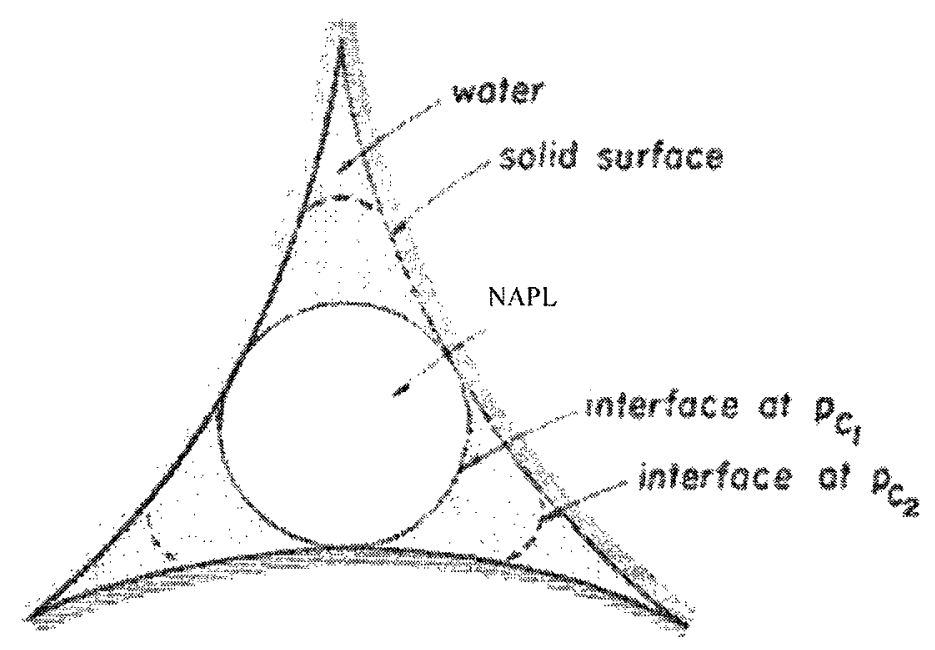

Figure 2.8: Pore Cross Section Containing Water and NAPL with Varying Water Saturations (Corey 1977). 
Increasing the capillary pressure on the NAPL/water interface from $P_{c 1}$ to $P_{c 2}$, by either increasing NAPL pressure or decreasing water pressure, the NAPL/water interface retreats to a position where its curvature increases. While that is occurring, NAPL saturation increases and water saturation decreases. This clarifies an important fact on the direct relationship between fluid saturations and the capillary pressure on the interface separating them.

\subsubsection{Contact Angle}

Contact angle is the angle formed when a liquid phase comes in contact with a solid surface. The magnitude of the contact angle depends primarily on the adhesive forces between the liquid and the solid surfaces, and the cohesive forces of the liquid. Contact angle is a measure of the system wettability (i.e., which of the fluids preferentially wet the solid surface). In order to calculate the contact angle, consider a cross section of a straight line contact between fluid and solid surfaces as shown in Figure 2.9. In order to achieve equilibrium, the summation of forces in the horizontal direction must add to zero.

$\Sigma$ Forces in the horizontal direction $=0$

$0=F_{s g^{-}} F_{s L^{-}} F_{L g} \cos \phi$

Where:

$F_{s g}:$ solid/gas surface force per unit length $=\sigma_{s g}$;

$F_{s L}:$ solid/liquid surface force per unit length $=\sigma_{s L} ;$ and

$F_{L g}:$ liquid/gas surface force per unit length $=\sigma_{L g}$.

Rewriting the above equation in terms of interfacial tensions, results in:

$\sigma_{s g}-\sigma_{s L}-\sigma_{L g} \cos \phi=0$ 
$\phi=\cos ^{-1}\left(\frac{\sigma_{s g}-\sigma_{s L}}{\sigma_{L g}}\right)$

This equation is known as Young-Laplace equation, which relates the contact angle and the three surface tensions (Selker et al. 1999).

(a)

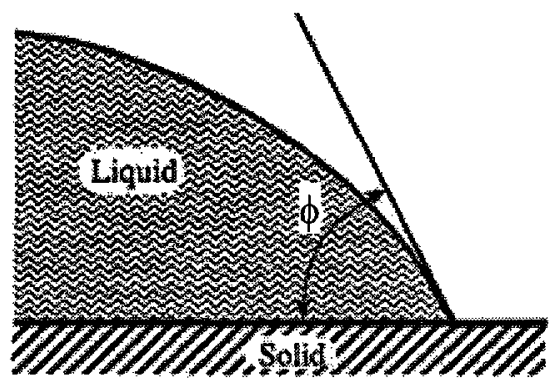

(b)

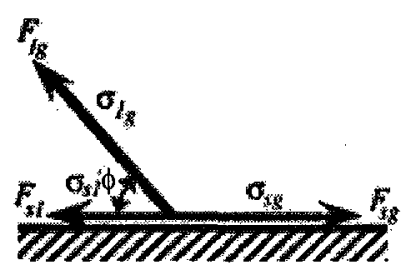

Figure 2.9: (a) Cross Section of a Line Contact between a Solid Surface with a Line of Liquid/ Air Contact at Equilibrium; (b) the Force Balance at the Point of Contact (Selker et al. 1999).

\subsection{4.a. Hysteresis in Contact Angle}

In order to observe the difference between the advancing and receding contact angles of a raindrop on a tilted surface, as shown in Figure 2.10, a magnified view of the point of contact at the receding and advancing points is needed.

For the advancing case (Figure 2.11a), the microscopic contact angle $\left(\phi_{1}\right)$ is less than the macroscopic contact angle $\left(\phi_{2}\right)$. This is due to the existence of a scratch, smudge of oil, or any other contamination. Therefore, the drop cannot advance until the apparent contact angle is quite large. Similarly, upon retreat (Figure $2.11 \mathrm{~b}$ ), the microscopic 
contact angle $\left(\phi_{1}\right)$ will be more than the macroscopic contact angle $\left(\phi_{2}\right)$ (Selker et al. 1999).

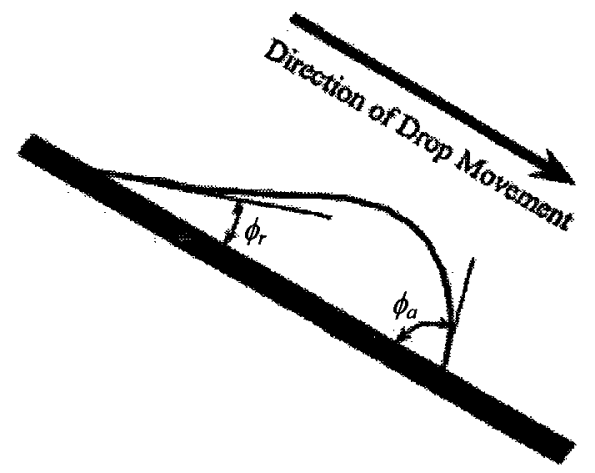

Figure 2.10: The Raindrop Effect of Contact Angle Hysteresis between Advancing and Receding Contact Angles (Selker et al. 1999).

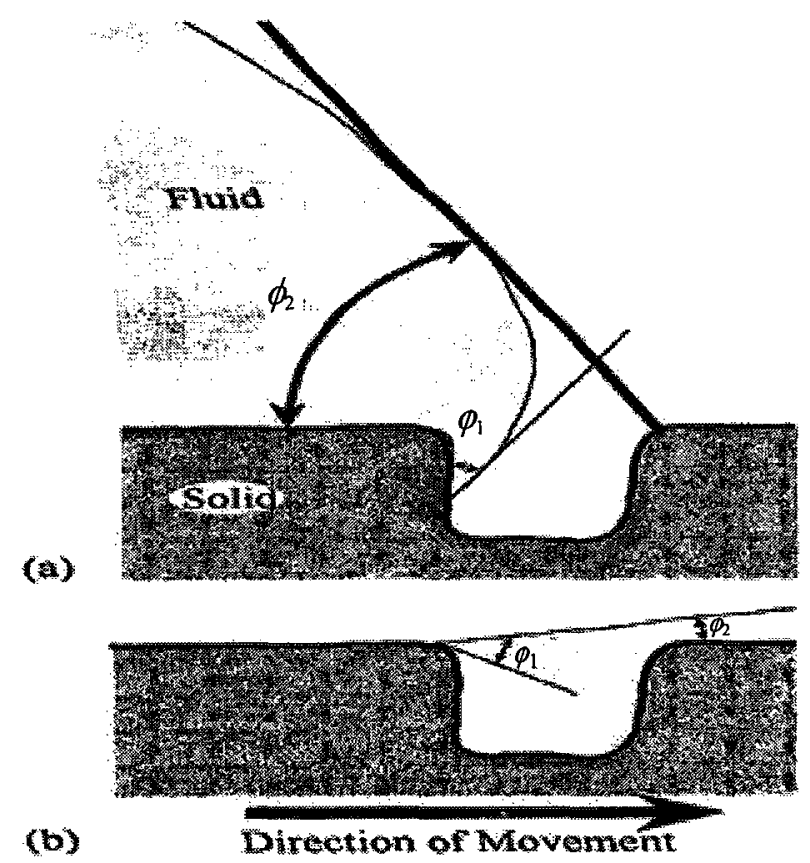

Figure 2.11: Illustration of the Effect of a Microscopic Defect on the Apparent Contact Angle for (a) Advancing and (b) Receding Fluid (Selker et al. 1999). 


\subsection{Flow of Immiscible Fluids}

Miscible fluids are completely soluble in one another, no interface separates the fluids, and they flow as one fluid phase. This is unlike the flow of immiscible fluids, which is characterized by simultaneous displacement of the different fluid phases at hand. In pores with two or more immiscible fluids, a distinct fluid/fluid interface exists between any two fluid phases where a non-zero interfacial tension along the curved interface results in a force. This force balances the capillary pressure force across the curved interface (Corey 1994, Bear 1988).

Due to the extremely large number of pores, the complexity of the pore geometry, different wettability conditions, and number of fluid phases present in every pore, the balance equations for the different fluid phases are difficult to apply on every pore. Instead, the balance equations are normally applied on a controlled volume, REV, for the different fluid phases present. A transfer of the pore scale effects to the REV scale is achieved by the aid of constitutive relations.

Reeves and Celia (1996) and Miller et al. (1998) and others stated that the required constitutive relationships are the $P_{c} \neg S$ relationship and the relative permeabilitysaturation $\left(k_{r}-S\right)$ relationship. Typically, the flow equations for fluid phases are written in terms of fluid phase pressures and saturations. These equations are linked by the $P_{c}-S$ relationship, which relates the difference in fluid phase pressures to the fluid saturations. In addition, the $k_{r} \neg S$ relationship is required for each fluid phase. The fluid saturations, which sum to unity, serve to link the two constitutive relationships.

\subsubsection{Mass Balance Equation}

A general mass balance equation for a fluid phase $\alpha$ is written as (Miller et al. 1998): 
$\frac{\partial}{\partial t}\left(S_{i} \rho_{i}\right)=-\nabla \cdot\left(S_{i} \rho_{i} v_{i}\right)+M_{i}+S S_{i}$

Where:

$v_{i}$ : Darcy velocity of phase $i$

$M_{i}$ : mass transfer of phase $i$; and

$S S_{i}$ : source or sink for phase $i$.

Equation 2.28 is valid for saturation values greater than a fluid saturation value at which fluid becomes discontinuous and flow ceases. As mentioned previously, this equation is applied for all fluid phases separately where Darcy's law representing a fluid phase velocity is incorporated in the above-mentioned equation. With the aid of the constitutive relationships, $P_{c}-S$ and $k_{r}-S$ relationships, and the fact that the sum of all fluid saturations has to add to unity, the resulting system of equations can be solved. Connecting flow of a fluid phase to its saturation, and hereby formation of its own flow pathways, makes it important to relate the flow of immiscible fluids to their effective or relative permeabilities (Bear 1988, Corey 1994, Helmig 1997).

\subsection{1.a. Assumptions for Developing Multiphase Flow Equations}

According to Hillel (1980) and Bear (1988), the multiphase flow equations are based on the following assumptions and simplifications:

1. Soil is incompressible (i.e., porosity does not change);

2. Water and NAPL densities are constant with respect to time and space;

3. Isothermal;

4. Flow is via the soil matrix only and not via macro pores or larger preferred pathways; and 
5. Soil is assumed to be isotropic.

\subsection{1.b. Development of Three-Phase, Air/NAPL/Water, Flow Equations}

Consider a soil element of dimensions $\Delta x, \Delta y$, and $\Delta z$ in $x, y$, and $z$ directions as shown in Figure 2.12 .

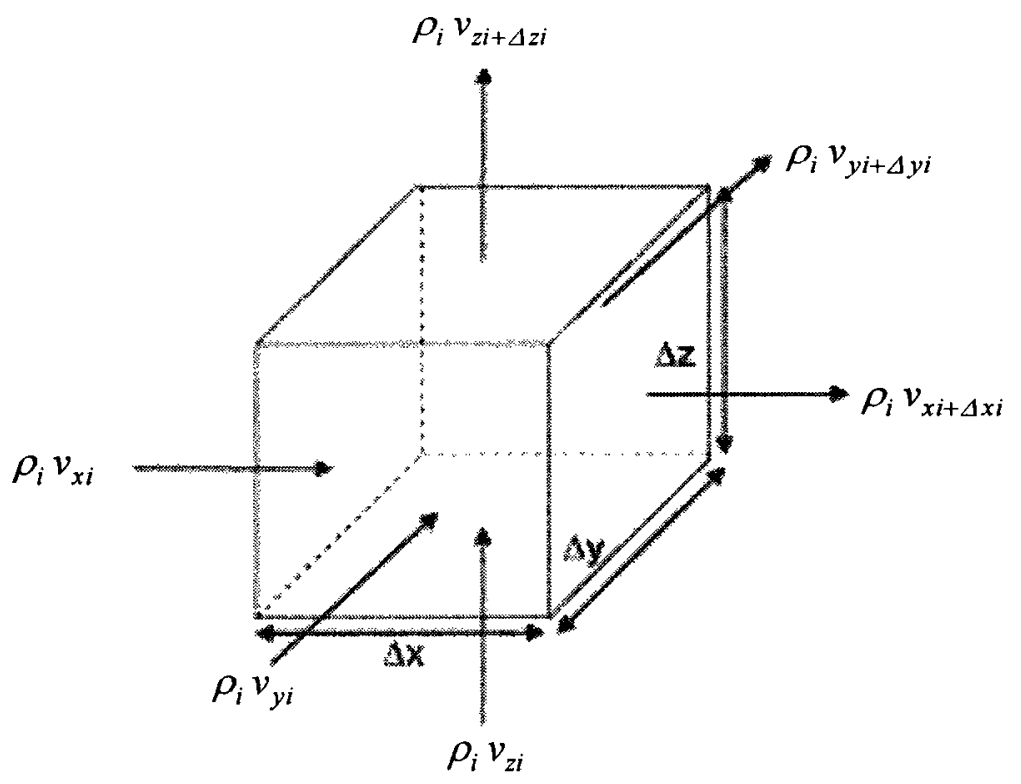

Figure 2.12: A Soil Element of Dimensions $\Delta x, \Delta y$, and $\Delta z$ in $x, y$, and $z$ Directions.

Conservation of mass for a phase $(i)$ :

Change in storage $=$ Mass $_{\text {in }}-$ Mass $_{\text {out }}$

$$
\begin{aligned}
\left(S_{i} \rho_{i} \Delta x \Delta y \Delta z \eta\right)^{t+\Delta t}-\left(S_{i} \rho_{i} \Delta x \Delta y \Delta z \eta\right)^{t} & =\rho_{i} v_{x i} \Delta y \Delta z-\rho_{i} v_{x i+\Delta x i} \Delta y \Delta z+ \\
& \rho_{i} v_{y i} \Delta x \Delta z-\rho_{i} v_{y i+\Delta y i} \Delta x \Delta z+ \\
& \rho_{i} v_{z i} \Delta x \Delta y-\rho_{i} v_{z i+\Delta z i} \Delta x \Delta y
\end{aligned}
$$

Where:

$\Delta x$ : infinitesimal distance in $x$-direction;

$\Delta y$ : infinitesimal distance in $y$-direction; 
$\Delta \mathrm{z}$ : infinitesimal distance in $\mathrm{z}$-direction;

$\Delta t:$ infinitesimal time increment;

$\mathrm{t}$ : time;

$v_{x i}$ : Darcy flux for fluid $i$ in $x$-direction at distance $x$;

$v_{x i+\Delta x i}$ : Darcy flux for fluid $i$ in $x$-direction at distance $x+\Delta x$;

$v_{y i}$ : Darcy flux for fluid $i$ in $y$-direction at distance $y$;

$v_{y i+\Delta y i}$ : Darcy flux for fluid $i$ in $y$-direction at distance $y+\Delta y$;

$v_{z i}$ : Darcy flux for fluid $i$ in $z$-direction at distance $z$; and

$v_{z i+\Delta z i}$ : Darcy flux for fluid $i$ in $z$-direction at distance $z$.

Dividing by $\Delta x \Delta y \Delta z \Delta t$ :

$$
\frac{\partial}{\partial t}\left(\eta S_{i} \rho_{i}\right)=-\frac{\partial}{\partial x}\left(\rho_{i} v_{x i}\right)-\frac{\partial}{\partial y}\left(\rho_{i} v_{y i}\right)-\frac{\partial}{\partial z}\left(\rho_{i} v_{z i}\right)
$$

- For water (wetting phase) in x-direction:

$$
q_{x w}=-K_{w}\left(\theta_{w}\right) \frac{\partial h_{w}}{\partial x}=\frac{k_{r w} k \rho_{w} g}{\mu_{w}} \frac{\partial h_{w}}{\partial x}
$$

Where:

$q_{x w}$ : Darcy flux of water in x-direction;

$K_{w}\left(\theta_{w}\right)$ : unsaturated hydraulic conductivity of water as a function of water content;

$k_{r w}$ : relative permeability of water; and

$\mu_{w}$ : dynamic viscosity of water.

- For NAPL (intermediate phase) in x-direction:

$$
q_{x n}=-K_{n}\left(\theta_{n}\right) \frac{\partial h_{n}}{\partial x}=\frac{k_{r n} k \rho_{n} g}{\mu_{n}} \frac{\partial h_{n}}{\partial x}
$$


Where:

$q_{x n}:$ Darcy flux of NAPL in x-direction;

$K_{n}\left(\theta_{n}\right)$ : unsaturated hydraulic conductivity of NAPL as a function of water content;

$k_{r n}$ : relative permeability of NAPL;

$k$ : intrinsic permeability; and

$\mu_{n}$ : dynamic viscosity of NAPL.

- Assuming the air pressure is zero and the air is infinitely mobile.

Using,

$v_{x i}=-K_{x}\left(\theta_{i}\right) \frac{\partial h_{i}}{\partial x}$

$v_{y i}=-K_{y}\left(\theta_{i}\right) \frac{\partial h_{i}}{\partial y}$

$v_{z i}=-K_{z}\left(\theta_{i}\right) \frac{\partial h_{i}}{\partial z}$

$K_{x}\left(\theta_{i}\right)=K_{y}\left(\theta_{i}\right)=K_{z}\left(\theta_{i}\right)=K\left(\theta_{i}\right)$

Where:

$K_{x}\left(\theta_{i}\right):$ unsaturated hydraulic conductivity of fluid $i$ in $x$-direction;

$K_{y}\left(\theta_{i}\right)$ : unsaturated hydraulic conductivity of fluid $i$ in $y$-direction;

$K_{z}\left(\theta_{i}\right)$ : unsaturated hydraulic conductivity of fluid $i$ in $z$-direction; and

$K\left(\theta_{i}\right)$ : unsaturated hydraulic conductivity of fluid $i$.

Therefore:

Water flow equation in 3-D:

$$
\frac{\partial}{\partial t}\left(\eta S_{w} \rho_{w}\right)=\frac{\partial}{\partial x}\left(\rho_{w} \frac{k k_{r w} \rho_{w} g}{\mu_{w}} \frac{\partial h_{w}}{\partial x}\right)+\frac{\partial}{\partial y}\left(\rho_{w} \frac{k k_{r w} \rho_{w} g}{\mu_{w}} \frac{\partial h_{w}}{\partial y}\right)+\frac{\partial}{\partial z}\left(\rho_{w} \frac{k k_{r w} \rho_{w} g}{\mu_{w}} \frac{\partial h_{w}}{\partial z}\right)(2
$$


NAPL flow equation in 3-D:

$$
\frac{\partial}{\partial t}\left(\eta S_{n} \rho_{n}\right)=\frac{\partial}{\partial x}\left(\rho_{n} \frac{k k_{r n} \rho_{n} g}{\mu_{n}} \frac{\partial h_{n}}{\partial x}\right)+\frac{\partial}{\partial y}\left(\rho_{n} \frac{k k_{r n} \rho_{n} g}{\mu_{n}} \frac{\partial h_{n}}{\partial y}\right)+\frac{\partial}{\partial z}\left(\rho_{n} \frac{k k_{r n} \rho_{n} g}{\mu_{n}} \frac{\partial h_{n}}{\partial z}\right)(2
$$

\subsubsection{Constitutive Relationships}

Constitutive relationships, also called closure relations, are used to supplement the mass balance equations with additional equations required to solve for the system unknowns (i.e., capillary pressure and saturation). Although constitutive relationships increase the number of unknown quantities, they supply the system with additional equations since capillary pressure, saturation, and relative permeability are expressed in terms of accessible parameters. In mathematical terms, they are called scaling relations because they serve as projection operators from pore scale to REV scale (Miller et al. 1998). Constitutive relationships are the means by which physical measurements, either in pore scale or REV scale, are incorporated into mathematical and numerical models (Reeves and Celia 1996). They are empirically based and hence the solution with them is approximate. Constitutive relationships are based on certain assumptions; the solid matrix is rigid, the solid phase is inert, and local chemical equilibrium exists between different fluid phases (Corey 1977, Bear 1988, Miller et al. 1998).

The constitutive relationships of concern to this research are those relating capillary pressure, relative permeability, and saturation of fluid phases. These relations are of central importance for multiphase system modelling. The various constitutive relationships, although they are different in form, agree on transferring the pore scale effects to the REV scale by ignoring pore scale details. 


\subsection{3. $P_{c}-S$ Relationship}

Multiphase fluid flow in the subsurface requires that the $P_{c}-S$ relationships are known for all fluid pairs. These relationships can be obtained directly by measurement or indirectly by applying scaling theories and Leverett's assumption where the $P_{c}-S$ relationship for only one fluid pair is measured. The existing scaling theories are based on some specific assumptions; the solid matrix is strongly water wet, deformation in solid matrix is negligible, NAPL is the intermediate wetting phase in a three-phase system of air/NAPL/water, and this intermediate wetting phase exists everywhere and all the time as a continuous layer between water and air (Lenhard and Parker 1988).

\subsection{3.a. Development of $P_{c}-S$ Relationship}

In order to show the relationship between capillary pressure and saturation in soil, the following experiment is used. Two fluids considered in this experiment are water and air; however, it can be applied to any immiscible fluid pair. Before presenting the experiment, an explanation of the grain size distribution as well as distribution of pore filling pressure heads corresponding to pore sizes are given.

For the grain size distribution of the soil sample shown in Figure 2.13a, the pore size distribution takes a similar shape as the grain size distribution, resulting in distribution of pores with radii between " $r_{\min }$ " to " $r_{\max }$ " as shown in Figure $2.13 \mathrm{~b}$. The relationship between pore size radius " $r$ " and its filling capillary pressure head is:

$$
\begin{aligned}
& \therefore h_{f}=-\frac{2 \sigma_{a w}}{\rho_{w} g r} \\
& r=r_{\text {min }} \quad \therefore h_{f \max }=-\frac{2 \sigma_{a w}}{\rho_{w} g r_{\text {min }}}
\end{aligned}
$$


$r=r_{\text {max }} \quad \therefore h_{f \min }=-\frac{2 \sigma_{a w}}{\rho_{w} g r_{\max }}$

Where

$h_{f}:$ the filling pressure.

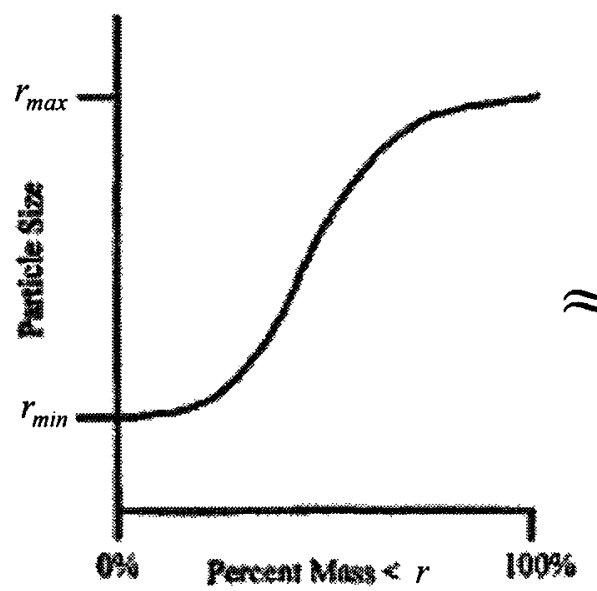

(a)

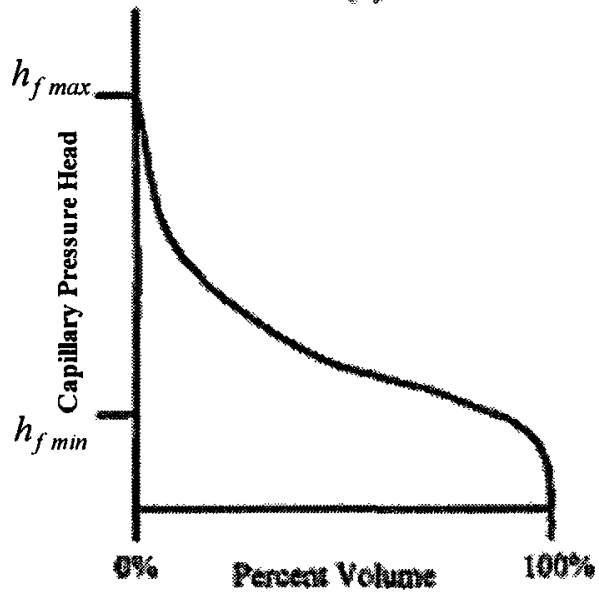

(c)

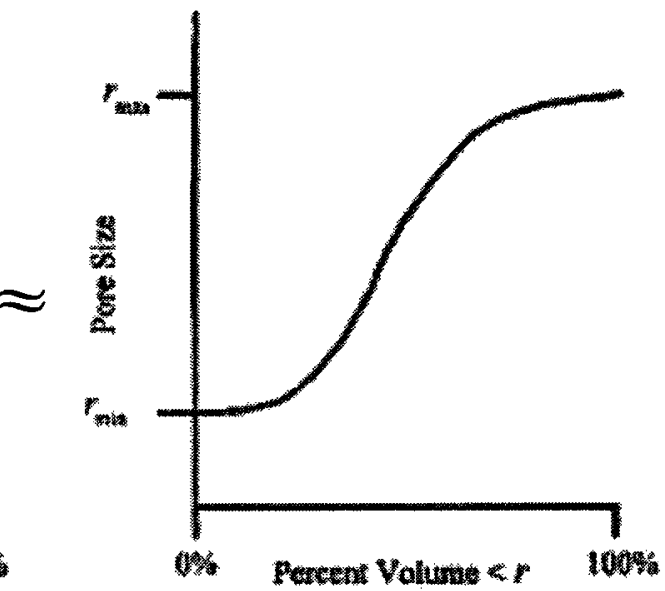

(b)

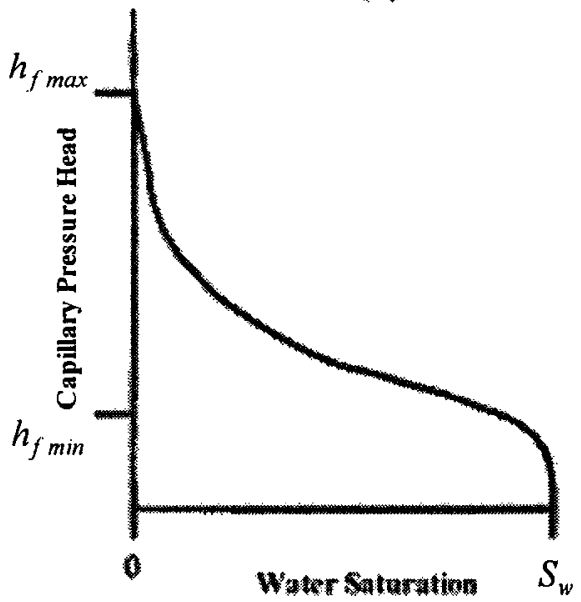

(d)

Figure 2.13: Conceptual Relationship between (a) Particle Size Distribution; (b) Pore Size Distribution; (c) Pore Volume Occupied Versus Pore Filling Pressure; and (d) the Characteristic Wetting Curve (Reproduced from Selker et al. 1999).

Therefore, the plot of filling capillary pressure head of pores and percentage volume of pores with radii less than $r$ is shown in Figure 2.13c. The capillary pressure head at which all pore sizes with radius less than or equal to $r$, corresponding to 
this capillary pressure head, will be filled with water. Keeping that in mind, water saturation corresponding to known capillary pressure head can be calculated and therefore the relationship between water saturation and capillary pressure head can be drawn as shown in Figure 2.13d (Selker et al. 1999).

To determine the previously mentioned two-phase $P_{c} S$ relationship, a Buchner funnel or Tempe cell is used. For both methods, the soil is in contact with a porous plate of a defined non-wetting phase entry pressure. The wetting phase pressure within the porous plate, which must remain less than the equivalent non-wetting phase entry pressure of the porous plate, can be controlled to control the fluid pressure within the soil sample in contact with the plate.

The experiment can be implemented over three stages: (1) wetting (2) draining, and (3) rewetting. For the wetting stage, the pressure of the wetting fluid phase is increased from an initially high suction to zero. For each increment, the system has to come to a steady state condition. In the wetting stage, involving the entry of water and displacement of air, the capillary pressure head is decreased until the maximum water saturation is reached, where capillary pressure head reaches zero. In the drainage stage, involving air entering the soil matrix to replace the water that is draining, the water suction is incrementally increased and the water saturation is decreased until the maximum negative pressure head applied to the water in soil sample is reached. At this point, water saturation is known as the irreducible water saturation. At this saturation, water exists as a thin film on water surfaces and fills small pore throats. Finally, in the rewetting stage, the water suction is decreased incrementally as in stage 1 (wetting stage) and the water saturation increases. By plotting the three stage results, the relationship 
between capillary pressure head and its corresponding water saturation can be developed and is shown in Figure 2.14 (Selker et al. 1999).

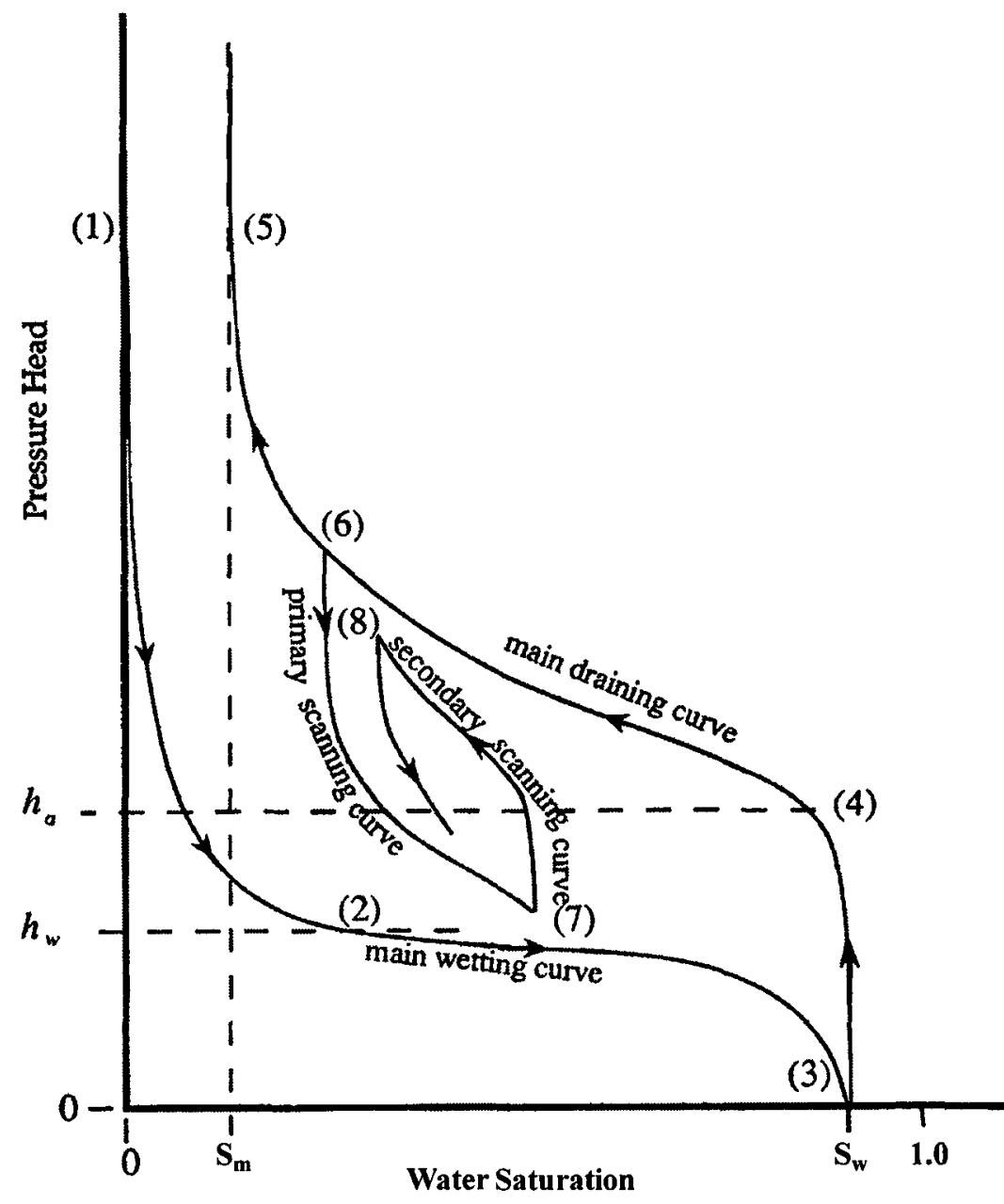

Figure 2.14: The Main Characteristic Curves for Wetting, Draining, and One Set of Scanning Curves (Reproduced from Selker et al. 1999).

\subsection{3.b. $P_{c}-S$ Hysteresis}

It is noticed from Figure 2.14 that the $P_{c}-S$ relationship is nonlinear. It is also noticed that at the lowest and highest values of water saturations, any small change in water saturation is accompanied by large change in capillary pressure head. The relation behaviour at low water saturations shows that the soil never loses all its water. Moreover, 
the relationship between capillary pressure and saturation depends on soil saturation history. It is clear that at intermediate water saturations, every single water saturation value can correspond to a range of different capillary pressure values. Therefore, saturation corresponding to a specific capillary pressure value cannot be estimated in the hysteresis zone unless the saturation path is known (Leverett 1941).

Figure 2.15 illustrates a simplified $P_{c} \neg S$ curve for wetting and draining paths. The wetting process combines stages (1) through (3). The wetting process is governed by pore bodies and therefore it is held up until the pressure is sufficient to fill the largest pores and hence water pressure head continues to increase from $\left(-2 \sigma_{a w} / \rho_{w} g r_{n}\right)$ to $\left(-2 \sigma_{a w} / \rho_{w} g r_{b}\right)$ to zero where maximum water saturation is achieved. In stage (1), no water enters the soil matrix until the water pressure head becomes greater than $\left(-2 \sigma_{a w} / \rho_{w} g r_{b}\right)$. Once this pressure is achieved, the pores will suddenly fill as shown in stage (2). On the other hand, the draining process is illustrated in stages (4) through (7). The system cannot drain significantly until the water pressure becomes sufficiently negative to pull the water out of the pore throats, and therefore the draining process is controlled by pore throats. First the outer pores will drain. Then, as water pressure continues to decrease until it reaches ($2 \sigma_{a w} / \rho_{w} g r_{n}$ ), all but the isolated pores (shown as a horizontal line, i.e., stage (6)) will suddenly drain as air can finally enter the pore bodies. Therefore between pressure heads $\left(-2 \sigma_{a w} / \rho_{w} g r_{n}\right)$ and $\left(-2 \sigma_{a w} / \rho_{w} g r_{b}\right)$, water saturation does not depend only on capillary pressure head, but also on wetting history. As the variability in pores sizes increases, the primary wetting and primary draining curves get closer to each other (Selker et al. 1999). 


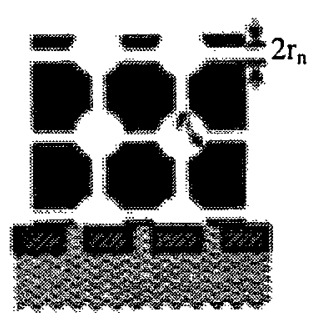

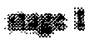

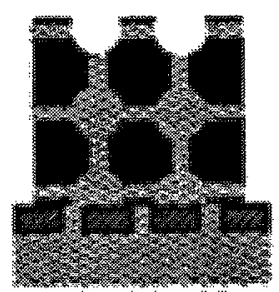

(3)

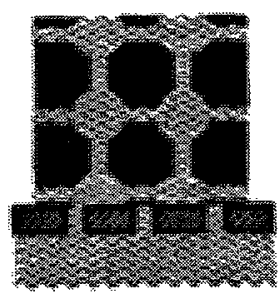

(x)
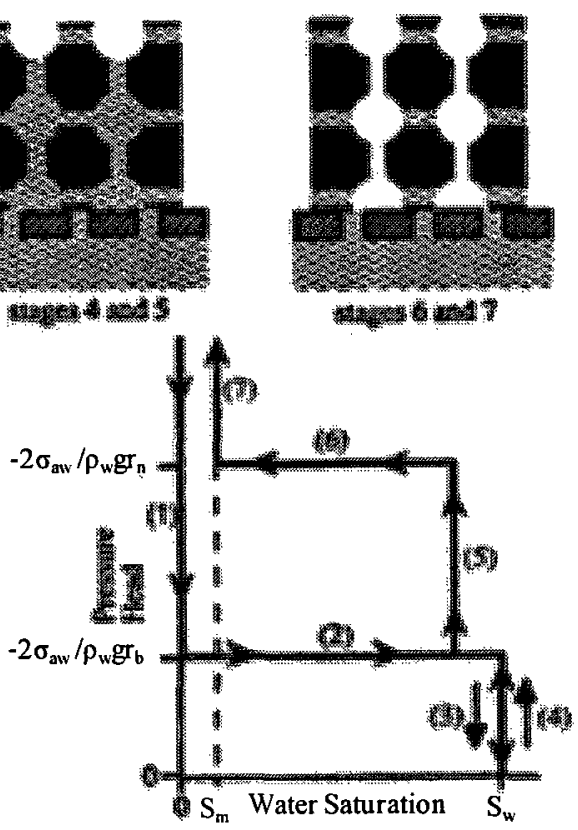

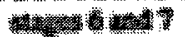

Figure 2.15: Illustration of a Capillary Pressure Head-Saturation Cycles for a Set of Equal-Size Pores with Equal-Size Throats Connecting Them (Reproduced from Selker et al. 1999).

\subsection{3.c. $P_{c} \rightarrow S$ Curves for Certain Types of Soils}

As explained previously, the $P_{c} \neg$ curve, sometimes called the water retention curve or soil moisture curve, depends on several factors; among them are the saturation history of the soil and the grain size distribution of the soil. Water-retention curves for three soils with different grain size distributions are shown in Figure 2.16.

Two factors affect the $P_{c} \rightarrow S$ curve shape. The first factor is the variation in pore sizes and the second is the values of the largest and smallest pore sizes. For a grain size 
distribution that is more uniform, the curve is flatter in its middle part at a height controlled by the pore size. As shown in Figure 2.16, the uniform sand is flatter in its middle part compared to silt loam with broad grain sizes. Also air entry pressure is a function of the pore size. The air entry pressure is the highest in silt loam soil which has the smallest pore size.

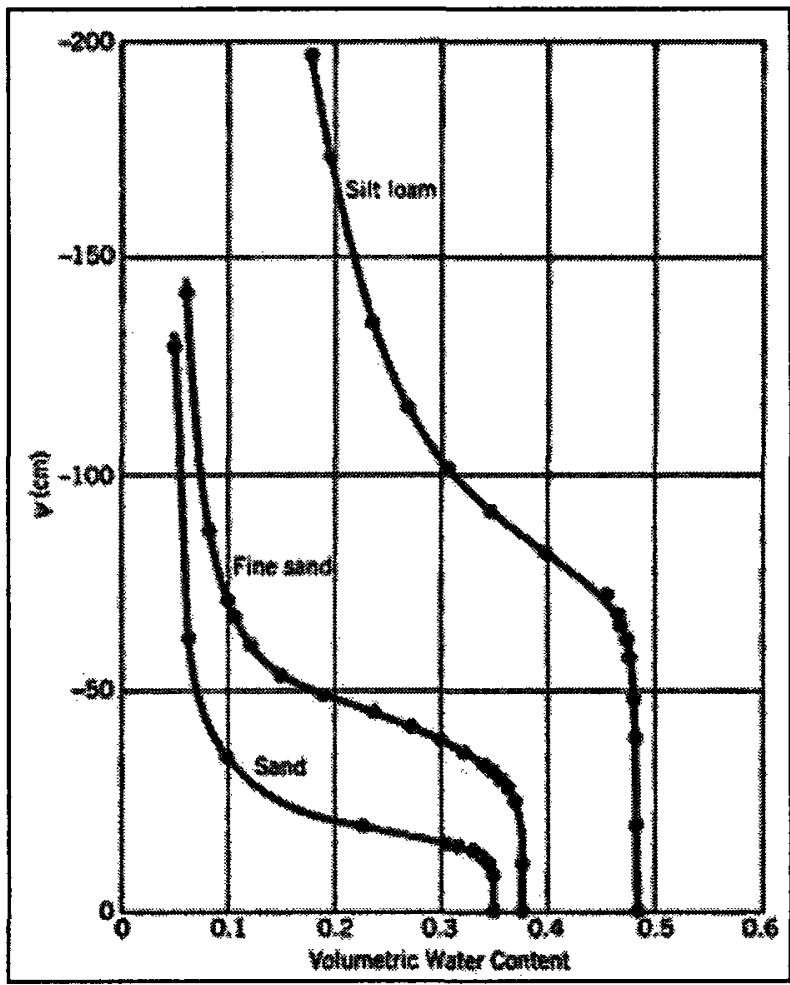

Figure 2.16: Water Retention Curves for Sand, Fine Sand, and Silt Loam (Brooks and Corey 1966).

\subsection{3.d. Common Constitutive Relationships Representing $\boldsymbol{P}_{c} \rightarrow \boldsymbol{S}$ Curve}

Among the most commonly used formulas representing a $P_{c}-S$ relationship are Brooks-Corey and Van Genuchten relationships. These relationships are written in terms of effective wetting phase saturation $(\bar{S})$. 
Brooks-Corey Equation:

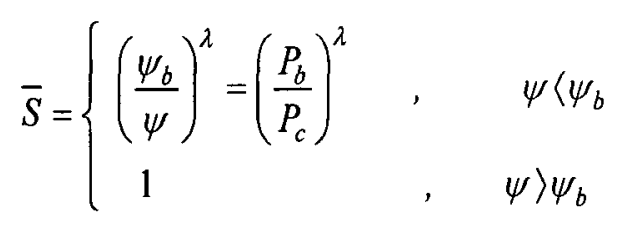

Where:

世. matrix suction head;

$\Psi_{b}$ : air entry pressure head, it is the suction head required to desaturate the largest soil pores;

$\lambda:$ pore size distribution index (control the curve slope); and $P_{b}$ : bubbling pressure.

As the range of grain (pore) sizes for a soil becomes larger (i.e., well graded soil), the curve is sloped (i.e., gradual changes in saturation due to changes in capillary pressure) and $\lambda$ is small. When the range of soil grain (pore) sizes reduces (i.e., uniform soil), the curve is flatter and $\lambda$ is higher. The height of the curve at $\bar{S}=1.0$ and $\Psi=\Psi_{b}$ is controlled by the largest pore size.

\section{Van Genuchten Equation:}

$\bar{S}=\frac{1}{\left(1+\left(\alpha P_{c}\right)^{n}\right)^{m}}$

Where:

$\alpha, n$, and $m$ are fitting parameters;

$\alpha$ : controls the height of the curve (soil/fluids parameter); the curve shifts upwards for finer grained soils or for fluid pairs with higher interfacial tensions; and 
$n$ : controls the slope of the curve; the slope of the curve increases as grain size distribution becomes more graded.

$m=1-\frac{1}{n} \quad$ (Van Genuchten and Nielsen 1985)

\subsection{3.e. $P_{c} \rightarrow S$ Curve Parameters}

Entry Pressure: Leverett (1941) defined the entry pressure as the capillary pressure required to displace water from a water saturated soil. McWhorter and Kueper (1996) stated that DNAPL cannot enter a pore throat unless it exceeds a threshold value of capillary pressure. More simply, Dane et al. (1992) defined the entry pressure of nonwetting fluid phase as the difference in pressure, to the favour of the non-wetting phase, such that it enters the porous media and displaces the wetting fluid phase.

Residual Saturation: Residual saturation is defined as the fluid phase saturation at which the relative permeability of that fluid phase reaches zero. Moreover, it is the fluid phase saturation at which transition from continuous to discontinuous fluid phase

distribution may take place (Helmig 1997). Zalidis et al. (1998) defined the residual NAPL saturation as the NAPL saturation that is either entrapped by the other fluid phases or that drains at very slow rate.

For clarity in the discussion that follows, an entrapped saturation will refer to the non-wetting fluid saturation trapped when displaced by the wetting fluid (e.g., a DNAPL in the saturated zone or an LNAPL above a rising water table) and a residual saturation will refer to the NAPL saturation that remains after the NAPL drains and is replaced by a non-wetting fluid (i.e., NAPL drains in the unsaturated zone and is replaced by air). 
These definitions of residual and entrapped saturations will be used in this thesis. Lenhard et al. (2004) stated that residual NAPL can be discontinuous and/or continuous throughout the pore spaces, but entrapped NAPL is always discontinuous.

Helmig (1997) stated that deriving theoretical expressions for residual and entrapped saturations are difficult to achieve. Instead, the residual and entrapped saturations can be considered as model parameters which are used to fit the experimental data. The estimated value of residual and entrapped saturations differs from case to case because it depends on number of parameters, which will be discussed in later sections.

\subsection{3.f. Two-Phase $P_{c}-S$ Relationship}

For both air/water and air/NAPL two-phase systems, air is considered as the nonwetting fluid (Taber 1968). Leverett (1941), Leverett and Lewis (1941), and Lenhard and Parker (1988) stated that $P_{c}-S$ relationships for two fluid pairs (i.e., NAPL/water and air/NAPL) can be scaled from a measured third fluid pair (i.e., air/water) for the same porous medium.

Scaling Relationships: Under conditions pertaining to rigid porous medium, Parker et al. (1987) developed a scaling technique for $P_{c}-S$ relationships. The scaling technique is very effective since it minimizes the experimental burden in a way that the $P_{c}-S$ relationship is measured experimentally for only one fluid pair then the $P_{c}-S$ relations for other fluid pairs can be predicted. They claimed that their technique is general and can be applied to many retention functions. This technique is based on the assumption that the $P_{c}-S$ relationship is a porous medium-fluid pair property. If the porous medium is rigid such that its structure and its pore size distribution are kept unchanged, then the 
$P_{c} \neg$ relations for different fluid pairs is a function of fluid-dependent scaling factors of the respective fluid pairs. Recall equation (2.20), Laplace equation of capillarity, the relationship between capillary pressure, interfacial tension, and interfacial curvature at the pore scale for any fluid pair $(i, j)$ is as follows:

$P_{i j}=\frac{2 \sigma_{i j}}{R_{i j}}=\rho_{w} g h_{i j}$

Where:

$h_{i j}$ : capillary pressure head on the fluid pair $i / j$ interface.

Parker et al. (1987) and Lenhard and Parker (1987a) stated that, at the REV scale, the pore size distribution can be predicted from the Laplace equation and $P_{c}-S$ data for a monotonic drainage saturation pathway. Conversely, $P_{c}-S$ relationship for a fluid pair can be predicted from pore size distribution and interfacial tension of that pair. Therefore, for the different fluid pairs (i.e., air/water, air/NAPL, and NAPL/water), the $P_{c}-S$ relationships for these fluid pairs could be defined as:

$S_{w}\left(2 \sigma_{a w} / P_{a w}\right)=S(R)$

$S_{w}\left(2 \sigma_{n w} / P_{n w}\right)=S(R)$

$S_{n}\left(2 \sigma_{a n} / P_{a n}\right)=S(R)$

Where:

$\sigma_{a w}:$ air/water interfacial tension;

$\sigma_{a n}:$ air/NAPL interfacial tension;

$P_{a w}$ : capillary pressure on the air/water interface;

$P_{n w}$ : capillary pressure on the NAPL/water interface; 
$P_{a n}$ c capillary pressure on the air/NAPL interface; and

$S(R)$ : capillary pressure-saturation relationship.

Leverett et al. (1942) stated that the scaling factors could be predicted as the ratio of interfacial tensions of the different fluid pairs. Leverett scaling theory refers to the prediction of $P_{c}-S$ relationships for different fluid pairs by using interfacial tension ratios as scaling factors. According to Parker et al. (1987), $P_{c} \rightarrow S$ relations for air/water, air/NAPL, and NAPL/water can be scaled such that a unique capillary pressure-saturation relationship, $S^{*}\left(R^{*}\right)$, for a porous medium can be obtained (equations (2.39.a-c)).

$$
\begin{aligned}
& \bar{S}_{w}\left(\beta_{a w} h_{a w}\right)=S^{*}\left(R^{*}\right) \\
& \bar{S}_{w}\left(\beta_{n w} h_{n w}\right)=S^{*}\left(R^{*}\right) \\
& \bar{S}_{n}\left(\beta_{a n} h_{a n}\right)=S^{*}\left(R^{*}\right)
\end{aligned}
$$

Where:

$\beta_{a w}:$ capillary pressure scaling factor for air/water;

$\beta_{n w}:$ capillary pressure scaling factor for NAPL/water;

$\beta_{a n}:$ capillary pressure scaling factor for air/NAPL;

$h_{a w}:$ capillary pressure head on the air/water interface;

$h_{m w}:$ capillary pressure head on the NAPL/water interface; and

$h_{a n}:$ capillary pressure head on the air/NAPL interface.

At a given wetting phase saturation and from equations (2.39.a-c), the scaling factors, $\beta_{i j}$, and the capillary pressures, $P_{i j}$, as defined in equation (2.37), on air/water, air/NAPL, and NAPL/water interfaces are related as follows: 
$\beta_{a w} P_{a w}=\beta_{n w} P_{n w}=\beta_{a n} P_{a n}$

From equations (2.39):

$P_{a w} / \sigma_{a w}=P_{n w} / \sigma_{n w}=P_{a n} / \sigma_{a n}$

Parker et al. (1987) chose the air/water system as a reference system with $\beta_{a w}=1$. Substituting into equations (2.40) and (2.41) results in:

$$
\begin{aligned}
& \beta_{n w}=\sigma_{a w} / \sigma_{n w} \\
& \beta_{a n}=\sigma_{a w} / \sigma_{a n}
\end{aligned}
$$

This scaling theory is based on the assumption that NAPLs are spreading. The situation of non-spreading NAPLs violates their assumption. However, White et al. (2004) proposed formulas to calculate the scaling coefficients for non-spreading NAPLs. These formulas did not account for saturation hysteresis (i.e., based on a constant contact angle for imbibition and drainage flow paths).

\subsection{3.g. $P_{c}-S$ Relationship in Three-Phase System}

It is difficult to measure fluid phase pressures and saturations in three-phase systems compared to two-phase systems due to the complexity of fluid phase interactions. Therefore, the most commonly used procedure for prediction of three fluid phase system behaviour is to consider the three-phase system as two two-phase systems. This conceptual model was developed by Leverett (1941) and is known as the Leverett assumption.

Leverett's Assumption: Leverett (1941) stated that, for a monotonic displacement process, $P_{c} \neg$ relationships in a three-phase system, air/NAPL/water, can be predicted from a measured pair of $P_{c} \neg$ relationships in two-phase systems, air/NAPL and 
NAPL/water. He based his assumption on certain assumptions; porous medium is strongly water wet, fluid wettabilities follow the order water $>$ NAPL $>$ air, the intermediate fluid phase (i.e., NAPL) forms a continuous layer spreading between the most wetting fluid and the least wetting fluid (i.e., air/water interface does not exist in the system as long as NAPL exists), and the porous medium is rigid. Leverett assumed that the effective total liquid (water + NAPL) saturation is a function of capillary pressure on the air/NAPL interface regardless of the number of liquid phases present in the porous medium. Hence he further stated that capillary pressure-effective total liquid saturation in a three-phase, air/NAPL/water, system can be conceptualized as capillary pressureeffective NAPL saturation in two-phase, air/NAPL, system as shown in equation (2.44). The second part of Leverett assumption is that the effective water saturation in threephase, air/NAPL/water, system is a function of the capillary pressure on the water/NAPL interface. He further stated that capillary pressure-effective water saturation in threephase, air/NAPL/water, system is equivalent to capillary pressure-effective water saturation in two-phase, NAPL/water, system as shown in equation (2.45).

$$
\begin{aligned}
& \bar{S}_{t}^{a n w}\left(h_{a n}^{a n w}\right)=\bar{S}_{n}^{a n}\left(h_{a n}^{a n}\right) \\
& \bar{S}_{w}^{a n w}\left(h_{n w}^{a n w}\right)=\bar{S}_{w}^{n w}\left(h_{n w}^{n w}\right)
\end{aligned}
$$

Where:

$\bar{S}_{t}^{a n w}$ : effective total liquid saturation in air/NAPL/water three-phase system;

$\bar{S}_{w}^{a n w}$ : effective water saturation in air/NAPL/water three-phase system;

$\bar{S}_{w}{ }_{w}$ : effective water saturation in NAPL/water two-phase system; 
$\bar{S}_{n}^{a n}:$ effective NAPL saturation in air/NAPL two-phase system;

$h_{a n}^{a n w}:$ capillary pressure head on the air/NAPL interface in air/NAPL/water three-phase system;

$h_{a n}^{a n}$ : capillary pressure head on the air/NAPL interface in air/NAPL two-phase system; and

$h_{m w}^{m w}$ : capillary pressure head on the NAPL/water interface in NAPL/water two-phase system.

Although this assumption is widely used in multiphase flow modeling, very little effort has been exerted to verify the validity of this assumption. Lenhard and Parker (1988) verified the applicability of this assumption for strongly water-wet porous media and spreading NAPLs.

Hofstee et al. (1997) argued that Leverett's assumption applies only for a part of the $P_{c} \rightarrow S$ curve of the air/NAPL two-phase system. At lower NAPL saturations, discontinuity in NAPL may occur in a three-phase system. NAPL saturation at this point is referred to as the critical NAPL saturation. Therefore, to correctly model the system, the constitutive theory has to be modified in order to account for that. Zalidis et al. (1998) showed that at a capillary pressure value on air/NAPL interface, as initial water saturation increases in a three-phase system, NAPL saturation decreases with the same magnitude, resulting in a constant total liquid saturation, such that equation (2.44) can be applied. However, there is a value for initial water saturation after which the total liquid saturation in air/NAPL/water three-phase system does not equal to the NAPL saturation in air/NAPL two-phase system. This value is referred to as critical initial water saturation. 
In air/NAPL/water three-phase system, at critical initial water saturation, NAPL saturation reaches its minimum value. It is shown in Figure 2.17 that increasing initial water saturation above the critical initial water saturation, results in a corresponding increase in total liquid saturation although the NAPL saturation does not decrease. Therefore, equation (2.44) no longer applies. Zalidis et al. (1998) suggested that further studies are required to determine the mechanisms governing total liquid saturations at higher initial water saturations (higher than critical initial water saturation for three-phase system) and therefore inapplicability of Leverett's assumption.

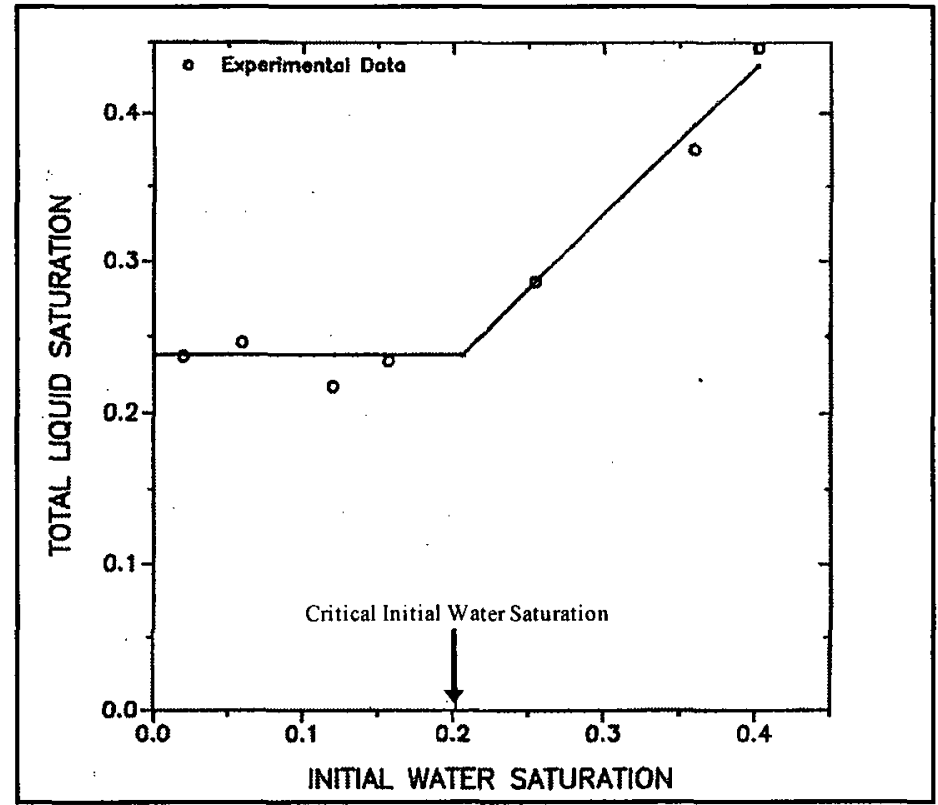

Figure 2.17: Influence of Initial Water Saturation on Total Liquid Saturation (Modified from Zalidis et al. 1998).

\subsection{4. $k_{r}-S$ Relationship}

\subsection{4.a. Effective Permeability}

Effective permeability of a fluid phase in a porous medium depends on the porous medium properties (i.e., pore size distribution, compaction, mechanical alteration of structure, leaching, and clay swelling), medium permeability to a single fluid, porous 
medium wettability, and the fluid phase saturation (Bear 1988, Corey 1994, Helmig 1997). However, Collins (1961) stated that for unconsolidated materials such as sand, compaction does not largely affect the porous medium porosity or structure and hence permeability is not affected. On the other hand, mechanical alteration of soil structure due to flow of viscous fluids may change the porous medium permeability. Also, permeability depends on the direction along which it is measured.

\subsection{4.b. Relative Permeability}

Relative permeability is the ratio of the effective permeability of a fluid phase to the medium permeability to a single fluid phase. Relative permeability of a fluid phase depends on the porous medium wettability for the flowing fluids and the nature of the porous medium (preferential pathways of the fluid of concern) as well as the fluid saturation (Bear 1988). Unlike relative permeability of gas and NAPL, which depends on all fluid phase saturations, the relative permeability of the most wetting phase, water, in a water wet porous medium depends only on water saturation (Leverett and Lewis 1941). As water saturation approaches its irreducible saturation, water may not be continuous providing a relative permeability of water close to zero. On the other hand, NAPL relative permeability increases as water saturation increases for the same NAPL saturation. This happens because NAPL is shifted towards the central portion of pores while gas leaves the system and therefore NAPL moves with less resistance (Leverett and Lewis 1941). 


\subsection{4.c. Common Constitutive Relationships Representing Non-hysteretic Air/Water} $k_{r}-S$ Relationship

In the unsaturated zone, where two or more flowing fluid phases are present, the concept of relative permeability is very useful in defining the relationship between hydraulic conductivity and capillary pressure head (Bear 1988, Selker et al. 1999).

$k_{r}=\frac{K(\theta)}{K}$

Where:

$k_{r}$ : relative permeability $\left(0 \leq k_{r} \leq 1\right)$;

$K$ : saturated hydraulic conductivity; and

$K(\theta)$ : unsaturated hydraulic conductivity $(0 \leq K(\theta) \leq K)$.

\section{Brooks-Corey and Burdine:}

- Relative permeability for the wetting fluid (water)

$$
k_{n w}=\left(\bar{S}_{w}\right)^{\frac{2+3 \lambda}{\lambda}}
$$

- Relative permeability for non-wetting fluid (air)

$$
k_{r a}=\left(1-\bar{S}_{w}\right)^{2}\left(1-\bar{S}_{w} \frac{2+\lambda}{\lambda}\right)
$$

For a given effective water saturation, an increase in $\lambda$ reflects a more uniform grain size distribution and hence relative permeability increases for the water and decreases for the air. 


\section{Van Genuchten and Mualem:}

- Relative permeability for the wetting fluid (i.e., water) in air/water two-phase system

$$
k_{r w}=\bar{S}_{w}^{1 / 2}\left(1-\left(1-\bar{S}_{w}^{1 / m}\right)^{m}\right)^{2}
$$

- Relative permeability for the non-wetting fluid (air)

$$
k_{r a}=\left(1-\bar{S}_{w}\right)^{1 / 2}\left(1-\bar{S}_{w}^{1 / m}\right)^{2 m}
$$

For a given water saturation, as $m$ increases (more uniform grain size distribution), relative permeability increases for the water and hence decreases for the air.

Gardner (1955) stated that relative permeability of the water decreases rapidly with little decrease in water saturation. This is because the larger pores have the highest contribution to the relative permeability and they are the first pores to drain (e.g., when water saturation is reduced by $50 \%$ of its value, relative permeability becomes much lower than $50 \%$ of its value as shown in Figure 2.18. In other words, $k_{r w}$ drops very quickly in response to a decline in water saturation since:

1. Larger pores empty first and these have the least resistance to flow;

2. Flow paths increase in length as water saturation decreases; and

3. Cross sectional area for flow decreases. 


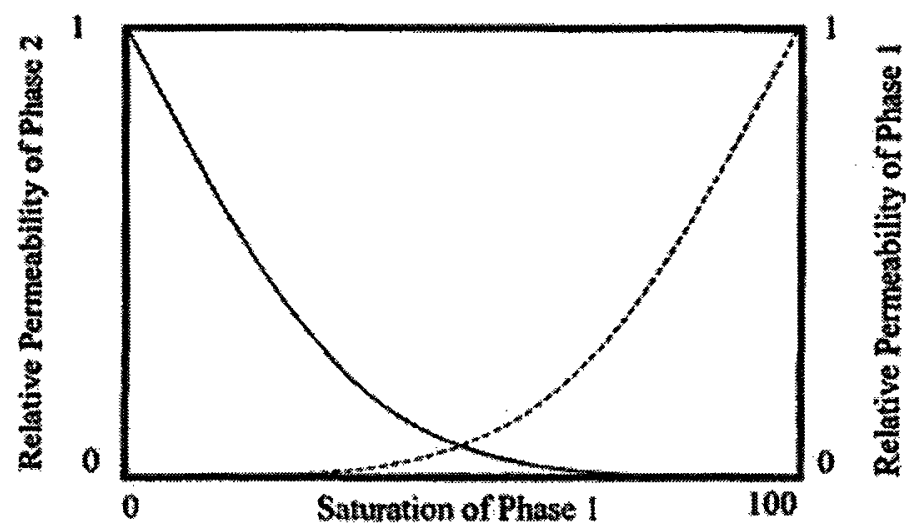

Figure 2.18: Relative Permeability of Water and Air in Porous Media (Selker et al. 1999).

\subsection{4.d. Two-Phase Hysteretic Air/Water $k_{r}-S$ Relationship:}

Lenhard and Parker (1987b) stated that the analysis of multiphase flow in the vadose zone requires knowledge of two systems; air/water system and air/NAPL/water system. The first system represents fluid phases before a NAPL spill occurs while the second case represents a situation after a NAPL spill takes place. Hysteresis in relative permeability is assumed to occur due to non-wetting phase entrapment. They stated that, at a given water saturation, relative permeability of water is higher for imbibing pathway than drainage pathway. The reason is that entrapped air bubbles tend to displace the water to larger pores with a higher conductance to the water. They developed $k_{r}-S$ relationships for water and air. The water or air relative permeability is a function of water and air saturations and can be found in Lenhard and Parker (1987b).

\subsection{4.e. Three-Phase, Air/NAPL/Water, Hysteretic $k_{r}-S$ Relationships:}

In their development of $k_{r}-S$ relationships for air, water, and NAPL, Lenhard and Parker (1987b) assumed that the water is the most wetting fluid, followed by NAPL, and then by air. Where water occupies the smallest pores and air occupies the largest pores. 
Air could be entrapped in the water or NAPL and NAPL could be entrapped in the water. They developed $k_{r}-S$ relationships for water, NAPL, and air at different water and total liquid saturation pathways. These relations are too numerous to state here and can be found in Lenhard and Parker (1987b).

\subsection{Pore Scale Models}

Many models developed to describe the physical processes occurring at the pore scale are aimed at creating a clear understanding of these processes before moving to the REV scale. Some of these models show the development of capillary rise and capillary rise hysteresis; others have been developed to replicate or verify the constitutive relationships.

\subsubsection{Describing $P_{c}-S-k_{r}$ Relationships}

\subsection{1.a. Capillary Tube Bundle}

The capillary tube bundle model is a relatively simple model that simulates the soil as a collection of capillary tubes of different sizes. This concept allows simple analytical formulas to be derived such as relative permeability as well as infiltration rates. However, the main problem with this concept is that it neglects the interconnectedness of pore space and it also neglects the pore bodies and pore throats which form the pore space in real soil. In addition, it cannot account for saturation hysteresis or fluid entrapment. According to Corey's (1994) definition of a porous medium, the capillary tube bundle does not represent a porous medium due to its lack of interconnectedness. 


\subsection{1.b. Network Models}

The first development of network models was based on the idea of representing the pore space as an interconnected network of capillary tubes with radii representing the dimensions of the porous medium pores. The microscopic physical processes are simulated within the pore network to model pore by pore displacement of immiscible fluids. Using the network models, the gap between pore scale and REV scale can be bridged since at the pore scale level, all the fluid to fluid interfaces can be delineated and therefore the macroscopic variables such as fluid saturations and interfacial areas of fluid interfaces per volume can be calculated (Reeves and Celia 1996).

In order to achieve this task, the network models have to be well defined and highly structured. The first development of network models was composed of pore elements with simple geometric shapes arranged in a regular lattice as shown in Figure 2.19. Then complexity to network models was introduced to better simulate a real porous medium. This complexity involved using spherical pore bodies connected by varying radii pore throats as shown in Figure 2.20. With the pore network models, drainage and imbibition pathways of the $P_{c}-S$ curve can be constructed (Reeves and Celia 1996). 


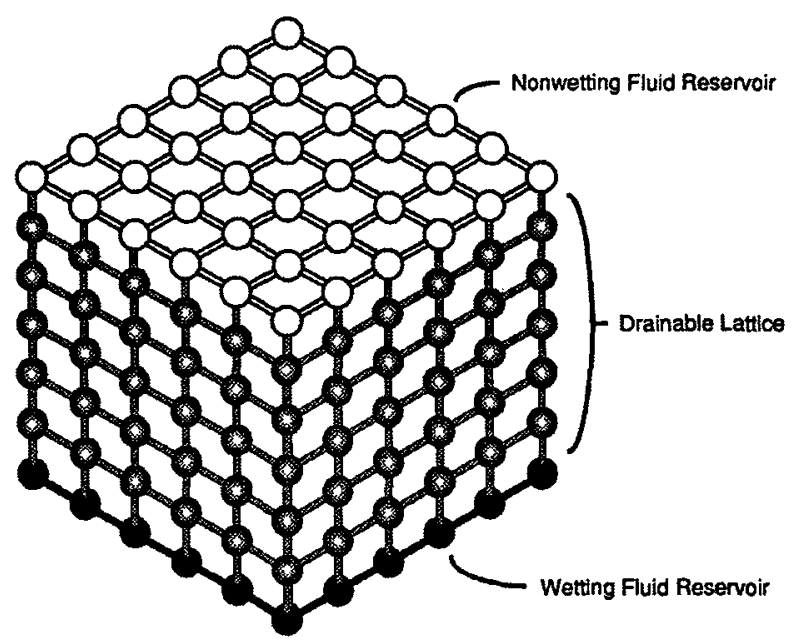

Figure 2.19: Cubic Pore Network (Reeves and Celia 1996).

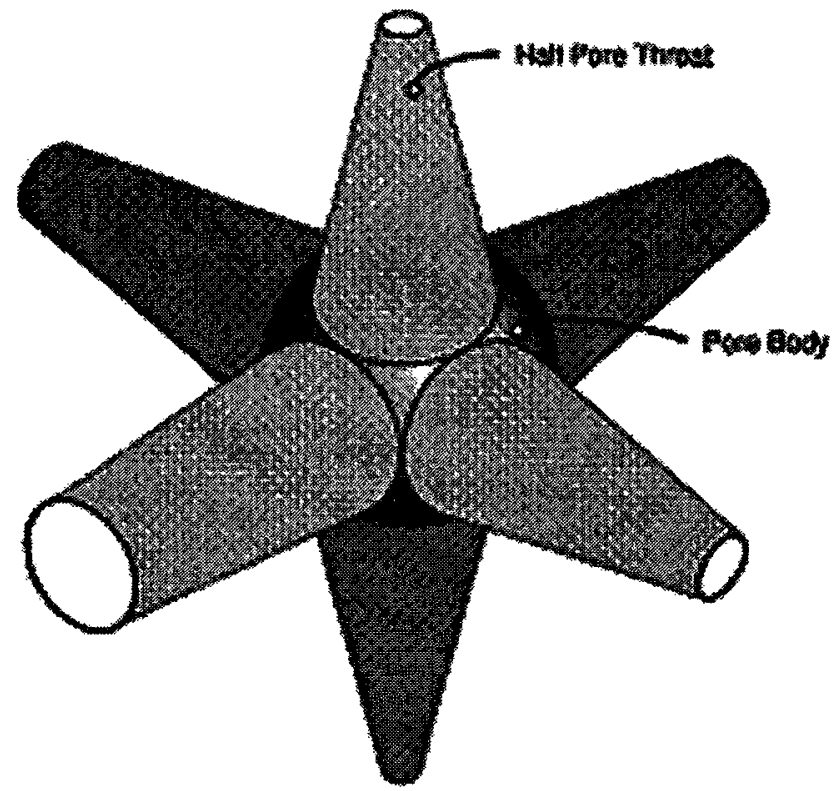

Figure 2.20: Typical Spherical Pore Body with its Associated Bi-Conical Pore Throats (Reeves and Celia 1996).

The network is defined by the domain size and distribution of pore bodies given a probability distribution for pore body radii. Each pore body is connected to its nearest pore body via a pore throat. The number of throats that meet at a pore body is defined as the coordination number. Hence, the distribution of pore throat radii can be assigned 
stochastically and then each pore throat length can be calculated (Lowry and Miller 1995).

\subsubsection{Describing $P C \neg S$ Hysteresis}

\subsection{2.a. Ink Bottle Effect}

The ink bottle effect or concept is a simple model showing the difference between capillary rise in an irregular capillary tube for filling and emptying processes. As shown in Figure 2.21, a capillary tube filling is governed by pore body radius " $r_{b}$ " while emptying is governed by pore throat (neck) radius " $r_{n}$ ". Filling pressure head $\left(h_{f}\right)$ is less than emptying pressure head $\left(h_{e}\right)$ (Rose 1966).

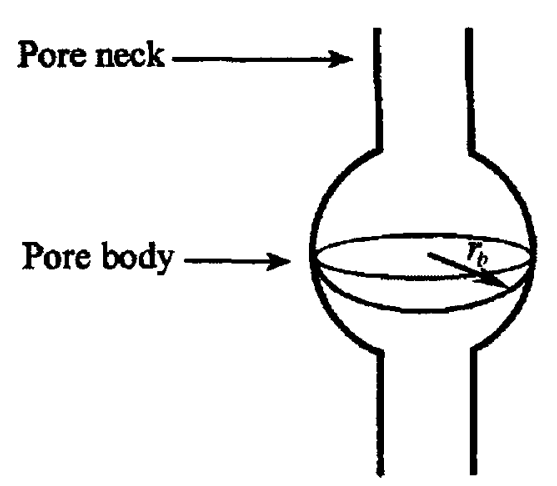

Filling

$$
h_{f}=\frac{2 \sigma}{\rho g r_{b}}
$$

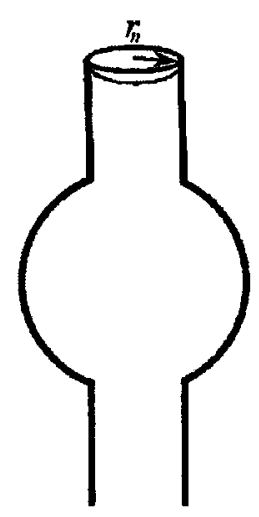

Emptying

$$
h_{e}=\frac{2 \sigma}{\rho g r_{n}}
$$

Figure 2.21: A Schematic Drawing of the Characteristic Radii for a Pore and the Characteristic Filling and Emptying Pressures of that Pore (Rose 1966).

\subsection{2.b. Rain Drop Effect}

This very simple model shows the difference between the wetting and drainage behaviour of contact angle (Figure 2.10). Although some authors correctly claim that there is no difference between advancing and receding contact angles based on 
thermodynamics and they attribute this to the surface defects, contact angle hysteresis is often given as a contributing factor for saturation hysteresis (Selker et al. 1999).

\subsection{2.c. Independent Domain Model}

Selker et al. (1999) presented the assumptions upon which the independent domain model is based on, as follows:

1. The soil is considered as a network of pore bodies connected by pore throats.

2. Each pore size corresponds to a pressure change independently and is not affected by the number or distribution of pore throats connected to it. Hence, the independent domain model does not account for connectivity between pores.

3. Filling is governed by pore bodies since these need to be filled before pore bodies and pore throats above can be filled. This is shown in Figure 2.21.

4. Draining is governed by pore throats since once the air entry pressure of a pore throat is exceeded, the pore throat and pore body will drain until a pore throat of narrower diameter is reached.

5. Independent domain model assumption breaks down for the isolated pores since they cannot drain regardless of the pressure exerted on the sample.

By considering the pore body and pore throat radii " $r_{b}$ " and " $r_{n}$ ", the porous medium could be described as a group of pore bodies and throats connected to each other. The fluid pair considered here is air/water but the same applies to any fluid pair. The filling pressure head $\left(h_{f}\right)$ can be calculated corresponding to the radii of the pore bodies $\left(r_{b}\right)$ and the draining pressure head $\left(h_{e}\right)$ can be calculated corresponding to the radii of the pore throats $\left(r_{n}\right)$ : 


$$
\begin{aligned}
& h_{f}=\frac{2 \sigma_{a w}}{\rho_{w} g r_{b}} \\
& h_{e}=\frac{2 \sigma_{a w}}{\rho_{w} g r_{n}}
\end{aligned}
$$

Based on the above assumptions, a porous medium is described as a collection of independent pores each having a characteristic filling pressure head $\left(h_{f}\right)$ controlled by pore body radii and draining pressure head $\left(h_{e}\right)$ controlled by pore throat radii. Hence, porous medium will have a range of magnitudes for $\left(h_{f}\right)$ and $\left(h_{e}\right)$, corresponding to pore body and pore throat radii respectively. The distribution of filling and draining pressure heads can be described by a joint probability density function as shown in Figure 2.22.

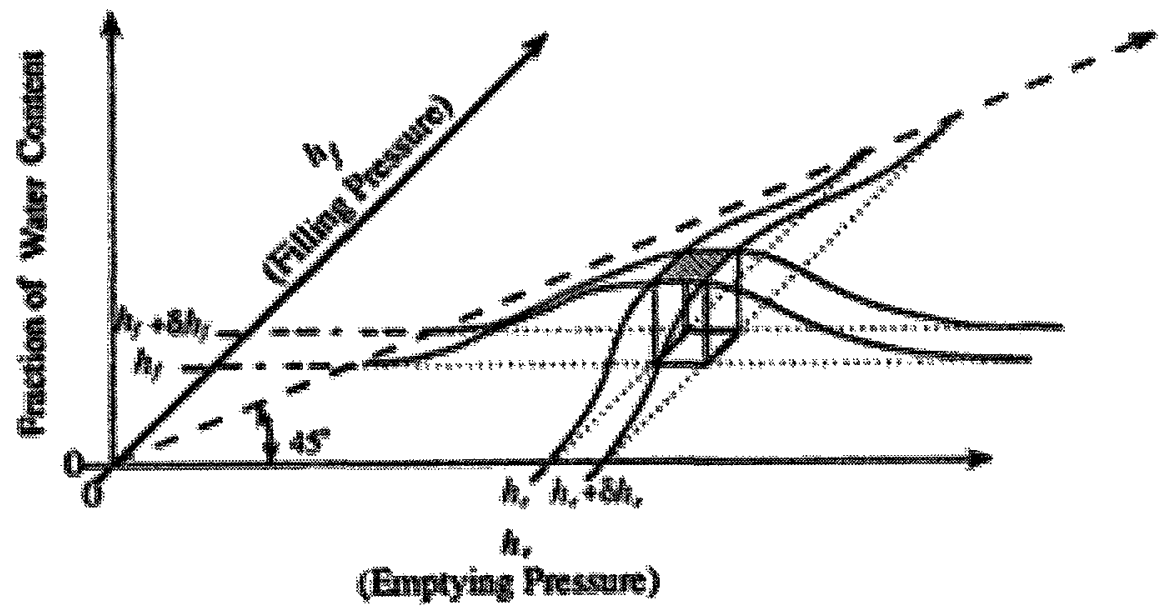

Figure 2.22: Illustration of the Probability Density Function Water Content as a Function of Filling and Draining Pressures (Selker et al. 1999).

The volume under the dashed surface in Figure 2.22 represents the fraction of the void space consisting of pore bodies which fill between pressures $h_{f}$ and $h_{f}+\delta h_{f}$ that are connected to pore throats which drain between pressures $h_{e}$ and $h_{e}+\delta h_{e}$. The total 
volume under the surface should be equal to unity and reflects the porous medium porosity.

Therefore, for a given pore body of filling pressure head, $h_{f}$, it could be connected to a range of pore throats with draining pressure head, $h_{e}$, starting from $h_{e}=h_{f}$ until $h_{e m a x}$. The reason $h_{f}$ is less than or equal to $h_{e}$ is because pore throats with $h_{e}$ less than $h_{f}$ are already considered as pore bodies. Therefore, the region of the surface will be limited to the right of the line with $45^{\circ}$. Hence, Figure 2.22 could be reduced to Figure 2.23 . By examining Figure 2.23, wetting, draining, and rewetting stages could be illustrated as was shown in Figure 2.14.

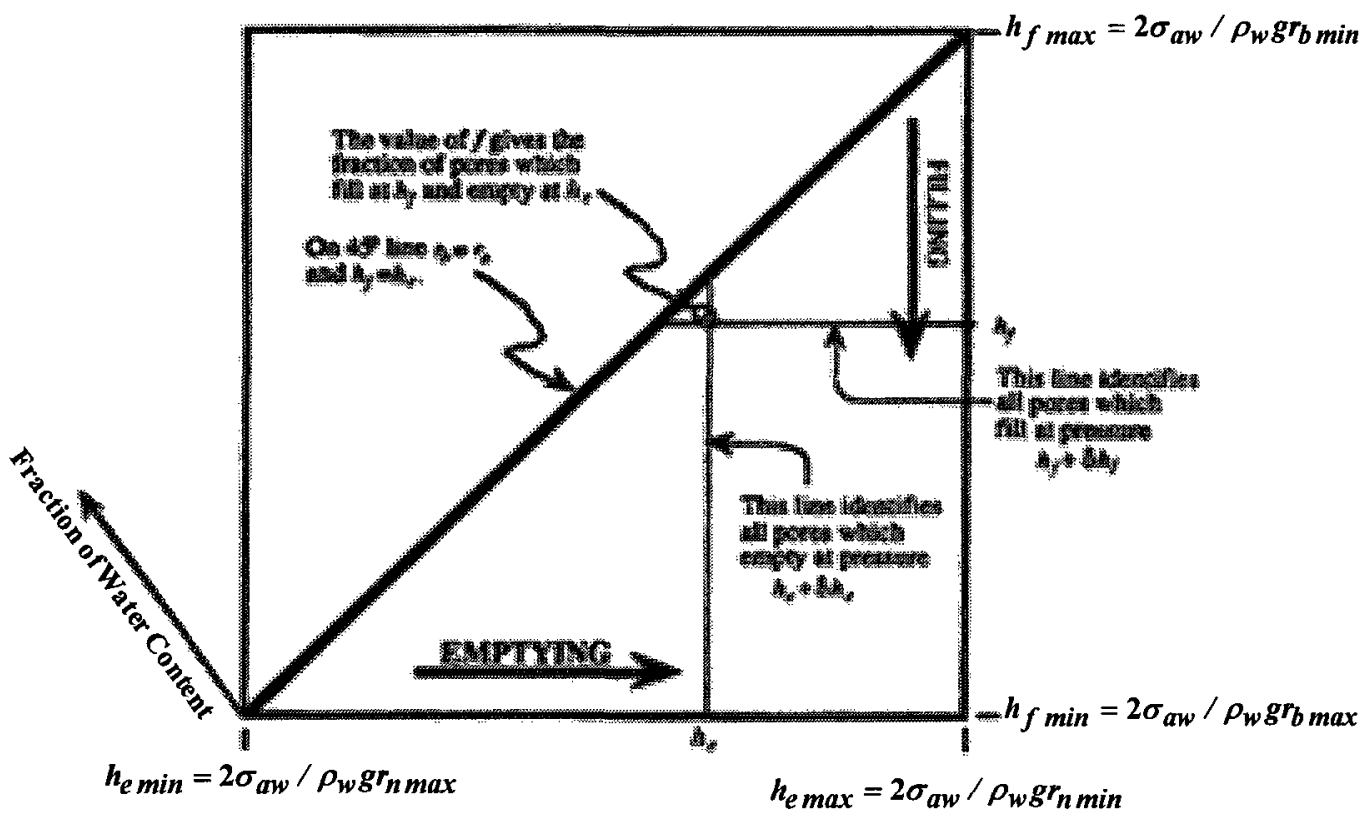

Figure 2.23: Features of the Joint Density Function Given in Figure 2.22 (Selker et al. 1999).

Main Wetting Curve: The main wetting curve is defined such that the capillary pressure head moves from smallest pore bodies with filling pressure head $\left(h_{f \max }=2 \sigma_{a w} / \rho_{w} g r_{b \min }\right)$ to the largest pore bodies with filling pressure head $\left(h_{\text {fmin }}=2 \sigma_{a w} / \rho_{w} g r_{b \max }\right)$ as shown in 
Figure 2.23. Figure 2.24a illustrates filling of pore bodies and throats from point (1) to point (2) as given in Figure 2.14. The curve starts at dry conditions $\left(h_{f}=h_{f \max }=2 \sigma_{a w} / \rho_{w} g r_{b \min }\right)$. Then the system continues filling until capillary pressure head equals to $\left(h_{f}=h_{f \min }=2 \sigma_{a w} / \rho_{w} g r_{b \max }\right)$.

At this point, the system is saturated and that defines point (3) in Figure 2.14 as shown in Figure 2.24.b. In other words, the water will be drawn up into the smaller pore throats and pore bodies. The number of the water-filled pores will be governed by the pore bodies since the water must fill these to get to the next pores until saturation is achieved (entrapment is ignored here in this explanation). Water content can be calculated using the joint density function $\theta(h)=\int_{a}^{h^{h}} \int_{0} f\left(h_{e}, h_{f}\right) d h_{e} h_{f}$.

Main Draining Curve: The main draining curve is defined such that the capillary pressure head moves from the largest pore throat with draining pressure head $\left(h_{e m i n}=2 \sigma_{a w} / \rho_{w} g r_{\text {max }}\right)$ to the smallest pore throats with draining pressure head $\left(h_{e m a x}=2 \sigma_{a w} / \rho_{w} g r_{n \min }\right)$ as shown in Figure 2.23.

Figure 2.24.c illustrates draining of pore throats and connected pore bodies from point (3) to point (6) of Figure 2.14. If draining continues, then capillary pressure head continues increasing until it equals to $\left(h_{e \max }=2 \sigma_{a w} / \rho_{w} g r_{n \min }\right)$, which corresponds to point (5) in Figure 2.14. In other words, the water will drain from the largest pore throats first as the small pore throats remain filled because their air entry pressure head has to be exceeded first, and the water content can be calculated using the joint density function as follows: 
$\theta(h)=1-\int_{0}^{h_{f}} \int_{0} f\left(h_{e}, h_{f}\right) d h_{f} d h_{e}$
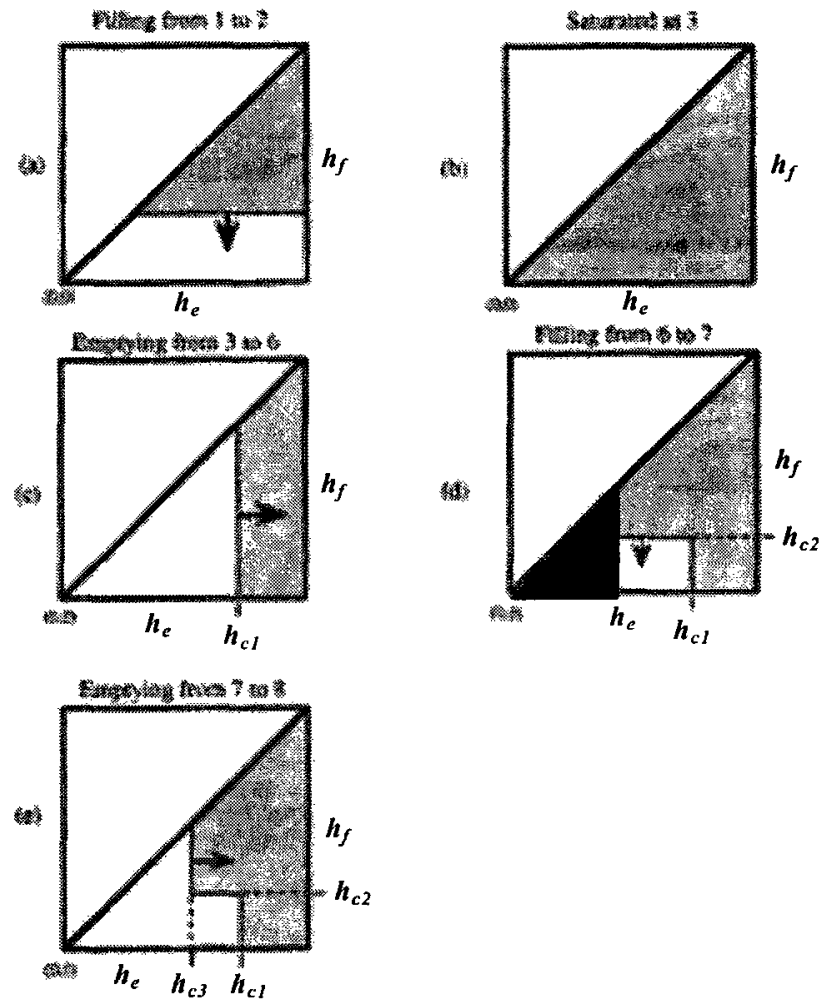

Figure 2.24: Mapping of the Water-Filled Pores through the Sequence of Wetting and Draining Characteristic Curves Presented in Figure 2.14 (Selker et al. 1999).

Rewetting Curve (Primary Scanning Curve): A scanning curve is described by movement from point (6) to point (7) as given in Figure 2.14. The pore bodies and their connected pore throats with filling pressure less than $h_{f}=h_{\mathrm{c} 2}$ will fill (as shown in Figure 2.24.d by the triangle defined between $h_{f}=h_{c 2}, h_{e}=h_{c 1}$, and the $45^{\circ}$ line). On the other hand, pore bodies and their connected pore throats defined by $h_{e}>h_{\mathrm{c} 1}$ will remain saturated since their capillary drainage pressure head is not reached, and the water content can be calculated using the joint density function as follows: 
$\theta(h)=1-\int_{0}^{h_{\mathrm{cl}}} \int_{0}^{h_{e}} f\left(h_{e}, h_{f}\right) d h_{f} h_{e}+\int_{h_{c 1}}^{h} \int_{f}^{h_{c 1}} f\left(h_{e}, h_{f}\right) d h_{e} d h_{f}$.

Redraining Curve (Secondary Scanning Curve): A secondary scanning curve is described as movement from point (7) to point (8) as given in Figure 2.14. The pore throats and their connected pore bodies will drain until the draining pressure head is reached at point (8) which correspond to $h_{e}=h_{\mathrm{c} 3}$ in Figure 2.24.e. At the same capillary pressure head $\left(h_{c}\right)$ a different set of pores are filled or drained depending on the saturation path. Therefore, the $P_{c} \rightarrow S$ relationship should not be defined by a curve but by an area. Instead, the boundary of this area lies between the primary wetting and primary draining curves where all the scanning curves will lay in between.

\subsubsection{Describing NAPL Entrapment Mechanisms}

\subsection{3.a. Glass Micro Model}

In single pore-throat model experiments, Wardlaw (1982) examined the effects of varying medium geometry, fluid/fluid properties such as viscosity and interfacial tension, and fluid/medium interaction such as wettability on NAPL entrapment. In examining the effect of pore-throat aspect ratio on NAPL entrapment, Wardlaw used a glass micro model with elements of different pore-throat aspect ratios. For a water-wet medium, NAPL and air are entrapped in water/NAPL and water/air fluid pairs, respectively, for high pore-throat aspect ratios. Snap-off is the main mechanism for high pore-throat aspect ratio. But for intermediate wettability, entrapment was found to be less. The reason behind that is that the curvature of a fluid/fluid interface, which is a function of the 
contact angle and the geometry of the surface contacted, determines the potential for entrapment.

Wardlaw (1982) showed that until the midpoint of the pore (location of maximum interfacial area), while the interface is moving from the entry throat, the interfacial area increases and the process is reversible, such that if the pressure decreases, the interface recedes. However, beyond that point, the advance process becomes irreversible, which is known as Haine's jump or Rheon, and entrapment occurs at this stage depending on other factors such as wettability, viscosity, and interfacial tension.

Fluid interfaces tend to be at minimum surface area. When a NAPL blob moves in a throat, it takes the throat shape (cylinder). It will break up and split into two spheres when the cylinder length is larger than its circumference. However, in Wardlaw's experiments in the single pore-throat model, it was shown that varying throat length with respect to its diameter was not an important parameter affecting entrapment of nonwetting phase.

\subsection{3.b. Microscopic Visualization of NAPL Entrapment in Glass Bead Soil}

In an effort to show the NAPL behaviour at the pore scale, Schwille (1988) used a $10 \mathrm{~cm} \times 20 \mathrm{~cm} \times 0.3 \mathrm{~cm}$ glass box filled with water-wet glass beads of 0.49 to $1.23 \mathrm{~mm}$ radii. The tetrachloroethylene were dyed with a red dye and water with a blue dye for better distinguishing between water, NAPL and beads since all of them are primarily transparent. The NAPL and water forms were viewed under a microscope in two-phase systems. Although Schwille's work was very simple in terms of homogeneity and wettability conditions, it resulted in illustrations of water wetting the glass beads in air/water two-phase system (Figure 2.25), NAPL wetting the glass beads in air/NAPL 
two-phase system (Figure 2.26), and distrilbution of NAPL in a water-wet NAPL/water two-phase system (Figure 2.27).

Figure 2.25: Water Wetting Glass Beads Surfaces in Air/Water Two-Phase System (Schwille 1988).

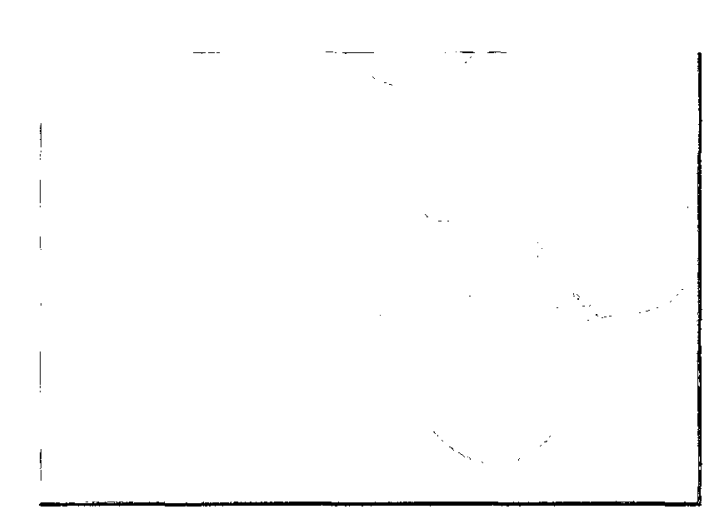

Figure 2.26: NAPL Wetting Glass Beads Surfaces in Air/NAPL Two-Phase System (Schwille 1988).

Figure 2.27: Water Replacing NAPL on Glass Beads Surfaces which Were Initially Saturated by NAPL in NAPL/Water Two-Phase System (Schwille 1988). 


\subsection{Porous Medium Wettability}

Craig (1971), Corey (1977) and Bradfrod and Leij (1995a) defined the porous medium wettability as the tendency of one fluid phase, in the presence of other immiscible fluids, to spread or adhere to the solid surface. These fluids are distributed on soil grains to achieve the lowest energy state as defined by the porous medium wettability.

According to Bear (1988), Corey (1994), Bradford et al. (1999), and Meinardus et al. (2002), in oil reservoirs, the soil is either-water wet, NAPL-wet, or mixed-wet (partially wet by water and NAPL). The same applies in the unsaturated zone after a NAPL spill. The governing flow equations, the NAPL/water distribution in the soil matrix, and the effectiveness of site remediation may differ if the soil is NAPL-wet in comparison to a soil that is water-wet or mixed-wet. Figure 2.28 illustrates fluid (NAPL and water) distribution in a water-wet porous medium and in a NAPL-wet porous medium for a two-phase, NAPL/water system.

A porous medium composed of water-wet and NAPL-wet particles is frequently described by two terms; mixed wettability or fractional wettability. When the porous medium wettability is a function of the particles sizes, it is referred as a mixed wettability porous medium. A fractional wettability porous medium is composed of particles with different surface wetting properties regardless of the particle size ( $\mathrm{O}^{\prime}$ Carroll et al. 2005). In this research, fractional wettability is used to describe a porous medium that contains particles with different surface wetting properties. 


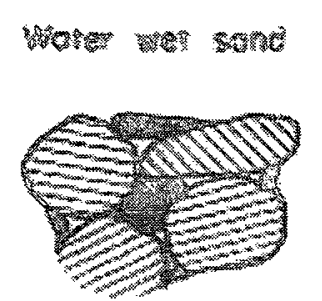

(1)

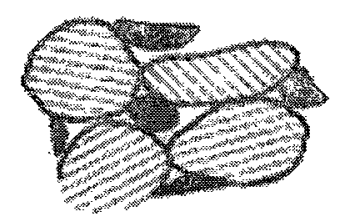

.

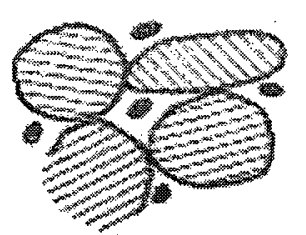

(a)

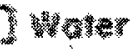

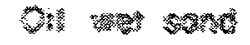

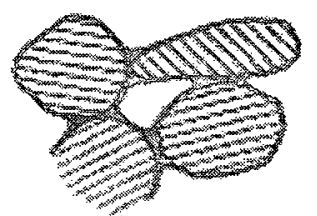

(a)

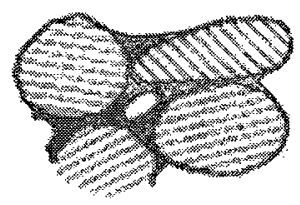

7

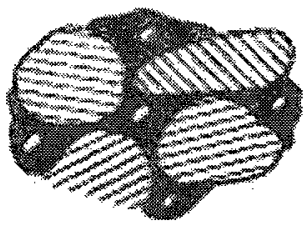

to

(4)

Figure 2.28: Possible Fluid Saturation States under Different Wettability Conditions (Bear 1988).

Bear (1988) and Corey (1994) stated that, for any two (or more) fluid phases in contact with a solid surface, the fluid which spreads on the solid surface is known as the wetting fluid while the other(s) is known as non-wetting fluid(s). Helmig (1997) stated that the non-wetting fluid phase is not in contact with the solid matrix, while the wetting phase is always in contact with the solid matrix. Bear (1988), Corey (1994) and Helmig (1997) stated that the universal measure of wettability is the contact angle and it is measured (or calculated) when the balance between the interfacial tension forces is achieved. Figure 2.29 shows NAPL and water which are in contact with a solid surface and the corresponding interfacial tensions on the interfaces. Young's equation studies the balance of this system as shown in Figure 2.29. 


\section{Non-Wetting Fluid}

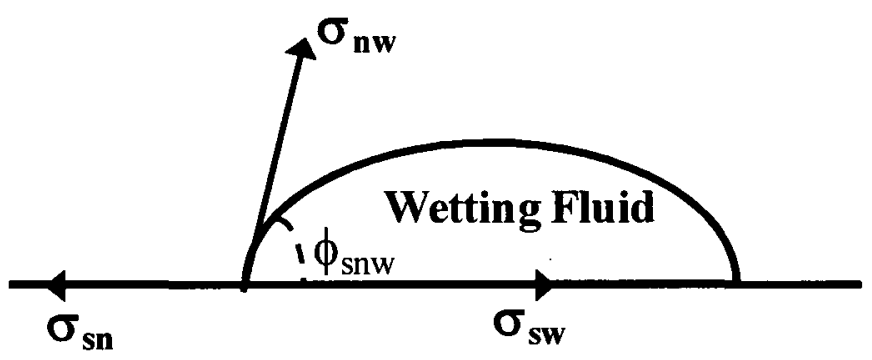

Solid

Figure 2.29: Hypothetical Distribution of Fluids and Interfacial Tensions at a Solid (s)-Wetting Fluid (w)-Non-Wetting Fluid (n) Contact Line (Modified from Bradford and Leij 1995a).

$\sigma_{n w} \cos \left(\phi_{s n w}\right)=\sigma_{s n}-\sigma_{s w}$

$\cos \left(\phi_{s n w}\right)=\left(\sigma_{s n}-\sigma_{s w}\right), \sigma_{n w}$

Where:

$\sigma_{s n}:$ interfacial tension between solid and NAPL;

$\sigma_{s w}:$ interfacial tension between solid and water;

$\phi_{s n w}$ : angle between NAPL/water interface and the solid/water interface, measured through the water;

$\cos \left(\phi_{s n w}\right):$ ratio between the energy released when a unit area of solid/water interface is formed to the energy required to form a unit area of water/NAPL interface; and $\sigma_{n w} \cos \left(\phi_{s n w}\right):$ adhesion tension.

The system is said to be strongly water-wet, when the contact angle that is measured through the water approaches $0^{\circ}$. On the other hand, the system is said to be 
strongly NAPL-wet, when the contact angle that is measured through the water approaches $180^{\circ}$ (O'Carroll et al. 2004, O'Carroll et al. 2005). The adhesion tension determines which fluid adheres to the solid surface and preferentially wets it. Zero adhesion tension refers to equal affinity of both fluids to wet the solid surface. Powers and Tamblin (1995) stated that the most important factor which determines the fluid affinity to wet a solid surface is the chemical composition of the fluid and the solid surface. Leverett (1941) found that, once a fluid covers the soil particles, which is the case in natural petroleum reservoirs, the contact angle of this fluid is zero.

\subsubsection{Evidence of Spatial Wettability Variations}

Adamson (1997) reported that the spatial wettability of porous media is due to the presence of immobile adsorbed liquid layers on soil particles. Anderson (1987) cited that the materials in the subsurface vary in their wettability. Coal, graphite, sulphur, talc, talclike silicates, and many sulfides have probably a neutral wettability or are NAPL-wet; while common aquifer materials, such as quartz, carbonates, and sulfates, are strongly water-wet. Powers and Tamblin (1995) stated that the wettability of originally water-wet porous media might change to NAPL-wet due to adsorption of polar compounds or due to deposition of organic materials. O'Carroll et al. (2004) reported that wettability variations in contaminated subsurface environments are common. Such variations affect NAPL migration and persistence in natural settings.

\subsubsection{Importance of Wettability on Governing Equations of Multiphase Flow}

The current constitutive relationships and scaling theories are based on the assumption that the water is always wetting the surface followed by NAPL, if present, and followed by air. This assumption is not always valid since a porous medium might be 
NAPL-wet or might contain NAPL-wet lenses. Bradford and Leij (1995a, b) stated that the assumption of continuous layer of intermediate wetting fluid in a three-phase system, air/NAPL/water, is not correct in fractional or NAPL-wet systems. They found that air/water, air/NAPL, and NAPL/water interfaces occur due to the absence of a continuous intermediate wetting fluid. Therefore, they stated that the capillary pressure-saturations cannot be predicted with the existing models.

NAPL fate in the subsurface is governed by gravitational, viscous, and capillary forces (Pennell et al. 1996). These forces, such as capillary forces, are governed by soil texture, the contact angle, and fluid properties. Therefore, any changes in medium texture and wettability affect the $P_{c}-S$ relationships (Bradford and Leij 1995a, O'Carroll et al. 2004, O'Carroll et al. 2005). Results of NAPL infiltration simulations showed that the spatial variations in wettability could lead to pronounced capillary barriers at the location of NAPL-wet lenses (Bradford et al. 1998).

In order to simulate multiphase fluid flow in the subsurface, $P_{c}-S$ relationships of the porous media have to be known for all fluid pairs (Bradford and Leij 1996). Therefore, by considering spatial wettability variations, the equations governing $P_{c}-k_{r}-S$ relationships should consider such changes in wettability and hence care should be taken when using these relationships in multiphase flow equations in terms of identifying the wetting and non-wetting phases.

\subsubsection{Multiphase Flow Equations with Variable Wettability}

The traditional way of simulating multiphase flow in porous media is based on the assumption that the soil is water-wet everywhere in the domain. Then both empirical $P_{c} \rightarrow S$ relationships and $k_{r}-S$ relationships are used in conjunction with mass balance 
equations to solve for fluid saturations and pressures. This assumption may not be true in every case since even the distribution of fluids in soil pores depends on whether the soil particles are water-wet or NAPL-wet. Bradford et al. (1999) stated that water occupies the smaller pores and NAPL occupies the larger pores in water-wet sands. While for NAPL-wet sands, water occupies the larger pores and NAPL occupies the smaller pores.

Therefore, in order to include the effect of variable wettability on multiphase flow equations, the empirical models which define $P_{c}-S$ and $k_{r}-S$ relationships should be modified to account for wettability variations.

\subsection{3.a. Two-Phase $\boldsymbol{P}_{c} \rightarrow S$ Relationships in Fractionally-Wet Porous Media:}

As mentioned in the previous sections, the scaling theory used to predict $P_{c} \neg S$ relationships for two fluid pairs from a third fluid pair in water-wet porous media cannot be applied for fractionally-wet porous media. Leverett et al. (1942), Lenhard and Parker (1987a), and Parker et al. (1987) used the ratios of interfacial tensions to predict NAPL/water and air/NAPL capillary pressures-saturation data from already measured air/water $P_{c} \neg S$ data in strongly water-wet porous media where all capillary pressure values were positive. Bradford and Leij (1996) measured negative and positive capillary pressures on the NAPL/water interface in a fractional wettability system. Therefore, they modified the scaling approach to take into account the fractional wettability effects such that:

$$
P_{n w}\left(\bar{S}_{w}^{n w}\right)=\frac{\sigma_{n w}}{\sigma_{a n}} P_{a n}\left(\bar{S}_{n}^{a n}\right)-\xi
$$

The difference between the scaling relation in water-wet media and fractionallywet media is the introduction of a capillary pressure shifting parameter ' $\xi$ '. The shifting 
parameter allows predictions of positive and negative NAPL/water capillary pressures from the absolutely positive air/NAPL capillary pressures. It is worth mentioning that Bradford and Leij (1996) and O'Carroll et al. (2005) were creating a fractionally-wet medium by mixing a known volume of treated NAPL-wet sand with a known volume of water-wet sand such that they had a homogeneous medium, in terms of grain size, with a specific fraction of NAPL-wet sand. They stated that the shifting parameter is a function of NAPL-wet sand fraction, interfacial tensions of fluid pairs, and the porous medium pore size distribution. In addition, it is hysteretic (i.e., the relationship between the shifting parameter and the NAPL-wet fraction differs in drainage than in imbibition). They intended to measure the air/NAPL (instead of air/water) relationship and used that to predict the NAPL/water capillary pressure for reasons such as $\sigma_{a n}<\sigma_{a w}$, which leads to a smaller range of pressures, and $\sigma_{a n}$ is less susceptible to contamination than $\sigma_{a w}$.

O'Carroll et al. (2005) stated that the previous models failed to predict $P_{c} \neg S$ relationships in two-phase systems under fractional wettability conditions. They developed a model based on an extension of Leverett scaling theory, known as the Leverett-Cassie model. This model allows the estimation of a $P_{c} \rightarrow S$ relationship for fluid pair $a$ and $b$ in a fractionally-wet porous medium containing a number $(N)$ of surfaces of different wetting properties with surface area fractions $\left(f_{I}\right)$. The estimated fractional $P_{c}-S$ relationship is a weighted, scaled function of the $P_{c}-S$ relationships of fluid pair $A$ and $B$ measured in a number $(N)$ of reference porous media $(I)$. According to their model, a reference porous medium $(I)$ should have the same pore structure as in the fractionallywet porous medium but it has one wettability value represented by a contact angle $\left(\phi_{a, l}\right)$. The contact angle $\left(\phi_{a, I}\right)$ is the angle that the $a / b$ interface makes with the solid surface $(I)$, 
measured through the wetting fluid $a . P_{c, I}^{A B}\left(S_{W, I}^{a p p}\right)$ is the capillary pressure as a function of apparent wetting phase saturation $(A)$, measured in a reference porous medium $(I)$. The contribution of each $A / B P_{c} \neg S$ relationship corresponding to a contact angle $\left(\phi_{a, I}\right)$ and fraction $\left(f_{l}\right)$, to $a / b \quad P_{c}-S$ relationship for the fractionally-wet medium is scaled by interfacial tension ratio $\left(\sigma^{a b} / \sigma^{A B}\right)$. The summation of the scaled contributions equals to the predicted $a / b P_{c}-S$ relationship in the fractionally-wet porous medium as shown in Equation (2.58).

$P_{c}^{a b}\left(S_{a}^{a p p}\right)=\frac{\sigma^{a b}}{\sigma^{A B}}\left[\sum_{I=1}^{N} f_{I} \cos \left(\phi_{a, I}\right) P_{c, I}^{A B}\left(S_{W, I}^{a p p}\right)\right]$

\subsection{3.b. $P_{c}-S$ Relationships for Three-Phase System in Fractionally-Wet Porous Media:}

Bradford and Leij (1996) stated that the distribution of fluids in a pore space of a fractionally-wet porous medium is different than that of a water-wet porous medium. Also, this distribution is a function of liquid saturations. Bradford and Leij (1996) and O'Carroll et al. (2005) stated that the existing models used to predict $P_{c} \neg$ relationships for three-phase systems in fractionally-wet porous media and NAPL-wet porous media are inadequate to apply since they are based on Leverett scaling theory. Moreover, Bradford and Leij (1995b) found that air/water, NAPL/water, and air/NAPL interfaces exist in three-phase, air/NAPL/water, systems in fractionally-wet and NAPL-wet porous media. They claimed that an interface capillary pressure dependence on fluid saturation is the same for fractionally-wet and NAPL-wet porous media. For both fractionally-wet or NAPL-wet porous media, they found that capillary pressure on the NAPL/water interface 
depends only on water saturation, capillary pressure on the air/NAPL interface depends largely on NAPL saturation and to a lesser extent on water saturation, and capillary pressure on the air/water interface depends mainly on water saturation and to a lesser extent on NAPL saturation. Since the NAPL/water capillary pressure is a function of water saturation in two-phase, NAPL/water, systems and three-phase, air/NAPL/water, systems, Bradford and Leij (1996) claimed that Leverett's approach can still be implemented in the sense of predicting NAPL/water capillary pressure in the three-phase system from that of the two-phase system (Equation 2.59) for a fractionally-wet or NAPL-wet porous media. Therefore, Leverett's approach is true for a NAPL/water interface regardless of the medium wettability such that the NAPL/water capillary pressure is positive when water is the most wetting fluid, while it is negative when water is the intermediate wetting fluid. Bradford and Leij (1996) could not apply Leverett's approach to predict the capillary pressure-total liquid saturation in an air/NAPL/water three-phase system from the capillary pressure-NAPL saturation in air/NAPL two-phase system for either fractionally-wet or NAPL-wet porous media.

$$
P_{n w}\left(\bar{S}_{w}^{a n w}\right)=P_{n w}\left(\bar{S}_{w}^{n w}\right)
$$

\subsection{Residual Non-Wetting Phase Saturation in Porous Media}

As NAPL migrates in the downward direction through the unsaturated zone, its distribution is a function of NAPL, air, and water pressures, pore size distribution, and the porous medium porosity and wettability. A fraction remains in the soil after NAPL flow ceases and is known as residual NAPL saturation (Mercer and Cohen 1990). 
Zalidis et al. (1998) stated that the saturation-capillary pressure curve is vertical at residual NAPL saturation (i.e., $d S_{r} / d P_{a n}=0$ ). Therefore, the residual NAPL either moves at an imperceptible rate or is incapable to move. Wilson and Conrad (1984) stated that the residual NAPL exists in unsaturated zone as films, pendular rings, wedges, filling throats, and residual blobs. Their description of residual NAPL forms was verified by a pore scale micro model using flow visualization techniques.

\subsubsection{Factors Contributing to the Residual NAPL Saturation in Porous Media}

\subsection{1.a. Spreading Coefficient Effect}

Kalaydjian and Tixier (1991) stated that residual saturation decreases with increasing spreading coefficient. Isolated blobs form because non-spreading liquids form less stable films in porous media. According to Adamson (1997), NAPL lenses and isolated blobs are formed due to less spreading of NAPL on the air/water interface.

\subsection{1.b. Initial Water Saturation}

Leverett (1941) assumed that the total liquid saturation in a three-phase system, where wettability follows the sequence water $>$ NAPL $>$ air and the air only contacts the NAPL, equals to the NAPL saturation in two-phase air/NAPL system. Based on this assumption, Zalidis et al. (1998) showed that the limits where Leverett's assumption was based need to be identified and therefore it was shown that the initial water saturation affects the residual NAPL saturation. In this regard, a relationship between initial water saturation and residual NAPL saturation was constructed.

It was shown that this relationship could be divided into two regions. The boundary of these two regions is the critical initial water saturation. In the first region, the 
relationship between the residual NAPL saturation and the initial water saturation is linear. In this region, Leverett's assumption applies such that when initial water saturation increases, the residual NAPL saturation decreases with the same magnitude in order to maintain a constant total liquid saturation. In the second region, the residual NAPL saturation does not decrease with the increase of the initial water saturation, although it remains constant at a certain value called minimum residual NAPL saturation in a three-phase system and the total liquid saturation increases with the increase of the initial water saturation.

Zalidis et al. (1998) further stated that Leverett's assumption does not apply after the critical initial water saturation. The reasons of its inapplicability are many factors other than the capillary pressure. Among these factors are NAPL sorption and isolation mechanisms. Moreover, the residual NAPL saturation is not continuous at its minimum residual saturation. Furthermore, the value of the critical initial water saturation and the minimum residual NAPL saturation are related to the wettability history of the soil matrix and fluid/fluid interfacial tension. It was recommended that more study should be performed to better understand the responsible mechanisms for the residual saturation in the second region.

\subsubsection{Conceptual Models Describing Residual NAPL Saturation}

Van Geel and Roy (2002) developed a conceptual model to include residual NAPL saturation in the hysteretic $P_{c} \neg S$ relationship. To help build the model, define, and determine its parameters, they ran two-phase and three-phase $P_{c}-S$ experiments. In the three-phase experiments, water was held at irreducible, $20 \%$, and $40 \%$ water saturations, respectively, while NAPL (e.g., heptane) imbibes and subsequently drains. They found 
that as the maximum heptane saturation reached prior to drainage increases, the residual heptane saturation increases. They observed that most of the experimental data points (residual heptane saturation-maximum heptane saturation) fall within an envelope bounded by linear and logarithmic relationships as shown in Figure 2.30.

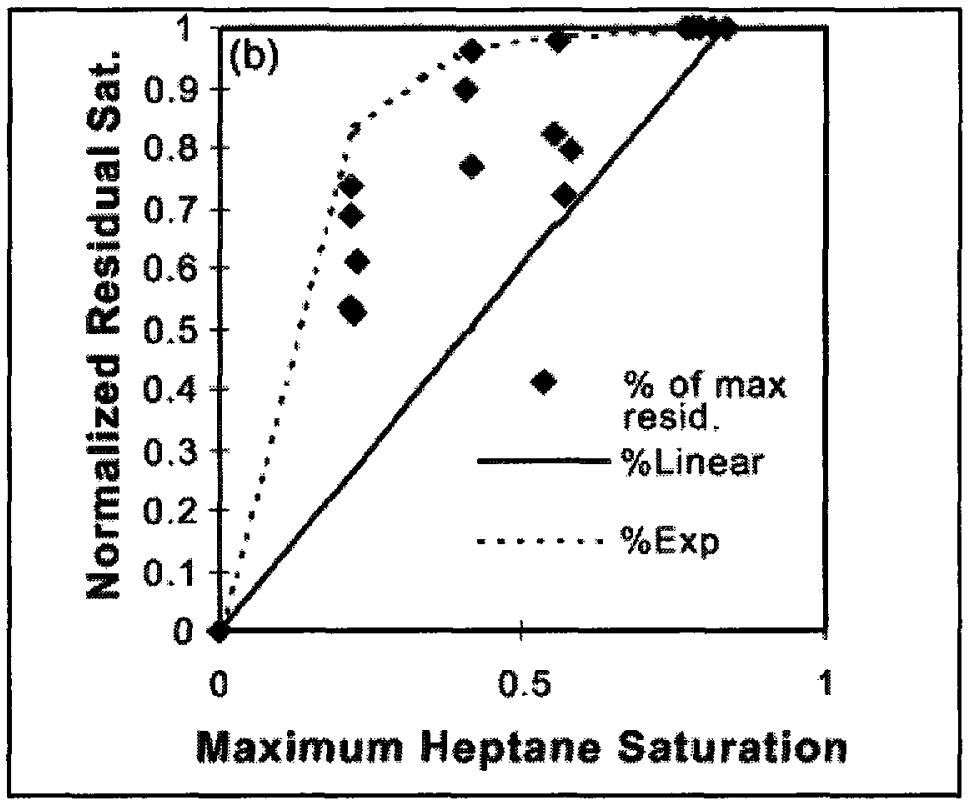

Figure 2.30: Maximum Heptane Saturation-Residual Heptane Saturation (Van Geel and Roy 2002).

Van Geel and Roy (2002) proposed four models for the residual heptane saturation, namely; a linear model, a constrained exponential model, an unconstrained exponential model, and a model based on the entrapment model of Land (1968). These models are based on the knowledge of an absolute maximum residual NAPL saturation, the apparent total liquid saturation, and the apparent total liquid saturation at the saturation path reversal from the primary wetting $P_{c} \checkmark S$ curve to a drainage scanning curve. Their conceptual models, which estimate the apparent maximum residual NAPL 
saturation obtained following drainage from an apparent total liquid saturation, were incorporated into the hysteretic model of Lenhard and Parker (1987b).

In their conceptual model to include residual NAPL saturation in the hysteretic $P_{c} \rightarrow S$ relationship, Lenhard et al. (2004) claimed that residual NAPL saturation depends on two factors; the volume of pore space occupied by NAPL and the size of the pores containing NAPL. These two factors are to scale the maximum residual NAPL saturation to predict effective residual NAPL saturation for given conditions. Using a spreading NAPL, they showed that their model is capable of predicting the residual NAPL saturation for varying initial water and NAPL saturations.

Van Geel and Roy (2002) assumed that the residual NAPL saturation starts to form as NAPL is draining from the system such that it reaches its maximum value, corresponding to an initial NAPL saturation, when NAPL flow ceases. While Lenhard et al. (2004) assumed that residual NAPL starts to build up as NAPL is imbibing into the system. They assumed that residual NAPL saturation reaches its maximum value, corresponding to a maximum NAPL saturation, once NAPL resides in all possible small pores and pore wedges, and resides as lenses on thin water films adsorbed to solid particles. Also, Van Geel and Roy (2002) and Lenhard et al. (2004) argued that water saturation is an important variable to take into account and should be included when predicting residual NAPL saturation. The difference between these two models is that at the same apparent saturation path, they give different relative permeability values.

\subsection{Summary}

In light of the presented multiphase flow literature, there are deficiencies in the assumptions on which the multiphase flow governing equations are based (i.e., soil is 
strongly water-wet and residual NAPL term is ignored). Also, the distribution of residual NAPL under different wettability conditions is not clearly described. Therefore, a revision to and modification of the Leverett assumption and scaling relations such that they can be applied under different wettability conditions are part of the research objectives. Pore scale study of the residual NAPL distribution and REV study of the magnitude of the residual NAPL saturation under different wettability conditions are important for improvement of the current constitutive relationships such that they include the residual NAPL term under these conditions. 


\section{Chapter 3. Methodology}

\subsection{Introduction}

In order to understand and describe the flow process of immiscible fluids in the unsaturated zone, it is important to have a clear picture in terms of flow equations, the location and form of the fluid phases located in the pore spaces, and estimates of residual non-wetting phase saturations and the physical factors that contribute to formation of the residual non-wetting phase saturation. As described earlier, this research focused on the effect of spatial wettability variations, for spreading NAPLs, on the constitutive relationships, residual NAPL distribution, and conceptual models of the residual NAPL saturation. In brief, the following areas were covered in this research:

1. A study of the effects of changing the wettability conditions on the formation and distribution of residual NAPL at the pore scale and REV scale;

2. Development of conceptual models to include the residual NAPL term in the constitutive relationships under different wettability conditions for spreading NAPL;

3. Validation of the scaling relations and Leverett assumption under different wettability conditions with spreading NAPL; and

4. Development of conceptual models to include porous medium wettability effects on the constitutive relations.

The research was divided into two main parts, an experimental part and an analytical part. For the experimental component, the porous media were exposed to various saturation pathways in a Buchner funnel, a modified Tempe cell, and in a glass box apparatus. Two sands with different grain size distributions, one uniform and one 
well graded were used in both the Tempe cell and the Buchner funnel. A porous medium with a grain size distribution similar to the uniform sand was generated from glass beads. The purpose of the glass bead medium and the glass box apparatus was to visually view the NAPL distribution at selected saturation values under the different wettability conditions. In the analytical part, the data obtained from the experimental part was analysed and conceptual models for $P_{c}-S$ relationships in two and three-phase systems, in a water-wet porous medium with a NAPL-wet soil lens, were developed and then the Leverett assumption and scaling relations were validated. In addition, conceptual models for the residual NAPL term were developed. The pore scale study facilitated the development of the conceptual models. Finally, the research focused on including the wettability effects, and residual NAPL saturation in the multiphase flow equations (i.e., the constitutive relationships).

The conceptual model for the soil studied in this research consisted of a water-wet porous medium with a NAPL-wet soil lens. For the experiments, the wettability of the porous medium was changed from experiment to experiment such that for each porous medium, the $P_{c} \neg S$ curve was constructed for a water-wet porous medium and water-wet porous medium with different size NAPL-wet soil lenses. The NAPL used was heptane, which corresponded to a spreading LNAPL. As for the residual NAPL study, each porous medium wettability configuration tested in the Tempe cell and glass box apparatus was exposed to various saturation paths to evaluate the magnitude and distribution of the residual NAPL term. A comparison between the NAPL distribution under different wettability conditions was made and then conceptual models explaining the effects of wettability on residual NAPL distribution were developed. 


\subsection{Experimental Plan}

The experiments were divided into two groups; two-phase experiments and threephase experiments. All experiments were conducted in duplicate for each wettability configuration.

\subsubsection{Two-Phase Experiments}

Under variable wettability conditions, two-phase $P_{c}-S$ relationships were constructed from the experimental data collected from the Buchner funnel experiments for the two sand types. The two-phase experiments included NAPL/water, air/NAPL, and air/water. The two-phase experiments provided some insight related to the magnitude of the irreducible saturation for the wetting fluid and the magnitude of the entrapped saturation for the non-wetting fluid. These experiments were also used to evaluate the applicability of the Leverett assumption and scaling theory to generate the $P_{c}-S$ relationship for one fluid pair from another fluid pair, under variable wettability conditions.

\subsubsection{Three-Phase Experiments}

$P_{c} S$ relationships and residual NAPL saturation were studied in three-phase systems in modified Tempe cells for the two sands. For the uniform sand experiments conducted in the Tempe cells, a parallel experiment was conducted, whenever possible, using the glass box apparatus. As mentioned previously, the grain size distribution for the uniform sand used in the Tempe cell was similar to the grain size distribution of the glass beads used in the glass box apparatus. In order to ensure similar porosity values for every 
soil type in the different experiments, a unique procedure was followed for filling the Buchner funnel, Tempe cell, and glass box apparatus with the porous media.

\subsection{2.a. Three-Phase Experiments Intended for Validation of Leverett Assumption}

In order to develop a conceptual model for $P_{c} \neg$ relationships in a three-phase system and to check the validity of Leverett assumption, capillary pressure-total liquid saturation experiments were conducted in a water-wet porous medium and in a porous medium that consisted of $60 \%$ (by volume) water-wet soil fraction with $40 \%$ NAPL-wet soil lens. These experiments were conducted using the well graded sand. In these experiments, water saturation was fixed at an initial water saturation as heptane drained from and imbibed into the porous medium. The experiments were conducted at two different initial water saturations. The first was conducted at the lowest initial water saturation (i.e., irreducible water saturation in the water-wet soil fraction) while the second was conducted at an initial water saturation of $45 \%$. These experiments were conducted in modified Tempe cells. Three transducers were connected to each cell; two transducers to measure heptane pressure and one transducer to measure water pressure. A transducer was connected to a cell such that it measured a liquid phase pressure head at an REV mid height. In the water-wet porous medium, the two transducers measured heptane pressure head at two locations in the porous medium. In the water-wet porous medium with the NAPL-wet soil lens, one transducer measured the heptane pressure head in the water-wet soil fraction and the second transducer measured the heptane pressure head in the NAPL-wet soil lens. The third transducer was connected to the cell such that it measured the water pressure head in the water-wet soil fraction. The transducers were connected to a data acquisition system. In addition to these REV scale experiments, pore 
scale experiments for the three-phase system were conducted to facilitate the development of the conceptual model.

\subsection{2.b. Three-Phase Experiments Intended for the Development of Conceptual Models for the Residual NAPL Saturation Term}

The three-phase experiments in the glass box apparatus provided insight related to the form and distribution of the residual NAPL saturation at the pore scale. The modified Tempe cell experiments provided insight into the magnitude of the residual NAPL saturation at various points along a saturation pathway. The data collected from the Tempe cell experiments, the data collected and the insight gained from the two-phase Buchner funnel experiments, and the insight gained from the glass box experiments were used to propose and develop new conceptual models for the residual NAPL saturation term under different wettability conditions. These conceptual models estimate the residual NAPL saturation term and account for wettability. The conceptual understanding gained from this approach provides needed knowledge when evaluating remedial alternatives for NAPL contaminated sites.

Three experimental data sets were generated. Experimental data set (1) and (2) include measurement of the residual heptane saturations left in a water-wet porous medium when a certain volume of heptane imbibes into the porous medium and subsequently drains. Both sand types were considered in these experiments. In experimental data set (1), the water saturation was fixed throughout each experiment and increasingly larger volumes of heptane were allowed to imbibe and drain from the soil. At the end of each drainage pathway, the residual heptane saturation was recorded by tracking the heptane volumes added and drained. The initial heptane volume added 
corresponds to $10 \%$ heptane saturation and this was increased by $10 \%$ for each successive imbibition cycle until a $100 \%$ apparent total liquid saturation was reached. The water saturations were fixed at irreducible water saturation and $20 \%, 30 \%, 40 \%$, $50 \%, 60 \%, 70 \%, 80 \%$, and $90 \%$ actual water saturations for each experiment. The residual NAPL saturations measured at fixed water saturation and different imbibing NAPL saturations illustrated the impacts of water saturation and NAPL saturation on the magnitude of the residual NAPL saturation. Based on these data, a relationship between the residual NAPL saturation, the NAPL saturation, and the water saturation was developed.

In experimental data set (2), the water saturation was initially fixed at a certain saturation equivalent to $45 \%$ and different volumes of heptane were allowed to imbibe and subsequently drain from the porous medium. At the end of the heptane drainage pathway, the water saturation was reduced to $30 \%$ and the heptane imbibition and drainage were repeated. Again, at the end of the heptane drainage pathway, the water saturation was reduced to irreducible and the heptane imbibition and drainage sequence was repeated. At each water saturation, the heptane saturation was increased from $20 \%$, to $30 \%$, and to a value corresponding to an apparent total liquid saturation of $100 \%$. The objective of this part was to show the applicability of the conceptual model under different water saturations within the same experiment.

The experimental procedure for experimental data set (3) was similar to that of experimental data set (2), except the soil wettability was changed. Four porous media wettability configurations were used; corresponding to $90 \%$ water-wet porous medium with a $10 \%$ NAPL-wet soil lens, $80 \%$ water-wet porous medium with a $20 \%$ NAPL-wet 
soil lens, $70 \%$ water-wet porous medium with a $30 \%$ NAPL-wet soil lens, and $60 \%$ water-wet porous medium with a $40 \%$ NAPL-wet soil lens in both uniform and well graded sand types. The objective of this data set was to validate the conceptual model predicting the residual NAPL saturation in a water-wet porous medium with a NAPL-wet soil lens.

\subsection{Materials}

\subsubsection{Porous Media}

The porous media properties for the uniform and well graded sands and the corresponding uniform glass beads were measured.

\subsection{1.a. Porous Medium Properties}

The soil porosity and dry density were determined experimentally. The porosity was determined by measuring the volume of pores contained in a soil volume as explained in Corey (1977). The dry density was measured by weighing a known volume of dry soil. Each of these soil properties was measured in five independent replicates for every soil pair. The average and standard deviation (i.e., standard error based on 5 replicates) of the porosity were 0.374 and 0.001 , respectively, for uniform sand. The corresponding values for well graded sand were 0.333 and 0.002 , respectively. The average and standard deviation (i.e., standard error based on 5 replicates) of the dry density were $1.658 \mathrm{~g} / \mathrm{cm}^{3}$ and 0.0018 , respectively, for uniform sand. The corresponding values for well graded sand were 1.767 and 0.004 , respectively. 


\subsection{1.b. Sands and Glass Beads Grain Size Distribution}

As mentioned earlier, two grain size distributions were tested in the REV scale experiments; uniform and well graded. The grain size distribution of the glass beads used in the pore scale experiments was uniform. United States Department of Agriculture (USDA) classified a soil sample as sand if the sample grain sizes range between $0.05 \mathrm{~mm}$ to $2 \mathrm{~mm}$. Soil is classified as well graded if at least $50 \%$ passes U.S. sieve number 4 $(4.76 \mathrm{~mm})$ with no more than $5 \%$ fines. It should also have a uniformity coefficient greater than 6 and a gradation coefficient between 1 and 3. These coefficients are defined as follows:

Coefficient of Grading $\left(C_{g}\right)=\left(d_{30}\right)^{2} /\left(d_{10} * d_{60}\right)$

Coefficient of Uniformity $\left(C_{u}\right)=d_{60} / d_{10}$

Where:

$d_{10}$ : maximum particle size of the smallest $10 \%$ of the sample;

$d_{30}$ : maximum particle size of the smallest $30 \%$ of the sample; and

$d_{60}$ : maximum particle size of the smallest $60 \%$ of the sample.

The well graded sand used in this research was a mixture of $30 \%$ coarse sand (retained on U.S. sieve number $30(0.6 \mathrm{~mm})), 50 \%$ medium sand $(25 \%$ retained on U.S. sieve number $40(0.425 \mathrm{~mm})$ and $25 \%$ retained on U.S. sieve number $70(0.212 \mathrm{~mm}))$, and $20 \%$ fine sand (retained on U.S. sieve number $120(0.125 \mathrm{~mm}))$. The uniform sand or glass beads was composed of one sieve size representing a medium sand (100\% passing U.S. sieve number 30 and retained on U.S. sieve number 40 ). The grain size distributions of the well graded and uniform soils are shown in Table 3.1 and Figure 3.1. The coefficients of grading and uniformity for the well graded sand are shown in Table 3.2. 
Although well graded sand has a uniformity coefficient of 3.8 , which is less than 6 , in the scope of this research, this soil was referred to as well graded sand.

Table 3.1: Grain Size Distribution of Well Graded and Uniform Soils.

\begin{tabular}{|l|l|c|c|c|c|c|}
\hline \multicolumn{2}{|l|}{ Sieve Size (Number), mm } & $\begin{array}{c}1.19 \\
(16)\end{array}$ & $\begin{array}{c}0.6 \\
(30)\end{array}$ & $\begin{array}{c}0.425 \\
(40)\end{array}$ & $\begin{array}{c}0.212 \\
(70)\end{array}$ & $\begin{array}{c}0.125 \\
(120)\end{array}$ \\
\hline Passing & Well Graded & 100 & 70 & 45 & 20 & 0 \\
\cline { 2 - 7 }$(\%)$ & Uniform & 100 & 100 & 0 & 0 & 0 \\
\hline
\end{tabular}

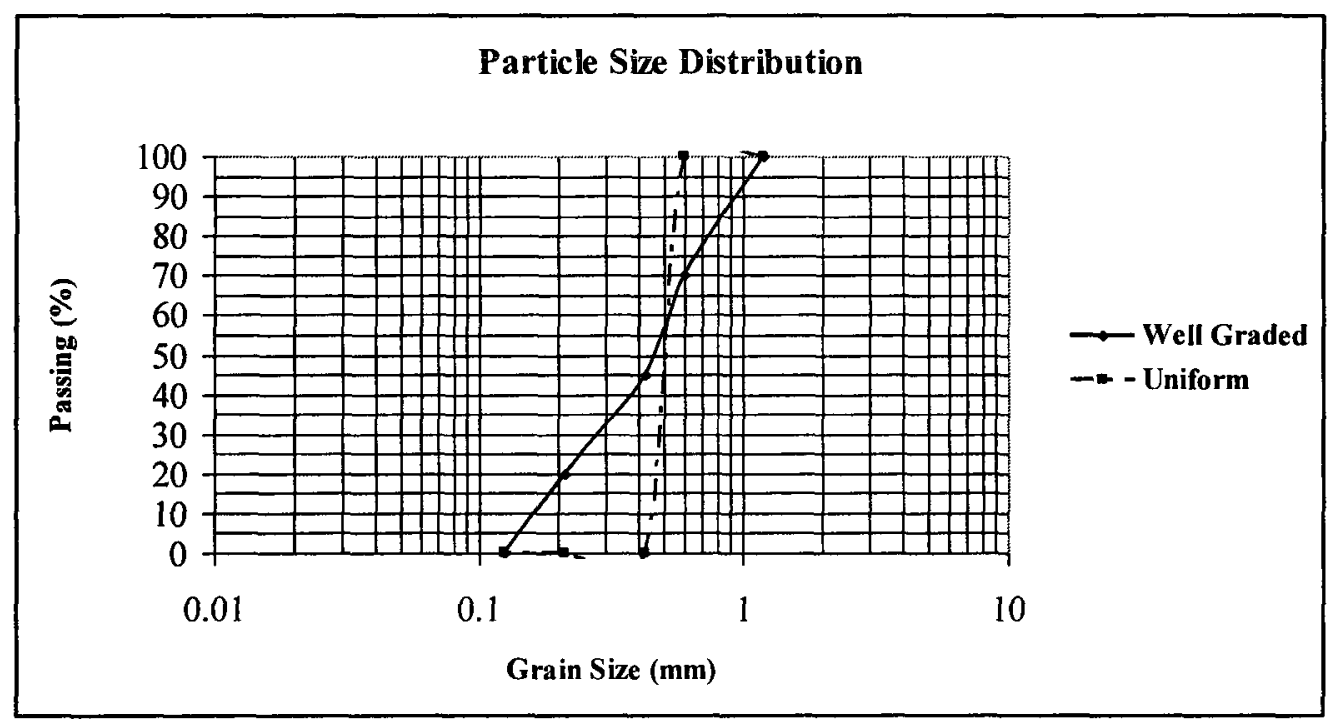

Figure 3.1: Grain Size Distribution of Well Graded and Uniform Soils.

Table 3.2: Coefficients of Grading and Uniformity for Well Graded Sand.

\begin{tabular}{|l|c|c|c|c|c|}
\cline { 2 - 6 } \multicolumn{1}{c|}{} & $\boldsymbol{d}_{\mathbf{1 0}}(\mathbf{\mathrm { mm }})$ & $\boldsymbol{d}_{\mathbf{3 0}}(\mathrm{mm})$ & $\boldsymbol{d}_{\mathbf{6 0}}(\mathbf{m m})$ & $C_{g}$ & $C_{u}$ \\
\hline Well Graded Sand & 0.14 & 0.28 & 0.53 & 1.06 & 3.80 \\
\hline
\end{tabular}

\subsection{1.c. Soil Homogenization}

In order to achieve soil homogeneity, the cone-and-quartering technique was used when making the well graded soils as explained in Schumacher et al. (1990). 


\subsection{1.d. Soil Specific Surface Area}

The specific surface areas of the uniform sand and the well graded sand were measured using the nitrogen adsorption technique and showed that they have similar values. The specific surface area was measured in duplicate for each sand type. The average specific surface area and the standard deviation for the uniform sand were $1030.25 \mathrm{~cm}^{2} / \mathrm{cm}^{3}$ and $21.6 \mathrm{~cm}^{2} / \mathrm{cm}^{3}$, respectively. The corresponding values for the well graded sand were $1063.36 \mathrm{~cm}^{2} / \mathrm{cm}^{3}$ and $30.4 \mathrm{~cm}^{2} / \mathrm{cm}^{3}$, respectively.

\subsection{1.e. Saturated Hydraulic Conductivity}

The saturated hydraulic conductivity for the uniform and well graded sands was measured using the constant head test. The saturated hydraulic conductivity was measured for five samples for each of the uniform and well graded sands. The average and the standard deviation of the saturated hydraulic conductivity for the uniform sand were $0.046 \mathrm{~cm} / \mathrm{sec}$ and $0.007 \mathrm{~cm} / \mathrm{sec}$, respectively. The corresponding values for the well graded sand were $0.011 \mathrm{~cm} / \mathrm{sec}$ and $0.0013 \mathrm{~cm} / \mathrm{sec}$, respectively.

\subsection{1.f. Wettability Control of Sands and Glass Beads}

Water-Wet Sands or Glass Beads: Sands and glass beads are normally water-wet and they do not need treatment.

NAPL-Wet Sands or Glass Beads: NAPL-wet sands and glass beads were achieved by making the sands and glass beads NAPL-wet. Sands and glass beads were treated with organosilane (octadecyltrichlorosilane, OTS). A sample was treated by placing it in a 5\% (by volume) solution of OTS in ethanol with continuous mixing in a shaker for five hours followed by rinsing using a modified rinsing procedure which is described in Chapter 4 . 


\subsubsection{Fluids}

The fluids used in the experiments were distilled water, heptane as a spreading NAPL (solubility in water is $3 \mathrm{mg} / \mathrm{L}$ at $20^{\circ} \mathrm{C}$ ), and air.

\subsection{2.a. Fluid Properties}

The fluid properties such as density, solubility limit of the heptane in water, and the interfacial tensions for all fluid pairs (heptane/water, air/water, and air/heptane) were determined from the literature. The interfacial tensions were also measured using the Du Nouy ring method. In order to limit the mass transfer between the water and heptane phases during the experiments and therefore to avoid the reduction of heptane saturation and change in interfacial tension, water used in the three-phase experiments was presaturated with heptane. Contact angle was not measured because it was assumed that either of the fluids used was completely wetting the porous medium and according to Mayer and Miller (1992), the contact angle in this case is zero.

\subsection{2.b. Dying of Liquids}

Water Dying for Pore Scale Experiments: Water was dyed with a methylene blue since it is not soluble in heptane but soluble in water. Dying water with methylene blue makes water appear blue instead of clear, therefore distinguishing glass beads and water.

NAPL Dying: Sudan III, red dye, was used to dye the heptane used in the REV scale experiments to distinguish water from heptane since Sudan III is soluble in heptane but insoluble in water. As for the pore scale experiments, heptane was dyed with Sudan IV, which is a darker red dye. The concentration of the dye in heptane was $0.25 \mathrm{~g} / \mathrm{L}$. 


\subsection{2.c. Interfacial Tensions}

The $\mathrm{Du}$ Nouy ring method was used to measure the interfacial tension of air/distilled water, air/heptane, and heptane/distilled water fluid pairs. The distilled water, used in interfacial tension measurement of heptane/distilled water fluid pair, was saturated with heptane. To ensure that the measured interfacial tensions reflect the interfacial tensions of the fluid pairs used in the $P_{c}-S$ or residual heptane experiments, the liquids used were collected from these experiments. The heptane used in the $P_{c} \neg$ and residual heptane experiments was Sudan III-dyed heptane. The heptane used in the interfacial tension measurements was Sudan III-dyed heptane and undyed heptane. The interfacial tension was measured for the latter to compare it with the literature values. The interfacial tension was measured ten times for each fluid pair at room temperature $\left(22^{\circ} \mathrm{C}\right)$. The average and standard deviation values of interfacial tensions for the different fluid pairs are shown in Table 3.3. Table 3.3 also shows the literature values of the interfacial tensions of the fluid pairs. The measured interfacial tensions are consistent with corresponding values found in the literature. It is important to note the impact of the Sudan III on the interfacial tension of heptane/distilled water fluid pair.

Table 3.3: Interfacial Tension Values.

\begin{tabular}{|l|c|c|c|c|}
\hline \multirow{2}{*}{ Fluid Pair } & \multicolumn{2}{c|}{ Measured Values } & \multicolumn{2}{c|}{ Literature Values } \\
\cline { 2 - 5 } & $\begin{array}{c}\text { Average } \\
(\text { dyne/cm })\end{array}$ & $\begin{array}{c}\text { St. Deviation } \\
(\text { dyne/cm })\end{array}$ & $\begin{array}{c}\text { Average } \\
(\text { dyne/cm })\end{array}$ & $\begin{array}{c}\text { St. Deviation } \\
(\text { dyne/cm })\end{array}$ \\
\hline Air/distilled water & 73.26 & 0.37 & $72.94^{*}$ & N.A. \\
\hline Air/ undyed heptane & 21.00 & 0.49 & $20.14^{*}$ & N.A. \\
\hline Air/ Sudan III-dyed heptane & 20.40 & 0.33 & N.A. & N.A. \\
\hline Undyed heptane/distilled water & 46.90 & 0.82 & $50.80^{* *}$ & N.A. \\
\hline Sudan III-dyed heptane/distilled water & 23.50 & 0.50 & N.A. & N.A. \\
\hline
\end{tabular}

${ }^{*}$ Adamson 1997 (at $20^{\circ} \mathrm{C}$ ), ** Aveyard et al. 1986 (at $25^{\circ} \mathrm{C}$ ), N.A.: Not available in literature 


\subsection{Apparatuses}

As mentioned previously, three apparatuses were used in this research; Buchner funnel, modified Tempe cell (or simply Tempe cell), and glass box apparatus.

\subsubsection{Buchner Funnel}

The two-phase $P_{c}-S$ curves for air/heptane, air/water, and heptane/water were constructed using a Buchner funnel. The Buchner funnel consists of $350 \mathrm{~mL}$ glass filter funnel equipped with a fritted glass disc with average pore size of $4-5.5 \mu \mathrm{m}$. For the air/water and heptane/water experiments, this disc was water-wet. While for air/heptane experiments, this disc was heptane-wet. Only the wetting fluid can move freely through the discs while the non-wetting fluid cannot as long as the entry pressure of the disc is not exceeded. Keeping that in mind, the non-wetting fluid flow is only restricted to sand pores and hence the change in wetting fluid volume is assumed to occur due to nonwetting fluid imbibition into and drainage from sand. The Buchner funnel with its other components is shown in Figure 3.2 and Figure 3.3.

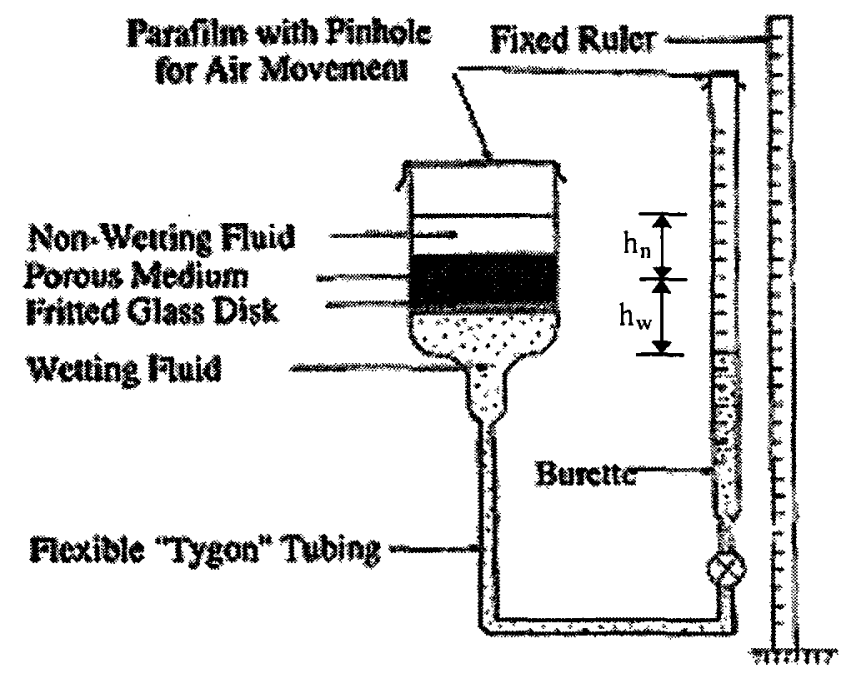

Figure 3.2: Buchner Funnel (Modified from Van Geel and Sykes 1994). 


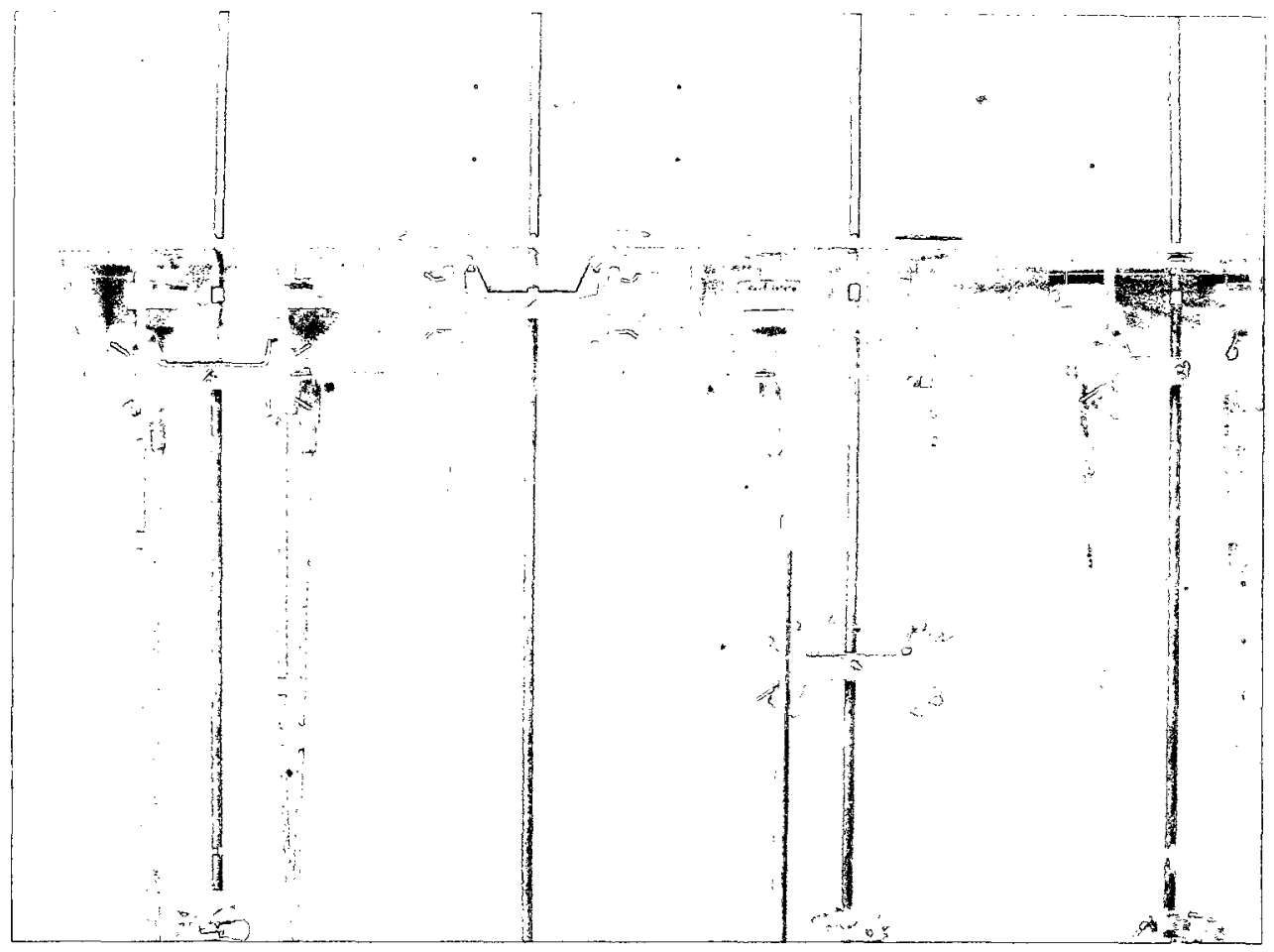

Figure 3.3: Two-Phase $P_{c}-S$ Experimental Setup.

Prior to conducting the experiments using the Buchner funnel, the porous plate and soil were flushed with $\mathrm{CO}_{2}$ at a rate of $3700 \mathrm{~cm}^{3} / \mathrm{min}$ for three minutes to replace the air, as $\mathrm{CO}_{2}$ more readily dissolves in water or heptane. The porous plate and soil were then saturated with water or heptane at $0.05 \mathrm{~cm}^{3} / \mathrm{sec}$. It was important that at least three pore volumes of the wetting fluid pass through the soil to ensure that any entrapped $\mathrm{CO}_{2}$ was dissolved. After setting up an experiment, the top of the funnel and burette were covered by parafilm with a small hole punctured through the centre of each in order to allow for the movement of air while reducing evaporation. Then the soil was subjected to different drainage and wetting pathways.

For drainage, after saturating the soil with the wetting fluid for air/water and air/heptane cases, the wetting fluid level in the burette was lowered to achieve a zero capillary pressure head. This was the starting point for the two-phase $P_{c}-S$ curve. Wetting 
fluid level in the burette was then lowered in steps. At each step, the wetting fluid pressure was decreased creating an increase in the capillary pressure which allows the non-wetting fluid to replace the wetting fluid. As wetting fluid was drained from sand pores, it moved through the porous disc and it was collected in the burette. For wetting fluid imbibition, the previous procedure was reversed and the burette was raised allowing wetting fluid to enter the porous medium and displace the non-wetting fluid. In the case of heptane/water, a volume of heptane was placed on top of the water-saturated soil sample. The initial heptane volume had to be large enough to ensure that a layer of heptane remained above the soil surface at all times during the experiment. The burette was lowered in steps and this caused the water pressure in the sand to decrease and hence the capillary pressure on the heptane/water interface increased. As water was drained from sand pores and replaced by heptane, it moved through the porous plate and it was collected in the burette. For water imbibition, the previous procedure was reversed and the burette was raised allowing water to enter the porous medium and displace the heptane.

\subsubsection{Modified Tempe Cell}

The three-phase air/heptane/water experiments were conducted in a modified Tempe cell by exposing the porous medium to various saturation paths. The cell body was made of aluminum and mainly composed of two parts; an upper rectangular plate and a lower part. The lower part was composed of the soil holder and the base. The upper and lower parts were connected by bolts. The dimensions of the different components of the modified Tempe cell are shown in Figure 3.4. 

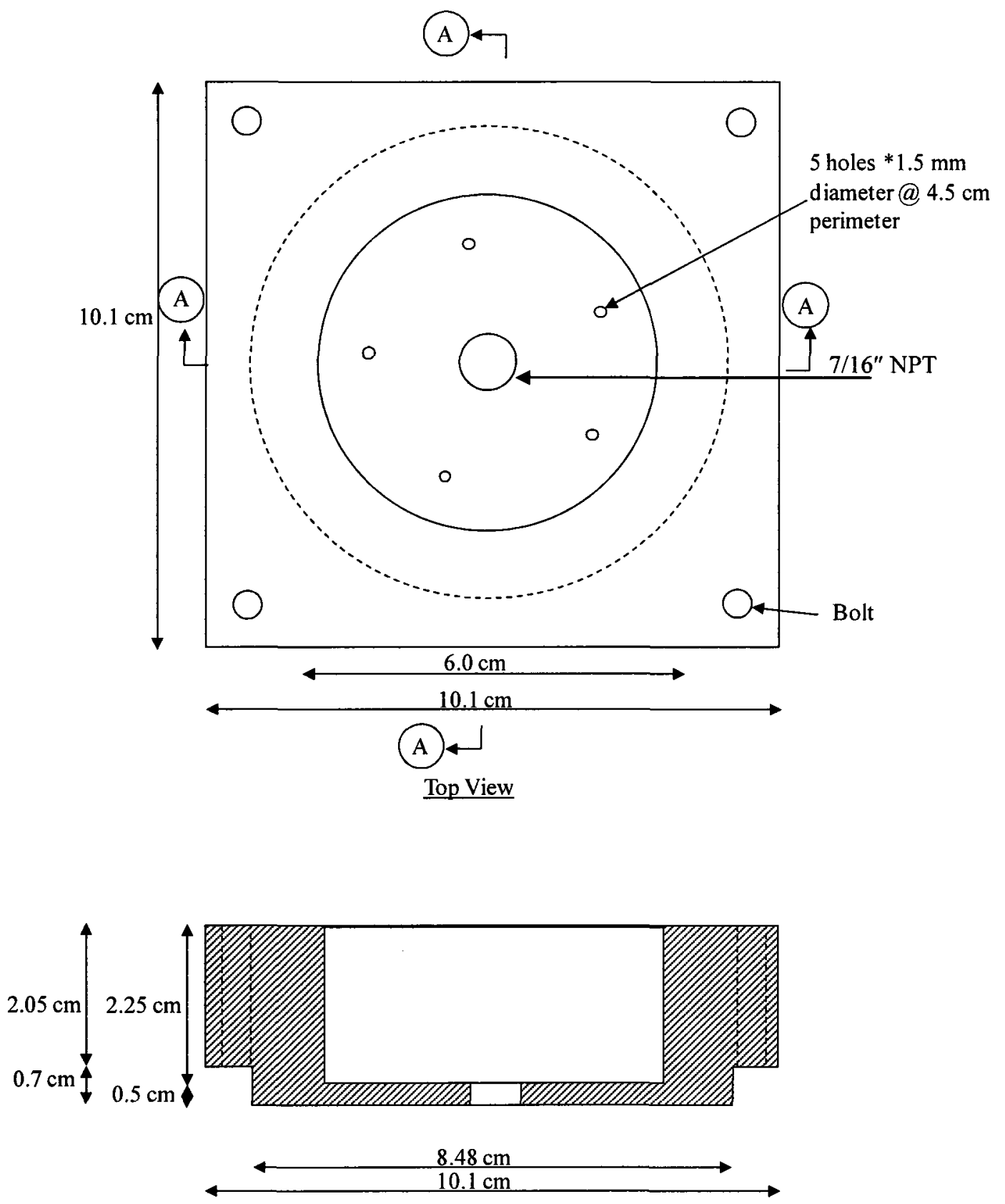

$\underline{\text { Section A-A }}$

Figure 3.4a: Upper Plate: Plan View and Side Cross Section. 


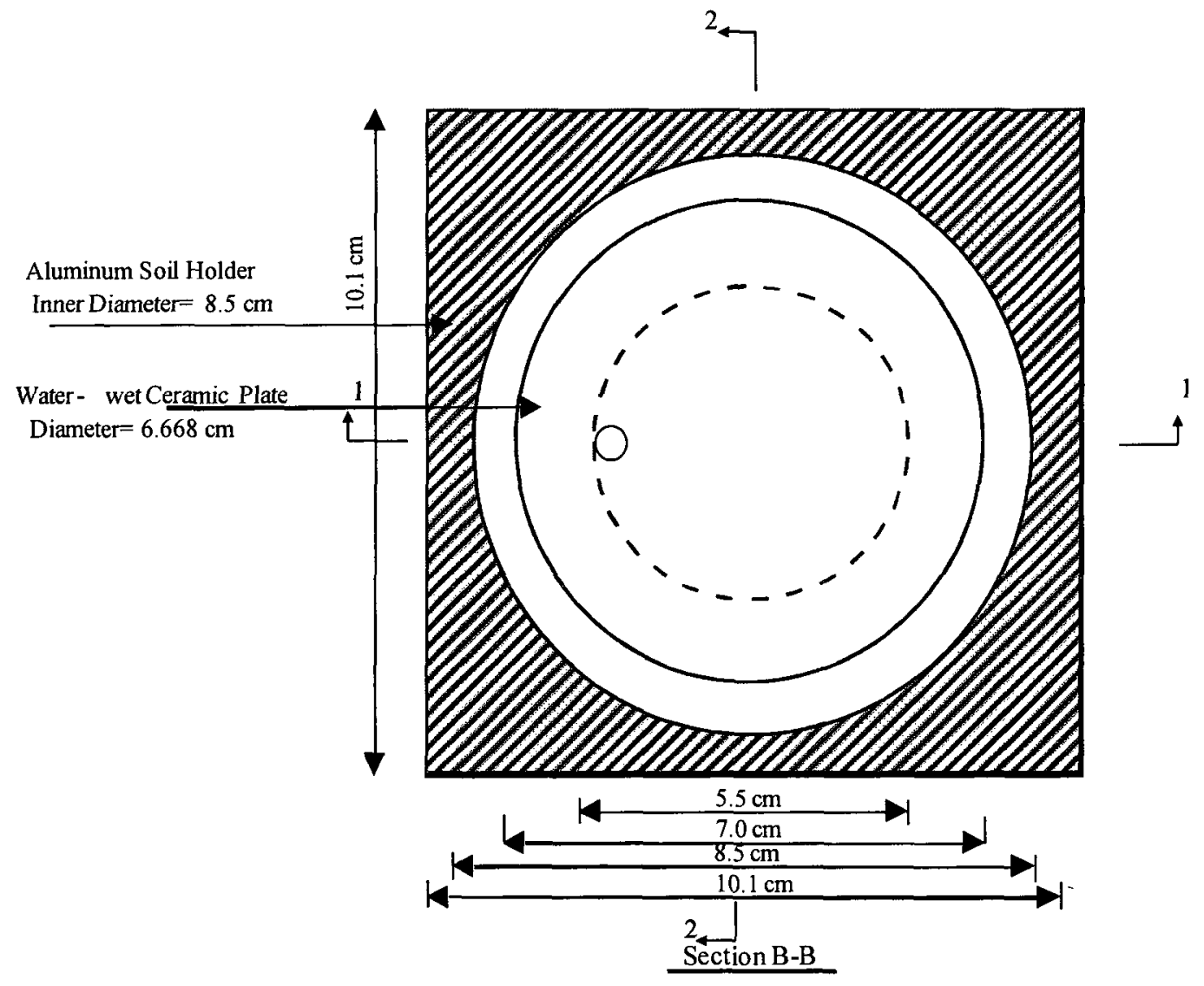

Figure 3.4b: Soil Holder and Bottom Plate: Cross Section (B-B). 

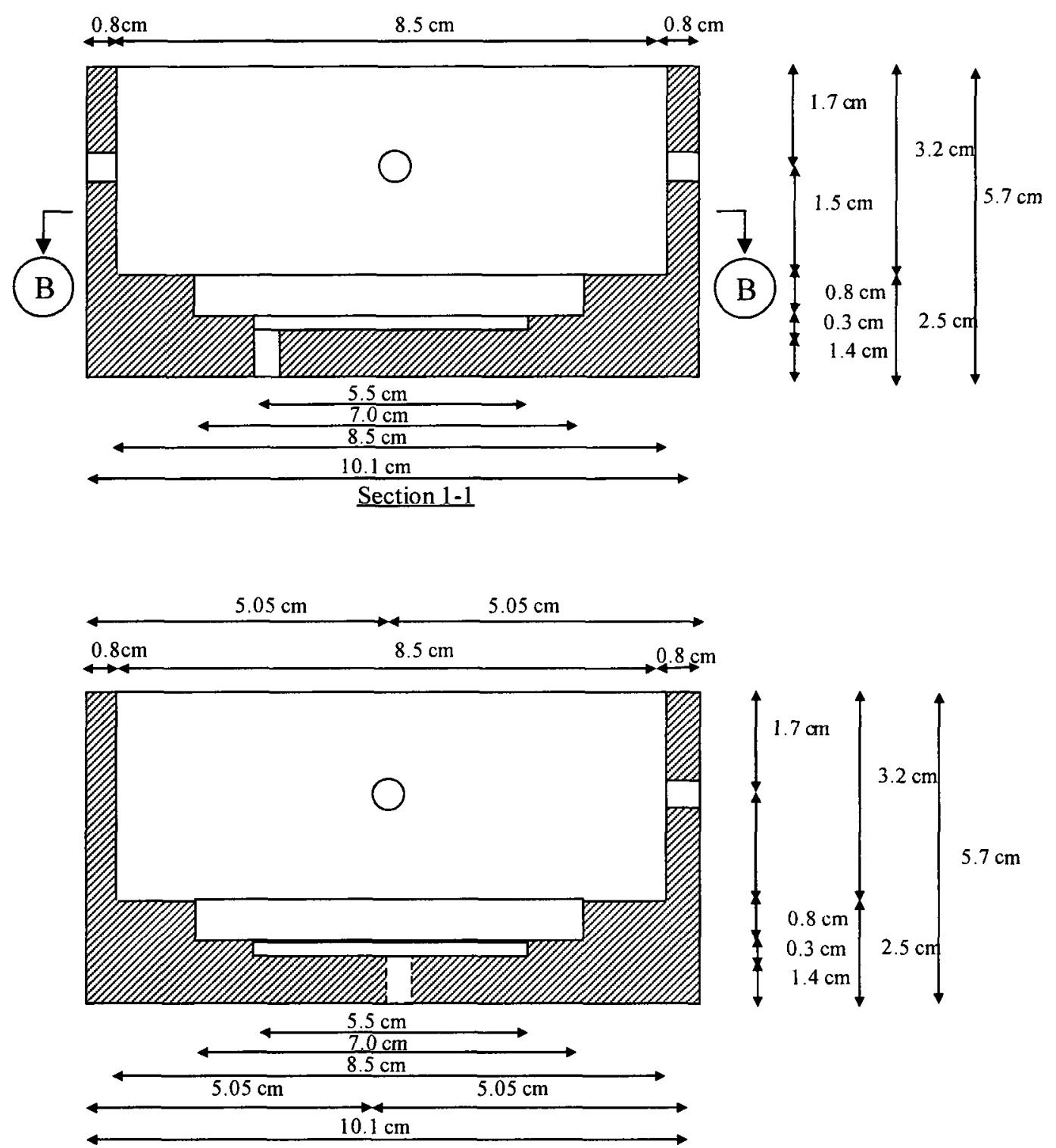

Section 2-2

Figure 3.4c: Soil Holder and Bottom Plate: Cross Sections (1-1) and (2-2). 


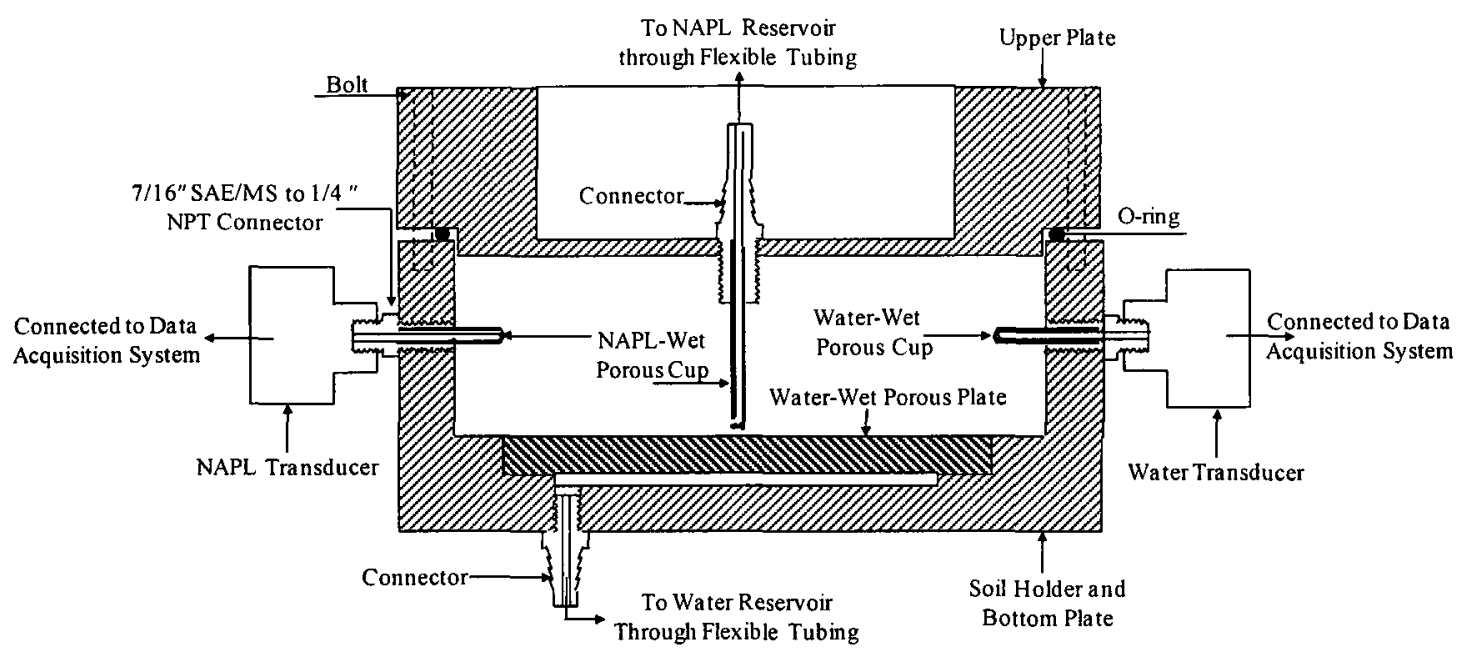

Figure 3.4d: Schematic of the Cross Section in the Assembled Cell.

Figure 3.4: Modified Tempe Cell.

In the upper aluminum plate, using a stainless steel connector that was composed of a hose on one side and a thread on the other side, the NAPL-wet ceramic porous cup was adjoined to the stainless steel connector, using epoxy, and the latter was connected to the upper aluminum plate. The hose of the stainless steel connector was connected to the heptane reservoir. The porous cup was extended over the entire depth of the soil sample to be placed in the lower aluminum plate.

A recession of $22.5 \mathrm{~mm}$ deep was created in the upper aluminum plate to allow collecting the water before initiating the main drainage pathway. At the bottom of the upper plate, a $7 \mathrm{~mm}$ deep recession with an $84.8 \mathrm{~mm}$ diameter was created to ensure a good fit when the cell is assembled. To minimize any potential water leakage when saturating the soil sample prior to initiating the main drainage pathway, an $85 \mathrm{~mm}$ diameter O-ring was placed between the upper and lower plates. Finally five $1.5 \mathrm{~mm}$ ports were opened to the atmosphere to allow air to freely move into or out of the cell in response to a change in water and heptane pressures. 
In the lower aluminum plate as shown in Figure 3.4, the water wet ceramic plate was placed on the upper side of the aluminum plate and adjoined to it using epoxy. A three millimetre recession existed below the plate to allow continuous flow of water. The water reservoir was connected to the cell just below the recession through the aluminum plate. Figure 3.5 shows the experimental setup for three-phase systems.

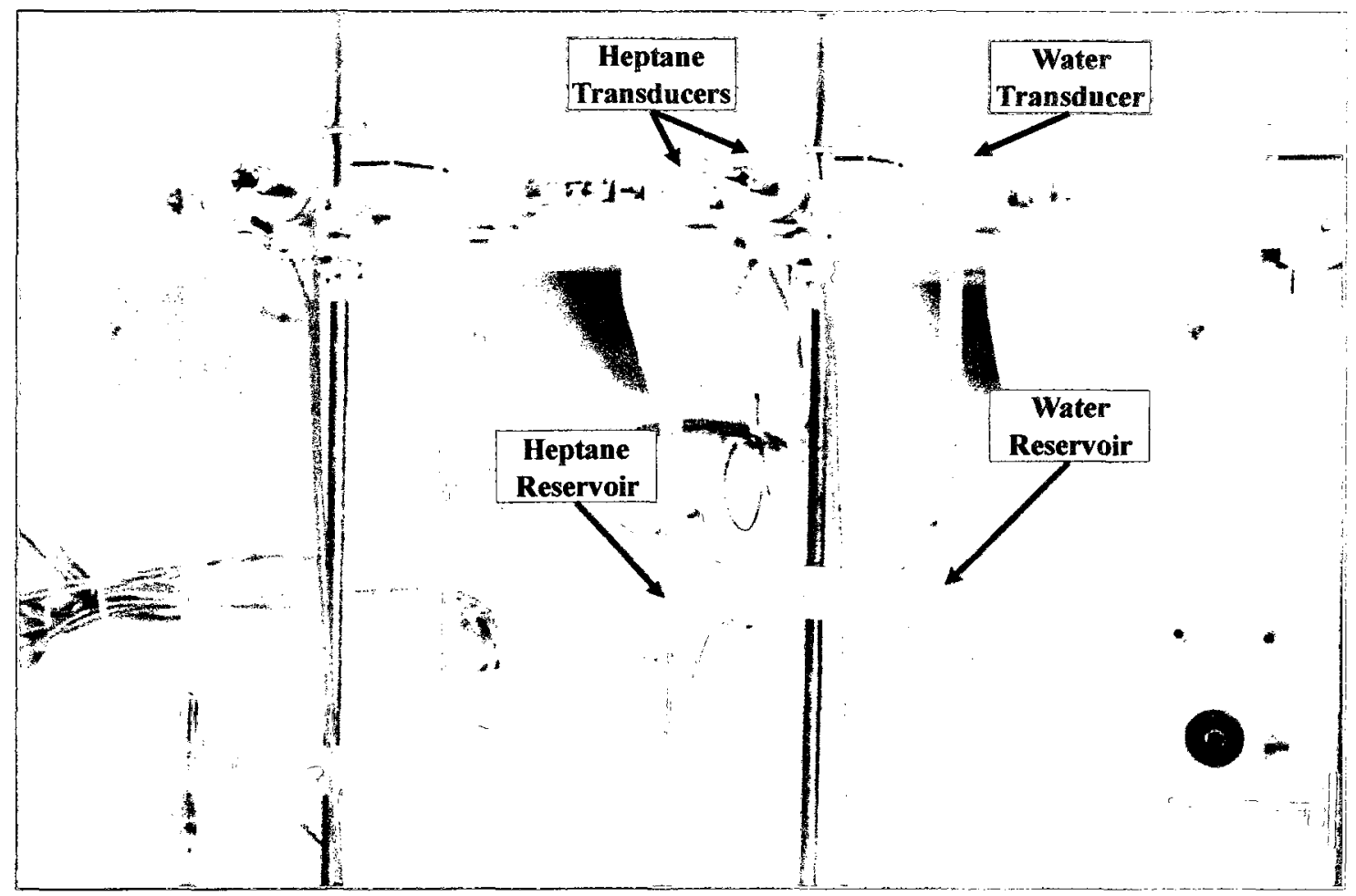

Figure 3.5: Experimental Setup for Three-Phase System.

For three-phase experiments intended to validate the Leverett assumption, the initial water saturation was fixed at its lowest value and at $45 \%$ actual water saturation. To set up these experiments, three transducers were connected to the cell after which the soil sample was placed. Then a NAPL-wet porous cup connected to a heptane burette, via flexible tubing, was connected to the upper aluminum plate. Using an O-ring and four 
bolts, the upper aluminum plate was connected to the cell. Through the water-wet ceramic plate, the soil sample was flushed with carbon dioxide for fifteen minutes and saturated allowing at least three pore volumes of water to pass through the soil sample. The water was collected in the cavity in the upper aluminum plate and was removed. Then the cavity in the upper aluminum plate was filled with heptane. Thereafter, as the water drained, it was replaced by heptane until the initial water saturation was reached.

At this point, the total liquid saturation in the porous medium was unity and any heptane that remained in the cavity of the upper plate was removed. Then the total liquid saturation main drainage pathway was initiated by lowering the heptane burette in steps. When the heptane burette was lowered, the heptane pressure decreased and this allowed the heptane to drain from the porous medium causing air to be drawn into the porous medium. Once the heptane transducer reached a steady heptane pressure, the heptane reading in the burette was recorded and the heptane saturation in the porous medium was calculated. This process was repeated in six to seven steps until the heptane present in the porous medium was at residual saturation. At this point, the heptane burette was raised in seven to eight steps. In each step, the heptane saturation was calculated until a $100 \%$ apparent total liquid saturation was reached.

For three-phase experiments intended to develop conceptual models for residual NAPL saturation study, initial water saturation was fixed at different values as explained earlier for experimental data sets (1), (2), and (3). To set up these experiments, the transducer ports were plugged and the soil sample was placed in each cell. Then a NAPLwet porous cup connected to a heptane burette was connected to the upper aluminum plate. Using an O-ring and four bolts, the upper aluminum plate was connected to the 
cell. Through the water-wet ceramic plate, the soil sample was flushed with carbon dioxide for fifteen minutes and saturated with water allowing at least three pore volumes of water to pass through the cell. Then water was drained from the porous medium and held constant at an initial value while heptane was introduced to the porous medium at different saturations through the porous cup connected to the heptane burette. After the heptane was introduced into the porous medium, the heptane burette was lowered in steps which allowed the heptane to drain until it reached the residual saturation. At the completion of this stage of the experiment, a volume of heptane sufficient to raise the heptane saturation to an upper target value was added to the porous medium. The drainage procedure was repeated to determine a second saturation path and final residual heptane saturation. This process (i.e., heptane imbibition and drainage) was repeated until the apparent total liquid saturation was equal to unity, then heptane was drained, and the residual heptane saturation was determined. This procedure was applied for the three experimental data sets discussed earlier.

The Tempe cells were also used to measure air/water $P_{c}-S$ curves in uniform and well graded NAPL-wet sands. To set up these experiments, the transducer ports were plugged. Then, the water-wet ceramic plate was flushed with carbon dioxide for fifteen minutes and saturated with water allowing at least three pore volumes of water to pass through the ceramic plate. The excess water was removed and the soil sample was placed in each cell. Then the holes in the upper aluminum plate were plugged and a NAPL-wet porous cup was connected to the upper aluminum plate. Using an O-ring and four bolts, the upper aluminum plate was connected to the cell. Then, the water surface in the burette was brought to a level corresponding to the REV midpoint. At this point, the 
porous medium was dry and capillary pressure head was zero, which represented the starting point of the water main imbibition pathway. Then, the water burette was raised in steps, maintaining negative capillary pressure, until the porous medium became apparently water-saturated. For the water main drainage pathway, the burette was lowered in steps, maintaining positive capillary pressure, until the water saturation reached an irreducible value.

\subsection{2.a. Porous Ceramic Plates}

Choosing the porous ceramic plate pore sizes was a major step in selecting the appropriate ceramic plates to control fluid flow in a multiphase flow experiment. The size of the largest pore in a ceramic plate was selected such that the external capillary pressure should not exceed the plate entry pressure. This way it was ensured that only the fluid phase saturating the ceramic plate flows through its corresponding plate. The entry pressure was a function of the tested fluids and ceramic plate maximum pore size. It was also important to take into account that the time required to reach a steady state condition after every external pressure increment is a function of the ceramic plate pore sizes and the corresponding hydraulic conductivity of the plate. Steady state conditions can be achieved more rapidly for ceramic plates with larger pore sizes.

Ceramic Plate Used: The part number for the ceramic plates used in the Tempe cell experiments was 0604D02.5-B0.5M2 from Soilmoisture Equipment Corp. It is a high flow ceramic plate with diameter of $6.668 \mathrm{~cm}$ and thickness of $0.714 \mathrm{~cm}$. Its air entry pressure is one half bar.

Ceramic Cup Used: The part number for the ceramic cup was 0652X03-B01M3 from Soilmoisture Equipment Corp. It is a high flow porous cup with an outer diameter of 
$0.635 \mathrm{~cm}$, length of $2.858 \mathrm{~cm}$, and wall thickness of $0.16 \mathrm{~cm}$. Its air entry pressure is one bar.

NAPL-Wet Ceramic Plates and Porous Cups: Ceramic plates and porous cups were treated to render them NAPL-wet. The treatment procedure is described in detail in Chapter 4.

\subsection{2.b. Pressure Transducers Calibration}

Ten transducers were calibrated, although six transducers were used in the threephase experiments intended to validate Leverett assumption. Three transducers were attached to each Tempe cell; one transducer measured the water pressure and two transducers measured heptane pressure. The transducer measuring the water pressure was connected to a water-wet porous ceramic cup and the transducers measuring the heptane pressures were connected to NAPL-wet porous ceramic cup. In order to ensure that the transducer cavities were filled with water or heptane, the procedure explained in Van Geel and Sykes (1994) was followed. Prior to use in the Tempe cell, the transducers were calibrated and checked for accuracy.

All the transducers were calibrated to determine their calibration curves and to verify linearity, accuracy, and drift. The calibration procedure was achieved by attaching the transducers to a horizontal manifold through threaded connectors and Teflon tape to create a water tight seal. After attaching the pressure transducers to a horizontal manifold, the latter was attached to a water reservoir through flexible tubing and slowly filled with water. A procedure was followed when filling the manifold with water to ensure that the system was air free. Water suction head was changed by raising and lowering the water reservoir. It is worth mentioning that transducers were calibrated before and after a three- 
phase experiment to ensure stability of transducers' readings. In each time, three cycles of raising and lowering of the water reservoir were performed. A cycle was achieved by raising and lowering the water reservoir in a suction head range from $-140 \mathrm{~cm}$ to zero and from zero to $-140 \mathrm{~cm}$, respectively. Each raising or lowering of the water reservoir was performed in eight steps. Transducers were connected to a data acquisition system which gave a voltage reading for each transducer at each applied suction head value. The transducers were powered by a $10-\mathrm{V}$ power supply.

The relationship between the voltage reading and the applied suction head was linear for all transducers. Table 3.4 shows transducer calibration equations together with the maximum absolute error and the standard error. Transducers were also checked for drift which showed no detected trend for voltage reading change at a zero suction head.

Table 3.4: Transducers Calibration Equations, Maximum Absolute Error, and Standard Error.

\begin{tabular}{|c|c|c|c|c|c|}
\hline Transducer & Slope & Intercept $(\mathbf{c m})$ & $\boldsymbol{R}^{\mathbf{2}}$ & $\begin{array}{c}\text { Maximum } \\
\text { Absolute Error } \\
(\mathbf{c m})\end{array}$ & $\begin{array}{c}\text { Standard } \\
\text { Error }(\mathbf{c m})\end{array}$ \\
\hline $\mathbf{T}_{\mathbf{1}}$ & -14.61 & -10.95 & 0.99999 & 0.53 & 0.04 \\
\hline $\mathbf{T}_{\mathbf{2}}$ & -14.26 & -14.49 & 0.99999 & 0.59 & 0.03 \\
\hline $\mathbf{T}_{\mathbf{3}}$ & -14.25 & -3.992 & 0.99999 & 0.51 & 0.02 \\
\hline $\mathbf{T}_{\mathbf{4}}$ & -14.38 & -4.944 & 0.99999 & 0.74 & 0.03 \\
\hline $\mathbf{T}_{\mathbf{5}}$ & -14.26 & -12.18 & 0.99999 & 0.57 & 0.03 \\
\hline $\mathbf{T}_{\mathbf{6}}$ & -14.11 & 7.762 & 0.99999 & 0.58 & 0.03 \\
\hline $\mathbf{T}_{\mathbf{7}}$ & -14.47 & -3.502 & 0.99999 & 0.45 & 0.03 \\
\hline $\mathbf{T}_{\mathbf{8}}$ & -14.24 & -0.48 & 0.99999 & 0.52 & 0.03 \\
\hline $\mathbf{T}_{\mathbf{9}}$ & -14.45 & -4.998 & 0.99999 & 0.38 & 0.03 \\
\hline $\mathbf{T}_{\mathbf{1 0}}$ & -14.21 & -15.3 & 0.99999 & 0.58 & 0.03 \\
\hline
\end{tabular}

Number of data points for each transducer $=48$ 


\subsubsection{Evaporation Control Cell}

In order to correct for evaporation in the Tempe cell and Buchner funnel experiments, control cells were set up. A control cell was composed of a liquid-filled Buchner funnel, flexible tubing, and a burette. The geometry and dimensions of the control cell, shown in Figure 3.6 were similar to those used in the $P_{c}-S$ or residual NAPL saturation experiments. Three control cells were used; the first was filled with water, the second was filled with heptane, and the third was filled with decane. Then the Buchner funnel and the burette were covered by parafilm with a small hole punctured through the centre of each in order to allow for the movement of air while reducing evaporation (i.e., similar to two-phase and three-phase experiments). The evaporated liquid volume was measured each time the corresponding liquid saturation was measured in the $P_{c}-S$ or residual NAPL saturation experiments. Then the liquid saturation was corrected for evaporation. 


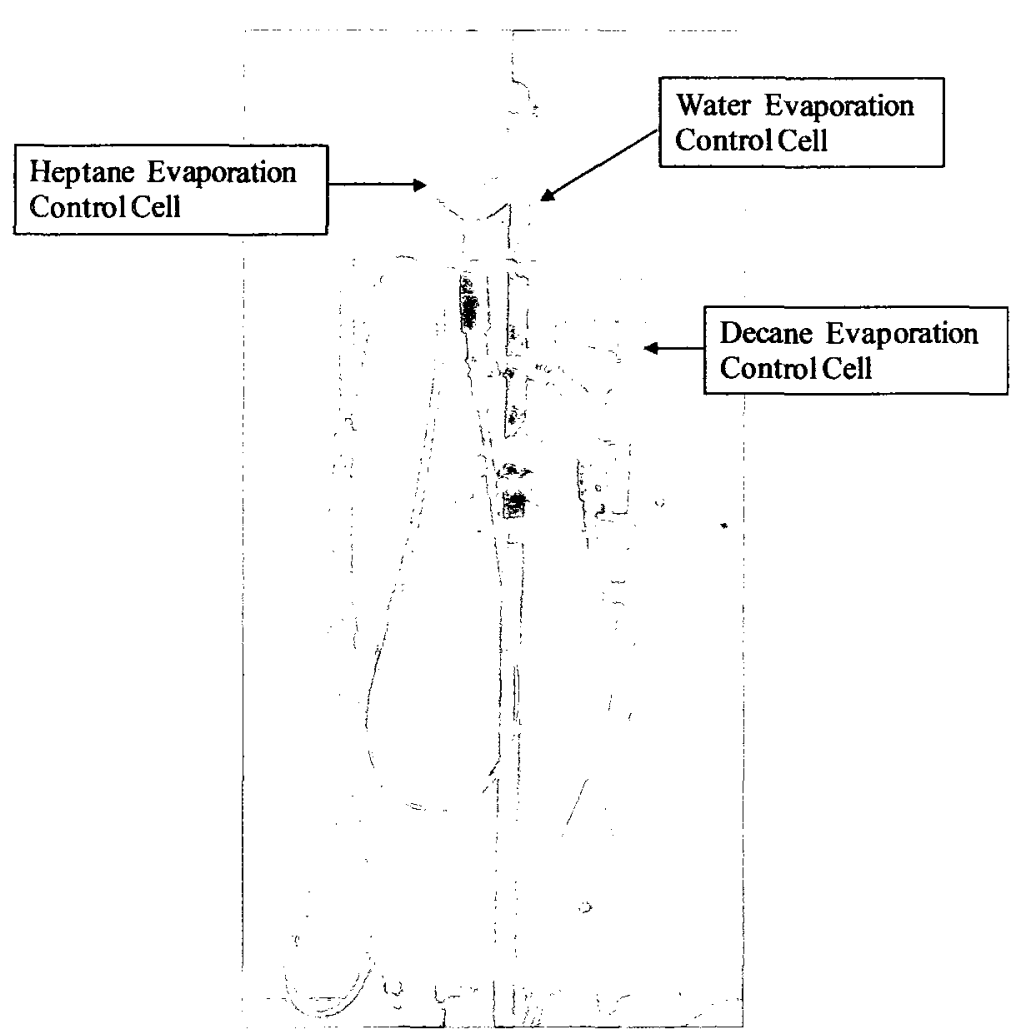

Figure 3.6: Evaporation Control Cells Setup.

\subsubsection{Glass Box Apparatus}

For the pore scale study, pressure cells assembled from glass plates as front, rear, and side walls, and an aluminum plate as a base were filled with glass beads as shown in Figure 3.7. Two-phase, air/water and heptane/water, and three-phase, air/NAPL/water, experiments were conducted in the glass box apparatus for the glass beads with uniform grain size distribution under different wettability conditions. 
(A) 7

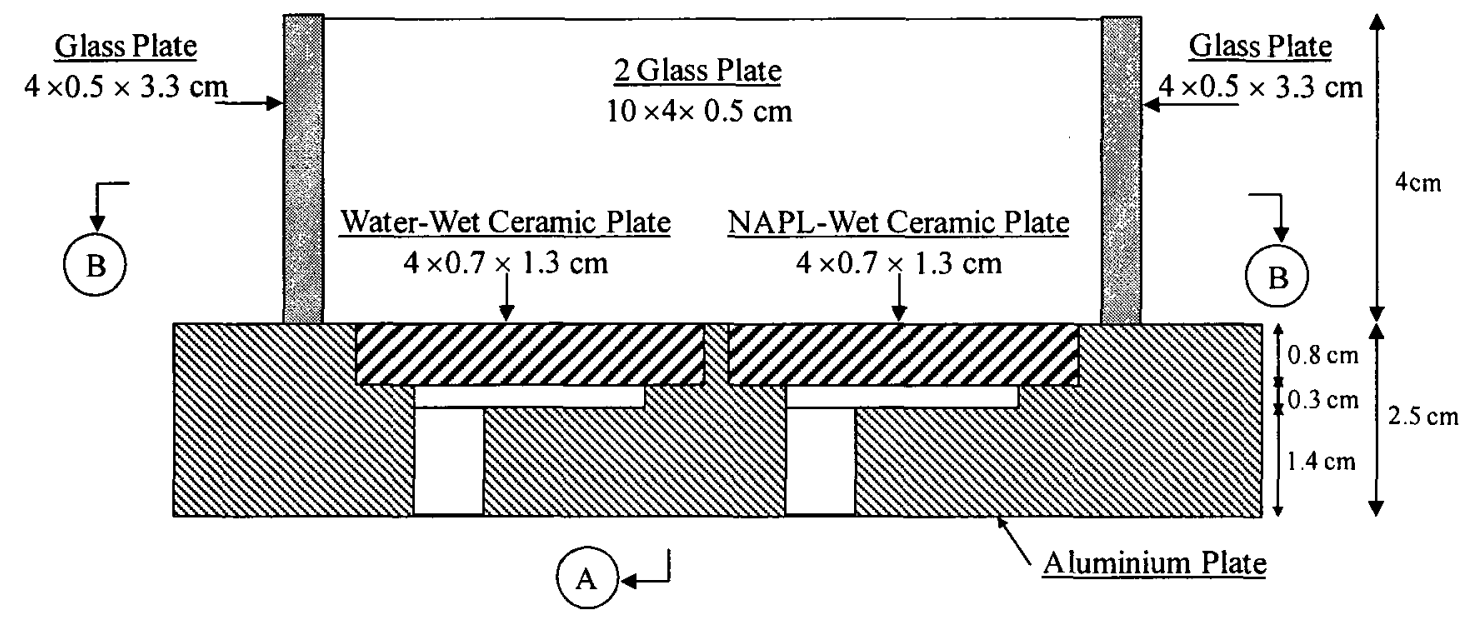

Figure 3.7a: Front View.

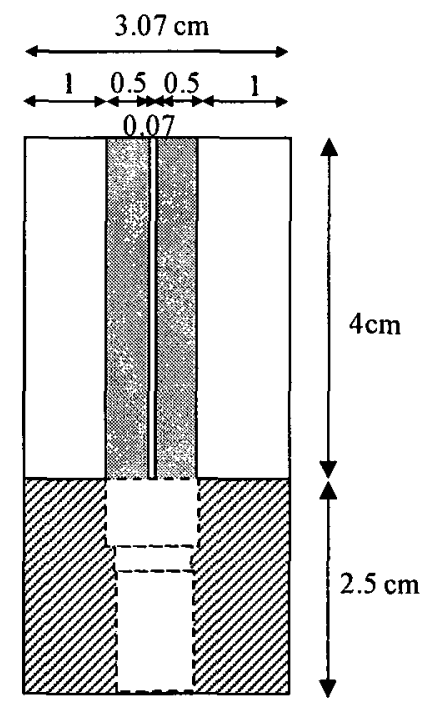

Figure 3.7b: Section A-A. 


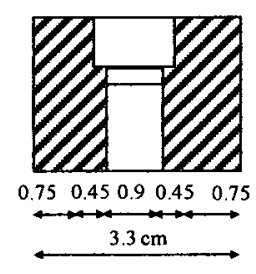

Section 2-2

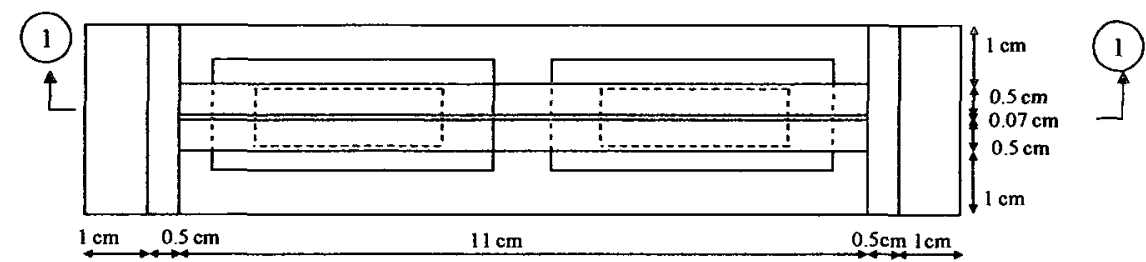

(2) $4.5 \mathrm{~cm}$

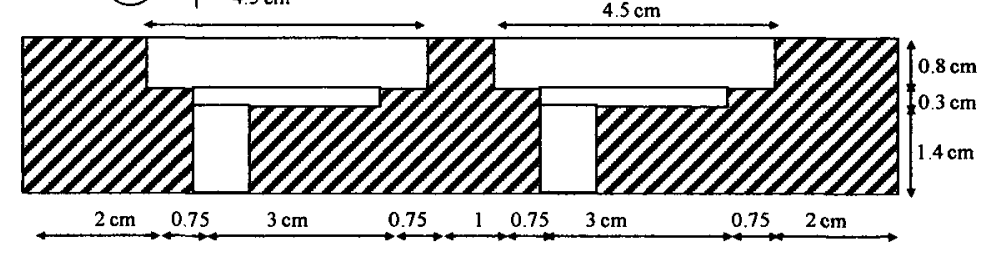

$14 \mathrm{~cm}$
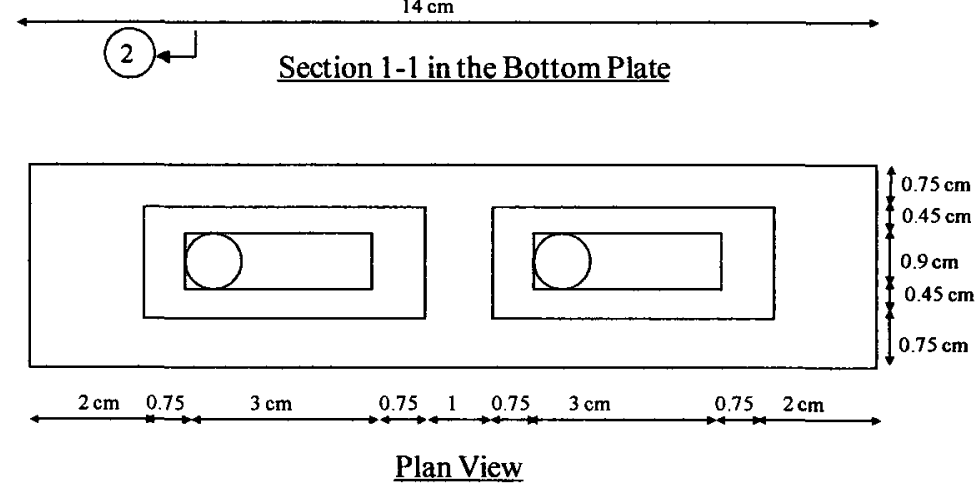

Figure 3.7c: Additional Cross Sections.

Figure 3.7d: Assembled Glass Box.

Figure 3.7: Glass Box Apparatus. 
The test procedure for the glass box pressure cell was similar to the one explained for the modified Tempe cell or the Buchner funnel. The difference between them lies, first, in using glass beads in the glass box pressure cell, and second, both of the water-wet and NAPL-wet ceramic plates were placed in the aluminum plate. The ceramic plates required for the glass box pressure cells were high flow with air entry pressure of one half bar. Their dimensions are $4 \mathrm{~cm} \times 1.3 \mathrm{~cm} \mathrm{x} 0.7 \mathrm{~cm}$. Pictures of the fluid distributions at the pore scale were taken using a digital camera attached to a stereomicroscope (Olympus, SZH10), as shown in Figure 3.8.

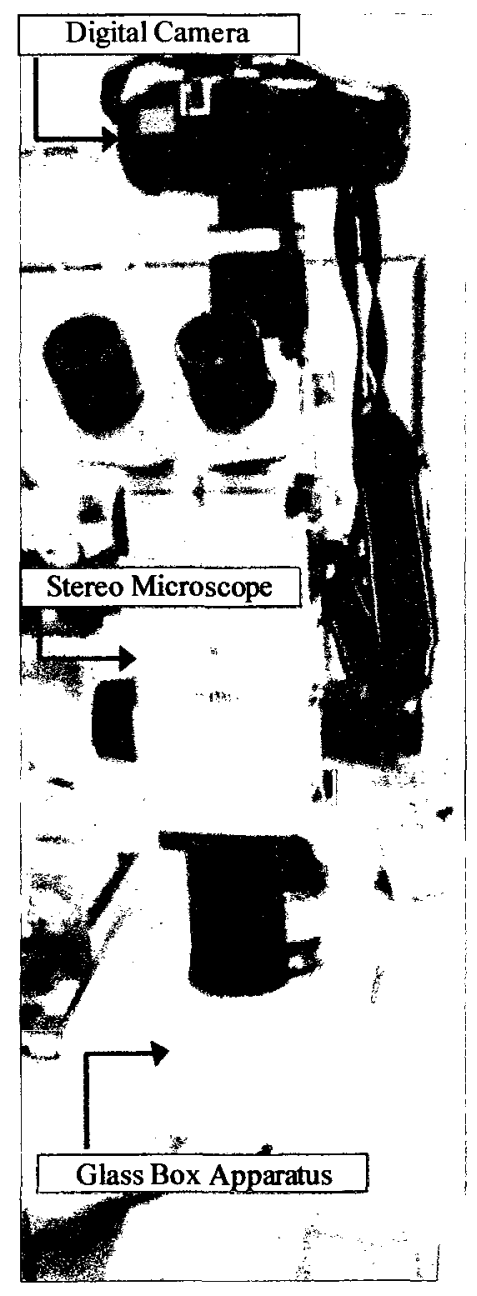

Figure 3.8: Pore Scale Experimental Setup. 


\subsection{Measurement Error}

There are different sources of errors in $P_{c} \rightarrow S$ experiments or in residual NAPL saturation experiments, such as the following:

1. Precision of the burette reading $(0.05 \mathrm{~mL})$;

2. Precision of the ruler reading (measuring water or heptane pressure heads) $(0.05$ $\mathrm{cm})$;

3. Precision of the calliper $(0.01 \mathrm{~cm})$;

4. Accuracy of the pressure transducers;

5. Accuracy of the scale for weighing soil and liquid, $(0.05 \mathrm{~g})$; and

6. Potential contamination which might result in changes in interfacial tensions.

\subsubsection{Potential Error in Measuring Capillary Pressure Head}

For two-phase experiments, the error associated with measuring capillary pressure head can be calculated for the different fluid pairs as follows:

- Air/water two-phase system: The measurement error $=0.05 \mathrm{~cm}$ (maximum error in locating the soil sample centreline) $+0.05 \mathrm{~cm}$ (maximum error in locating water level in the burette) $= \pm 0.1 \mathrm{~cm}$.

- Air/heptane two-phase system: The measurement error $=0.68$ (heptane specific gravity) $\times(0.05 \mathrm{~cm}$ (maximum error in locating the soil sample centreline) +0.05 $\mathrm{cm}$ (maximum error in locating heptane level in the burette) $)= \pm 0.068 \mathrm{~cm}$.

- Heptane/water two-phase system: The measurement error $=0.05 \mathrm{~cm}$ (maximum error in locating the soil sample centreline) $+0.05 \mathrm{~cm}$ (maximum error in locating water level in the burette) $+0.05 \mathrm{~cm}$ (maximum error in locating heptane depth above a soil sample) $x 0.68$ (heptane specific gravity) $= \pm 0.134 \mathrm{~cm}$. 
For three-phase experiments, the air/heptane capillary pressure head was measured with the aid of transducers. The transducers were calibrated several times and resulted in a standard error (i.e., standard deviation) range of $\pm 0.02 \mathrm{~cm}$ to $\pm 0.04 \mathrm{~cm}$, depending on the transducer, as shown in Table 3.4.

\subsubsection{Potential Error in Measuring a Liquid Saturation}

Fluid phase saturation is calculated as follows:

$$
S=\frac{\text { volume of liquid stored in pore space } \pm \text { volume of liquid drained or imbibed }}{\text { volume of voids }}
$$

In order to estimate the value of the measurement error, the method by which each term in Equation (3.1) was measured, is discussed. Then the error associated with each term was estimated. The saturation measurement error was calculated for two cases corresponding to drainage and imbibition. The volume of voids in the soil sample was determined from the soil sample volume and porosity. It may also be determined using the mass balance of the wetting fluid. For a water-wet soil or for an air/heptane two-phase system, it was found that the volume of voids calculated using the soil sample volume and porosity, or using the wetting fluid mass balance method was the same. In a waterwet porous medium with NAPL-wet soil lenses or a NAPL-wet porous medium for air/water or heptane/water two-phase system, or three-phase system, the volume of voids was calculated using the soil sample volume and porosity. In this case, the mass balance method was used to determine the water stored in the pore space at the beginning of the $P_{c} \rightarrow S$ experiment or residual NAPL saturation experiment.

Volume of voids $=($ porosity + porosity standard error $) x($ volume + error in volume $)$ 
The average and standard deviation (i.e., standard error based on 5 replicates) of the porosity were 0.374 and 0.001 , respectively, for the uniform sand. The corresponding values for the well graded sand were 0.333 and 0.002 , respectively. The average and standard deviation (i.e., standard error based on 5 replicates) of the dry density were $1.658 \mathrm{~g} / \mathrm{cm}^{3}$ and 0.0018 , respectively, for the uniform sand. The corresponding values for the well graded sand wer $1.767 \mathrm{~g} / \mathrm{cm}^{3}$ and 0.004 , respectively. Using the soil sample dry density, the mass of the soil sample volume was determined and weighed. Therefore:

$$
\begin{aligned}
V_{v}= & \eta V \pm \frac{\left(\eta^{*} \text { scale precision }\right)}{\text { (sand dry density } \pm \text { density standard error })} \\
& \pm \eta \text { standard error } * \mathrm{~V}+\frac{(\eta \text { standard error } * \text { scale precision })}{\text { (sand dry density } \pm \text { density standard error })}
\end{aligned}
$$

\section{Main Drainage Pathway}

$$
S=\frac{\text { initial volume of liquid stored in pore space }- \text { volume of liquid drained }}{\text { volume of voids }}
$$

\section{Main Imbibition Pathway}

$$
S=\frac{\text { final volume of liquid stored in pore space }+ \text { volume of liquid imbibed }}{\text { volume of voids }}
$$

Initial volume of liquid stored in void space was calculated using the mass balance method, and it represented the change in liquid storage in the soil sample. This change in storage corresponded to liquid volume needed to saturate a dry soil sample. As shown in Figure 3.3 and Figure 3.5, a Buchner funnel or a Tempe cell was connected to the wetting phase reservoir (i.e., burette) through flexible tubing. During the set-up of a $P_{c} \neg S$ or a residual NAPL saturation experiment, the wetting phase was brought to a level 
right below the porous disc. At this point, the flexible tubing was filled with the wetting phase and the burette was nearly filled and it was at this point that the volumes of fluids entering and leaving the cell were tracked. For the calculations that followed, a beaker, initially filled with the wetting fluid, was used to add wetting fluid to the burette. The error associated with the volume of liquid stored in the void space was the error that might potentially result when weighing the liquid mass before and after the soil sample becomes saturated and due to the precision of the measurement tools.

Initial volume of liquid stored in pore space $=$ weight of the beaker before saturating the soil sample - weight of the beaker after the soil sample was saturated + burette reading after the soil sample was saturated - burette reading before the soil sample was saturated - volume of space under the porous disc + weight of the Buchner funnel (Tempe cell) with water-saturated porous disc - weight of the Buchner funnel (Tempe cell) with dry porous disc $=$ Actual volume of liquid stored in pore space $+6 * 0.05+$ measurement error in volume of space under the porous disc.

The space under the porous disc had a circular cross section of eight centimetre diameter. This space was extended to a depth that was measured in each experiment using a calliper. The maximum value of this depth was $0.5 \mathrm{~cm}$. The precision of the calliper was $0.01 \mathrm{~cm}$.

Initial volume of liquid stored in pore space $=$ Actual volume of liquid stored in pore space $\pm 6^{*} 0.05 \pm\left(0.5^{*}(\pi / 4)^{*}(8.01)^{2}-0.5^{*}(\pi / 4)^{*}(8)^{2}\right) \pm 0.01 *(\pi / 4)^{*}(8)^{2}=$ Actual volume of liquid stored in pore space $\pm 0.9 \mathrm{~mL}$. 
The final volume of liquid stored in pore space was zero with an error of $0.9 \mathrm{~mL}$. The error associated with the volume of liquid drained or imbibed was $0.05 \mathrm{~mL}$ (i.e., the precision of the burette).

To be able to assess the measurement error, the saturations were calculated at known values. These known values correspond to saturated and dry soil samples. For a saturated soil sample, the volume of liquid stored in the soil sample was similar to the volume of voids. The volume of liquid drained or imbibed was zero. In contrast, there was no liquid stored in a dry soil sample. For this case, the volume of liquid drained was the volume of voids and the volume of liquid imbibed as zero. The measurement error in liquid saturations was calculated for the uniform and well graded sands. It was found that the magnitude of the measurement error depended on the liquid saturation such that it equalled $0.1 \%$ when the sample was saturated by the liquid in either sand. In contrast, in a dry soil sample, the measurement error equalled $\pm 1.0 \%$ in uniform sand and $\pm 1.2 \%$ in well graded sand. Based on these error calculations, it was clear that the errors were small and hence were disregarded in the corresponding $P_{c} \rightarrow S$ or residual NAPL saturation data. 


\section{Chapter 4. Concerns Related to Using Octadecyltrichlorosilane in Rendering Soils and Porous Ceramics Hydrophobic}

\subsection{Background}

Significant research efforts have been directed towards evaluating the movement and distribution of NAPL in intermediate-wet and NAPL-wet soils and these studies have been limited to the laboratory. A summary of these research efforts follows. In all cases, the materials used to reflect intermediate-wet or NAPL-wet conditions were not natural soils but were originally water-wet soils that were rendered hydrophobic or NAPL-wet through the use of a treatment process, typically using octadecyltrichlorosilane (OTS) solution in ethanol followed by triplicate rinses in fresh ethanol and then air-dried as proposed by Anderson et al. (1991). This treatment process is referred to as the traditional OTS treatment procedure and is the focus of this chapter.

Bradford and Leij (1995b) conducted a number of $P_{c}-S$ experiments with "fractional wettability" soils (i.e., soils that contained a fraction of water-wet soil and a fraction of NAPL-wet soil) and demonstrated that the NAPL-wet soil fraction shifted the $P_{c} S$ relationship to negative capillary pressures (i.e., higher water pressure than NAPL pressure, contrary to the 'ideal' systems typically employed). Using air, soltrol 220, and water, Bradford and Leij (1995b) constructed primary drainage and wetting $P_{c}-S$ curves for air/water, and NAPL/water in two-phase systems and capillary pressure-total liquid saturation curves in three-phase systems for $0 \%, 25 \%, 50 \%, 75 \%$, and $100 \%$ OTS-treated fractions. The OTS-treated soils were shaken in a $5 \%$ (by volume) OTS solution in ethanol for five hours after which the treated soil was air-dried. The authors reference the 
work of Anderson et al. (1991) for the treatment procedure and hence it is assumed that the soils were rinsed in ethanol as per the traditional treatment procedure.

Bradford et al. (1999) conducted an experimental study to evaluate the effects of hydrophobic silica sand fractions on the magnitude and dissolution of entrapped perchloroethylene (PCE) in porous media. They used OTS-treated Ottawa sands to generate fractionally wet sands with hydrophobic fractions corresponding to $0 \%, 10 \%$, $25 \%, 50 \%, 75 \%$, and $100 \%$. The sands were exposed to different saturation pathways for PCE/water fluid pair and the magnitude of the entrapped PCE as a function of the hydrophobic sand fraction was evaluated. The Ottawa silica sand was rendered hydrophobic using the traditional OTS treatment procedure.

O'Carroll et al. (2004) used PCE as the NAPL and Milli-Q water as the aqueous phase in two-dimensional experiments intended to quantify spatial wettability variations on DNAPL movement and entrapment. The porous medium was composed of hydrophilic Ottawa silica sand with one layer of hydrophobic sand and two hydrophobic sand lenses. One-dimensional columns were used to measure $P_{c}-S$ relationships for each of the hydrophilic and hydrophobic porous media. In addition, O'Carroll et al. (2005) conducted a series of two-phase $P_{c} \neg S$ experiments to validate their scaling approach known as the Leverett-Cassie model. They measured water/PCE primary drainage and wetting $P_{c}-S$ pathways in fractionally wet porous media. In both studies, the Ottawa silica sands were treated using the traditional OTS treatment procedure.

To delineate the forms of the entrapped NAPL and the pore space network and to obtain the relationships between the pore network geometry and the entrapped NAPL characteristics, Al-Raoush and Wilson (2005) used synchrotron X-ray tomography to 
capture pore scale images. Hydrophobic glass beads were packed in plastic columns of 5 $\mathrm{mm}$ inner diameter and $7 \mathrm{~cm}$ length, Iodononane-doped soltrol 220 functioned as the $\mathrm{NAPL}$, and $\mathrm{NaBr}$-doped water represented the aqueous phase. The glass beads were rendered hydrophobic using the same traditional OTS treatment procedure.

In summary, to investigate the impacts of soil wettability on the constitutive relationships and distribution of NAPL in multiphase systems, the above studies treated the soil and ceramics to render them hydrophobic using a $5 \%$ (by volume) solution of OTS in ethanol followed by triplicate rinsing in ethanol and air drying as proposed by Anderson et al. (1991). As stated previously, this treatment process is the focus of this chapter as outlined in the motivation for this work which follows.

\subsection{Motivation}

As explained in Chapters 1 and 3, the original motivation for this research was to study the impacts of soil wettability on the $P_{c} \neg S$ relationship and the formation and distribution of residual NAPL in variably wet porous media. Preliminary experiments in this area generated some unexpected results which led to the focus of this chapter. Twophase $P_{c} \neg S$ experiments were set up for a hydrophilic silica sand porous medium with an OTS-treated NAPL-wet soil lens. The sands were treated using traditional OTS treatment procedure. During the experiments, the wetting saturation pathways resulted in significantly larger entrapped NAPL saturations than expected. It was hypothesized that excess unbound OTS on the treated soil surface was dissolved in the NAPL and subsequently impacted the wettability of the surrounding hydrophilic silica sand, which in turn increased the entrapped NAPL saturations. This chapter presents the results of the initial two-phase $P_{c}-S$ experiments and verifies the hypothesis that unbound OTS remains 
on surfaces treated using the traditional OTS treatment procedure, and that this unbound OTS dissolves into a wide range of solvents used in the literature to investigate the impacts of soil wettability on the constitutive relationships and distribution of NAPL in multiphase systems. In addition, this study presents a treatment procedure which reduces the excess unbound OTS on OTS-treated hydrophobic solids.

\subsection{Conceptual Description of Rendering Hydrophilic Solids Hydrophobic and the}

\section{Existence of Excess OTS}

Octadecyltrichlorosilane, also known as n-Octadecyltrichlorosilane, Trichlorooctadecylsilane, and Stearyltrichlorosilane or simply OTS, is a colorless liquid, flammable, and reacts violently with water. Its density is $0.984 \mathrm{~kg} / \mathrm{L}$, boiling point is $223^{\circ} \mathrm{C}$, and structural chemical formula is $\mathrm{CH}_{3}\left(\mathrm{CH}_{2}\right)_{17} \mathrm{SiCl}_{3}$. OTS is an organometallic compound, since it contains organic (i.e., carbon) and metallic (i.e., silicon) elements. It is also an amphiphilic compound consisting of a long chain alkyl group $\left(\mathrm{Cl}_{8} \mathrm{H}_{3} \tau\right)$, which is hydrophobic, and a polar head group $\left(\mathrm{SiCl}_{3}-\right)$, which is hydrophilic. In order to accelerate the OTS reaction with hydrophilic solids, a diluted solution of OTS in ethanol was first prepared where OTS was catalyzed in ethanol forming a derivative of OTS with a chemical formula of $\mathrm{CH}_{3}\left(\mathrm{CH}_{2}\right)_{17} \mathrm{Si}\left(\mathrm{OC}_{2} \mathrm{H}_{5}\right)_{3}$ (Schreiber 2000, Yoshida et al. 2001). When this OTS derivative comes in contact with hydrophilic solids, the OTS derivative's polar head group $\left(\mathrm{Si}\left(\mathrm{OC}_{2} \mathrm{H}_{5}\right)_{3}\right)$ may react with the hydrophilic solid while the OTS derivative's long chain alkyl group $\left(\mathrm{CH}_{3}\left(\mathrm{CH}_{2}\right)_{17}\right)$ provides the hydrophobic property of the treated solids. This reaction results in the formation of a thin film of Self-Assembled Monolayer (SAM) on the treated solids. The hydrophilic solids could be oxidic substrates such as aluminum (III) oxide $\left(\mathrm{Al}_{2} \mathrm{O}_{3}\right)$ and silicon dioxide $\left(\mathrm{SiO}_{2}\right)$. The formation of the 
SAM is known as silanation of the hydrophilic solids (Maoz and Sagiv 1984, Silberzan et al. 1991). It is hypothesized that part of the catalyzed OTS compound in ethanol may not react with the hydrophilic solids and exists as free or excess derivative of OTS present but not bonded to the treated solids.

For convenience, the expression "unbound OTS" or "excess OTS" will be used instead of "excess unbound derivative of OTS". Figure 4.1 illustrates the conceptual understanding for the existence of the derivative of OTS molecules, those bonded to the silica sand particles and those present as excess OTS molecules. Under ambient pressure and temperature conditions, the bond of the OTS derivative to the treated solids is very stable in most solvents. Kluth et al. (1997) and Cha and Kim (2001) stated that the chemical bond between the derivative of OTS and silica becomes unstable for temperatures higher than $100^{\circ} \mathrm{C}$ at ambient pressure. It is hypothesized that excess OTS molecules exist on the treated surface and that the excess OTS molecules may dissolve in a solvent when the solvent comes in contact with these hydrophobic solids.
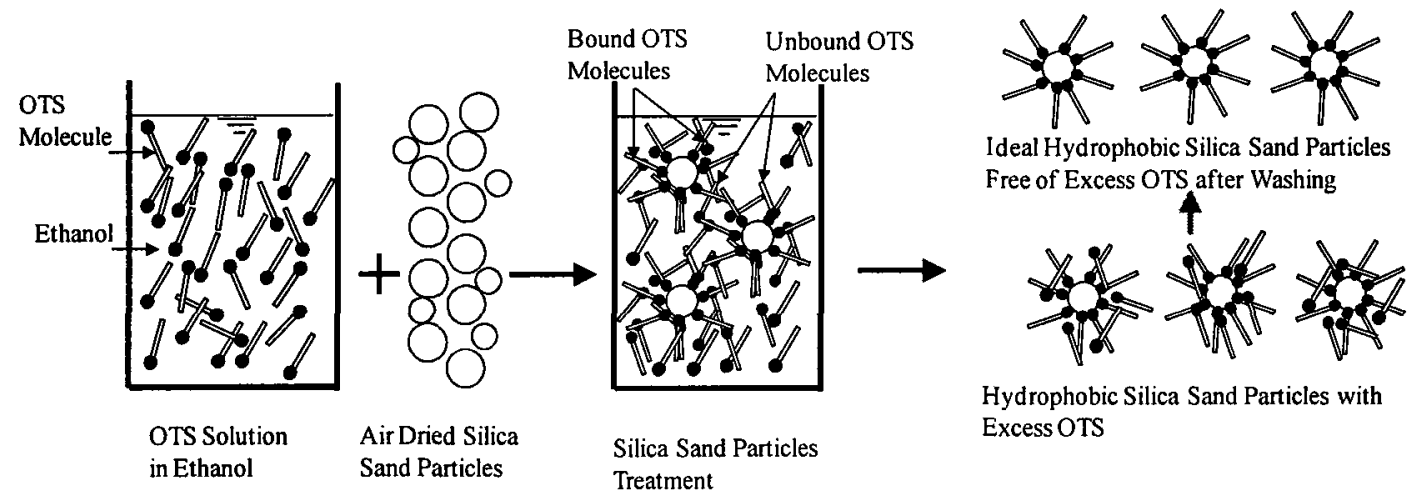

Hydrophobic Silica Sand Particles with Excess OTS

Figure 4.1: Conceptual Illustration of Bound and Unbound OTS Molecules to Silica Sand Particles. 


\subsection{Materials and Methods}

\subsubsection{Basic Soil Treatment}

The soil used in these experiments was Ottawa silica sand (Opta Minerals Inc., Waterdown, Ontario) with $98 \% \mathrm{SiO}_{2}$ (weight basis) which was composed of one sieve size representing a medium sand $(100 \%$ passing U.S. sieve number 30 and retained on U.S. sieve number 40). In order to render a silica sand sample hydrophobic, a specified amount of OTS (90+\%, Sigma-Aldrich, Inc., 3050 Spruce Street, St. Louis, USA) was mixed with either ethanol (Anhydrous Ethyl Alcohol, Commercial Alcohols Inc., Brampton, Ontario, Canada, $78.3^{\circ} \mathrm{C}$ boiling point) or tetrahydrofuran $(99+\%$, Fisher Scientific, Fair Lawn, New Jersey 07410, USA) for three minutes; this mixture is referred to as the OTS solution and results in catalyzed OTS. The specified amount of OTS was a variable considered in this study. A soil sample was then added to a container containing the OTS solution such that the OTS solution saturated and covered the soil sample. The container was sealed and placed in a shaker for 12 hours after which the excess OTS solution was drained and the soil was rinsed a number of times with either ethanol, tetrahydrofuran, or hexane (spectranalyzed 99.9\%, Fisher Scientific, Fair Lawn, New Jersey 07410 , USA, $62-69^{\circ} \mathrm{C}$ boiling point) and air-dried. The number of times the treated soil was rinsed with solvent was also a variable considered in this study.

\subsection{2. $P_{c} \rightarrow S$ Curves}

Initially, a hydrophilic silica sand sample was rendered hydrophobic using $5 \%$ (by volume) OTS solution in ethanol followed by rinsing two or eight times with ethanol and then air-dried (similar to the traditional OTS treatment procedure). Two-phase $P_{c} \rightarrow S$ 
curves were constructed using a Buchner funnel for decane/water and heptane/water fluid pairs. The Buchner funnel and its other components were shown previously in Figure 3.2. Initially two experiments were conducted for a porous medium composed of $75 \%$ (by volume) hydrophilic silica sand with $25 \%$ hydrophobic silica sand in the form of a lens. The hydrophobic sand lens used for decane/water experiment was taken from the treated sand that had been washed two times with ethanol while the hydrophobic sand lens used for heptane/water experiment was taken from the treated sand that had been washed eight times with ethanol. A second set of duplicate experiments were conducted for heptane/water for a porous medium composed of $80 \%$ (by volume) hydrophilic silica sand with $20 \%$ hydrophobic silica sand lens; the hydrophobic silica sand was rinsed eight times in ethanol before air drying. In addition, two-phase $P_{c}-S$ relationships for heptane/water were measured for a porous medium composed of $80 \%$ (by volume) hydrophilic silica sand with $20 \%$ hydrophobic silica sand. The hydrophobic silica sand was generated using an enhanced soil washing procedure proposed as part of this study. A schematic of the Buchner funnel with the $80 \%$ hydrophilic and $20 \%$ hydrophobic silica sand is shown in Figure 4.2. Also, heptane/water and decane/water $P_{c}-S$ curves were measured for a hydrophilic porous medium. 


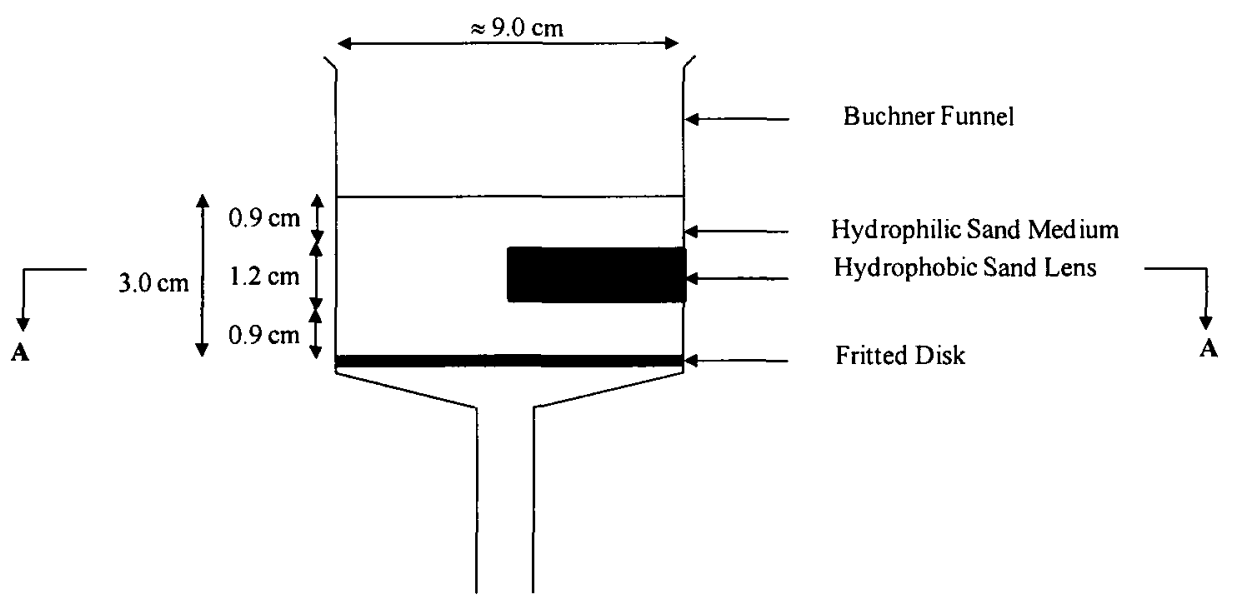

Section B - B

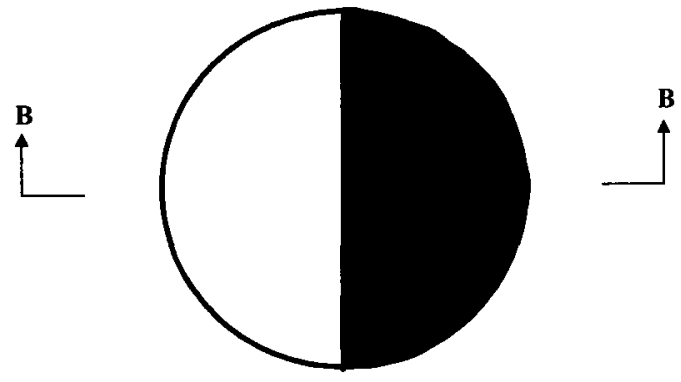

Section A-A

Figure 4.2: Schematic Illustrating the Location and Extent of a Hydrophobic Sand Lens in a Hydrophilic Sand Medium.

\subsubsection{Enhanced Soil Washing Procedure}

In terms of additional soil washing to remove the excess OTS on the hydrophobic silica sands, a soxhlet extractor was used. A soxhlet extractor is an apparatus used for extraction of soluble components from a soil sample by repetitive washing with a solvent (Luque de Castro and Garcia-Ayuso 1998). In this study, the soluble component was the excess OTS. A soxhlet extractor (Figure 4.3) is composed of three main components: condenser, distillation chamber, and flask, which is placed on a heat source. The soil sample is placed in a thimble, made of thick filter paper, in the distillation chamber. The 
extraction solvent is poured into the flask, which is then connected to the distillation chamber containing the soil sample to be washed, and the condenser is connected to the distillation chamber. When all parts of the extractor are connected, the heat source below the flask is turned on and upon reaching the boiling point of the solvent, the solvent vapour travels up through the distillation arm, and then condensates in the condenser and distils in the distillation chamber. As the solvent distils in the distillation chamber, the chamber slowly fills up with the solvent and the excess OTS partitions from the soil sample to the solvent. The solvent temperature in the distillation chamber is close to its boiling point.

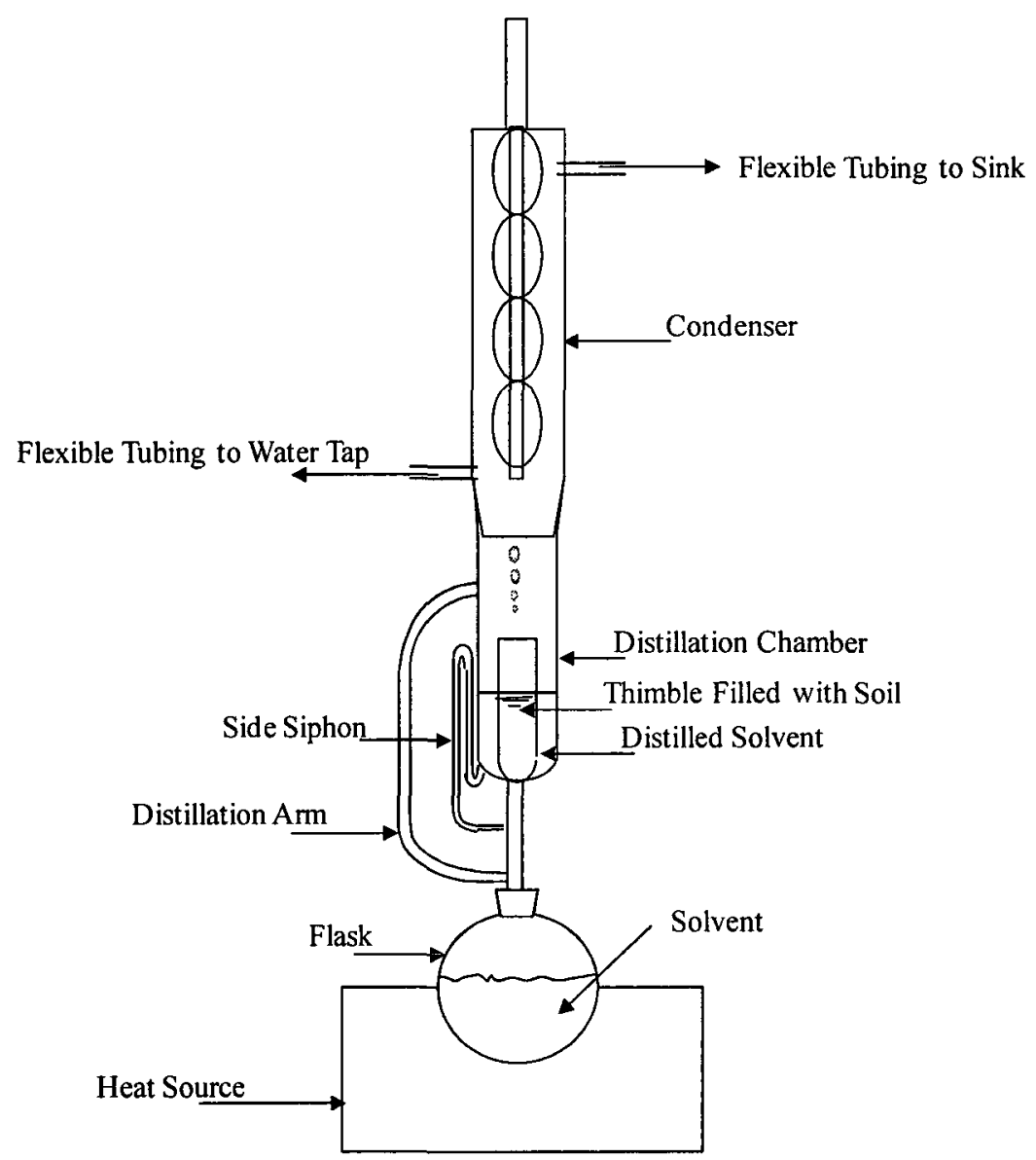

Figure 4.3: Soxhlet Extractor. 
Once the distilled solvent reaches a certain level in the distillation chamber, the latter is automatically emptied through the siphon side arm. This process is continuous and allows for successive washings of the soil sample by a distilled solvent that is free of excess OTS. The excess OTS that is washed from the soil sample remains in the flask as its boiling point is well above that of the solvent. Hence, the soxhlet extractor allows the soil sample to be washed many times to remove the excess OTS. This is important as the goal is to generate hydrophobic sand without any excess OTS. For this study, the solvents used for soil sample washing in the soxhlet extractor were ethanol, hexane, heptane (reagent grade, Fisher Scientific, Fair Lawn, New Jersey 07410 , USA, $98^{\circ} \mathrm{C}$ boiling point), and decane (certified 99.9\%, Fisher Scientific, Fair Lawn, New Jersey 07410 , USA, $174-175^{\circ} \mathrm{C}$ boiling point).

\subsubsection{Verification of Excess OTS Dissolution in a Solvent}

In order to verify the removal of the excess OTS from the hydrophobic sand sample, the following procedure was followed. An OTS-treated sand sample of $20 \mathrm{~g}$ was placed in a beaker containing $15 \mathrm{~mL}$ of clean solvent. With the aid of a magnetic stirrer, the soil sample and the solvent were mixed for 1.5 hours after which, the solvent was drained and poured into another beaker containing $20 \mathrm{~g}$ of a hydrophilic sand sample. Using a magnetic stirrer, the solvent and the hydrophilic sand sample were mixed for another 1.5 hours after which the solvent was drained and the sand was oven-dried for one hour at $95^{\circ} \mathrm{C}$. The wettability condition of the originally hydrophilic sand sample was then checked. If the sand sample remained hydrophilic, it was assumed that no excess OTS remained on the treated sand, and if the sand sample became hydrophobic, it was assumed that excess OTS was still present on the OTS-treated sand. The solvents used to 
check the potential partitioning of excess OTS from the OTS-treated soil samples were; hexane, heptane, decane, toluene (spectro grade 99.5\%, Caledon Laboratories Ltd, Georgetown, Ontario, Canada), soltrol 100 (100\%, Chevron Philips Chemical Company LLC, 10001 Six Pines Drive, the Woodlands, TX 77380, USA), soltrol 220 (99\%, Chevron Philips Chemical Company LLC, 10001 Six Pines Drive, the Woodlands, TX 77380, USA ), and trichloroethylene (TCE) (99.8\%, BDH Inc., Toronto, Ontario, Canada). Each test was performed in triplicate.

\subsubsection{Identifying the Excess OTS Using Nuclear Magnetic Resonance and Infra Red}

\section{Spectroscopy}

In order to determine the chemical structure of the excess OTS that was being dissolved in a solvent (e.g., heptane), Nuclear Magnetic Resonance (NMR) spectroscopy (Bruker Avance $300 \mathrm{MHZ}$ ) and Infra Red (IR) spectroscopy (Varian $1000 \mathrm{FT}$-IR) techniques were used. In the NMR spectroscopy, a sample of the tested substance (i.e., excess OTS) was dissolved in a deuterated chloroform $\left(\mathrm{CDCl}_{3}\right)$ and placed in a glass probe (PABBO BB-1H/D Z-GRD) and then tested in the NMR spectrometer. In the IR spectroscopy, a sample of the tested substance was dissolved in chloroform. Then the dissolved sample was placed on one side of a potassium bromide $(\mathrm{KBr})$ pellet and airdried for 5 minutes to allow the chloroform to vaporize. The $\mathrm{KBr}$ pellet with the tested substance was then tested in the IR spectrometer. The NMR and IR spectra provide information on the number and type of chemical bonds and functional groups in the tested substance. To determine the presence of excess OTS in a solvent, the NMR and IR spectra for tested samples were compared against the NMR and IR spectra for an excess 
OTS standard. Additional details related to the excess OTS standard and the sample preparation are provided in Section 4.5.2.c.

\subsubsection{Determination of Soil Wettability}

The wettability condition of a sand sample was determined by dripping distilled water droplets on the dried sand sample. If the sand sample absorbed the water droplets, then the sand was considered hydrophilic and if the water formed droplets on the surface and was not drawn into the sand, then the sand was considered hydrophobic.

\subsection{Results and Discussion}

\subsection{1. $P_{c} \rightarrow S$ Experimental Results}

The results for two-phase experiments for decane/water and heptane/water fluid pairs are provided in Figure 4.4 and Figure 4.5, respectively. Note the hydrophobic sand lens consisted of sand treated with 5\% (by volume) OTS solution in ethanol and rinsed two and eight times for the decane/water and heptane/water experiments, respectively, followed by air drying (similar to the traditional OTS treatment procedure). For decane/water and heptane/water fluid pairs, the water drainage pathways followed the expected path in a hydrophilic porous medium with a hydrophobic soil lens (i.e., by increasing capillary pressure, water continued to drain and was replaced by either decane or heptane). The initial water saturations are less than unity as the hydrophobic soil lens is not saturated as will be discussed in Chapter 6 . The maximum capillary pressure heads reached were approximately 70 and $45 \mathrm{~cm}$ of water for the decane/water and heptane/water fluid pairs, respectively. The irreducible water saturation for the decane/water fluid pair was 0.075 and the irreducible water saturations for the 
heptane/water fluid pairs were $0.062,0.065$, and 0.075 . In contrast, a problem arose when constructing the water imbibition pathways. There was unexpected resistance to water entering the porous media to replace decane or heptane. When the burettes were raised in steps up to a level corresponding to a capillary pressure head of zero, the actual water saturation in the decane/water experiment was 0.212 corresponding to an entrapped saturation of 0.788 (Figure 4.4). The actual water saturations in the heptane/water experiments at a capillary pressure head of zero were $0.432,0.467$, and 0.471 corresponding to entrapped saturations of $0.568,0.533$, and 0.529 , respectively (Figure 4.5). These entrapped saturations were well above the entrapped saturations measured for the hydrophilic soil ( 0.15 and 0.09 for the decane/water and heptane/water fluid pairs, respectively) and well above typical values published in the literature, even after adjusting for the hydrophobic sand lens. In a hydrophilic silica sand medium, Van Geel and Sykes (1994) reported an entrapped heptane saturation in a heptane/water two-phase system of 0.15 ; while Hofstee et al. (1998) reported an entrapped PCE saturation of 0.125. It is hypothesized that the significantly higher entrapped saturations were due to excess OTS on the hydrophobic soil lens altering the wettability of the surrounding hydrophilic soil. It was also thought that increased washings with fresh ethanol may reduce the excess OTS. The higher entrapped saturation values for decane may be due to two reasons. First, the increased number of washings with ethanol may have removed more of the excess OTS from the OTS-treated sand. In the heptane/water experiment, the OTS-treated sand was washed eight times compared to two washings for the OTS-treated sand used in the decane/water experiment; therefore the heptane/water setup may have had less excess OTS compared to the decane/water setup. Second, excess OTS may be 
more soluble in decane than in heptane. In either case, it was hypothesized that decane and heptane dissolved part of the excess OTS from the OTS-treated sand within the lens and rendered the adjacent hydrophilic sands hydrophobic. This was confirmed by taking samples from the originally hydrophilic sands packed in the funnels, drying them in an oven, and determining their wettability conditions as described in Section 4.4.6. As hypothesized, the hydrophilic sands had become hydrophobic. In order to confirm whether excess OTS had dissolved in decane and heptane, two samples of clean hydrophilic sand were taken and mixed for 1.5 hours with the decane and heptane which had previously been used in the two-phase experiments. After mixing, the sand samples were removed, oven-dried, and their wettability were tested as described in Section 4.4.6. As expected, the originally hydrophilic sand became hydrophobic.

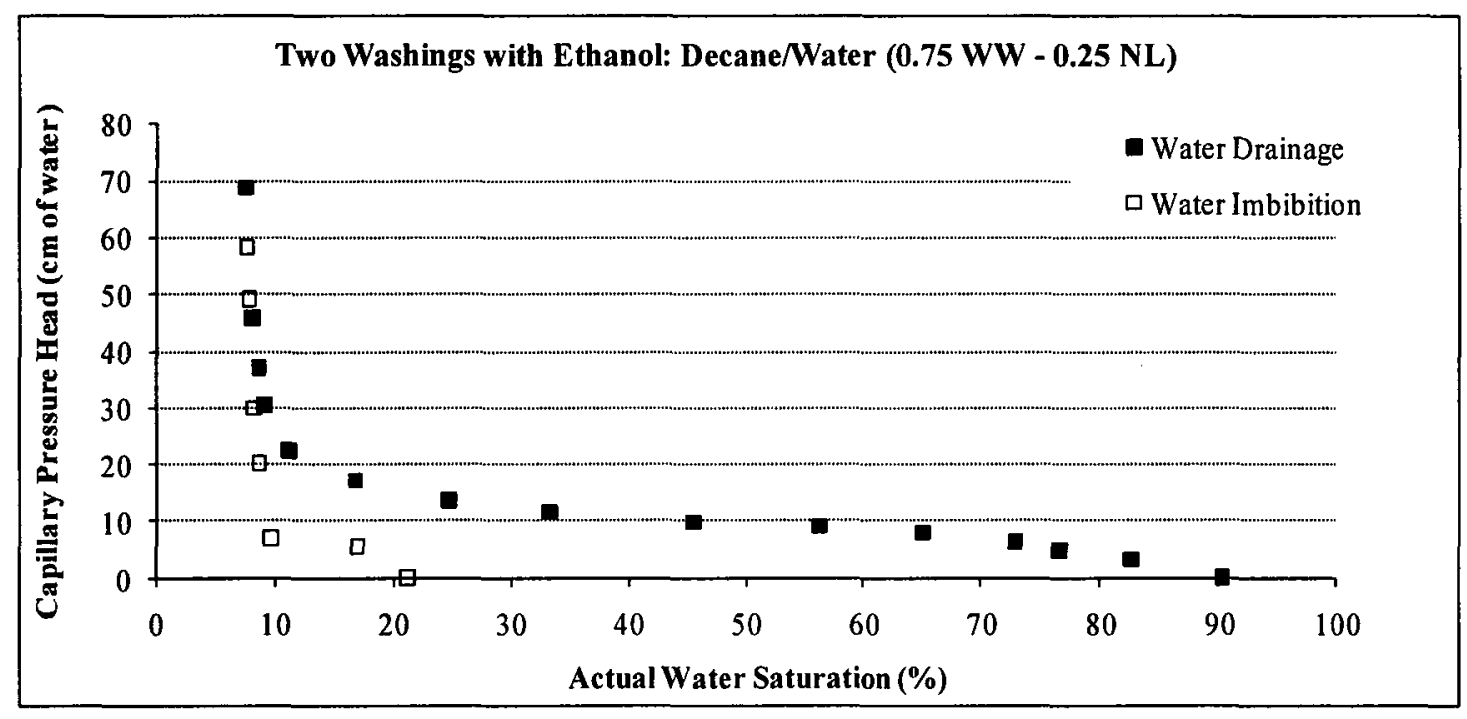

Figure 4.4: Decane/Water Capillary Pressure Head-Actual Water Saturation Pathways. 


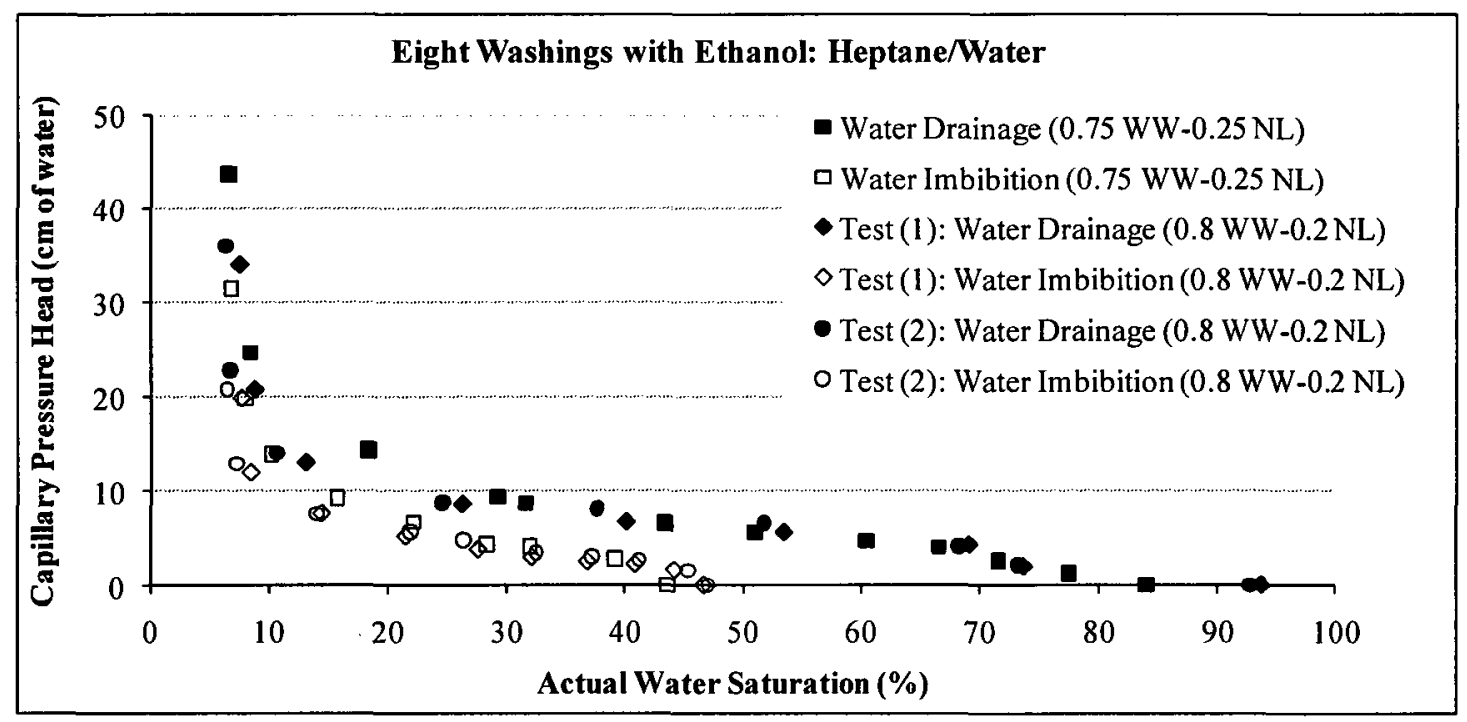

Figure 4.5: Heptane/Water Capillary Pressure Head-Actual Water Saturation Pathways.

\subsubsection{Assessment of OTS Treatment Procedure}

\subsection{2.a. Concentration of OTS Solution}

OTS solutions in ethanol were made at different OTS/ethanol ratios corresponding to $1 \%, 2 \%$, and $5 \%$ (by volume). An air dry silica sand sample was placed in a container with a screw type lid and then the desired OTS solution in ethanol was poured onto the soil until it was immersed. The container was sealed and placed in a shaker for 12 hours after which the OTS solution in ethanol was drained and the soil sample was washed with ethanol. The number of soil sample washings with ethanol varied from two to thirty. For each washing, the ethanol was drained and replaced with fresh ethanol. After the final washing, the soil samples were air-dried in a fume hood (i.e., after two, eight, and thirty washings). This method of treatment using OTS solution in ethanol followed by washing with ethanol is referred to as case (A). The above mentioned procedure was also completed using tetrahydrofuran instead of ethanol and is 
referred to as case (B). Since ethanol is known to be a stronger solvent, for OTS, it was hypothesized that the use of tetrahydrofuran may result in less excess OTS after soil washing. Samples of air dry silica sand treated and washed according to cases (A) and (B) were then washed twelve times in hexane which represents cases (A-C) and (B-C).

The wettability of air-dried OTS-treated sand samples corresponding to cases (A), (B), (A-C), and (B-C) were tested as described in Section 4.4.6. It was shown that all sand samples treated according to cases (A), (B), (A-C), and (B-C) for 5\% OTS/ethanol (tetrahydrofuran) ratios were hydrophobic. In contrast, the sand samples treated with $1 \%$ and $2 \%$ OTS/ethanol (or tetrahydrofuran) ratios remained hydrophilic. Therefore, the OTS in the latter solutions (i.e., $1 \%$ and $2 \%$ ) was more diluted and preferred to remain in solution to such an extent that they did not render the hydrophilic sand samples hydrophobic.

\subsection{2.b. Use of Different Solvents to Dissolve Excess OTS}

Air-dried hydrophobic sand samples, treated according to cases (A), (B), (A-C), and (B-C) using $5 \%$ (by volume) OTS solution, were placed in a beaker containing either heptane, decane, soltrol 100, soltrol 220 , toluene, or TCE. Using a magnetic stirrer, the soil and the solution were stirred for 1.5 hours after which the heptane, decane, soltrol 100 , soltrol 220 , toluene, and TCE were drained and placed in new beakers containing hydrophilic sand samples and were stirred for 1.5 hours. After the stirring was completed, the heptane, decane, soltrol 100 , soltrol 220 , toluene, or TCE were drained into a waste container. The treated hydrophobic and originally hydrophilic silica sand samples were dried in an oven at $95^{\circ} \mathrm{C}$ for one hour and placed in desiccators to cool down to room temperature. The wettability conditions of these sand samples were determined as 
described in Section 4.4.6. The results of which showed that all the hydrophobic sand samples remained hydrophobic and the hydrophilic sand samples became hydrophobic. In order to determine whether the clean NAPLs rendered the hydrophilic sand samples hydrophobic or if dissolved OTS in these NAPLs rendered the sand hydrophobic, clean hydrophilic sand samples were stirred for 1.5 hours in beakers containing clean heptane, decane, soltrol 100, soltrol 220, toluene, or TCE. Then these samples were oven-dried for one hour at $95^{\circ} \mathrm{C}$ and placed in desiccators to cool down to room temperature. The wettability conditions of these sand samples were determined as described in Section 4.4.6. The results of which showed no changes in their wettability condition (i.e., they remained hydrophilic). These results suggest that dissolved excess OTS was the reason behind rendering the hydrophilic sand samples hydrophobic.

In the previous tests, dry sand samples were exposed to the OTS-impacted NAPLs. However, in multiphase flow experiments, hydrophilic sand particles may be coated by a layer of water and this water layer between the NAPL and the solid may restrict the movement of OTS to the solid surface. To evaluate any possible effects, a similar procedure as explained earlier, was conducted with sand samples at different water saturations exposed to OTS-impacted NAPLs. For this test, the NAPL with dissolved OTS was exposed to hydrophilic sand samples at water saturations corresponding to $0.1,0.3$, and 0.5 instead of dry hydrophilic sand samples. For all NAPLs, after being exposed to the OTS-impacted NAPLs, the hydrophilic sand samples became hydrophobic at all water saturations. Hence, it appears that the derivatized OTS was able to migrate from the NAPL through the water before chemically bonding to the sand particles. 
It may be argued that the previous procedure of actively mixing (stirring) the solvents with the hydrophobic and hydrophilic sand samples does not reflect what is typically occurring in laboratory multiphase flow experiments. To address this, air-dried hydrophobic sand samples treated according to case (A) with $5 \%$ OTS/ethanol ratio were placed in heptane, decane, soltrol 100 , soltrol 220 , toluene, or TCE for one day (the typical time for $P_{c} \neg S$ experiment is several days). Then the heptane, decane, soltrol 100, soltrol 220 , toluene, or TCE were drained and placed in beakers containing hydrophilic sand samples and left for another day. Heptane, decane, soltrol 100, soltrol 220, toluene, or TCE were drained into a waste container. The hydrophobic and hydrophilic silica sand samples were dried in an oven at $95^{\circ} \mathrm{C}$ for one hour and placed in desiccators to cool down to room temperature. The wettability conditions of these sand samples were determined as described in Section 4.4.6. The results showed that all the hydrophobic sand samples remained hydrophobic and the hydrophilic sand samples became hydrophobic suggesting that the method of sample preparation (i.e., the mixing of the samples using the magnetic stirrer) had no effect.

\subsection{2.c. Use of NMR and IR Spectroscopy to Evaluate the Excess OTS}

First a sample of the excess OTS in heptane was prepared. In order to prepare this sample, a $100 \mathrm{~g}$ of hydrophobic silica sand sample, treated according to the traditional treatment procedure, was placed in a beaker containing $100 \mathrm{~mL}$ of clean heptane. With the aid of a magnetic stirrer, the soil sample and the heptane were mixed for 1.5 hours. After mixing, the heptane was drained and passed through a fine filter (Whatman, Grade $5,2.5 \mu \mathrm{m})$ and collected in a clean flask. The filtered heptane sample was allowed to evaporate by placing the flask in an oven at $98^{\circ} \mathrm{C}$ for 6 hours. As a control, a volume of 
clean heptane equivalent to the volume of the filtered sample was poured in a flask and placed in an oven at $98^{\circ} \mathrm{C}$ for 6 hours. The flasks were taken out of the oven, covered, and placed in desiccators to cool down to room temperature. At this point, the heptane had evaporated from the flasks. No substance or residue was found in the flask that contained clean heptane. However, a wax-like, off-white, and odourless substance remained in the flask that contained the heptane exposed to the OTS-treated sand. It was suspected that this wax-like substance was excess OTS.

In order to verify that the wax-like substance suspected to be excess OTS was the same as the derivative of OTS $\left(\mathrm{CH}_{3}\left(\mathrm{CH}_{2}\right)_{17} \mathrm{Si}\left(\mathrm{OC}_{2} \mathrm{H}_{5}\right)_{3}\right)$, which results from the reaction of OTS and ethanol, a sample of OTS solution in ethanol was prepared. To prepare the sample, $5 \mathrm{~mL}$ of OTS was added to $95 \mathrm{~mL}$ of ethanol in a clean flask, and they were mixed using a magnetic stirrer for 15 minutes. The flask was then placed in an oven at $98^{\circ} \mathrm{C}$ for 6 hours. As a control, a volume of clean ethanol equivalent to the volume of the prepared sample (i.e., OTS + ethanol) was poured in a flask and placed in the oven at $98^{\circ} \mathrm{C}$ for 6 hours. At this point, all the ethanol evaporated from the flasks and consequently what was left in the prepared sample (i.e., OTS + ethanol) flask was assumed to be the derivative of OTS. The flasks were taken out of the oven, covered, and placed in desiccators to cool down to room temperature. No substance or residue was found in the flask that contained clean ethanol. The derivative of OTS that was left in the flask was wax-like, off-white, and odourless substance.

The wax-like substance from the heptane that was in contact with the OTS-treated sand and the wax-like substance or the OTS derivative from the OTS solution in ethanol were compared using NMR and IR. The NMR spectra for the wax-like substance (from 
the heptane) are shown in Figure 4.6 and Figure 4.7. Figure 4.6 shows the decoupling ${ }^{13} \mathrm{C}$ NMR spectrum $\left(\mathrm{CDCl}_{3}\right)$ while Figure 4.7 shows ${ }^{1} \mathrm{H}$ NMR spectrum $\left(\mathrm{CDCl}_{3}\right)$. The IR spectrum $(\mathrm{KBr})$ is shown in Figure 4.8. The NMR spectra for the OTS derivative are shown in Figure 4.9 and Figure 4.10. Figure 4.9 shows the decoupling ${ }^{13} \mathrm{C}$ NMR spectrum $\left(\mathrm{CDCl}_{3}\right)$ while Figure 4.10 shows ${ }^{1} \mathrm{H}$ NMR spectrum $\left(\mathrm{CDCl}_{3}\right)$. The IR spectrum $(\mathrm{KBr})$ is shown in Figure 4.11.

1.D carbon with proton decoupling

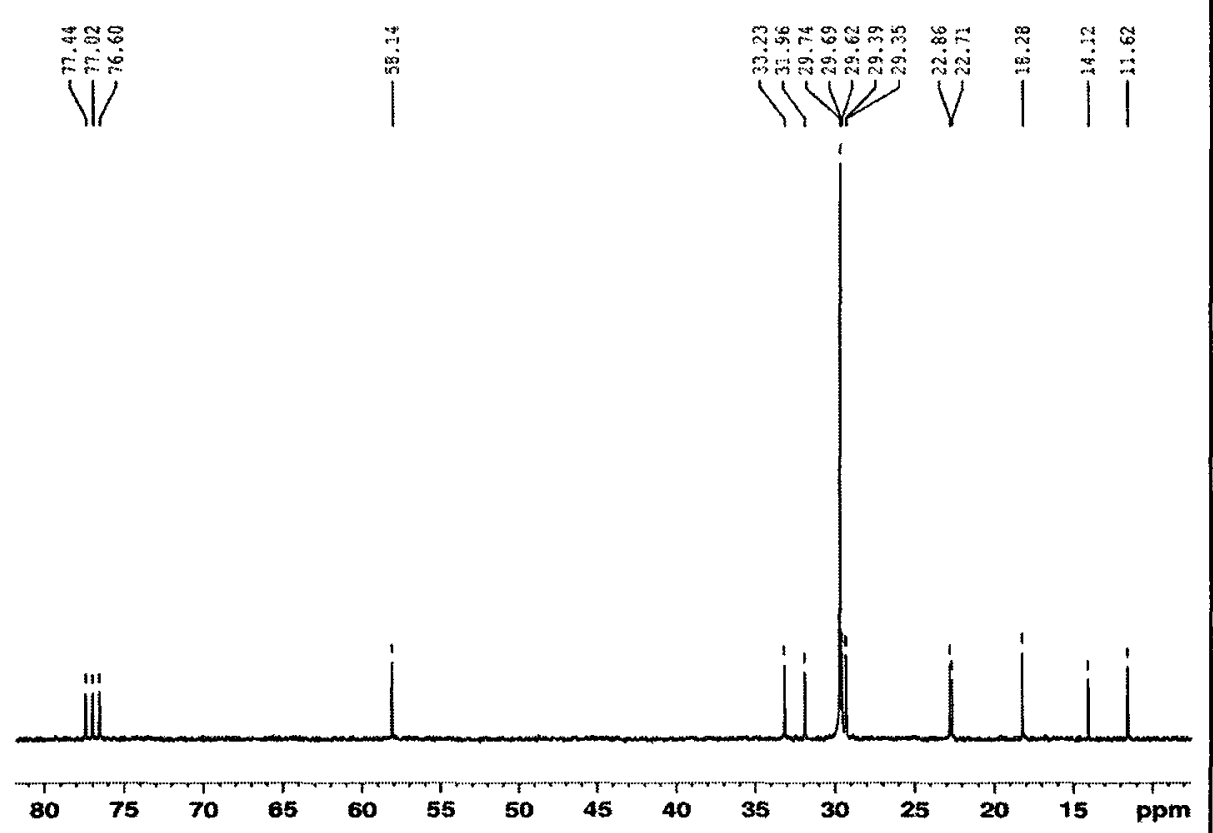

Figure 4.6: Decoupling ${ }^{13} \mathrm{C}$ NMR Spectrum $\left(\mathrm{CDCl}_{3}\right)$ for Excess OTS. 


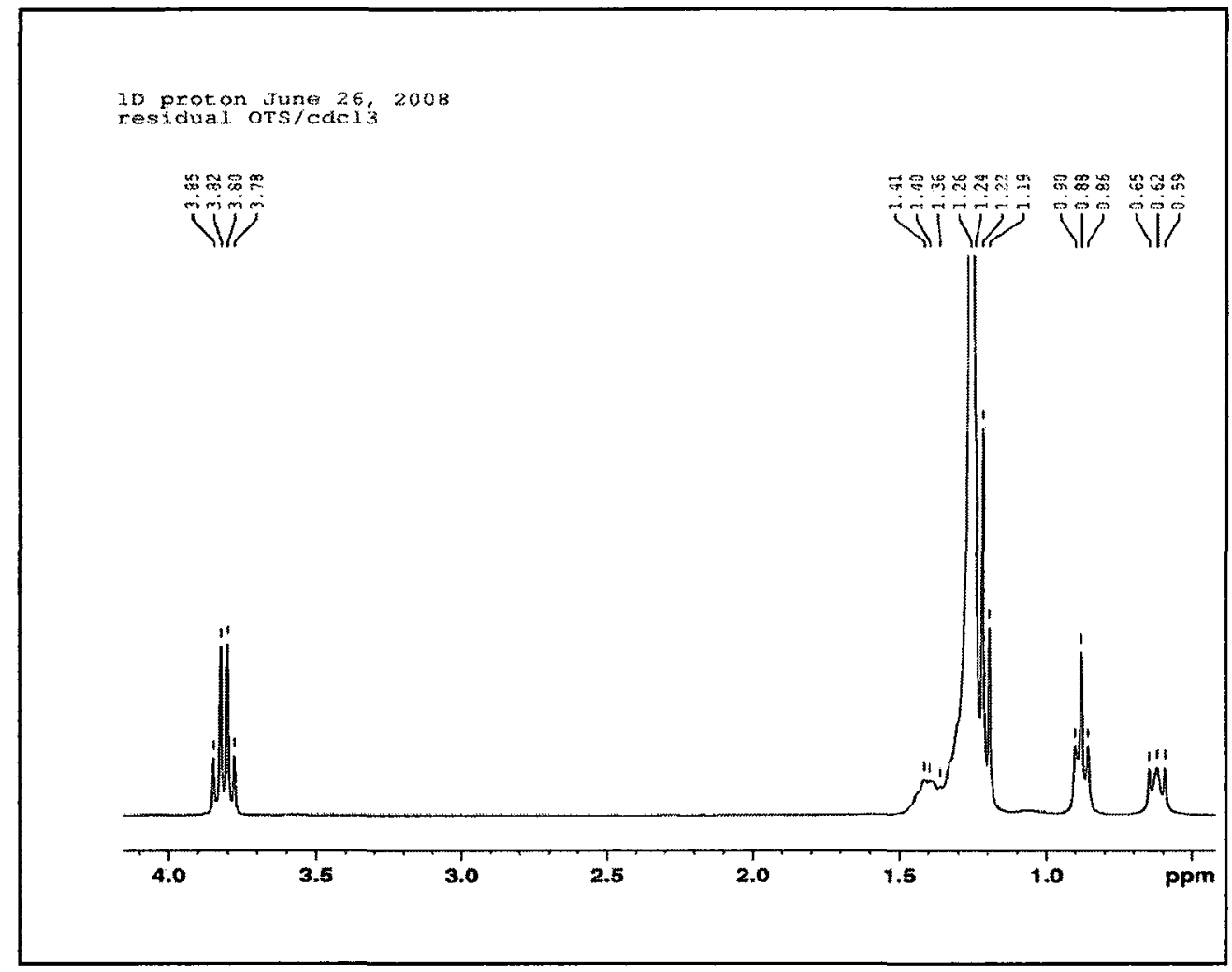

Figure 4.7: ${ }^{1} \mathrm{H}$ NMR Spectrum $\left(\mathrm{CDCl}_{3}\right)$ for Excess OTS.

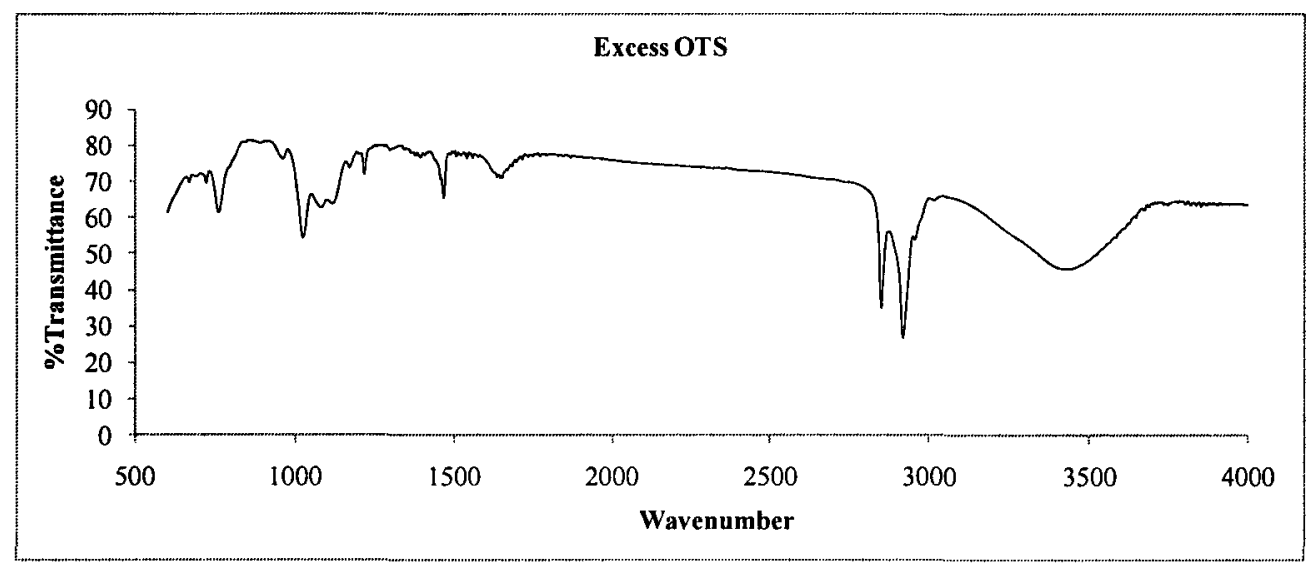

Figure 4.8: FT-IR Spectrum for Excess OTS. 


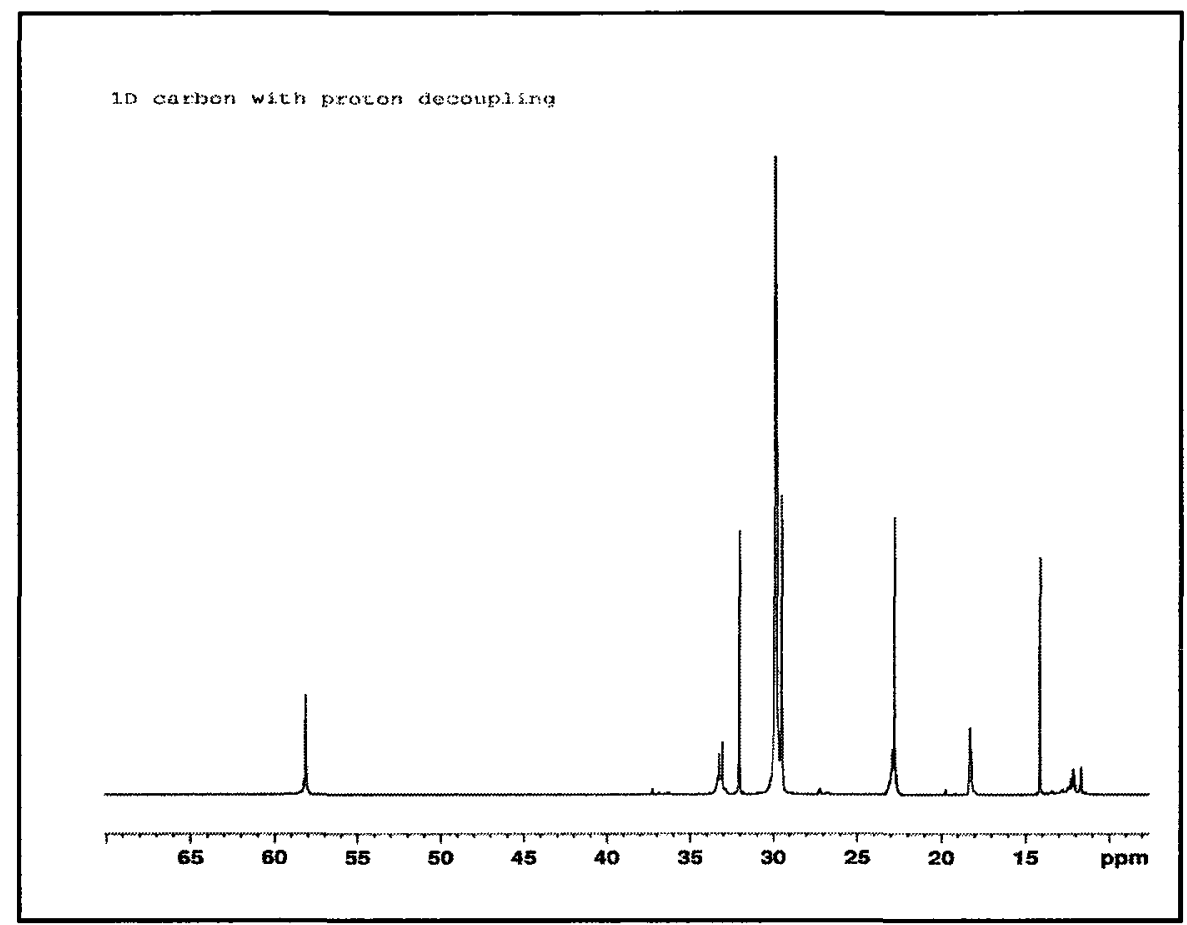

Figure 4.9: Decoupling ${ }^{13} \mathrm{C} \mathrm{NMR}$ Spectrum $\left(\mathrm{CDCl}_{3}\right)$ for the Derivative of OTS.

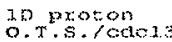

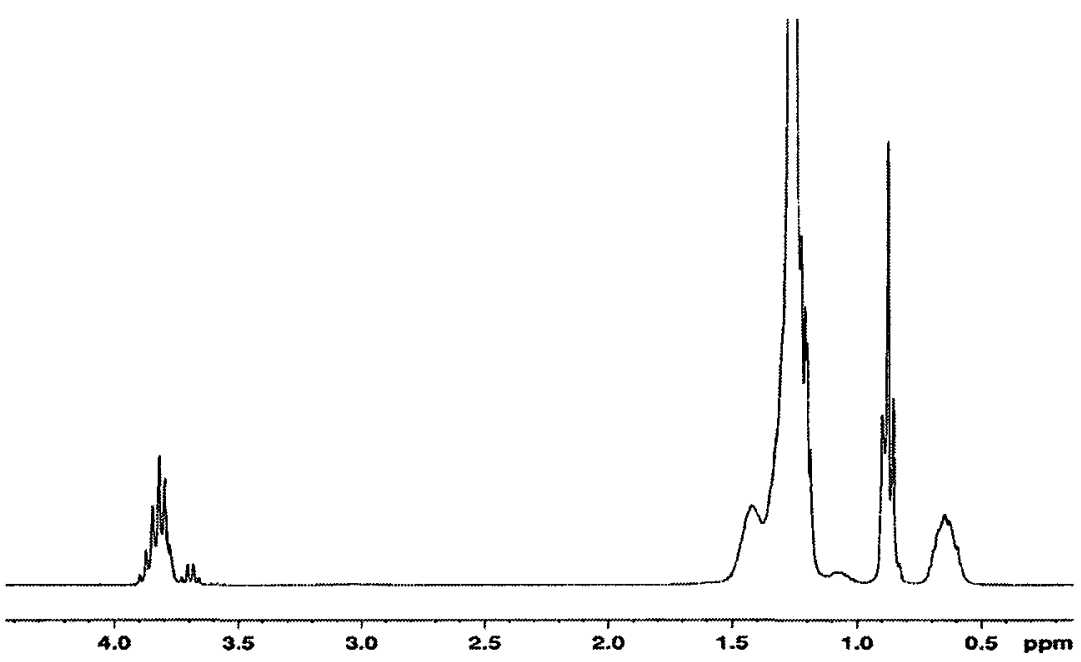

Figure 4.10: ${ }^{1} \mathrm{H}$ NMR Spectrum $\left(\mathrm{CDCl}_{3}\right)$ for the Derivative of OTS. 


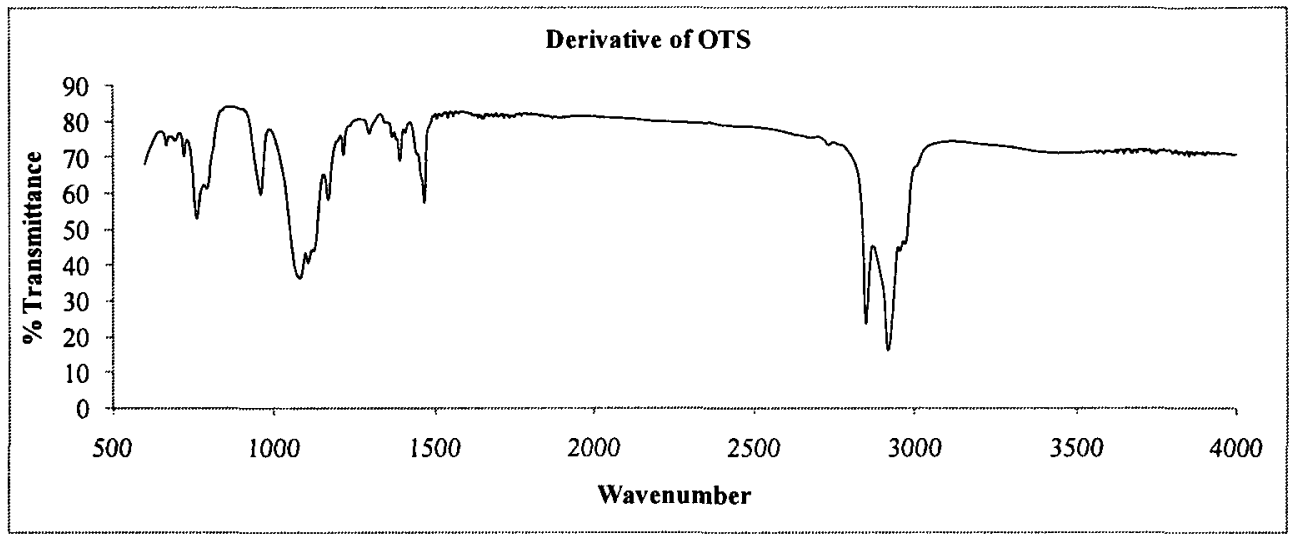

Figure 4.11: FT-IR Spectrum for the Derivative of OTS.

One way to confirm that the wax-like substance left in the heptane flask was similar to the OTS derivative in the ethanol solution was to compare both the number of peaks and the associated chemical shifts in the wax-like substance's NMR spectrum with those in the derivative of OTS's spectrum.

Table 4.1 provides a summary of the comparison between the chemical shifts for the carbon with proton decoupling, Table 4.2 illustrates this comparison between the chemical shifts for the proton, and Table 4.3 shows the comparison between the wave numbers for the major bands in the wax-like substance's spectrum and the derivative of OTS spectrum.

Table 4.1: Chemical Shifts from Decoupling ${ }^{13} \mathrm{C}$ NMR Spectra $\left(\mathrm{CDCl}_{3}\right)$.

\begin{tabular}{|c|c|}
\hline Excess OTS & Derivative of OTS \\
\hline 11.620 & 11.930 \\
\hline 14.120 & 14.160 \\
\hline 18.280 & 18.275 \\
\hline 22.785 & 22.904 \\
\hline 29.370 & 29.461 \\
\hline 29.683 & 29.725 \\
\hline 31.960 & 32.074 \\
\hline 33.230 & 33.186 \\
\hline 58.140 & 58.167 \\
\hline
\end{tabular}


Table 4.2: Chemical Shifts from ${ }^{1} \mathrm{H}$ NMR Spectra $\left(\mathrm{CDCl}_{3}\right)$.

\begin{tabular}{|c|c|}
\hline Excess OTS & Derivative of OTS \\
\hline 0.620 & 0.638 \\
\hline 0.880 & 0.878 \\
\hline 1.220 & 1.220 \\
\hline 1.390 & 1.420 \\
\hline 3.813 & 3.822 \\
\hline
\end{tabular}

Table 4.3: FT-IR Spectra for the Excess OTS and the Derivative of OTS.

\begin{tabular}{|l|l|l|l|}
\cline { 2 - 4 } \multicolumn{1}{c|}{} & Excess OTS & Derivative of OTS & Attribution \\
\hline First band & $2918.6,2850.6$ & $2919.9,2851.1$ & C-O \\
\hline Second band & 1023.6 & 1079.5 & C-H \\
\hline
\end{tabular}

As shown in Table 4.1 and Table 4.2 , both the wax-like substance and the derivative of OTS spectra have the same number of signals and have similar chemical shifts. In addition, as shown in Table 4.3 both the wax-like substance and the derivative of OTS FT-IR spectra have the same functional group (ether group). This confirms that the wax-like substance, which was assumed to be excess OTS, and the derivative of OTS in ethanol have similar chemical structures.

\subsubsection{Enhanced Soil Washing Technique}

It may be argued that the excess OTS on the hydrophobic sand surfaces is due to insufficient number of washings in ethanol. To determine the effects of numerous washing in ethanol on OTS-treated sands, two hydrophobic sand samples, which were treated by using $5 \%$ OTS solution in ethanol followed by twice washing in ethanol, were placed in two soxhlet extractors. One of the sand samples was placed in a soxhlet extractor containing ethanol and was allowed to run for four days at $80^{\circ} \mathrm{C}$, which was 
equivalent to approximately 280 washings (one ethanol cycle in the extractor was approximately twenty minutes). While the second sand sample was placed in a soxhlet extractor containing hexane and was allowed to run for four days at $70^{\circ} \mathrm{C}$, which was equivalent to approximately 380 washings (one hexane cycle in the extractor was approximately fifteen minutes). In addition to the large number of washings using the soxhlet extractor, the ethanol and hexane that distilled on the sand samples, were at temperatures close to their boiling points which may have enhanced the dissolution of excess OTS in these sand samples. These sand samples were oven-dried at $95^{\circ} \mathrm{C}$ for one hour and their wettability conditions were assessed to confirm they were hydrophobic. The samples were then divided and placed in separate beakers containing heptane, decane, or TCE and stirred for 1.5 hours. After the stirring, the heptane, decane, and TCE were drained and placed in clean beakers containing hydrophilic sand samples and stirred for 1.5 hours after which heptane, decane, and TCE were drained in a waste container. The originally hydrophobic and hydrophilic silica sand samples were dried in an oven at $95^{\circ} \mathrm{C}$ for one hour and placed in desiccators to cool down to room temperature. The wettability conditions of these sand samples were checked as described in Section 4.4.6 which showed that the hydrophobic sand samples washed with ethanol remained hydrophobic and the hydrophilic sand samples became hydrophobic, indicating that 280 washings in the soxhlet extractor with ethanol did not remove all the excess OTS. In contrast, the hydrophobic sand samples washed with hexane remained hydrophobic and the hydrophilic sand samples appeared to be less hydrophobic (i.e., impacted), indicating that the hexane was more effective in removing the excess OTS but removal was not complete. 
Despite the large number of successive washings of a hydrophobic sand sample in a soxhlet extractor with ethanol at an elevated temperature, the excess OTS was not all removed from the hydrophobic sand samples. The excess OTS on the hydrophobic sand samples easily dissolved in heptane, decane, and TCE. Therefore, in addition to the repetitive washings with a solvent at an elevated temperature, there were other parameters controlling the removal of the excess OTS from a hydrophobic sand sample. One of these parameters may have been the solvent type. As heptane, decane, and TCE are non-polar solvents, it was important to wash the soil, in the soxhlet extractor, with a non-polar solvent (e.g., hexane). Another parameter may have been that the excess OTS dissolves in different solvents to different degrees. Therefore, if a specific solvent will be exposed to a hydrophobic sand sample, this sand sample should be washed in the soxhlet extractor with that solvent.

\subsection{3.a. Impacts of Solvent Type on Soil Washing}

Two sand samples that were treated according to case (A) and washed eight times with ethanol were placed in the soxhlet extractor containing hexane to determine if the non-polar solvents were more effective in dissolving the excess OTS. The temperature of the hexane in the extractor was increased to $70^{\circ} \mathrm{C}$ for 24 hours. At one point from the start of washing, it was noticed that the hexane, in the flask, became cloudy, which might indicate dissolution of excess OTS in hexane. Therefore, for effective washing, it was important to replace the used hexane with fresh hexane after 12 hours. After washing the sand samples with hexane, they were oven-dried at $95^{\circ} \mathrm{C}$ for one hour, and then they were placed in separate soxhlets for 24 hours. One of the soxhlets contained heptane, for which the temperature was set to $95^{\circ} \mathrm{C}$, and the other one contained decane, with the 
temperature set to $175^{\circ} \mathrm{C}$. After removing the samples from the soxhlet extractors, both samples were oven-dried for one hour at $95^{\circ} \mathrm{C}$. Two sub-samples from the hydrophobic sand washed with heptane were placed in two separate beakers, one of which contained heptane and the other contained decane. One sample from the hydrophobic sand washed with decane was placed in a beaker containing decane. All were stirred for 1.5 hours after which the heptane and decane were drained and placed in clean beakers containing hydrophilic sand samples and stirred for another 1.5 hours. Heptane and decane were drained in a waste container. The originally hydrophobic and hydrophilic silica sand samples were dried in an oven at $95^{\circ} \mathrm{C}$ for one hour and placed in desiccators to cool down to room temperature. The wettability condition of these sand samples were checked as described in Section 4.4.6. These experimental sets were repeated in triplicate, starting from washing the soil samples in the soxhlet extractors with heptane and decane.

The hydrophobic sand samples washed in the soxhlet extractor with hexane followed by heptane and then placed in contact with heptane remained hydrophobic and the hydrophilic sand sample remained hydrophilic. In contrast, the hydrophobic sand samples washed in the soxhlet extractor with hexane followed by heptane and then placed in contact with decane remained hydrophobic and the hydrophilic sand samples appeared to be less hydrophobic (i.e., impacted). While the hydrophobic sand samples washed in the soxhlet extractor with hexane followed by decane and then placed in contact with decane remained hydrophobic and the hydrophilic sand samples became hydrophobic. These results indicate that the heptane used in the soxhlet extractor washed all the excess OTS that could dissolve in heptane from the hydrophobic sand samples. However, a fraction of excess OTS remained that could dissolve in decane and could not be removed 
by heptane. On the other hand, due to the high boiling point of decane in the soxhlet extractor, the chemical bond between OTS and silica sand may have decomposed in the washed hydrophobic sand samples. The latter was confirmed by taking a decane sample from the soxhlet distillation chamber after 24 hours (i.e., distilled decane used in the final washing). This decane sample was poured on a hydrophilic sand sample in a beaker and stirred for 1.5 hours. Decane was drained into a waste container. The sand sample was then oven-dried at $95^{\circ} \mathrm{C}$ for one hour and placed in desiccators to cool down to room temperature. After cooling, the wettability condition of the sand sample was checked as described in Section 4.4.6 which indicated that the hydrophilic sand sample became hydrophobic. This indicated that even after approximately 70 washings with decane, excess OTS was still being removed which rendered the hydrophilic soil sample hydrophobic. This is either due to excess OTS still present on the soil or within the recycled decane or due to the bond breakage. The latter agrees with Kluth et al. (1997) and Cha and Kim (2001) who stated that the chemical bond between OTS derivative and silica becomes unstable for temperatures higher than $100^{\circ} \mathrm{C}$.

Based on the results presented, a new soil washing procedure is proposed. Soils treated with OTS and rinsed in ethanol should be placed in a soxhlet extractor and rinsed with hexane for 24 hours, replacing the hexane with new hexane after approximately 12 hours, followed by 12 hours of rinsing in a soxhlet extractor with the NAPL to be used for the experimental work. Note, this procedure is only applicable for NAPLs with boiling points below $100^{\circ} \mathrm{C}$ as at temperature above this value; the OTS derivative bond with the sand surface may be broken and impact the wettability of the hydrophilic soil fraction. 


\subsection{3.b. Repeated $P_{c}-S$ Experiments after Using the Enhanced Soil Washing Technique}

As discussed previously, it was not possible to have hydrophobic sands free of excess OTS when decane was the NAPL but it was possible to have hydrophobic sands free of excess OTS when heptane was the NAPL. Therefore, the heptane/water $P_{c}-S$ experiments in a hydrophilic porous medium with a hydrophobic soil lens were repeated twice. The OTS-treated sands used in these experiments were washed using the proposed enhanced soil washing technique. Figure 4.12 shows the heptane/ water $P_{c} S$ main drainage and imbibition pathways in an $80 \%$ hydrophilic porous medium with a $20 \%$ hydrophobic soil lens, washed in a soxhlet extractor using the proposed enhanced washing technique. As shown in Figure 4.12, at the end of the main imbibition pathway, equivalent to zero capillary pressure, the water saturations were 0.748 and 0.764 compared to 0.467 and 0.470 for the hydrophobic soil lens rinsed in ethanol and air-dried (Figure 4.5). At zero capillary pressure, the entrapped heptane saturations in the hydrophilic soil fractions were 0.052 and 0.034 as shown in Figure 4.12 compared to 0.333 and 0.330 as shown in Figure 4.5. The entrapped heptane saturations in Figure 4.12 are lower than the typical values in the literature and agree with the entrapped heptane saturations measured in the hydrophilic porous medium. 


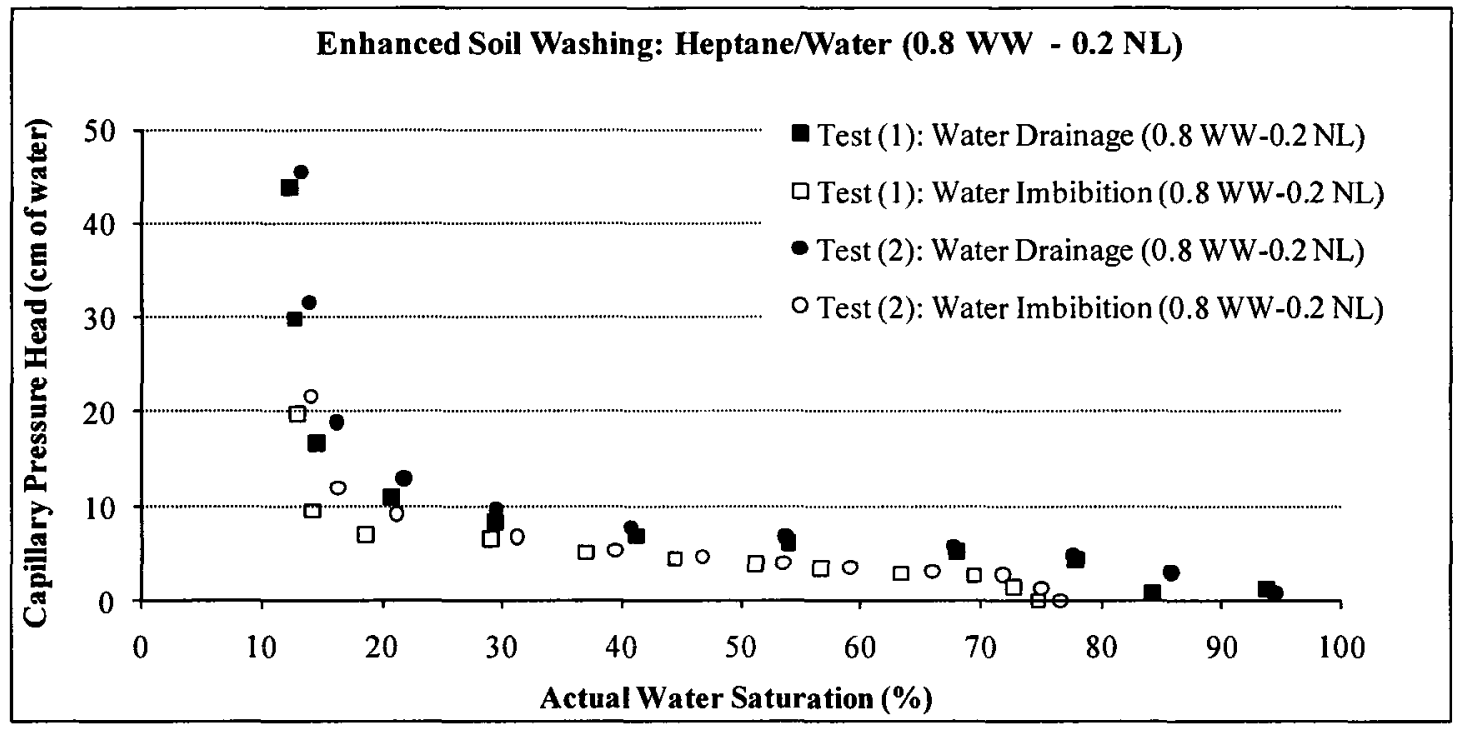

Figure 4.12: Heptane/Water Capillary Pressure Head-Actual Water Saturation Pathways Using Hydrophobic Sands Free of Excess OTS.

\subsubsection{Porous Ceramic Plates Treatment and Washing}

Porous ceramic plates (0604D02.5-B0.5M2, Soilmoisture Equipment Corp, Santa Barbara, CA, USA) are originally hydrophilic. In order to render a porous ceramic plate hydrophobic, it was treated according to case (A) followed by eight washings in ethanol. After placing five hydrophobic porous ceramic plates in decane for three days, $15 \mathrm{~mL}$ samples of the decane were collected. These samples were then placed in clean beakers containing hydrophilic sand samples, $20 \mathrm{~g}$ each, and mixed for 1.5 hours. After mixing, the decane was drained into a waste container and the sand samples were dried in an oven at $95^{\circ} \mathrm{C}$ for one hour. Thereafter, the wettability conditions of the sand samples were checked as described in Section 4.4.6 which indicated that the hydrophilic sand samples became hydrophobic. This indicated that the treated hydrophobic porous ceramic plates contained excess OTS that dissolved in the rinsing decane and, hence, there was a need for additional washing to remove the excess OTS from the plates. 
Due to the size of the porous ceramic plates, the setup shown in Figure 4.13 was used to wash the plates to remove excess OTS. The key objective was to wash the plates with hexane at an elevated temperature as in the soxhlet extractor. After six hours of washing with hexane, the ceramic plates were oven-dried at $65^{\circ} \mathrm{C}$ for three hours and then they were rinsed in decane and heptane separately for three days. Four sand samples, $20 \mathrm{~g}$ each, were placed in separate beakers, each containing $15 \mathrm{~mL}$ of decane or heptane that was previously used to rinse the ceramic plates. The samples and NAPLs were stirred for 1.5 hours, after which the decane and heptane were drained into a waste container and the sand samples were oven-dried for one hour at $95^{\circ} \mathrm{C}$. Then the wettability condition of each sample was checked as described in Section 4.4.6 which indicated that the hydrophilic sand samples remained hydrophilic, which in turn indicated that the porous ceramic plates were free of excess OTS. In addition, the wettability conditions of the washed porous ceramic plates, after they were oven-dried, were checked as described in Section 4.4.6 and they remained hydrophobic.

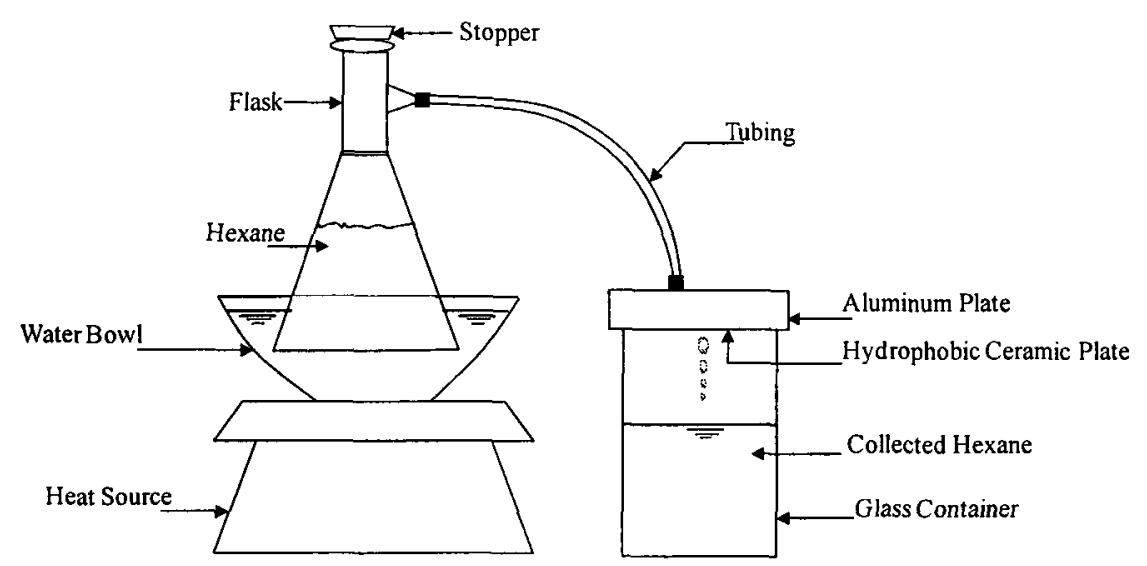

Figure 4.13: Ceramic Plate Washing. 


\subsection{Summary}

This chapter illustrated that the traditional procedure that has been widely published in the literature to render the hydrophilic silica sands and the porous ceramics hydrophobic is insufficient and may result in excess unbound OTS on the treated surfaces. Experimental results indicated that treatment with a 5\% (by volume) OTS solution in ethanol followed by rinsing in ethanol resulted in hydrophobic porous media, but also resulted in excess OTS (i.e., present but not bonded to silica sands or porous ceramics) which dissolved in most solvents used in multiphase flow experiments. When the OTS-impacted solvents contacted hydrophilic sands, the wettability of these sands was altered and they became hydrophobic. Excess OTS was found to dissolve in heptane, decane, soltrol 100, soltrol 220, toluene, and TCE. The dissolved OTS migrates by advection and/or diffusion from hydrophobic sands to adjacent hydrophilic sands rendering them hydrophobic. It was shown that OTS-impacted solvents rendered both water-coated and dry hydrophilic silica sands hydrophobic.

An enhanced washing technique for hydrophobic silica sands and porous ceramics was presented. Hydrophilic silica sands were rendered hydrophobic according to the traditional treatment procedure (i.e., using a 5\% OTS solution in ethanol and rinsing three to five times with ethanol). The silica sands are then placed in hexane in a soxhlet extractor for 24 hours. It was important to replace the used hexane with fresh hexane after 12 hours, since remaining 24 hours in the soxhlet extractor results in 50-100 washings of the sand with the solvent in the extractor. After drying the treated sand sample, it should be washed in the soxhlet extractor with the NAPL type that will be used in the multiphase flow experiments for 12 hours. The only restriction on using this procedure is that it 
cannot be used for solvents with boiling points higher than $100^{\circ} \mathrm{C}$ as the OTS derivative bond may be broken at these temperatures. A similar procedure was followed for washing hydrophobic porous ceramic plates. The hydrophobic porous ceramic plates were washed many times in fresh ethanol, then they were dried and washed for six hours in hexane at an elevated temperature using an apparatus ensuring continuous supply of the OTS-free hexane (i.e., similar to the one used in the previous section where the solvent is vaporized, condensed and the hot condensate is allowed to flow through the treated porous ceramic). It was found that washing the hydrophobic porous ceramics with hexane at an elevated temperature was enough to remove the excess OTS that may dissolve in heptane or decane. The enhanced washing technique, presented in this chapter, was used for the hydrophobic soils and ceramics used in the experimental work presented in this thesis. 


\section{Chapter 5. Capillary Pressure-Saturation Relationships in Two-Phase Systems under Different Soil Wettability Configurations}

\subsection{Introduction}

A $P_{c}-S$ relationship for two fluid phases sharing an interface is essential for modeling flow of these fluid phases in porous media. Scaling theory is frequently used for a water-wet porous medium to obtain the $P_{c}-S$ relationships for two fluid pairs (e.g., air/NAPL and NAPL/water) from a measured $P_{c}-S$ relationship for a known fluid pair (e.g., air/water). In a water-wet porous medium with a NAPL-wet soil lens, the scaling theory may not be applicable. The purpose of this chapter is to verify the applicability of the scaling theory in a water-wet porous medium with a NAPL-wet soil lens. In addition, conceptual models for $P_{c}-S$ relationships, in a water-wet porous medium with a NAPLwet soil lens, are developed to refine the scaling theory to allow modellers to simulate subsurface systems with NAPL-wet soil lenses. In these conceptual models, the rigid porous medium assumption is valid for all wettability configurations.

A water-wet porous medium with a NAPL-wet soil lens consists of two parts; a water-wet soil fraction and a NAPL-wet soil lens. For this research study, the NAPL-wet soil lens is the same granular soil as the water-wet soil fraction except it has been treated to render it NAPL-wet. The approach taken in this research is to consider the water-wet porous medium with the NAPL-wet soil lens as the field scale REV and to derive constitutive relationships for this REV scale based on our knowledge of the constitutive relationships of each soil fraction (water-wet and NAPL-wet). Once this relationship is established, then one might only need to determine the percentage volume of the NAPLwet soil lens in a porous medium to establish the $P_{c}-S$ relationships in that medium with 
the knowledge of a fluid pair $P_{c}-S$ relationship in a water-wet and NAPL-wet porous medium.

This chapter is divided into three main sections to address the development of conceptual models for the $P_{c}-S$ relationships for air/heptane (Section 5.2), air/water (Section 5.3), and heptane/water (Section 5.4) in a porous medium consisting of a waterwet soil fraction and a NAPL-wet soil lens. To support the development of the conceptual models, each section initially presents the results of a series of two-phase experiments for a range of soil wettability configurations. In addition, pore scale images are captured at selected points on $P_{c}-S$ drainage and imbibition pathways. Based on REV scale twophase data and supported by pore scale images, conceptual models to describe the $P_{c} S$ relationship for an REV consisting of a water-wet porous medium with a NAPL-wet soil lens are formulated. Then, the proposed conceptual models are validated using the $P_{c}-S$ data.

\subsection{Air/Heptane $\boldsymbol{P}_{c}-S$ Relationship}

The discussion of the two-phase air/heptane $P_{c}-S$ relationships is divided into three sections. The first section discusses the experimental data for two-phase tests with a water-wet porous medium, water-wet porous medium with different size NAPL-wet soil lenses, and a NAPL-wet porous medium to address the impact of wettability on the $P_{c}-S$ relationship for air/heptane, where heptane is the wetting fluid in all cases. The second section demonstrates the conceptual model for $P_{c}-S$ relationship under different soil wettability configurations. This conceptual model is validated in the third section. 


\subsubsection{Capillary Pressure Head-Actual Heptane Saturation Data in Different Wettability Porous Media}

The air/heptane capillary pressure head-actual heptane saturation drainage and imbibition pathways in water-wet, $80 \%$ water-wet- $20 \%$ NAPL-wet, and NAPL-wet porous media are shown in Figure 5.1 and Figure 5.2 for uniform sand and well graded sand, respectively. Table 5.1, for uniform sand, and Table 5.2, for well graded sand, show the irreducible heptane saturations, and the entrapped air saturations at the end of the main imbibition pathways for the three porous media. For the three porous media data presented in Figure 5.1 and Figure 5.2, each porous medium reaches 100\% actual heptane saturation prior to initiating drainage (i.e., a main drainage pathway starts at $100 \%$ actual heptane saturation). For the same grain size distribution, it is noticed that the heptane drainage pathways from the three porous media follow the same pathway leaving approximately the same irreducible saturations in these porous media. In addition, heptane imbibition pathways for the three porous media follow the same imbibition pathway. It is also noticed that the entrapped air saturations are similar in the uniform sand and slightly different in the well graded sand for the three soil wettability configurations. 


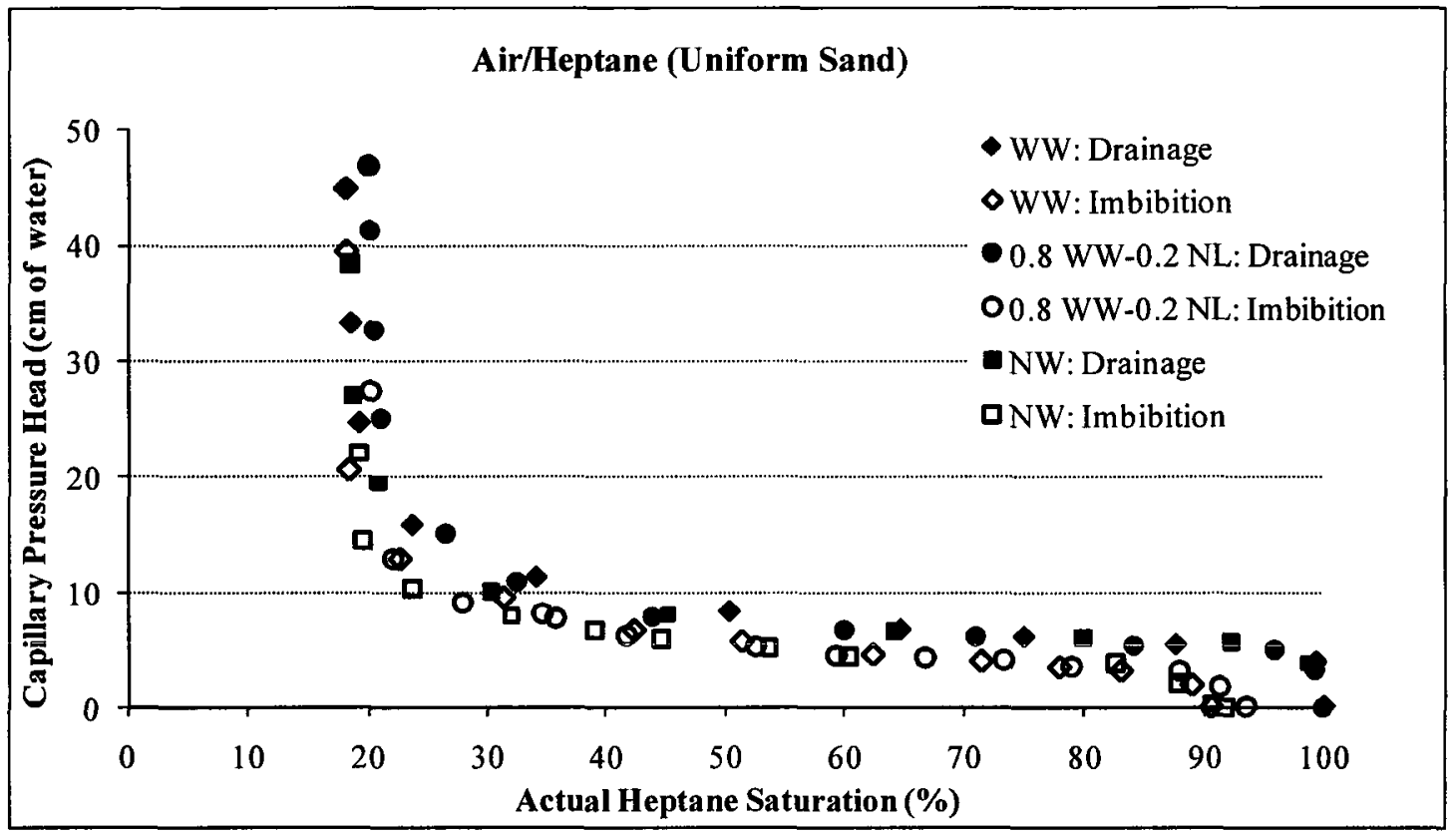

Figure 5.1: Capillary Pressure Head - Actual Heptane Saturation in Different Wettability Porous Media.

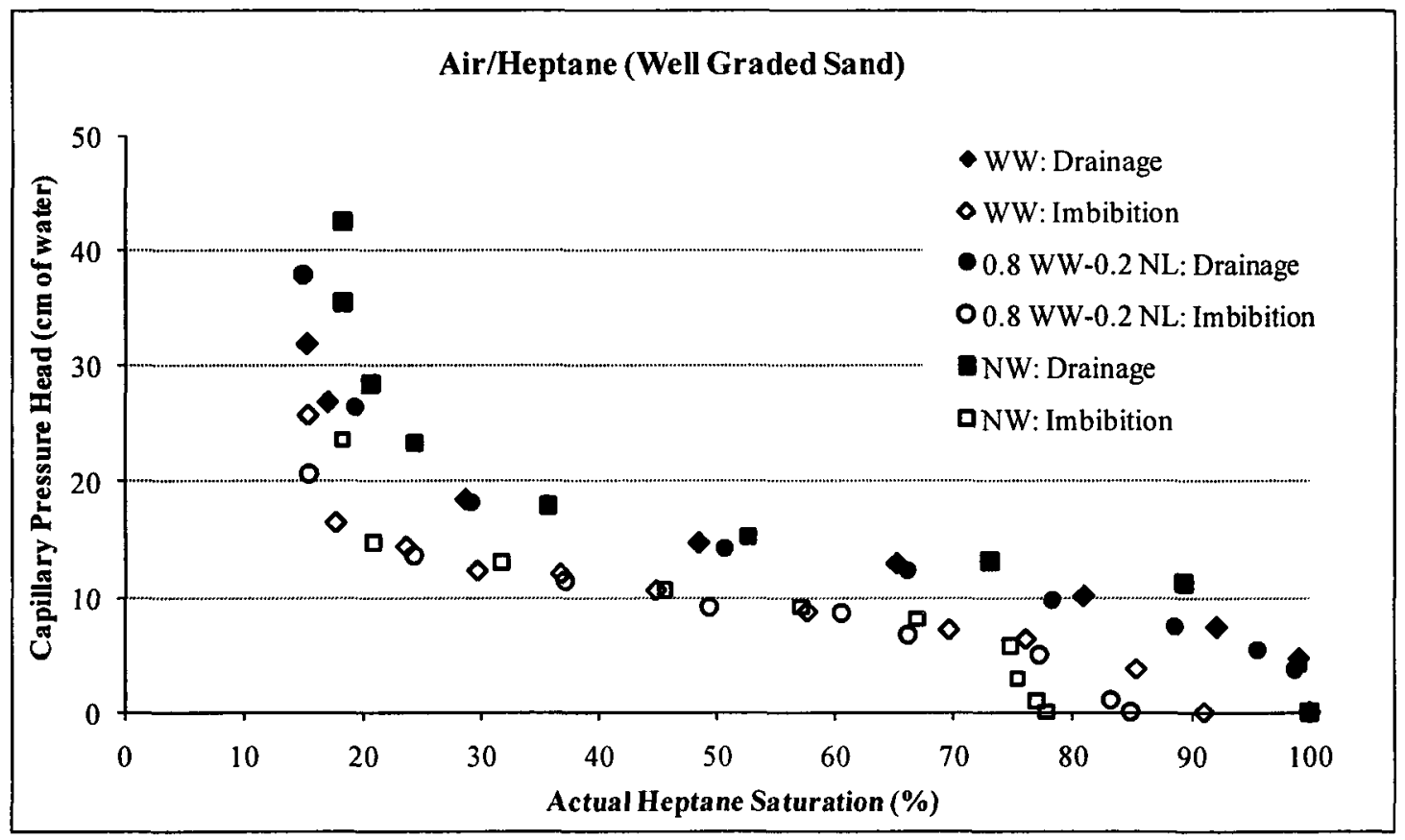

Figure 5.2: Capillary Pressure Head-Actual Heptane Saturation in Different Wettability Porous Media. 
Table 5.1: Irreducible Heptane and Entrapped Air Saturations in Uniform Soil.

\begin{tabular}{|c|c|c|c|c|}
\hline \multirow{2}{*}{$\begin{array}{c}\text { Wettability } \\
\text { Configuration }\end{array}$} & \multicolumn{2}{|c|}{$\begin{array}{c}\text { Irreducible Heptane } \\
\text { Saturation }\end{array}$} & \multicolumn{2}{c|}{$\begin{array}{c}\text { Entrapped Air } \\
\text { Saturation }\end{array}$} \\
\cline { 2 - 5 } & Test (1) & Test (2) & Test (1) & Test (2) \\
\hline WW & 19.10 & 18.10 & 5.68 & 9.40 \\
\hline $\mathbf{0 . 9}$ WW - 0.1 NL & 19.31 & 19.39 & 7.26 & 5.93 \\
\hline $\mathbf{0 . 8}$ WW - 0.2 NL & 19.97 & 17.26 & 6.43 & 11.77 \\
\hline $\mathbf{0 . 7}$ WW - 0.3 NL & 20.27 & 20.18 & 13.25 & 12.23 \\
\hline $\mathbf{0 . 6}$ WW - 0.4 NL & 22.16 & 22.79 & 12.74 & 13.58 \\
\hline NW & 18.24 & 18.38 & 4.93 & 8.30 \\
\hline
\end{tabular}

Table 5.2: Irreducible Heptane and Entrapped Air Saturations in Well Graded Soil.

\begin{tabular}{|c|c|c|c|c|}
\hline \multirow{2}{*}{$\begin{array}{c}\text { Wettability } \\
\text { Configuration }\end{array}$} & \multicolumn{2}{|c|}{$\begin{array}{c}\text { Irreducible Heptane } \\
\text { Saturation }\end{array}$} & \multicolumn{2}{c|}{$\begin{array}{c}\text { Entrapped Air } \\
\text { Saturation }\end{array}$} \\
\cline { 2 - 5 } & Test (1) & Test (2) & Test (1) & Test (2) \\
\hline WW & 15.1 & 14.84 & 13.12 & 9.01 \\
\hline $\mathbf{0 . 9}$ WW - 0.1 NL & 15.82 & 16.67 & 11.93 & 13.15 \\
\hline $\mathbf{0 . 8}$ WW - 0.2 NL & 14.84 & 15.54 & 15.1 & 13.45 \\
\hline $\mathbf{0 . 7}$ WW - 0.3 NL & 18.74 & 17.02 & 8.18 & 8.8 \\
\hline $\mathbf{0 . 6}$ WW - 0.4 NL & 17.72 & 17.81 & 14.68 & 12.18 \\
\hline NW & 14.82 & 18.15 & 19.96 & 22.2 \\
\hline
\end{tabular}

\subsubsection{Conceptual Model for $P_{c} S$ Relationship under Different Wettability Configurations}

For a given porous medium wettability configuration (water-wet porous medium, water-wet porous medium with a NAPL-wet soil lens, or NAPL-wet porous medium), heptane is the wetting fluid and air is the non-wetting fluid. Given that the only difference between the soils tested is the wettability configuration, the only parameter that might impact the $P_{c}-S$ relationship is the contact angle. Therefore the difference between heptane behaviour in a water-wet porous medium versus in a NAPL-wet porous medium 
may be the contact angle. Given the consistency in the results for the three porous media, it can be assumed that the contact angles for the water-wet and NAPL-wet media are similar.

\subsubsection{Validation of the Conceptual Model}

In this section, the Van Genuchten relationship was fitted to the main drainage and imbibition pathways in a water-wet porous medium, in a water-wet porous medium with a NAPL-wet soil lens, and in a NAPL-wet porous medium. For each porous medium of the two sand types (i.e., uniform sand and well graded sand), Van Genuchten parameters were calculated for two scenarios; unconstrained $n$ and $\alpha$, and unconstrained $\alpha$ and constrained $n$. The first scenario refers to calculating $n$ and $\alpha$ by fitting air/heptane $P_{c} \rightarrow S$ drainage or imbibition pathway data to the Van Genuchten equation. In this scenario, there is no restriction in estimating these parameters. The second scenario refers to calculating $\alpha$ by fitting air/heptane $P_{c}-S$ drainage or imbibition pathway data to the Van Genuchten equation. In this scenario, $n$ is calculated for the water-wet porous medium and used for the other porous media. The reason for constraining $n$ and not constraining $\alpha$ is that $n$ controls the slope of the Van Genuchten curve while $\alpha$ controls the height of the Van Genuchten curve. The slope of the Van Genuchten curve depends on the grain size distribution. The height of the curve depends on the fluid pair, the porous medium grain size distribution, and the fluid/porous medium interaction. Since the grain size distributions are similar under different wettability configurations within the same sand type, $n$ should not vary and any variations should be seen in $\alpha$.

Van Genuchten parameters $(n, \alpha)$ for the two scenarios are summarized in Table 5.3, for the uniform sand, and Table 5.4, for the well graded sand. The tables also show 
the average and standard deviation for $n$ and $\alpha$ for each two-phase system. For a given scenario, standard deviation for $n$ or $\alpha$ for a given pathway is small. The variations in $n$ and $\alpha$ values may be attributed to experimental errors. In addition, $n$ and $\alpha$ were fit to the pooled data (i.e., all the imbibition data for the two-phase experiments). The values for $n$ and $\alpha$ for the pooled data are close to the average values from the individual data sets. The fitted Van Genuchten main drainage and imbibition pathways for the first and the second scenarios and the $P_{c}-S$ main drainage and imbibition pathway data for the six porous media are shown in Figure 5.3 through Figure 5.14. As shown in Figure 5.3 through Figure 5.14, the data points fit the Van Genuchten curves in the first and the second scenarios. As shown in the figures, when $n$ was constrained, a Van Genuchten relationship better fits the $P_{c}-S$ data. Figure 5.15 and Figure 5.16 show the $P_{c}-S$ main drainage and imbibition pathways for the pooled data in uniform sand and well graded sand, respectively. These two figures show the fitted Van Genuchten main drainage and imbibition pathways. The main drainage and imbibition pathway data fit the Van Genuchten curves in the first and the second scenarios. Therefore, the conceptual model is valid. 
Table 5.3: Van Genuchten Parameters for Uniform Sand.

\begin{tabular}{|c|c|c|c|c|c|c|c|c|}
\hline \multirow{2}{*}{$\begin{array}{c}\text { Wettability } \\
\text { Configuration }\end{array}$} & \multicolumn{3}{|c|}{ Unconstrained $\boldsymbol{n}, \boldsymbol{c}$} & \multicolumn{3}{c|}{ Unconstrained $\boldsymbol{\alpha}$, Constrained $\boldsymbol{n}$} \\
\cline { 2 - 9 } & $\begin{array}{c}\text { Drainage } \\
\text { Pathway }\end{array}$ & $\begin{array}{c}\text { Imbibition } \\
\text { Pathway }\end{array}$ & \multicolumn{2}{c|}{$\begin{array}{c}\text { Drainage } \\
\text { Pathway }\end{array}$} & \multicolumn{2}{c|}{$\begin{array}{c}\text { Imbibition } \\
\text { Pathway }\end{array}$} \\
\cline { 2 - 9 } & $\boldsymbol{n}$ & $\boldsymbol{\alpha}$ & $\boldsymbol{n}$ & $\boldsymbol{\alpha}$ & $\boldsymbol{n}$ & $\boldsymbol{\alpha}$ & $\boldsymbol{n}$ & $\boldsymbol{\alpha}$ \\
\hline WW & 4.527 & 0.152 & 4.181 & 0.218 & 4.527 & 0.152 & 4.181 & 0.218 \\
\hline $\mathbf{0 . 9}$ WW - 0.1 NL & 5.887 & 0.157 & 4.100 & 0.236 & 4.527 & 0.161 & 4.181 & 0.235 \\
\hline $\mathbf{0 . 8}$ WW - 0.2 NL & 5.524 & 0.153 & 3.970 & 0.219 & 4.527 & 0.154 & 4.181 & 0.217 \\
\hline $\mathbf{0 . 7}$ WW - 0.3 NL & 6.240 & 0.164 & 4.066 & 0.235 & 4.527 & 0.168 & 4.181 & 0.233 \\
\hline $\mathbf{0 . 6}$ WW - 0.4 NL & 6.151 & 0.167 & 4.609 & 0.235 & 4.527 & 0.171 & 4.181 & 0.237 \\
\hline NW & 5.970 & 0.140 & 4.049 & 0.205 & 4.527 & 0.142 & 4.181 & 0.203 \\
\hline Average & $\mathbf{5 . 7 1 7}$ & $\mathbf{0 . 1 5 5}$ & $\mathbf{4 . 1 6 3}$ & $\mathbf{0 . 2 2 4}$ & $\mathbf{4 . 5 2 7}$ & $\mathbf{0 . 1 5 8}$ & $\mathbf{4 . 1 8 1}$ & $\mathbf{0 . 2 2 4}$ \\
\hline Standard Deviation & $\mathbf{0 . 6 3 4}$ & $\mathbf{0 . 0 1 0}$ & $\mathbf{0 . 2 2 9}$ & $\mathbf{0 . 0 1 3}$ & $\mathbf{0 . 0 0 0}$ & $\mathbf{0 . 0 1 1}$ & $\mathbf{0 . 0 0 0}$ & $\mathbf{0 . 0 1 3}$ \\
\hline Pooled Data & 5.433 & 0.155 & 4.040 & 0.225 & 4.527 & 0.157 & 4.181 & 0.224 \\
\hline
\end{tabular}

Table 5.4: Van Genuchten Parameters for Well Graded Sand.

\begin{tabular}{|c|c|c|c|c|c|c|c|c|}
\hline \multirow{2}{*}{$\begin{array}{c}\text { Wettability } \\
\text { Configuration }\end{array}$} & \multicolumn{3}{|c|}{ Unconstrained $\boldsymbol{n}, \boldsymbol{c}$} & \multicolumn{3}{c|}{ Unconstrained $\boldsymbol{\alpha}$, Constrained $\boldsymbol{n}$} \\
\cline { 2 - 9 } & $\begin{array}{c}\text { Drainage } \\
\text { Pathway }\end{array}$ & $\begin{array}{c}\text { Imbibition } \\
\text { Pathway }\end{array}$ & \multicolumn{2}{c|}{$\begin{array}{c}\text { Drainage } \\
\text { Pathway }\end{array}$} & \multicolumn{2}{c|}{$\begin{array}{c}\text { Imbibition } \\
\text { Pathway }\end{array}$} \\
\cline { 2 - 9 } & $\boldsymbol{n}$ & $\boldsymbol{\alpha}$ & $\boldsymbol{n}$ & $\boldsymbol{\alpha}$ & $\boldsymbol{n}$ & $\boldsymbol{\alpha}$ & $\boldsymbol{n}$ & $\boldsymbol{\alpha}$ \\
\hline WW & 4.749 & 0.081 & 4.193 & 0.125 & 4.749 & 0.081 & 4.193 & 0.125 \\
\hline $\mathbf{0 . 9}$ WW - 0.1 NL & 5.050 & 0.085 & 4.970 & 0.121 & 4.749 & 0.086 & 4.193 & 0.125 \\
\hline $\mathbf{0 . 8}$ WW - 0.2 NL & 4.631 & 0.084 & 4.322 & 0.120 & 4.749 & 0.084 & 4.193 & 0.121 \\
\hline $\mathbf{0 . 7}$ WW - 0.3 NL & 5.193 & 0.094 & 4.399 & 0.130 & 4.749 & 0.095 & 4.193 & 0.132 \\
\hline $\mathbf{0 . 6}$ WW - 0.4 NL & 4.912 & 0.091 & 5.498 & 0.120 & 4.749 & 0.091 & 4.193 & 0.126 \\
\hline NW & 6.720 & 0.070 & 6.326 & 0.101 & 4.749 & 0.073 & 4.193 & 0.107 \\
\hline Average & $\mathbf{5 . 2 0 9}$ & $\mathbf{0 . 0 8 4}$ & $\mathbf{4 . 9 5 1}$ & $\mathbf{0 . 1 2 0}$ & $\mathbf{4 . 7 4 9}$ & $\mathbf{0 . 0 8 5}$ & $\mathbf{4 . 1 9 3}$ & $\mathbf{0 . 1 2 3}$ \\
\hline Standard Deviation & $\mathbf{0 . 7 6 7}$ & $\mathbf{0 . 0 0 8}$ & $\mathbf{0 . 8 3 2}$ & $\mathbf{0 . 0 1 0}$ & $\mathbf{0 . 0 0 0}$ & $\mathbf{0 . 0 0 8}$ & $\mathbf{0 . 0 0 0}$ & $\mathbf{0 . 0 0 8}$ \\
\hline Pooled Data & 4.872 & 0.084 & 4.647 & 0.120 & 4.749 & 0.084 & 4.193 & $\mathbf{0 . 1 2 2}$ \\
\hline
\end{tabular}




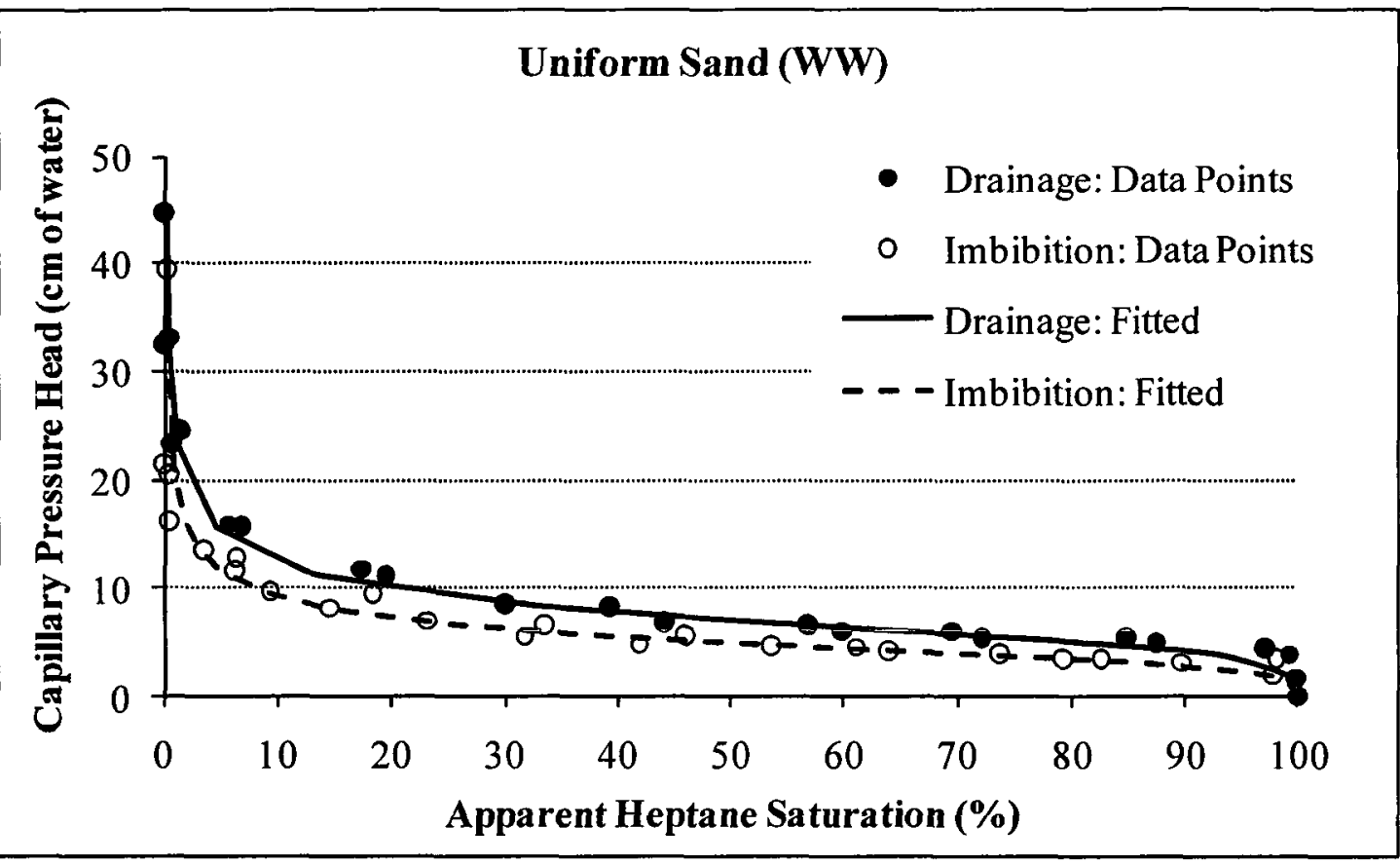

Figure 5.3: Capillary Pressure Head-Apparent Heptane Saturation Main Drainage and Imbibition Pathways in a Water-Wet Porous Medium in Uniform Sand.

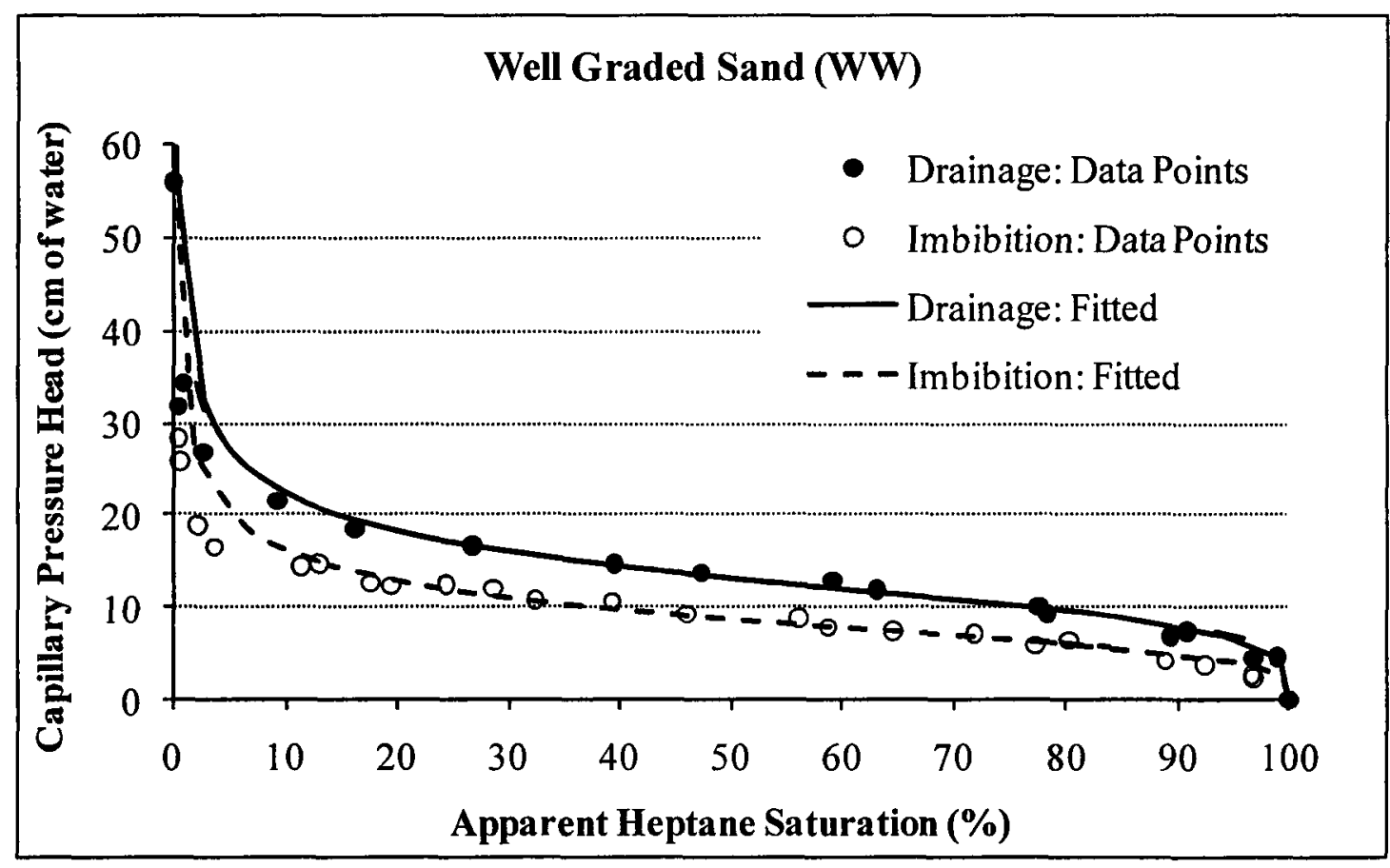

Figure 5.4: Capillary Pressure Head-Apparent Heptane Saturation Main Drainage and Imbibition Pathways in a Water-Wet Porous Medium in Well Graded Sand. 


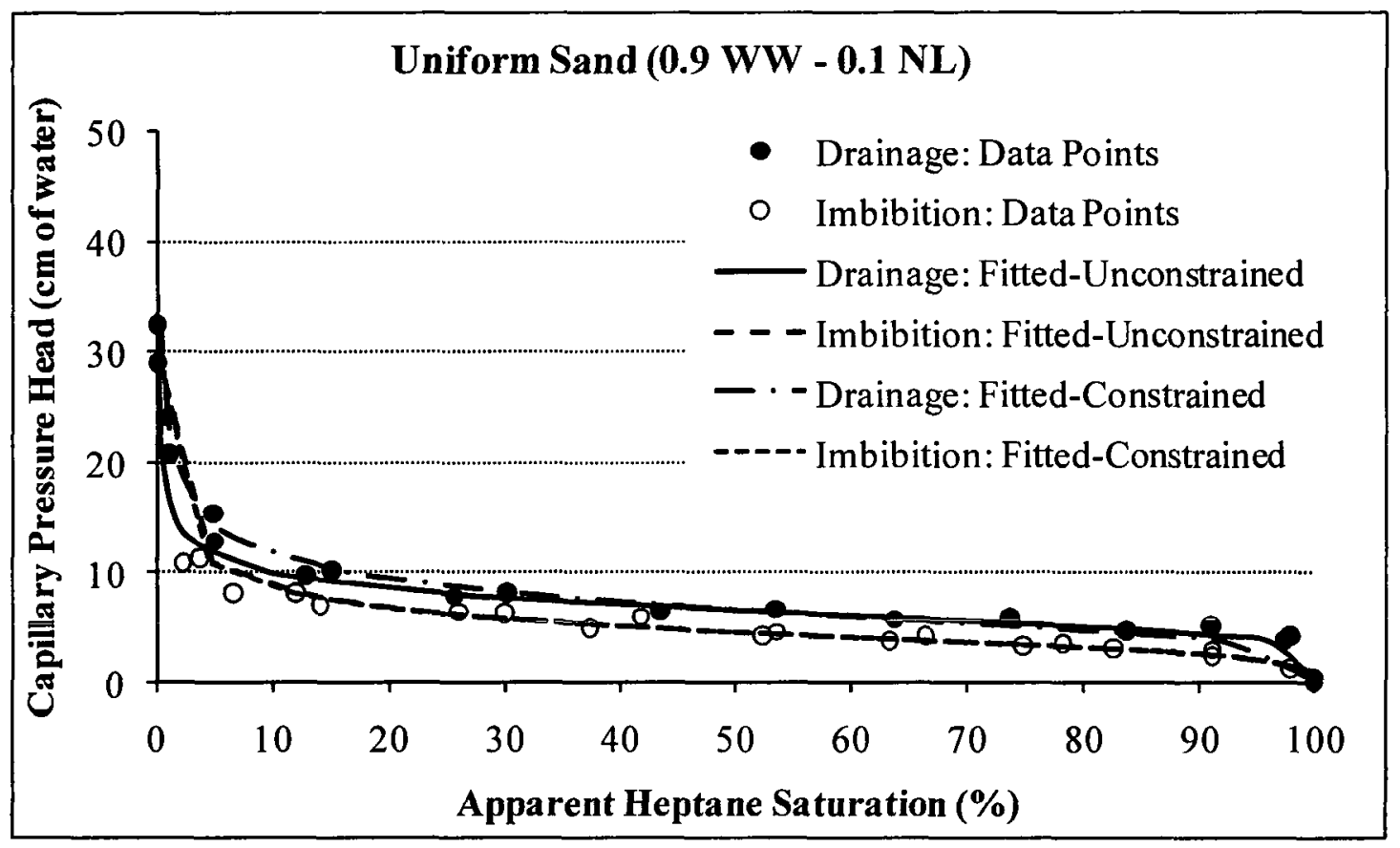

Figure 5.5: Capillary Pressure Head-Apparent Heptane Saturation Main Drainage and Imbibition Pathways in a $90 \%$ Water-Wet-10\% NAPL-Wet Porous Medium in Uniform Sand.

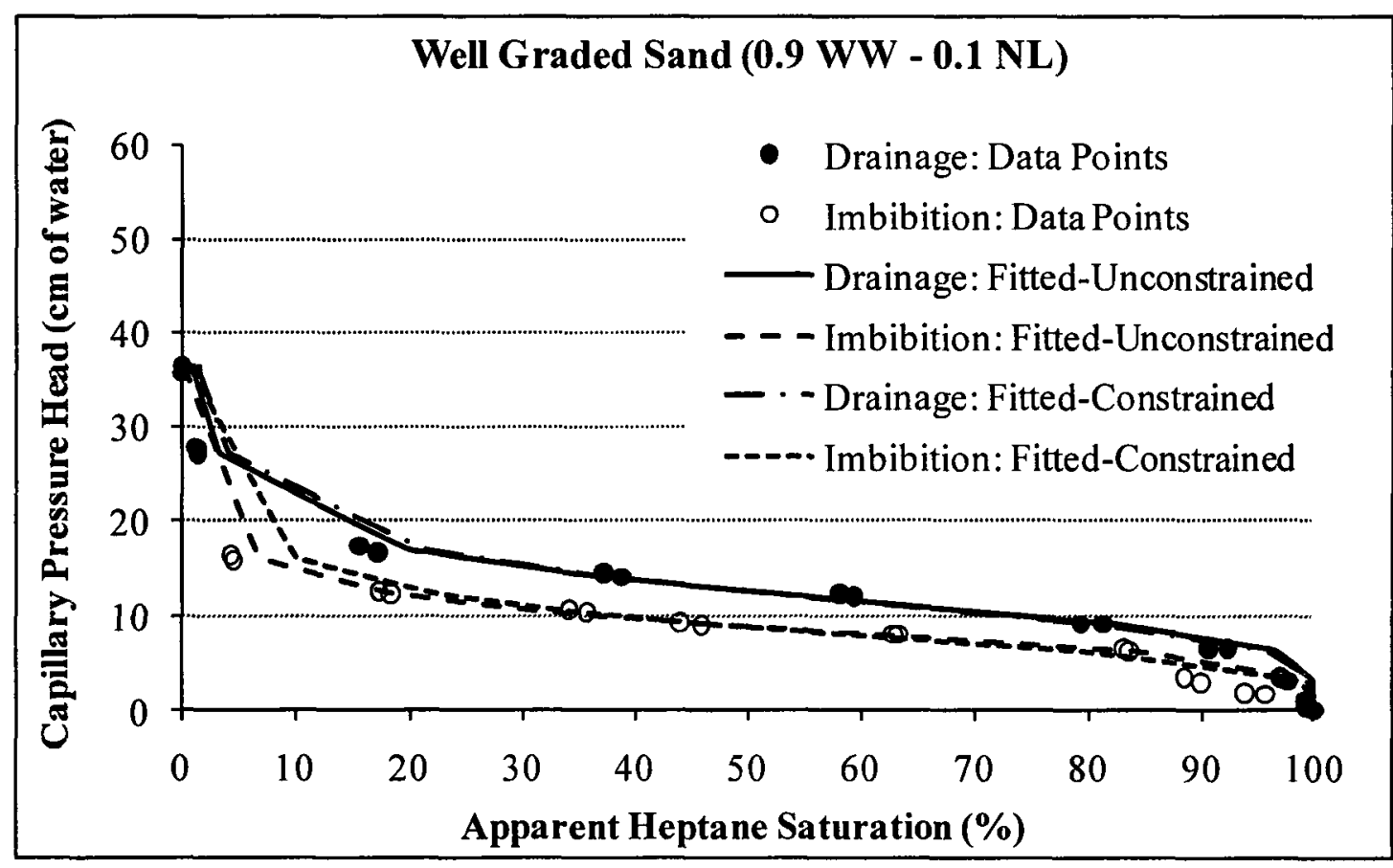

Figure 5.6: Capillary Pressure Head-Apparent Heptane Saturation Main Drainage and Imbibition Pathways in a $90 \%$ Water-Wet-10\% NAPL-Wet Porous Medium in Well Graded Sand. 


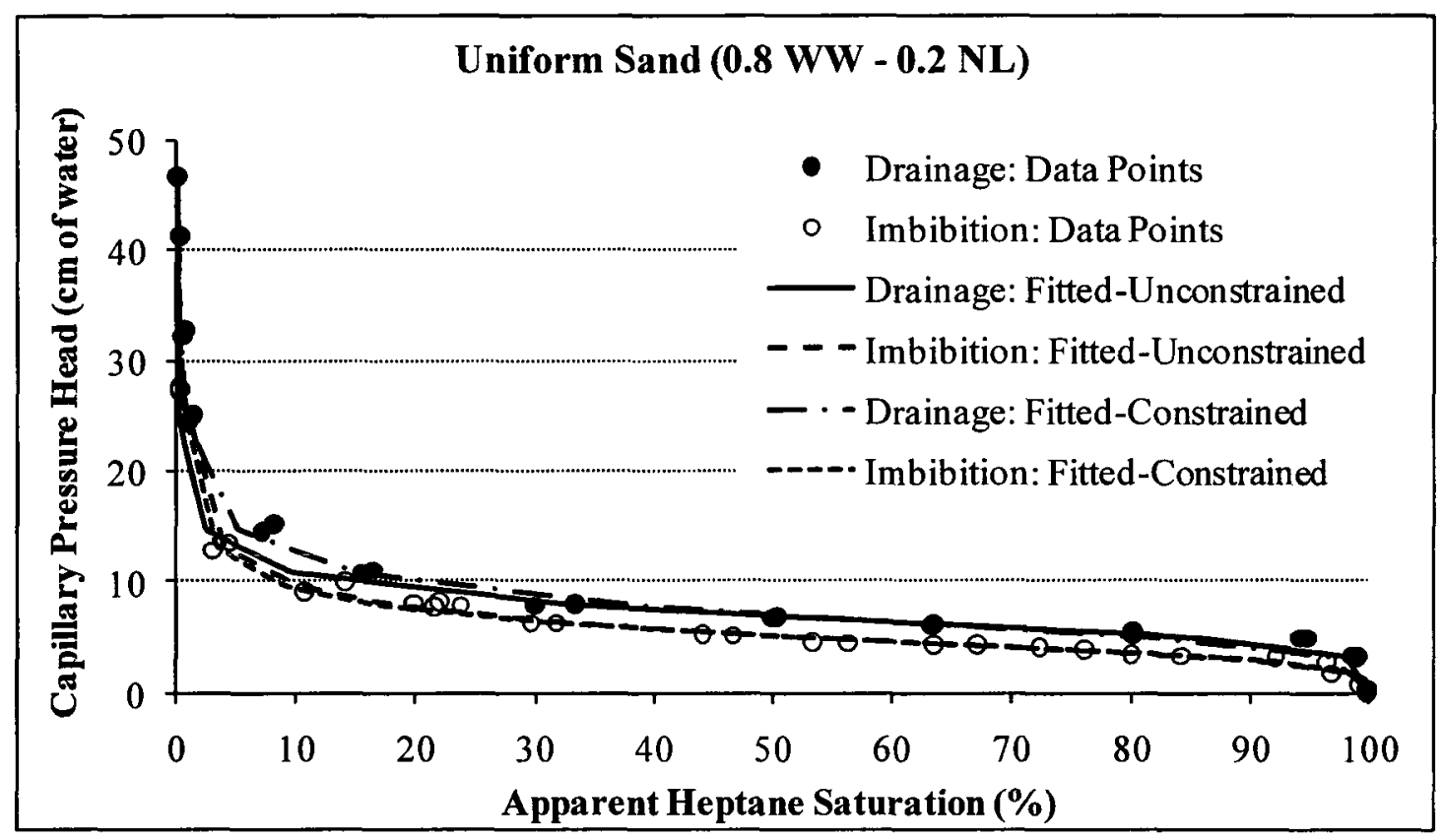

Figure 5.7: Capillary Pressure Head-Apparent Heptane Saturation Main Drainage and Imbibition Pathways in an $80 \%$ Water-Wet-20\% NAPL-Wet Porous Medium in Uniform Sand.

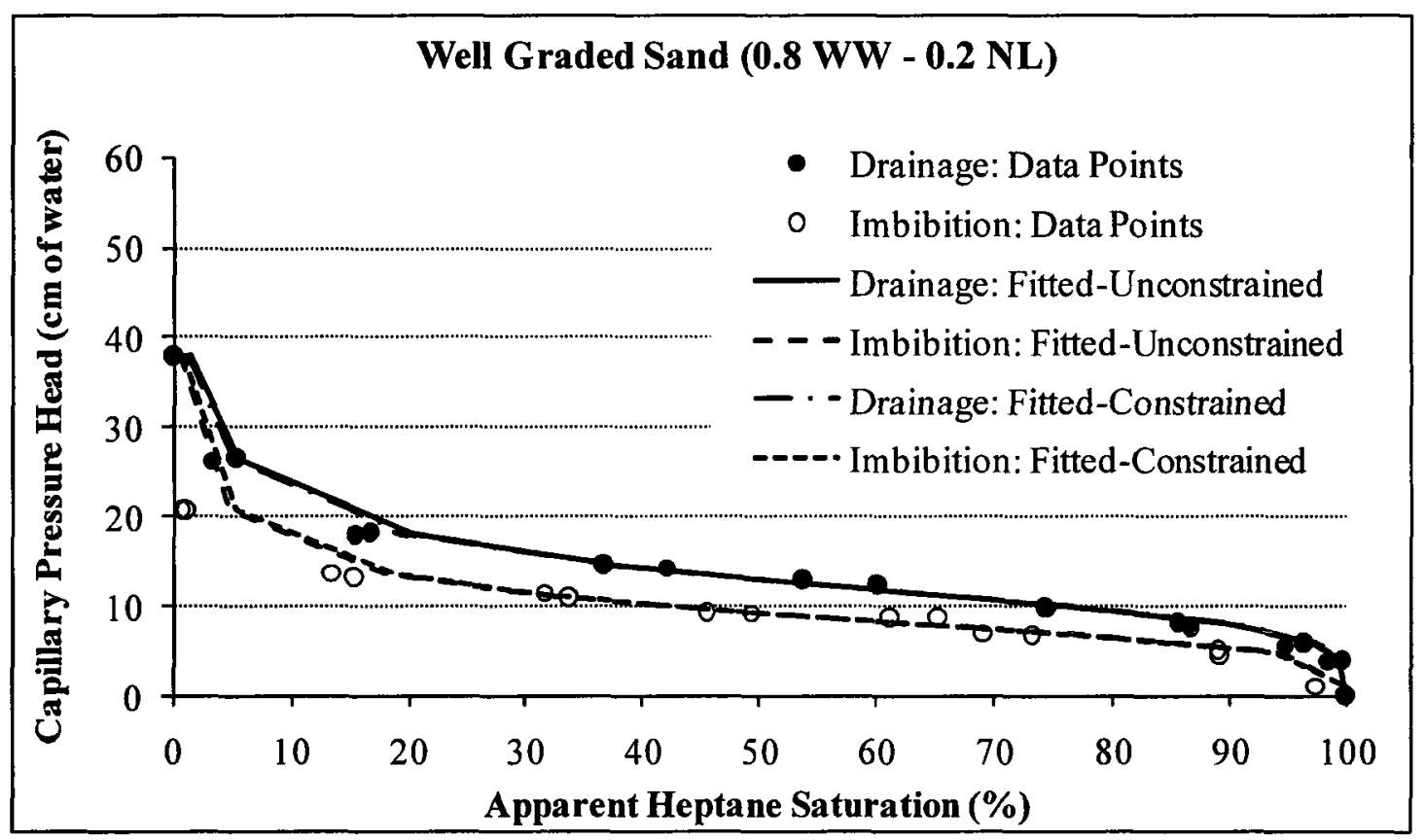

Figure 5.8: Capillary Pressure Head-Apparent Heptane Saturation Main Drainage and Imbibition Pathways in an 80 \% Water-Wet-20\% NAPL-Wet Porous Medium in Well Graded Sand. 


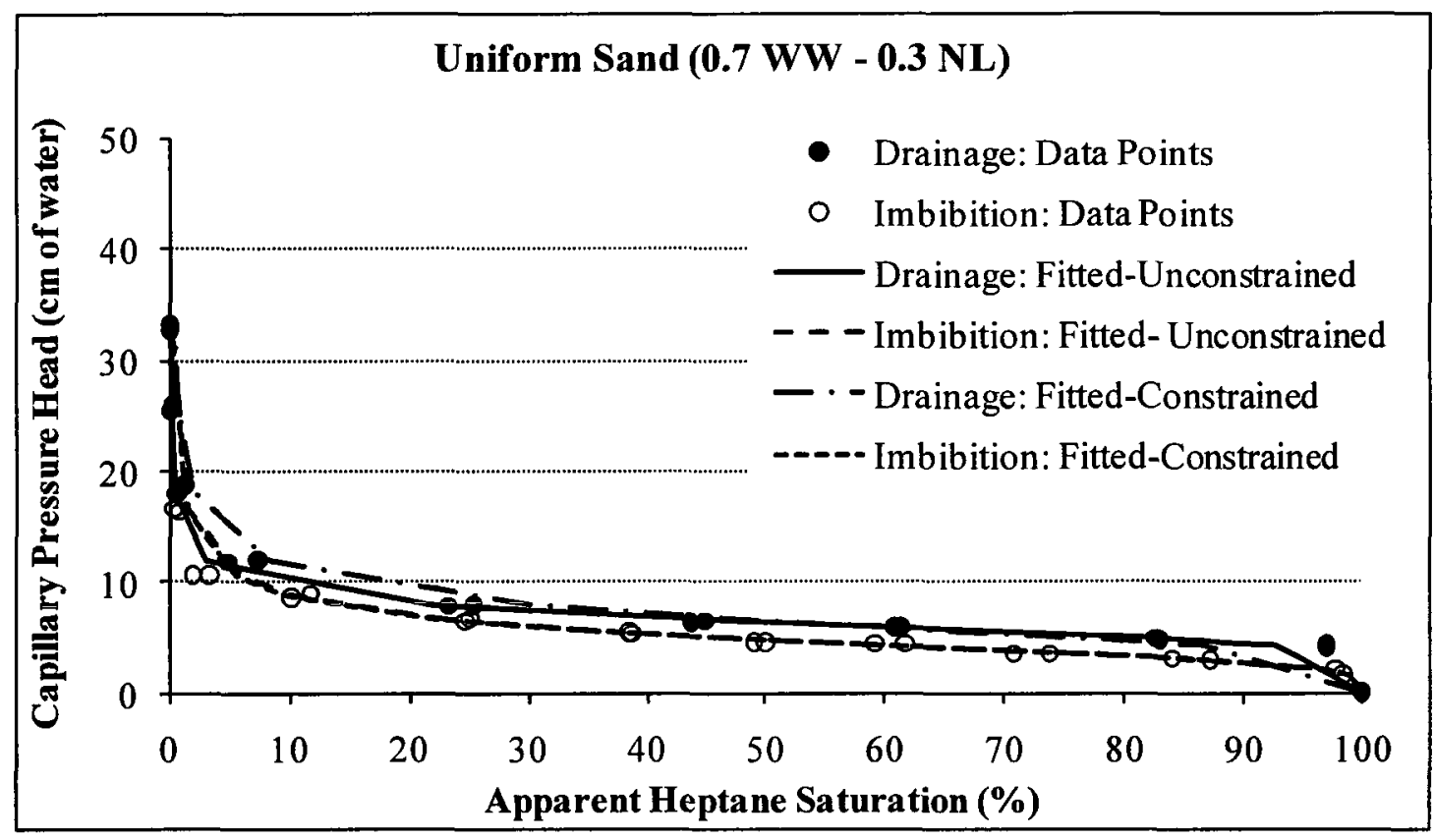

Figure 5.9: Capillary Pressure Head-Apparent Heptane Saturation Main Drainage and Imbibition Pathways in a $70 \%$ Water-Wet-30\% NAPL-Wet Porous Medium in Uniform Sand.

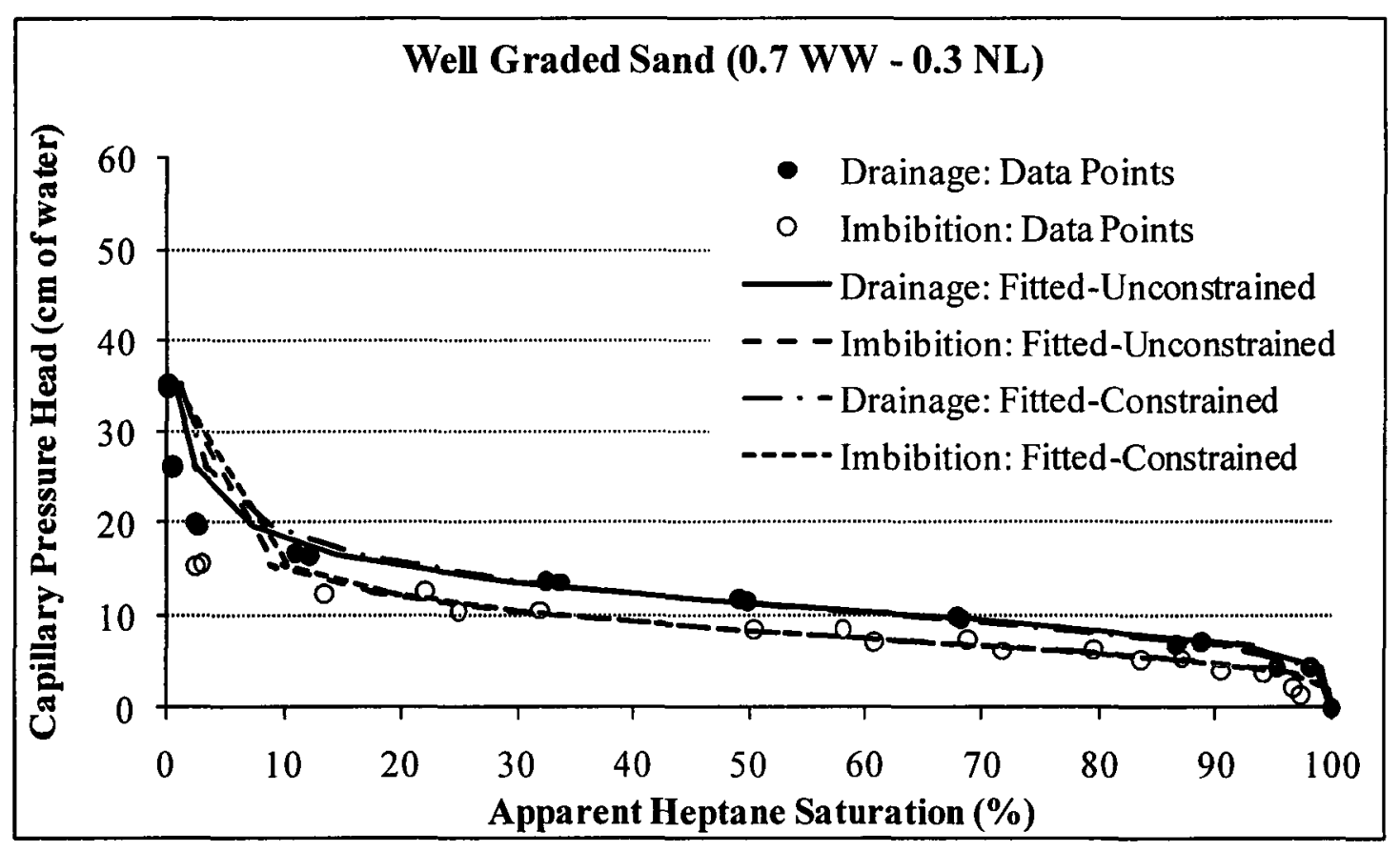

Figure 5.10: Capillary Pressure Head-Apparent Heptane Saturation Main Drainage and Imbibition Pathways in a 70 \% Water-Wet-30\% NAPL-Wet Porous Medium in Well Graded Sand. 


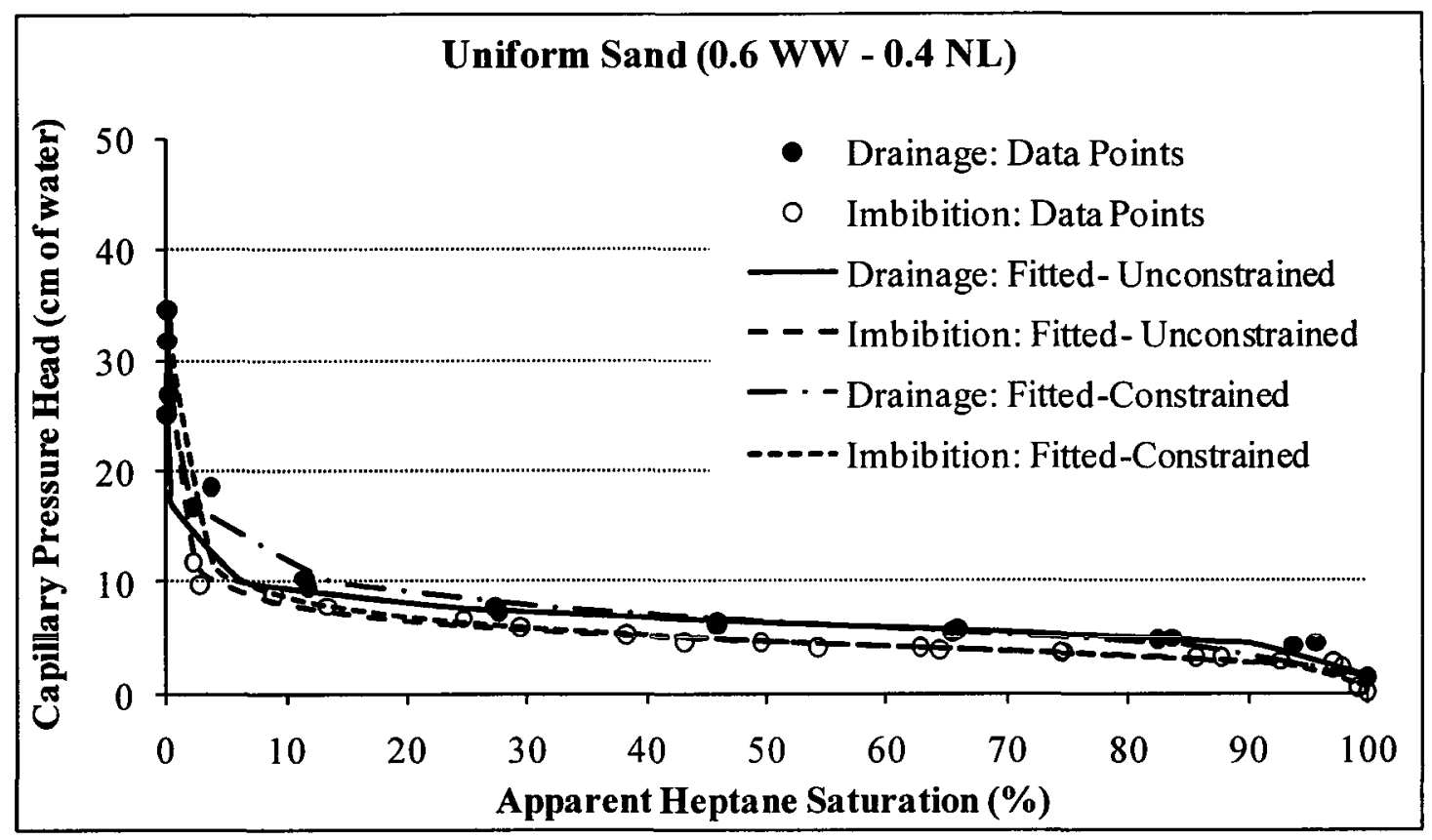

Figure 5.11: Capillary Pressure Head-Apparent Heptane Saturation Main Drainage and Imbibition Pathways in a $60 \%$ Water-Wet-40\% NAPL-Wet Porous Medium in Uniform Sand.

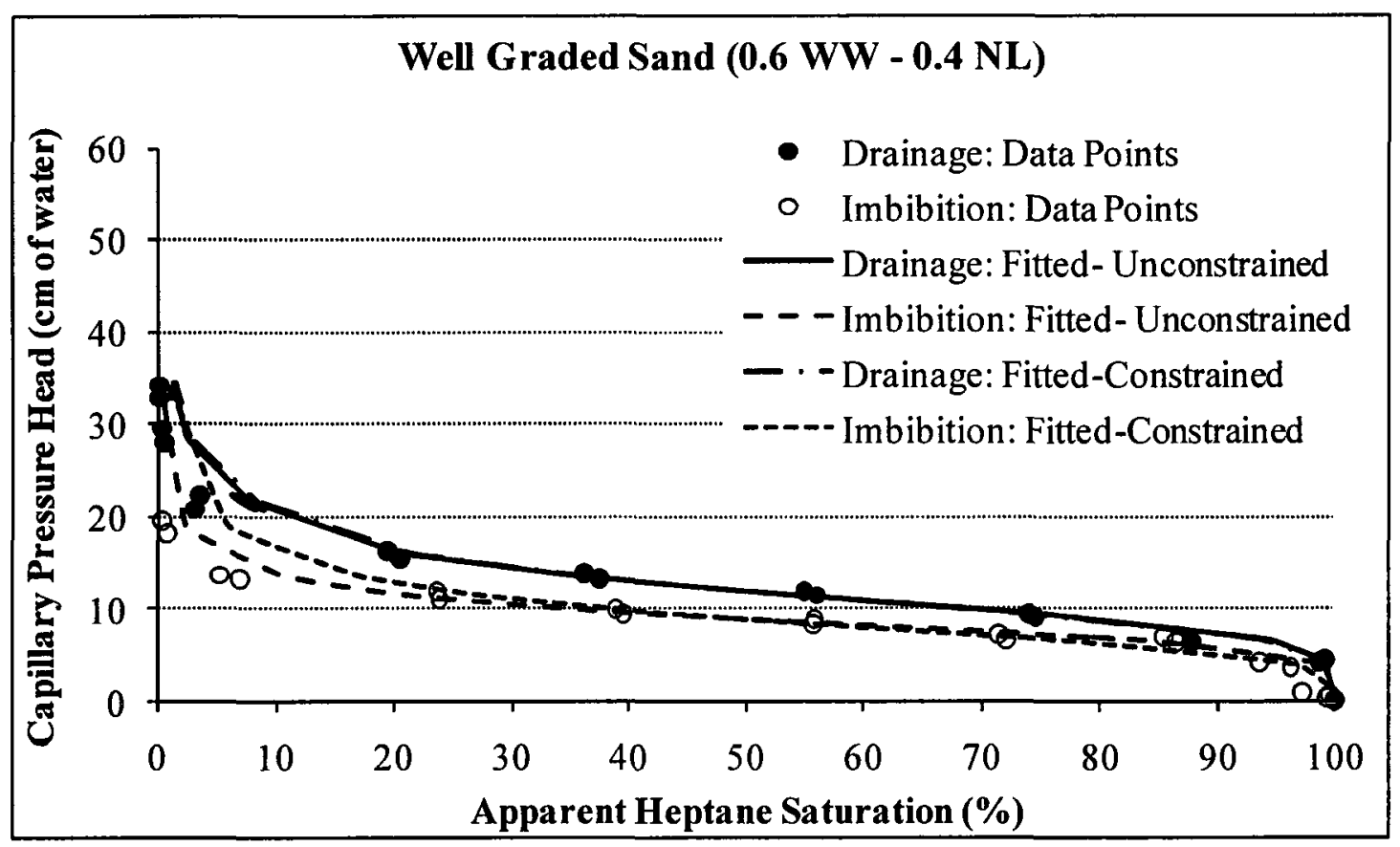

Figure 5.12: Capillary Pressure Head-Apparent Heptane Saturation Main Drainage and Imbibition Pathways in a $60 \%$ Water-Wet-40\% NAPL-Wet Porous Medium in Well Graded Sand. 


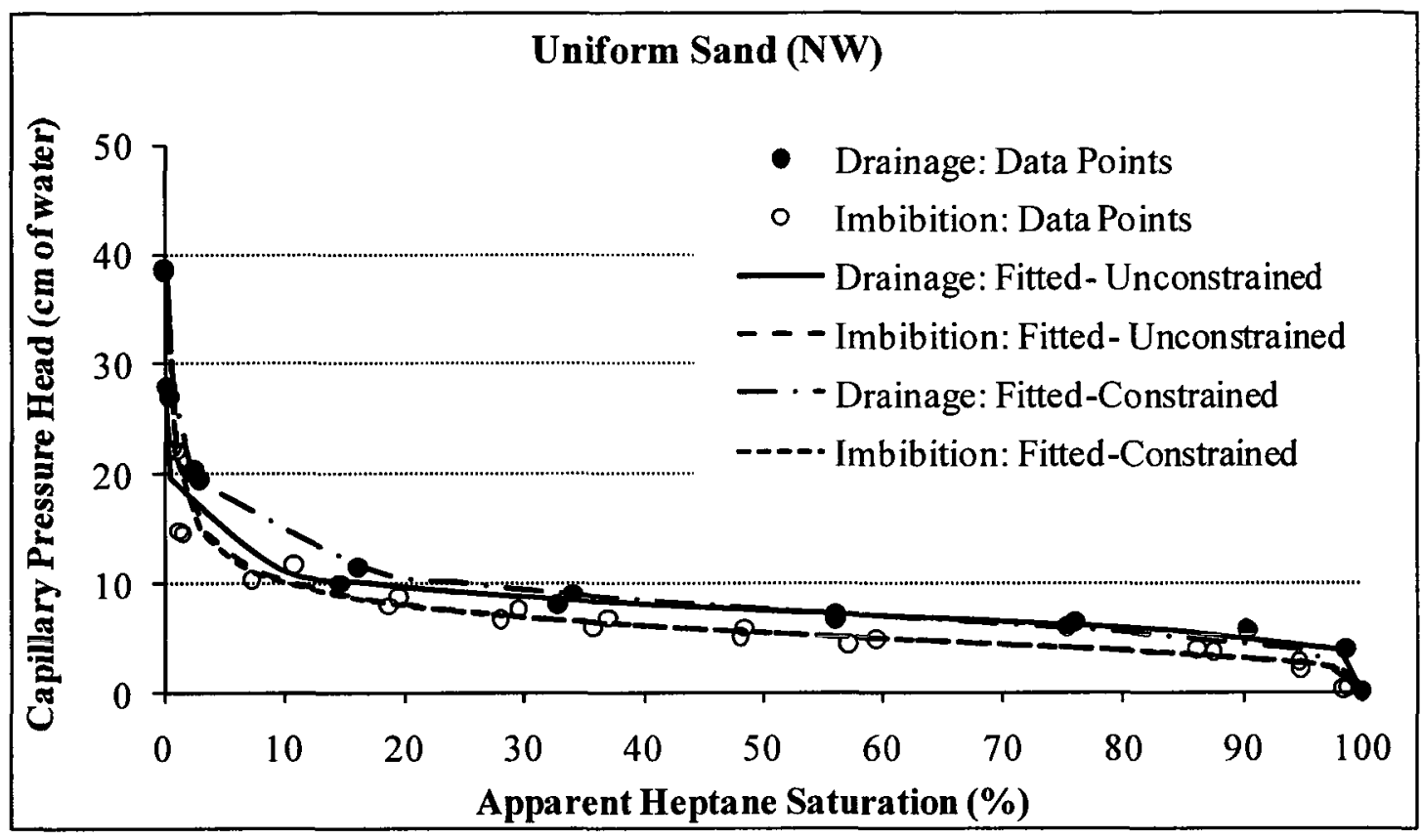

Figure 5.13: Capillary Pressure Head-Apparent Heptane Saturation Main Drainage and Imbibition Pathways in a NAPL-Wet Porous Medium in Uniform Sand.

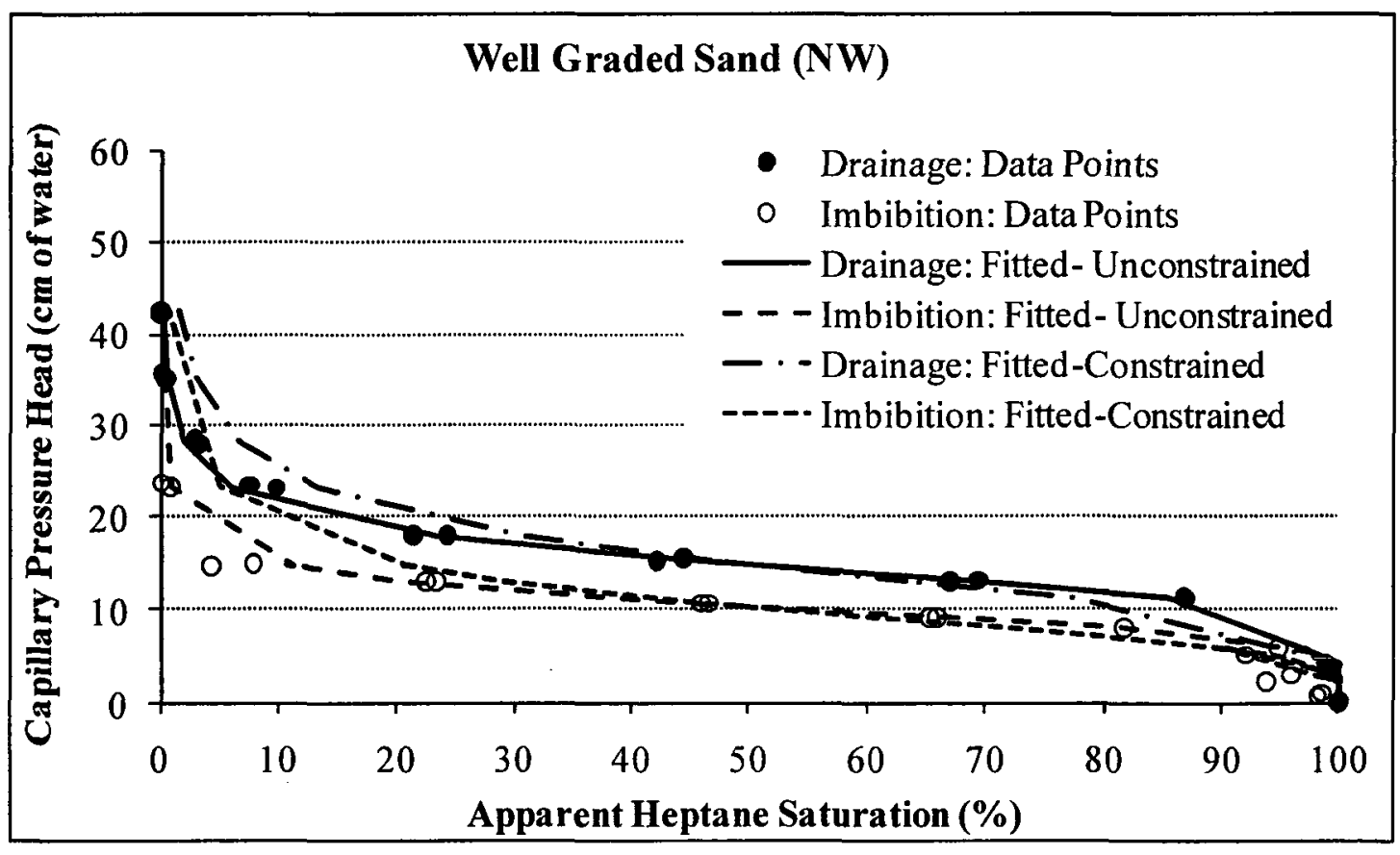

Figure 5.14: Capillary Pressure Head-Apparent Heptane Saturation Main Drainage and Imbibition Pathways in a NAPL-Wet Porous Medium in Well Graded Sand. 


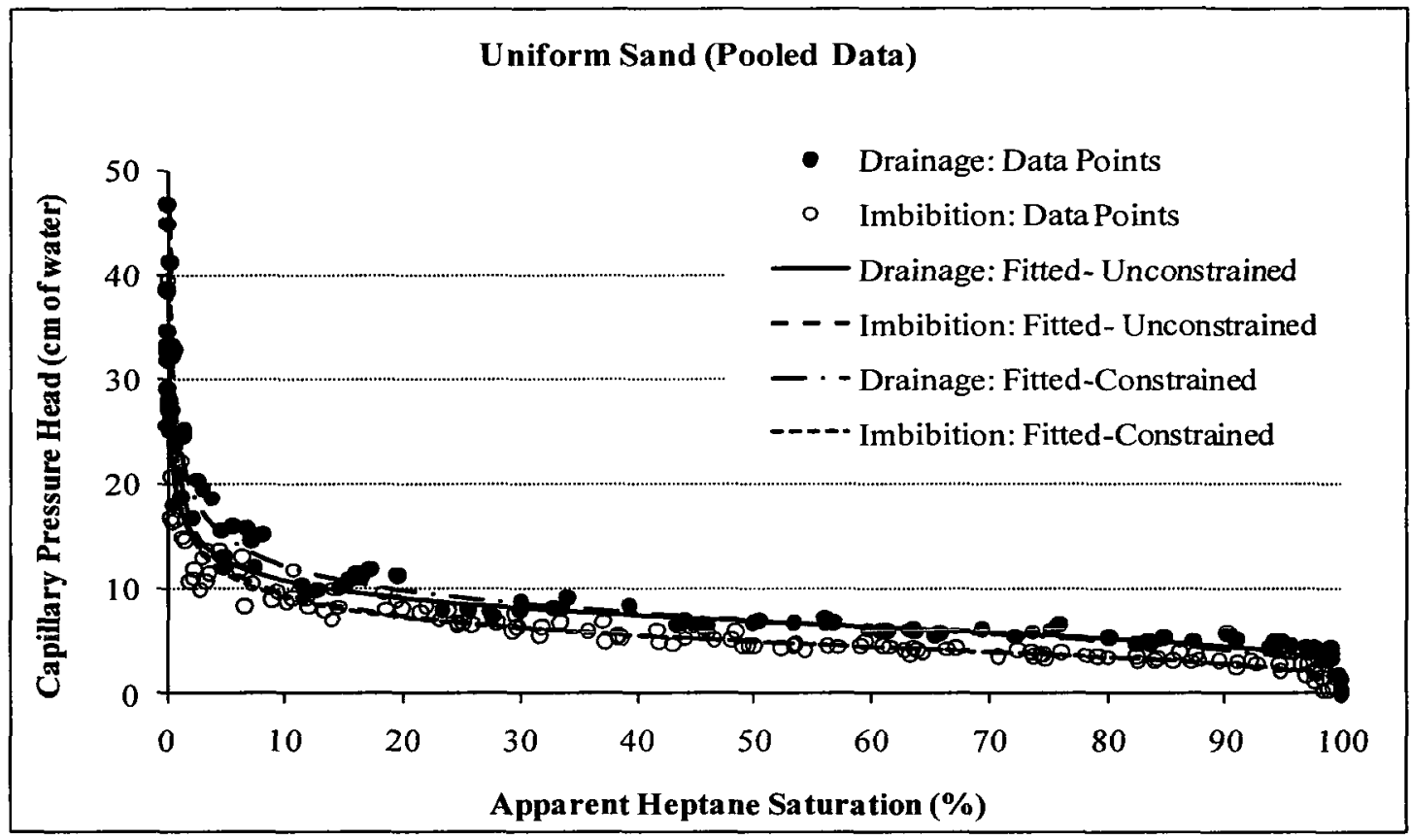

Figure 5.15: Capillary Pressure Head-Apparent Heptane Saturation Main Drainage and Imbibition Pathways Using Pooled Data In Uniform Sand.

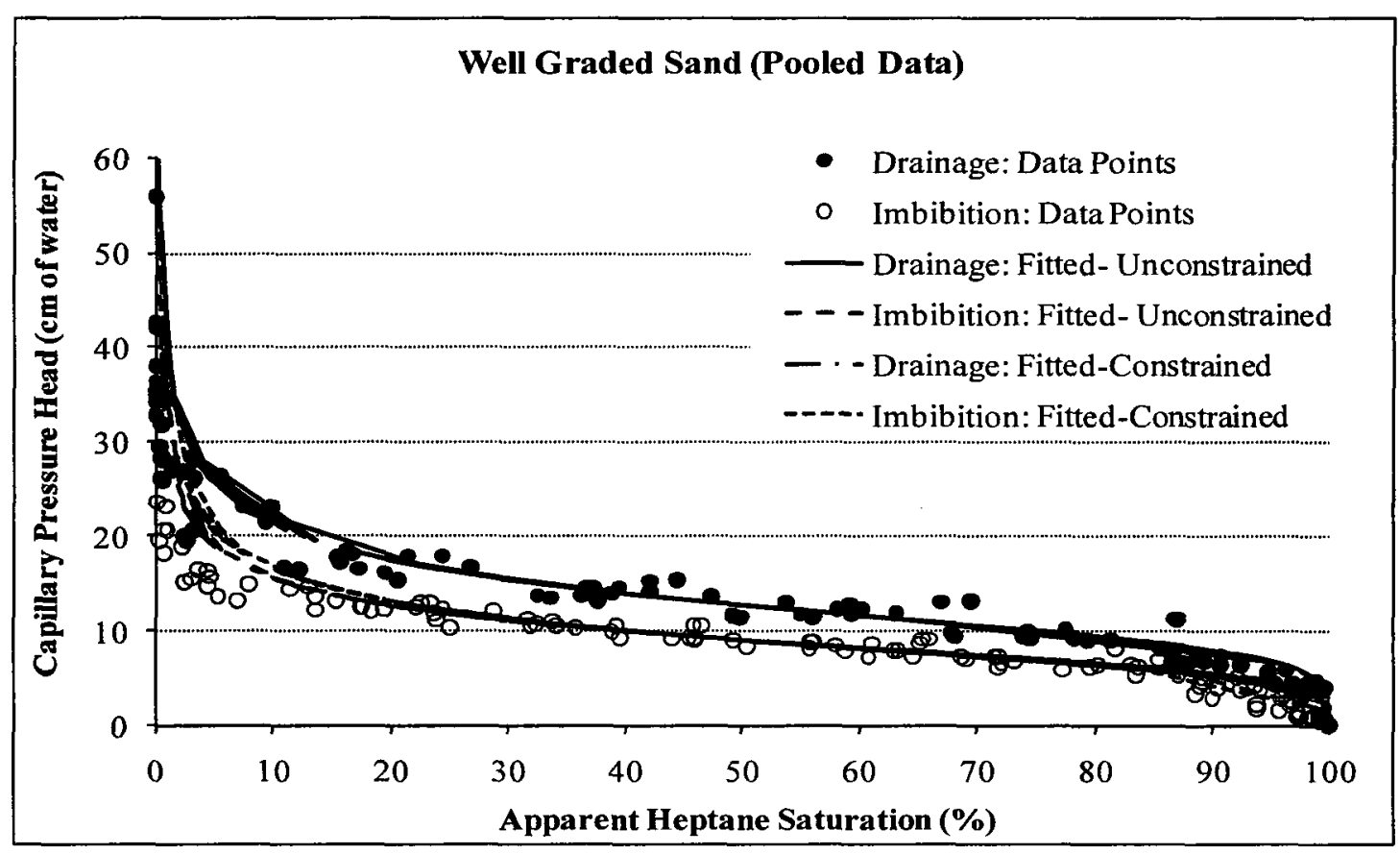

Figure 5.16: Capillary Pressure Head-Apparent Heptane Saturation Main Drainage and Imbibition Pathways Using Pooled Data in Well Graded Sand. 


\subsection{Air/Water $\boldsymbol{P}_{c} \rightarrow S$ Relationship}

The discussion of the air/water $P_{c} \rightarrow S$ relationships is divided into four sections; Section 5.3.1 presents the data for the experiments conducted in a water-wet porous medium and in water-wet porous media with different size NAPL-wet soil lenses. Unlike the air/heptane experiments where the heptane was always the wetting fluid, water is the wetting fluid in the water-wet porous media and both water and air are neutrally wetting fluids in the NAPL-wet soil lenses. Hence, Sections 5.3.2 and 5.3.3 discuss the expected saturation history for air/water in a water-wet porous medium, and a NAPL-wet porous medium, respectively. The final Section 5.3.4 then proposes a conceptual model to construct a $P_{c} \checkmark S$ relationship for an REV consisting of a water-wet porous medium with a NAPL-wet soil lens based on the relationships in each medium, and provides some validation of the proposed model.

\subsubsection{Capillary Pressure Head-Actual Water Saturation Data}

Figure 5.17 and Figure 5.19 show the main drainage and imbibition pathways in uniform sand, respectively for the three porous media scenarios considered; namely a water-wet porous medium, an $80 \%$ water-wet porous medium with $20 \%$ NAPL-wet soil lens, and a $60 \%$ water-wet porous medium with $40 \%$ NAPL-wet soil lens. Similarly, Figure 5.18 and Figure 5.20 show these data in well graded sand. Table 5.5, for uniform sand, and Table 5.6, for well graded sand, show the entrapped gas (i.e., $\mathrm{CO}_{2}$ ) saturation prior to initiating the main drainage pathway, the irreducible water saturation at the end of the main drainage pathway, and the entrapped air saturation at the end of the main imbibition pathway for five porous media; namely a water-wet porous medium, a $90 \%$ water-wet porous medium with $10 \%$ NAPL-wet soil lens, an $80 \%$ water-wet porous 
medium with $20 \%$ NAPL-wet soil lens, a $70 \%$ water-wet porous medium with $30 \%$ NAPL-wet soil lens, and a $60 \%$ water-wet porous medium with $40 \%$ NAPL-wet soil lens.

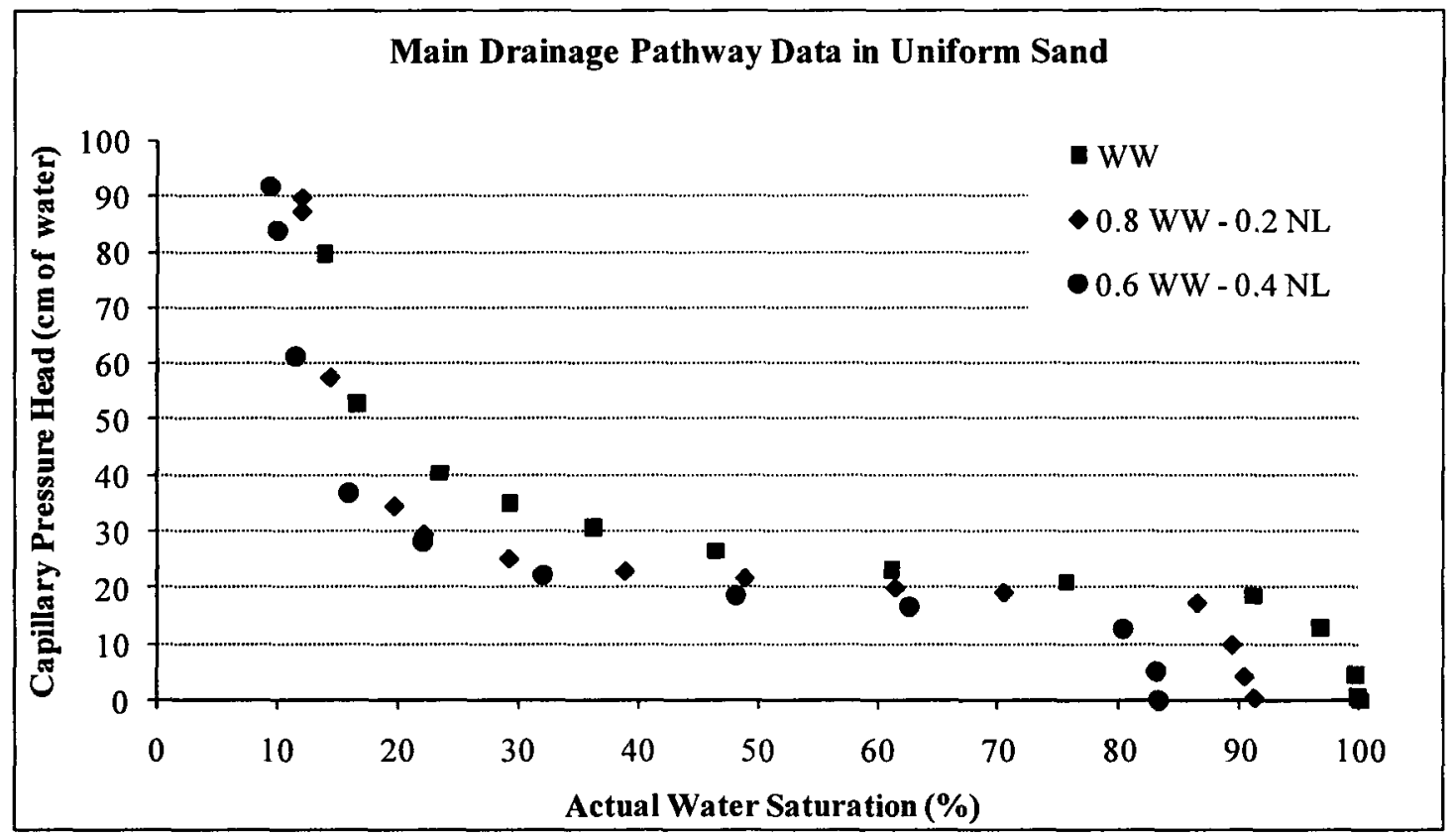

Figure 5.17: Main Drainage Pathway in Different Wettability Porous Media in Uniform Sand.

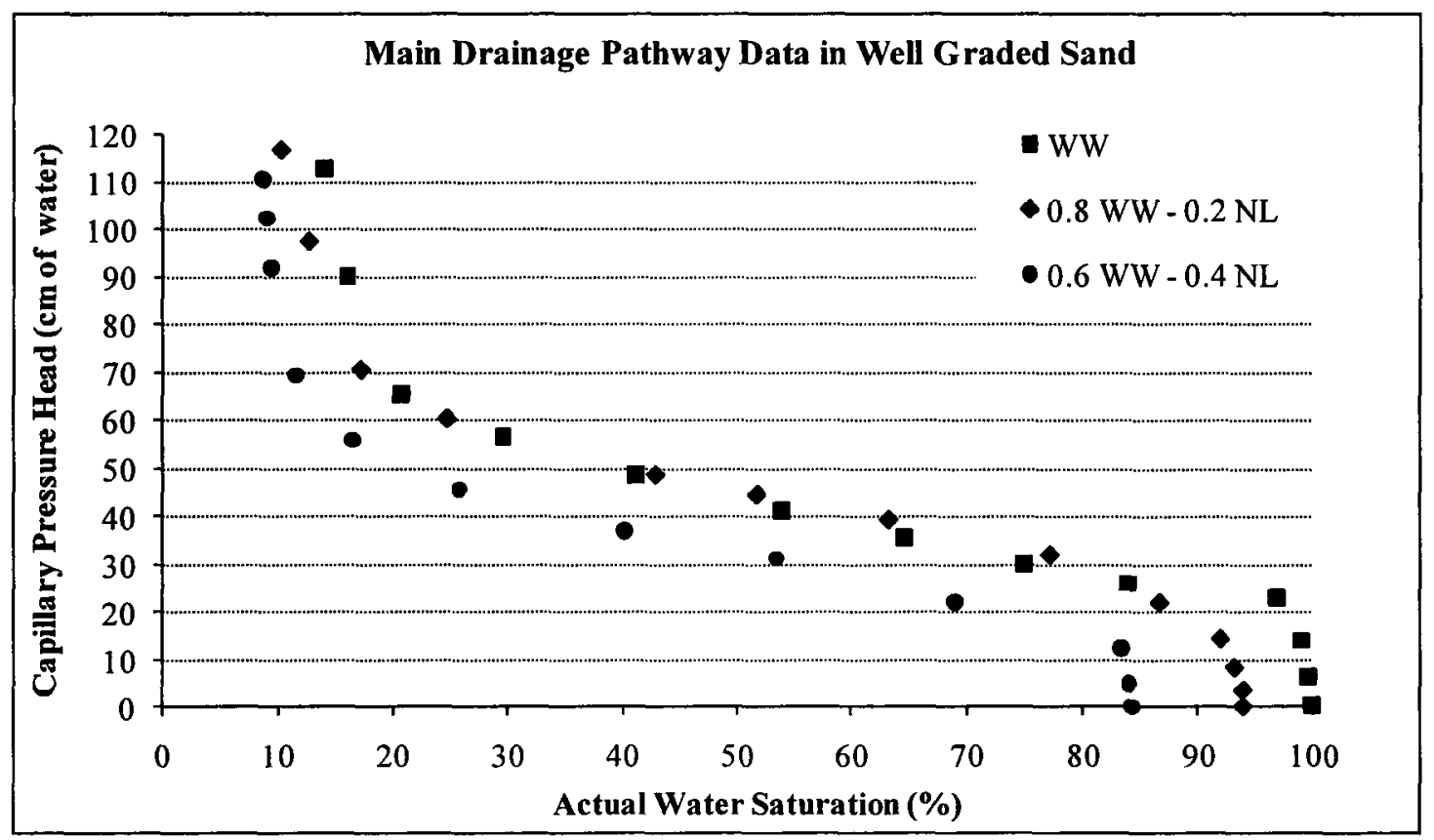

Figure 5.18: Main Drainage Pathway in Different Wettability Porous Media in Well Graded Sand. 


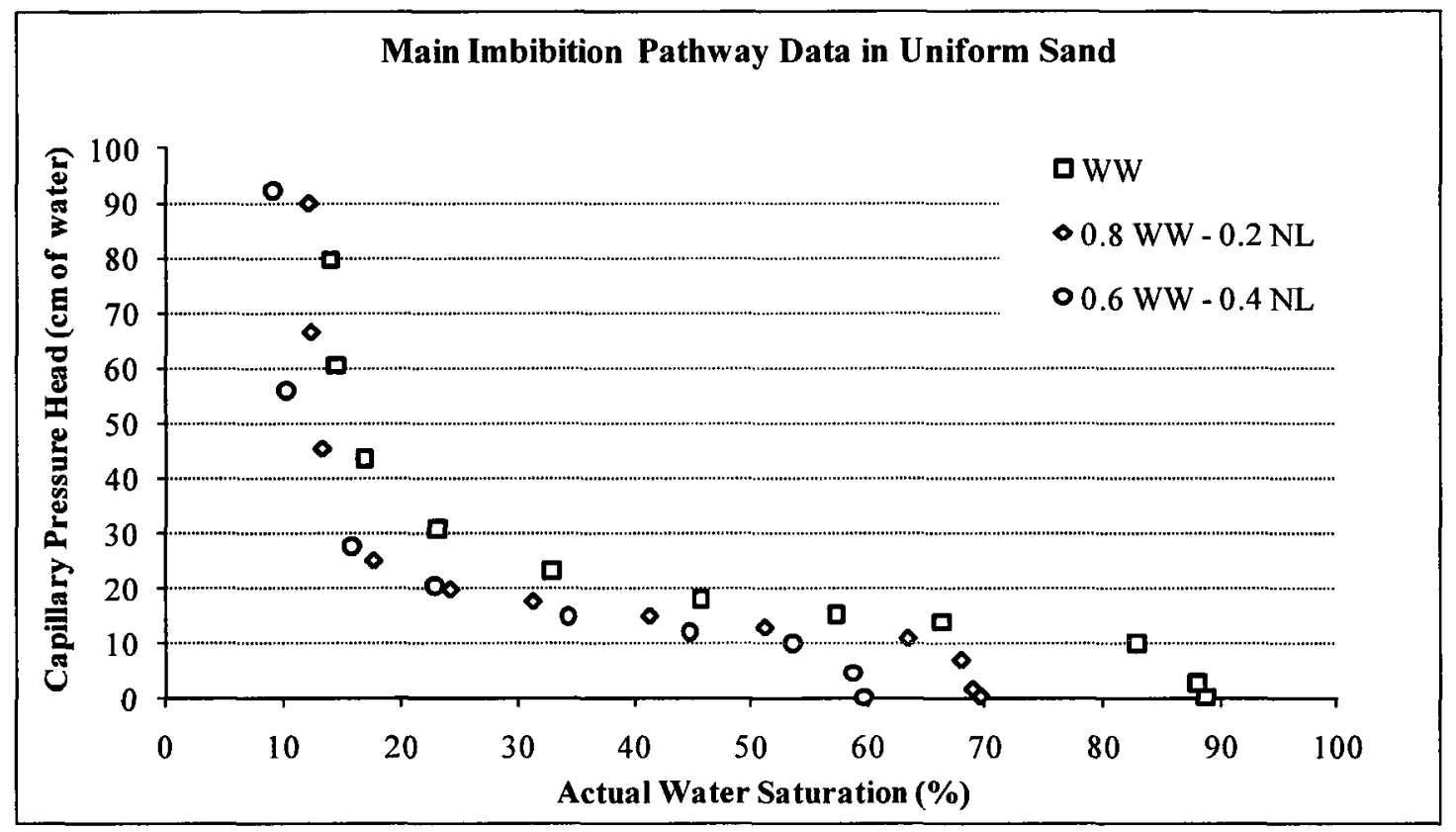

Figure 5.19: Main Imbibition Pathway in Different Wettability Porous Media in Uniform Sand.

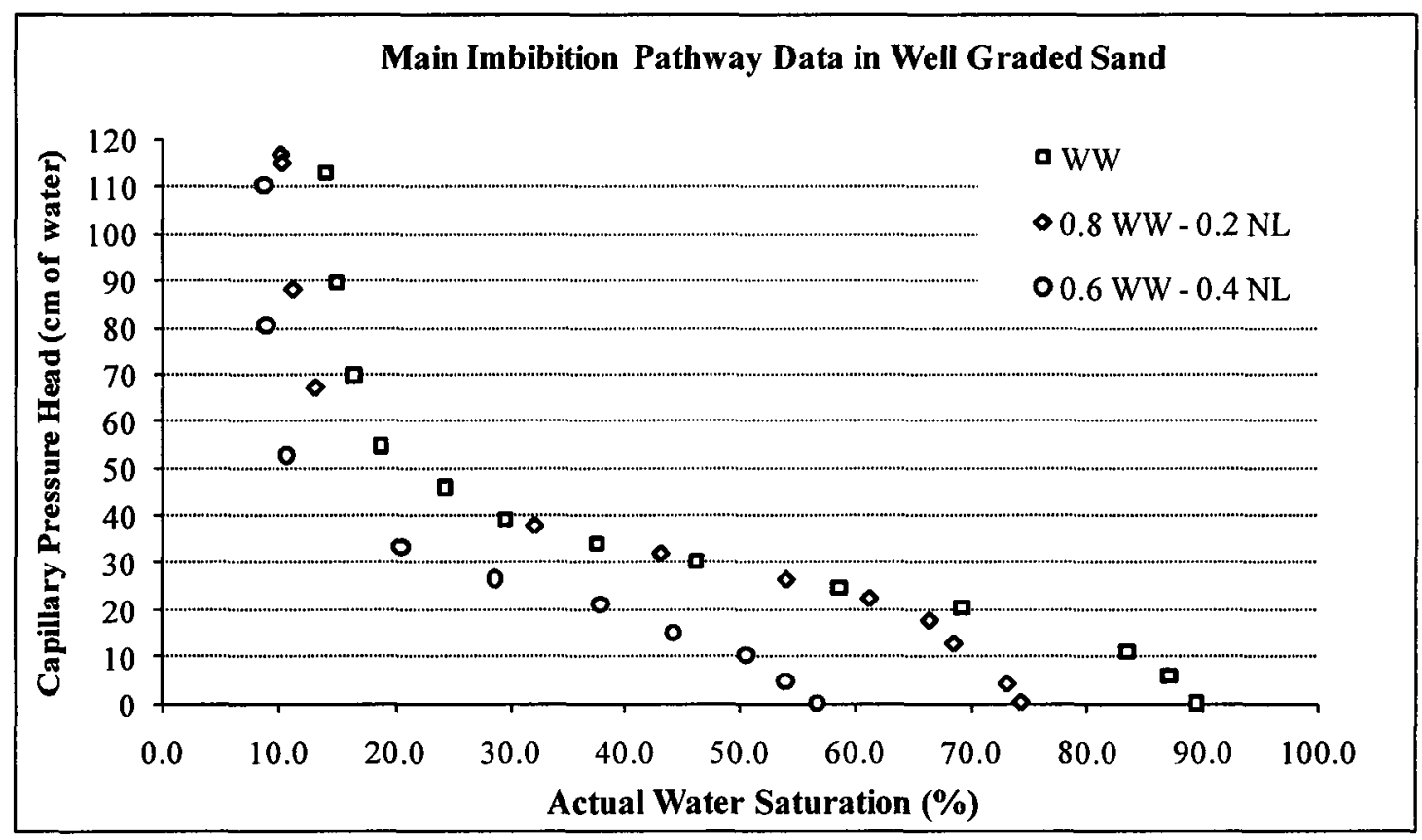

Figure 5.20: Main Imbibition Pathway in Different Wettability Porous Media in Well Graded Sand. 
Table 5.5: Irreducible Water and Entrapped Air Saturations in Uniform Soil.

\begin{tabular}{|c|c|c|c|c|c|c|c|c|}
\hline \multirow{2}{*}{$\begin{array}{c}\text { Wettability } \\
\text { Configuration }\end{array}$} & \multicolumn{4}{|c|}{ Drainage Pathway } & \multicolumn{3}{c|}{ Imbibition Pathway } \\
\cline { 2 - 9 } & \multicolumn{2}{|c|}{ Irreducible } & \multicolumn{2}{c|}{ Entrapped } & \multicolumn{2}{c|}{ Irreducible } & \multicolumn{2}{c|}{ Entrapped } \\
\cline { 2 - 9 } & Test (1) & Test (2) & Test (1) & Test (2) & Test (1) & Test (2) & Test (1) & Test (2) \\
\hline WW & 13.90 & 14.99 & 0.00 & 0.00 & 13.90 & 14.99 & 11.27 & 11.80 \\
\hline $\mathbf{0 . 9}$ WW - 0.1 NL & 14.05 & 14.58 & 5.10 & 2.46 & 14.05 & 14.58 & 20.72 & 19.19 \\
\hline $\mathbf{0 . 8}$ WW - 0.2 NL & 11.72 & 12.09 & 7.18 & 8.67 & 11.72 & 12.09 & 28.23 & 30.48 \\
\hline $\mathbf{0 . 7}$ WW - 0.3 NL & 10.26 & 10.84 & 7.30 & 9.70 & 10.26 & 10.84 & 33.90 & 34.19 \\
\hline $\mathbf{0 . 6}$ WW - 0.4 NL & 9.09 & 9.50 & 30.20 & 16.77 & 9.09 & 9.50 & 40.38 & 40.33 \\
\hline
\end{tabular}

Table 5.6: Irreducible Water and Entrapped Air Saturations in Well Graded Soil.

\begin{tabular}{|c|c|c|c|c|c|c|c|c|}
\hline \multirow{2}{*}{$\begin{array}{c}\text { Wettability } \\
\text { Configuration }\end{array}$} & \multicolumn{4}{|c|}{ Drainage Pathway } & \multicolumn{3}{c|}{ Imbibition Pathway } \\
\cline { 2 - 9 } & \multicolumn{2}{|c|}{ Irreducible } & \multicolumn{2}{|c|}{ Entrapped } & \multicolumn{2}{c|}{ Irreducible } & \multicolumn{2}{c|}{ Entrapped } \\
\cline { 2 - 9 } & Test (1) & Test (2) & Test (1) & Test (2) & Test (1) & Test (2) & Test (1) & Test (2) \\
\hline WW & 13.48 & 13.92 & 0.00 & 0.00 & 13.48 & 13.92 & 10.59 & 10.54 \\
\hline $\mathbf{0 . 9}$ WW - 0.1 NL & 11.62 & 12.30 & 3.80 & 4.57 & 11.62 & 12.30 & 19.91 & 19.31 \\
\hline $\mathbf{0 . 8 ~ W W ~ - ~ 0 . 2 ~ N L ~}$ & 10.00 & 10.22 & 7.38 & 5.98 & 10.00 & 10.13 & 24.74 & 25.68 \\
\hline $\mathbf{0 . 7}$ WW - 0.3 NL & 9.98 & 8.56 & 11.82 & 11.51 & 9.98 & 8.56 & 33.25 & 33.12 \\
\hline $\mathbf{0 . 6}$ WW - 0.4 NL & 8.61 & 9.28 & 15.68 & 12.18 & 8.61 & 9.28 & 43.25 & 43.20 \\
\hline
\end{tabular}

As shown in Figure 5.17 and Figure 5.18, and Table 5.5 and Table 5.6, the main drainage pathway in the water-wet porous medium starts at $100 \%$ actual water saturation while the main drainage pathways in the other porous media start at an actual water saturation less than unity with an entrapped gas in each medium. When attempting to saturate the porous medium, water saturates the entire water-wet soil fraction and a fraction of the NAPL-wet soil lens; hence gas is entrapped in the NAPL-wet soil lens. The magnitude of the entrapped gas, within the same sand type, is higher in the $60 \%$ water-wet porous medium with $40 \%$ NAPL-wet soil lens than in the $80 \%$ water-wet 
porous medium with $20 \%$ NAPL-wet soil lens. In other words, the magnitude of the entrapped gas increases with the increase of the NAPL-wet soil lens size. By examining the irreducible water saturations, it is noticed that the irreducible water saturation decreases with the existence or increase in volume of the NAPL-wet soil lens in a waterwet porous medium.

As shown in Figure 5.19 and Figure 5.20, and Table 5.5 and Table 5.6, each of the main imbibition pathways starts at its corresponding irreducible saturation and ends at actual water saturation less than unity, leaving an entrapped air in each medium. The entrapped air saturation increases with the existence or the increase in volume of the NAPL-wet soil lens. As shown in Figure 5.19 and Figure 5.20, the main imbibition pathways for the three porous media are comparable within the same sand type.

\subsubsection{Water-Wet Porous Medium}

In a water-wet porous medium, water is the wetting fluid and air is the nonwetting fluid. When water drains from the porous medium, it starts to drain from pore body centres and is replaced by air. As water continues drainage, air continues filling the pore bodies and the large pore throats until water reaches its irreducible saturation value. At this point, water is located in small pore throats and as thin films coating soil particles (as shown in Figure 5.21). When water imbibes into the porous medium, it starts filling the pore throats and small pore bodies as it replaces air. By continuing imbibition, water fills the larger pore bodies where some air is entrapped. 


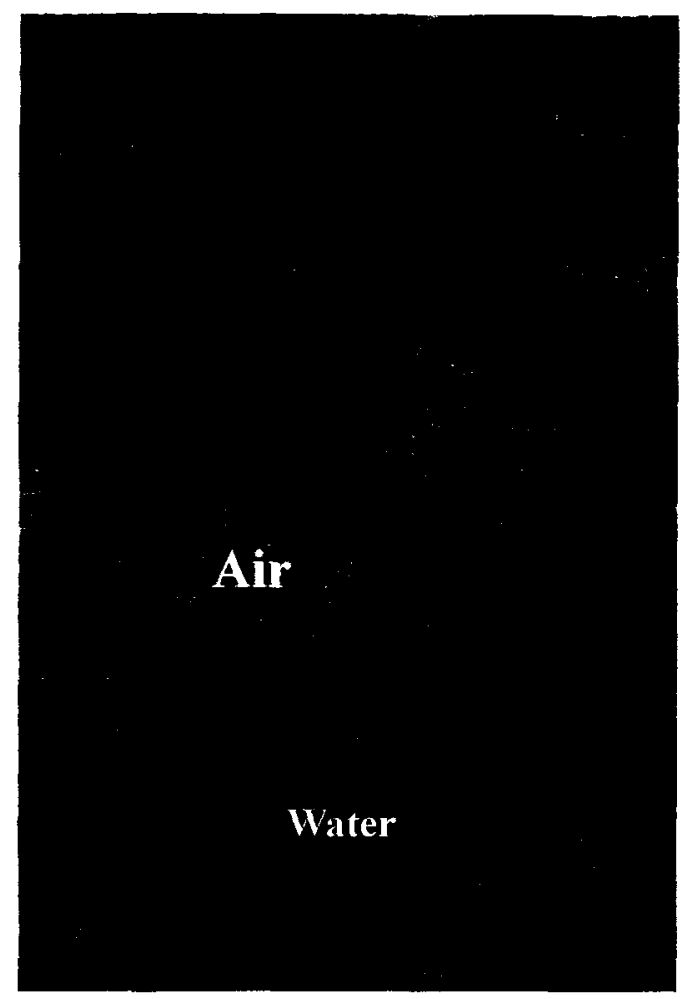

Figure 5.21: Air and Water Distributions at Irreducible Water Saturation.

\subsection{2.a. Water Main Drainage Pathway}

The main drainage pathway starts at an actual water saturation of unity and capillary pressure head of zero. The capillary pressure head has to be higher than the air entry pressure head before water starts to drain. As capillary head increases, the actual water saturation decreases until it reaches irreducible water saturation $\left(S_{m}\right)$ (as shown in Figure 5.22). In a way to scale the actual saturations between $S_{m}$ and unity over an effective saturation between zero and unity, Parker and Lenhard (1987) defined the effective saturation as a scaled saturation that does not take into account other entrapped fluids in it. Therefore, it includes only one fluid phase. Parker and Lenhard (1987) defined an apparent saturation as a scaled fluid saturation that takes into account other entrapped fluids in it and enables scaling the effective saturations between zero and unity. 
Therefore, it includes multiple fluid saturations in one apparent fluid saturation term. The apparent fluid saturation reflects the location of the wetting-non-wetting fluid interface which is not impacted by the presence of the entrapped fluids. The effective saturations and apparent saturations are equal along the main drainage pathway (as shown in Figure 5.22).

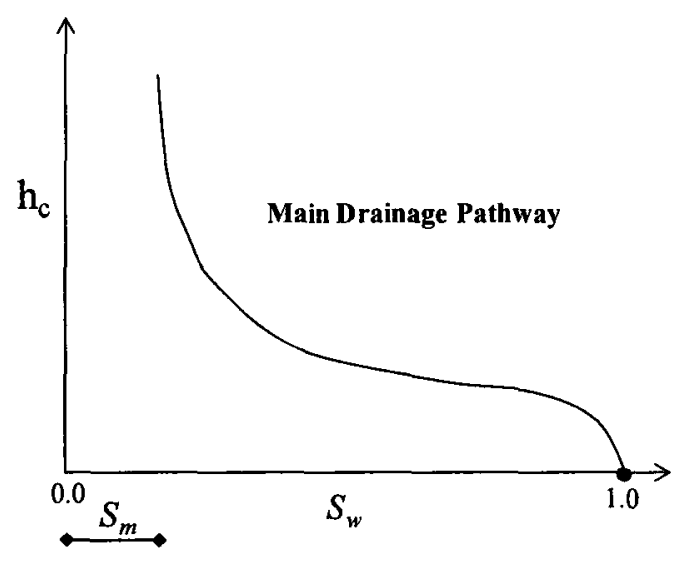

a. Actual Saturation

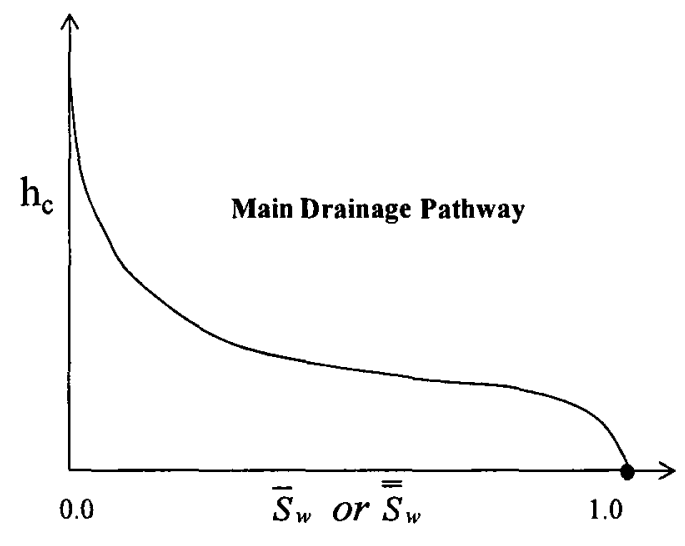

b. Effective or Apparent Saturation

Figure 5.22: Capillary Pressure Head -Saturation Main Drainage Pathway.

\subsection{2.b. Water Main Imbibition Pathway}

The main imbibition pathway starts at the irreducible water saturation and it ends at a capillary pressure head of zero where the actual water saturation is less than unity due to the entrapped air (as shown in Figure 5.23a). Figure 5.23b and Figure 5.23c show the capillary pressure head - effective saturation and - apparent saturation, respectively. 


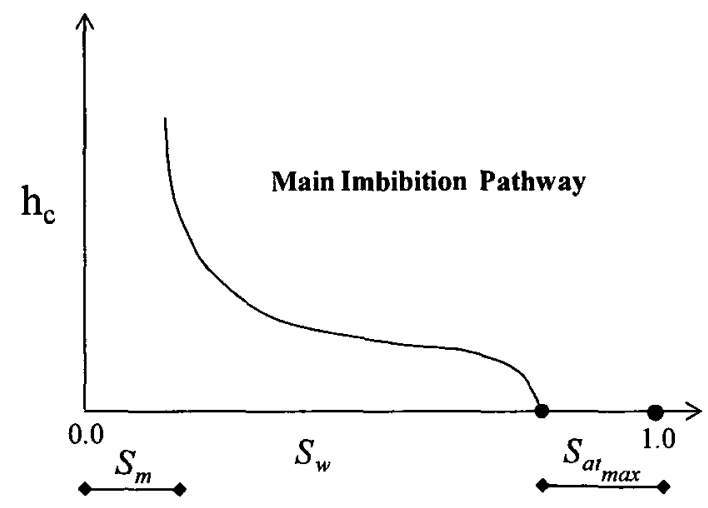

a. Actual Saturation

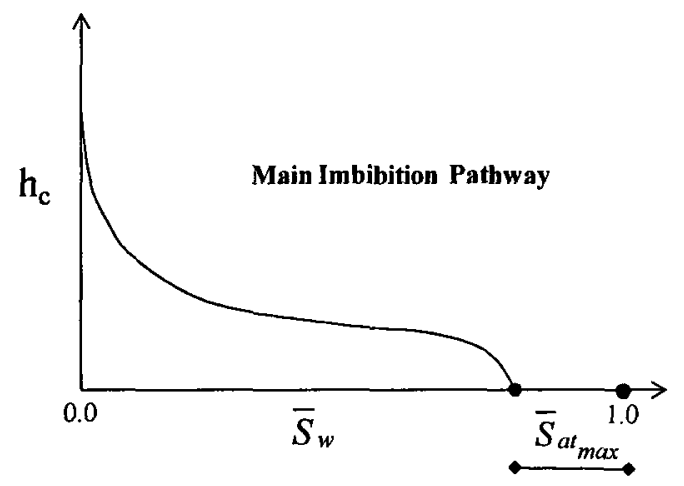

b. Effective Saturation

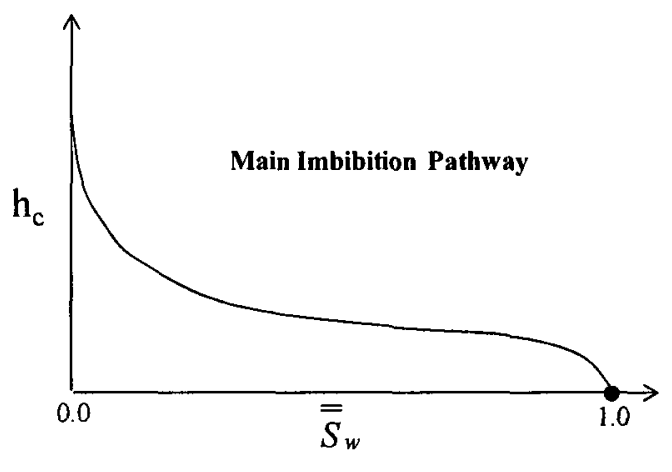

c. Apparent Saturation

\section{Figure 5.23: Capillary Pressure Head-Saturation Main Imbibition Pathway.}

Where:

$S_{a t_{\max }}:$ maximum actual entrapped air saturation;

$\bar{S}_{a t_{\max }}:$ maximum effective entrapped air saturation; and

$S_{a t}$ : actual entrapped air saturation corresponding to actual water saturation $S_{w}$ on the main imbibition pathway. 
$\bar{S}_{a t}=\frac{\bar{S}_{w}}{\left[\left(1 / \bar{S}_{a_{\text {max }}}\right)-1\right]}$

(Lenhard 1992)

$\overline{\bar{S}}_{w}=\bar{S}_{w}+\bar{S}_{a t}$

$\overline{\bar{S}}_{w}=\frac{1}{\left[1+\left(\alpha h_{c}\right)^{n}\right]^{m}}$ for $h_{c}>0.0$ or $\quad h_{c}=\frac{1}{\alpha}\left[\left(\overline{\vec{S}}_{w}\right)^{-1 / m}-1\right]^{1 / n}$ for $\overline{\bar{S}}_{w}<1.0$

$\overline{\bar{S}}_{w}=1.0$ when $h_{c}=0.0 \quad$ or $\quad h_{c}=0.0$ when $\overline{\bar{S}}_{w}=1.0$.

\subsubsection{NAPL-Wet Porous Medium}

In a NAPL-wet porous medium, both air and water are neutrally wetting phases and hence the distribution of air and water in the pore space is difficult to assess. Neutrally wetting means that if the porous medium is saturated with one fluid, then the second fluid enters the porous medium and replaces the first fluid only if suction is applied on the first fluid or pressure is applied on the second fluid. An air/water $P_{c}-S$ experiment in a NAPL-wet porous medium starts with an air-saturated soil sample. In order for the water to enter the porous medium, the capillary pressure has to drop to a value less than the water entry pressure. The water may enter into and move in a porous medium under its pressure or under the air suction. Since the air pressure is zero, then, in a NAPL-wet porous medium, the water pressure has to be positive along the water imbibition pathway in order to force the water to move into the porous medium and increase its saturation. On the other hand, along the water drainage pathway, air pressure has to exceed the water pressure in order for the air to replace the water. This implies that the capillary pressure has to be positive. 
One way to conceptually illustrate the imbibition and drainage of water in a NAPL-wet porous medium is to look at the pore space as a collection of NAPL-wet capillary tubes. It is hypothesized that the contact angle of water, in the presence of air, with the inner surfaces of these tubes is slightly larger than $90^{\circ}$ for the water imbibition and it is slightly less than $90^{\circ}$ for the water drainage. The air/water capillary pressure head may be calculated using Equation (2.17). This implies that, water saturation in a NAPL-wet porous medium is less than water saturation in a water-wet porous medium, if both media are exposed to the same external capillary pressure head along their main drainage pathways. This conclusion will be used to develop the conceptual model for air/water two-phase system in a water-wet porous medium with a NAPL-wet soil lens. The following sections describe the water main imbibition and drainage pathways in a NAPL-wet porous medium.

\subsection{3.a. Water Main Imbibition Pathway}

The water main imbibition pathway or the air main drainage pathway starts at an actual air saturation of unity, and zero water pressure. As mentioned earlier, the water pressure has to exceed the water entry pressure before air starts to drain and is replaced by water. As water pressure head increases, the actual air saturation decreases until it reaches irreducible air saturation $\left(S_{a m}\right)$ (as shown in Figure 5.24). Therefore, along the water main imbibition pathway, actual water saturation in a NAPL-wet porous medium should be more than zero and less than $1-S_{a m}$. 


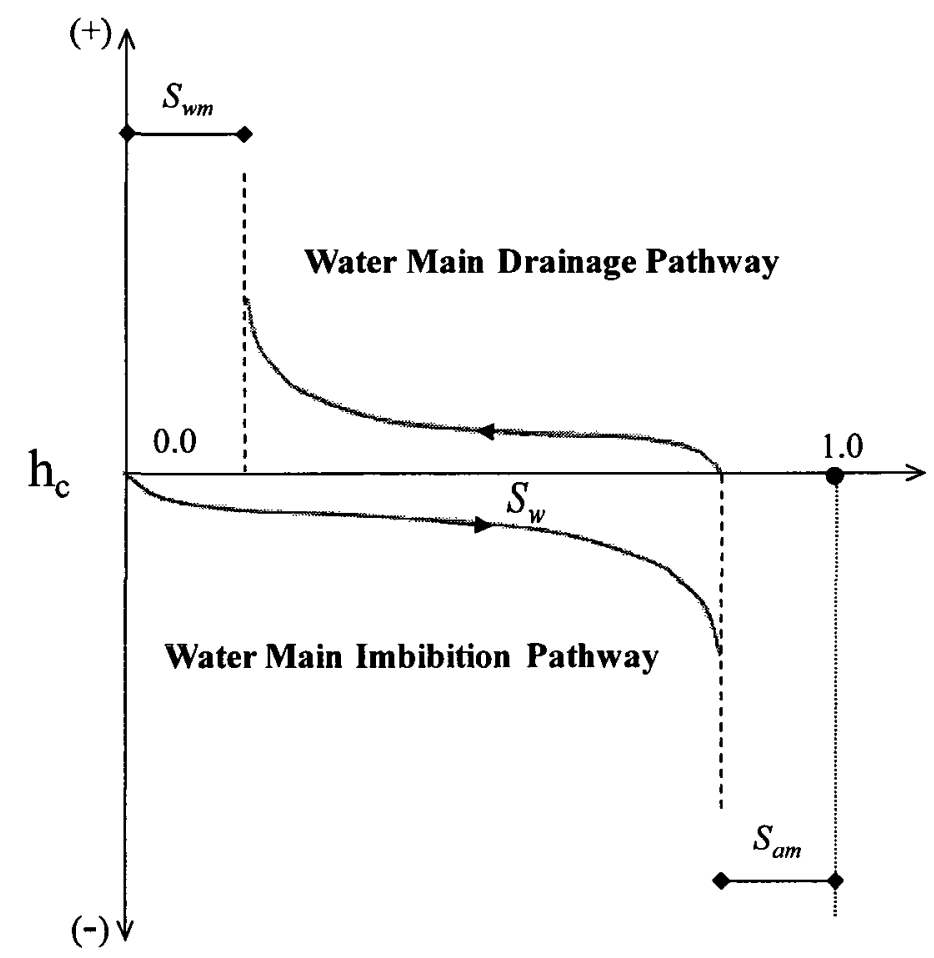

Figure 5.24: Capillary Pressure Head-Actual Water Saturation Main Imbibition and Drainage Pathways in a NAPL-Wet Porous Medium.

\subsection{3.b. Water Main Drainage Pathway}

The water main drainage pathway or the air main imbibition pathway starts at an actual water saturation of $1-S_{a m}$ and irreducible air saturation $\left(S_{a m}\right)$. As mentioned earlier and shown in Figure 5.24, in order for the water to drain from the porous medium and to be replaced by air, water has to be under suction if air pressure is zero. As water suction increases, air imbibes into the porous medium and water is displaced. This continues to happen (i.e., as water suction increases, air imbibes into the porous medium and displaces the water) until water saturation reaches its irreducible value (i.e., an actual saturation of $S_{w m}$ ). At this point, the air exists at actual air saturation less than unity (as shown in Figure 5.24). The air/water $P_{c}-S$ curves were measured in the uniform and well graded 
NAPL-wet porous media and shown in Figure 5.25 and Figure 5.26. These data validate the conceptual description of an air/water $P_{c}-S$ relationship in a NAPL-wet porous medium.

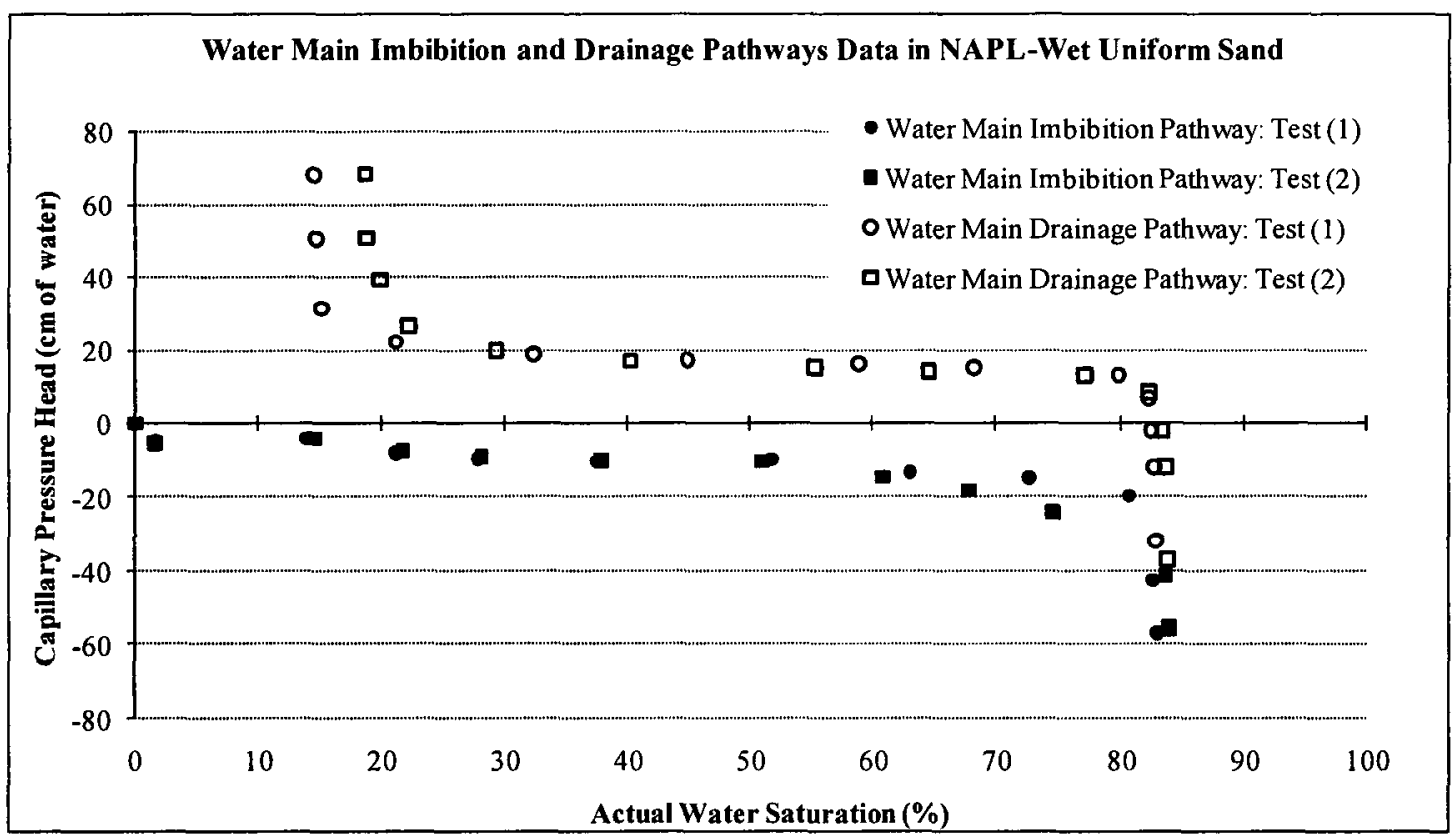

Figure 5.25: Capillary Pressure Head-Actual Water Saturation Main Imbibition and Drainage Pathways in a Uniform NAPL-Wet Porous Medium. 


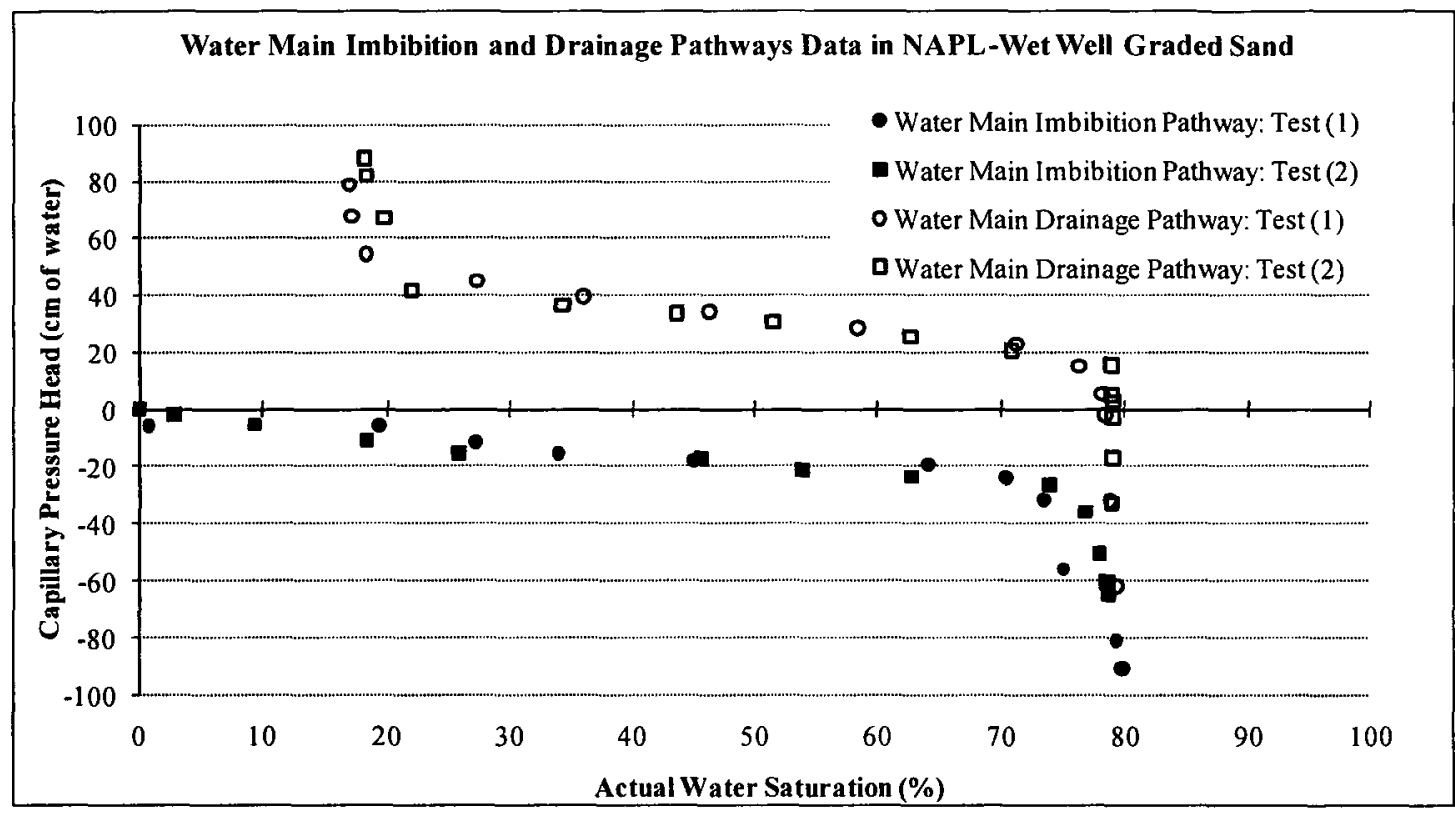

Figure 5.26: Capillary Pressure Head-Actual Water Saturation Main Imbibition and Drainage Pathways in a Well Graded NAPL-Wet Porous Medium.

Therefore, there are some points that have to be highlighted. First, a NAPL-wet porous medium cannot normally reach $100 \%$ water saturation. Instead, air remains in the porous medium at an irreducible saturation. The water drains from the porous medium under positive capillary pressure. At the end of the water drainage pathway, water remains in the porous medium at an irreducible saturation.

\subsubsection{Water-Wet Porous Medium with a NAPL-Wet Soil Lens}

In a water-wet porous medium or a water-wet porous medium with a NAPL-wet soil lens, prior to initiating the water main drainage pathway, a certain procedure is followed to set up the experiment. This procedure was explained in detail in Chapter 3 . In this procedure, the tested soil sample was flushed with carbon dioxide. Then, in order to ensure saturating the soil sample with water, it was flushed with at least three pore volumes of water under a negative capillary pressure (i.e., positive water pressure). 
Following this procedure resulted in a water-saturated water-wet porous medium while in a water-wet porous medium with a NAPL-wet soil lens, water preferentially flowed in the water-wet soil fraction, leaving the NAPL-wet soil lens either dry or partially water-saturated. The degree of water saturation in the NAPL-wet soil lens depends on the water pressure in the lens. Figure 5.27 shows the water drainage and imbibition pathways in the water-wet porous medium and in the NAPL-wet porous medium representing the water main drainage and imbibition pathways in the water-wet soil fraction and in the NAPL-wet soil lens, respectively. The primary imbibition pathways in the water-wet soil fraction and in the NAPL-wet soil lens start at points 1 as shown in Figure 5.27a and b, respectively. In the water-wet soil fraction, this point corresponds to zero water saturation and high positive capillary pressure. In the NAPLwet soil lens, at point 1 , the water saturation and the capillary pressure head are zero. As explained in Chapter 3, the porous medium is saturated as capillary pressure is decreased (i.e., water pressure is increased). To ensure $100 \%$ water saturation, the porous medium is flushed with at least three pore volumes of water. During this time, the porous medium is exposed to small positive water pressures, which creates a slight negative capillary pressure. Therefore, the water saturation and the corresponding capillary pressure head in the water-wet soil fraction and in the NAPL-wet soil lens correspond to point 2 in Figure 5.27. At this point, the water-wet soil fraction is $100 \%$ water-saturated. The water saturation in the NAPL-wet soil lens at point 2 is calculated by knowing the water main imbibition pathway in the NAPL-wet porous medium and the capillary pressure head or the water pressure head for zero air pressure. This water pressure head is slightly more 
than the water depth above the lens in the Buchner funnel prior to the end of the experimental setup before initiating the $P_{c}-S$ experiment.

As explained in Chapter 3, the excess water collected above the soil sample in the Buchner funnel is removed prior to initiating the water main drainage pathway. At this point, the capillary pressure head is zero. The water saturations in the water-wet soil fraction and in the NAPL-wet soil lens are represented by point 3 in Figure 5.27a and $b$, respectively. At this point, the water-wet soil fraction is $100 \%$ water-saturated. The water saturation in the NAPL-wet soil lens can be obtained from the drainage scanning path $2 \rightarrow 3$, as shown in Figure $5.27 \mathrm{~b}$. As the capillary pressure increases, the water saturations in the water-wet soil fraction and in the NAPL-wet soil lens decrease. In the water-wet soil fraction, water drainage follows the water main drainage pathway, as shown in Figure 5.27a. In the NAPL-wet soil lens, the water drains following the water drainage scanning path $3 \rightarrow 5$, as shown in Figure $5.27 \mathrm{~b}$. After this point, as capillary pressure increases, water drains from the NAPL-wet soil lens until the water saturation equals to the irreducible water saturation for the scanning curve $2 \rightarrow 5$ in a neutrally wet porous medium (i.e., $S_{w m}$ at point 5 in Figure 5.27b). The lowest water saturation in the water-wet soil fraction is the irreducible water saturation in a water-wet porous medium (i.e., at point 5 in Figure 5.27a). 


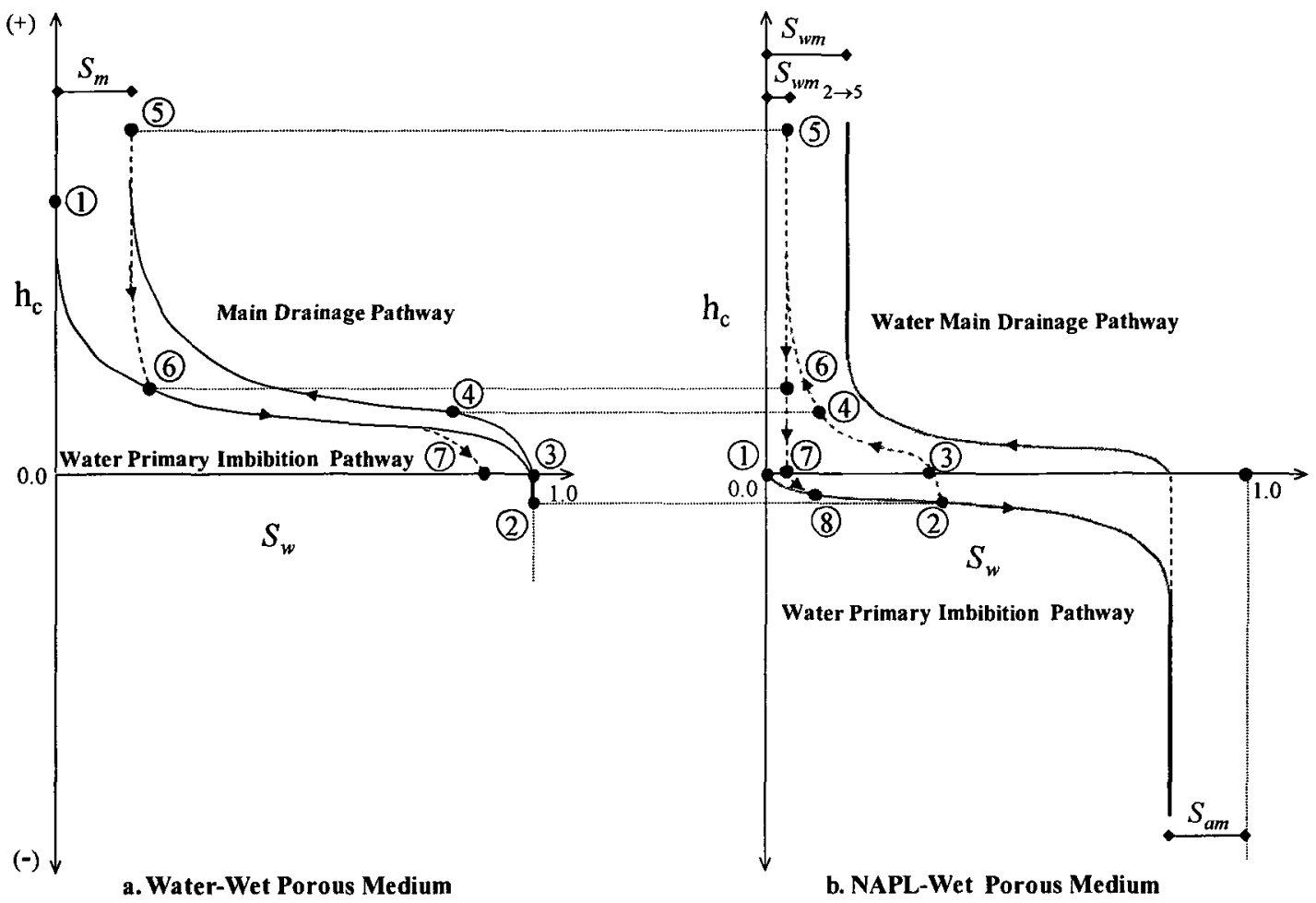

Figure 5.27: Conceptual Model for Air/Water Capillary Pressure Head-Actual Water Saturation Imbibition and Drainage Pathways in a Water-Wet Soil Fraction and in a NAPL-Wet Soil Lens.

If the capillary pressure decreases, the water saturation in the water-wet soil fraction increases following the main imbibition pathway, as described by the path $5 \rightarrow 6$ in Figure 5.27a. In contrast, the water saturation in the NAPL-wet soil lens remains constant at its irreducible saturation. It follows the path $5 \rightarrow 6$ shown in Figure $5.27 \mathrm{~b}$. At zero capillary pressure, the water saturation in the water-wet soil fraction is less than unity (point 7 shown in Figure 5.27a). The water saturation in the NAPL-wet soil lens remains constant at its irreducible saturation (point 7 shown in Figure 5.27b). If the capillary pressure is further decreased, the water saturation in the water-wet soil fraction remains constant at a water saturation corresponding to point 7 in Figure $5.27 \mathrm{a}$. While in 
the NAPL-wet soil lens, water saturation increases following a scanning curve towards the water primary imbibition pathway $7 \rightarrow 8$, shown in Figure $5.27 \mathrm{~b}$.

The previously described conceptual model for air/water $P_{c} \neg S$ relationship in a water-wet porous medium with a NAPL-wet soil lens clarifies certain points. The waterwet soil fraction is $100 \%$ water-saturated prior to initiating the main drainage pathway. The water saturation in the NAPL-wet soil lens prior to initiating the water drainage from the porous medium depends on the capillary pressure head to which the sample is exposed during the experimental setup. For the experimental data presented here, at zero capillary pressure head, the water saturation in the NAPL-wet soil lens is well below unity. Therefore, the water saturation at the beginning of the main drainage pathway in an REV consisting water-wet porous medium with a NAPL-wet soil lens is less than unity. The deficit from unity within the REV increases as the NAPL-wet soil fraction increases, given that the porous media are exposed to the same capillary pressure during the experimental setup. This agrees with the data shown in Figure 5.17 and Figure 5.18, and listed in Table 5.5 and Table 5.6. According to these data, for a water-wet porous medium with a NAPL-wet soil lens, prior to initiating the main drainage pathway, the water-wet soil fraction is water-saturated while the NAPL-wet soil lens is partially watersaturated.

Moreover, it is obvious from Figure 5.27 that at low capillary pressure heads, which are higher than the air entry pressure of the water-wet soil fraction, most of the water drains from the NAPL-wet soil lens, leaving the NAPL-wet soil lens at irreducible saturation. At this low capillary pressure head, the water saturation in the water-wet soil fraction is much larger than the irreducible water saturation. After this point and 
maintaining positive capillary pressure heads, the water-wet soil fraction is the medium where water drains and imbibes. In contrast, the water saturation in the NAPL-wet soil lens remains constant at its irreducible saturation.

Along the main imbibition pathway, water imbibes into the water-wet soil fraction as the capillary pressure head decreases in a way consistent to imbibition of water into a water-wet porous medium when exposed to the same capillary pressure on the main imbibition pathway. As for the NAPL-wet soil lens, water saturation remains at the irreducible saturation.

\subsection{4.a. Conceptual Model for Water Main Drainage Pathway}

The $P_{c}-S$ main drainage pathway starts at zero capillary pressure and actual water saturation less than unity. For water to drain from the water-wet soil fraction, the capillary pressure has to be higher than the air entry pressure. As capillary pressure increases, water drains from the water-wet soil fraction in a way consistent to the drainage of water from a water-wet porous medium when exposed to the same capillary pressure on the main drainage pathway. As for the NAPL-wet soil lens, as described earlier, most of the water drains from the lens at low capillary pressures leaving the water saturation in the NAPL-wet soil lens at an irreducible value. When the porous medium is exposed to an initial positive capillary pressure, the reduction in water saturation in the NAPL-wet soil lens is larger than that in the water-wet soil fraction, as previously explained in Section 5.3.4. This means that the existence or increase in volume of the NAPL-wet soil lens in a water-wet porous medium shifts the $P_{c} \rightarrow S$ curve for the porous medium. The data presented in Figure 5.17 and Figure 5.18 supports this finding. 
As capillary pressure head increases, the actual water saturation decreases until it reaches irreducible water saturation $\left(S_{m}\right.$ and $\left.S_{w m}\right)$ in the water-wet soil fraction and in the NAPL-wet soil lens, respectively (as shown in Figure 5.28). The water saturation at the end of the main drainage pathway depends on the volume fraction of the water-wet soil fraction and the NAPL-wet soil lens, and the degree of saturation reached in the NAPLwet soil lens prior to initiating the main drainage pathway.

As mentioned above, prior to initiating the main drainage pathway, the water saturation in a water-wet porous medium with a NAPL-wet soil lens is less than unity. At this point, the water-wet soil fraction is saturated while the NAPL-wet soil lens is partially saturated. Therefore, the initial water saturation deficit from unity is a function of the NAPL-wet soil lens degree of saturation. The soil sample degree of saturation lies between two limits. The lower limit represents a case when the NAPL-wet soil lens is dry (case (A)). The upper limit represents a case when the NAPL-wet soil lens is apparently water-saturated (case (C)). The general case represents a case when the NAPL-wet soil lens is partially water-saturated (case (B)). Therefore, the main drainage pathway for each case is defined according to Figure 5.28. 


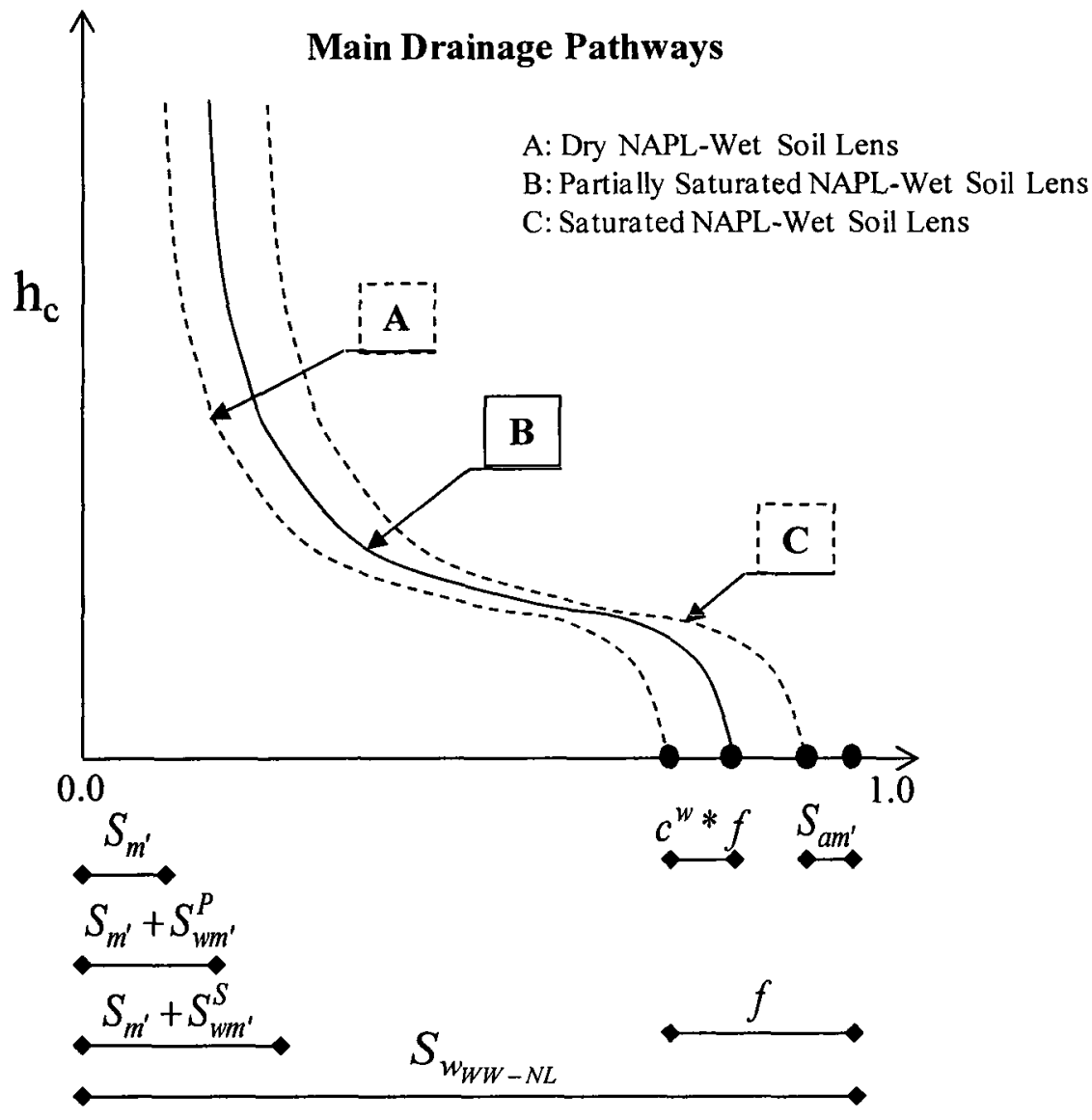

Figure 5.28: Capillary Pressure Head-Saturation Main Drainage Pathway in a Water-Wet Porous Medium with a NAPL-Wet Soil Lens.

Where:

$S_{w_{W W-N L}}:$ actual water saturation in a water-wet porous medium with a NAPL-wet soil lens;

$c_{w}$ : degree of water saturation in the NAPL-wet soil lens prior to initiating the main drainage pathway;

$f=\frac{\text { Volume of the } N A P L-\text { wet soil lens }}{\text { Total porous medium volume }}$

$S_{m} \cdot=S_{m} *(1-f)$ 
$S_{m}:$ irreducible water saturation in the water-wet soil fraction;

$S_{w m^{\prime}}^{P}=S_{w m_{2 \rightarrow 5}} * f$

$S_{w m^{\prime}}^{S}=S_{w m}^{* f}$

$S_{u m^{\prime}}^{P}:$ irreducible water saturation in a partially saturated NAPL-wet soil lens;

$S_{u m^{\prime}}^{S}$ : irreducible water saturation in a saturated NAPL-wet soil lens;

$S_{w m_{2 \rightarrow 5}}:$ irreducible water saturation for the scanning curve $2 \rightarrow 5$ in a neutrally wet porous medium;

$S_{a m^{\prime}}=S_{a m} * f$

$S_{a m}:$ irreducible air saturation in the NAPL-wet soil lens;

Volume of water-filled pores, at $h_{c}=0.0$, in case $(\mathrm{A})=(1-f)^{*} \eta_{w w} * V$

Volume of water-filled pores, at $h_{c}=0.0$, in case (B) $=(1-f)^{*} \eta_{w w}{ }^{*} V+f^{*} c^{w *} \eta_{w w}{ }^{*} V(5.10)$

Volume of water-filled pores, at $h_{c}=0.0$, in case (C) $=\eta_{w w} * V-S_{a m} * \eta_{n w} * V$

$\eta_{w w}:$ water-wet porous medium porosity; and

$\eta_{n w}:$ NAPL-wet porous medium porosity.

The difference between the three cases shown in Figure 5.28 is the water saturation at the beginning of the main drainage pathway. In case (A), the water saturation at the beginning of the main drainage pathway is the lowest since the waterwet soil fraction is saturated while the NAPL-wet soil lens is dry. By examining the main drainage pathway in this case, water drains from the water-wet soil fraction with a dry NAPL-wet lens. Therefore, if one considers the water-wet soil fraction alone for the main 
drainage pathway, then the main drainage pathway should be similar to the main drainage pathway in a water-wet porous medium.

In case (B), the water saturation at the beginning of the main drainage pathway is less than unity. At this point, the water-wet soil fraction is water-saturated while the NAPL-wet soil lens is partially water-saturated. The water drains from the water-wet soil fraction following the water main drainage pathway in a water-wet porous medium. As explained in Section 5.3.4, the water drains from the NAPL-wet soil lens following a drainage scanning curve in a NAPL-wet porous medium. Moreover, at low capillary pressures, most of the water drains from the NAPL-wet soil lens leaving the water saturation in the lens at irreducible saturation. At a given capillary pressure head, the water saturation in the water-wet porous medium with the NAPL-wet soil lens is the summation of the volume-weighted water saturations in the water-wet soil fraction and in the NAPL-wet soil lens.

In case $(C)$, the water saturation at the beginning of the main drainage pathway is slightly less than unity. At this point, the water-wet soil fraction is water-saturated and the NAPL-wet soil lens is apparently water-saturated. The water drains from the waterwet soil fraction following the water main drainage pathway in a water-wet porous medium. In the NAPL-wet soil lens water drains from the lens following the water main drainage pathway in a NAPL-wet porous medium. At a given capillary pressure head, the water saturation in the water-wet porous medium with the NAPL-wet soil lens is the summation of the volume-weighted water saturations in the water-wet soil fraction and in the NAPL-wet soil lens. 


\section{Actual Water and Air Saturations for Case (A) (Dry NAPL-wet soil lens)}

If the water-wet soil fraction is considered alone, the actual, effective, and apparent water saturations in the water-wet soil fraction in case (A) should be similar to those shown in Figure 5.22. The actual water saturation in a water-wet porous medium with a NAPL-wet soil lens is shown in Figure 5.28. Therefore, one should calculate the apparent, effective, and actual saturations in a water-wet porous medium. Then, using an actual water saturation in the water-wet porous medium and knowing the volume fraction of the NAPL-wet soil lens, the following equations are used to calculate the actual water and air saturations in the water-wet porous medium with the NAPL-wet soil lens:

$$
\begin{aligned}
& S_{w_{W W-N L}}=S_{w_{W W}} *(1-f) \\
& S_{a_{W W-N L}}=S_{a_{W W}} *(1-f)+f
\end{aligned}
$$

Where:

$S_{w_{W W}}$ actual water saturation in the water-wet porous medium;

$S_{a_{W W-N L}}:$ actual air saturation in the water-wet porous medium with the NAPL-wet soil lens; and

$S_{a_{W W}}:$ actual air saturation in the water-wet porous medium.

II. Actual Water and Air Saturations for Case (B) (Partially saturated NAPL-wet soil lens)

The water main drainage pathway in the water-wet porous medium with the NAPL-wet soil lens, shown in Figure 5.28, can be obtained by knowing the water main 
drainage pathway in the water-wet soil fraction (i.e., in the water-wet porous medium) and the water drainage pathway (i.e., a drainage scanning curve) in the NAPL-wet soil lens (i.e., in the NAPL-wet porous medium). In addition, the apparent, effective, and actual water and air saturations should be calculated in the two media (i.e., the water-wet and NAPL-wet porous media). Then, at a given capillary pressure head and using the corresponding actual water saturations in the water-wet and the NAPL-wet porous media and knowing the volume fraction of the NAPL-wet soil lens, the following equations are used to calculate the actual water and air saturations in the water-wet porous medium with the NAPL-wet soil lens:

$$
\begin{aligned}
& S_{w_{W W-N L}}=S_{w_{w W}} *(1-f)+S_{w_{N W}} * f \\
& S_{a_{W W-N L}}=S_{a_{W W}} *(1-f)+S_{a_{N W}} * f
\end{aligned}
$$

Where:

$S_{w_{N W}}$ : actual water saturation in the NAPL-wet porous medium; and

$S_{a_{N W}}:$ actual air saturation in the NAPL-wet porous medium.

\section{Actual Water and Air Saturations for Case (C) (Saturated NAPL-wet soil lens)}

Similarly, the water main drainage pathway in the water-wet porous medium with the NAPL-wet soil lens, shown in Figure 5.28, can be obtained by knowing the water main drainage pathway in the water-wet soil fraction (i.e., in the water-wet porous medium) and in the NAPL-wet soil lens (i.e., in the NAPL-wet porous medium). In addition, the apparent, effective, and actual water and air saturations should be calculated in the two media (i.e., the water-wet and NAPL-wet porous media). Then, at a given capillary pressure head and using the corresponding actual water saturations in the water- 
wet and the NAPL-wet porous media and knowing the volume fraction of the NAPL-wet soil lens, Equations 5.14 and 5.15 could be used to calculate the actual water and air saturations in the water-wet porous medium with the NAPL-wet soil lens.

\subsection{4.b. Conceptual Model Validation for Water Main Drainage Pathway}

The capillary pressure head-actual water saturation main drainage pathway data (i.e., Figure 5.17 and Figure 5.18) in the water-wet porous media with the NAPL-wet soil lenses indicate that the water saturations in these media could be represented by the conceptual model presented in case (B). In these data, the measured saturation pathways are the main drainage pathways in the water-wet porous media and in the water-wet porous media with the NAPL-wet soil lenses.

The conceptual model presented in case (B) implies that the actual water saturation in a water-wet porous medium with a NAPL-wet soil lens is less than unity at the beginning of the main drainage pathway. The conceptual model presented in case (B) states that the capillary pressure-actual water saturation relationship in a water-wet porous medium with a NAPL-wet soil lens is the volume-weighted summation of the capillary pressure-actual water saturation relationship in the water-wet porous medium and the capillary pressure-actual water saturation relationship in the NAPL-wet porous medium. In addition, the capillary pressure-actual water saturation pathway in a waterwet porous medium with a NAPL-wet soil lens is lower than in the water-wet porous medium and higher than in the water-wet porous medium with a dry NAPL-wet soil lens. Therefore, the conceptual model is considered valid if the capillary pressure-actual water saturation data in a water-wet porous medium with a NAPL-wet soil lens is bounded by the capillary pressure-actual water saturation relationship for a water-wet porous medium 
and the capillary pressure-actual water saturation relationship for a water-wet porous medium with a dry NAPL-wet soil lens. Figure 5.29 and Figure 5.30 show the fitted and the measured main drainage pathway in water-wet uniform and well graded sands.

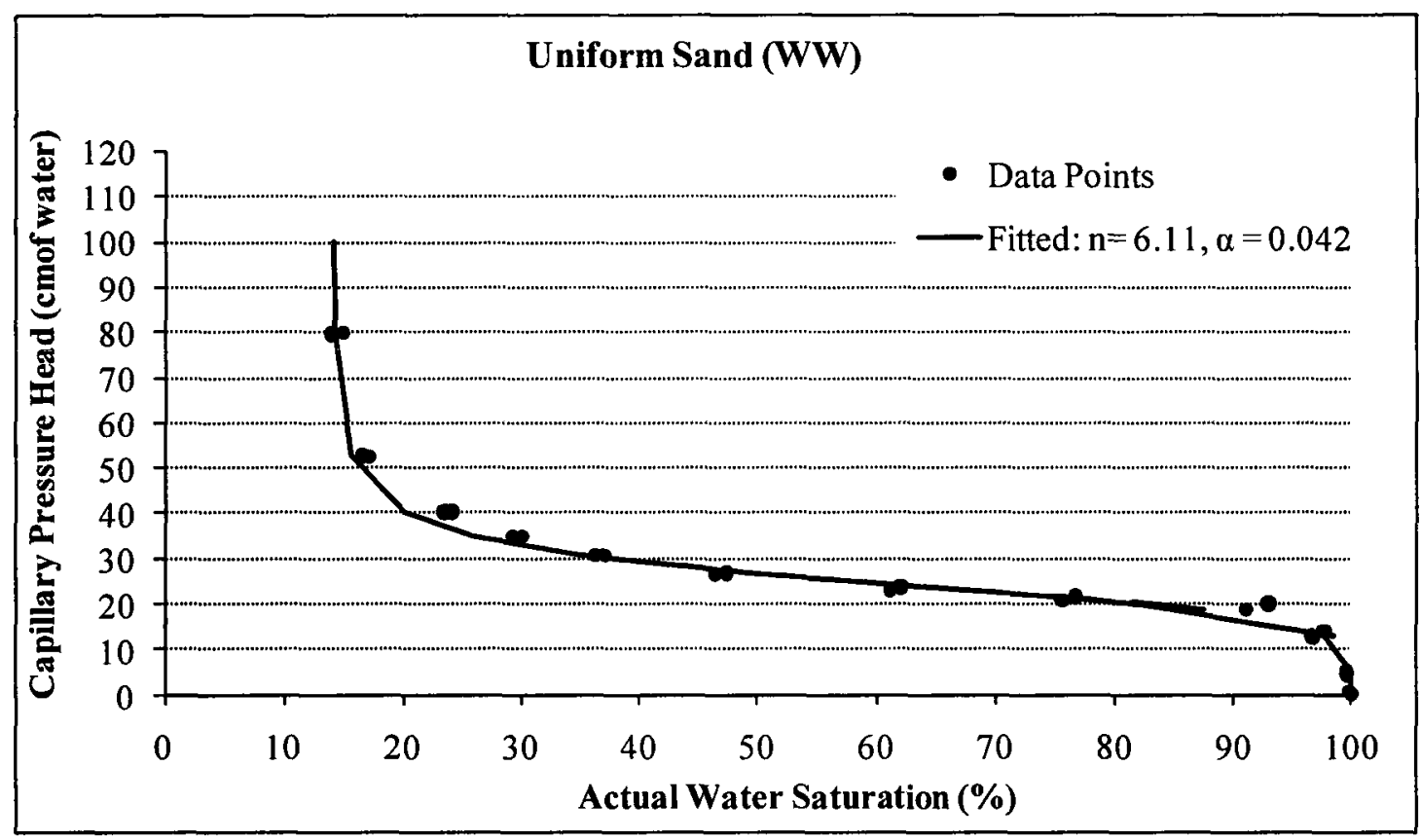

Figure 5.29: Capillary Pressure Head-Actual Water Saturation Main Drainage Pathway in a Water-Wet Porous Medium in Uniform Sand. 


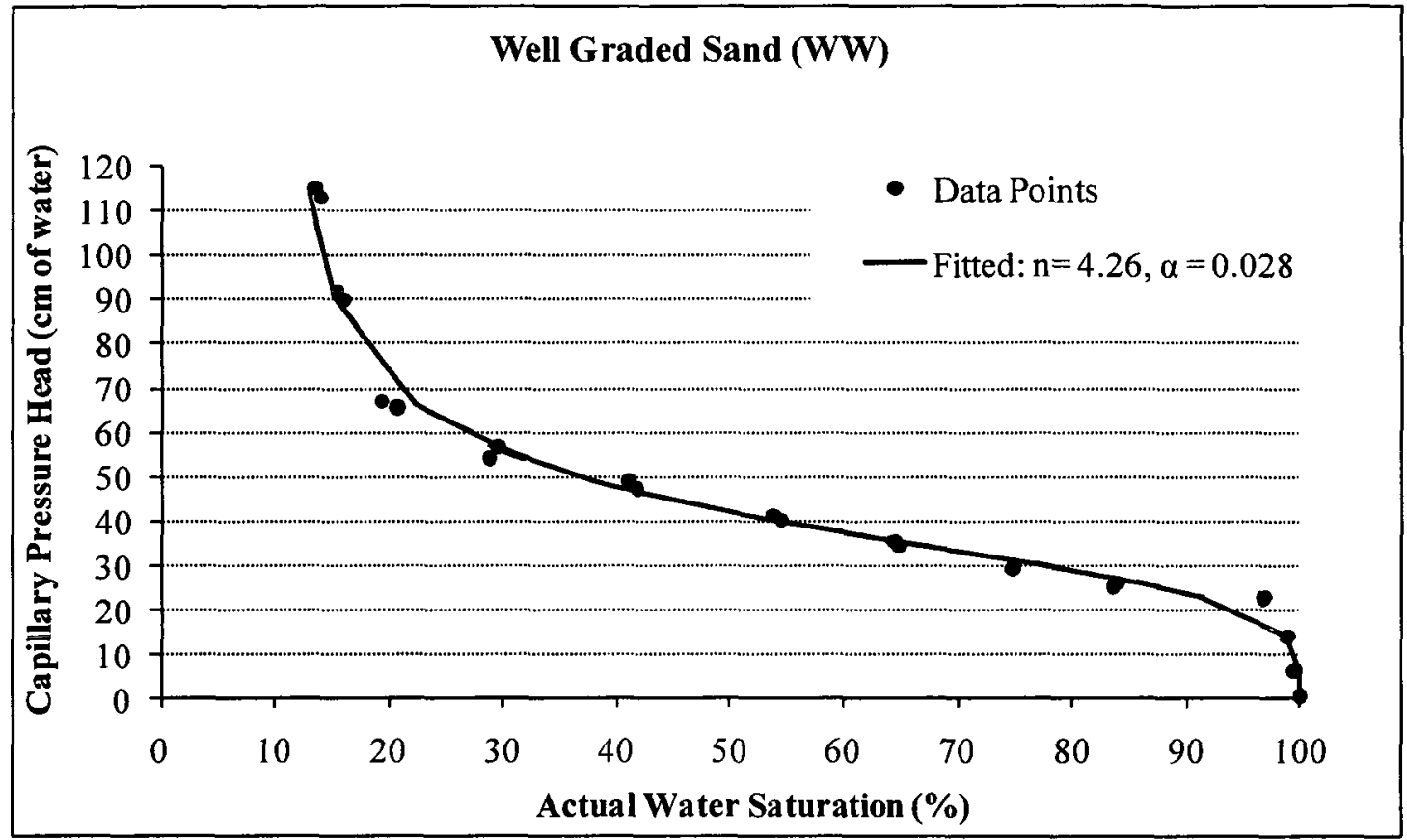

Figure 5.30: Capillary Pressure Head-Actual Water Saturation Main Drainage Pathway in a Water-Wet Porous Medium in Well Graded Sand.

Figure 5.31 through Figure 5.38 show the main drainage pathways data points in water-wet porous media with NAPL-wet soil lenses, $(1-f) \mathrm{WW}-f \mathrm{NL}$, in uniform and well graded sands. These figures also show fitted Van Genuchten main drainage pathway in a water-wet porous medium, Fitted_WW, which represent the upper limit of the main drainage pathways in water-wet porous media with NAPL-wet soil lenses. In addition to the capillary pressure head-actual water saturation relationship, Scaled_WW, which represent the lower limit of the main drainage pathways in water-wet porous media with dry NAPL-wet soil lenses. A Scaled_WW is obtained by scaling the water saturations of the fitted Van Genuchten main drainage pathway with $(1-f)$. The figures show that the measured main drainage pathways in the water-wet porous media with the NAPL-wet soil lenses start at actual water saturations less than unity. In addition, the measured main drainage pathways in these media are bounded by the capillary pressure-actual water 
saturation relationship for a water-wet porous medium and the capillary pressure-actual water saturation relationship for a water-wet porous medium with a dry NAPL-wet soil lens, in most cases, and this validates the conceptual model. The deviation might be ascribed to variations in soil placement and compaction.

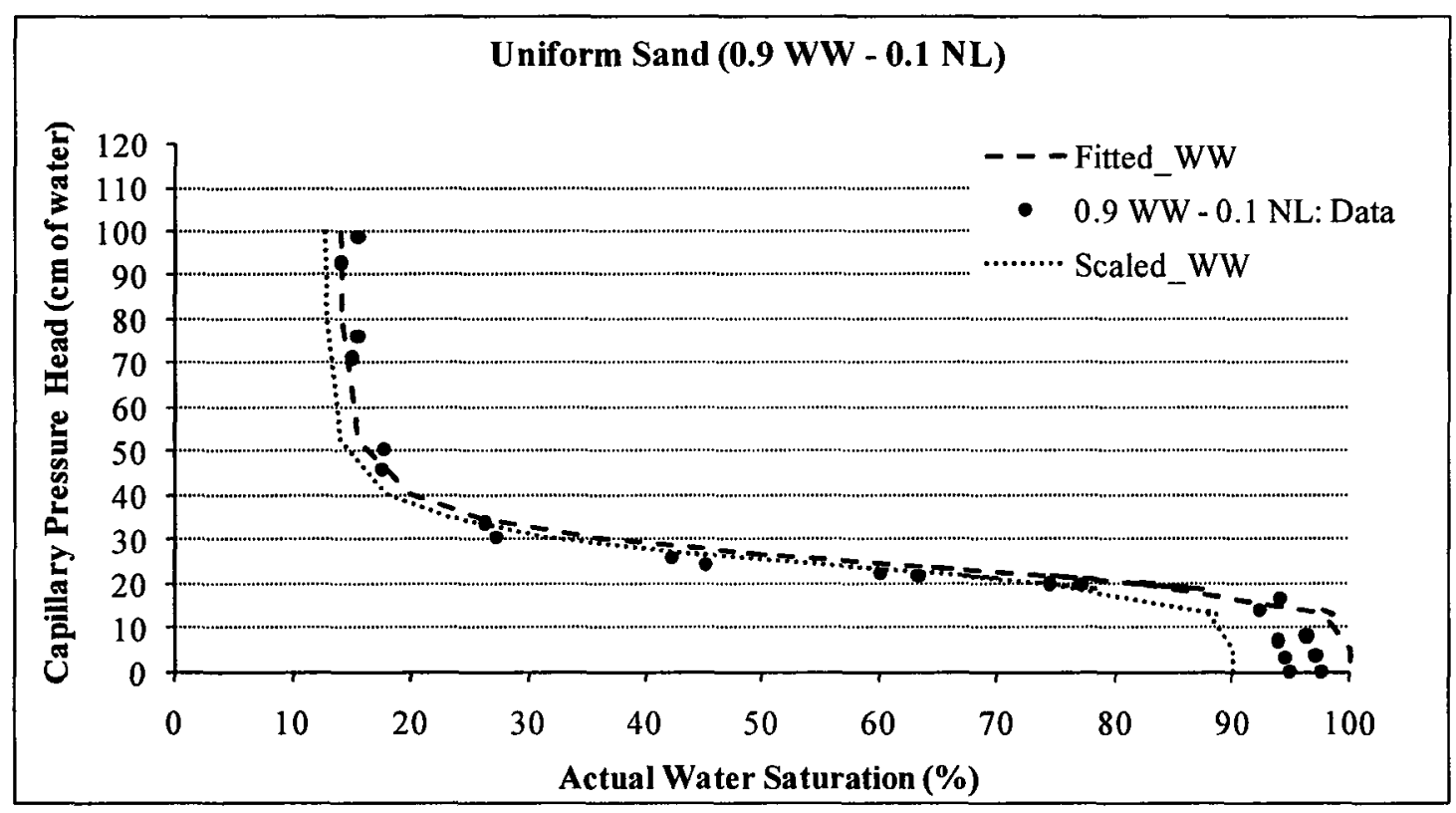

Figure 5.31: Capillary Pressure Head-Actual Water Saturation Main Drainage Pathway in a 90\% Water-Wet-10\% NAPL-Wet Porous Medium in Uniform Sand. 


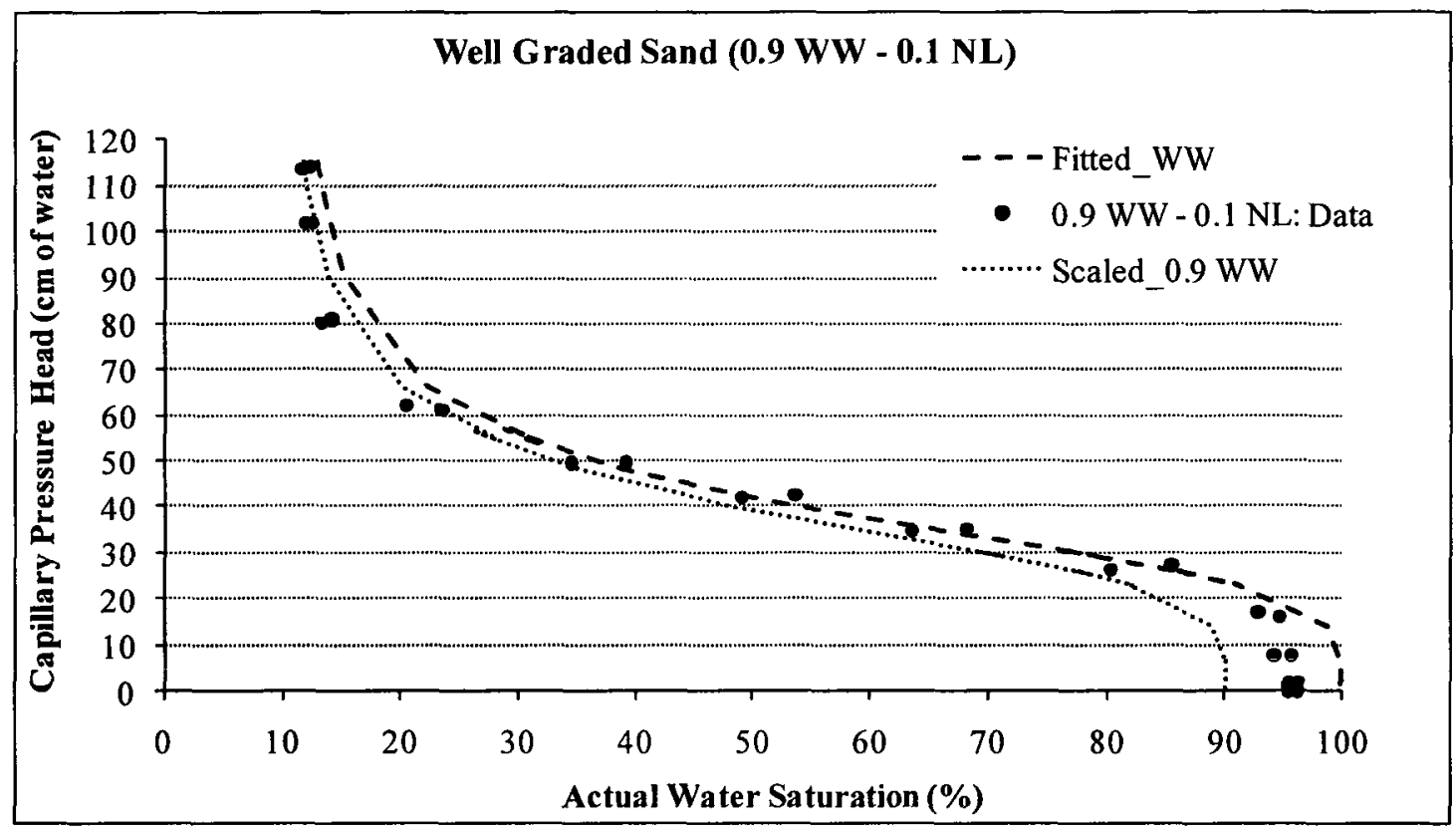

Figure 5.32: Capillary Pressure Head-Actual Water Saturation Main Drainage Pathway in a 90\% Water-Wet-10\% NAPL-Wet Porous Medium in Well Graded Sand.

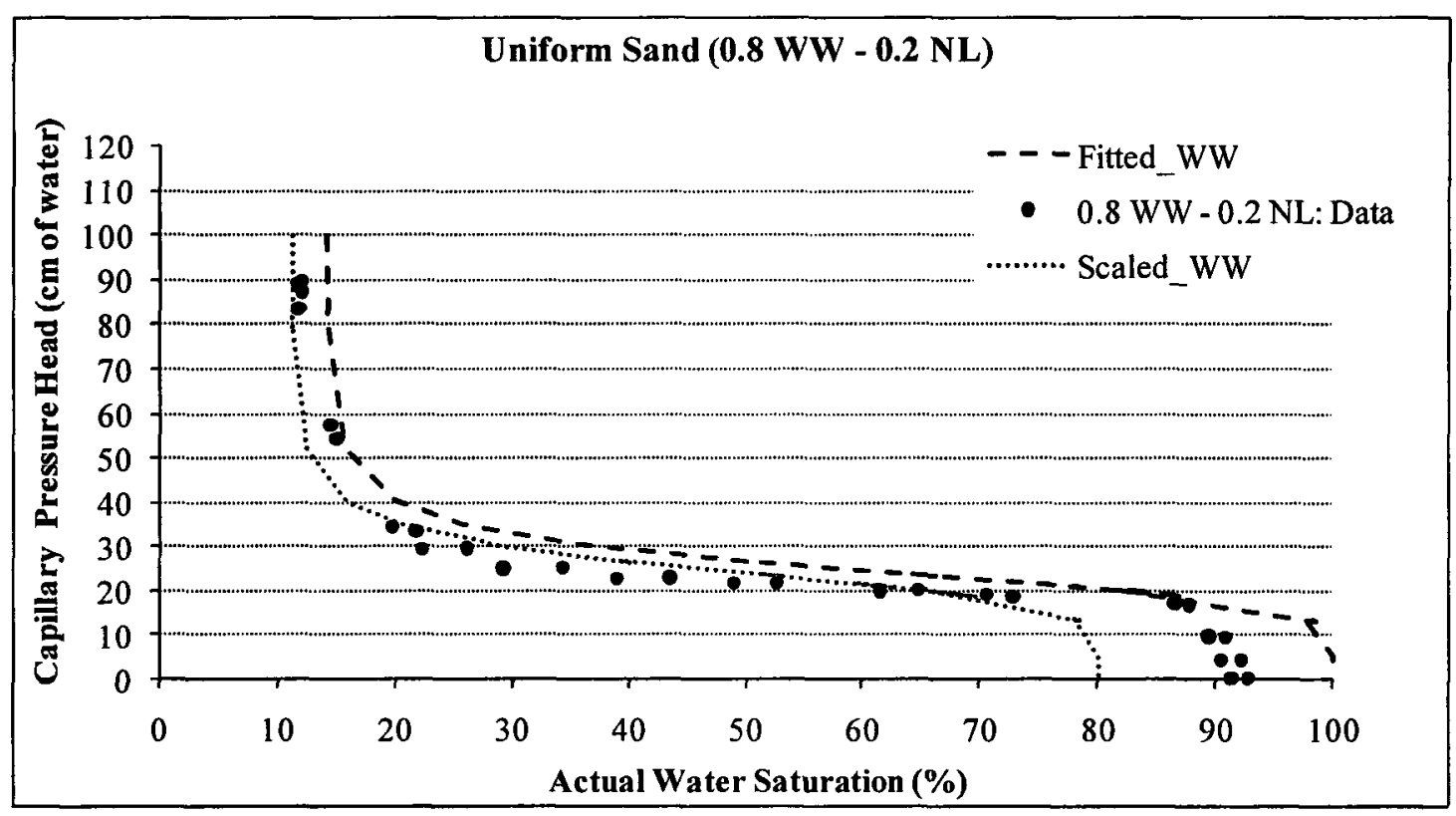

Figure 5.33: Capillary Pressure Head -Actual Water Saturation Main Drainage Pathway in an 80\% Water-Wet-20\% NAPL-Wet Porous Medium in Uniform Sand. 


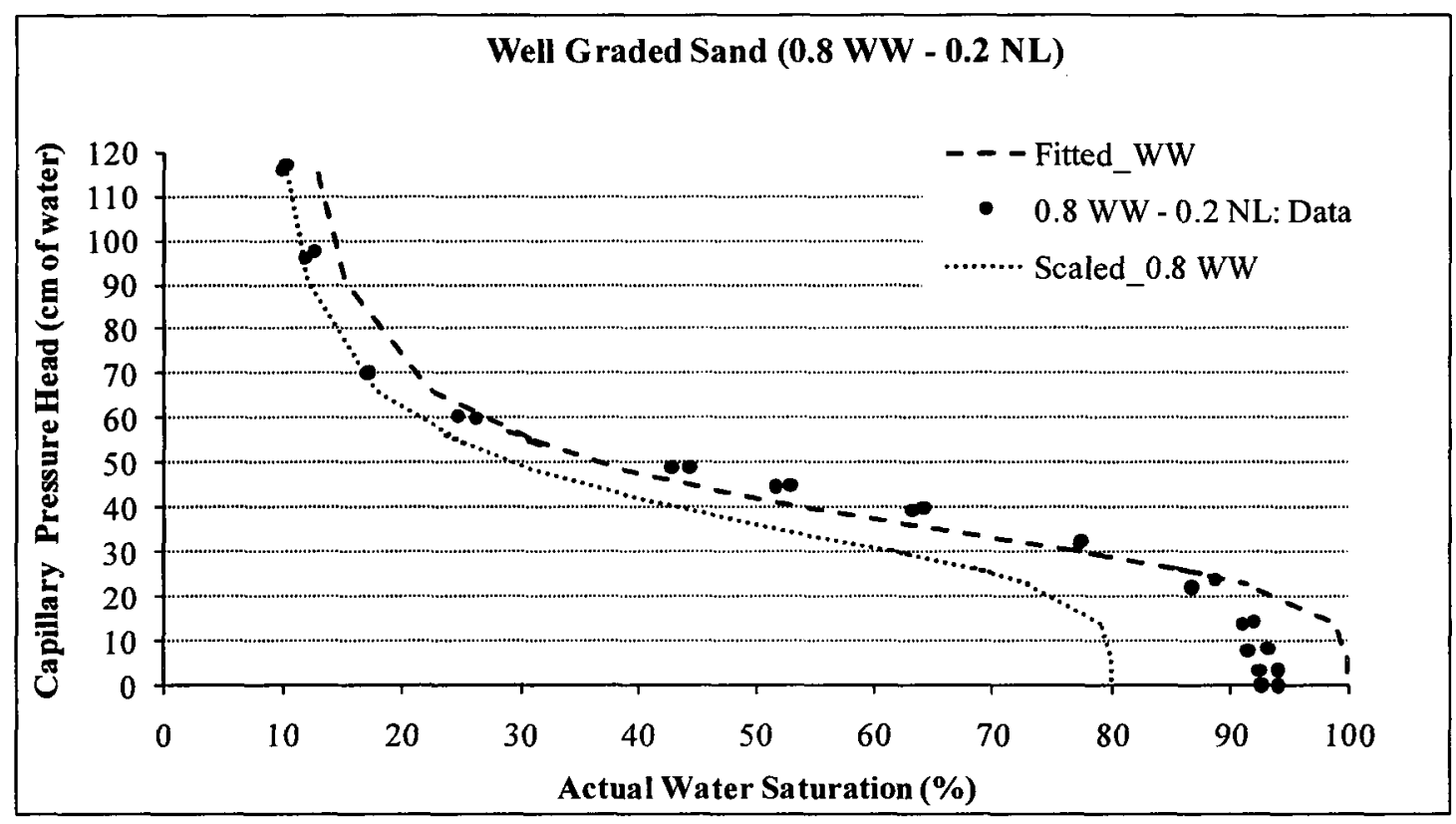

Figure 5.34: Capillary Pressure Head -Actual Water Saturation Main Drainage Pathway in an $\mathbf{8 0 \%}$ Water-Wet-20\% NAPL-Wet Porous Medium in Well Graded Sand.

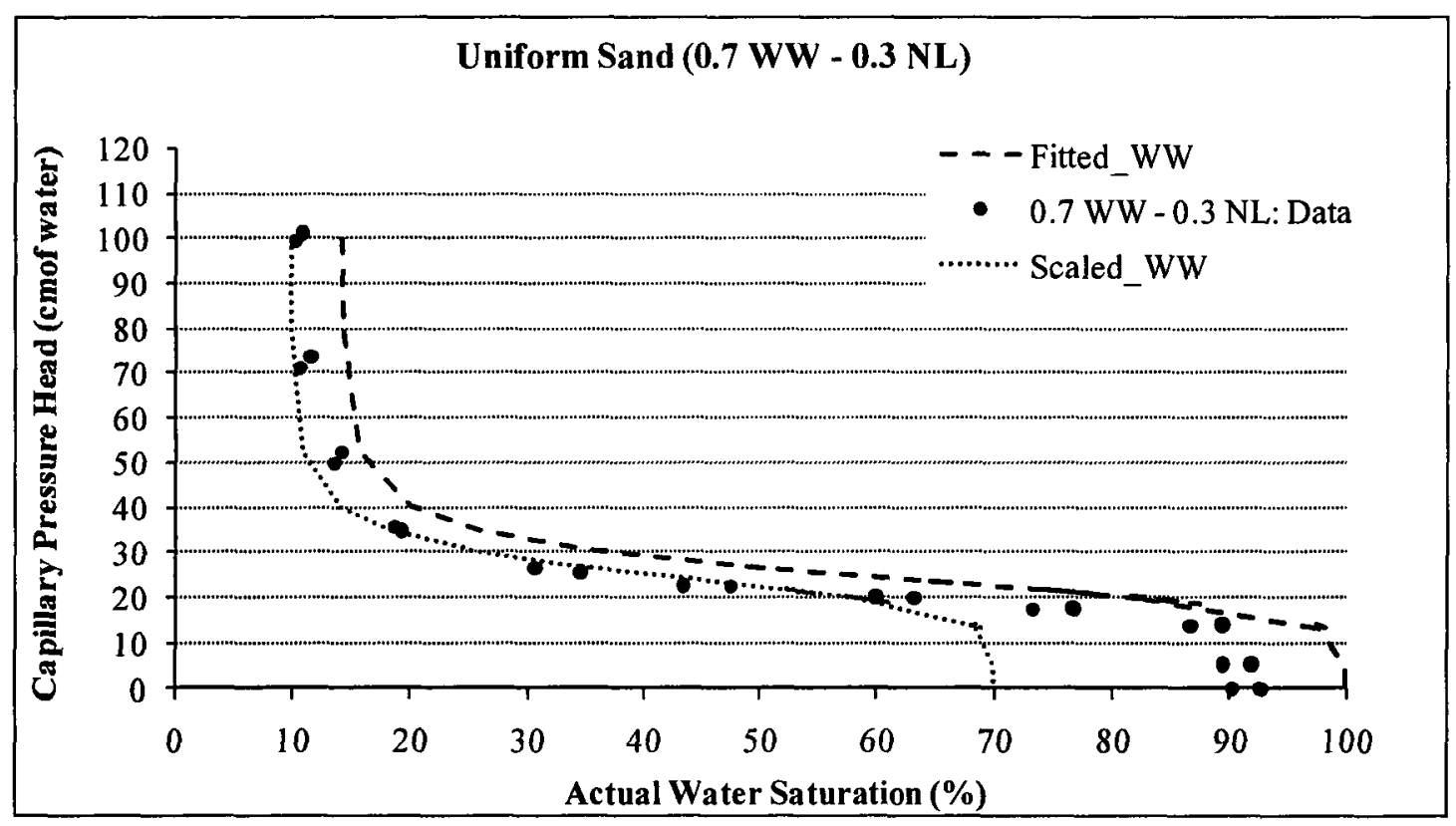

Figure 5.35: Capillary Pressure Head -Actual Water Saturation Main Drainage Pathway in a 70\% Water-Wet-30\% NAPL-Wet Porous Medium in Uniform Sand. 


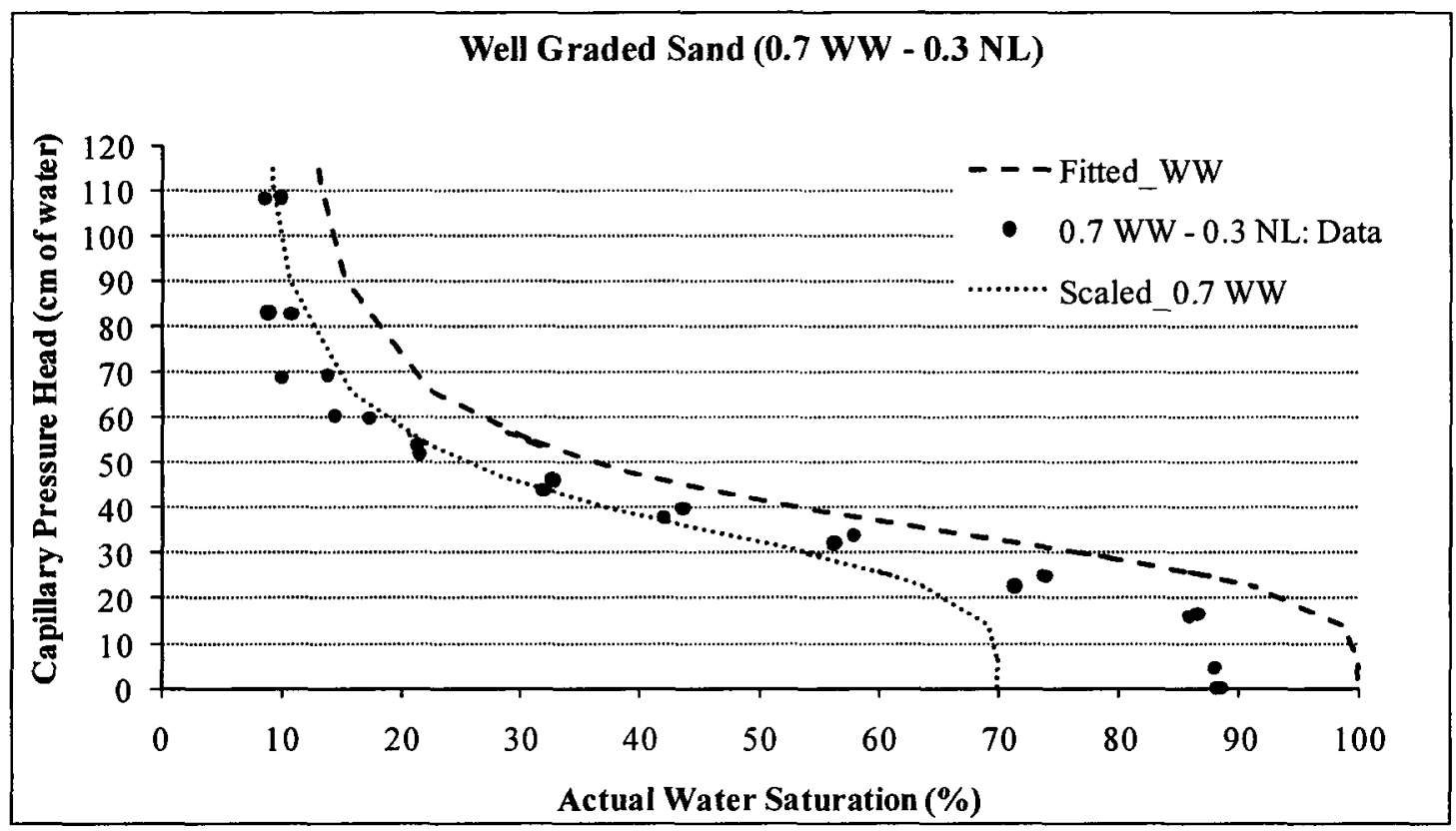

Figure 5.36: Capillary Pressure Head -Actual Water Saturation Main Drainage Pathway in a 70\% Water-Wet-30\% NAPL-Wet Porous Medium in Well Graded Sand.

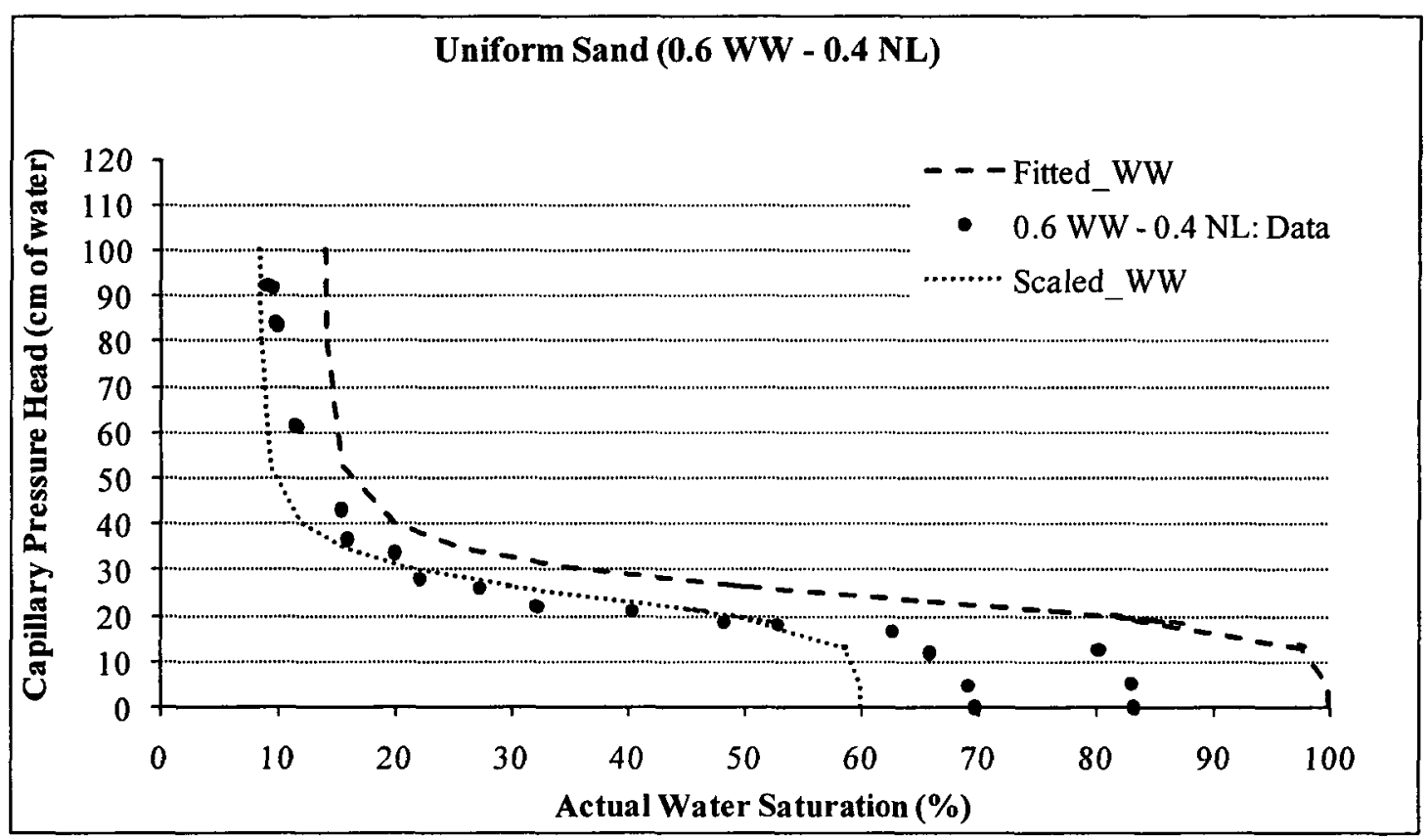

Figure 5.37: Capillary Pressure Head -Actual Water Saturation Main Drainage Pathway in a $60 \%$ Water-Wet-40\% NAPL-Wet Porous Medium in Uniform Sand. 


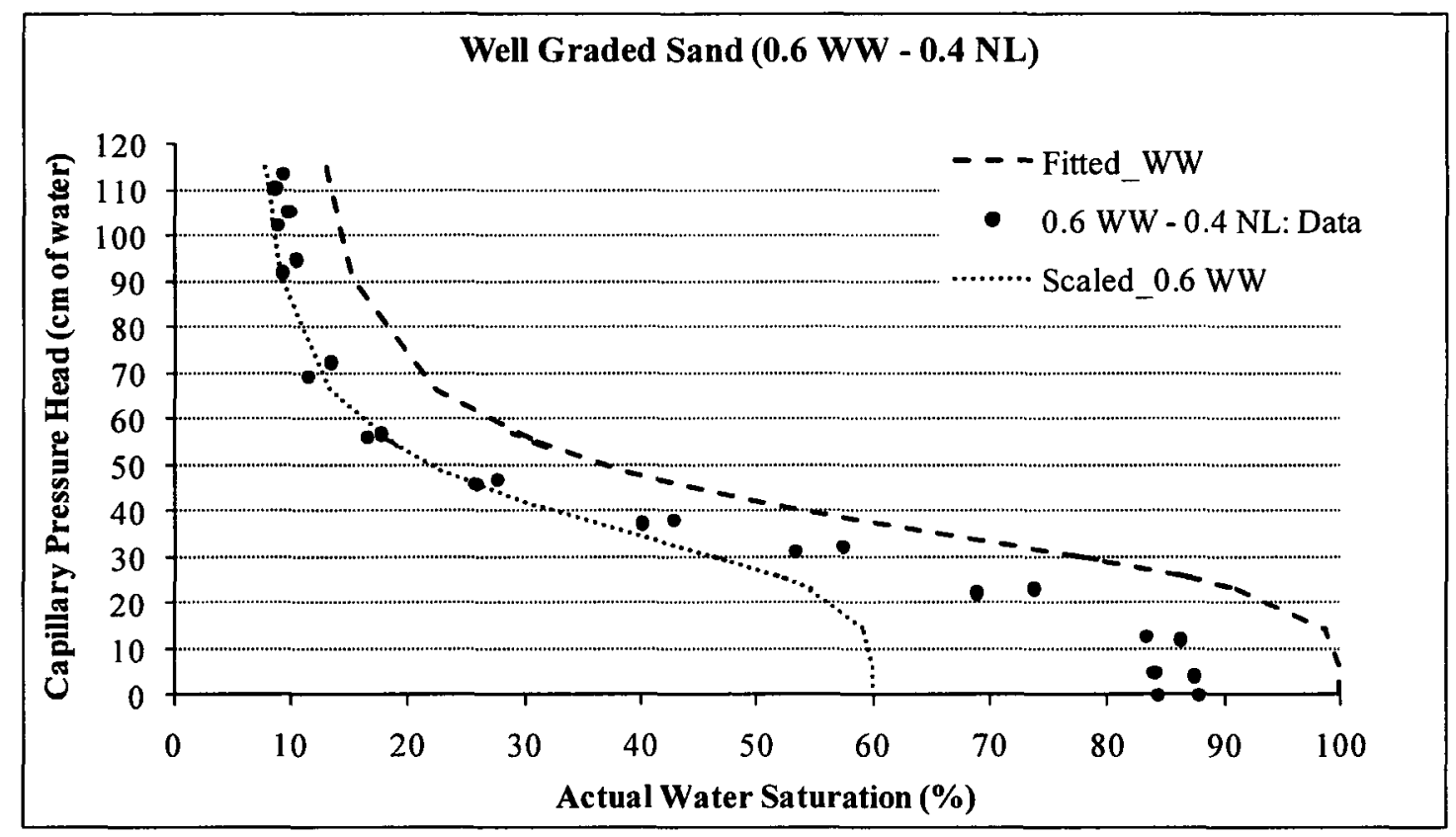

Figure 5.38: Capillary Pressure Head -Actual Water Saturation Main Drainage Pathway in a $60 \%$ Water-Wet-40\% NAPL-Wet Porous Medium in Well Graded Sand.

\subsection{4.c. Conceptual Model for Water Main Imbibition Pathway}

The $P_{c}-S$ main imbibition pathway starts where the main drainage pathway ends. As capillary pressure decreases, water imbibes into the water-wet soil fraction while it does not imbibe into the NAPL-wet soil lens. Therefore, the water saturation in the NAPL-wet soil lens remains zero (i.e., case (A)) or at an irreducible saturation (i.e., cases (B) and (C)).

In case (A), the main imbibition pathway starts at water saturation of $S_{m}$. (i.e., $S_{m}$ in the water-wet porous medium) and dry NAPL-wet soil lens. In case (B), the main imbibition pathway starts at water saturation of $S_{m}$ (i.e., $S_{m}$ in the water-wet porous medium) and an irreducible water saturation of $S_{w m^{\prime}}^{P}$ in the NAPL-wet soil lens (i.e., $S_{w m_{2 \rightarrow 5}}$ in the NAPL-wet porous medium). For case (C), the main imbibition pathway 
starts at water saturation of $S_{m}$ (i.e., $S_{m}$ in the water-wet porous medium) and an irreducible water saturation of $S_{w m^{\prime}}^{S}$ in the NAPL-wet soil lens (i.e., $S_{w m}$ in the NAPLwet porous medium). At any capillary pressure head, the water saturation in case (A) is less than the water saturation in case (B) or (C) by $S_{w m^{\prime}}^{P}$ or $S_{w m^{\prime}}^{S}$, respectively. Therefore, the main imbibition pathways for the two cases are shifted as shown in Figure 5.39.

As water imbibes into the water-wet soil fraction, air is entrapped. At the end of the main imbibition pathway (i.e., capillary pressure head equals to zero), water is apparently saturating the water-wet soil fraction with a maximum entrapped air saturation of $S_{a t_{\text {max }}}$. The air and the irreducible water saturations in the NAPL-wet soil lens depend on the case considered (i.e., A, B or C). 


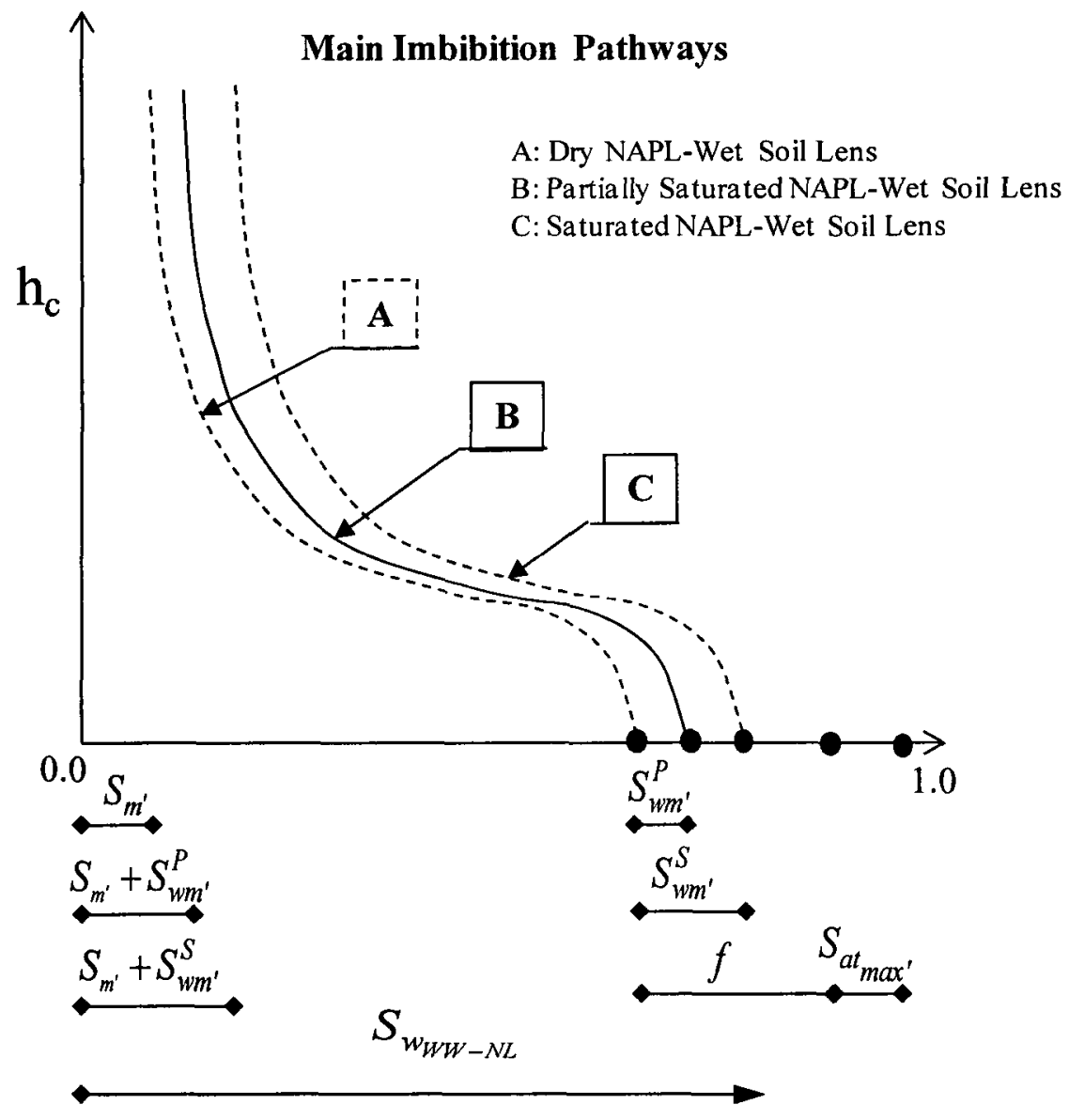

Figure 5.39: Capillary Pressure Head-Saturation Main Imbibition Pathway in a Water-Wet Porous Medium with a NAPL-Wet Soil Lens.

\section{Actual Water and Air Saturations for Case (A) (Dry NAPL-wet soil lens)}

As mentioned above, the NAPL-wet soil lens remains dry along the main imbibition pathway. This means that the NAPL-wet soil lens does not have an effect on the imbibition of water into the medium. Therefore, if the NAPL-wet soil lens has been removed from the REV and the pore volume of the water-wet soil fraction is used in water saturation calculations, one should obtain the same main imbibition pathway as in a water-wet porous medium. Hence, the effective and apparent water saturations in this 
case are similar to those shown in Figure 5.23b and Figure 5.23c. In order to calculate the actual water and air saturations in a water-wet porous medium with a NAPL-wet soil lens, one should calculate the apparent, effective, and actual saturations in a water-wet porous medium. Then, the following equations are used to calculate the actual water and air saturations for case $(\mathrm{A})$ :

$$
\begin{aligned}
& S_{w_{W W-N L}}=S_{w_{W W}} *(1-f) \\
& S_{a_{W W-N L}}=S_{a_{W W}} *(1-f)+f
\end{aligned}
$$

II. Actual Water and Air Saturations for Case (B) (Partially saturated NAPL-wet soil lens)

It has been explained that the water saturation in the water-wet soil fraction follows the same pathway as in a water-wet porous medium, while the water saturation in the NAPL-wet soil lens remains, at all times, at an irreducible saturation of $S_{w m^{\prime}}^{P}$. In order to obtain air and water saturations, the procedure used in case (A) is followed in this case. The main imbibition pathway in the water-wet soil fraction is constructed, which should be similar to the main imbibition pathway in a water-wet porous medium. Therefore, one might calculate the apparent, effective, and actual water and air saturations in a water-wet porous medium, and then the following equations are used to calculate the actual water and air saturations for case (B):

$$
\begin{aligned}
& S_{w_{W W-N L}}=S_{w_{W W}} *(1-f)+S_{w m^{\prime}}^{P} \\
& S_{a_{W W-N L}}=S_{a_{W W}} *(1-f)+f-S_{w m^{\prime}}^{P}
\end{aligned}
$$




\section{Actual Water and Air Saturations for Case (C) (Saturated NAPL-wet soil lens)}

Case (C) is similar to case (B) except that the irreducible water saturation in the NAPL-wet soil lens is $S_{w m^{\prime}}^{S}$ instead of $S_{w m^{\prime}}^{P}$. Therefore, one might calculate the apparent, effective, and actual water and air saturations in a water-wet porous medium, and then the following equations are used to calculate the actual water and air saturations for case (C):

$$
\begin{aligned}
& S_{w_{W W-N L}}=S_{w_{w W}} *(1-f)+S_{w m^{\prime}}^{S} \\
& S_{a_{W W-N L}}=S_{a_{W W}} *(1-f)+f-S_{w m^{\prime}}^{S}
\end{aligned}
$$

\subsection{4.d. Conceptual Model Validation for Water Main Imbibition Pathway}

It was shown that the measured main drainage pathways are represented by the conceptual model presented in case (B), as described in Section 5.3.4.b, and hence the measured main imbibition pathways should be represented by the conceptual model presented in case (B), as described in Section 5.3.4.c. Figure 5.40, for uniform sand, and Figure 5.41, for well graded sand, show the capillary pressure head-actual water saturation for the five porous media. As shown in these figures, as the NAPL-wet soil lens size increases, the initial and final water saturations on the main imbibition pathway decrease. It is noticed that the main imbibition pathways for the five porous media are, to some extent, similar to one another but shifted. 


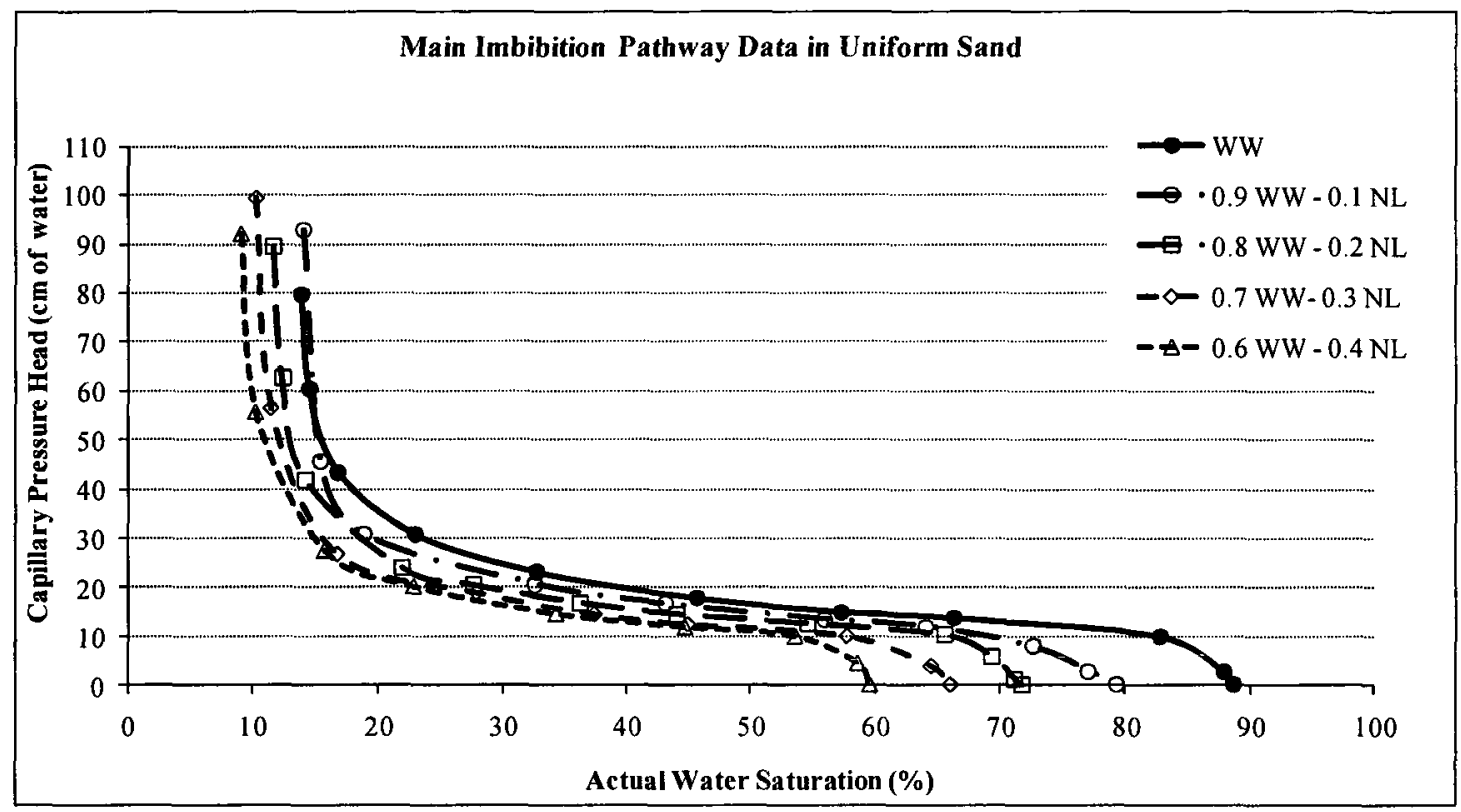

Figure 5.40: Capillary Pressure Head-Actual Water Saturation in Different Wettability Porous Media in Uniform Sand.

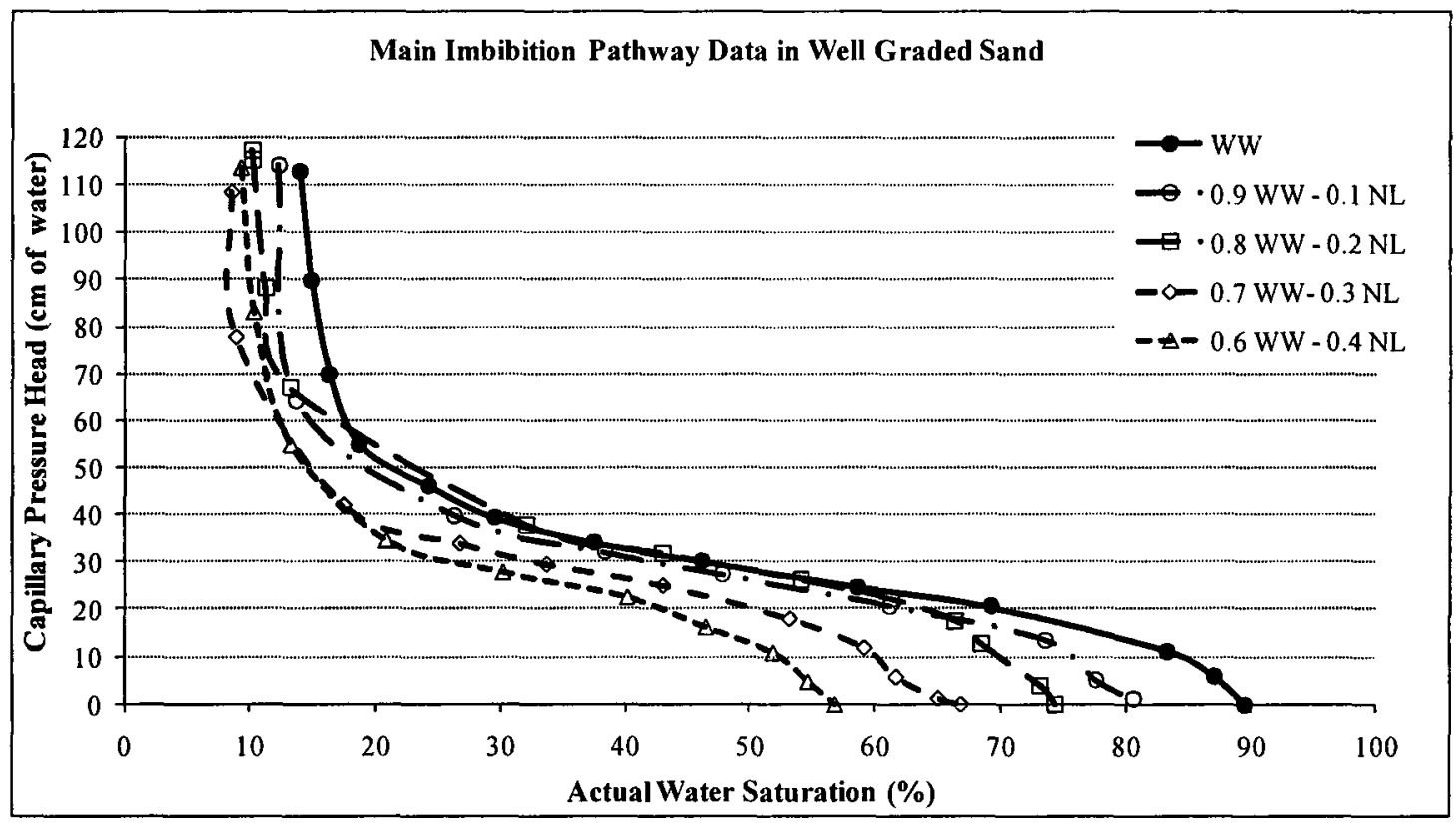

Figure 5.41: Capillary Pressure Head-Actual Water Saturation in Different Wettability Porous Media in Well Graded Sand.

It was explained above that the main imbibition pathway for case (B) can be predicted by summing the contribution of the main imbibition pathway in the water-wet 
soil fraction and in the NAPL-wet soil lens, when each is considered alone, respectively. It was described earlier that the main imbibition pathway in a water-wet soil fraction, when considered alone, is similar to the main imbibition pathway in the water-wet porous medium. Moreover, the water saturation in the NAPL-wet soil lens remains unchanged at an irreducible water saturation of $S_{w m^{\prime}}^{P}$. Therefore, in order to obtain the main imbibition pathway in a water-wet porous medium with a NAPL-wet soil lens, one should know the main imbibition pathway in a water-wet porous medium, the volume of the NAPL-wet soil lens in a given REV, and the irreducible water saturation in the NAPL-wet soil lens $\left(S_{w m^{\prime}}^{P}\right)$. Given these inputs, a model as presented in case (B) may be used to calculate the main imbibition pathway in a water-wet porous medium with a NAPL-wet soil lens.

A possible way to check the validity of this model is first; to find the irreducible water saturation in the NAPL-wet soil lens, second; to subtract that saturation from the water saturations of the main imbibition pathway in the water-wet porous medium with the NAPL-wet soil lens. The resulting water saturations are the water saturations in the water-wet soil fraction. If the latter are divided by $(1-f)$, the capillary pressure-water saturation relationship for the water-wet soil fraction should be similar to the capillary pressure-water saturation relationship in a water-wet porous medium.

It is known that the main imbibition pathway in a water-wet porous medium with a NAPL-wet soil lens starts at a water saturation of $S_{m^{\prime}}+S_{w m^{\prime}}^{P}$. This water saturation is the summation of the irreducible water saturations in the water-wet soil fraction and the NAPL-wet soil lens. Therefore, the irreducible water saturation in the NAPL-wet soil lens can be calculated if the irreducible water saturation in the water-wet soil fraction is known. Pertaining to the rigid porous medium assumption and assuming that the 
irreducible water saturation in a water-wet porous medium is constant, the irreducible water saturation in the water-wet porous medium is used as a basis for the irreducible water saturation calculation in the water-wet soil fraction. Therefore, if the irreducible water saturation in the water-wet porous medium is known, the irreducible water saturation in the water-wet soil fraction may be calculated using Equation 5.5.

Table 5.7, for uniform sand, and Table 5.8, for well graded sand, show the water saturation at the beginning of the main imbibition pathways $\left(\mathrm{S}_{m}\right.$ or $\left.S_{m^{\prime}}+S_{w m^{\prime}}^{P}\right)$, the irreducible water saturation in the water-wet soil fractions $\left(S_{m}\right)$, and the irreducible water saturation in the NAPL-wet soil lenses $\left(S_{w m^{\prime}}^{P}\right)$. The $P_{c} \neg$ experiment, for each soil wettability configuration, has been conducted in duplicate. It is noticed that the water saturations at the beginning of the main imbibition pathways in the first and second experiments are different. Therefore, as shown in Table 5.7 and Table 5.8, the data were organized in two sets. Experiment set (1) refers to the $P_{c}-S$ experiments with smaller water saturation at the beginning of their main imbibition pathways. Experiment set (2) refers to the $P_{c} \neg S$ experiments with larger water saturation at the beginning of their main imbibition pathways. When the irreducible water saturation in the water-wet porous medium is used to calculate the irreducible water saturation in the water-wet soil fraction, both should be from the same experimental set. 
Table 5.7: Irreducible Water Saturations in Uniform Sand.

\begin{tabular}{|c|c|c|c|c|c|c|c|c|}
\hline \multirow{2}{*}{$\begin{array}{c}\text { Wettability } \\
\text { Configuration }\end{array}$} & \multicolumn{4}{|c|}{ Experiment Set (1) } & \multicolumn{4}{c|}{ Experiment Set (2) } \\
\cline { 2 - 10 } & $\begin{array}{c}S_{m} \text { or } \\
\left(S_{m^{\prime}}+S_{w m^{\prime}}^{P}\right)\end{array}$ & $\begin{array}{c}\text { Expected } \\
S_{m^{\prime}}\end{array}$ & $\begin{array}{c}\text { Actual } \\
S_{m^{\prime}}\end{array}$ & $S_{w m^{\prime}}^{P}$ & $\begin{array}{c}S_{m} \text { or } \\
\left(S_{m^{\prime}}+S_{w m^{\prime}}^{P}\right)\end{array}$ & $\begin{array}{c}\text { Expected } \\
S_{m^{\prime}}\end{array}$ & $\begin{array}{c}\text { Actual } \\
S_{m^{\prime}}\end{array}$ & $S_{w m^{\prime}}^{P}$ \\
\hline WW & 13.90 & 13.9 & 13.90 & 0.00 & 14.99 & 14.99 & 14.99 & 0.00 \\
\hline $\mathbf{0 . 9}$ WW - 0.1 NL & 14.05 & 12.51 & 12.51 & 1.54 & 14.58 & 13.49 & 13.49 & 1.09 \\
\hline $\mathbf{0 . 8}$ WW - 0.2 NL & 11.72 & 11.12 & 11.12 & 0.60 & 12.09 & 11.99 & 11.99 & 0.10 \\
\hline $\mathbf{0 . 7}$ WW - 0.3 NL & 10.26 & 9.73 & 9.73 & 0.53 & 10.84 & 10.49 & 10.49 & 0.35 \\
\hline $\mathbf{0 . 6}$ WW - 0.4 NL & 9.09 & 8.34 & 8.34 & 0.75 & 9.50 & 8.99 & 8.99 & 0.51 \\
\hline
\end{tabular}

Table 5.8: Irreducible Water Saturations in Well Graded Sand.

\begin{tabular}{|c|c|c|c|c|c|c|c|c|}
\hline \multirow{2}{*}{$\begin{array}{c}\text { Wettability } \\
\text { Configuration }\end{array}$} & \multicolumn{4}{|c|}{ Experiment Set (1) } & \multicolumn{3}{c|}{ Experiment Set (2) } \\
\cline { 2 - 10 } & $\begin{array}{c}S_{m} \text { or } \\
\left(S_{m^{\prime}}+S_{w m^{\prime}}^{P}\right)\end{array}$ & $\begin{array}{c}\text { Expected } \\
S_{m^{\prime}}\end{array}$ & $\begin{array}{c}\text { Actual } \\
S_{m^{\prime}}\end{array}$ & $S_{w m^{\prime}}^{P}$ & $\begin{array}{c}S_{m} \text { or } \\
\left(S_{m^{\prime}}+S_{w m^{\prime}}^{P}\right)\end{array}$ & $\begin{array}{c}\text { Expected } \\
S_{m^{\prime}}\end{array}$ & $\begin{array}{c}\text { Actual } \\
S_{m^{\prime}}\end{array}$ & $S_{w m^{\prime}}^{P}$ \\
\hline WW & 13.48 & 13.48 & 13.48 & 0.00 & 13.92 & 13.92 & 13.92 & 0.00 \\
\hline $\mathbf{0 . 9}$ WW - 0.1 NL & 11.62 & 12.13 & 11.62 & 0.00 & 12.30 & 12.53 & 12.30 & 0.00 \\
\hline $\mathbf{0 . 8}$ WW - 0.2 NL & 10.00 & 10.78 & 10.00 & 0.00 & 10.22 & 11.14 & 10.22 & 0.00 \\
\hline $\mathbf{0 . 7}$ WW - 0.3 NL & 8.56 & 9.44 & 8.56 & 0.00 & 9.98 & 9.74 & 9.74 & 0.24 \\
\hline $\mathbf{0 . 6}$ WW - 0.4 NL & 8.61 & 8.09 & 8.09 & 0.52 & 9.28 & 8.35 & 8.35 & 0.93 \\
\hline
\end{tabular}

In a given water-wet porous medium with a NAPL-wet soil lens, subtracting $S_{w m^{\prime}}^{P}$ from the water saturations of the main imbibition pathway, results in the water saturations in the water-wet soil fraction. Once these saturations are divided by $(1-f)$ and plotted versus the corresponding capillary pressure heads, the new main imbibition pathway should be similar to the main imbibition pathway in the water-wet porous medium. Figure 5.42, for uniform sand, and Figure 5.43, for well graded sand, show the capillary pressure head-actual water saturation in the water-wet porous media and the water-wet soil fractions. The figures show that the data are aligned which validates the previously proposed conceptual model. 


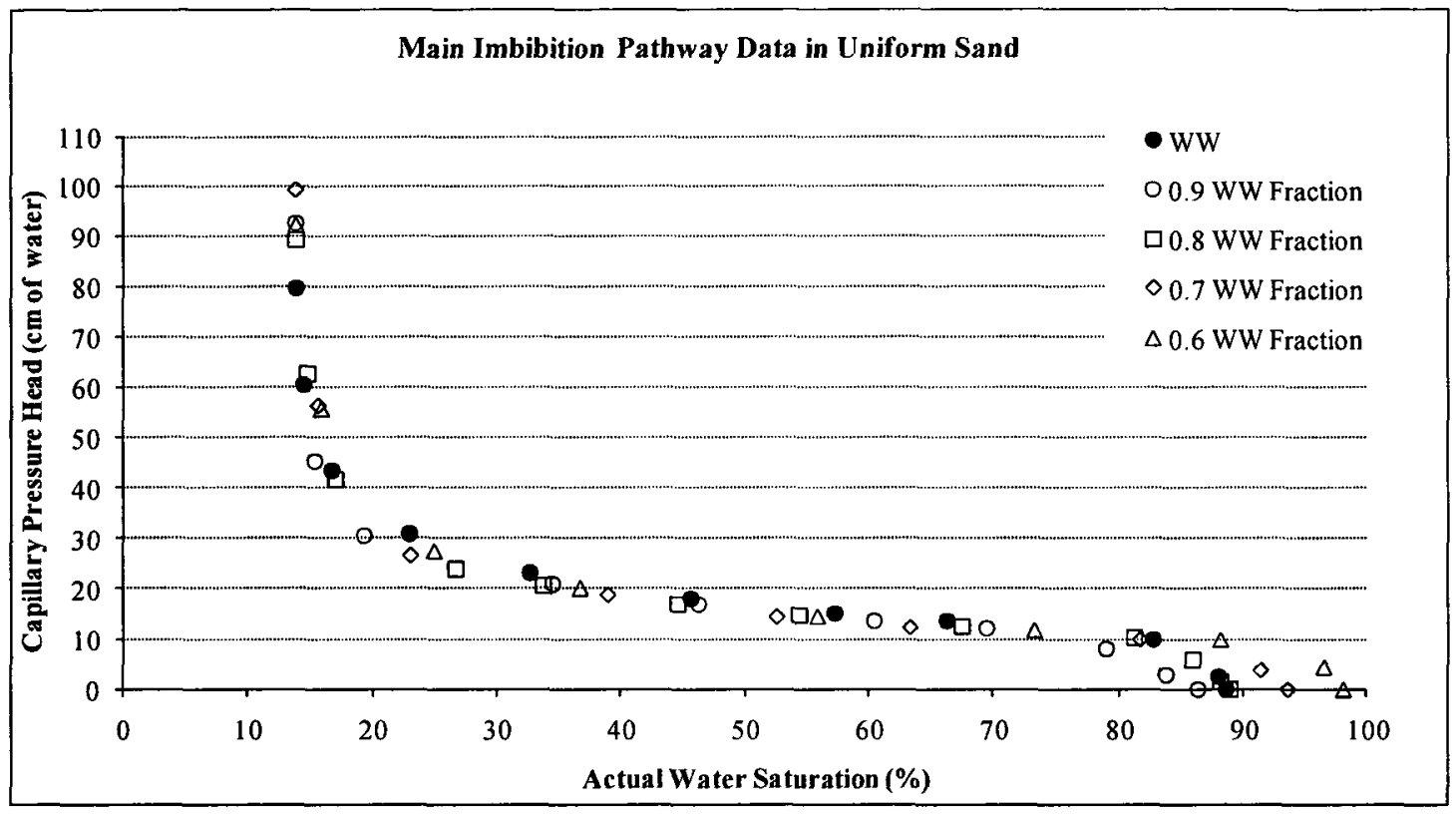

Figure 5.42: Capillary Pressure Head-Actual Water Saturation Data in the WaterWet Porous Medium and the Water-Wet Soil Fractions in Uniform Sand.

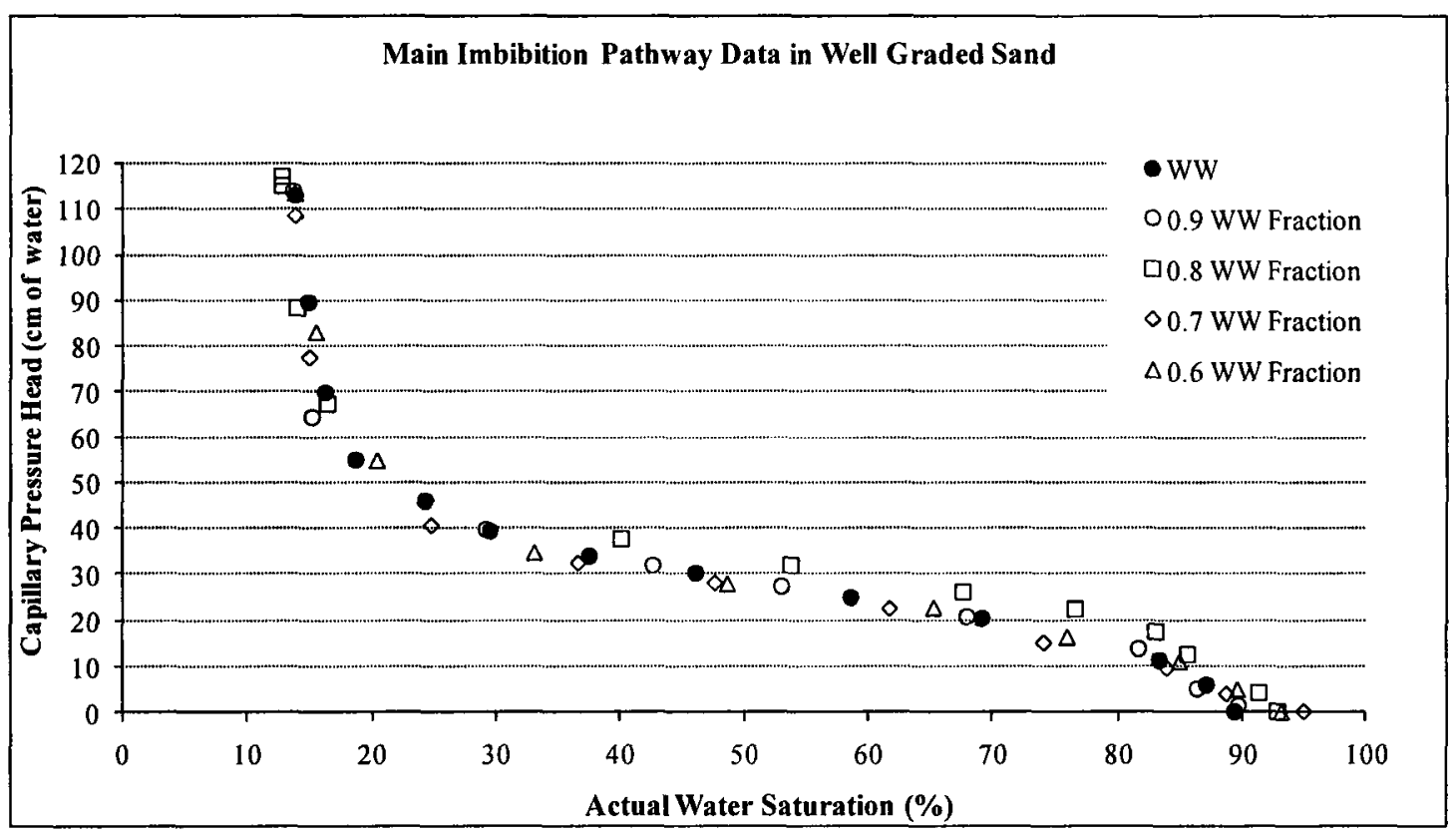

Figure 5.43: Capillary Pressure Head-Actual Water Saturation Data in the WaterWet Porous Medium and the Water-Wet Soil Fractions in Well Graded Sand. 
Figure 5.44, for uniform sand, and Figure 5.45, for well graded sand, show the capillary pressure head-apparent water saturation data for the water-wet porous media and the water-wet soil fractions, and the fitted Van Genuchten main imbibition pathway in the water-wet porous media. The fitted Van Genuchten main imbibition pathway in each water-wet porous medium, in each sand type, fits the capillary pressure headapparent water saturation data in the water-wet soil fractions. In the uniform sand, the fitted Van Genuchten curve appears to be slightly higher than the capillary pressure head-apparent water saturation data in the water-wet soil fractions. While for the well graded sand, the data for the water-wet soil fractions which are adjacent to smaller NAPL-wet soil lenses (i.e., 0.1 NL and 0.2 NL) lie above the fitted Van Genuchten main drainage pathway and the data for the water-wet soil fractions which are adjacent to larger NAPL-wet soil lenses (i.e., 0.3 NL and 0.4 NL) lie below the fitted Van Genuchten main drainage pathway. The reason might be ascribed to variations in soil placement and compaction. 


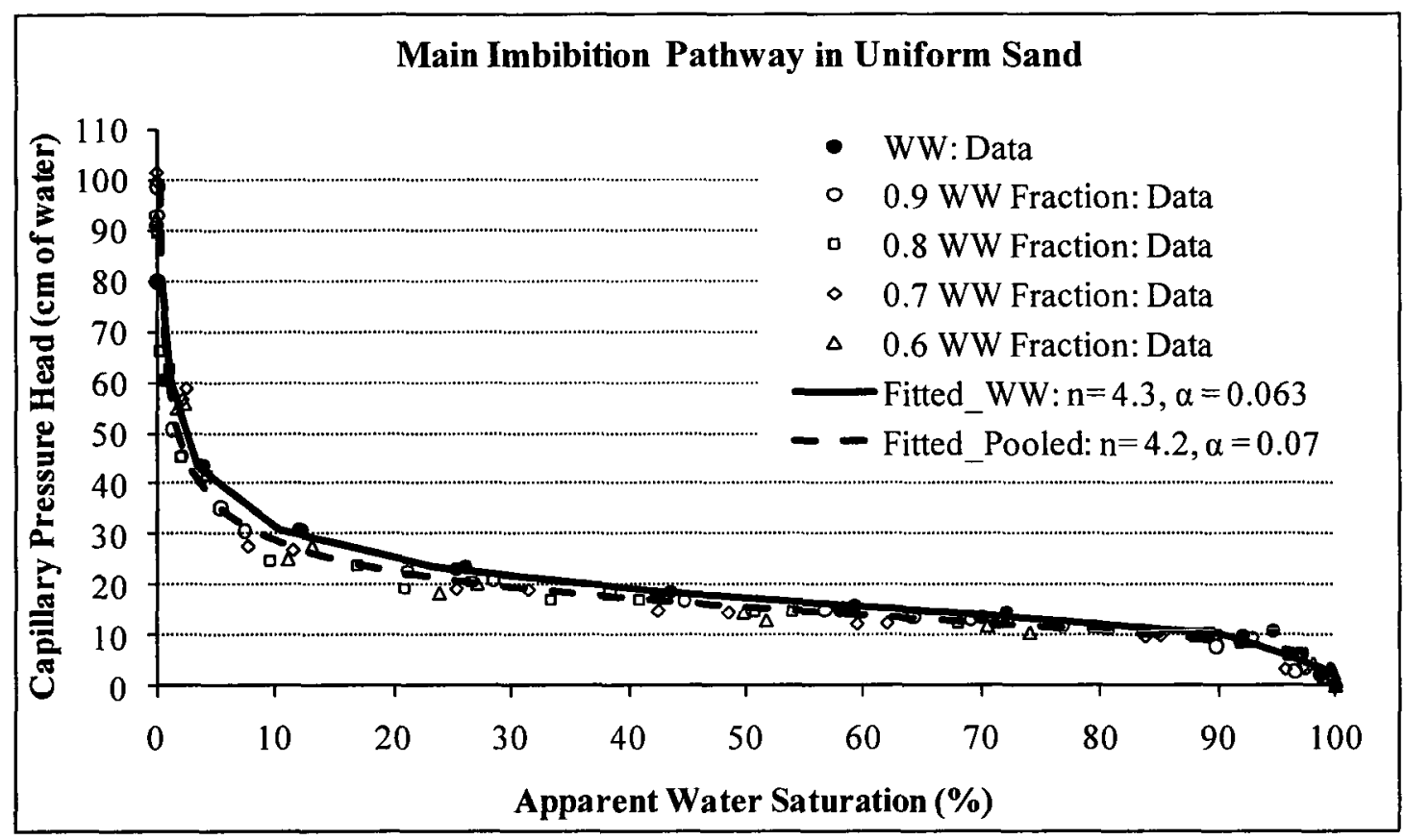

Figure 5.44: Capillary Pressure Head-Apparent Water Saturation in the WaterWet Porous Medium and the Water-Wet Soil Fractions in Uniform Sand.

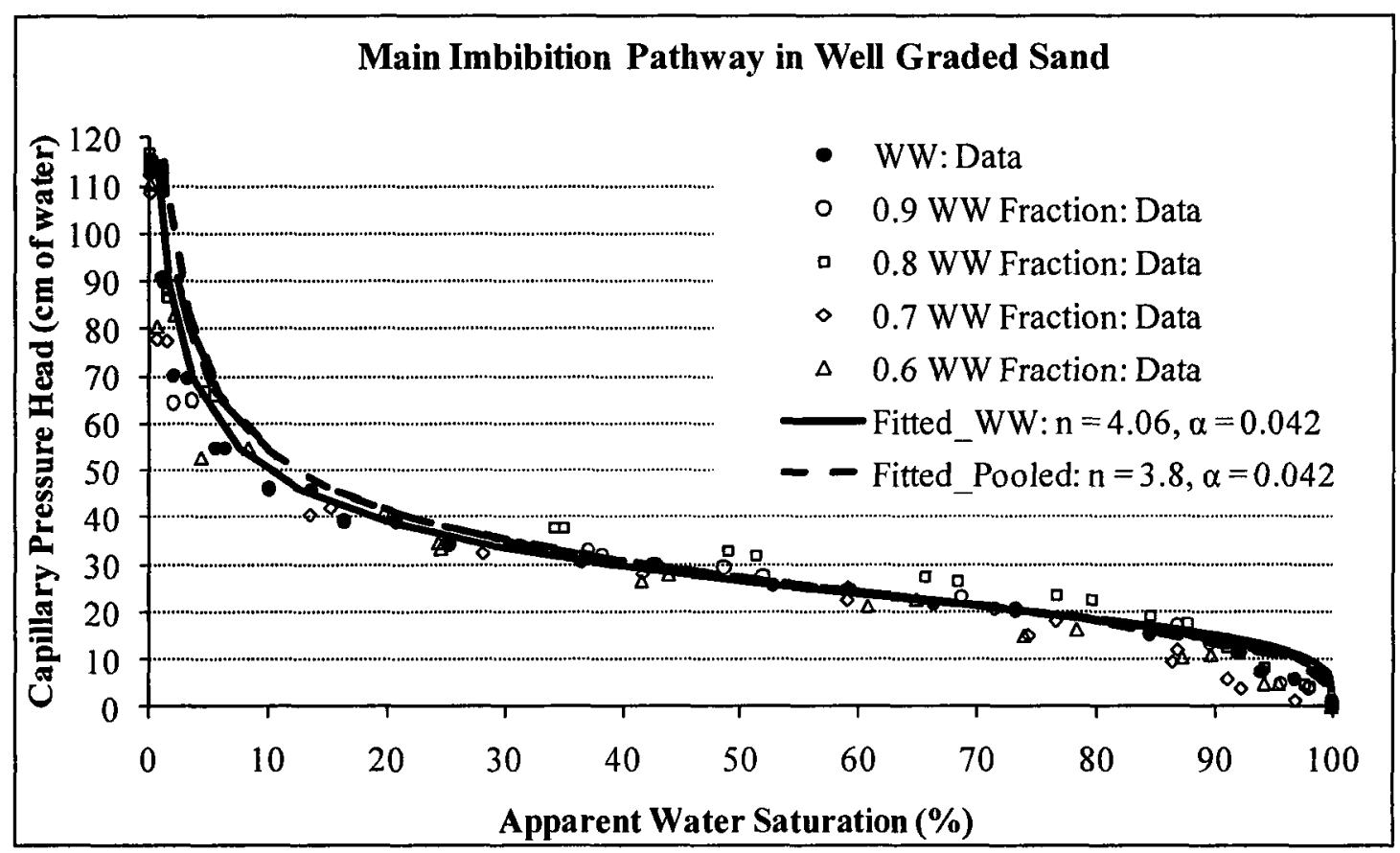

Figure 5.45: Capillary Pressure Head-Apparent Water Saturation in the WaterWet Porous Medium and the Water-Wet Soil Fractions in Well Graded Sand. 


\subsection{Heptane/Water $P_{c}-S$ Relationship}

This section starts with a description of the experimental data of the heptane/water capillary pressure-actual water saturation main drainage and imbibition pathways in uniform sand and well graded sand in a water-wet porous medium, an $80 \%$ water-wet porous medium with $20 \%$ NAPL-wet soil lens, and a $60 \%$ water-wet porous medium with $40 \%$ NAPL-wet soil lens. Also, a table summarizing the irreducible water and entrapped heptane and gas (i.e., $\mathrm{CO}_{2}$ ) saturations in each sand type in a water-wet porous medium, a $90 \%$ water-wet porous medium with $10 \%$ NAPL-wet soil lens, an $80 \%$ waterwet porous medium with $20 \%$ NAPL-wet soil lens, a $70 \%$ water-wet porous medium with $30 \%$ NAPL-wet soil lens, and a $60 \%$ water-wet porous medium with $40 \%$ NAPLwet soil lens, are provided. Then, the $P_{c}-S$ conceptual model formulation for a water-wet porous medium with a NAPL-wet soil lens is presented. The section ends by validation of the proposed conceptual model.

\subsubsection{Capillary Pressure Head-Actual Water Saturation Data}

Figure 5.46 and Figure 5.48 show the main drainage and imbibition pathways in uniform sand, respectively. Figure 5.47 and Figure 5.49 show the main drainage and imbibition pathways in well graded sand, respectively. As shown in Figure 5.46 and Figure 5.47 , the main drainage pathways for the water-wet porous media start at $100 \%$ actual water saturation while the main drainage pathways for the other porous media start at an actual water saturation less than unity, leaving an entrapped gas in each medium. When attempting to saturate the porous medium, given a negative capillary pressure during the experimental setup, water saturates the entire water-wet soil fraction and a fraction of the NAPL-wet soil lens; hence gas is entrapped in the NAPL-wet soil lens. 


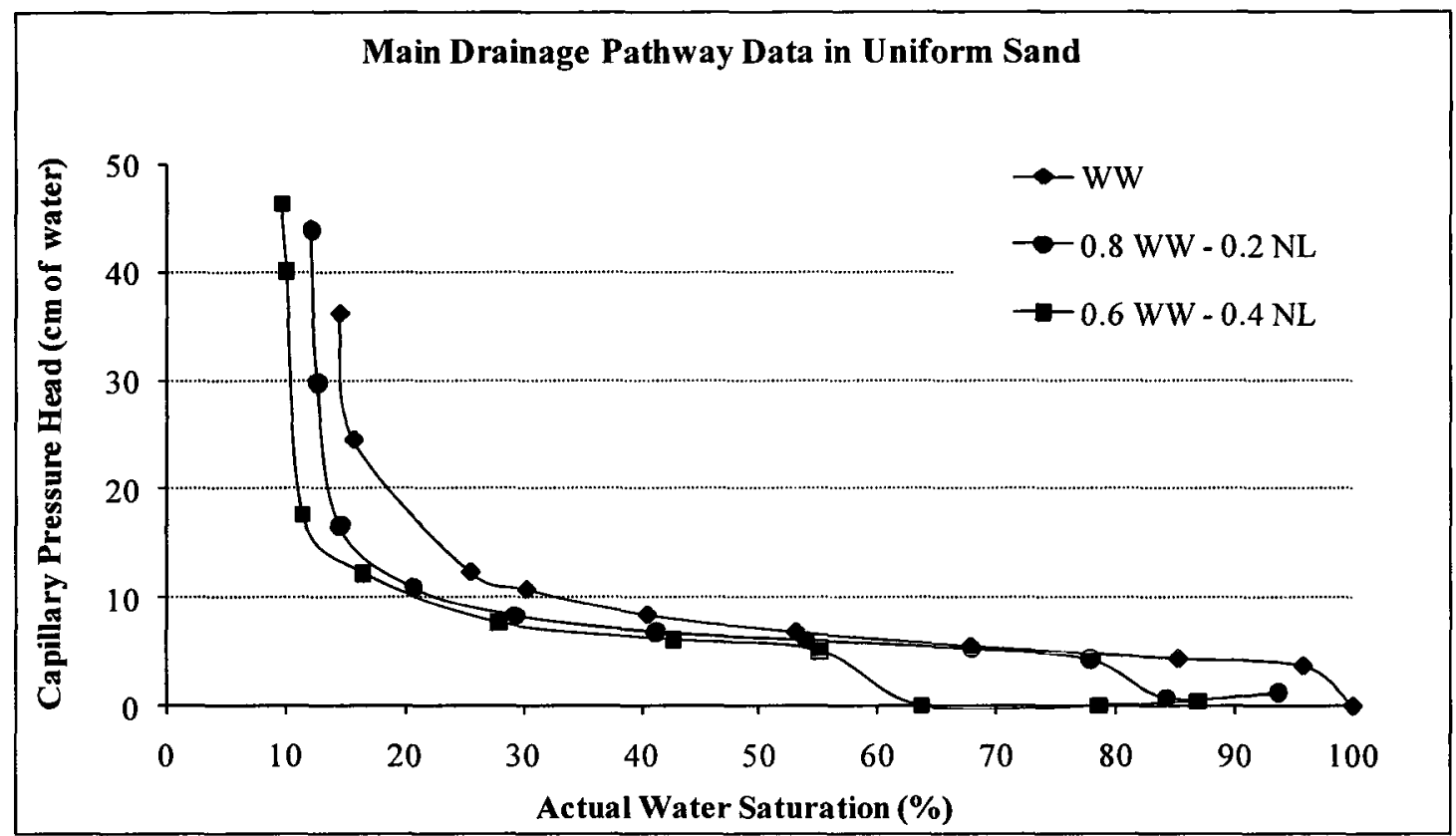

Figure 5.46: Main Drainage Pathways in Different Wettability Porous Media in Uniform Sand.

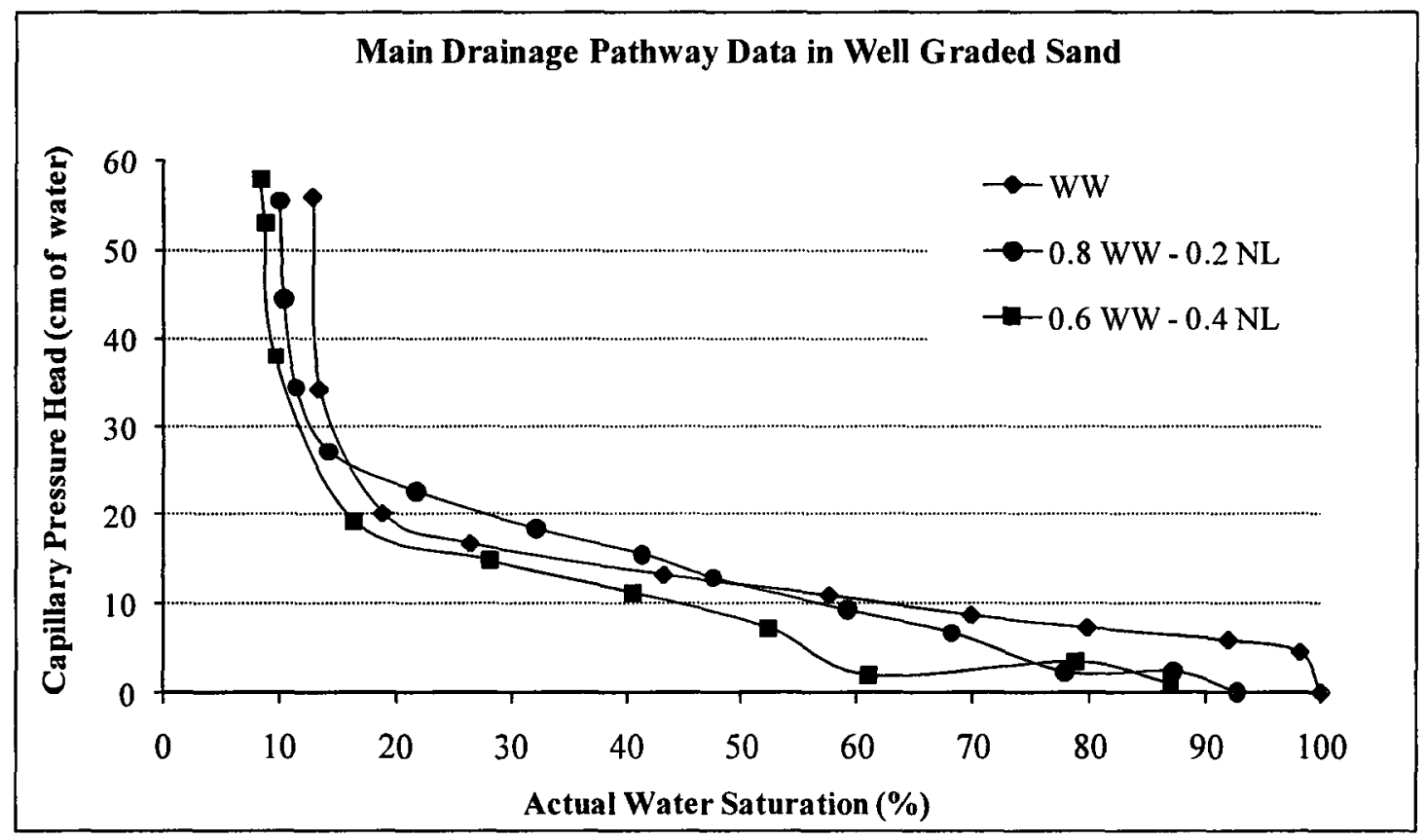

Figure 5.47: Main Drainage Pathways in Different Wettability Porous Media in Well Graded Sand. 


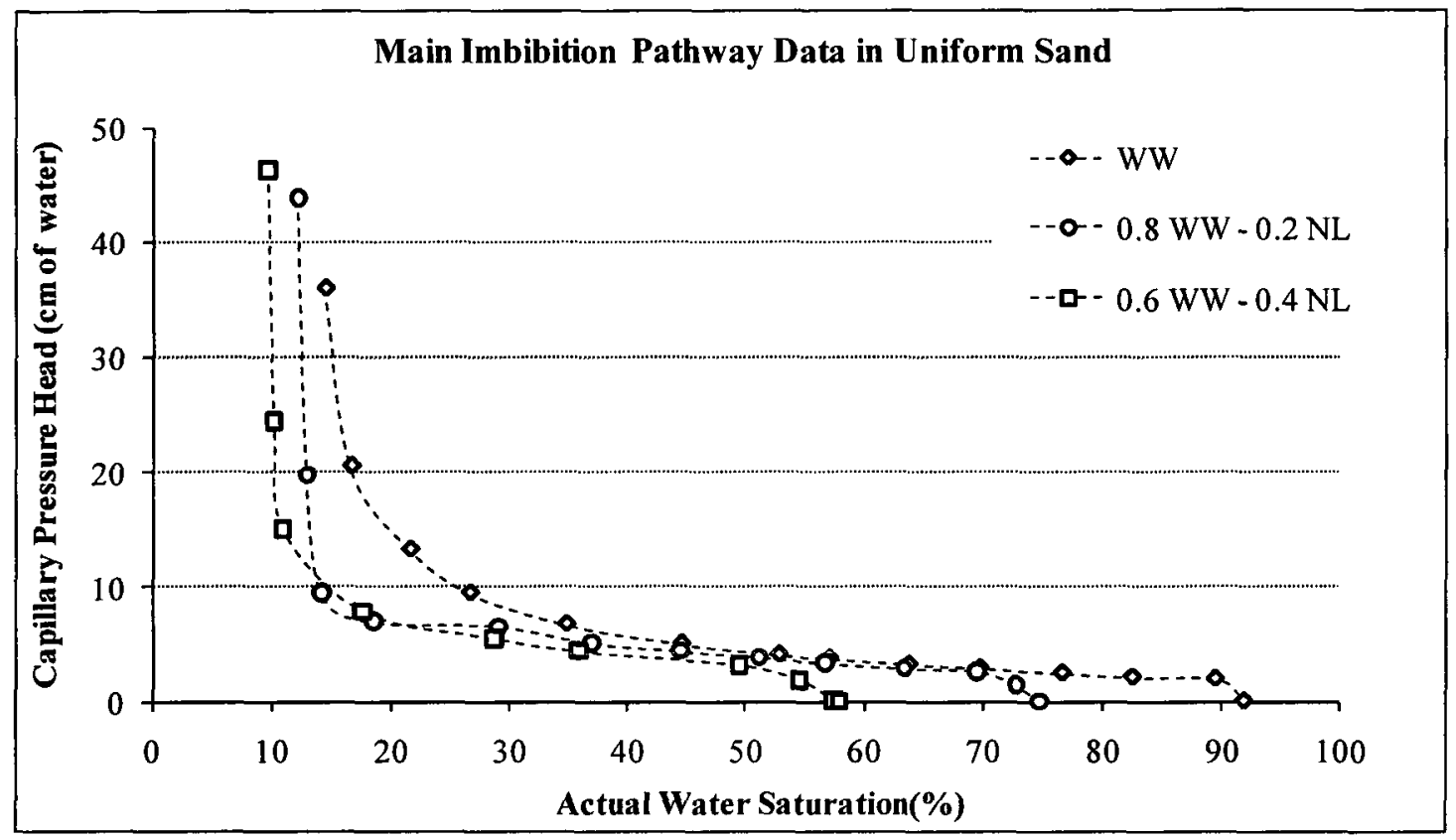

Figure 5.48: Main Imbibition Pathways in Different Wettability Porous Media in Uniform Sand.

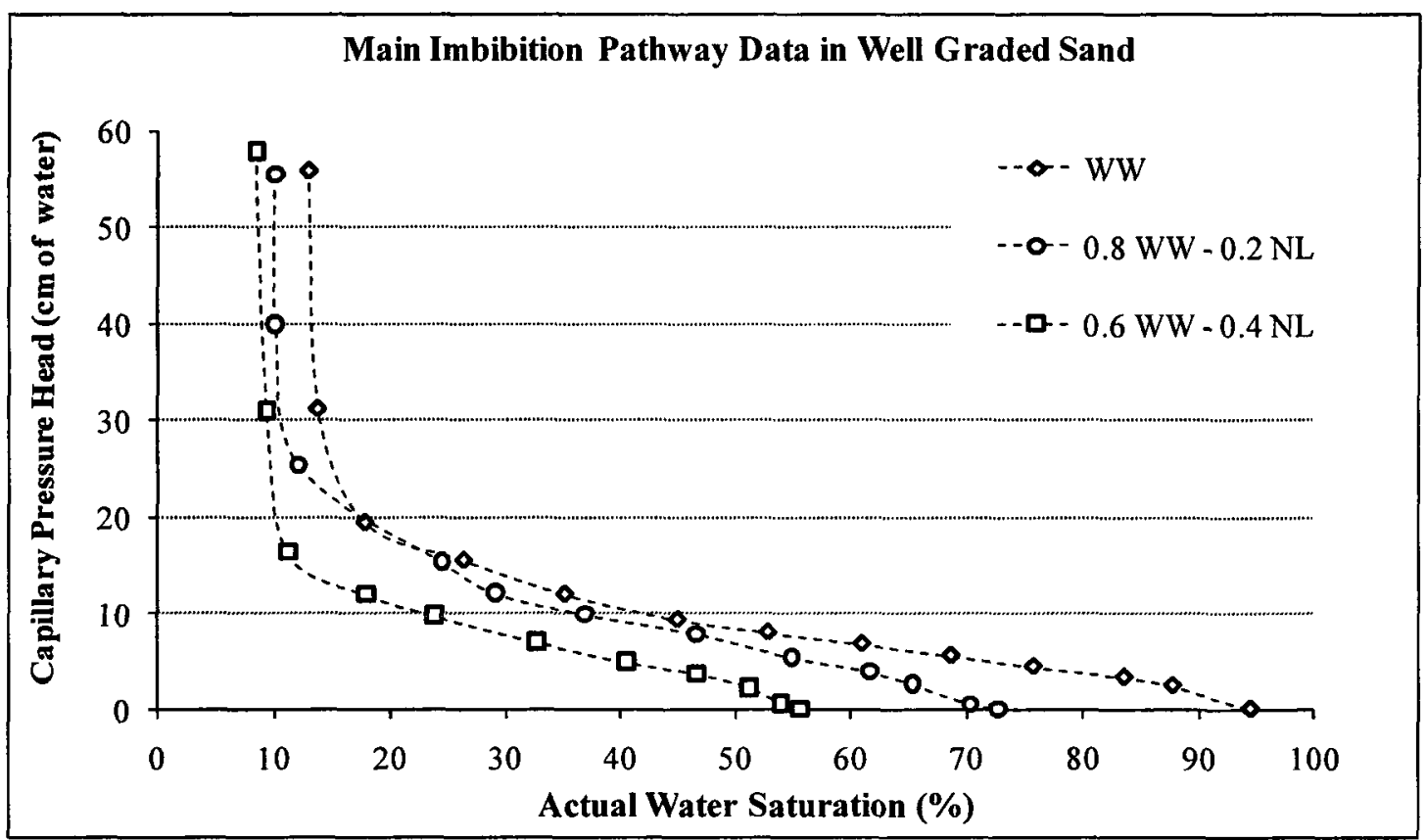

Figure 5.49: Main Imbibition Pathways in Different Wettability Porous Media in Well Graded Sand. 
It is noticed from Figure 5.46 and Figure 5.47 that in a water-wet porous medium with a NAPL-wet soil lens, in the first part of the main drainage pathway, water drains from the soil sample and is being replaced by heptane, even though the capillary pressure is zero or slightly higher than zero. This continues to happen until water saturation becomes close to $(1-f)$. Afterwards, water drains as capillary pressure increases until water saturation reaches its minimum value. Table 5.9, for uniform sand, and Table 5.10, for well graded sand, show the entrapped gas saturations in the NAPL-wet soil lenses before initiating the main drainage pathways and the minimum or irreducible water saturations at the end of the main drainage pathways. It is noticed that the entrapped gas saturation increases as the NAPL-wet soil lens size increases and the results are consistent with the starting saturations for the air/water experiments discussed in Section 5.3.1. The minimum or irreducible water saturation at the end of the main drainage pathway decreases as the NAPL-wet soil lens size increases.

Table 5.9: Irreducible and Entrapped Saturations in Uniform Sand.

\begin{tabular}{|c|c|c|c|c|c|c|c|c|}
\hline \multirow{2}{*}{$\begin{array}{c}\text { Wettability } \\
\text { Configuration }\end{array}$} & \multicolumn{4}{|c|}{ Drainage Pathway } & \multicolumn{3}{c|}{ Imbibition Pathway } \\
\cline { 2 - 9 } & Irreducible & \multicolumn{2}{c|}{ Entrapped } & Irreducible & \multicolumn{2}{c|}{ Entrapped } \\
\cline { 2 - 9 } & $\begin{array}{c}\text { Test } \\
(\mathbf{1})\end{array}$ & $\begin{array}{c}\text { Test } \\
(\mathbf{2})\end{array}$ & $\begin{array}{c}\text { Test } \\
(\mathbf{1})\end{array}$ & $\begin{array}{c}\text { Test } \\
(\mathbf{2})\end{array}$ & $\begin{array}{c}\text { Test } \\
(\mathbf{1})\end{array}$ & $\begin{array}{c}\text { Test } \\
(\mathbf{2})\end{array}$ & $\begin{array}{c}\text { Test } \\
(\mathbf{1})\end{array}$ & $\begin{array}{c}\text { Test } \\
(\mathbf{2})\end{array}$ \\
\hline WW & 14.42 & 15.63 & 0.00 & 0.00 & 14.42 & 15.63 & 8.00 & 5.52 \\
\hline $\mathbf{0 . 9}$ WW - 0.1 NL & 13.27 & 14.28 & 4.88 & 4.27 & 13.27 & 14.28 & 18.15 & 18.71 \\
\hline $\mathbf{0 . 8}$ WW - 0.2 NL & 12.16 & 13.16 & 6.27 & 5.57 & 12.16 & 13.16 & 25.24 & 23.36 \\
\hline $\mathbf{0 . 7}$ WW - 0.3 NL & 10.62 & 11.40 & 5.50 & 8.36 & 10.62 & 11.40 & 32.49 & 33.84 \\
\hline $\mathbf{0 . 6}$ WW - 0.4 NL & 9.59 & 10.45 & 13.13 & 11.86 & 9.59 & 10.45 & 42.08 & 42.90 \\
\hline
\end{tabular}


Table 5.10: Irreducible and Entrapped Saturations in Well Graded Sand.

\begin{tabular}{|c|c|c|c|c|c|c|c|c|}
\hline \multirow{2}{*}{$\begin{array}{c}\text { Wettability } \\
\text { Configuration }\end{array}$} & \multicolumn{4}{|c|}{ Drainage Pathway } & \multicolumn{3}{c|}{ Imbibition Pathway } \\
\cline { 2 - 10 } & Irreducible & \multicolumn{2}{c|}{ Entrapped } & \multicolumn{1}{|c|}{ Irreducible } & \multicolumn{2}{c|}{ Entrapped } \\
\cline { 2 - 10 } & $\begin{array}{c}\text { Test } \\
(\mathbf{1})\end{array}$ & $\begin{array}{c}\text { Test } \\
(\mathbf{2})\end{array}$ & $\begin{array}{c}\text { Test } \\
(\mathbf{1})\end{array}$ & $\begin{array}{c}\text { Test } \\
(\mathbf{2})\end{array}$ & $\begin{array}{c}\text { Test } \\
(\mathbf{1})\end{array}$ & $\begin{array}{c}\text { Test } \\
(\mathbf{2})\end{array}$ & $\begin{array}{c}\text { Test } \\
(\mathbf{1})\end{array}$ & $\begin{array}{c}\text { Test } \\
(\mathbf{2})\end{array}$ \\
\hline WW & 12.94 & 12.88 & 0.00 & 0.00 & 12.94 & 12.88 & 8.87 & 5.58 \\
\hline $\mathbf{0 . 9}$ WW - 0.1 NL & 11.44 & 11.4 & 4.28 & 2.96 & 11.44 & 11.4 & 20.94 & 20.69 \\
\hline $\mathbf{0 . 8}$ WW - 0.2 NL & 9.97 & 9.43 & 7.22 & 8.45 & 9.97 & 9.43 & 27.33 & 28.63 \\
\hline $\mathbf{0 . 7}$ WW - 0.3 NL & 9.48 & 9.21 & 10.04 & 11.02 & 9.48 & 9.21 & 32.92 & 39.93 \\
\hline $\mathbf{0 . 6}$ WW - 0.4 NL & 9.41 & 8.37 & 15.77 & 13.04 & 9.41 & 8.37 & 42.07 & 44.38 \\
\hline
\end{tabular}

As shown in Figure 5.48 and Table 5.9, for uniform sand, and Figure 5.49 and Table 5.10, for well graded sand, each of the main imbibition pathways starts at its corresponding minimum or irreducible water saturation and ends at an actual water saturation less than unity, leaving entrapped heptane and gas saturations in each medium. The entrapped saturations increase with the existence or the increase of the NAPL-wet soil lens size. Figure 5.48 and Figure 5.49 show that the main imbibition pathways for the three porous media for each sand type are comparable.

\subsubsection{Water-Wet Porous Medium}

It was explained in Chapter 2 that both air and heptane are non-wetting to a waterwet porous medium in the presence of water. Moreover, the air should behave in a similar manner as the heptane. Therefore, instead of repeating the content of Section 5.3.2, the heptane will simply replace the air in the text, equations, and figures with the following terms introduced:

$S_{n t} \quad$ : actual entrapped heptane saturation corresponding to actual water saturation $S_{w}$ on the main imbibition pathway;

$S_{m_{\max }}:$ maximum actual entrapped heptane saturation; and 
$\bar{S}_{n t_{\max }}$ : maximum effective entrapped heptane saturation.

In addition to that, the following images were captured at the pore scale. Figure 5.50 shows the heptane located in pore body centres and surrounded by water. Figure 5.51 shows the water forms at irreducible water saturation.

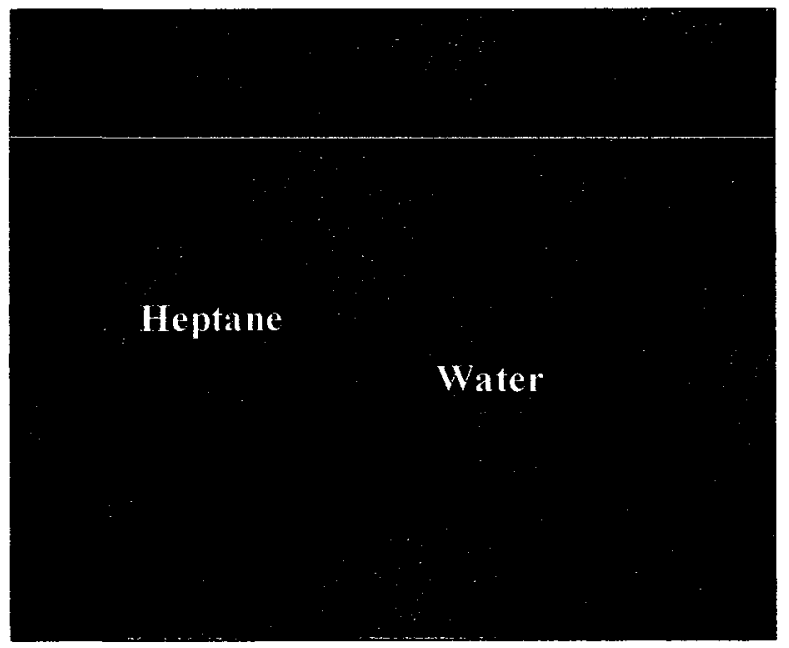

Figure 5.50: Heptane Located at Pore Body Centres and Surrounded by Water.

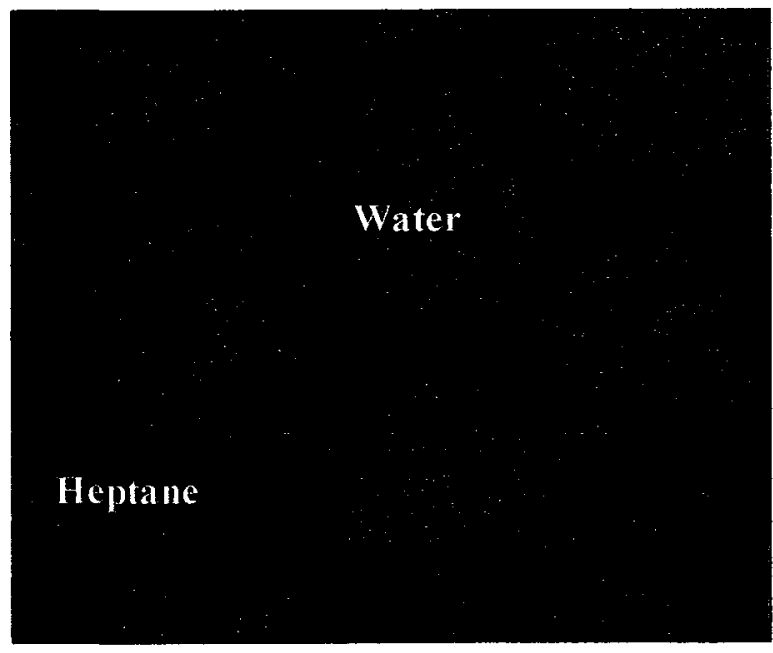

Figure 5.51: Water Forms at Irreducible Saturation. 


\subsubsection{NAPL-Wet Porous Medium}

In a NAPL-wet porous medium, heptane is the wetting phase and water is the non-wetting phase. A water/heptane $P_{c}-S$ experiment in a NAPL-wet porous medium starts with a heptane-saturated soil sample. In order for the water to enter the porous medium, the capillary pressure has to exceed the water entry pressure. A non-wetting phase may enter into and move in a porous medium under its pressure or under the wetting phase suction. In a NAPL-wet porous medium, water enters the porous medium and replaces heptane only at negative capillary pressure. Note that for consistency in the discussion, the capillary pressure is always defined as the NAPL pressure minus the water pressure. A similar approach was used by Bradford and Leij (1996) and O'Carroll et al. (2005). Therefore, along a water imbibition pathway, if heptane pressure is set to zero, water pressure has to increase in order to force the water to move and increase its saturation in the porous medium. Along the water drainage pathway, the capillary pressure has to increase in order for the water to move out of the medium and decrease its saturation.

Similar to the drainage of water from an initially water-saturated water-wet soil sample where water starts to drain from the larger soil pores as heptane replaces the water, in an initially heptane-saturated NAPL-wet soil sample, heptane starts to drain from the larger pore bodies as water replaces the heptane. As water is forced to move into the porous medium, heptane continues to drain and is being replaced by water until the soil sample reaches the irreducible heptane saturation. At this point, heptane exists as a thin layer coating the soil particles and fills the small pore throats. When heptane is allowed to imbibe into the medium, water is displaced from the smaller pores. At a 
capillary pressure head of zero (or greater), water may be entrapped within the centre of individual pores or as ganglia in series of connected pores.

\subsection{3.a. Water Main Imbibition Pathway}

The water main imbibition pathway or the heptane main drainage pathway starts at an actual heptane saturation of unity and zero capillary pressure. As mentioned before, the water pressure has to exceed the water entry pressure before heptane starts to drain and is replaced by water. As water pressure increases, the actual heptane saturation decreases until it reaches irreducible heptane saturation $\left(S_{n m}\right)$ (as shown in Figure 5.52). Therefore, along the water main imbibition pathway, actual water saturation in a NAPLwet porous medium should be more than zero and less than $1-S_{n m}$. Pertaining to a rigid porous medium assumption and according to O'Carroll et al. (2005), the shape of the water main imbibition pathway in a NAPL-wet porous medium should be similar to the shape of the water main drainage pathway in a water-wet porous medium. 


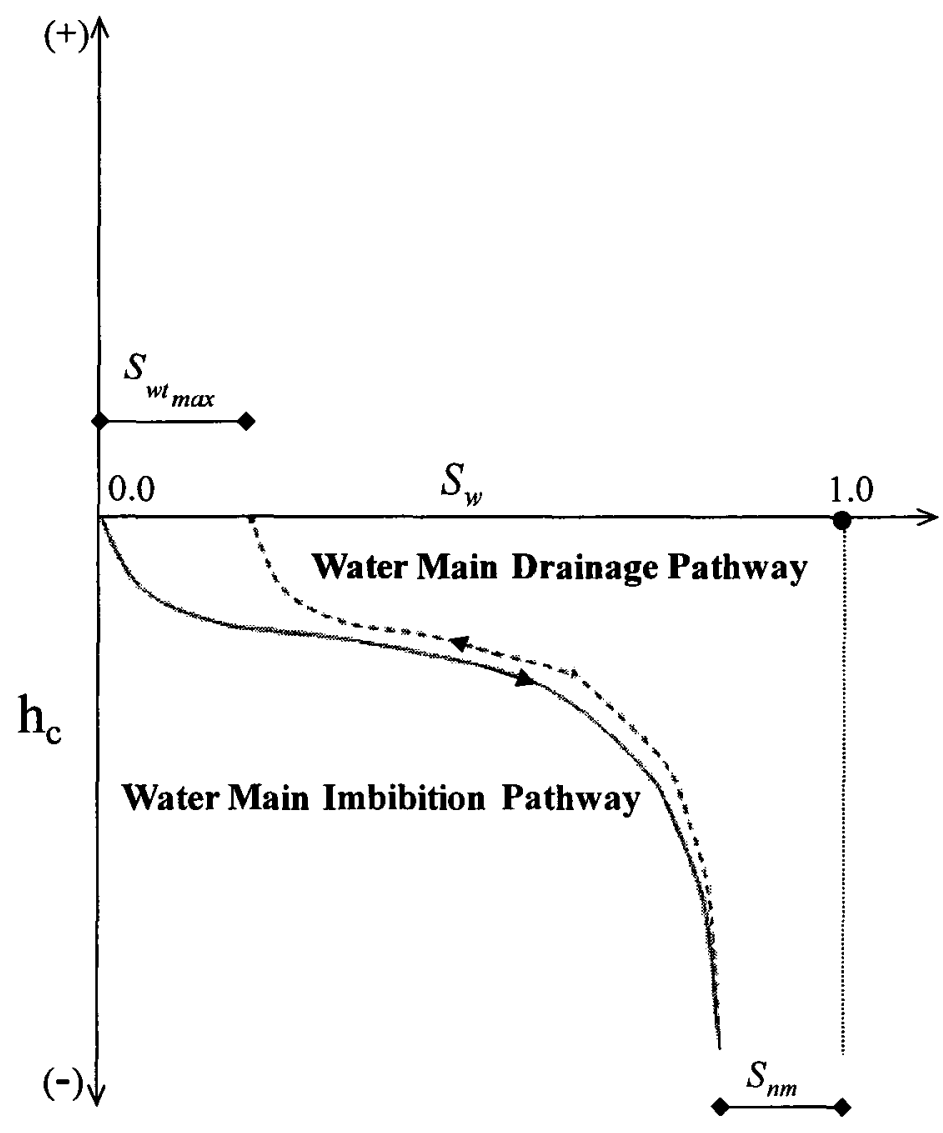

Figure 5.52: Capillary Pressure Head-Actual Water Saturation Main Imbibition and Drainage Pathways in a NAPL-Wet Porous Medium.

\subsection{3.b. Water Main Drainage Pathway}

The water main drainage pathway or the heptane main imbibition pathway starts at an actual water saturation of $1-S_{n m}$ and irreducible heptane saturation $\left(S_{n m}\right)$. As capillary pressure increases, heptane imbibes into the porous medium and water is displaced. As this is happening, heptane entraps the water that is disconnected. This continues to happen (i.e., as capillary pressure increases, heptane imbibes into the porous medium and entraps the disconnected water) until capillary pressure becomes zero. At 
this point, water exists as an entrapped phase at an actual saturation of $S_{w t_{\max }}$ and heptane exists at an actual heptane saturation less than unity (as shown in Figure 5.52). According to O'Carroll et al. (2005), the shape of the water main drainage pathway in a NAPL-wet porous medium should be similar to the shape of the water main imbibition pathway in a water-wet porous medium.

Therefore, there are some points that have to be highlighted. First, a NAPL-wet porous medium cannot normally reach $100 \%$ water saturation. Instead, heptane remains in the porous medium at an irreducible saturation of $S_{n m}$. As heptane imbibes into the porous medium and replaces water, it entraps part of the water. At the end of the heptane main imbibition pathway, water remains in the porous medium at the maximum entrapped saturation (i.e., $S_{w t_{\max }}$ ). Therefore, even for a positive capillary pressure head, water should exist in a NAPL-wet porous medium at the maximum entrapped value. Figure 5.53 shows the entrapped water located at the centre of a pore body and it is surrounded by heptane.

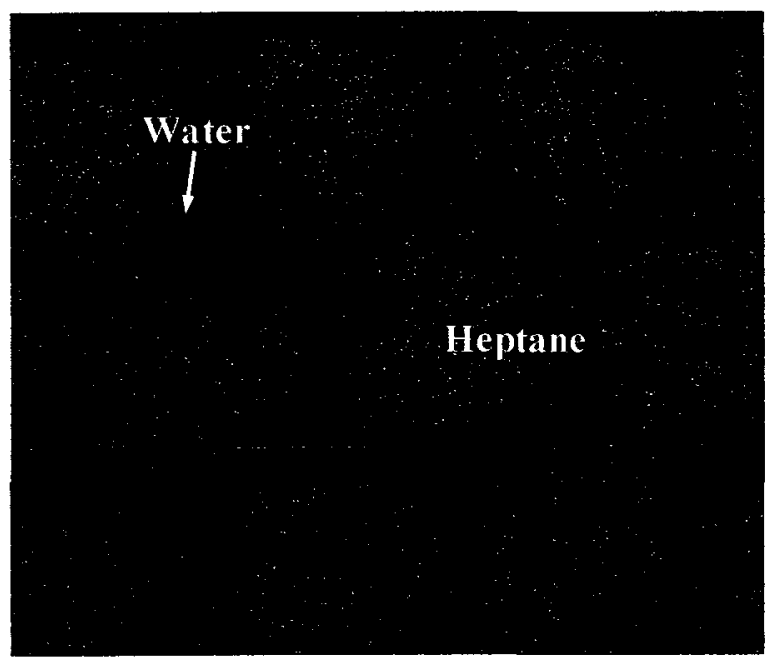

Figure 5.53: Entrapped Water Located at the Centre of Pore Bodies and Surrounded by Heptane in a NAPL-Wet Porous Medium. 


\subsubsection{Water-Wet Porous Medium with a NAPL-Wet Soil Lens}

In a water-wet porous medium, using the procedure followed in Chapter 3 to set up the heptane/water experiments, the porous medium is water-saturated prior to initiating the water main drainage pathway. While for a water-wet porous medium with a NAPL-wet soil lens, a 100\% saturation condition may not be achieved. The experimental setup for heptane/water and air/water experiments is similar. This implies that, during the experimental setup, the system is a two-phase air/water system. Hence, the water imbibition and drainage pathways in the water-wet soil fraction and in the NAPL-wet soil lens are similar to those presented in Section 5.3.4, up to point 3 shown in Figure 5.27. At this point, the water-wet soil fraction is water-saturated while the NAPL-wet soil lens is partially water-saturated.

Figure 5.54 shows the water imbibition pathway for air/water two-phase system (i.e., prior to introducing the heptane to the system), and the water main drainage and imbibition pathways for heptane/water two-phase system in the water-wet and NAPL-wet porous media. In this figure, the water-wet and NAPL-wet porous media represent the water-wet soil fraction and the NAPL-wet soil lens in the REV, respectively. As shown in Figure 5.54, point 3 is the beginning of the water main drainage pathway in the water-wet porous medium with the NAPL-wet soil lens. At this point, heptane is introduced to the system. As shown in Figure 5.54b, once heptane reaches the NAPL-wet soil lens, heptane apparently saturates the lens as heptane displaces the water and gas phases. This is because the capillary pressure head is positive (i.e., positive heptane head and negative water head) on the NAPL-wet soil lens. Part of the water is entrapped by heptane in the lens. As for the gas phase, it is either entrapped in the centre of pore bodies or leaves the 
soil sample which is vented to the atmosphere. Figure 5.55 shows the gas and water entrapped in heptane in the NAPL-wet soil lens.

In this case, the water drainage follows the path $3 \rightarrow 4$ as shown in Figure $5.54 \mathrm{~b}$ and it stops at point 4 . At this point, in the NAPL-wet soil lens, the water saturation is the entrapped saturation $\left(S_{w t}\right)$ and the heptane saturation is $1-S_{w t}$. The water and heptane saturations in the NAPL-wet soil lens are fixed at these values as long as the capillary pressure is positive (i.e., at points 4,5 , and 6 ). If the capillary pressure decreases to a negative value, the water re-enters the lens following the path $6 \rightarrow 7$ shown in Figure $5.54 \mathrm{~b}$.

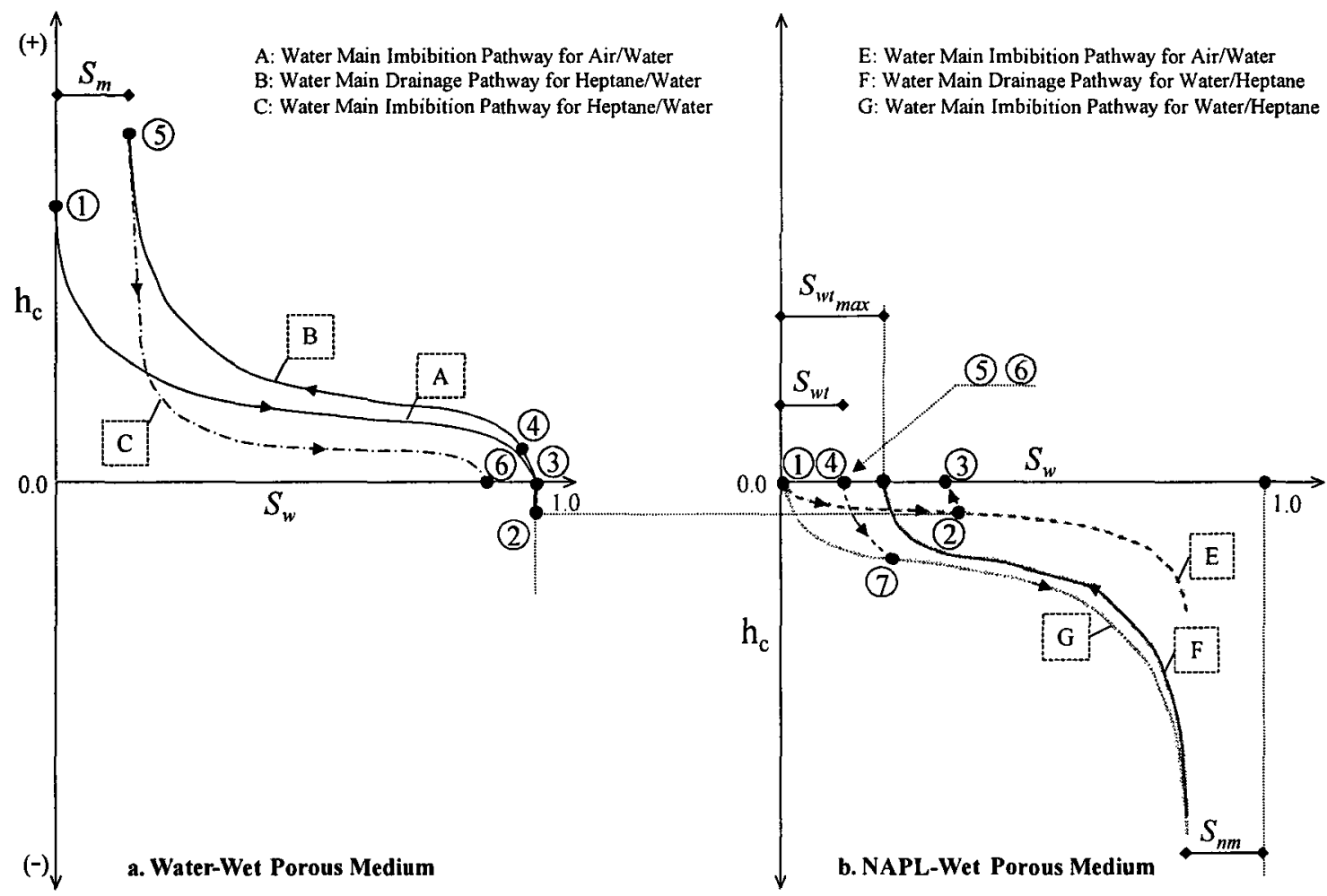

Figure 5.54: Conceptual Model for Heptane/Water Capillary Pressure Head-Actual Water Saturation Imbibition and Drainage Pathways in a Water-Wet Soil Fraction and in a NAPL-Wet Soil Lens. 


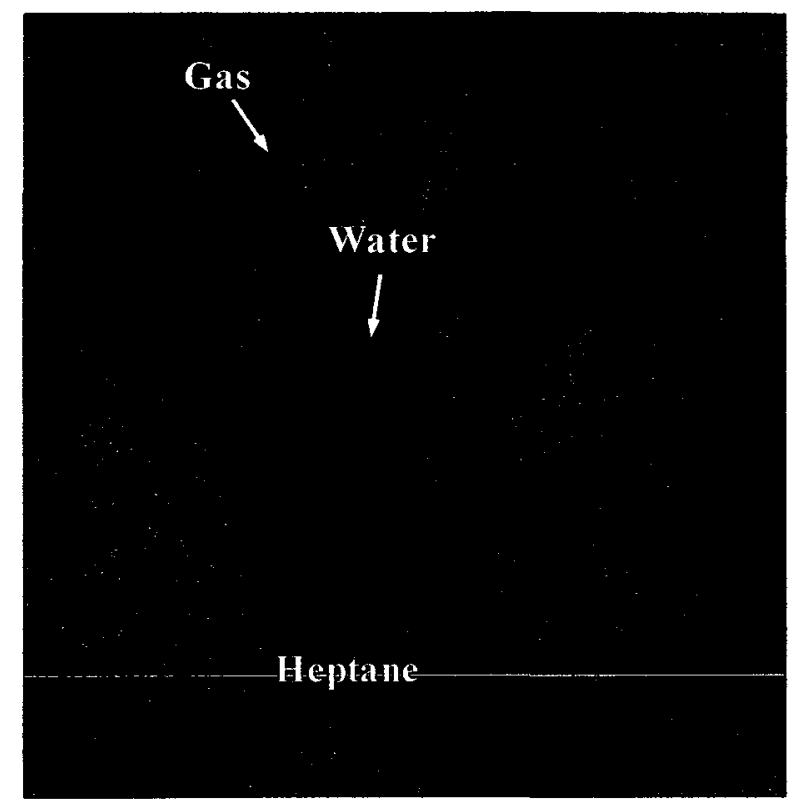

Figure 5.55: Water and Gas Entrapped in Heptane in a NAPL-Wet Soil Lens.

In the water-wet soil fraction, as shown in Figure 5.54a, the water main drainage pathway starts at point 3 . The water drains from the water-wet soil fraction as the capillary pressure increases, following the path $3 \rightarrow 4 \rightarrow 5$. The water saturation at point 5 is the irreducible saturation. At this point, if the capillary pressure decreases, water imbibes into the porous medium following the path $5 \rightarrow 6$.

In the water-wet porous medium with the NAPL-wet soil lens, the imbibition of heptane into the NAPL-wet soil lens depends on the location of the lens in the medium. If the NAPL-wet soil lens is in contact with the top boundary of the REV (i.e., in direct contact with heptane when it is added), the NAPL-wet soil lens becomes apparently heptane-saturated at zero capillary pressure. While, if the lens is embedded in the porous medium, then the beginning of heptane imbibition into the lens implies that the capillary pressure has to exceed the heptane entry pressure of the water-wet soil fraction. This is represented by point 4 in Figure 5.54. At this point, heptane imbibed into the lens, and 
displaced and entrapped water and gas. As long as the capillary pressure remains positive, water drains from and imbibes into the water-wet soil fraction while the water saturation in the NAPL-wet soil lens remains constant at an entrapped value. This is represented by points 5 and 6 in Figure 5.54 .

\subsection{4.a. Conceptual Model for Water Main Drainage Pathway}

The $P_{c}-S$ main drainage pathway starts at zero capillary pressure and actual water saturation less than unity. The capillary pressure has to be higher than the heptane entry pressure before water starts to drain and is replaced by heptane. At this point, as described earlier, the NAPL-wet soil lens becomes apparently heptane-saturated entrapping part of the water. The magnitude of the entrapped water saturation in the NAPL-wet soil lens depends on the water saturation reached prior to the end of the experimental setup (i.e., point 2 in Figure 5.54b). At this point, the NAPL-wet soil lens degree of saturation depends on whether the lens is dry, partially water-saturated, or apparently water-saturated. This implies that the entrapped water saturation in the lens could be zero (i.e., dry NAPL-wet soil lens), $S_{w t^{\prime}}^{P}$ (i.e., partially water-saturated NAPLwet soil lens), or $S_{w t^{\prime}}^{S}$ (i.e., apparently water-saturated NAPL-wet soil lens). The heptane and the entrapped water saturations in the lens remain constant as the capillary pressure remains positive. While in the water-wet soil fraction, as the capillary pressure increases (i.e., after point 3 in Figure 5.54a), water drains from it in a way consistent with the drainage of water from a water-wet porous medium when exposed to the same capillary pressure on the main drainage pathway.

Therefore, the water main drainage pathway in the water-wet porous medium with the NAPL-wet soil lens is the summation of the scaled main drainage pathway in the 
water-wet soil fraction and the scaled entrapped water saturation in the NAPL-wet soil lens. The water main drainage pathway in the water-wet porous medium with the NAPLwet soil lens lies between two limits. The lower limit represents a case when the NAPLwet soil lens is dry (case (A)). The upper limit represents a case when the NAPL-wet soil lens is apparently water-saturated (case (C)). The general case represents a case when the NAPL-wet soil lens is partially water-saturated (case (B)). Therefore, the main drainage pathway for each case is defined according to Figure 5.56.

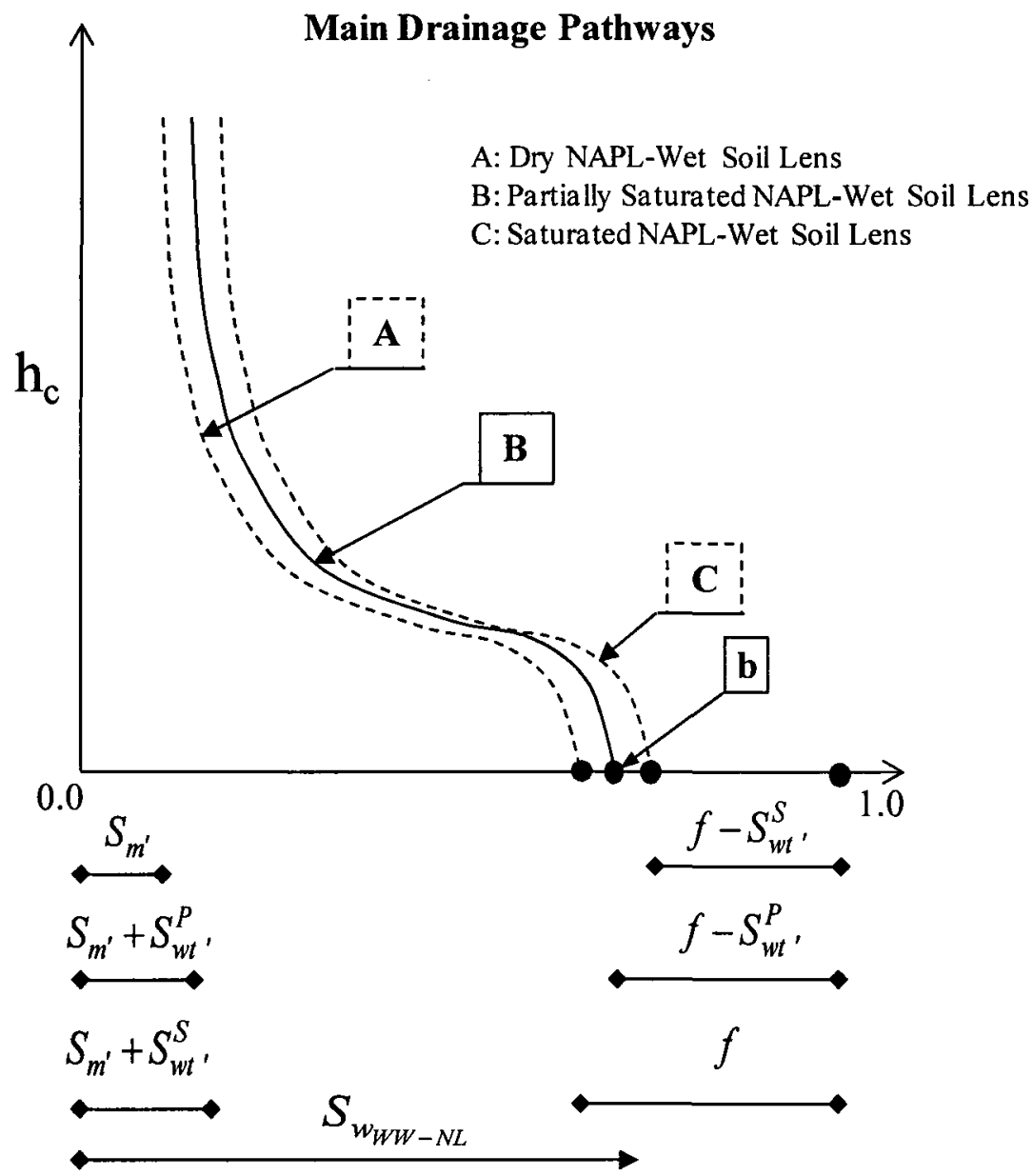

Figure 5.56: $P_{c}-S$ Main Drainage Pathway in a Water-Wet Porous Medium with a NAPL-Wet Soil Lens. 
The difference between these three cases is the entrapped water saturation in the NAPL-wet soil lens. Therefore, the water drainage pathways corresponding to the three cases are shifted. The water saturation at the end of the water drainage pathway is the summation of the irreducible water saturation in the water-wet soil fraction, which should be fixed in the three cases, and the entrapped water saturation in the NAPL-wet soil lens which depends on the case considered.

For any of the three cases, once the NAPL-wet soil lens becomes apparently heptane saturated, water drains only from the water-wet soil fraction with the increase of capillary pressure. Therefore, the water main drainage pathway within the water-wet soil fraction should be similar to the water main drainage pathway in a water-wet porous medium. Hence, one can predict water saturation in a water-wet porous medium with a NAPL-wet soil lens with the knowledge of the water main drainage pathway in a waterwet porous medium, the volume fraction of the NAPL-wet soil lens, and the entrapped water saturation in the NAPL-wet soil lens. The latter depends on the case considered (A, $\mathrm{B}$, or C).

As for the heptane, at any capillary pressure, heptane saturation is the summation of the weighted heptane saturation in the water-wet soil fraction and the heptane saturation in the NAPL-wet soil lens. The heptane saturation in the NAPL-wet soil lens depends on the entrapped gas and water saturations in the lens.

\section{Actual Water and Heptane Saturations for Case (A) (Dry NAPL-wet soil lens)}

Using the same analogy presented in Section 5.3.4.a.I, one might calculate the actual water and heptane saturations in case (A). In order to achieve that, one should first 
know the apparent, effective, and actual saturations in a water-wet porous medium. Then, using these actual saturations, the following equations are used to calculate the actual water and heptane saturations for case (A) as follows:

$S_{w_{W W-N L}}=S_{w_{w W}} *(1-f)$

$S_{n_{W W-N L}}=S_{n_{W W}} *(1-f)+f *\left(1-S_{a t_{N L}}\right)$

$S_{a t_{N L}}=\frac{V_{a t_{N L}}}{\eta_{n w}{ }^{*} V_{N L}}$

$S_{n_{W W}}:$ actual NAPL (e.g., heptane) saturation in a water-wet porous medium;

$S_{a_{t_{N L}}}:$ entrapped air saturation in the NAPL-wet soil lens, when considered alone, or in the NAPL-wet porous medium;

$V_{a t_{N L}}:$ volume of entrapped air in the NAPL-wet soil lens; and

$V_{N L}:$ NAPL-wet soil lens volume.

II. Actual Water and Heptane Saturations for Case (B) (Partially saturated NAPL-wet soil lens)

It was explained that, at the beginning of the water main drainage pathway, as capillary pressure is increased such that the heptane is allowed to reach the NAPL-wet soil lens, the heptane imbibes into the NAPL-wet soil lens. At this point, the heptane continues filling the NAPL-wet soil lens and the displaced water leaves the soil sample and is collected in the burette. As water drains from the NAPL-wet soil lens, the capillary pressure in the REV declines to zero or to a value close to zero. This continues to happen until the NAPL-wet soil lens is apparently heptane-saturated entrapping water and gas. At 
this point, the capillary pressure is zero, or at a value close to zero, and the water-wet soil fraction is water-saturated. Afterwards, as capillary pressure increases, the water drains from the water-wet soil fraction in the same manner as the water drains from a water-wet porous medium. This continues to occur until the end of the main drainage pathway where water saturation in the water-wet soil fraction is irreducible water saturation. Similar to case (A), with the knowledge of the water saturation in the water-wet porous medium, the volume fraction of the NAPL-wet soil lens, and the entrapped gas and water saturations in the NAPL-wet soil lens, one can calculate the actual water and heptane saturations for case (B) as follows:

$$
\begin{aligned}
& S_{w_{w W-N L}}=S_{w_{w W}} *(1-f)+S_{w t^{\prime}}^{P} \\
& S_{n_{w W-N L}}=S_{n_{w W}} *(1-f)+f^{*}\left(1-S_{a t_{N L}}\right)-S_{w t^{\prime}}^{P} \\
& S_{w t^{\prime}}^{P}=f^{*} S_{w t}
\end{aligned}
$$

Where:

$S_{w t}^{P}:$ entrapped water saturation in the initially partially water-saturated NAPL-wet soil lens.

III. Actual Water and Heptane Saturations for Case (C) (Saturated NAPL-wet soil lens)

Case (C) is similar to case (B) except that the entrapped water saturation in the NAPL-wet soil lens is $S_{w t}^{S}$,instead of $S_{w t^{\prime}}^{P}$. Therefore, the actual water and heptane saturations for case (C) can be calculated as follows: 


$$
\begin{aligned}
& S_{w_{w w-N L}}=S_{w_{w W}} *(1-f)+S_{w t^{\prime}}^{S} \\
& S_{n_{w w-N L}}=S_{n_{w w}} *(1-f)+f^{*}\left(1-S_{a t_{N L}}\right)-S_{w t^{\prime}}^{S} \\
& S_{w t^{\prime}}^{S}=f^{*} S_{w t_{\max }}
\end{aligned}
$$

Where:

$S_{w t^{\prime}}^{S}$ : entrapped water saturation in the initially apparently water-saturated NAPL-wet soil lens.

\subsection{4.b. Conceptual Model Validation for Water Main Drainage Pathway}

It is noticed from the capillary pressure head-water saturation data for the waterwet porous media with the NAPL-wet soil lenses that the water main drainage pathway in any of these media can be represented by case (B). Therefore, actual water and heptane saturations can be calculated as explained in case (B). This solution is based on an assumption that the water drainage from the water-wet soil fraction is similar to the drainage of water from the water-wet porous medium. In order to check the validity of this assumption, the water saturations and the corresponding capillary pressure heads in the water-wet soil fraction are extracted from the capillary pressure head-water saturation data measured in the water-wet porous medium with the NAPL-wet soil lens.

In order to extract these data, the start and end points of the main drainage pathway in a water-wet porous medium with a NAPL-wet soil lens are determined. The start point is the point after which water saturation decreases as capillary pressure increases. This point corresponds to point $b$ in Figure 5.56. The end point is the point corresponding to the minimum water saturation in the porous medium. The minimum 
water saturation in the porous medium is the summation of the irreducible water saturation in the water-wet soil fraction and the entrapped water saturation in the NAPLwet soil lens. If the curve (B) in Figure 5.56 is shifted to the left by a value equivalent to the entrapped water saturation in the NAPL-wet soil lens (i.e., the entrapped water saturation is subtracted from the water saturations), the remaining water saturations and their corresponding capillary pressure heads are those measured in the water-wet soil fraction. If these water saturations are divided by $(1-f)$ and plotted versus the corresponding capillary pressure heads, the resulting main drainage pathway should be equivalent to the main drainage pathway in a water-wet porous medium. In order to do that, the entrapped water saturation in the NAPL-wet soil lens is calculated. Using the same analogy explained in Section 5.3.4.d, the entrapped water saturation in the NAPLwet soil lens and the irreducible water saturation in the water-wet soil fraction may be calculated as shown in Table 5.11, for uniform sand, and Table 5.12, for well graded sand.

Table 5.11: Irreducible and Entrapped Water Saturations in Uniform Sand.

\begin{tabular}{|c|c|c|c|c|c|c|c|c|}
\hline \multirow{2}{*}{$\begin{array}{c}\text { Wettability } \\
\text { Configuration }\end{array}$} & \multicolumn{4}{|c|}{ Experiment Set (1) } & \multicolumn{4}{c|}{ Experiment Set (2) } \\
\cline { 2 - 10 } & $\begin{array}{c}S_{m} \text { or } \\
\left(S_{m^{\prime}}+S_{w t^{\prime}}^{P}\right)\end{array}$ & $\begin{array}{c}\text { Expected } \\
S_{m^{\prime}}\end{array}$ & $\begin{array}{c}\text { Actual } \\
S_{m^{\prime}}\end{array}$ & $S_{w t^{\prime}}^{P}$ & $\begin{array}{c}S_{m} \text { or } \\
\left(S_{m^{\prime}}+S_{w t^{\prime}}^{P}\right)\end{array}$ & $\begin{array}{c}\text { Expected } \\
S_{m^{\prime}}\end{array}$ & $\begin{array}{c}\text { Actual } \\
S_{m^{\prime}}\end{array}$ & $S_{w t^{\prime}}^{P}$ \\
\hline WW & 14.42 & 14.42 & 14.42 & 0.00 & 15.63 & 15.63 & 15.63 & 0.00 \\
\hline $\mathbf{0 . 9}$ WW - 0.1 NL & 13.27 & 12.98 & 12.98 & 0.29 & 14.28 & 14.07 & 14.07 & 0.21 \\
\hline $\mathbf{0 . 8} \mathbf{W W}-\mathbf{0 . 2}$ NL & 12.16 & 11.54 & 11.54 & 0.62 & 13.16 & 12.5 & 12.50 & 0.66 \\
\hline $\mathbf{0 . 7}$ WW - 0.3 NL & 10.62 & 10.09 & 10.09 & 0.53 & 11.40 & 10.94 & 10.94 & 0.46 \\
\hline $\mathbf{0 . 6} \mathbf{W W}-\mathbf{0 . 4} \mathbf{~ N L}$ & 9.59 & 8.65 & 8.65 & 0.94 & 10.45 & 9.38 & 9.38 & 1.07 \\
\hline
\end{tabular}


Table 5.12: Irreducible and Entrapped Water Saturations in Well Graded Sand.

\begin{tabular}{|c|c|c|c|c|c|c|c|c|}
\hline \multirow[b]{2}{*}{$\begin{array}{l}\text { Wettability } \\
\text { Configuration }\end{array}$} & \multicolumn{4}{|c|}{ Experiment Set (1) } & \multicolumn{4}{|c|}{ Experiment Set (2) } \\
\hline & $\begin{array}{c}S_{m} \text { or } \\
\left(S_{m^{\prime}}+S_{w t^{\prime}}^{P}\right)\end{array}$ & $\begin{array}{l}\text { Expected } \\
\qquad S_{m^{\prime}}\end{array}$ & $\begin{array}{c}\text { Actual } \\
S_{m^{\prime}}\end{array}$ & $S_{w t^{\prime}}^{P}$ & $\begin{array}{c}S_{m} \text { or } \\
\left(S_{m^{\prime}}+S_{w t^{\prime}}^{P}\right)\end{array}$ & $\begin{array}{l}\text { Expected } \\
\quad S_{m^{\prime}}\end{array}$ & $\begin{array}{c}\text { Actual } \\
S_{m^{\prime}}\end{array}$ & $S_{w t^{\prime}}^{P}$ \\
\hline Ww & 12.94 & 12.94 & 12.94 & 0.00 & 12.88 & 12.88 & 12.88 & 0.00 \\
\hline $0.9 \mathrm{WW}-0.1 \mathrm{NL}$ & 11.44 & 11.65 & 11.44 & 0.00 & 11.40 & 11.59 & 11.40 & 0.00 \\
\hline $0.8 \mathrm{WW}-0.2 \mathrm{NL}$ & 9.97 & 10.35 & 9.97 & 0.00 & 9.43 & 10.30 & 9.43 & 0.00 \\
\hline $0.7 \mathrm{WW}-0.3 \mathrm{NL}$ & 9.48 & 9.06 & 9.06 & 0.42 & 9.21 & 9.02 & 9.02 & 0.19 \\
\hline $0.6 \mathrm{WW}-0.4 \mathrm{NL}$ & 9.41 & 7.76 & 7.76 & 1.65 & 8.37 & 7.73 & 7.73 & 0.64 \\
\hline
\end{tabular}

Figure 5.57, for uniform sand, and Figure 5.58, for well graded sand, show the capillary pressure head-apparent water saturation data in the water-wet porous media and the water-wet soil fractions. It is evident that the effective and apparent saturations are similar along these pathways in each sand type. In each sand type, the figure also shows the fitted Van Genuchten relationship based on the water-wet porous medium data (i.e., Fitted_WW) and Van Genuchten relationship based on water-wet porous medium and water-wet soil fractions data (i.e., Fitted_Pooled). The latter was calculated to take into account the potential errors in different experiments (e.g., variations in soil placement and compaction, contamination). 


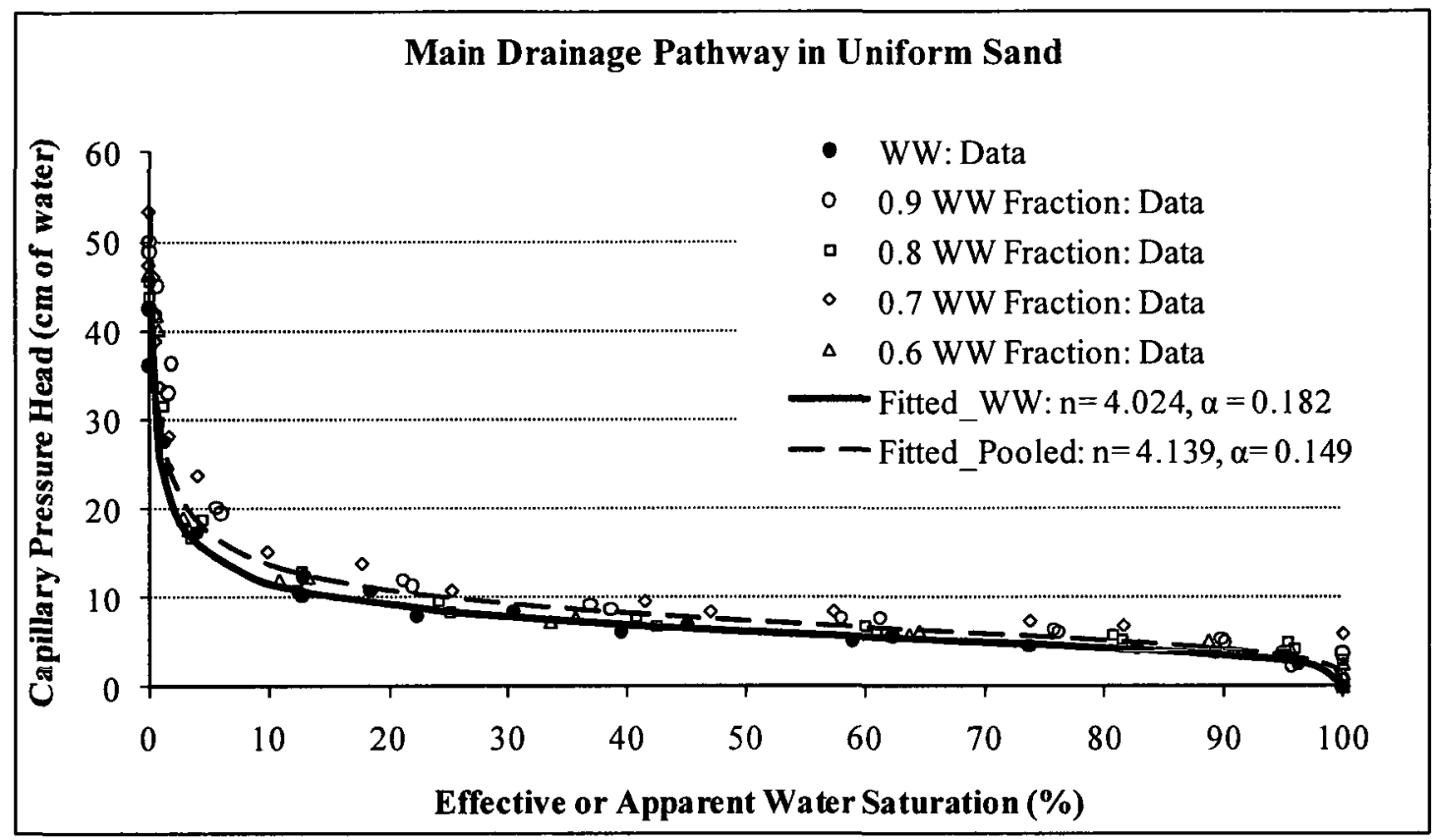

Figure 5.57: Capillary Pressure Head-Effective (Apparent) Water Saturation in Water-Wet Porous Medium and Water-Wet Soil Fractions in Uniform Sand.

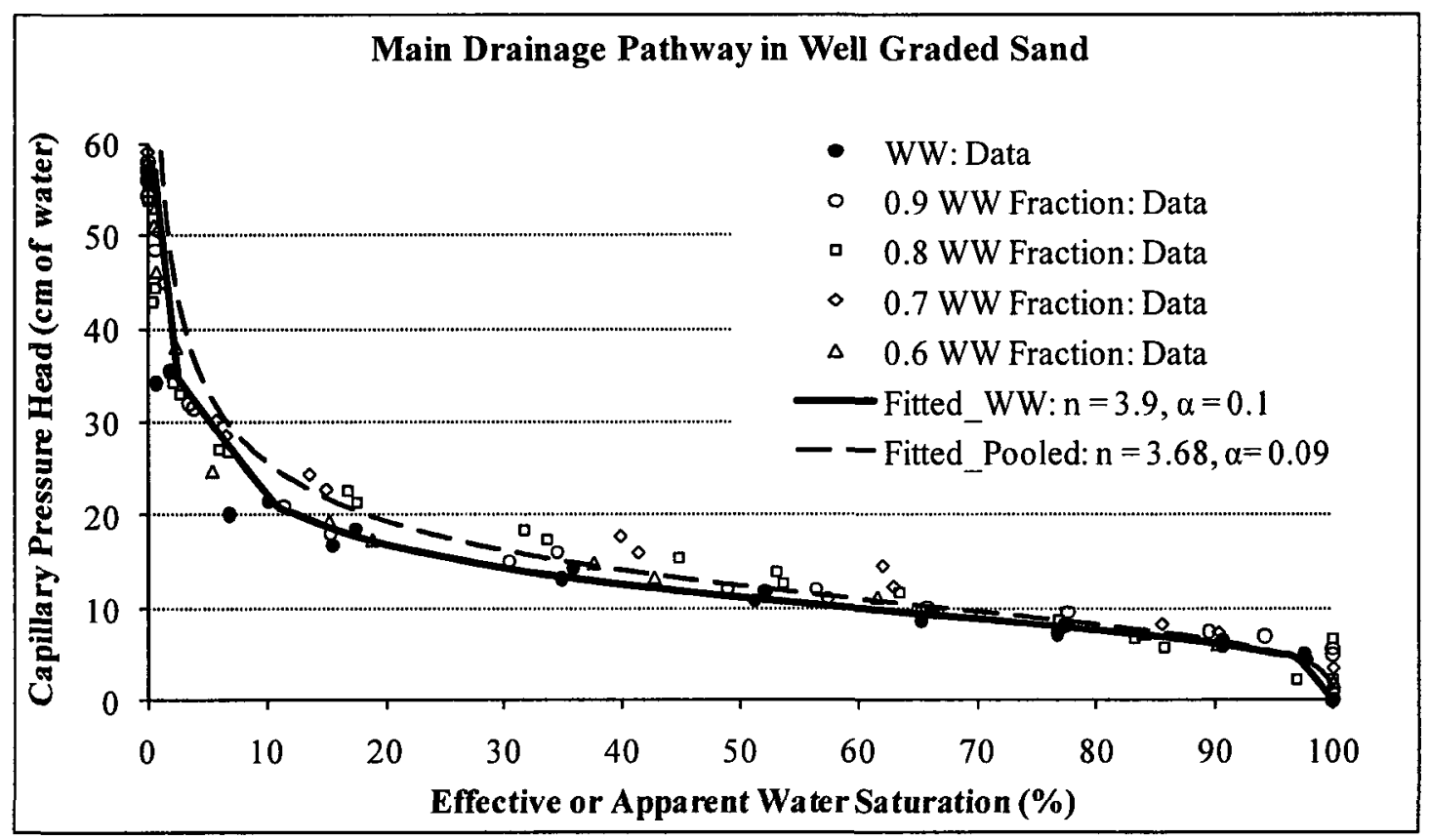

Figure 5.58: Capillary Pressure Head-Effective (Apparent) Water Saturation in Water-Wet Porous Medium and Water-Wet Soil Fractions in Well Graded Sand. 
It is noticed from Figure 5.57 and Figure 5.58 that the capillary pressure heads corresponding to apparent water saturations in the water-wet soil fractions are slightly higher than the capillary pressure heads corresponding to apparent water saturations based on the Fitted_WW relationship. In addition, at a given apparent water saturation, a capillary pressure head based on the Fitted_Pooled relationship is slightly higher than that based on Fitted_WW. It is evident that the pooled data fit the Fitted_Pooled relationship well and validates the proposed conceptual model.

\subsection{4.c. Conceptual Model for Water Main Imbibition Pathway}

The capillary pressure-water saturation main imbibition pathway in a water-wet porous medium with a NAPL-wet soil lens starts at the minimum water saturation. As capillary pressure decreases, the water imbibes into the water-wet soil fraction and replaces the heptane. At zero capillary pressure, the water-wet soil fraction is apparently water-saturated where part of the heptane is entrapped. Figure 5.59 shows the water main imbibition pathways for cases (A), (B), and (C). It is assumed that the magnitude of the entrapped heptane in the water-wet soil fraction does not depend on the case considered (i.e., it is the same in case (A), (B), or (C)). Along the water main imbibition pathway, the NAPL-wet soil lens remains apparently heptane saturated where gas and/or water may be entrapped, depending on the case considered (i.e., A, B, or C). Therefore, the existence of the NAPL-wet soil lens does not affect the water main imbibition pathway for the waterwet soil fraction. Hence, if one removes the lens with its entrapped water from the tested soil sample, the remaining soil will be the water-wet soil fraction. By considering the water-wet soil fraction alone, one should obtain a water main imbibition pathway similar to that in a water-wet porous medium. 


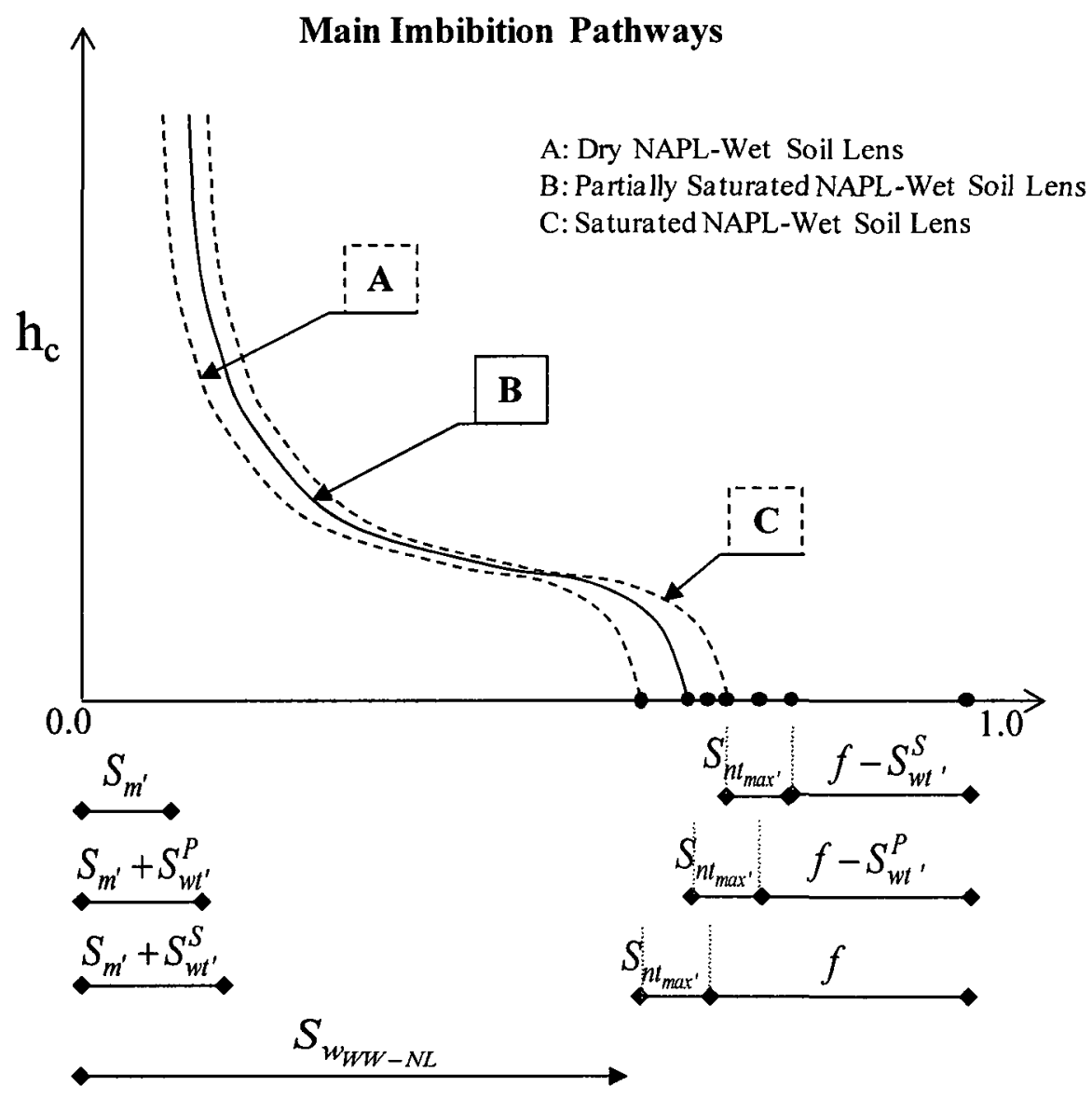

Figure 5.59: $P_{c}-S$ Main Imbibition Pathway in a Water-Wet Porous Medium with a NAPL-Wet Soil Lens.

Where:

$S_{n t_{\text {max }}}=(1-f)^{*} S_{n t_{\text {max }}}$

$S_{n t_{\max }}=\frac{V_{n t_{W W F}}}{\eta_{w w}{ }^{*} V_{W W F}}$

$S_{n_{\max }}:$ maximum actual entrapped heptane saturation in the water-wet soil fraction at the end of the water main imbibition pathway; 
$S_{n t_{\max }}$ : maximum actual entrapped heptane saturation in the water-wet soil fraction, when considered alone, or in the water-wet porous medium at the end of the water main imbibition pathway;

$V_{n t_{W W F}}:$ volume of the entrapped heptane in the water-wet soil fraction; and

$V_{W W F}$ : volume of the water-wet soil fraction.

As capillary pressure decreases, water imbibes into the water-wet soil fraction while it does not imbibe into the NAPL-wet soil lens. Therefore, the water saturation in the NAPL-wet soil lens remains at zero (i.e., case (A)) or at an entrapped saturation (i.e., cases (B) or (C)). In case (A), the main imbibition pathway starts at water saturation of $S_{m}$, (i.e., $S_{m}$ in a water-wet porous medium) and zero entrapped water saturation in the NAPL-wet soil lens. In case (B), the main imbibition pathway starts at water saturation of $S_{m}$, in the water-wet soil fraction and an entrapped water saturation of $S_{w t}^{P}$, in the NAPLwet soil lens. In case (C), the main imbibition pathway starts at water saturation of $S_{m}$, in the water-wet soil fraction and an entrapped water saturation of $S_{w t}^{S}$, in the NAPL-wet soil lens. At any capillary pressure head along the main imbibition pathway, the water saturation in case (A) is less than the water saturation in case (B) by $S_{w t}^{P}$, and less than the water saturation in case (C) by $S_{w t^{\prime}}^{S}$. Therefore, the main imbibition pathways for the three cases are shifted as shown in Figure 5.59.

As water imbibes into the water-wet soil fraction, heptane is being entrapped. At the end of the main imbibition pathway (i.e., capillary pressure head equals to zero), 
water is apparently saturating the water-wet soil fraction with a maximum entrapped heptane saturation of $S_{n t_{\max }}$ (i.e., $S_{n t_{\max }}$ in a water-wet porous medium). The water saturation and entrapped heptane saturation, in the water-wet porous medium with the NAPL-wet soil lens, are calculated according to the case considered (i.e., case (A), (B), or $(\mathrm{C}))$.

\section{Actual Water and Heptane Saturations for Case (A) (Dry NAPL-wet soil lens)}

As mentioned above, the NAPL-wet soil lens remains apparently heptanesaturated along the water main imbibition pathway. It has been explained earlier that the NAPL-wet soil lens does not have an effect on the imbibition of water into the medium. Therefore, if the NAPL-wet soil lens has been removed from the porous medium and the pore volume in the water-wet soil fraction is used in water saturation calculations, one should obtain the same main imbibition pathway as in a water-wet porous medium. Considering the water-wet soil fraction alone, the effective and apparent water saturations in this case are similar to those shown in Figure 5.23b and Figure 5.23c. In order to calculate the actual water and heptane saturations in a water-wet porous medium with a NAPL-wet soil lens, the actual water and heptane saturations in a water-wet porous medium should be known. Then, the actual water and heptane saturations for case (A) can be calculated as follows:

$$
\begin{aligned}
& S_{w_{W W-N L}}=S_{w_{W W}} *(1-f) \\
& S_{n_{W W-N L}}=S_{n_{W W}} *(1-f)+f *\left(1-S_{a t_{N L}}\right)
\end{aligned}
$$


II. Actual Water and Heptane Saturations for Case (B) (Partially saturated NAPL-wet soil lens)

Similar to case (A), the water saturation in the water-wet soil fraction, when considered alone, follows the same pathway as in a water-wet porous medium. While the water saturation in the NAPL-wet soil lens remains, all the time, at an entrapped saturation of $S_{w t}^{P}$. In order to obtain heptane and water saturations, the analogy used in case $(\mathrm{A})$ is followed in this case. Using the actual water and heptane saturations in a water-wet porous medium, the following equations are used to calculate the actual water and heptane saturations in case (B):

$S_{w_{W W-N L}}=S_{w_{w W}} *(1-f)+S_{w t^{\prime}}^{P}$

$S_{n_{W W-N L}}=S_{n_{W W}} *(1-f)+f^{*}\left(1-S_{a t_{N L}}\right)-S_{w t^{\prime}}^{P}$

\section{Actual Water and Heptane Saturations for Case (C) (Saturated NAPL-wet soil lens)}

Following the same analogy described in cases (A) and (B) and using the actual water and heptane saturations in a water-wet porous medium, the following equations are used to calculate the actual water and heptane saturations in case (C):

$$
\begin{aligned}
& S_{w_{w W-N L}}=S_{w_{w W}} *(1-f)+S_{w t^{\prime}}^{S} \\
& S_{n_{w W-N L}}=S_{n_{w W}} *(1-f)+f^{*}\left(1-S_{a t_{N L}}\right)-S_{w t^{\prime}}^{S}
\end{aligned}
$$




\subsection{4.d. Conceptual Model Validation for Water Main Imbibition Pathway}

It was shown in Sections 5.4.1, 5.4.4.b, and 5.4.4.c that the heptane/water main drainage and imbibition pathways in a water-wet porous medium with a NAPL-wet soil lens can be obtained as described in case (B). Figure 5.60, for uniform sand, and Figure 5.61, for well graded sand, show the water main imbibition pathways for the five porous media. As shown in each figure, as the NAPL-wet soil lens size increases, the initial and final water saturations on the main imbibition pathway decrease. It is noticed that the water main imbibition pathways for the five porous media are similar in shape but shifted.

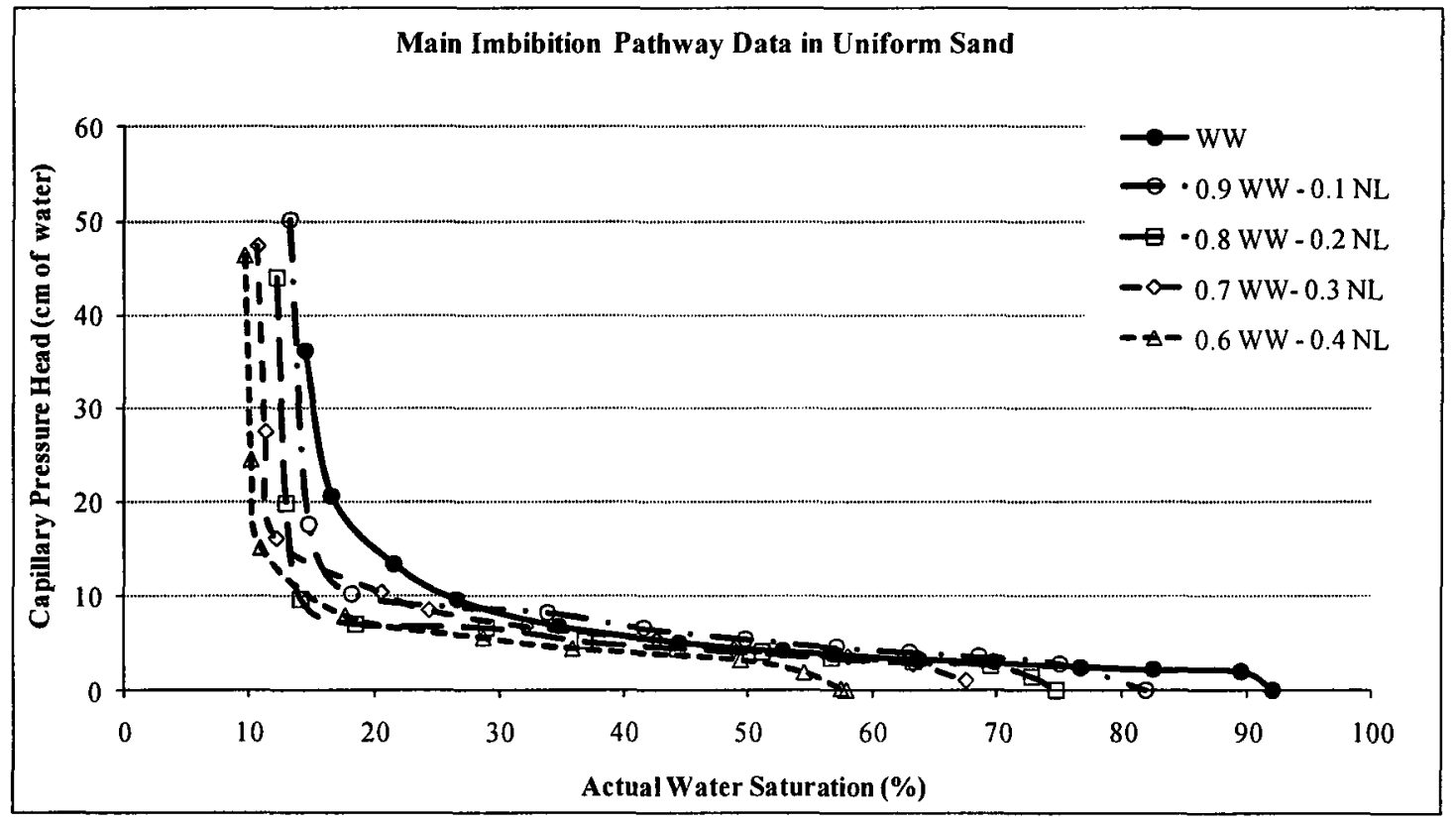

Figure 5.60: Capillary Pressure Head-Actual Water Saturation in Water-Wet Porous Medium and Water-Wet Porous Media with NAPL-Wet Soil Lenses in Uniform Sand. 


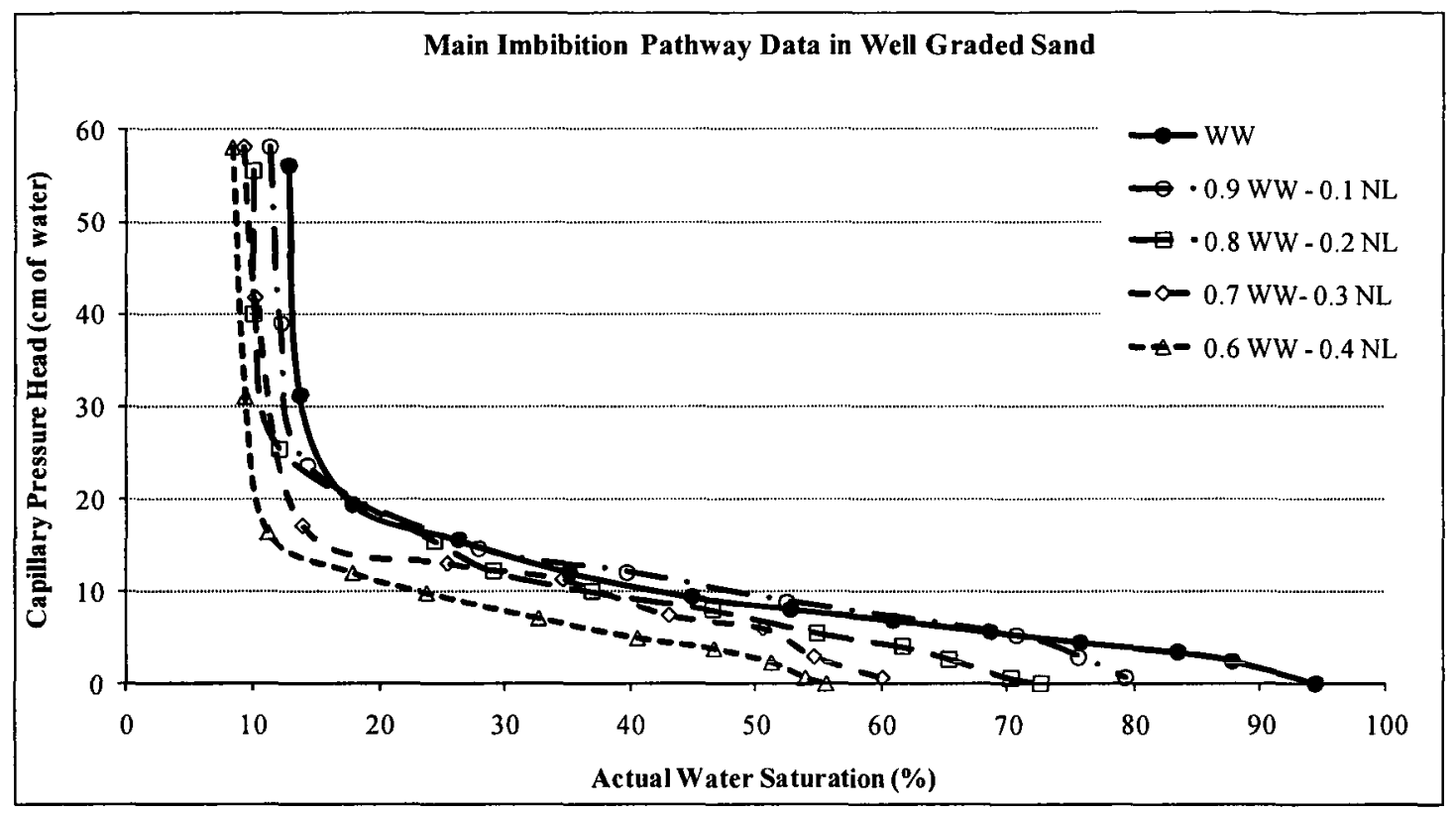

Figure 5.61: Capillary Pressure Head-Actual Water Saturation in Water-Wet Porous Medium and Water-Wet Porous Media with NAPL-Wet Soil Lenses in Well Graded Sand.

It was explained in Section 5.4.4.c that the main imbibition pathway for case (B) can be predicted by removing the NAPL-wet soil lens from the REV and considering only the water-wet soil fraction. It was described that when using the pore volume of this fraction in the water saturation calculations, one should obtain the same main imbibition pathway as in the water-wet porous medium. On the other hand, the water saturation in the NAPL-wet soil lens remains unchanged at an entrapped water saturation of $S_{w z^{\prime}}^{P}$. Therefore, in order to obtain the main imbibition pathway in a water-wet porous medium with a NAPL-wet soil lens, one should know the main imbibition pathway in a water-wet porous medium, the volume of the NAPL-wet soil lens in a given REV, and the entrapped water saturation in the NAPL-wet soil lens $\left(S_{w t^{\prime}}^{P}\right)$. Given these inputs, the 
conceptual model given in Section 5.4.4.c.II may be used to calculate the main imbibition pathway in a water-wet porous medium with a NAPL-wet soil lens.

In order to check the validity of this model, first, the entrapped water saturation, $S_{w t^{\prime}}^{P}$, in the NAPL-wet soil lens should be calculated. Second, that entrapped water saturation is subtracted from the water saturations of the main imbibition pathway in the water-wet porous medium with the NAPL-wet soil lens. The resulting water saturations are the water saturations in the water-wet soil fraction. If these water saturations are divided by $(1-f)$ and plotted versus the corresponding capillary pressure heads, the resulting water main imbibition pathway should be similar to the water main imbibition pathway in a water-wet porous medium. The entrapped water saturations in the NAPLwet soil lenses were given in Table 5.11, for uniform sand, and Table 5.12, for well graded sand. Figure 5.62, for uniform sand, and Figure 5.63, for well graded sand, show the capillary pressure head-actual water saturation in the water-wet porous media and the water-wet soil fractions for one test. Each figure shows that the data are in good agreement which validates the previously proposed model. 


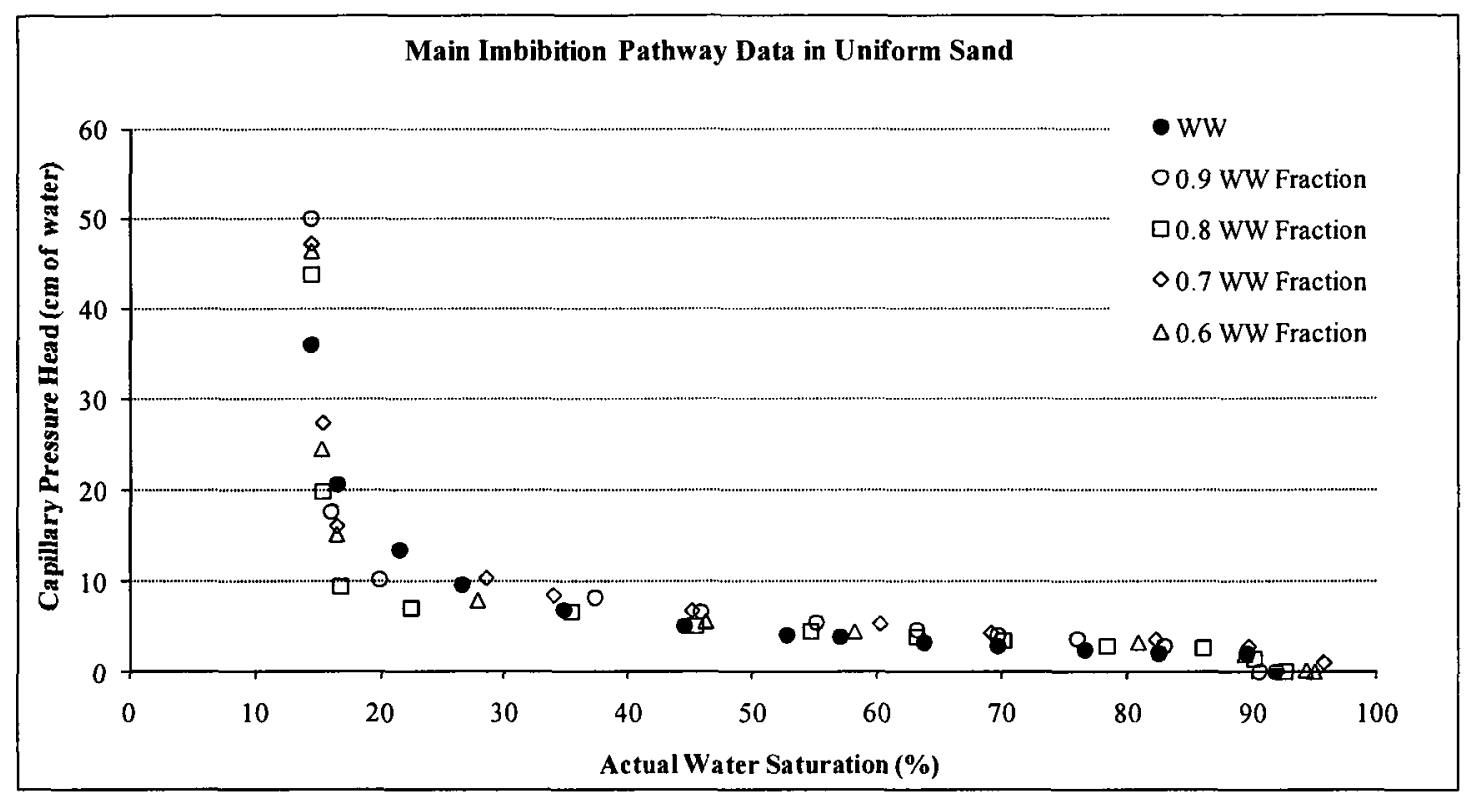

Figure 5.62: Capillary Pressure Head-Actual Water Saturation Data in Water-Wet Porous Medium and Water-Wet Soil Fractions in Uniform Sand.

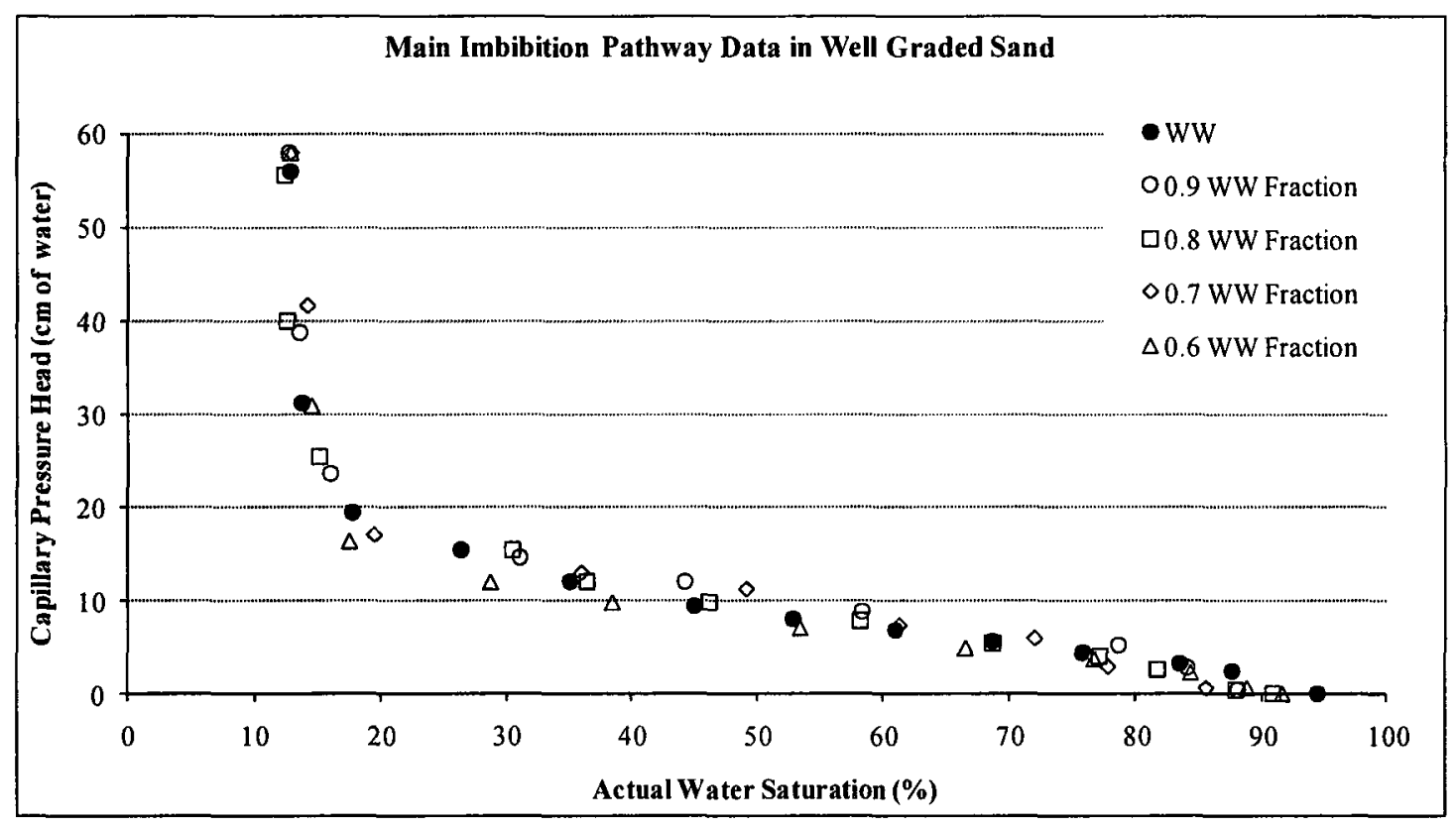

Figure 5.63: Capillary Pressure Head-Actual Water Saturation Data in Water-Wet Porous Medium and Water-Wet Soil Fractions in Well Graded Sand. 
Figure 5.64, for uniform sand, and Figure 5.65, for well graded sand, show the capillary pressure head-apparent water saturation data for the water-wet porous media and the water-wet soil fractions, for each test duplicates. Each figure also shows the fitted Van Genuchten curve based on the water-wet porous medium data (i.e., Fitted_WW), and the fitted Van Genuchten curve based on the water-wet porous medium and water-wet soil fractions data (i.e., Fitted_Pooled). It is noticed from Figure 5.64 and Figure 5.65 that the capillary pressure heads corresponding to apparent water saturations in the water-wet soil fractions are slightly higher than the capillary pressure heads corresponding to apparent water saturations based on the Fitted_WW relationship. These differences might be attributed to variations in soil placement and compaction and contamination. In order to accommodate these variations, the Van Genuchten Fitted_Pooled relationship was calculated for each sand type. Each figure shows that the Van Genuchten Fitted_Pooled curve and the Van Genuchten Fitted_WW curve are aligned. Therefore, this data validates the proposed conceptual model presented in Section 5.4.4.c.II. 


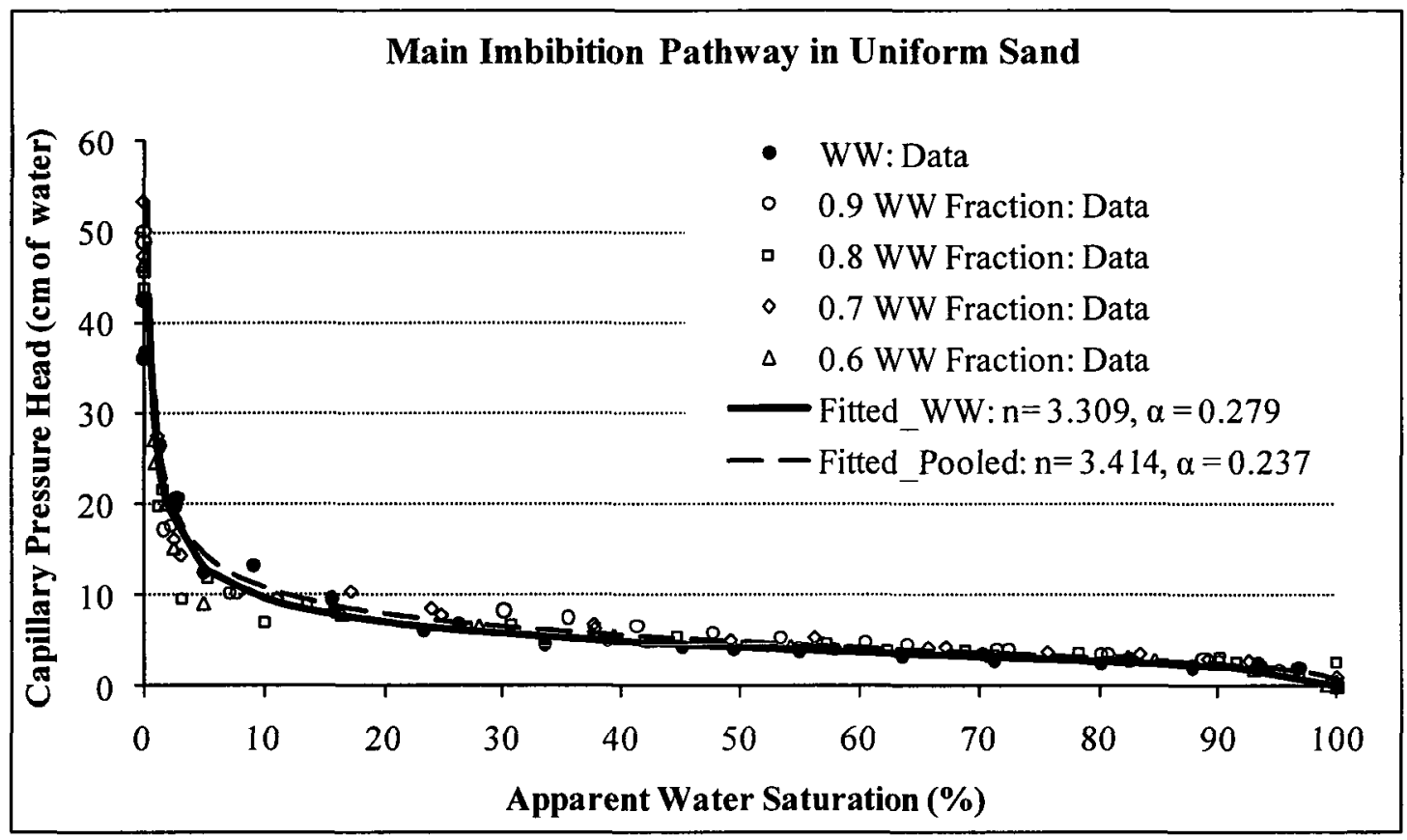

Figure 5.64: Capillary Pressure Head-Apparent Water Saturation in Water-Wet Porous Medium and Water-Wet Soil Fractions in Uniform Sand.

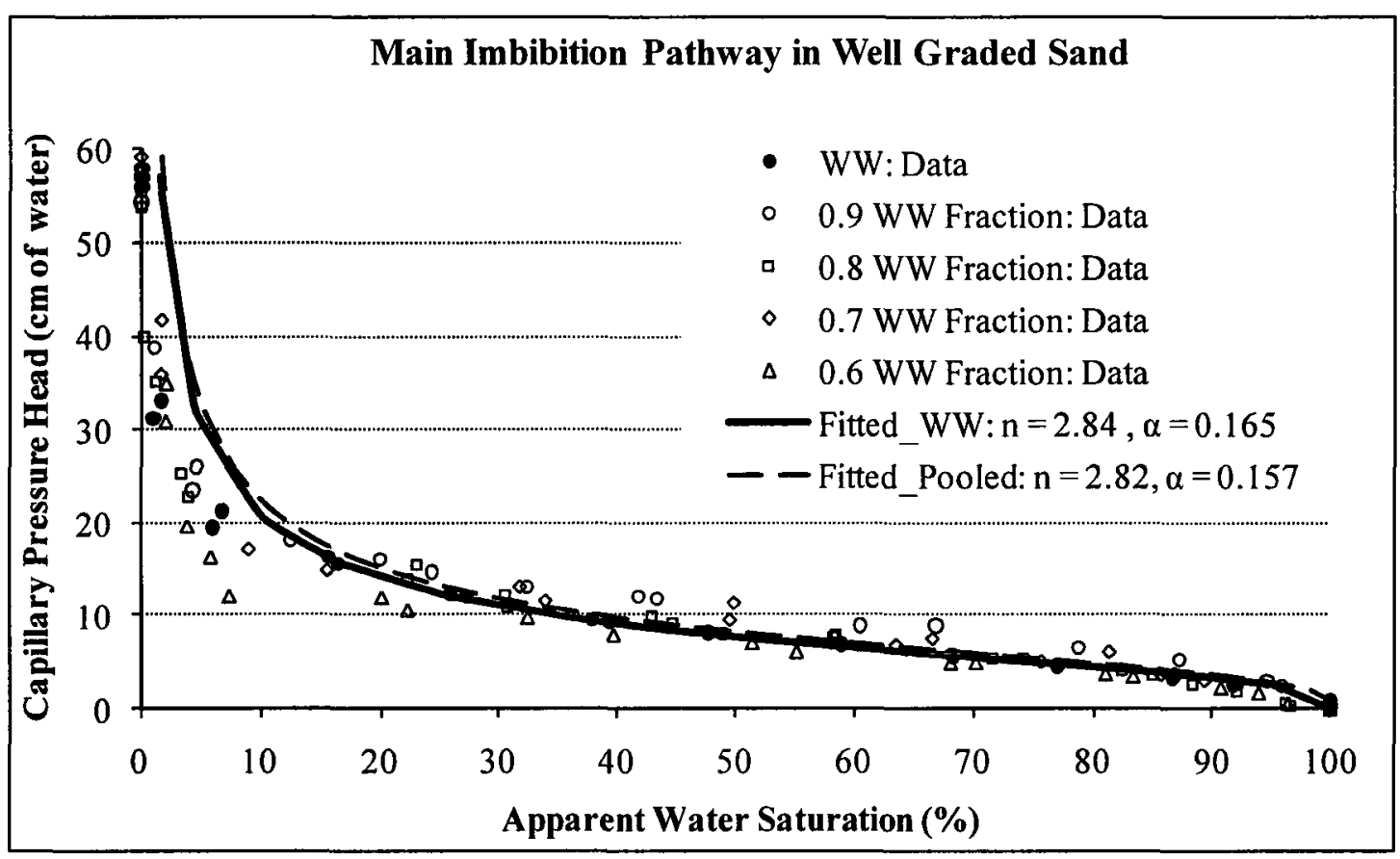

Figure 5.65: Capillary Pressure Head-Apparent Water Saturation in Water-Wet Porous Medium and Water-Wet Soil Fractions in Well Graded Sand. 


\subsection{Summary}

This chapter focused on the conceptual model development and validation for the $P_{c}-S$ relationships in a water-wet porous medium with a NAPL-wet soil lens for air/heptane, air/water, and heptane/water two-phase systems. It was shown that the NAPL-wet soil lens in a water-wet porous medium has no effect on the air/heptane $P_{c} \mathcal{S}$ relationship, since heptane is the wetting fluid in water-wet and NAPL-wet porous media. It was also noticed that the NAPL-wet soil lens does not affect the irreducible heptane or the entrapped air saturations in a porous medium. The NAPL-wet soil lens in a water-wet porous medium impacts the $P_{c}-S$ relationships in air/water and heptane/water two-phase systems. The conceptual models for air/water and heptane/water $P_{c}-S$ relationships depend on the size of the NAPL-wet soil lens, the water main imbibition and drainage pathways in the water-wet soil fraction and in the NAPL-wet soil lens, and the degree of water saturation in the NAPL-wet soil lens reached prior to initiating the water main drainage pathway. The NAPL-wet soil lens affects the irreducible water and entrapped air saturations in a porous medium for air/water two-phase system. Similarly, the NAPL-wet soil lens affects the irreducible and entrapped heptane saturations in a porous medium. 


\section{Chapter 6. Capillary Pressure-Saturation Relationships in Three-Phase Systems under Different Soil Wettability Configurations}

\subsection{Introduction}

This chapter is aimed to the development of conceptual models for the constitutive relationships for three-phase flow; specifically the capillary pressure-total liquid saturation and capillary pressure-water saturation in a water-wet porous medium with a NAPL-wet soil lens and to validate the Leverett assumption. The knowledge gained in the $P_{c} \neg$ relationships in two-phase systems in a water-wet porous medium with a NAPL-wet soil lens facilitates the development of these conceptual models. It was shown in Sections 5.3 and 5.4 of Chapter 5 that one can construct the $P_{c} \neg S$ relationships for an REV of a water-wet porous medium with a NAPL-wet soil lens based on the volume fraction of the NAPL-wet soil lens and the $P_{c}-S$ relationship for the water-wet and the NAPL-wet porous media. It is proposed in the following sections that a similar approach can be taken to develop the $P_{c} \neg S$ relationships for a three-phase system.

The flow in the vadose zone, if air, NAPL, and water are present, is a three-phase flow. In the vadose zone, the air is assumed to be infinitely mobile (i.e., the air pressure is zero) and the NAPL and water are typically under suction (i.e., water and NAPL pressures are lower than zero) unless perched groundwater or NAPL zones exist. It was shown in Section 5.4 of Chapter 5 that, if the upper limit of a water pressure is zero, the water saturation in a NAPL-wet porous medium (or NAPL-wet soil lens) should be zero or at an entrapped value. Figure 6.1 is a pore scale image in a water-wet soil fraction where water is the wetting phase, heptane is the non-wetting phase, and air is the least wetting phase. Figure 6.2 is a pore scale image in a NAPL-wet soil lens where heptane is 
the wetting phase, water is entrapped in heptane, and air is the non-wetting phase. Both figures show the air/heptane interface. Therefore, the flow in a NAPL-wet soil lens located in the vadose zone can be treated as a two-phase air/heptane system, and the flow in the neighbouring water-wet soil fraction is an air/heptane/water three-phase flow system.

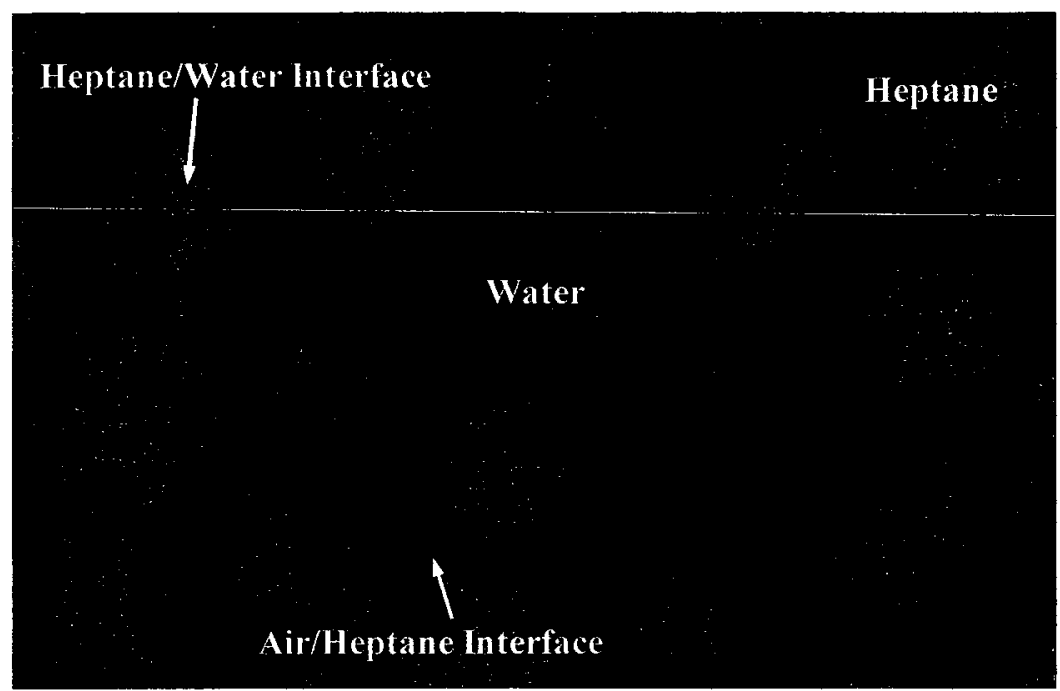

Figure 6.1: Water (Blue), Heptane (Light Red), and Air/Heptane Interface (Dark Line) in the Water-Wet Soil Fraction.

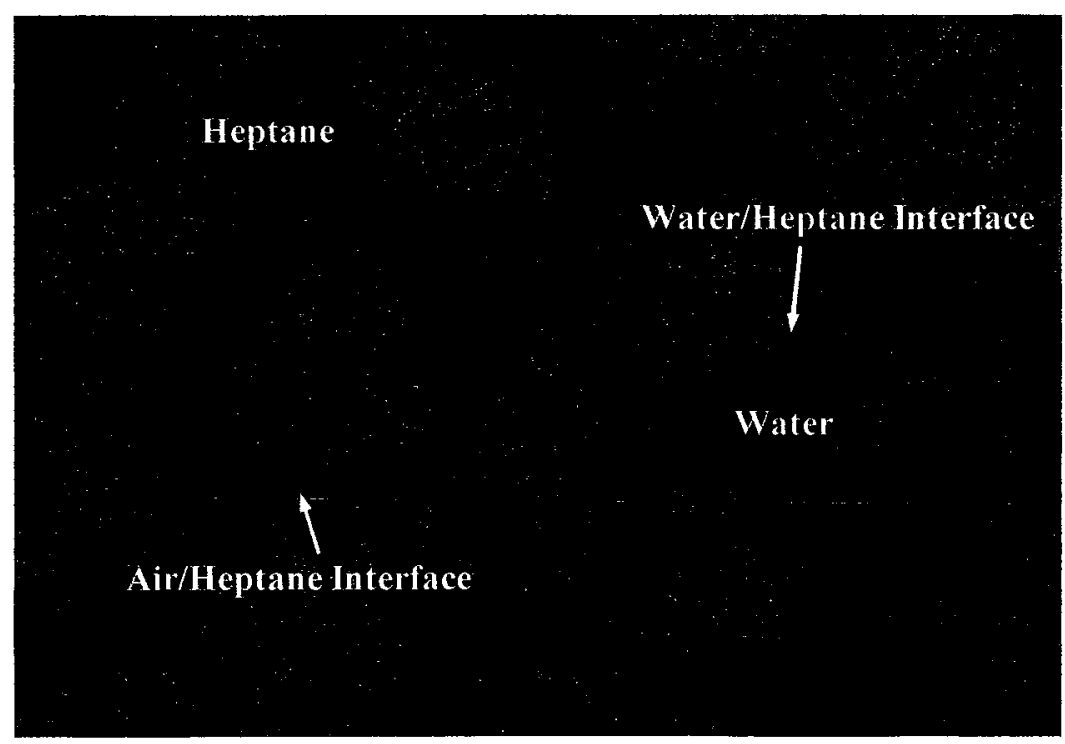

Figure 6.2: Heptane (Light Red), Entrapped Water (Blue), and Air/Heptane Interface (Dark Line) in the NAPL-Wet Soil Lens. 
The Leverett assumption, as described earlier in Chapter 2, states that $P_{c}-S$ relationships in a three-phase system may be obtained by considering the three-phase system as two two-phase systems. In this assumption, the total liquid saturation in a three-phase system is equivalent to the NAPL saturation in a similar air/NAPL two-phase system at the same air/NAPL capillary pressure. In addition, the water saturation in a three-phase system is equivalent to water saturation in a similar NAPL/water two-phase system at the same NAPL/water capillary pressure. The Leverett assumption is based on certain assumptions; the porous medium is rigid and strongly water wet, the fluid wettabilities follow the order water $>$ NAPL $>$ air, and the intermediate fluid (i.e., NAPL) forms a continuous layer spreading between the most wetting fluid (i.e., water) and the least wetting fluid (i.e., air). The latter assumption means that the air/water interface does not exist in the porous medium as long as the NAPL exists.

The total liquid saturation, in a water-wet porous medium with a NAPL-wet soil lens, might be obtained by considering the saturation pathways in the two soil fractions. The NAPL saturation pathway in the NAPL-wet soil lens should be similar to the NAPL saturation pathway in the air/NAPL two-phase system in a NAPL-wet porous medium, with the potential of entrapped air and water. The total liquid saturation in the water-wet soil fraction is the summation of the water and the NAPL saturations. If the Leverett assumption is applied in the water-wet soil fraction, the total liquid saturation pathway in the three-phase system should be equivalent to the NAPL saturation pathway in air/NAPL two-phase system in the water-wet porous medium. The relationship between the saturations in the two soil fractions along the drainage and imbibition pathways is discussed in Section 6.2. 


\subsection{Conceptual Model for Air/NAPL Capillary Pressure-Total Liquid Saturation Relationship in a Water-Wet Porous Medium with a NAPL-Wet Soil Lens}

Leverett states that, in a water-wet porous medium, the air/NAPL capillary pressure-total liquid saturation in a three-phase system is equivalent to the air/NAPL capillary pressure-NAPL saturation in an air/NAPL two-phase system. This assumption is based on the premise that the capillary pressure on the air/NAPL interface in a waterwet porous medium is a function of the liquid saturation behind that interface. The capillary pressure on the air/NAPL interface is the negative of the NAPL pressure. The liquid saturation behind the interface is the summation of the NAPL and water saturations.

In multiphase flow, at steady state conditions, a fluid phase pressure should be invariable over the REV. Therefore, in the water-wet porous medium with the NAPL-wet soil lens, the NAPL pressure in the water-wet soil fraction should be similar to the NAPL pressure in the NAPL-wet soil lens. This implies that, at a given NAPL pressure, the total liquid saturation in the water-wet soil fraction and the NAPL saturation in the NAPL-wet soil lens can be determined based on the air/NAPL interface, even though the water and NAPL saturations within the water-wet soil fraction and the NAPL-wet soil lens differ. In the REV, if the water saturation is known and the NAPL is allowed to drain (i.e., as the air/NAPL capillary pressure increases) from the porous medium, the total liquid saturation in the water-wet soil fraction may reach a lower limit equal to the summation of the water and the residual NAPL saturations. At this lower limit which is defined here as the total liquid saturation lower limit (TLSLL), the capillary pressure on the main drainage pathway is called the critical capillary pressure (CCP). 
Along the main drainage pathway, the total liquid saturation in the water-wet soil fraction and in the NAPL-wet soil lens starts at unity as shown in Figure 6.3 and Figure 6.4. As capillary pressure increases, the total liquid saturation in the water-wet soil fraction decreases and follows a pathway dependent on its grain size distribution and the NAPL type, as shown in Figure 6.3, until it reaches the TLSLL $\left(\overline{\bar{S}}_{w}+\bar{S}_{r}\right.$, where $\bar{S}_{r}$ is the effective residual NAPL saturation) in the water-wet soil fraction at the CCP (i.e., at point b). In contrast, in the NAPL-wet soil lens, as capillary pressure increases due to increased suction in the NAPL, the NAPL saturation decreases and two scenarios should be considered. Scenario (1) refers to an isolated (i.e., not connected to other NAPL-wet soil lenses or to the NAPL reservoir) NAPL-wet soil lens. Scenario (2) refers to a NAPLwet soil lens connected to other NAPL-wet soil lenses or a NAPL reservoir.

In both scenarios, a fluid saturation pathway in the REV is the summation of the scaled fluid saturation pathways in the water-wet soil fraction and in the NAPL-wet soil lens, respectively. In addition, it is important to recognize that the flow in the water-wet soil fraction is three-phase flow (i.e., air/NAPL/water), while the flow in the NAPL-wet soil lens is two-phase flow (i.e., air/NAPL). Sections 6.2.1 and 6.2.2 describe the fluid saturation pathways in both the water-wet soil fraction and NAPL-wet soil lens for isolated and connected NAPL-wet soil lenses, respectively. By keeping track of these pathways for each fluid saturation pathway, the following equations might be used to calculate water, NAPL, and air saturations in the REV:

$$
\begin{aligned}
& S_{w}=S_{w_{W W F}} *(1-f)+S_{w_{N L}} * f \\
& S_{n}=S_{n_{W W F}} *(1-f)+S_{n_{N L}} * f
\end{aligned}
$$


$S_{a}=1-\left(S_{w}+S_{n}\right)$

Where:

$S_{w_{W W F}}:$ actual water saturation in the water-wet soil fraction;

$S_{w_{N L}}$ : actual water saturation in the NAPL-wet soil lens;

$S_{n_{W W F}}:$ actual NAPL saturation in the water-wet soil fraction; and

$S_{n_{N L}}:$ actual NAPL saturation in the NAPL-wet soil lens.

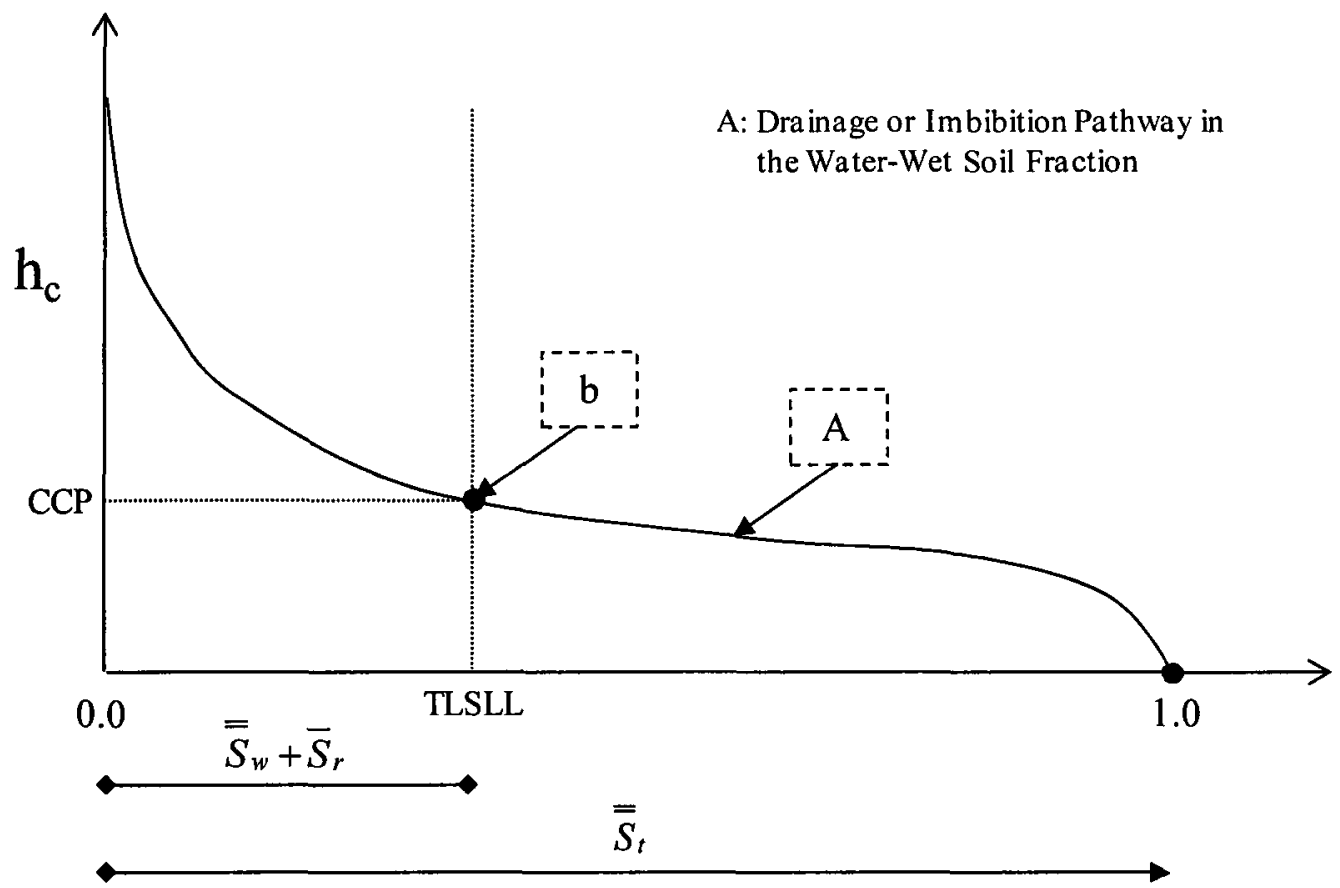

Figure 6.3: Capillary Pressure Head-Apparent Total Liquid Saturation Pathway in the Water-wet Soil Fraction. 


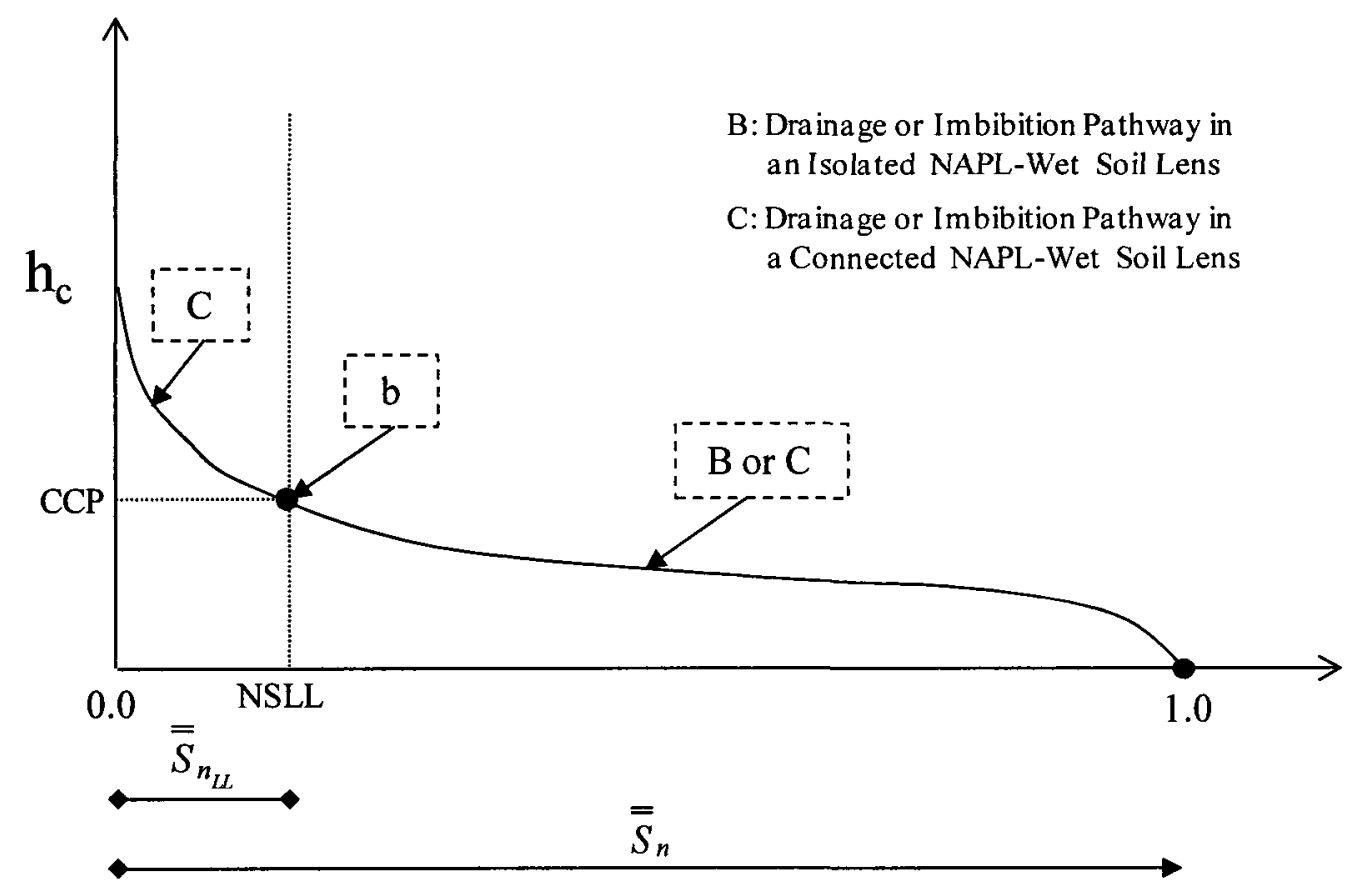

Figure 6.4: Capillary Pressure Head-Apparent NAPL Saturation Pathway in an Isolated or a Connected NAPL-wet Soil Lens.

\subsubsection{Scenario (1): Isolated NAPL-Wet Soil Lens of a Grain Size Distribution Different than the Water-Wet Soil Fraction}

The total liquid saturation main drainage pathway in the water-wet soil fraction follows the pathway A shown in Figure 6.3. If water saturation remains constant and the total liquid saturation in the water-wet soil fraction reaches the TLSLL, due to NAPL drainage; then the apparent total liquid saturation in the water-wet soil fraction becomes fixed at the TLSLL at point b, shown in Figure 6.3. The apparent TLSLL in the waterwet soil fraction corresponds to a CCP and it is equivalent to $\overline{\bar{S}}_{w}+\bar{S}_{r}$. In contrast, the NAPL saturation main drainage pathway in the isolated NAPL-wet soil lens follows the pathway B shown in Figure 6.4 until it reaches a lower limit of the NAPL saturation 
(NSLL) at point $b$. The apparent NSLL corresponds to the CCP and it is equivalent to $\overline{\bar{S}}_{n_{L}}$. For the NAPL saturation in the NAPL-wet soil lens to decrease, the water saturation in the water-wet soil fraction must decrease causing the $\mathrm{CCP}$ to increase. Assuming the water saturation in the water-wet soil fraction remains constant, the total liquid saturation imbibition pathways in the water-wet and NAPL-wet soil fractions start at the TLSLL and NSLL, respectively. As NAPL imbibes into the porous medium, it distributes itself such that the capillary pressure in the water-wet and NAPL-wet soil fractions have the same magnitude. Therefore, for an isolated NAPL-wet soil lens the total liquid saturation drainage or imbibition pathways, at constant water saturation in the water-wet soil fraction, should be similar to the drainage and imbibition pathways in a water-wet porous medium, at the same water saturation, respectively. In addition, the NAPL saturation drainage or imbibition pathways in the NAPL-wet soil lens should be similar to the drainage and imbibition pathways in a NAPL-wet porous medium, respectively.

If water is allowed to drain, the TLSLL decreases and the CCP increases. It is important to mention that the lowest NAPL saturation in the water-wet soil fraction is the residual NAPL saturation in a water-wet porous medium. This residual NAPL saturation, as will be discussed in Chapter 7, depends on water and NAPL saturations. If the NAPL is not allowed to drain or the rate of water drainage is larger than the rate of NAPL drainage, NAPL first flows from the NAPL-wet soil lens to the water-wet soil fraction to achieve an invariable capillary pressure in the REV and then, if allowed, NAPL drains from the porous medium. If NAPL drains from the porous medium, it drains until the total liquid and NAPL saturations in the water-wet and NAPL-wet soil fractions reach the 
TLSLL and NSLL, respectively. While, if NAPL imbibes into the porous medium, NAPL will distribute itself between the water-wet and NAPL-wet soil fractions such that the capillary pressure is the same in both fractions.

If water imbibes into the porous medium, the CCP decreases. In this case, if the NAPL is not allowed to imbibe or the rate of water imbibition is larger than the rate of NAPL imbibition, NAPL flows from the water-wet soil fraction to the NAPL-wet soil lens until the TLSLL is reached. If the TLSLL is reached, the relative permeability of the NAPL in the water-wet soil fraction surrounding the NAPL-wet soil lens is zero and the NAPL in the NAPL-wet soil lens is trapped at a saturation equal to or less than the NSLL. While, if the NAPL drains from the porous medium, the NAPL first flows from the water-wet soil fraction to the NAPL-wet soil lens such that the capillary pressure is invariable in the REV. Then NAPL might drain from the porous medium until the NAPL saturation in the NAPL-wet soil lens reaches the NSLL, after which, NAPL will not drain.

\subsubsection{Scenario (2): Connected NAPL-Wet Soil Lens of a Grain Size Distribution Different than the Water-Wet Soil Fraction}

The total liquid saturation main drainage pathway in the water-wet soil fraction follows the pathway A, shown in Figure 6.3. Hence, for a known water saturation, the lowest total liquid saturation in the water-wet soil fraction is the TLSLL (i.e., at point b in Figure 6.3), which corresponds to a CCP. In contrast, the NAPL saturation main drainage pathway in the NAPL-wet soil lens follows the pathway $C$ shown in Figure 6.4 because the NAPL-wet soil lenses are connected. This implies that the lowest NAPL saturation in 
the NAPL-wet soil lens is the irreducible NAPL saturation in a NAPL-wet porous medium.

If water saturation remains constant and the air/NAPL capillary pressure increases above the CCP on the main drainage pathway, the total liquid saturation in the water-wet soil fraction remains fixed at the TLSLL (point $b$ in Figure 6.3). But the NAPL saturation in the NAPL-wet soil lens decreases such that it follows the two-phase air/NAPL main drainage pathway (case $\mathrm{C}$ in Figure 6.4). If the air/NAPL capillary pressure in the NAPL-wet soil lens is lower than the CCP, the NAPL pressure is continuous between the two fractions. If the air/NAPL capillary pressure in the NAPLwet soil lens exceeds the CCP, the water-wet soil fraction will follow an air/water system with a residual NAPL and the NAPL-wet soil lens will follow an air/NAPL system with entrapped air and water.

If water is allowed to drain, the TLSLL decreases and the CCP increases. In this case, if the NAPL is not allowed to drain or the rate of water drainage is larger than the rate of NAPL drainage, NAPL first flows from the NAPL-wet soil lens into the water-wet soil fraction until the capillary pressure is the same in the REV and then, if allowed, NAPL drains from the REV. If the NAPL drains at a rate larger than water drainage, NAPL will not flow from the NAPL-wet soil lens into the water-wet soil fraction but it will drain from the REV until the NAPL reaches residual and irreducible saturations in the water-wet and NAPL-wet soil fractions, respectively. While, if NAPL imbibes into the porous medium, NAPL first imbibes into the NAPL-wet soil lens until the air/NAPL capillary pressure reaches the CCP, then, NAPL distributes itself between the water-wet 
soil fraction and the NAPL-wet soil lens such that the air/NAPL capillary pressure is invariable throughout the REV.

If water imbibes into the porous medium, the $\mathrm{CCP}$ decreases. In this case, if the NAPL is not allowed to imbibe or the rate of water imbibition is larger than the rate of NAPL imbibition, NAPL flows from the water-wet soil fraction to the NAPL-wet soil lens until the air/NAPL capillary pressure is invariable throughout the REV or the NAPL saturation in the NAPL-wet soil lens is less than or at the NSLL and then, if allowed, NAPL imbibes into the NAPL-wet soil lens or into the REV. While, if the NAPL drains from the porous medium, the NAPL first flows from the water-wet soil fraction to the NAPL-wet soil lens, then NAPL drains from the NAPL-wet soil lens until the NAPL saturation in the NAPL-wet soil lens reaches the irreducible NAPL saturation.

\subsection{2.a. Connected NAPL-Wet Soil Lens of a Grain Size Distribution Similar to the Water-Wet Soil Fraction}

A special case of scenario (2) is when the water-wet and NAPL-wet soil fractions have similar grain size distributions. In this scenario, the TLSLL and the NSLL are equivalent. The total liquid saturation main drainage and imbibition pathways for the water-wet and the NAPL-wet soil fractions are shown in Figure 6.5 as pathways D and E, respectively. These pathways are aligned for the total liquid saturations (or the NAPL saturations) in the NAPL-wet soil lens larger than the TLSLL. This is defined by the part of the pathways $\mathrm{D}$ and $\mathrm{E}$ on the right side of point $\mathrm{b}$, shown in Figure 6.5. At point $\mathrm{b}$, if air/NAPL capillary pressure increases, the total liquid saturation in the NAPL-wet soil lens continues to decrease until it reaches the irreducible NAPL saturation. The difference between the total liquid saturation in the water-wet soil fraction and the NAPL 
saturation in the NAPL-wet soil lens is called the NAPL saturation deficit. The NAPL saturation deficit is zero when the NAPL saturation in the NAPL-wet soil lens equals to the total liquid saturation in the water-wet soil fraction. As the NAPL saturation in the NAPL-wet soil lens decreases, the NAPL saturation deficit increases. The NAPL saturation deficit reaches a maximum value when the NAPL saturation in the NAPL-wet soil lens reaches irreducible NAPL saturation.

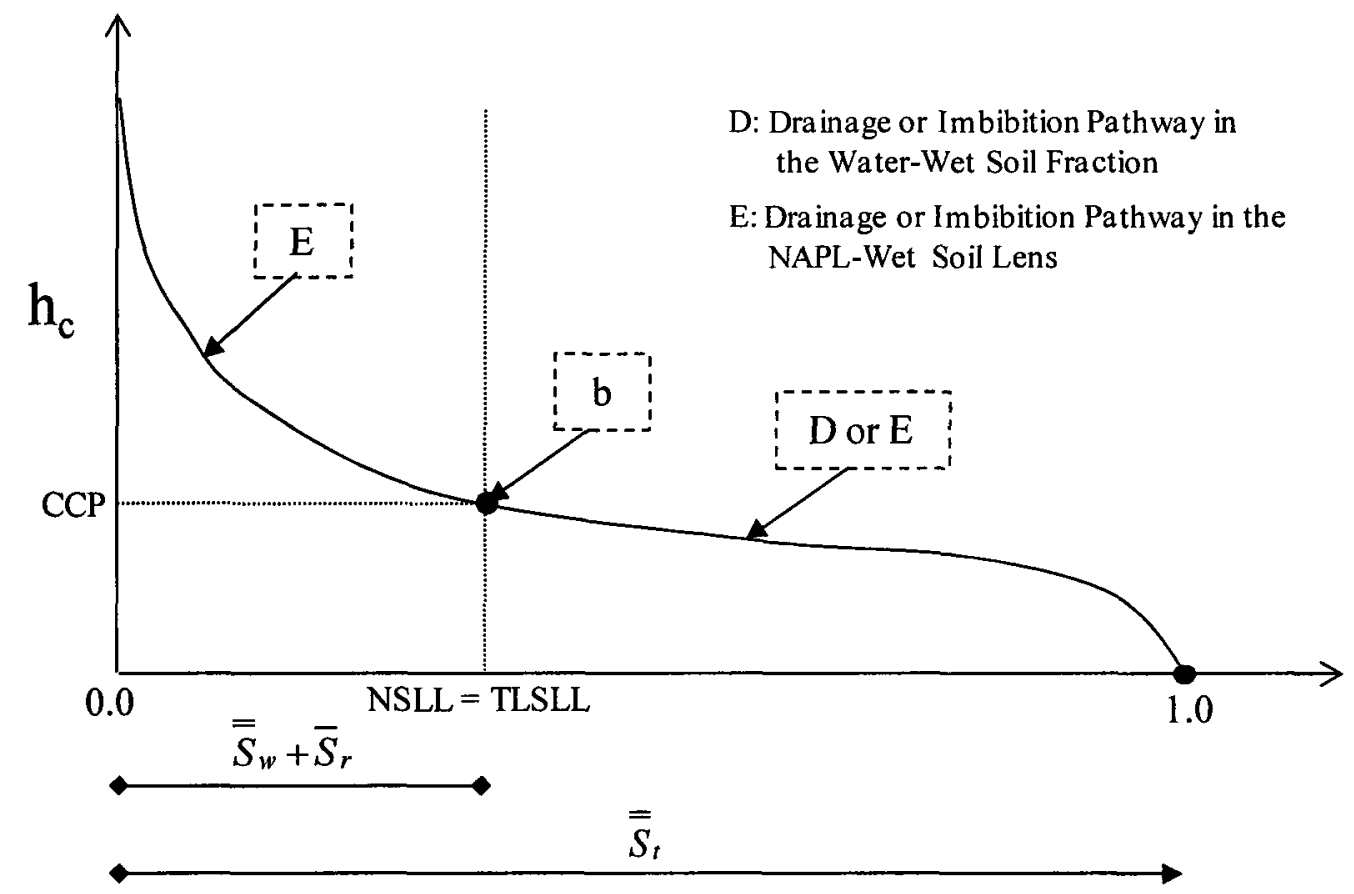

Figure 6.5: Capillary Pressure Head-Apparent Total Liquid Saturation Pathways in the Water-Wet Soil Fraction and in the Connected NAPL-Wet Soil Lens.

On the other hand, as the air/NAPL capillary pressure decreases, the NAPL imbibes into the NAPL-wet soil lens. As the NAPL imbibes into the NAPL-wet soil lens it reduces the NAPL saturation deficit and entraps air. At an air/NAPL capillary pressure, the apparent NAPL saturation in the NAPL-wet soil lens equals to the apparent NAPL 
saturation on the NAPL imbibition pathway in the air/ NAPL two-phase system. The NAPL essentially imbibes into the NAPL-wet soil lens until the apparent NAPL saturation in the NAPL-wet soil lens equals to the apparent TLSLL in the water-wet soil fraction, which is at the CCP and where the NAPL saturation deficit becomes zero. At this point, if the capillary pressure is decreased further, the NAPL imbibes into the entire REV. As the NAPL imbibes, it entraps air in the water-wet soil fraction and in the NAPL-wet soil lens. The NAPL imbibition pathway in the water-wet porous medium with the NAPL-wet soil lens follows the air/NAPL capillary pressure-apparent NAPL saturation imbibition pathway in the air/NAPL two-phase system.

Therefore, in a water-wet porous medium with a NAPL-wet soil lens, the Leverett assumption is valid for the total liquid saturation drainage pathway as long as the total liquid saturation is larger than the TLSLL or when the NAPL saturation deficit is zero. Similarly, the Leverett assumption is valid for the total liquid saturation imbibition pathway over the same total liquid saturation range.

The proposed conceptual model, for a connected NAPL-wet soil lens, states that Leverett assumption can be applied in a water-wet porous medium with a NAPL-wet soil lens as long as the CCP is not exceeded. After the CCP, the two soil fractions follow independent saturation paths. As the capillary pressure increases the total liquid saturation (or the NAPL saturation) in the NAPL-wet soil lens decreases. The NAPL drainage or imbibition pathway in the NAPL-wet soil lens should be equivalent to the NAPL drainage or imbibition pathway in an air/NAPL two-phase system, respectively. 


\subsubsection{Conceptual Model Validation for a Water-Wet Porous Medium with a Connected NAPL-Wet Soil Lens of Similar Grain Size Distribution}

In order to check the validity of the proposed conceptual model, capillary pressure-total liquid saturation experiments were conducted in a water-wet porous medium and in a porous medium that consisted of $60 \%$ water-wet soil fraction and $40 \%$ NAPL-wet soil lens. In these experiments, the water-wet and the NAPL-wet soil fractions had similar grain size distributions. In addition, water saturation was fixed at an initial water saturation as NAPL (i.e., heptane) drained from and imbibed into the porous medium. The experiments were conducted at two different initial water saturations. The first was conducted at the lowest initial water saturation (corresponding to irreducible water saturation in the water-wet soil fraction) while the second was conducted at an initial REV water saturation of $45 \%$. These experiments were conducted in the modified Tempe cells. Three transducers were connected to each cell; two heptane transducers and one water transducer. A heptane transducer refers to a transducer connected to a NAPLwet porous ceramic cup; the transducer cavity and the porous ceramic cup were filled with heptane. A water transducer refers to a transducer connected to a water-wet porous ceramic cup; the transducer cavity and the porous ceramic cup were filled with water. A transducer was connected to a cell such that it measured a liquid phase pressure head at an REV mid height. In a water-wet porous medium experiment, the two heptane transducers measured the heptane pressure head at two locations in the porous medium. In a water-wet porous medium with a NAPL-wet soil lens experiment, one heptane transducer measured the heptane pressure head in the water-wet soil fraction and the second heptane transducer measured the heptane pressure head in the NAPL-wet soil 
lens. The water transducer was connected to a cell such that it measured the water pressure head in the water-wet soil fraction. The transducers were connected to a data acquisition system.

To set up an experiment, as explained previously in Chapter 3, three transducers were connected to their corresponding cell after which the soil sample was placed. Then a NAPL-wet porous cup connected to a heptane burette was connected to the upper aluminum plate. Using an O-ring and four bolts, the upper aluminum plate was connected to the cell. The soil sample was then flushed with carbon dioxide for fifteen minutes through the water-wet ceramic plate followed by at least three pore volumes of water under positive water pressure. Then the cavity in the upper aluminum plate was filled with heptane. Thereafter, the water was drained and replaced by heptane until the desired initial water saturation was reached. During this stage, the total liquid saturation in the porous medium was unity. In the water-wet porous medium experiments, the water and heptane saturations were uniform throughout the medium. In the water-wet soil fraction with the NAPL-wet soil lens, the water and heptane saturations in the water-wet soil fraction were different than those in the NAPL-wet soil lens.

At a total liquid saturation of unity, in a water-wet porous medium with a NAPLwet soil lens, as explained in Section 5.4 of Chapter 5, it is known that the NAPL-wet soil lens is apparently heptane-saturated with water and gas entrapped. The entrapped water saturation is very small and may be ignored to facilitate this analysis. As mentioned above, two types of experiments, with respect to the initial water saturation, were conducted. The first type was conducted at lowest initial water saturation. The second type was conducted at initial water saturation of $45 \%$. In the first type, the water was 
drained and replaced by heptane until the lowest initial water saturation was reached. At this point, the water saturation in the NAPL-wet soil lens was considered zero and the water saturation in the water-wet soil fraction was equivalent to the irreducible water saturation. In the second type, the water saturation in the NAPL-wet soil lens was considered zero and hence the water saturation in the water-wet soil fraction was $75 \%$ to provide an REV water saturation of $45 \%$. In order to start a total liquid saturation drainage pathway, the heptane burette was lowered in increments. During each increment, heptane drained from the porous medium and was collected in the burette until the heptane pressure head in the REV equilibrated with the external heptane pressure head in the burette. The heptane pressure heads at two locations in the porous medium were measured by the heptane transducers. In order to start a total liquid saturation imbibition pathway, the heptane burette was raised in increments. During each increment, the heptane imbibed into the porous medium from the burette until the heptane pressure head in the REV equilibrated with the external heptane pressure head in the burette. Although it was found that two hours were enough to achieve such equilibrium, the heptane burette was lowered and raised every four hours. Pertaining to a fixed heptane/water capillary pressure head on the heptane/water interface, the water pressure head decreased when the heptane burette was lowered, and the water pressure head increased when the heptane burette was raised.

The first objective was to check that the Leverett assumption is valid if the heptane saturation deficit is zero. In order to do that, the residual heptane saturation and the initial water saturation were calculated and the TLSLL was calculated (as listed in Table 6.1). Then, the total liquid saturation drainage and imbibition pathway data points 
in the air/heptane/water three-phase system were plotted, in the same graph, with the heptane saturation drainage and imbibition pathways in air/heptane two-phase system, respectively. The total liquid saturation drainage (or imbibition) pathway data points should align the heptane saturation drainage (or imbibition) pathway in air/heptane twophase system as long as the heptane saturation deficit is zero.

The second objective was to check that the heptane saturation main drainage and imbibition pathways in the NAPL-wet soil lens are equivalent to the heptane saturation main drainage and imbibition pathways in the air/heptane two-phase system, respectively. The heptane main drainage and imbibition pathways in the NAPL-wet soil lens started at $100 \%$ apparent heptane saturations and ended at irreducible and zero actual and apparent heptane saturations, respectively.

Along the heptane main drainage pathway in the NAPL-wet soil lens, the heptane volume decreased as the capillary pressure head increased. At capillary pressure heads lower than or equal to the CCP, which corresponds to the TLSLL, for every incremental reduction in heptane volume in the water-wet porous medium with the NAPL-wet soil lens, $40 \%$ of this heptane volume reduction was due to heptane drainage from the NAPLwet soil lens and $60 \%$ was due to heptane drainage from the water-wet soil fraction, as expressed in Equation 6.2. At capillary pressure heads higher than the CCP, the incremental reduction in heptane volume was due to heptane drainage from the NAPLwet soil lens. Therefore, the heptane volume in the REV was calculated at the CCP or at the TLSLL. Then, the heptane volume and saturation in the NAPL-wet soil lens were recalculated at capillary pressure heads lower than, equal to, and higher than the CCP. Thereafter, the heptane saturation main drainage pathway data points in the NAPL-wet 
soil lens were plotted in the same graph with the heptane saturation main drainage pathway in the air/heptane two-phase system. If the conceptual model is correct, the experimental data points should follow the heptane saturation main drainage pathway in the air/heptane two-phase system.

The heptane saturation main imbibition pathway data points were obtained in a similar manner. As air/heptane capillary pressure decreased, the heptane imbibed only into the NAPL-wet soil lens. As heptane imbibed, it entrapped air. In the water-wet soil fraction, the total liquid saturation remained fixed at the TLSLL. This was the case until the apparent total liquid saturation in the NAPL-wet soil lens was equivalent to the apparent TLSLL. At this point, as capillary pressure decreased, the heptane imbibed into the water-wet and NAPL-wet soil fractions such that $40 \%$ of the imbibing heptane volume went to the NAPL-wet soil lens and $60 \%$ went to the water-wet soil fraction. The conceptual model was validated by comparing the results for the heptane saturation imbibition pathway.

Table 6.1: Residual Heptane and Initial Water Saturations, and TLSLL.

\begin{tabular}{|c|c|c|c|c|c|c|}
\hline & & & $S_{r}$ & $S_{w_{W W F}}$ & $S_{w_{N L}}$ & $S_{T L L}$ \\
\hline \multirow{4}{*}{3} & Test (1) & $S_{w^{v}}=S_{m}=13.6$ & 8.3 & 13.6 & 0.0 & N.A. \\
\hline & Test (2) & $S_{w}=S_{m}=17.4$ & 11.6 & 17.4 & 0.0 & N.A. \\
\hline & Test (1) & $S_{w}=45.0$ & 5.3 & 45.0 & 0.0 & N.A. \\
\hline & Test (2) & $S_{w}=45.0$ & 8.0 & 45.0 & 0.0 & N.A. \\
\hline \multirow{4}{*}{$\frac{\sum_{x}^{2}}{2}$} & Test (1) & $S_{w}=S_{l w}=15.1$ & 21.4 & 25.2 & 0.0 & 46.6 \\
\hline & Test (2) & $S_{w}=S_{b v}=22.9$ & 25.5 & 38.1 & 0.0 & 63.6 \\
\hline & Test (1) & $S_{w}=45.0$ & 16.8 & 75.0 & 0.0 & 91.8 \\
\hline & Test (2) & $S_{w}=45.0$ & 14.5 & 75.0 & 0.0 & 89.5 \\
\hline
\end{tabular}


Where:

WW: water-wet porous medium;

WWF/NL: $60 \%$ water-wet soil fraction-40\% NAPL-wet soil lens;

$S_{l w}$ : lowest initial water saturation in the water-wet porous medium with the NAPL-wet soil lens; and

$S_{T L L}$ : actual total liquid saturation lower limit.

\subsection{3.a. Conceptual Model Validation for the Main Drainage Pathways}

\section{Air/Heptane Capillary Pressure Head-Total Liquid Saturation at Lowest Initial} Water Saturation

Figure 6.6 shows the predicted Van Genuchten capillary pressure head-actual heptane saturation curve in air/heptane two-phase system and the capillary pressure head-actual total liquid saturation data for two tests, in the three-phase system, in a well graded water-wet porous medium at irreducible water saturation. Figure 6.7 shows the corresponding data plotted as apparent saturations. As shown in Figure 6.6 and Figure 6.7, apart from the data for the low total liquid saturations, the data points fit the predicted Van Genuchten heptane saturation main drainage pathway for the air/heptane two-phase system. In the remainder of this section, for convenience, the latter will be replaced by the predicted drainage pathway. 


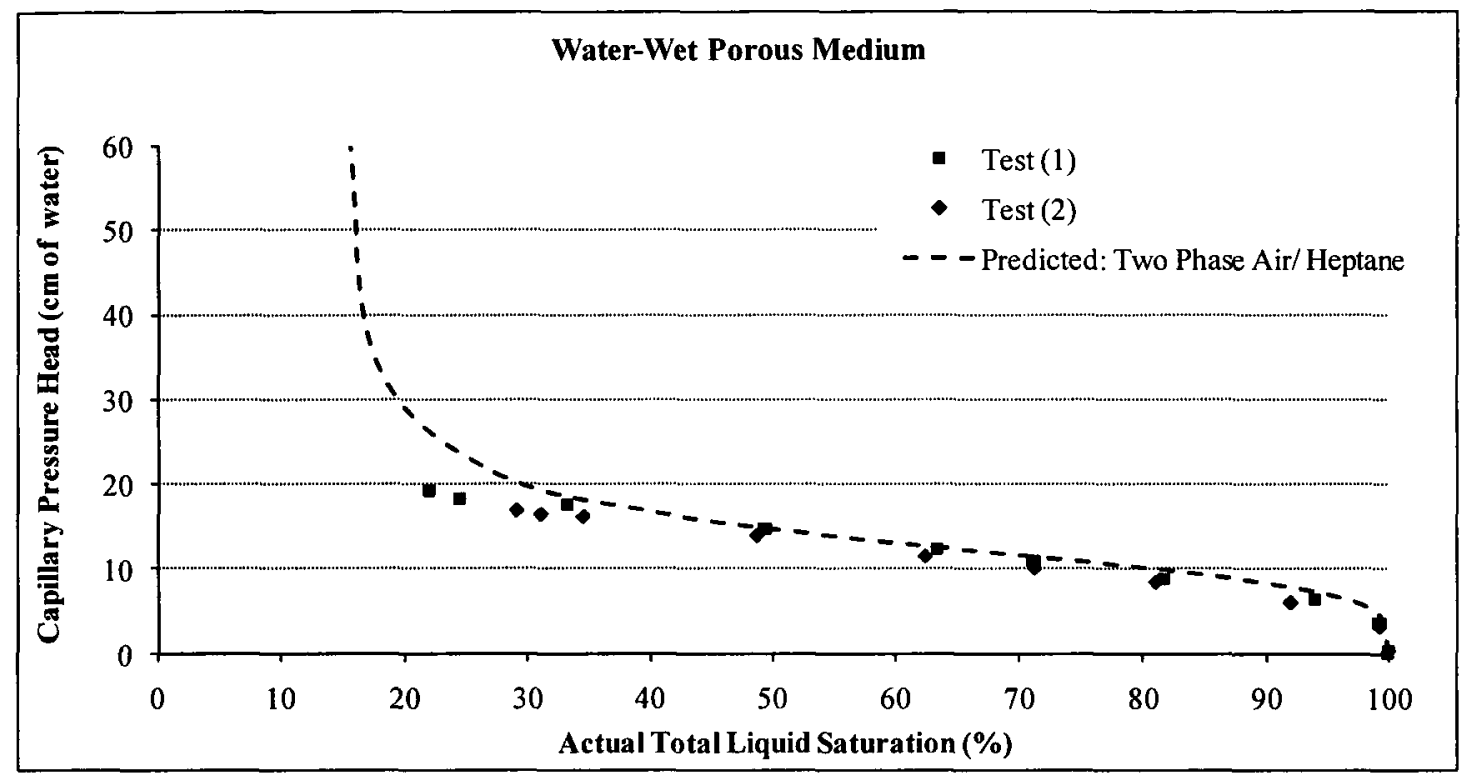

Figure 6.6: Air/Heptane Capillary Pressure Head-Actual Total Liquid Saturation Main Drainage Pathway at Irreducible Water Saturation.

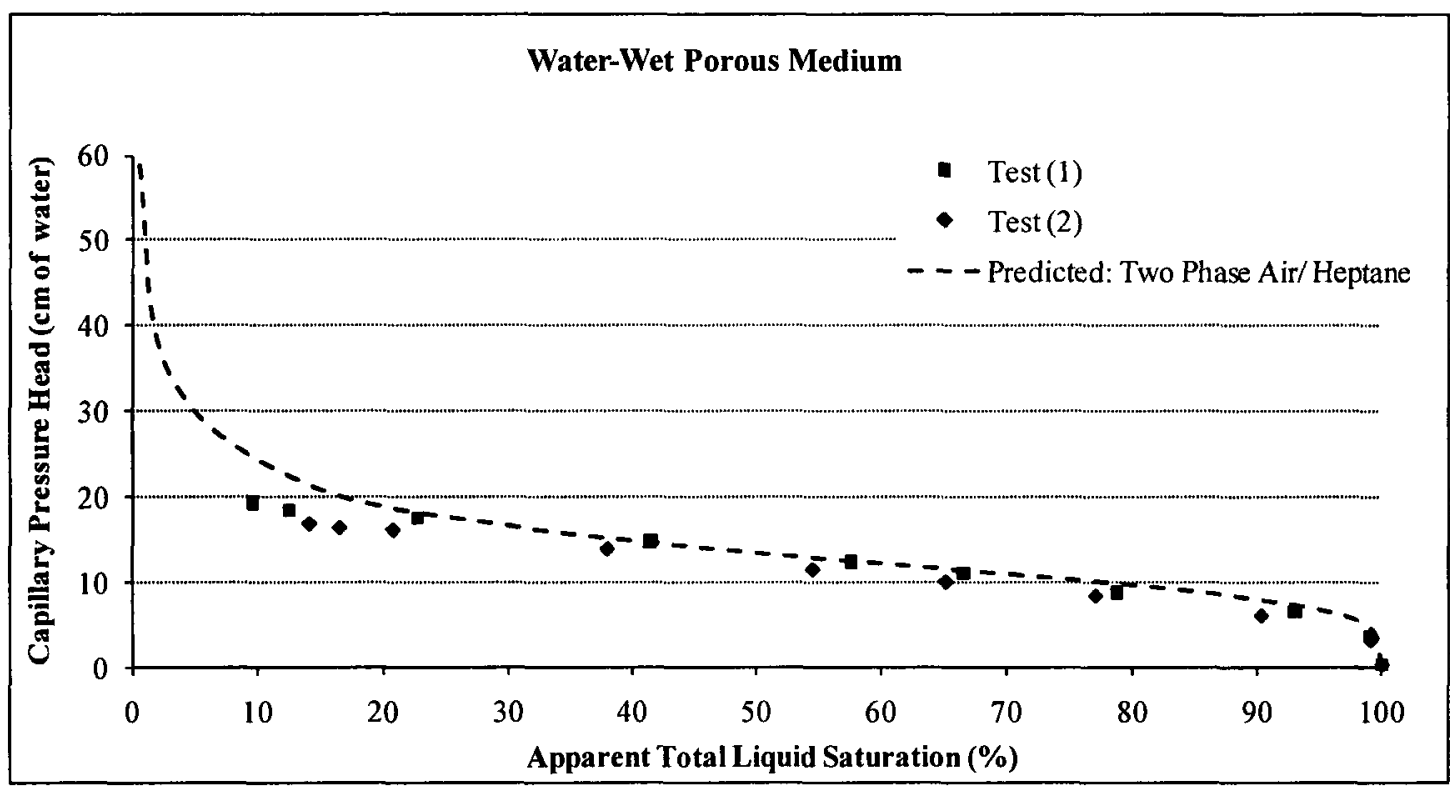

Figure 6.7: Air/Heptane Capillary Pressure Head-Apparent Total Liquid Saturation Main Drainage Pathway at Irreducible Water Saturation.

Capillary pressure heads at low total liquid saturation are lower than the capillary pressure heads in the predicted drainage pathway. As explained earlier in Chapter 2, 
heptane relative permeability, in a three-phase system, depends on the total liquid and heptane saturations. At low total liquid saturation, the heptane saturation approached its residual saturation and the relative permeability of the heptane was very low. It is important to note that for the three-phase experiments presented in this section, the fluid pressures were measured directly using the pressure transducers. The three-phase cells were also connected to burettes which were raised and lowered to adjust the fluid pressures and fluid flow into and out of the system. The transducer data were consistent with the fluid levels recorded in the burettes until one approached the irreducible or residual fluid saturations. At these low saturations, the relative permeabilities of the fluid phase were very low and hence a much greater time was needed before the transducer pressures equilibrate with the fluid levels in the burettes. Therefore, at low total liquid saturations, the heptane suction heads measured by the heptane transducers were lower than the capillary pressure heads on the predicted drainage pathway.

Figure 6.8 shows the predicted drainage pathway and the capillary pressure headactual total liquid saturation data for the two three-phase experiments conducted in the well graded sand. The plot includes the drainage pathway in the REV (i.e., the water-wet porous medium with the NAPL-wet soil lens), in the NAPL-wet soil lens, and in the water-wet soil fraction. The latter two drainage pathways were back-calculated from the REV data and using Equations 6.1 and 6.2. Figure 6.9 shows the corresponding data plotted as apparent saturations. As for the NAPL-wet soil lens, the data points fit the predicted drainage pathway. Similarly, due to the low heptane relative permeability at low heptane saturations, the data points deviate from the predicted drainage pathway at lower saturations. 


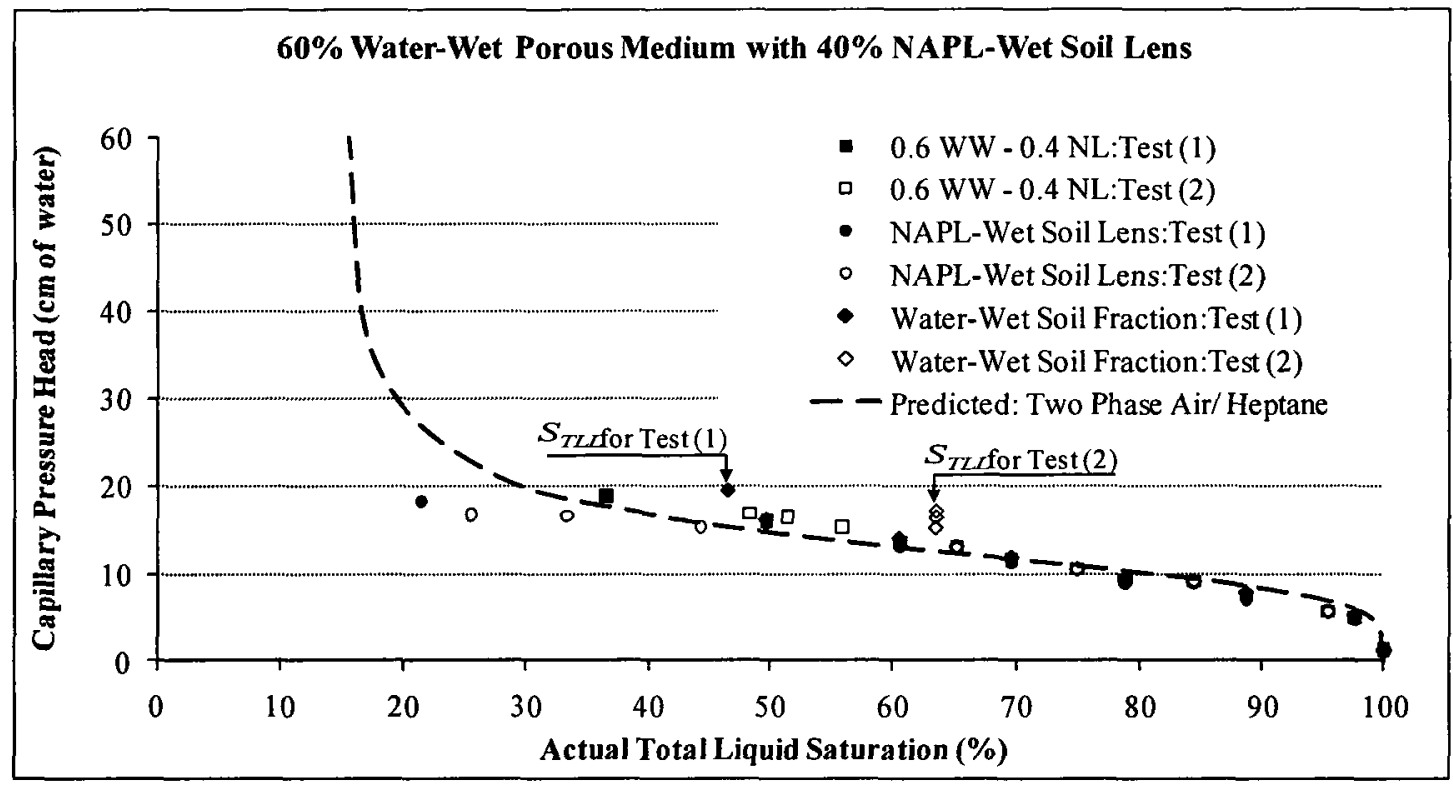

Figure 6.8: Air/Heptane Capillary Pressure Head-Actual Total Liquid Saturation Drainage Pathway at Lowest Initial Water Saturation in the REV.

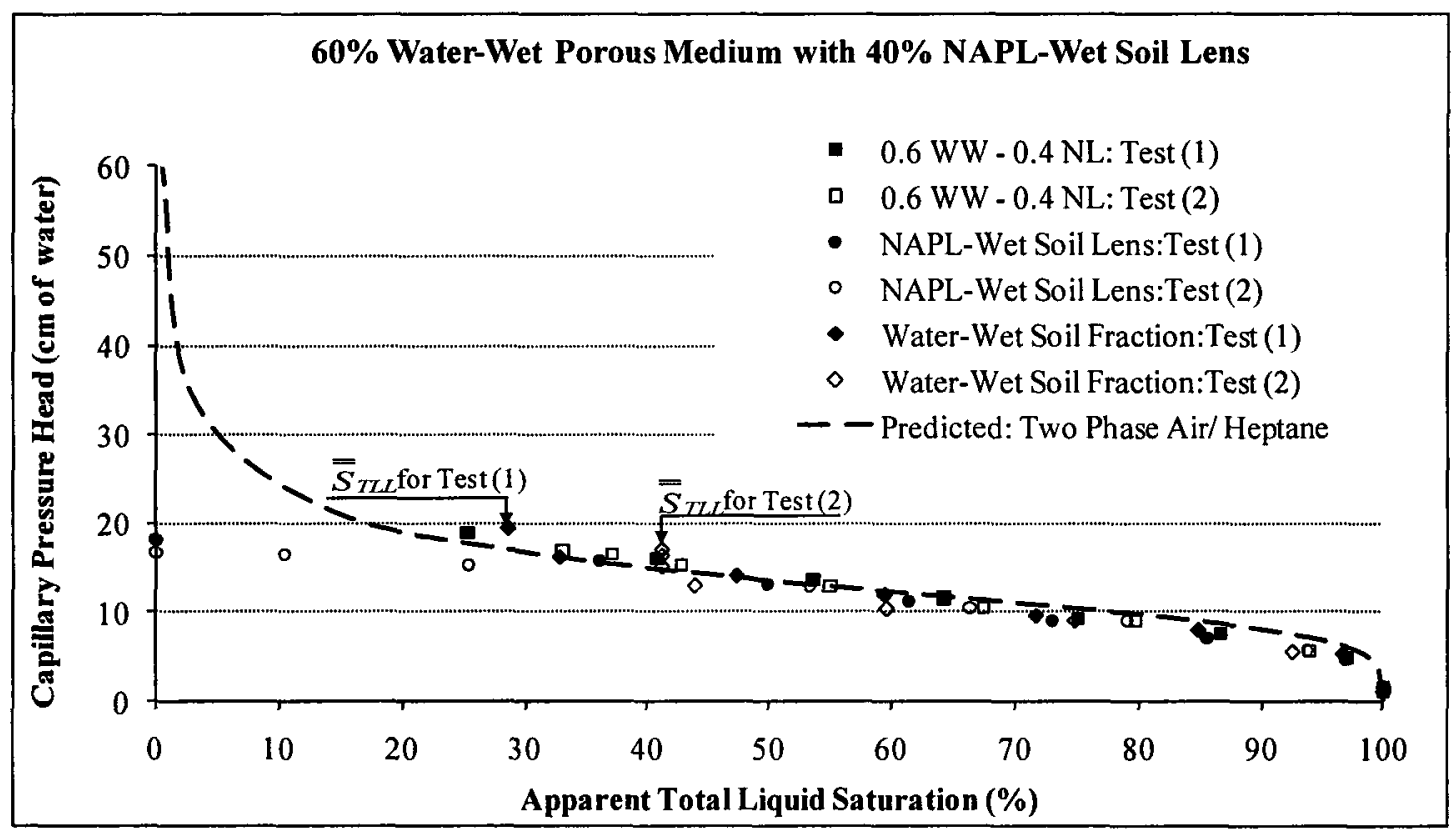

Figure 6.9: Air/Heptane Capillary Pressure Head-Apparent Total Liquid Saturation Drainage Pathway at Lowest Initial Water Saturation in the REV. 
As for the water-wet soil fraction, the test (1) data points, apart from the last point of the drainage pathway, fit the predicted drainage pathway. The total liquid saturation at the last point of the drainage pathway in test (1) is the TLSLL. The actual TLSLL is 46.6\%. At the TLSLL, the air/heptane capillary pressure heads in the water-wet soil fraction are higher than the capillary pressure heads in the predicted drainage pathway because the TLSLL (i.e., an actual saturation of $46.6 \%$, and apparent saturation of $28.6 \%$ ) was reached due to the high initial water saturation and the residual heptane saturation in the water-wet soil fraction. Therefore, even though the air/heptane capillary pressure head was increased, which resulted in insignificant reduction in heptane saturation, the total liquid saturation did not decrease. Similarly, test (2) data points fit the predicted drainage pathway until the TLSLL was reached. The actual TLSLL is $63.6 \%$ (apparent saturation is $41.2 \%$ ). Once this saturation was reached, the total liquid saturation, in the water-wet soil fraction, remained unchanged as the air/heptane capillary pressure head increased.

For the REV, the data points fit the predicted drainage pathway. Although these data points follow the predicted drainage pathway, at actual total liquid saturations lower than $46.6 \%$ and $63.6 \%$ in tests (1) and (2), respectively, the total liquid saturation in the water-wet soil fraction is different than that in the NAPL-wet soil lens. As a result, the saturation histories within the two soil fractions need to be tracked separately.

\section{Air/Heptane Capillary Pressure Head-Total Liquid Saturation at REV Actual Water Saturation of $\mathbf{4 5 \%}$}

Figure 6.10 shows the predicted drainage pathway and the capillary pressure headactual total liquid saturation data for two tests, in a three-phase system, in a well graded 
water-wet porous medium at an actual water saturation of $45 \%$. Figure 6.11 shows the corresponding data in terms of apparent saturations at an apparent water saturation of $33.5 \%$. Apparent total liquid saturations were calculated assuming an irreducible water saturation of 17.4\%. As shown in Figure 6.10 and Figure 6.11, apart from the low total liquid saturations, the data fit the predicted drainage pathway. The capillary pressure heads at the lower total liquid saturations are higher than the capillary pressure heads in the predicted drainage pathway. The reason is that the lowest total liquid saturation is the summation of a large initial water saturation (i.e., an actual water saturation of $45 \%$ ) and residual heptane saturation. Therefore, even though the air/heptane capillary pressure head was increased, which resulted in insignificant reduction in heptane saturation, the total liquid saturation did not decrease.

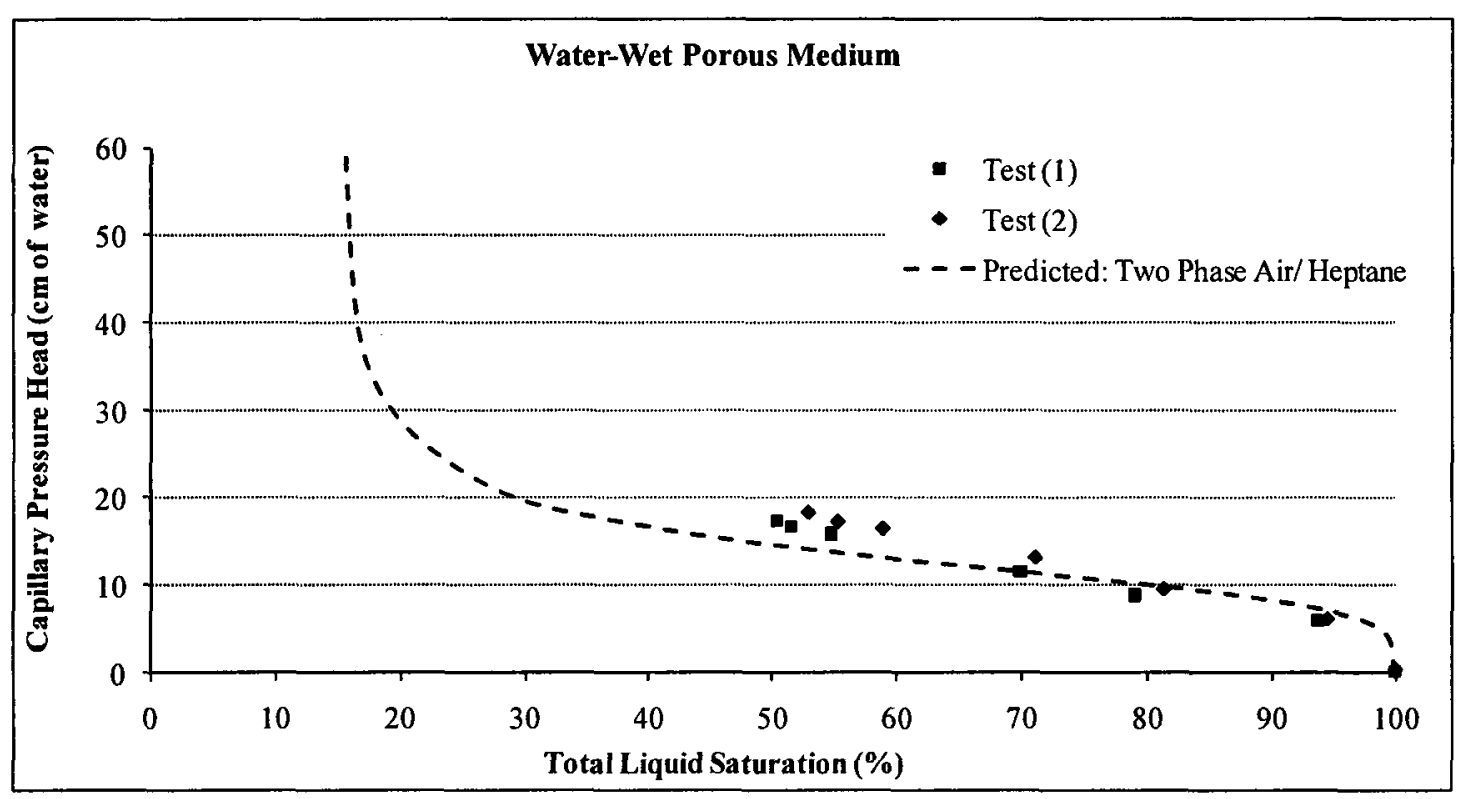

Figure 6.10: Air/Heptane Capillary Pressure Head-Actual Total Liquid Saturation Drainage Pathway at 45\% Actual Water Saturation. 



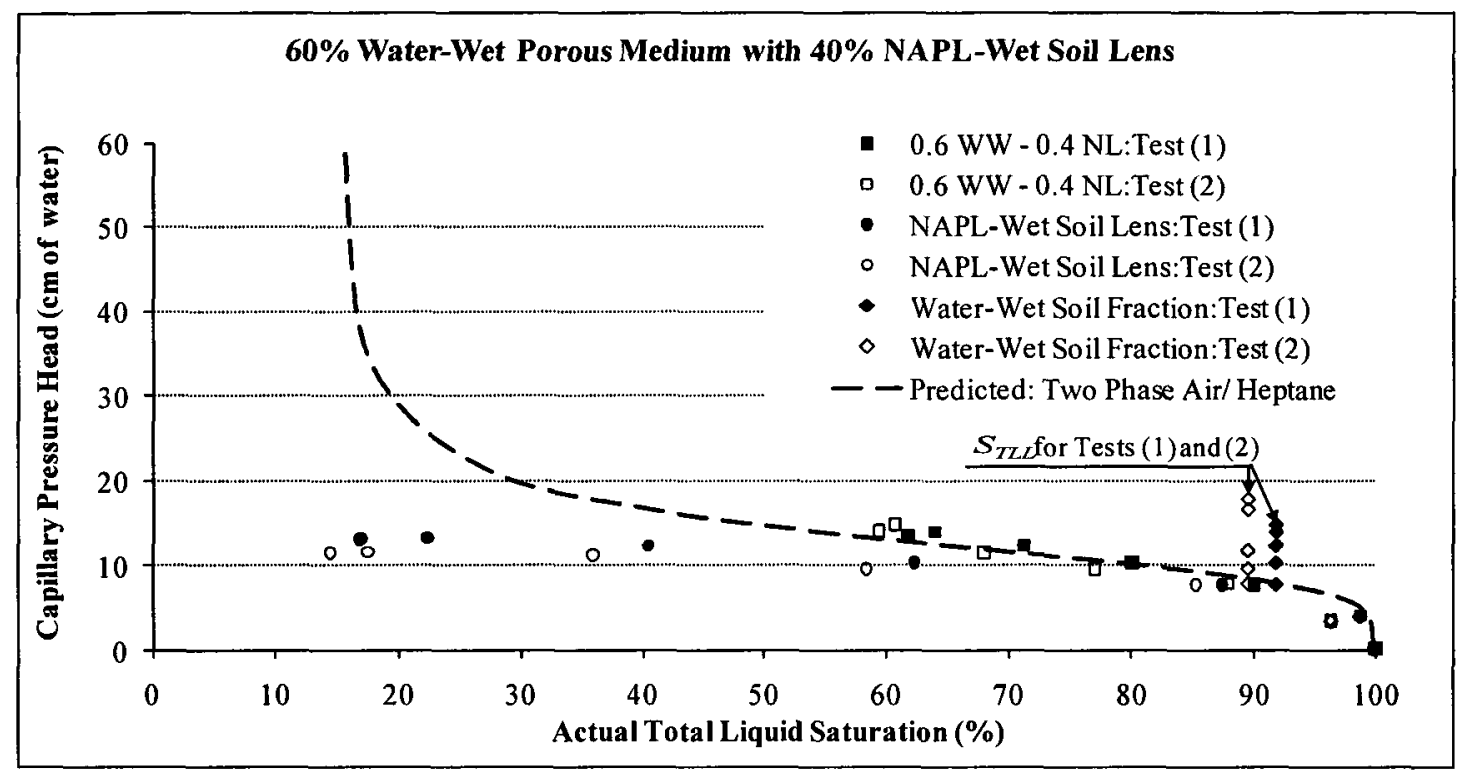

Figure 6.12: Air/Heptane Capillary Pressure Head-Actual Total Liquid Saturation Drainage Pathway at $45 \%$ Actual Water Saturation.

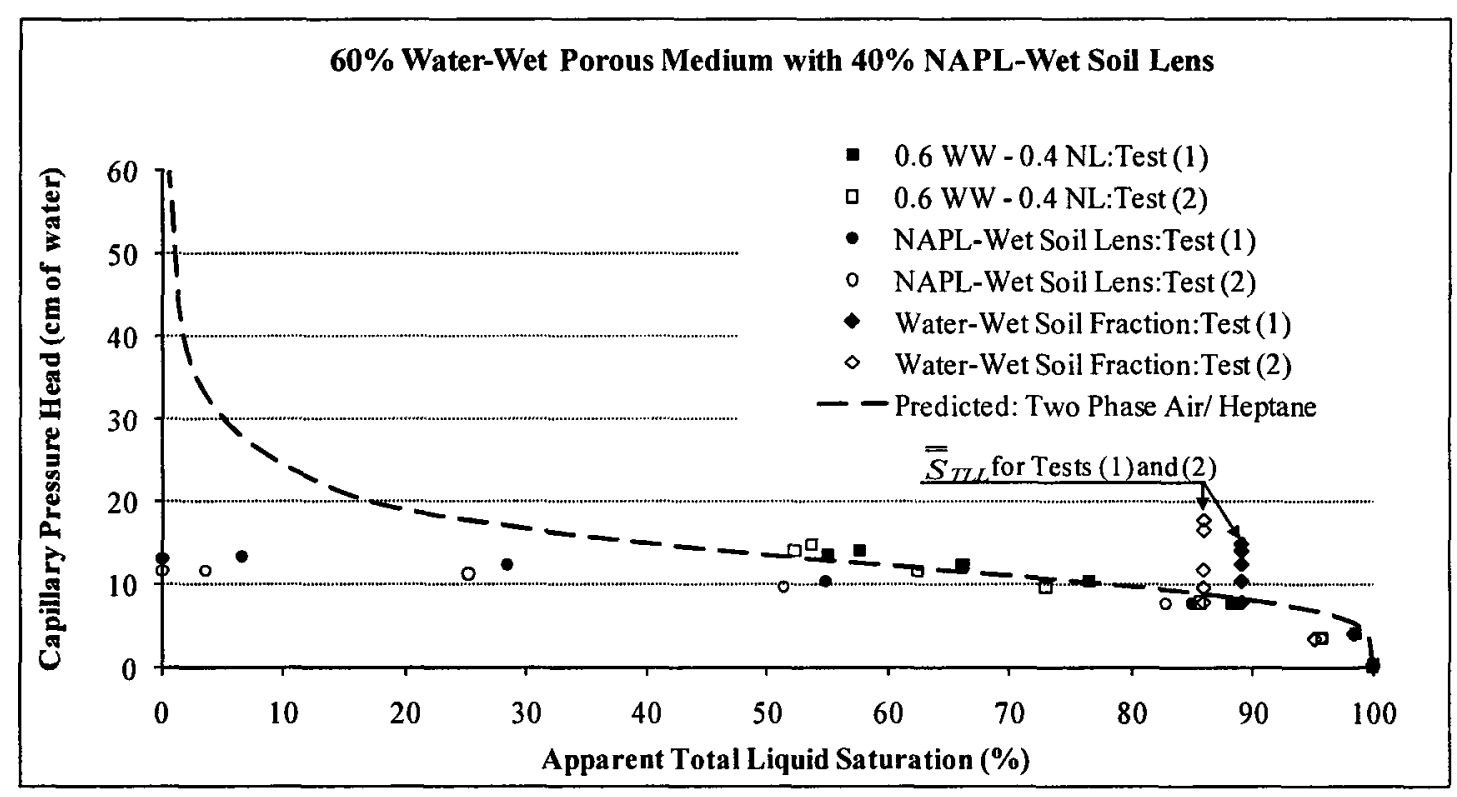

Figure 6.13: Air/Heptane Capillary Pressure Head - Apparent Total Liquid Saturation Drainage Pathway at $45 \%$ Actual Water Saturation.

As for the NAPL-wet soil lens, the data for actual heptane saturations larger than $60 \%$ fit the predicted drainage pathway. It is not expected that the data points deviate 
from the predicted drainage pathway at heptane saturations lower than $60 \%$. There are two possible reasons for this. The first reason is that there might be entrapped water and gas in heptane in the NAPL-wet soil lens. The entrapped fluid increases the total liquid saturation in the NAPL-wet soil lens, which in turn results in lower air/heptane capillary pressure heads. The second reason applies to the very low heptane saturations where heptane relative permeability is low at these saturations.

As for the water-wet soil fraction, the data for total liquid saturations higher than the TLSLL fit the predicted drainage pathway. The TLSLL in tests (1) and (2) are $91.8 \%$ and $89.5 \%$, respectively. At the TLSLL, the air/heptane capillary pressure head in the water-wet soil fraction is higher than the capillary pressure head in the predicted drainage pathway because the TLSLL is high due to the high initial water saturation and the residual heptane saturation in the water-wet soil fraction. Therefore, even though the air/heptane capillary pressure head increased, the total liquid saturation did not decrease.

For the REV, the data points fit the predicted drainage pathway. Although these data points follow the predicted drainage pathway, at actual total liquid saturations lower than $91.8 \%$ and $89.5 \%$ in tests (1) and (2), respectively, the total liquid saturation in the water-wet soil fraction is different than that in the NAPL-wet soil lens. As a result, the saturation histories within the two soil fractions need to be tracked separately.

\subsection{3.b. Conceptual Model Validation for the Imbibition Pathways}

I. Air/Heptane Capillary Pressure Head-Total Liquid Saturation at Lowest Initial Water Saturation

Figure 6.14 shows the predicted Van Genuchten main imbibition capillary pressure head-actual heptane saturation curve in air/heptane two-phase system and the 
capillary pressure head-actual total liquid saturation data for two tests, in three-phase system, in a well graded water-wet porous medium at irreducible water saturation. Figure 6.15 shows the corresponding data plotted as apparent saturations. Both figures show that the data fit the predicted Van Genuchten heptane main imbibition pathway in air/heptane two-phase system. In the remainder of this section, for convenience, the latter might be replaced by the predicted imbibition pathway.

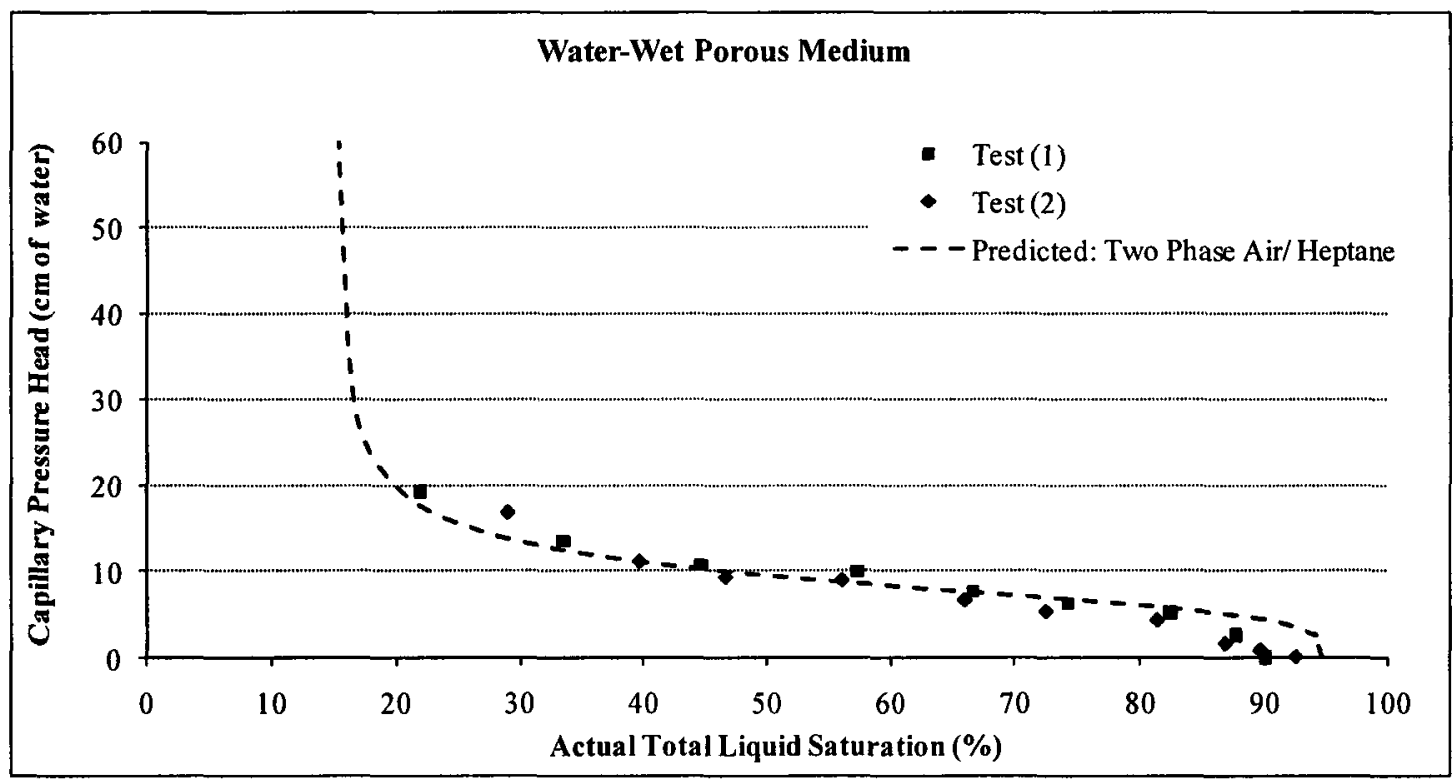

Figure 6.14: Air/Heptane Capillary Pressure Head-Actual Total Liquid Saturation Main Imbibition Pathway at Irreducible Water Saturation. 


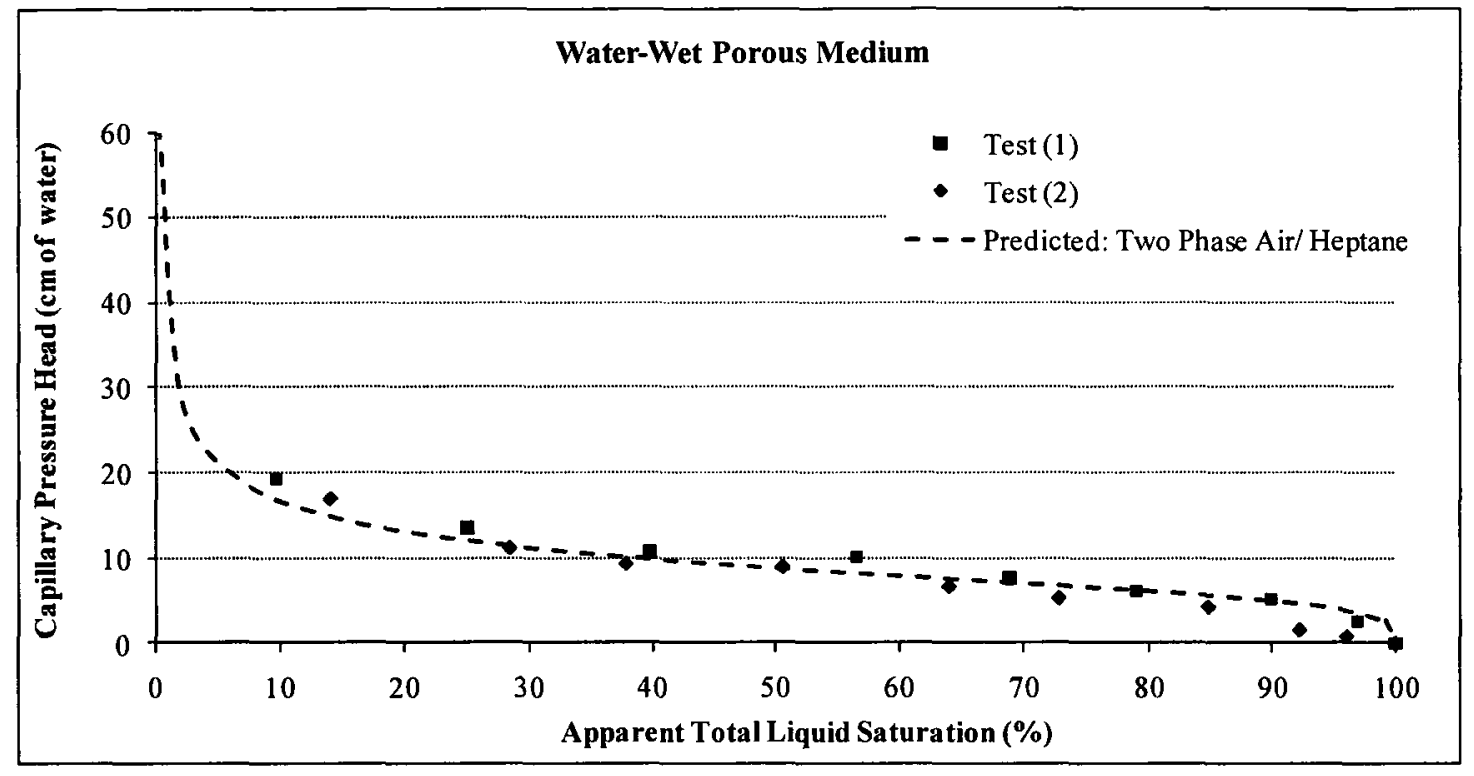

Figure 6.15: Air/Heptane Capillary Pressure Head-Apparent Total Liquid Saturation Main Imbibition Pathway at Irreducible Water Saturation.

Figure 6.16 shows the predicted drainage and imbibition pathways and the capillary pressure head-actual total liquid saturation data for the two three-phase experiments in the REV, in the NAPL-wet soil lens, and in the water-wet soil fraction at lowest initial water saturation. Figure 6.17 shows the corresponding data plotted as apparent saturations. In the NAPL-wet soil lens, Figure 6.16 shows that the data appear to follow the predicted imbibition pathway but deviate at higher saturations due to the entrapped air saturations. In Figure 6.17, apart from the low apparent heptane saturations, the data fit the predicted imbibition pathway. Due to the low heptane relative permeability at low apparent heptane saturations, the data deviate from the predicted imbibition pathway. 


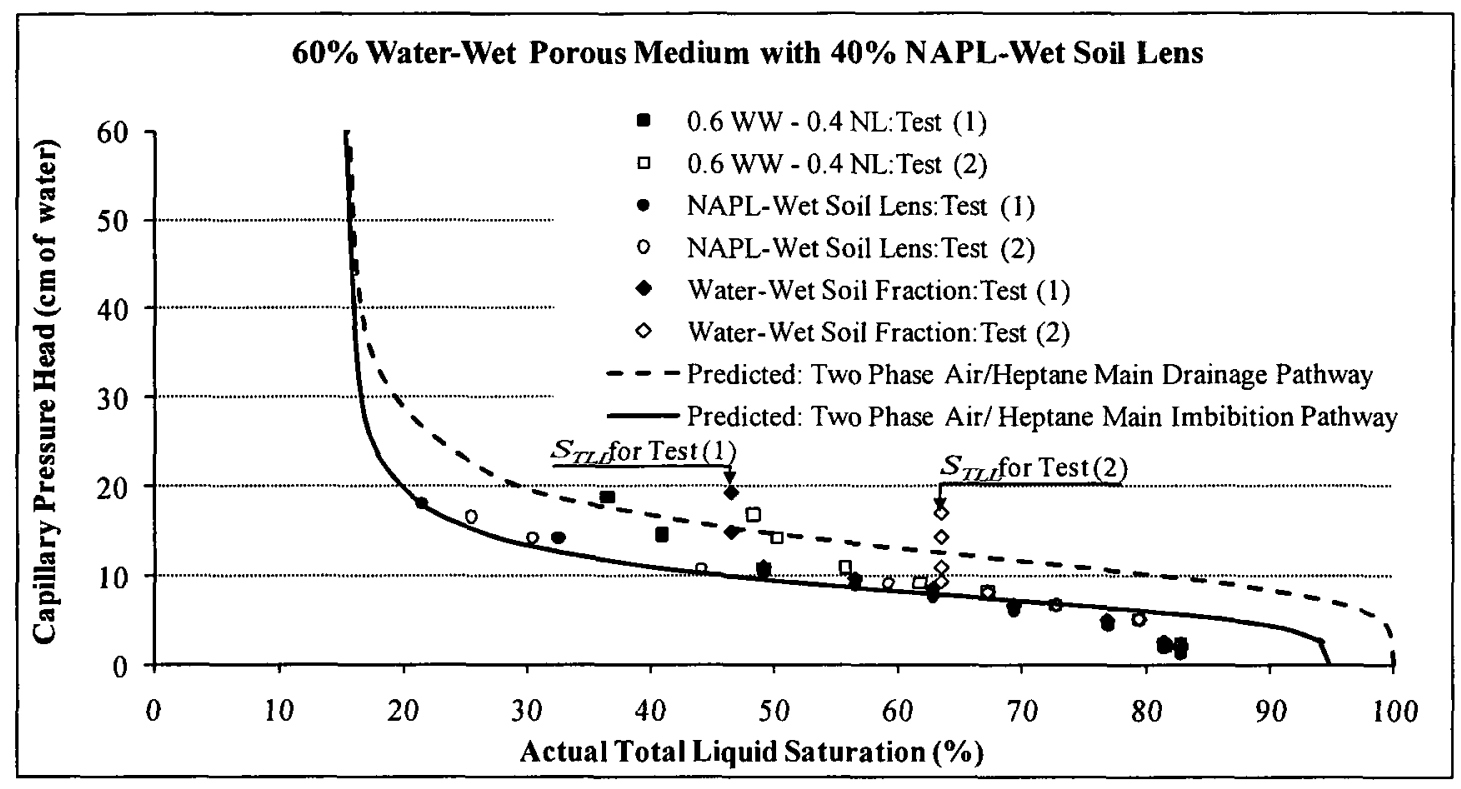

Figure 6.16: Air/Heptane Capillary Pressure Head-Actual Total Liquid Saturation Imbibition Pathway at Irreducible Water Saturation in the Water-Wet Soil Fraction.

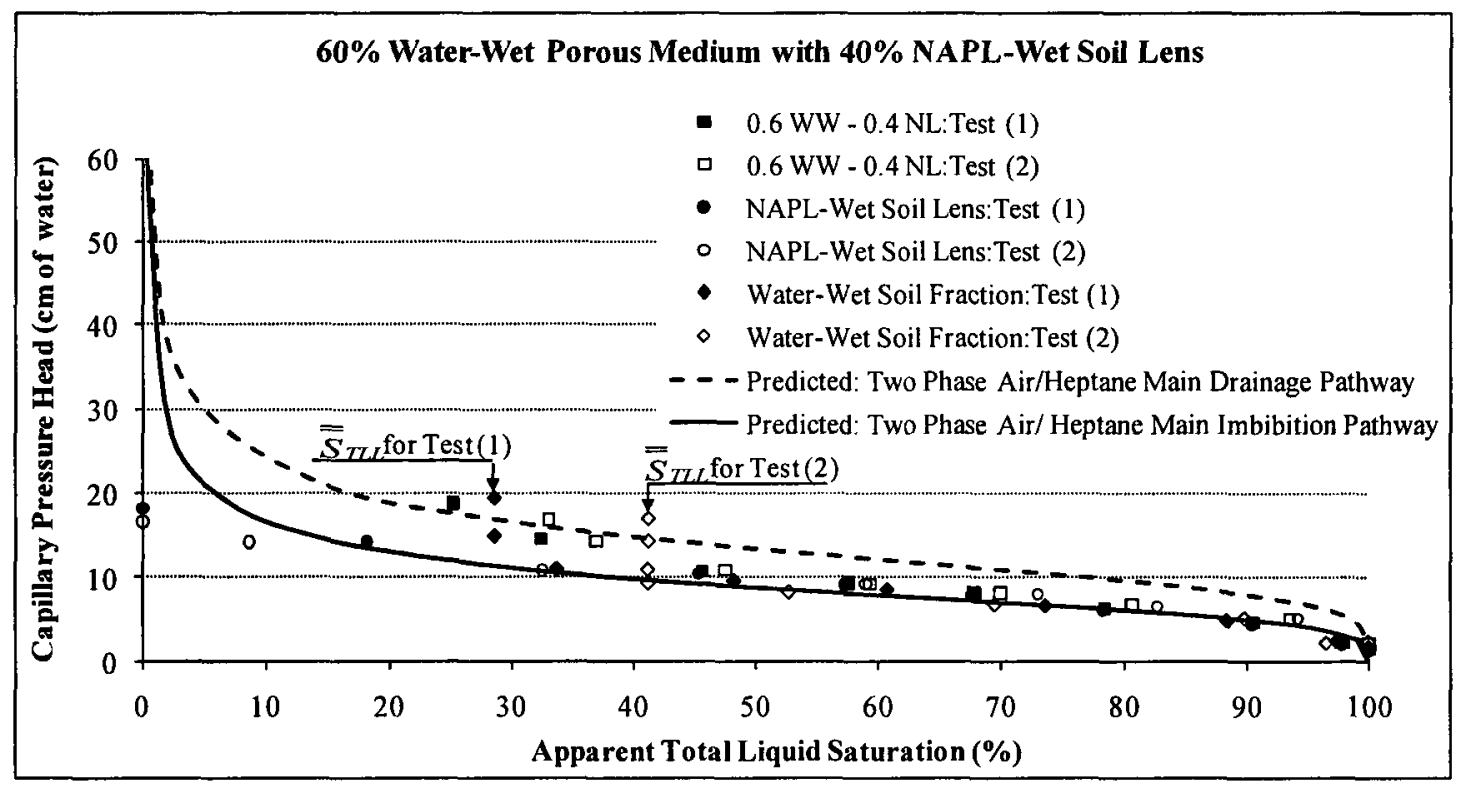

Figure 6.17: Air/Heptane Capillary Pressure Head-Apparent Total Liquid Saturation Imbibition Pathway at Irreducible Water Saturation in the Water-Wet Soil Fraction. 
As for the water-wet soil fraction, the apparent TLSLL in tests (1) and (2) are $28.6 \%$ and $41.2 \%$, respectively. As shown in Figure 6.17 , as capillary pressure head decreases, the apparent total liquid saturation in tests (1) or (2) remains fixed at the apparent TLSLL until the apparent heptane saturation in the NAPL-wet soil lens equals to the apparent TLSLL. At this point, as capillary pressure head decreases, the apparent total liquid saturation in the water-wet soil fraction increases. Figure 6.17 shows that the data fit the predicted imbibition pathway for apparent total liquid saturations higher than the apparent TLSLL. It is worth mentioning that the saturation path, in the water-wet soil fraction, follows a scanning curve in moving from the main drainage pathway to the main imbibition pathway, which is more noticeable in test (1) than in test (2).

In the water-wet porous medium with the NAPL-wet soil lens, as shown in Figure 6.17, for apparent total liquid saturations lower than the apparent TLSLL, the corresponding capillary pressure heads are higher than the capillary pressure heads of the predicted imbibition pathway. This is because the saturation path follows a scanning curve in moving from its main drainage pathway to its main imbibition pathway. In contrast, for apparent total liquid saturations higher than the apparent TLSLL, the data fit the predicted imbibition pathway. It is important to remember that for apparent total liquid saturations lower than the apparent TLSLL, the total liquid saturation in the waterwet soil fraction is different than that in the NAPL-wet soil lens. As a result, the saturation histories within the two soil fractions need to be tracked separately. 


\section{Air/Heptane Capillary Pressure Head-Total Liquid Saturation at REV Actual Water Saturation of $\mathbf{4 5 \%}$}

Figure 6.18 shows the predicted drainage and imbibition pathways and the capillary pressure head-actual total liquid saturation data for two tests, in three-phase system, in a water-wet porous medium at an REV initial actual water saturation of $45 \%$. Figure 6.19 shows the corresponding data in terms of apparent saturations at initial apparent water saturation of $33.5 \%$. Apparent total liquid saturations were calculated assuming an irreducible water saturation of $17.4 \%$. Figure 6.18 shows that the data do not follow the predicted imbibition pathway due to differences in entrapped air saturations between these data and the predicted imbibition pathway. As shown in Figure 6.19, apart from the lower total liquid saturations, the data fit the predicted imbibition pathway. Capillary pressure heads measured at the lower total liquid saturations are higher than the capillary pressure heads in the predicted imbibition pathway. The reason is that the saturation path follows a scanning curve in moving from the main drainage pathway to the main imbibition pathway. 


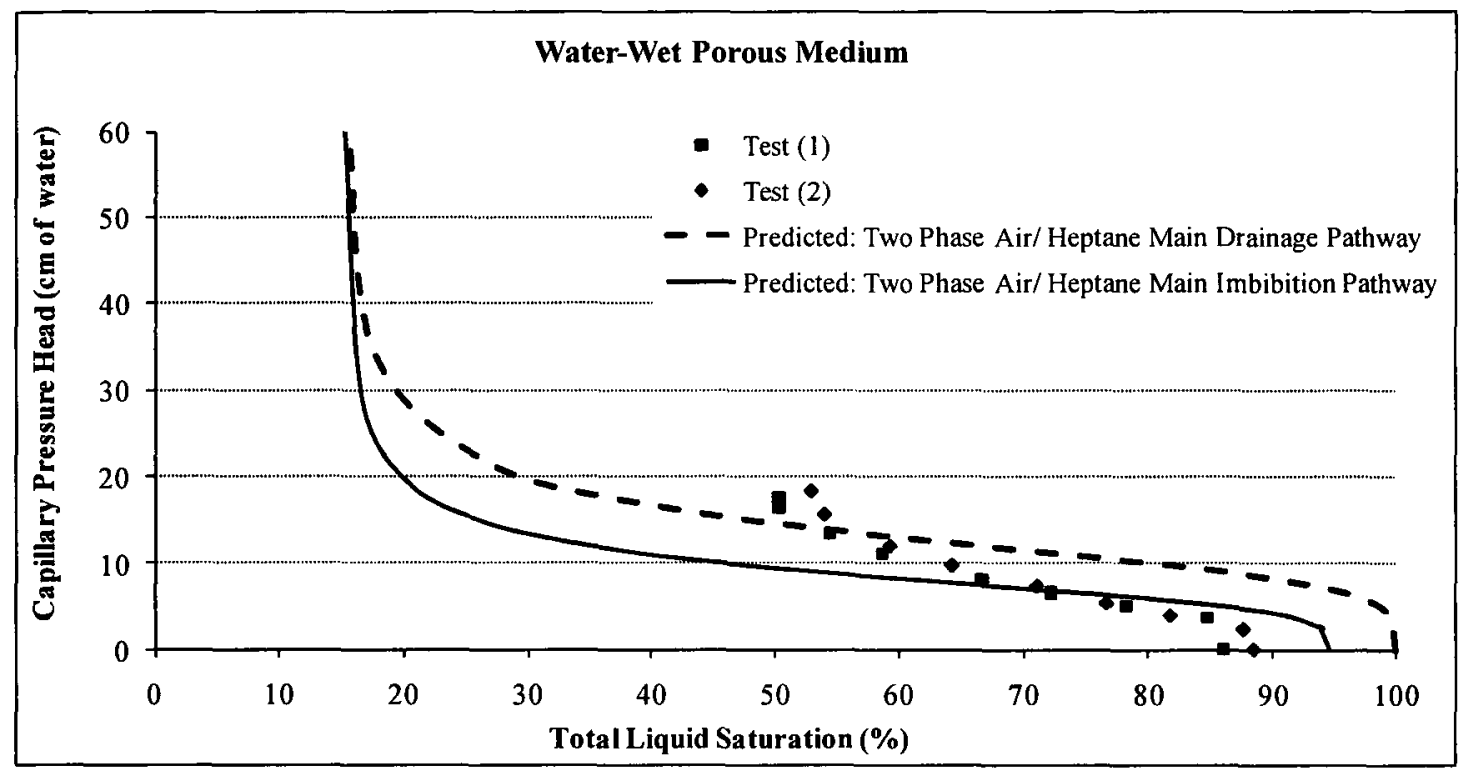

Figure 6.18: Air/Heptane Capillary Pressure Head-Actual Total Liquid Saturation Imbibition Pathway at $\mathbf{4 5 \%}$ Actual Water Saturation.

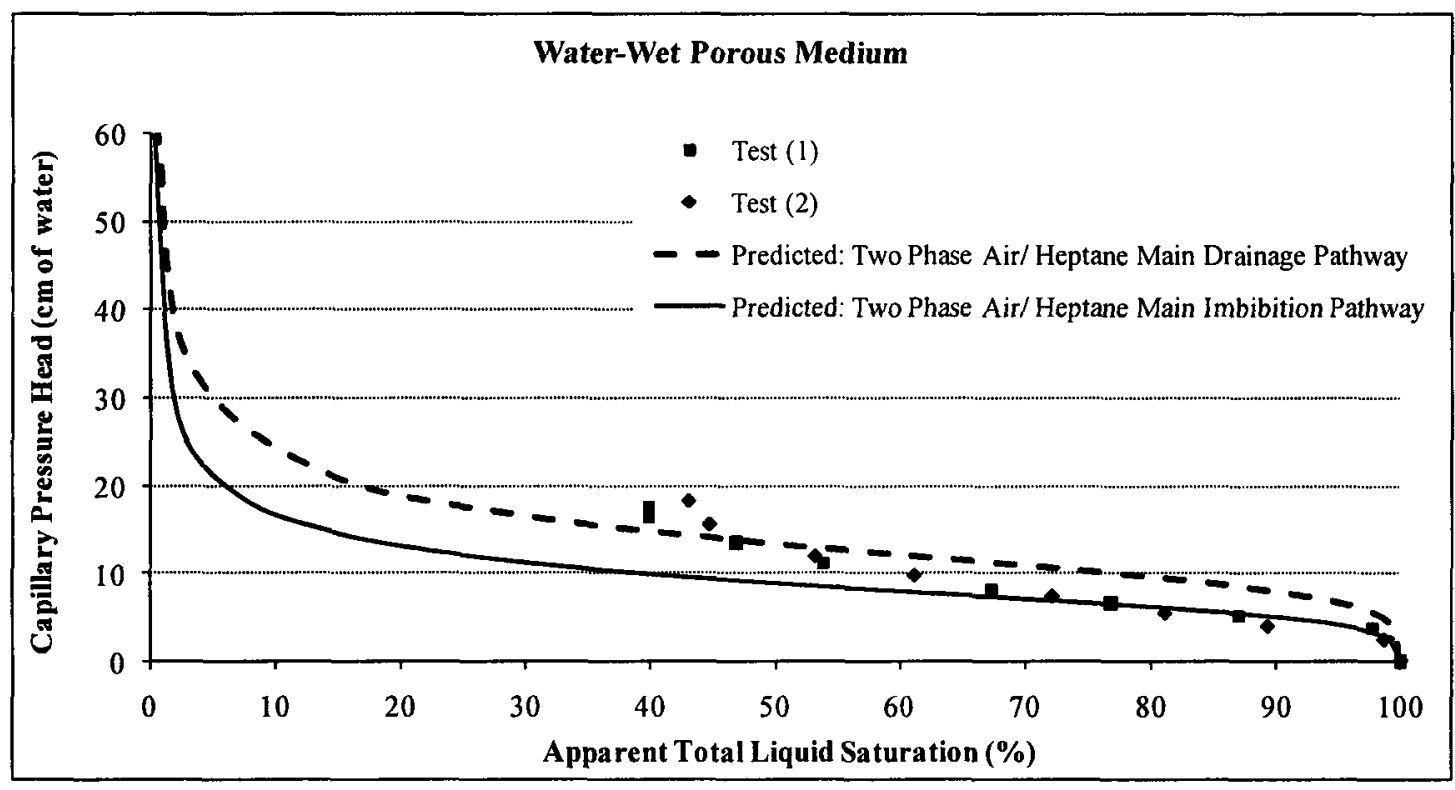

Figure 6.19: Air/Heptane Capillary Pressure Head-Apparent Total Liquid Saturation Imbibition Pathway at $\mathbf{4 5 \%}$ Actual Water Saturation. 
Figure 6.20 shows the predicted drainage and imbibition pathways and the capillary pressure head-actual total liquid saturation data for two tests, in three-phase system, in the REV, in the NAPL-wet soil lens, and in the water-wet soil fraction, at REV actual water saturation of $45 \%$. Figure 6.21 shows the corresponding data in terms of apparent saturations. In the NAPL-wet soil lens, Figure 6.20 shows that the data do not follow the predicted imbibition pathway due to differences in entrapped air saturations between the data points and the predicted imbibition pathway. In Figure 6.21, apart from the low apparent heptane saturations, the data points fit the predicted imbibition pathway. Due to the low heptane relative permeability at low apparent heptane saturations, the data points deviate from the predicted imbibition pathway.

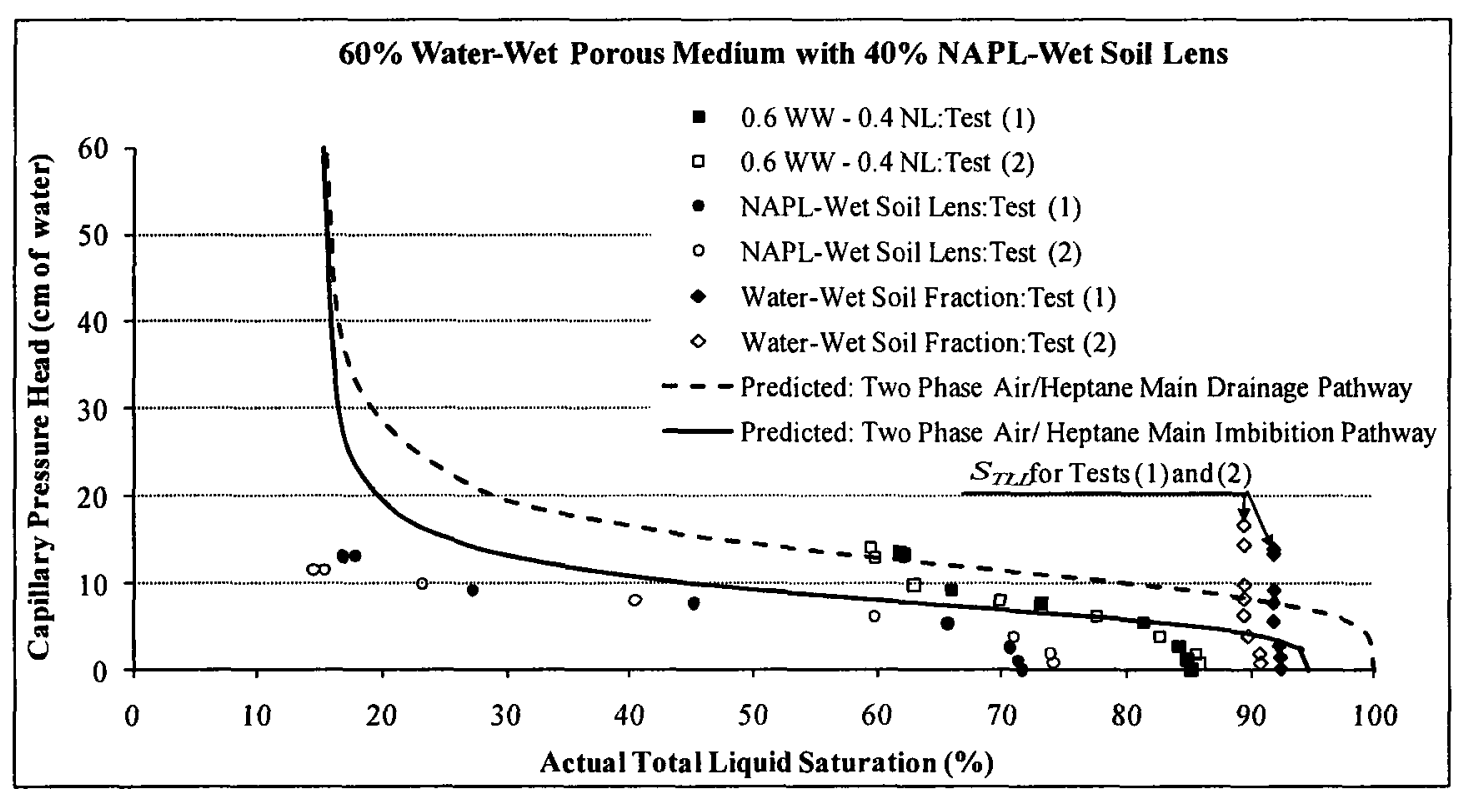

Figure 6.20: Air/Heptane Capillary Pressure Head-Actual Total Liquid Saturation Imbibition Pathway at $45 \%$ Actual Water Saturation. 


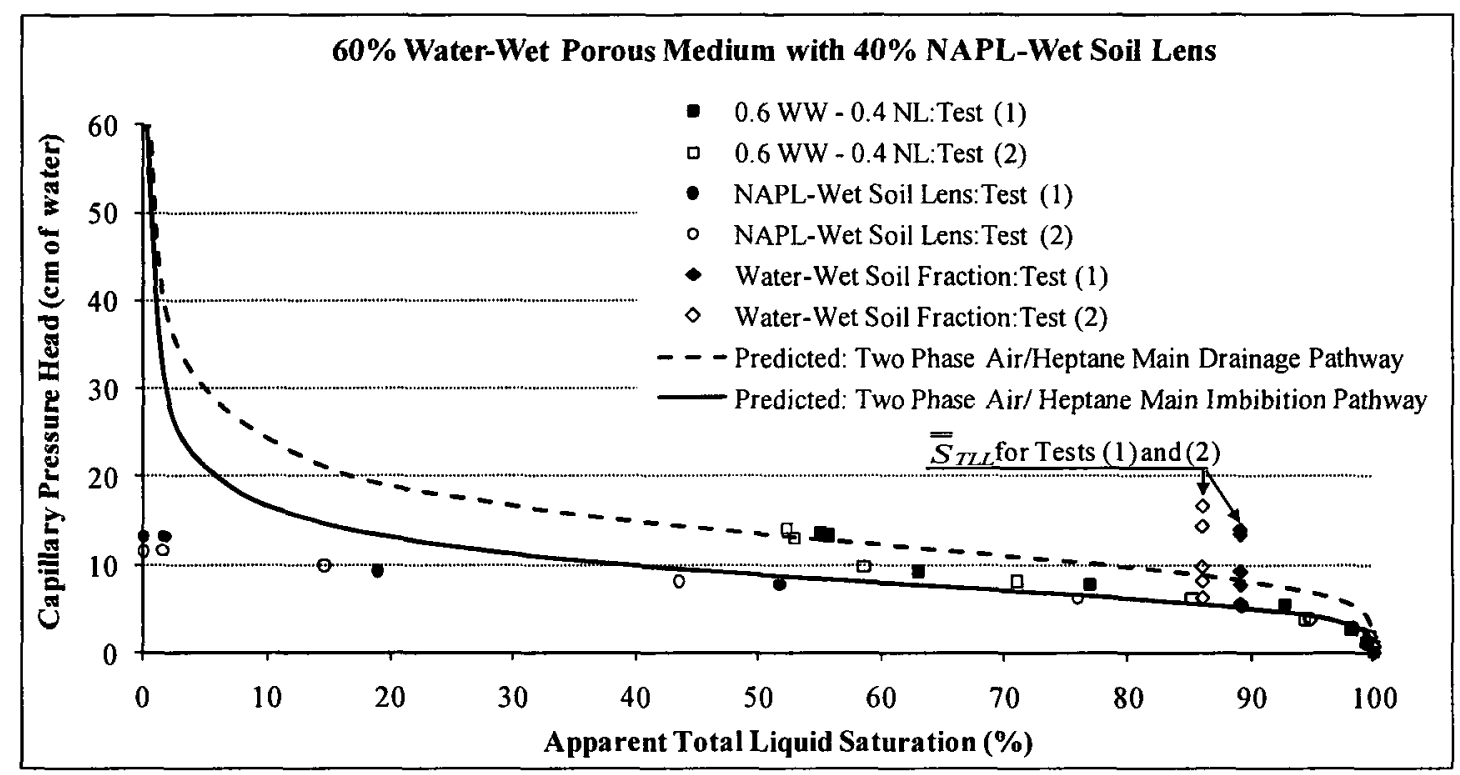

Figure 6.21: Air/Heptane Capillary Pressure Head-Apparent Total Liquid Saturation Imbibition Pathway at $\mathbf{4 5 \%}$ Actual Water Saturation.

As for the water-wet soil fraction, the apparent TLSLL in tests (1) and (2) are $89.1 \%$ and $85.9 \%$, respectively. As shown in Figure 6.21 , as capillary pressure head decreases, the apparent total liquid saturation in tests (1) or (2) remains fixed at the apparent TLSLL until the apparent heptane saturation in the NAPL-wet soil lens equals to the apparent TLSLL. At this point, as capillary pressure head decreases, the apparent total liquid saturation in the water-wet soil fraction increases. Figure 6.21 shows that at apparent total liquid saturations larger than the apparent TLSLL, the data fit the predicted imbibition pathway. It is worth mentioning that the saturation path, in the water-wet soil fraction, follows a scanning curve in moving from the main drainage pathway to the main imbibition pathway, which is more noticeable in test (2) than in test (1).

In the REV, as shown in Figure 6.21, the heptane suction heads at the lower apparent total liquid saturations are higher than the capillary pressure heads on the predicted imbibition pathway. This is because the saturation path follows a scanning 
curve in moving from its main drainage pathway to its main imbibition pathway. At larger apparent total liquid saturations, the data fit the predicted imbibition pathway. It is important to remember that at apparent total liquid saturations larger than the lowest total liquid saturations and less than the apparent TLSLL, the total liquid saturation in the water-wet soil fraction is different than that in the NAPL-wet soil lens. As a result, the saturation histories within the two soil fractions need to be tracked separately. Based on the previous discussion, the proposed conceptual model for a connected NAPL-wet soil lens is valid.

\subsection{Capillary Pressure Head-Water Saturation}

In a water-wet porous medium, Leverett stated that the water saturation main drainage and imbibition pathways in a three-phase system are equivalent to the water main drainage and imbibition pathways in heptane/water two-phase system. In a waterwet porous medium with a NAPL-wet soil lens, it was explained that the water imbibition and drainage are restricted to the water-wet soil fraction. In addition, the water saturation in the NAPL-wet soil lens is zero or an entrapped saturation. Therefore, if the water main drainage and imbibition pathways are measured for heptane/water two-phase system in a water-wet porous medium and the Leverett assumption is applied, the conceptual models developed in Sections 5.4.4.a.II and 5.4.4.c.II can be used to calculate the water main drainage and imbibition pathways in three-phase system in a water-wet porous medium with a NAPL-wet soil lens, respectively.

\subsection{Summary}

This chapter focused on the development of a conceptual model for air/heptane capillary pressure-total liquid saturation and heptane/water capillary pressure-water 
saturation in a water-wet porous medium with a NAPL-wet soil lens. This also included checking the validity of Leverett assumption in this porous medium. Two scenarios corresponding to isolated and connected NAPL-wet soil lenses were considered. Then the conceptual model was validated for air/heptane capillary pressure-total liquid saturation, assuming a connected NAPL-wet soil lens and constant water saturation. 


\section{Chapter 7. Residual NAPL Saturation under Different Soil Wettability Configurations}

\subsection{Introduction}

It was explained earlier in Chapters 1 and 2 that the residual NAPL saturation was not incorporated in multiphase flow simulators. Only recently, conceptual models have been proposed to incorporate a residual NAPL saturation term into the constitutive relationships in water-wet porous media (e.g., Wipfler and Van der Zee 2001, Van Geel and Roy 2002, Lenhard et al. 2004). However, the processes that impact the magnitude and distribution of the residual NAPL are not well understood. In addition, none of the existing constitutive relationships includes the effects of spatial wettability variations. Also, residual NAPL distribution has not been clearly demonstrated under different soil wettability conditions. Therefore, the objective of this chapter is to develop conceptual models for residual NAPL formation, distribution, and quantification in a water-wet porous medium and in a water-wet porous medium with a NAPL-wet soil lens. These conceptual models relate the residual NAPL saturation to water and NAPL saturations and porous medium-liquid specific parameters in a water-wet porous medium, in addition to the NAPL-wet lens size in a water-wet porous medium with a NAPL-wet soil lens, as will be described in Sections 7.2 and 7.3.

The first main section focuses on the development of a conceptual model to predict the residual NAPL saturation in a water-wet porous medium followed by a validation of the conceptual model using residual NAPL saturations measured in two experimental data sets; experimental data sets (1) and (2). This section also includes a comparison of the predictions of the developed conceptual model versus the predictions of Van Geel and Roy (2002) linear and Land models and Lenhard et al. (2004) model of 
the experimental data sets (1) and (2), and Van Geel and Roy (2002) experimental data set. The second main section focuses on the residual NAPL saturation in a water-wet porous medium with NAPL-wet soil lenses. This includes the development of a conceptual model to predict residual NAPL saturation in a water-wet porous medium with NAPL-wet soil lenses followed by a validation of the conceptual model using residual NAPL saturations measured in experimental data set (3), which is described below.

Experimental data sets (1) and (2) included measurement of residual NAPL saturations left in a water-wet porous medium when a certain volume of NAPL imbibed into the porous medium and subsequently drained, at known water saturation, in two sand types; uniform sand and well graded sand. Experimental data set (1) was comprised of eighteen setups (i.e., nine setup duplicates) for each sand type. For each setup the water saturation was fixed during the experiment. The fixed water saturations correspond to irreducible water saturation, $20 \%, 30 \%, 40 \%, 50 \%, 60 \%, 70 \%, 80 \%$, and $90 \%$ actual water saturations. In each experiment setup, the residual NAPL saturations that resided in the porous medium were measured when different volumes of NAPL, equivalent to actual NAPL saturation of $10 \%$ with $10 \%$ increments up to NAPL saturation corresponding to $100 \%$ apparent total liquid saturation, imbibed into the porous media and subsequently drained. Hence, the NAPL saturation was increased to $10 \%$ and allowed to drain to residual, then increased to $20 \%$ and allowed to drain to residual; this process was continued until $100 \%$ apparent total liquid saturation was achieved prior to a final drainage to residual NAPL saturation. The residual NAPL saturations measured at fixed water saturation and different imbibing NAPL saturations illustrated how the 
residual NAPL saturation varies with the increase in the imbibing NAPL saturation at known water saturation. Based on these data, a relationship between the residual NAPL saturation, the imbibing NAPL saturation, and the water saturation was developed. In this section, the terms; NAPL saturation and imbibing NAPL saturation are used interchangeably.

Experimental data set (2) was composed of five setups for each sand type. In each setup, the water saturation takes three values corresponding to actual water saturations of $45 \%$ and $30 \%$, and irreducible water saturation. At an actual water saturation of $45 \%$, different volumes of NAPL imbibed into the porous medium and subsequently drained to residual NAPL saturations. This process was repeated at lower water saturations. At each water saturation, the NAPL saturation was increased from $20 \%$, to $30 \%$, and to a value corresponding to an apparent total liquid saturation of unity. The objective of this part is to show the applicability of the conceptual model under different water saturations within the same experiment.

The experimental procedure of experimental data set (3) was similar to that of experimental data set (2). The difference between them was the porous medium wettability. Four porous media wettability configurations were used; corresponding to $90 \%$ water-wet- $10 \%$ NAPL-wet, $80 \%$ water-wet-20\% NAPL-wet, $70 \%$ water-wet- $30 \%$ NAPL-wet, and $60 \%$ water-wet-40\% NAPL-wet in two sand types; uniform sand and well graded sand. The objective of this data set is to validate the conceptual model predicting the residual NAPL saturation in a water-wet porous medium with a NAPL-wet soil lens. 


\subsection{Residual NAPL Saturation in a Water-Wet Porous Medium}

The discussion of residual NAPL saturation in a water-wet porous medium is divided into three sections. The first section discusses the experimental data for residual NAPL saturation measured at different water and NAPL saturations in two different porous media; uniform sand and well graded sand. The purpose of this section is to show the expected trend for the residual NAPL saturation with the variation of water and NAPL saturations. The second section demonstrates the development of a conceptual model for residual NAPL saturation in a water-wet porous medium. This conceptual model is validated in the third section.

\subsubsection{Residual Heptane Saturation Data in Uniform Sand and Well Graded Sand}

This section illustrates some selected experimental data for the residual heptane saturations measured at different water and heptane saturations. Two data sets are presented for each sand type. These data sets are discussed in Sections 7.2.1.a and 7.2.1.b.

\subsection{1.a. Effective Residual Heptane Saturation-Apparent Heptane Saturation}

This data set includes the residual heptane saturations that resided in the porous media when certain volumes of heptane, equivalent to $10 \%$ actual heptane saturation with $10 \%$ increments up to a heptane saturation corresponding to $100 \%$ apparent total liquid saturation, were allowed to imbibe into the porous media and subsequently drain. A subset of the data is presented here for constant water saturations corresponding to irreducible, $40 \%$, and $60 \%$ actual water saturations. This data set is shown in Figure 7.1 for uniform sand and in Figure 7.2 for well graded sand. The remaining data are provided in Section 7.2.3.a. 


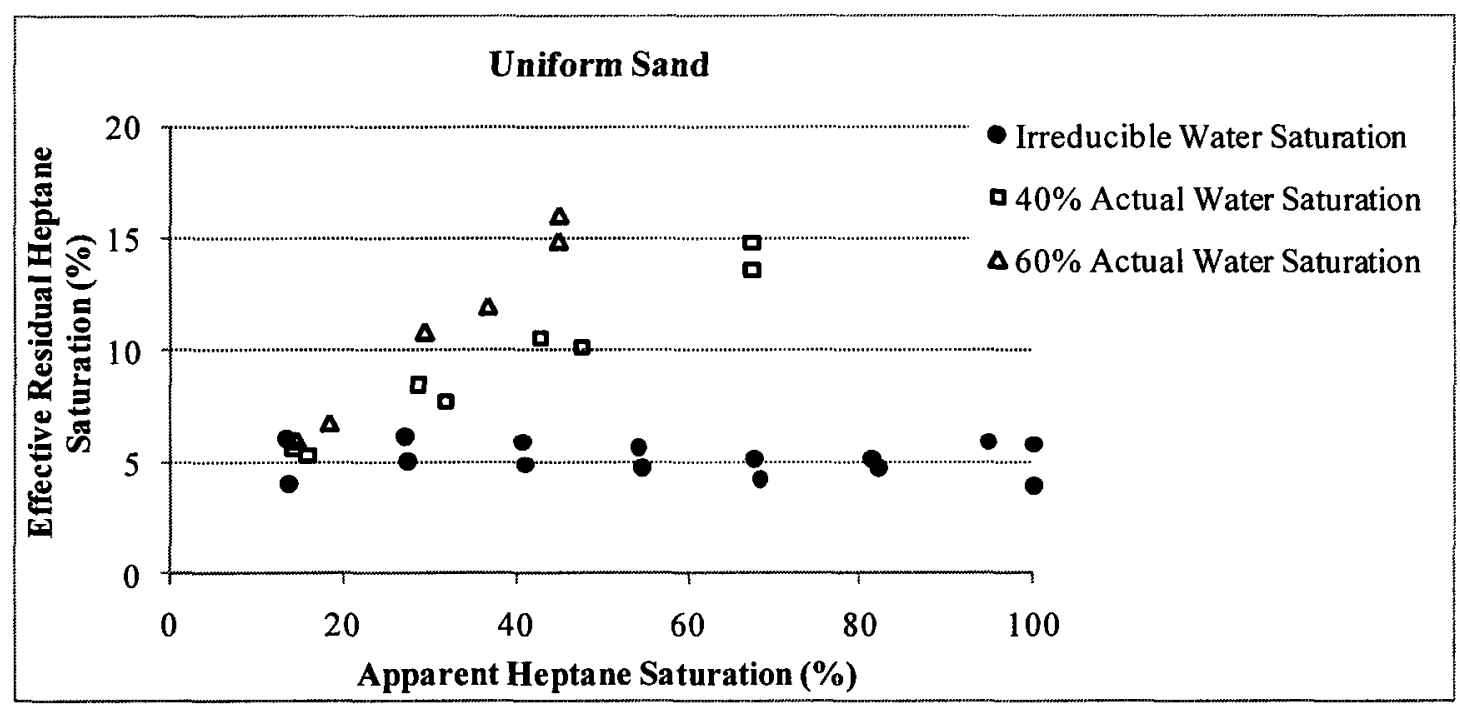

Figure 7.1: Effective Residual Heptane Saturation-Apparent Heptane Saturation in Uniform Sand.

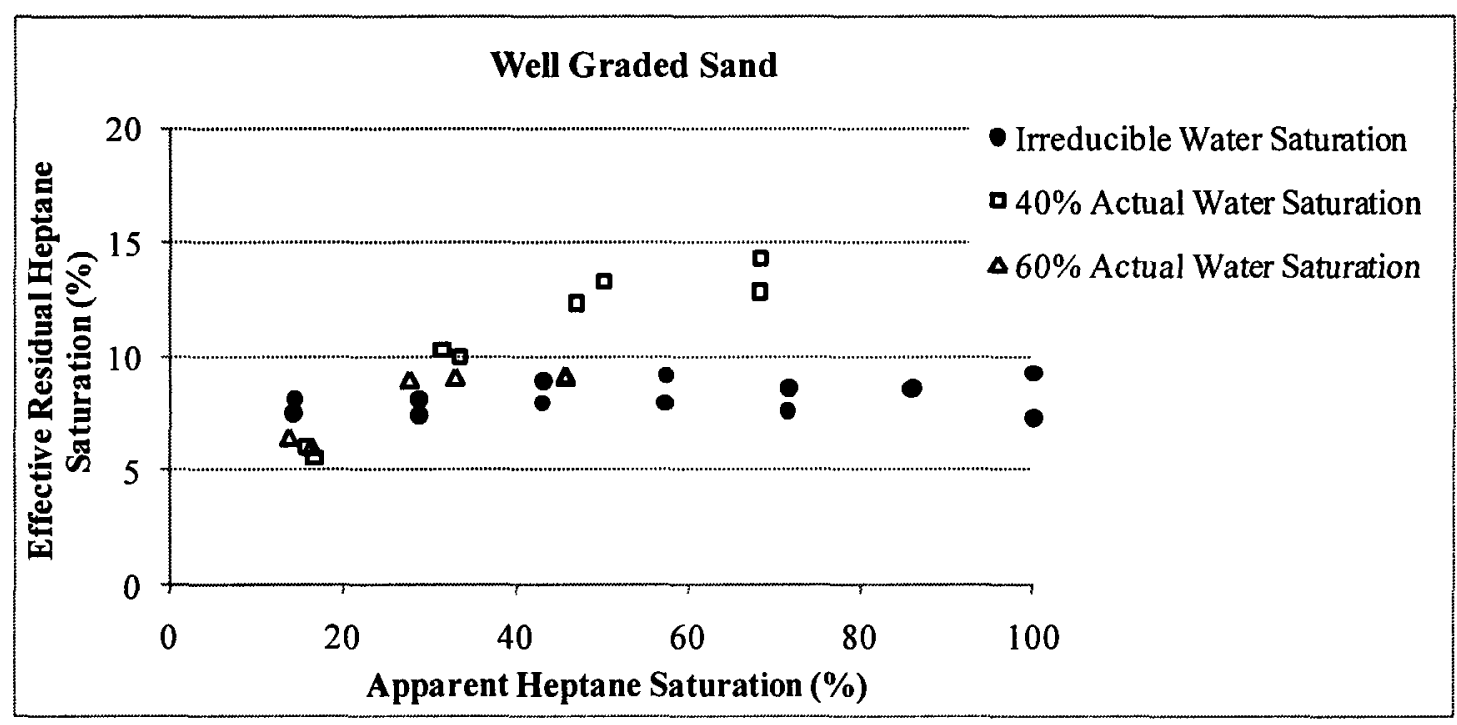

Figure 7.2: Effective Residual Heptane Saturation-Apparent Heptane Saturation in Well Graded Sand.

Figure 7.1 shows that the residual heptane saturation increases linearly as the heptane saturation increases for the same water saturation. This is clearly depicted for 
residual heptane saturations measured at $40 \%$ and $60 \%$ actual water saturations. While for residual heptane saturations measured at irreducible water saturation, the residual heptane saturation fluctuates around an average value (i.e., constant residual heptane saturation). In contrast, in well graded sand, Figure 7.2 shows that the residual heptane saturation increases nonlinearly as the heptane saturation increases for the same water saturation. At each water saturation, the residual heptane saturation increases as the heptane saturation increases until the heptane saturation approaches a certain value. If the heptane saturation increases above this value, the residual heptane saturation fluctuates around one value (i.e., the residual heptane saturation does not change). It is also noticed that the residual heptane saturations measured at $40 \%$ actual water saturation are larger than the residual heptane saturations measured at $60 \%$ actual water saturation.

\subsection{1.b. Effective Residual Heptane Saturation-Apparent Water Saturation}

Figure 7.3 for uniform sand and Figure 7.4 for well graded sand contain two data sets. The first data set is the residual heptane saturation that remained after heptane was allowed to imbibe to a heptane saturation of $10 \%$ and then subsequently drained to a residual value at different water saturations. The second data set represents the average residual heptane saturation after heptane was allowed to imbibe to saturations of $10 \%$ through $100 \%$ apparent total liquid saturation, in increments of $10 \%$, and then subsequently drained to residual heptane saturation at different water saturations.

Both figures show that the residual heptane saturation starts at a low value measured at irreducible water saturation. As water saturation increases, the residual heptane saturation increases until it reaches a maximum value after which the residual heptane saturation decreases as the water saturation increases. For uniform sand, the 
residual heptane saturations residing in the porous media, after $10 \%$ actual heptane saturation was allowed to imbibe into the porous media and subsequently drained, have one peak at $66.3 \%$ apparent water saturation. In contrast, there are two peaks for the well graded sand; at irreducible water saturation and $65.8 \%$ apparent water saturation.

For the uniform sand, the average residual heptane saturations have one peak at $54.4 \%$ apparent water saturation as shown in Figure 7.3. While the average residual heptane saturations residing in the well graded sands have one peak at $8.9 \%$ apparent water saturation as shown in Figure 7.4. It is noticed that the effective residual heptane saturation-apparent water saturation relationship in both sands is close to a quadratic relationship.

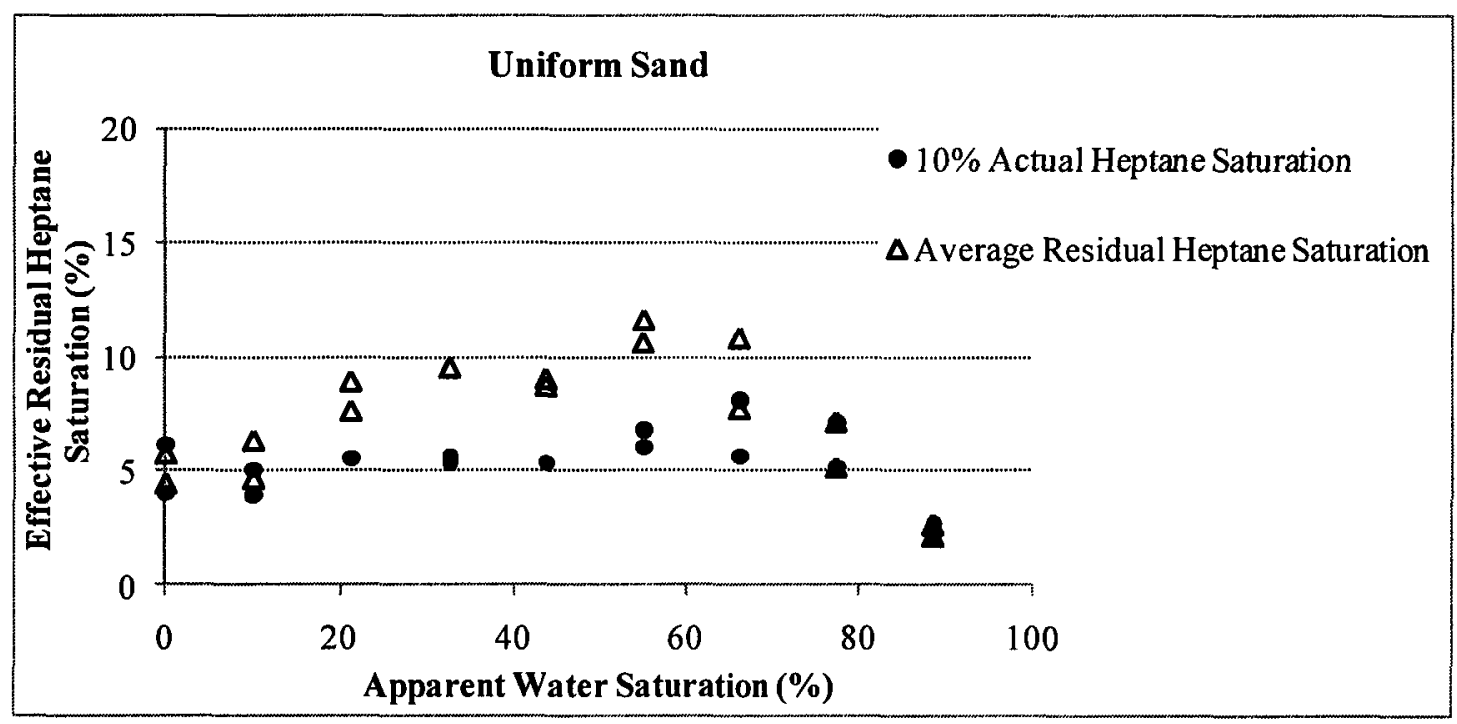

Figure 7.3: Effective Residual Heptane Saturation-Apparent Water Saturation in Uniform Sand. 


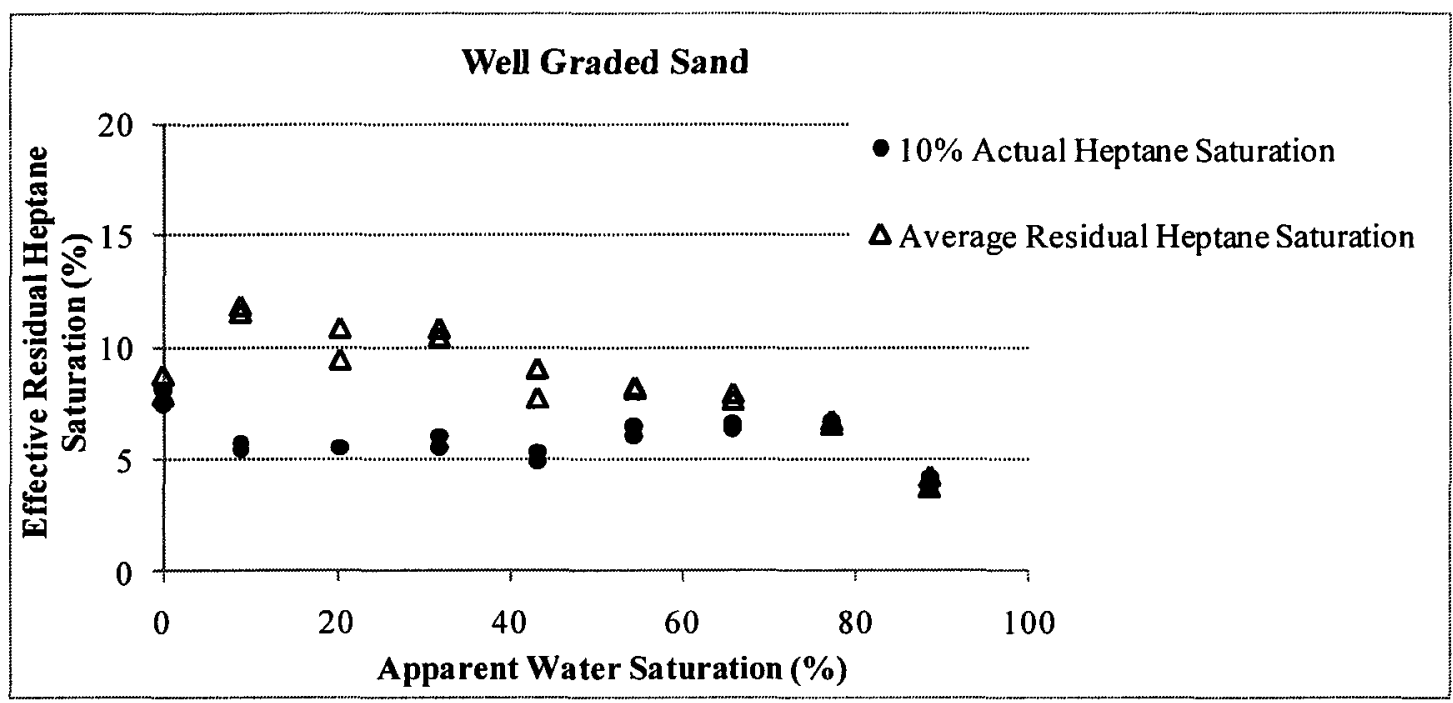

Figure 7.4: Effective Residual Heptane Saturation-Apparent Water Saturation in Well Graded Sand.

\subsubsection{Conceptual Model for Residual NAPL Saturation}

Lenhard et al. (2004) conceptually described the residual NAPL formation and distribution in a water-wet porous medium. According to their model, the residual NAPL starts to build up in the porous medium as the NAPL imbibes. They stated that the residual NAPL exists in the porous medium as a thin film on water surfaces, and fills

small pores or pore throats, and small pore wedges. Their conceptual description of residual NAPL formation and distribution in a water-wet porous medium did not take into account the water saturation effects. Instead, they focused on the residual NAPL forms and distribution at irreducible water saturation. There are some concerns with their conceptual description of the residual NAPL, which are discussed in this section.

Lenhard et al. (2004) stated that the residual NAPL in a water-wet porous medium might be considered as the summation of three components; film on water surfaces, filling small pores or pore throats, and filling pore wedges. It is hypothesized here that the NAPL film on water surfaces exists at any water saturation. The magnitude of this 
residual NAPL component depends on the NAPL/water interfacial area $\left(\mathrm{SA}_{\mathrm{NAPL}}\right.$ /Water $)$ and the thickness of the NAPL film. Only in an air/NAPL two-phase system, the irreducible NAPL fills the small pores or pore throats and pore wedges. It should not otherwise because these are filled or partially filled with water depending on their size. Figure 7.5 is a schematic of water and NAPL forms and distribution in the pore space both at low and intermediate water saturations.

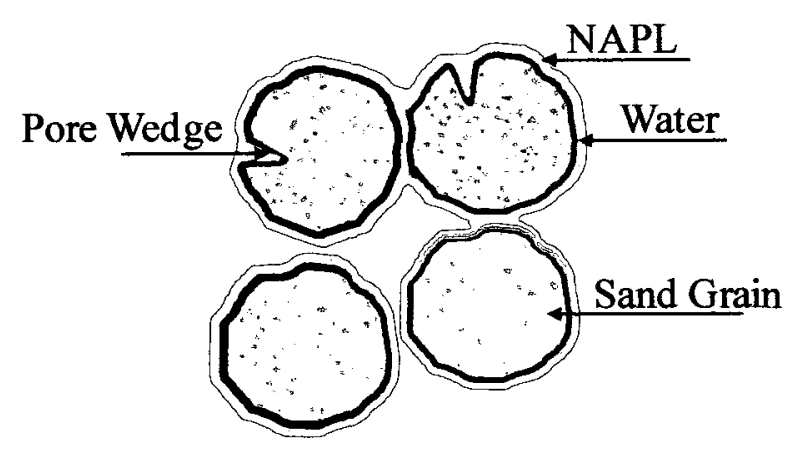

A. At Low Water Saturation

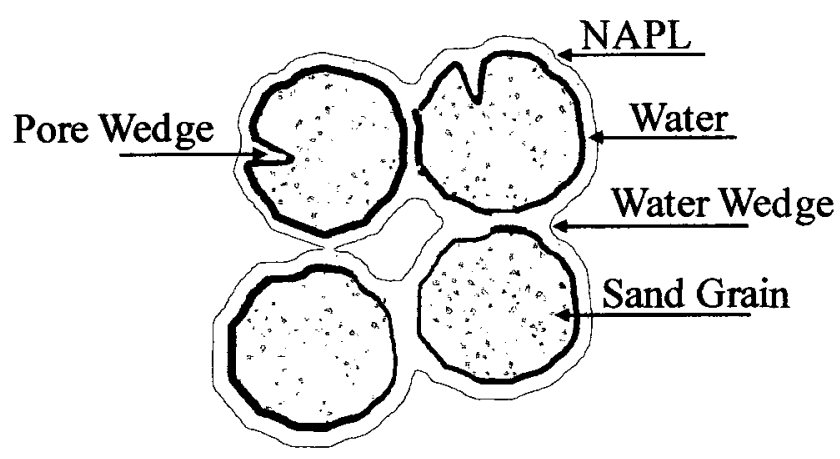

B. At Intermediate Water Saturation

Figure 7.5: Residual NAPL Forms; A. At Low Water Saturation and B. At Intermediate Water Saturation. 
It is conceptualized that, the residual NAPL might be considered as the summation of two components; NAPL film on water surfaces and NAPL present in wedges (i.e., either larger pore wedges as defined by Lenhard et al. (2004), which are partially filled with water, or water wedges at or near grain contacts). The larger pore wedges defined by Lenhard et al. (2004), which are partially filled with water, or the water wedges at or near grain contacts are referred to as water wedges in this text. A water wedge, as shown in Figure 7.6, is a part of the soil pore that is located at or near the contact points of two soil grains, or is positioned between the pore body and the pore throat, or the large pore wedge that is partially filled by water.

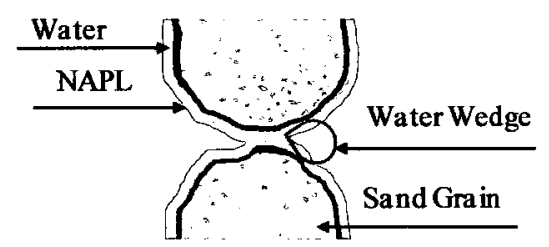

A. At Low Water Saturation

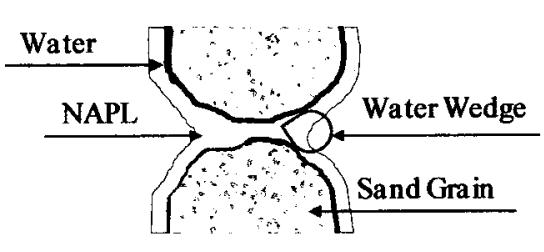

B. At Intermediate Water Saturation

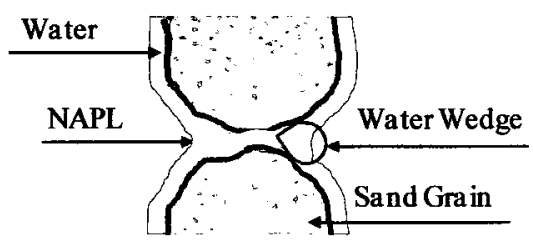

C. At Higher Water Saturation

\section{Figure 7.6: Water Wedge; A. At Low Water Saturation, B. At Intermediate Water Saturation, and C. At Higher Water Saturation.}

As proposed in Figure 7.5, at low water saturations in an air/NAPL/water threephase system, the residual NAPL is present as a film on water surfaces. Even in pore throats, at low water saturations, the residual NAPL is present as a film on water surfaces. 
At high water saturations, the water fills the small soil pores or pore throats and then the residual NAPL is present only in larger soil pores as a film on water surfaces and filling water wedges. It is conceptualized that the fraction of residual NAPL filling water wedges is proportional to water and imbibing NAPL saturations. The fraction of residual NAPL filling the water wedges is low at low water saturations (e.g., water saturations close to irreducible water saturation). At intermediate water saturations (e.g., from $20 \%$ up to $70 \%$ actual water saturations), the residual NAPL filling water wedges increases as water saturation increases. At higher water saturations (e.g., from $70 \%$ up to $100 \%$ actual water saturations), the residual NAPL filling water wedges decreases as water saturation increases. At any water saturation, it is conceptualized that the fraction of the residual NAPL filling the water wedges increases as the imbibing NAPL saturation increases. Moreover, it is conceptualized that the number and size of water wedges is a function of the porous medium grain size distribution. Therefore, the residual NAPL saturation component filling water wedges is a function of the porous medium grain size distribution. This explains why the residual NAPL saturation magnitude and trend are different in the uniform sand and the well graded sand, for the same water and imbibing NAPL saturation histories in both media. The uniform sand has pores of similar sizes but the well graded sand has more pores of dissimilar sizes. Hence, the distribution and magnitude of residual NAPL in water wedges is different in these sands for the same water and imbibing NAPL saturation histories. This conceptual description of residual NAPL forms and distribution explains the residual heptane saturation-heptane saturation data and the residual heptane saturation-water saturation data presented in Figure 7.1, Figure 7.2, Figure 7.3, and Figure 7.4. 
In summary, the residual NAPL is a summation of two components; NAPL film on water surfaces and NAPL filling water wedges. The NAPL film on water surfaces is constant at constant water saturation and it should not depend on the imbibing NAPL saturation as long as the imbibing NAPL volume is large enough to form the NAPL film on water surfaces. This residual NAPL component is discussed in Section 7.2.2.a. In contrast, the second residual NAPL component (i.e., the NAPL filling water wedges) depends on the imbibing NAPL and water saturations. As discussed in Section 7.2.1.a, the residual NAPL saturation increases linearly or nonlinearly with the increase of the imbibing NAPL saturation in the uniform sand or the well graded sand, respectively. Moreover, the data presented in Section 7.2.1.b shows that the relationship between the residual NAPL saturation and water saturation is close to quadratic. The second residual NAPL component is discussed in Sections 7.2.2.b and 7.2.2.c.

The conceptual model shown in Figure 7.7 is the proposed relationship between residual NAPL saturation and water saturation at a fixed imbibing NAPL saturation (e.g., $10 \%)$. This relationship could be represented by $C_{w}$. In addition, at point $\mathrm{A}$ on $C_{w}$, the relationship between the residual NAPL saturation and the imbibing NAPL saturation may be represented by $C_{n}$. The apparent NAPL saturation in $C_{n}$ may take any value between zero and $1-\overline{\bar{S}}_{w}$. The relationships $C_{w}$ and $C_{n}$ include the two residual NAPL saturation components and are discussed in the following sections. 


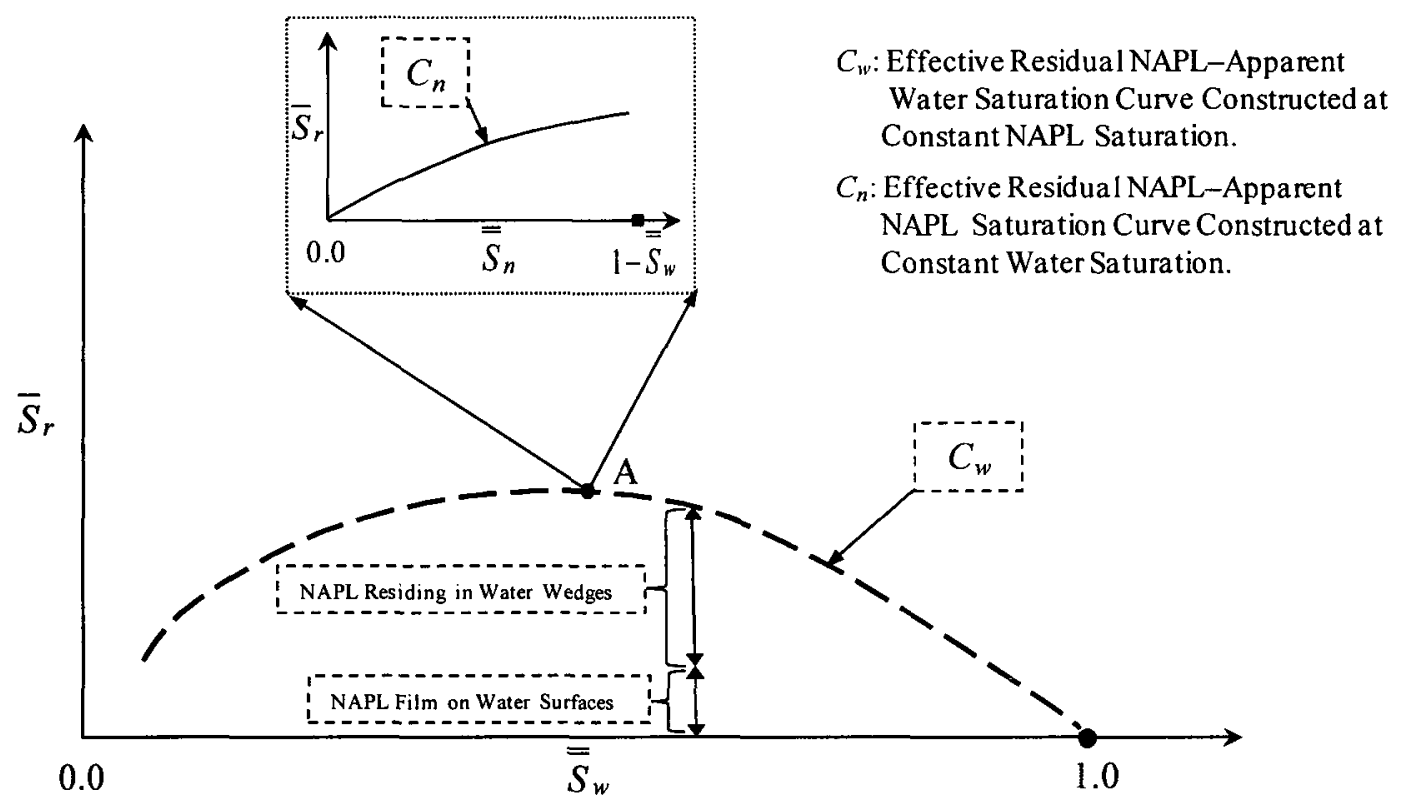

Figure 7.7: Conceptual Model for Residual NAPL Saturation-Water Saturation and Residual NAPL Saturation-NAPL Saturation Relationships.

\subsection{2.a. Estimation of First Residual NAPL Saturation Component; NAPL Film on}

\section{Water Surfaces}

The volume of the NAPL film on water surfaces is proportional to the NAPL/water interfacial area $\left(\mathrm{SA}_{\mathrm{NAPL}} /\right.$ water $)$ and the thickness of the NAPL film in the porous medium. The NAPL/water interfacial area is proportional to the water saturation. Cary (1994) developed a relationship to calculate two immiscible fluids interfacial area as a function of the wetting phase saturation, the soil grains surface area, and the $P_{c} \rightarrow S$ relationship. Figure 7.8 shows the relationship between the NAPL/water interfacial area and the water saturation. The water saturation at point $\mathrm{A}$ in Figure 7.8 is zero and the NAPL/water interfacial area is equivalent to the soil grains surface area. In contrast, at point $B$ in Figure 7.8 , the porous medium is $100 \%$ water-saturated and the NAPL/water interfacial area is zero. 


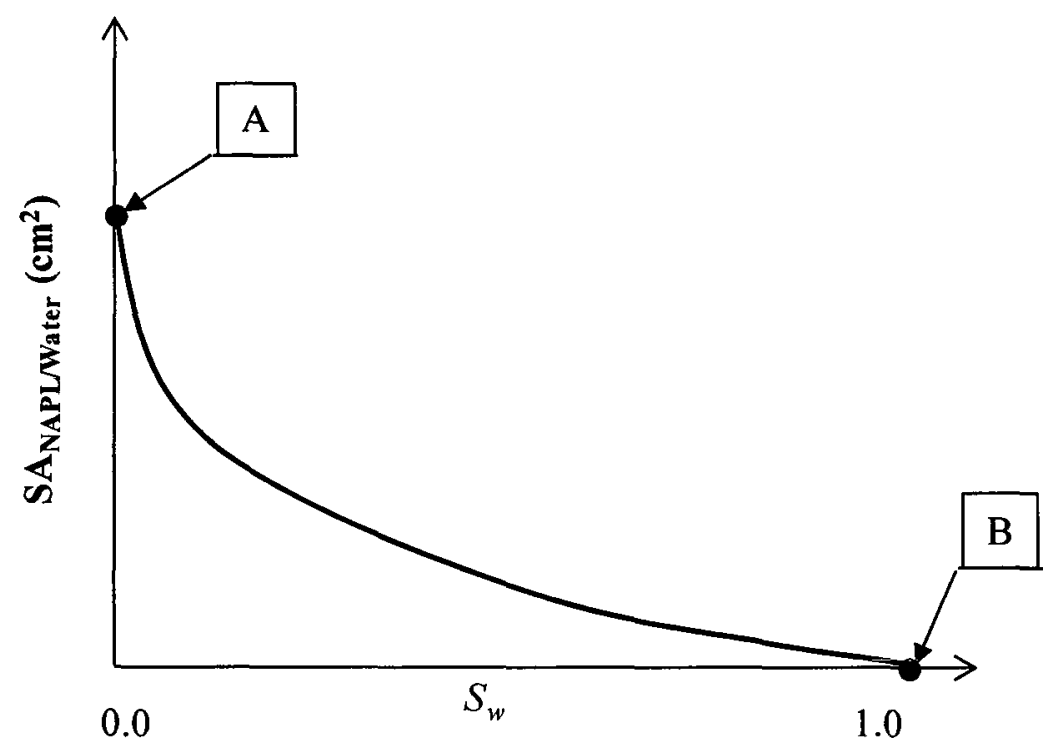

Figure 7.8: NAPL/Water Interfacial Area (SANAPL/Water)-Actual Water Saturation.

If the thickness of the NAPL film, at the residual NAPL saturation, does not change when the water saturation changes, then the volume of the NAPL film on water surfaces varies with the water saturation the same way the NAPL/water interfacial area varies. Figure 7.9 shows the relationship between the volume of the NAPL film and the water saturation. The porous medium is dry at point $\mathrm{C}$ in Figure 7.9 (i.e., air/NAPL twophase system) and the volume of the NAPL film is maximum. In contrast, point D in Figure 7.9 corresponds to a water-saturated porous medium and the volume of the NAPL film is zero. Similarly, for a fixed NAPL film thickness, the residual NAPL saturation component that takes the form of NAPL film on water surfaces should follow a trend similar to the trend shown in Figure 7.9. 


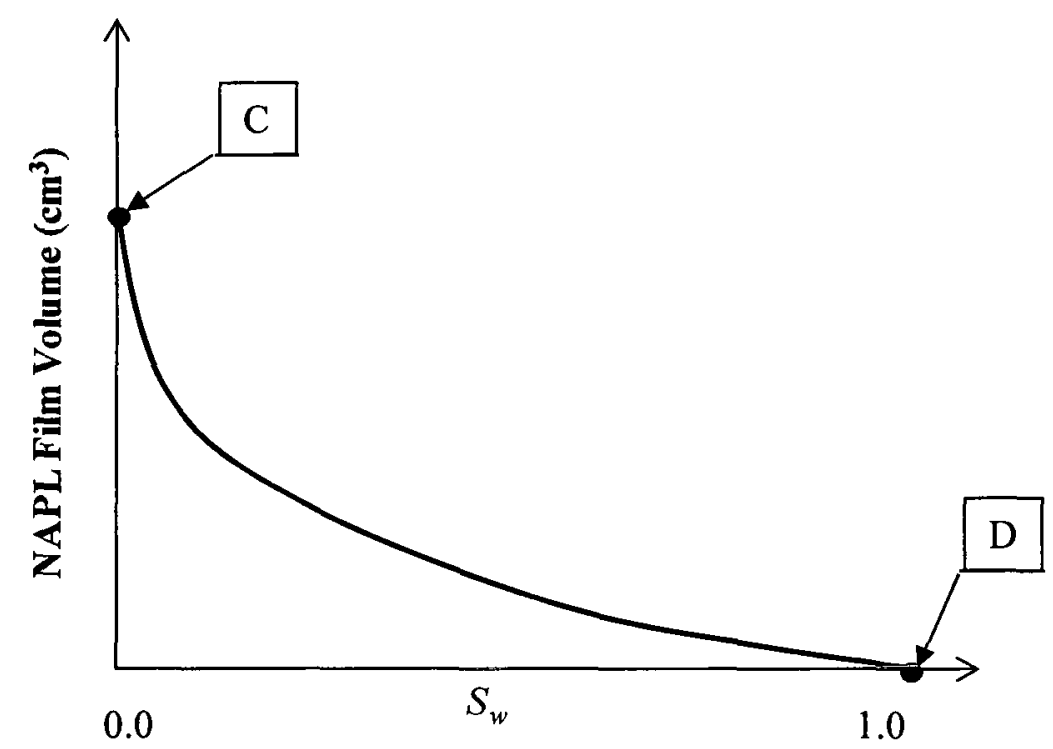

Figure 7.9: NAPL Film Volume-Actual Water Saturation.

In order to calculate the film component of the residual NAPL saturation, the volume of the NAPL film on water surfaces must be estimated. The volume of the film residual NAPL saturation component, at known water saturation, is the product of the NAPL film thickness and the NAPL/water interfacial area. The NAPL/water interfacial area is calculated according to Cary (1994) as follows:

$$
S A=\left(2 \eta^{-\lambda} / r_{0}\right)\left[\left(\Gamma \eta S_{\text {monolayer }} / \lambda\right)\left(\eta^{\lambda}-\left(\eta S_{w}\right)^{\lambda}\right)+(\lambda+1)^{-1}\left(\eta^{\lambda+1}-\left(\eta S_{w}\right)^{\lambda+1}\right)\right]
$$

Where:

$S A:$ NAPL/water specific interfacial area (interfacial area in $\mathrm{cm}^{2}$ per $\mathrm{cm}^{3}$ of the porous medium);

$r_{0}$ : pore radius that defines the NAPL entry value of the water-saturated porous medium; $S_{\text {monolayer }}$ actual water saturation corresponding to a monolayer of water on the porous medium grains; and 
$\Gamma$ : dry end fitting parameter; it is determined from the above equation by equating the NAPL/water specific interfacial area to the porous medium specific surface area at water saturation calculated for a monolayer of water.

One of the important parameters in this relationship, Equation 7.1, is the fitting parameter $\lambda$. This fitting parameter reflects the effect of porous medium grain size distribution on the NAPL/water interfacial area at a given water saturation, if the other parameters are fixed. Figure 7.10 shows the effect of variation of $\lambda$ on the NAPL/water interfacial area-actual water saturation relationship. It is noticed from the figure that for a more uniform and less graded porous medium (i.e., large $\lambda$ ), the relationship is flatter at low water saturations. This means that the NAPL/water interfacial area, and hence the film component of the residual NAPL saturation, does not change when water saturation increases at low water saturations. In contrast, in a more graded porous medium (i.e., small $\lambda$ ), the NAPL/water interfacial area-water saturation relationship is steeper. This means that the NAPL/water interfacial area, and hence the film component of the residual NAPL saturation, changes when water saturation increases over the entire water saturation range (i.e., from irreducible water saturation to a water saturation of unity). This is consistent with the tangible reduction in NAPL/water interfacial area in a well graded porous medium (i.e., $\lambda=0.25$, shown in Figure 7.10 ) when water saturation increases, as a large fraction of the small pores become filled with water which decreases the NAPL/water interfacial area. In contrast, in a uniform porous medium (i.e., $\lambda=3.0$, shown in Figure 7.10), when water saturation increases, the NAPL/water interfacial area advances towards pore centres as all the pores progressively fill and hence the reduction in the interfacial area is smaller. 


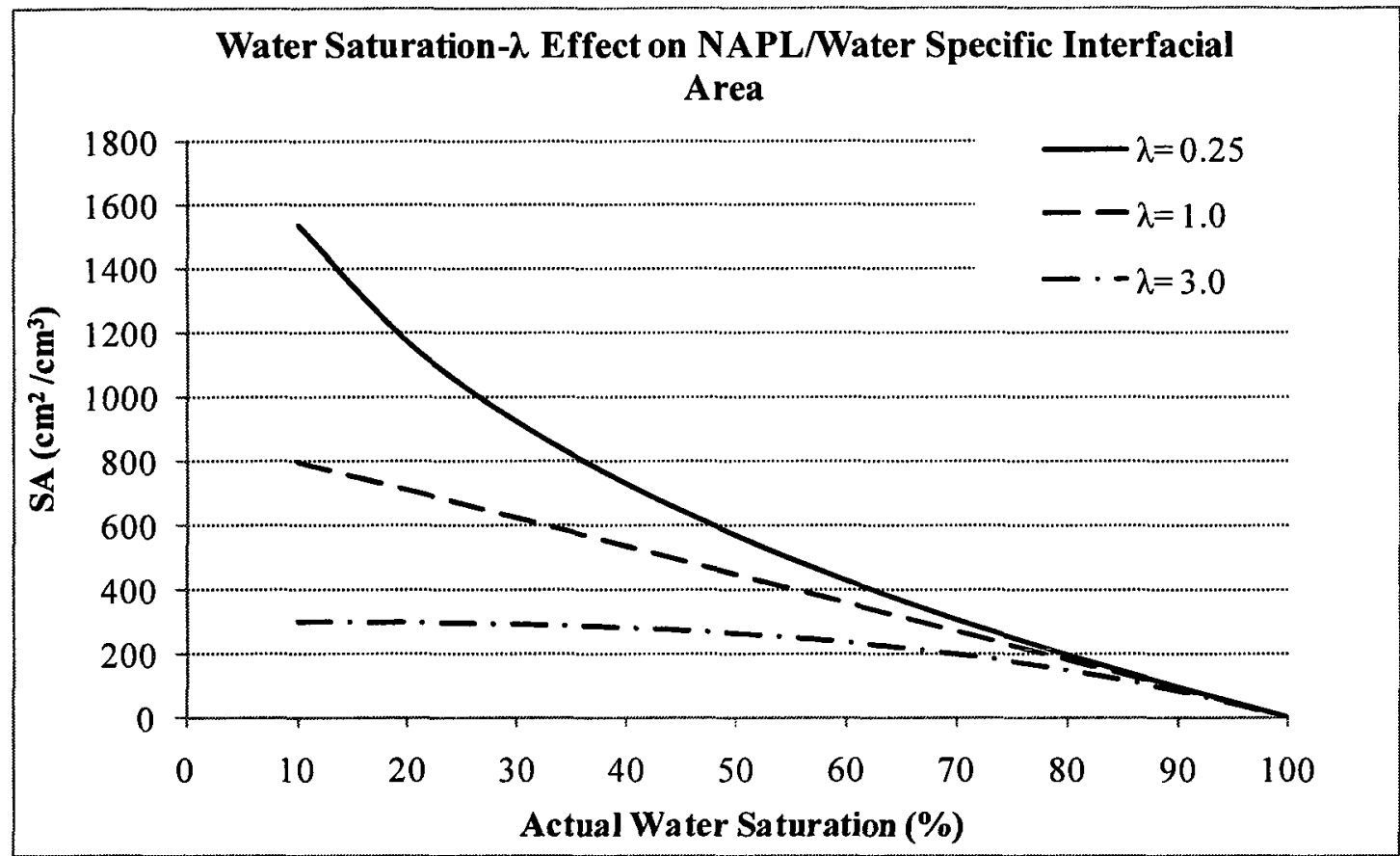

Figure 7.10: Fitting Parameter $\lambda$-Actual Water Saturation Effect on the NAPL/Water Specific Interfacial Area.

As explained in Chapter 3, the specific surface areas of the uniform sand and the well graded sand were measured using nitrogen adsorption. The results indicated that the uniform and well graded sands had similar specific surface areas. The heptane entry head and the fitting parameter $\lambda$ for the uniform sand and the well graded sand were calculated by fitting Brooks and Corey relationship to the heptane/water capillary pressure headactual water saturation data in these media, respectively, as shown in Figure 7.11 and Figure 7.12. After calculating or measuring the porous medium physical soil properties, Equation 7.1 was used to calculate the heptane/water specific interfacial area at a given water saturation. The other physical properties of the tested uniform and well graded sands are shown in Table 7.1. 


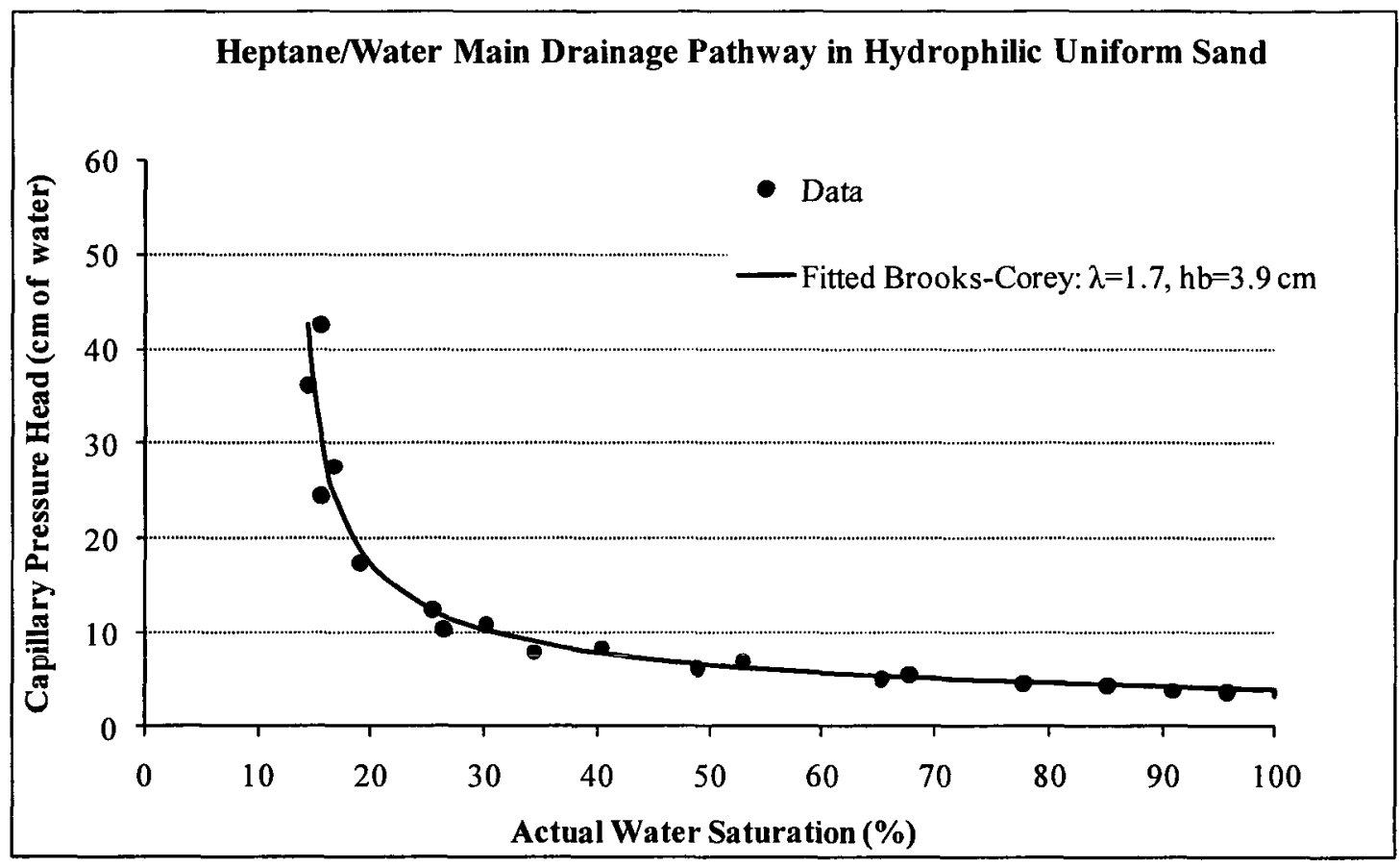

Figure 7.11: Capillary Pressure Head-Actual Water Saturation in Heptane/Water Two-Phase System in Uniform Sand.

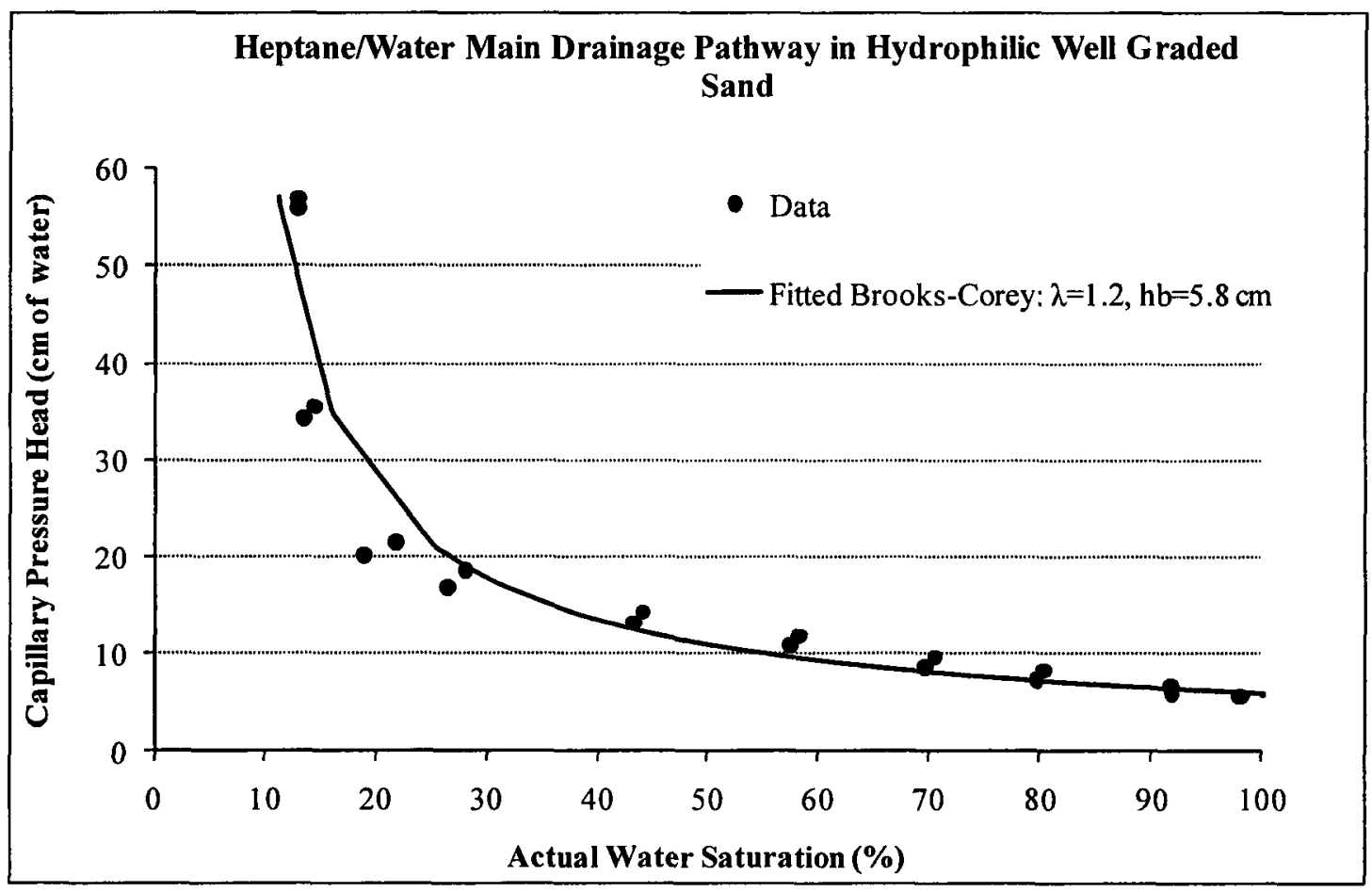

Figure 7.12: Capillary Pressure Head-Actual Water Saturation in Heptane/Water Two-Phase System in Well Graded Sand. 
Table 7.1: Physical Soil Properties.

\begin{tabular}{|l|c|c|}
\hline Sand Type & Uniform & Well Graded \\
\hline Specific Surface Area $\left(\mathrm{cm}^{2} / \mathrm{cm}^{3}\right)$ & 1030.3 & 1063.4 \\
\hline Heptane Entry Head $\left(h_{b}\right)(\mathrm{cm})$ & 3.9 & 5.8 \\
\hline Porous Medium Porosity $(\eta)$ & 0.374 & 0.34 \\
\hline Fitting Parameter $(\lambda)$ & 1.7 & 1.2 \\
\hline Monolayer of Water Saturation $\left(S_{\text {monolayer }}\right)$ & $8.5 \times 10^{-5}$ & $9.7 \times 10^{-5}$ \\
\hline Dry End Fitting Parameter $(I)$ & 329465.2 & 154255.0 \\
\hline$S A$ at Irreducible Water Saturation $\left(\mathrm{cm}^{2} / \mathrm{cm}^{3}\right)$ & 1009.7 & 985.7 \\
\hline
\end{tabular}

*Determined as explained in Cary (1994) with an assumed depth of water equivalent to the thickness of a water monolayer.

One possible way to estimate the heptane film thickness, and to determine the first component of the residual heptane at a given water saturation, was to consider the residual heptane measured at irreducible water saturation when a certain volume of heptane equivalent to $10 \%$ of the pore space imbibed into the porous medium and subsequently drained to take the form of a heptane film (i.e., $S_{r}=S_{r_{f} \text { flm }}$ at irreducible water saturation). In air/heptane/water three-phase system, at irreducible water saturation, the volumes of the heptane film on water surfaces in $158.95 \mathrm{~cm}^{3}$ volume of uniform sand and well graded sand were $2.87 \mathrm{~cm}^{3}$ and $4.04 \mathrm{~cm}^{3}$, respectively. These residual heptane volumes correspond to heptane film thicknesses of $0.18 \mu \mathrm{m}$ and $0.26 \mu \mathrm{m}$ in uniform and well graded sands, respectively. It is hypothesized that this residual heptane volume in the uniform sand (i.e., $2.87 \mathrm{~cm}^{3}$ ) is a heptane film on water surfaces. In contrast, the residual heptane volume in the well graded sand (i.e., $4.04 \mathrm{~cm}^{3}$ ) is partly a heptane film on water surfaces and partly a heptane residing in water wedges. Therefore, the residual heptane volume in the uniform sand was used to calculate the heptane film thickness, which corresponds to $0.18 \mu \mathrm{m}$. Alternatively, a NAPL film thickness on water surfaces may be obtained from literature. Knowledge of heptane/water interfacial area, calculated at a given water saturation, and the heptane film thickness were used to determine the 
first component of the residual heptane saturation. The NAPL film spreading on water surfaces is a function of water saturation and does not depend on the NAPL saturation as long as the NAPL saturation is large enough for this film to form on water surfaces. Figure 7.13 shows the effective saturation of the heptane film-apparent water saturation curves in the uniform and well graded sands.

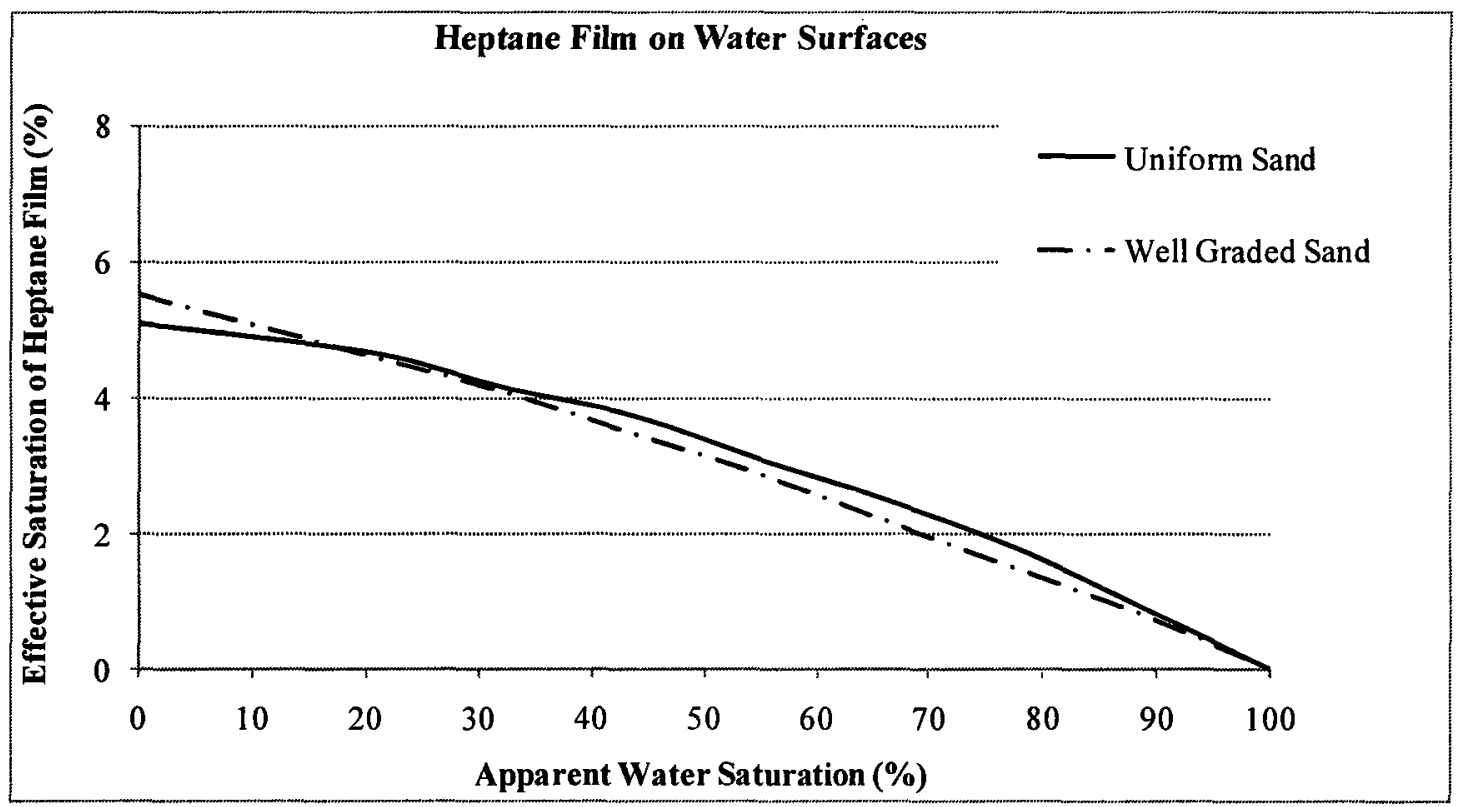

Figure 7.13: Effective Saturation of Heptane Film-Apparent Water Saturation in Well Graded and Uniform Sands.

\subsection{2.b. Water Saturation Effect on Residual NAPL Saturation}

It was described in Section 7.2.2.a how the water saturation affects the magnitude of the first component of the residual NAPL saturation. It was shown in Figure 7.3 and Figure 7.4 that the residual heptane saturation-water saturation data, at a fixed imbibing heptane saturation (e.g., 10\% actual heptane saturation), does not follow the same trend shown in Figure 7.13 (i.e., effective saturation of heptane film-apparent water saturation) 
which supports the hypothesis that there is a second component to the residual NAPL saturation. It is conceptualized, as explained in Section 7.2.2, that the water saturation affects the magnitude of residual NAPL saturation residing in the water wedges. It was explained in Section 7.2.2 that the residual heptane saturation-water saturation relationship was close to quadratic. The residual heptane saturation-water saturation data were fitted to some functions (including the quadratic function). It was found that the relationship of the NAPL saturation residing in water wedges-water saturation relationship has the following form:

$\bar{S}_{r_{-} \text {waterwedge_w }}=\left(\bar{S}_{r_{-} \max }-\bar{S}_{r_{-} f l m}\right) *\left(\bar{S}_{w}^{2 \lambda}-\overline{\bar{S}}_{w}^{3 \lambda}\right)$

Where,

$\bar{S}_{r_{-} \text {water wedge_w }}$ : effective residual NAPL saturation in water wedges;

$\bar{S}_{r_{-} \max }$ : maximum effective residual NAPL saturation in the porous medium;

$\bar{S}_{r_{-} \text {film }}$ : effective saturation of residual NAPL that takes the form of NAPL film on water surfaces; and

$\left(\bar{S}_{r_{-} \max }-\bar{S}_{r_{-} \text {flm }}\right):$ maximum effective residual NAPL saturation that could be present in water wedges.

This relationship (i.e., Equation 7.2) estimates the water saturation effect on the saturation of the NAPL residing in water wedges. The water saturation effect is a multiplier of the maximum effective residual NAPL saturation that could be present in water wedges. This multiplier is a function of the apparent water saturation and the fitting parameter $\lambda$. This fitting parameter reflects the effect of the porous medium grain size distribution on the multiplier at a given water saturation. As shown in Figure 7.14, the 
effective residual NAPL saturation-apparent water saturation relationship is close to quadratic. Depending on the value of $\lambda$, the peak of this relationship is shifted. For a well graded porous medium (i.e., $\lambda=0.25$ ), the peak is located at low water saturation, as the number and size of water wedges, within which the residual NAPL can reside, is larger than the number and size of water wedges at high water saturations. In contrast, for a uniform porous medium (i.e., $\lambda=3.0$ ), the size of water wedges at low water saturations is so large that it cannot hold residual NAPL. As water saturation increases, the size of the water wedges decreases which enhances the locations where NAPL can reside as residual in these wedges, and this shifts the peak to occur at high water saturation.

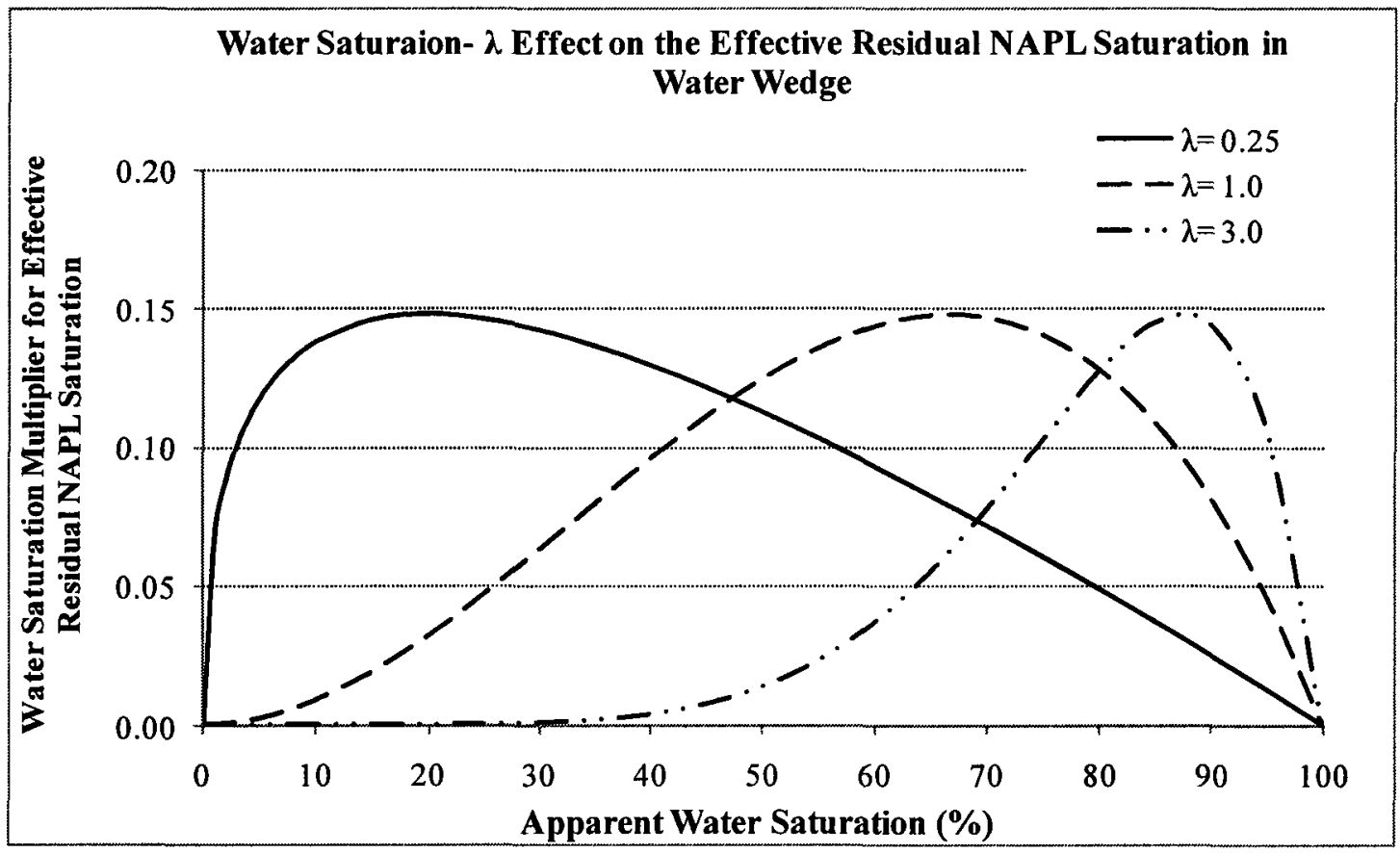

Figure 7.14: Water Saturation-Porous Medium Grain Size Distribution Effect on the Effective Residual NAPL Saturation in Water Wedges.

It is obvious that the saturation of NAPL residing in water wedges depends on the combined effect of water and NAPL saturations. Therefore, checking the validity of the 
water saturation effect solely is impossible. Instead, the combined effect of water and NAPL saturations is checked after the NAPL saturation effect is determined (i.e., at the end of Section 7.2.2.c).

\subsection{2.c. NAPL Saturation Effect on Residual NAPL Saturation}

It was described in Section 7.2.2.a that part of the imbibing NAPL resides in the porous medium as a NAPL film and a second part, as described in Section 7.2.2.b, resides in water wedges. The volume of NAPL making the film does not depend on the imbibing NAPL volume as long as the latter is larger than the NAPL film volume. The imbibing NAPL saturation affects the saturation of the NAPL residing in water wedges. It was shown in Section 7.2.1.a that the residual heptane saturation-imbibing heptane saturation relationship, at fixed water saturation, is close to linear in the uniform sand and nonlinear in the well graded sand. In either sand, the saturation of the heptane residing in water wedges increases as the imbibing heptane increases. The residual heptane saturationheptane saturation data were fitted to many functions. It was found that the relationship of the NAPL saturation residing in water wedges-NAPL saturation relationship has the following form:

$\bar{S}_{r_{-} \text {water wedge } n}=\left(\bar{S}_{r_{-} \max }-\bar{S}_{r_{-} \text {film }}\right)^{*}\left(\overline{\bar{S}}_{n}^{\text {hist } \max }-\frac{\left(\overline{\bar{S}}_{n}^{\text {hist }}{ }_{-}^{\max }\right)^{2}}{2}\right)$

Where,

$\overline{\bar{S}}_{n}^{\text {hist }}{ }^{\max }$ : historic maximum apparent NAPL saturation; and

$\bar{S}_{r_{-} \text {water wedge } n}$ : effective residual NAPL saturation in water wedges due to NAPL saturation effect. 
This relationship (i.e., Equation 7.3) estimates the NAPL saturation effect on the saturation of the NAPL residing in water wedges. The NAPL saturation effect is a second multiplier of the maximum effective residual NAPL saturation that could be present in water wedges. As shown in Figure 7.15, the effective residual NAPL saturation-apparent NAPL saturation relationship is nonlinear and the magnitude of the multiplier increases as NAPL saturation increases. This is conceptualized to be valid because as the NAPL saturation increases, the NAPL invades more pores and the NAPL volume that can reside in water wedges increases.

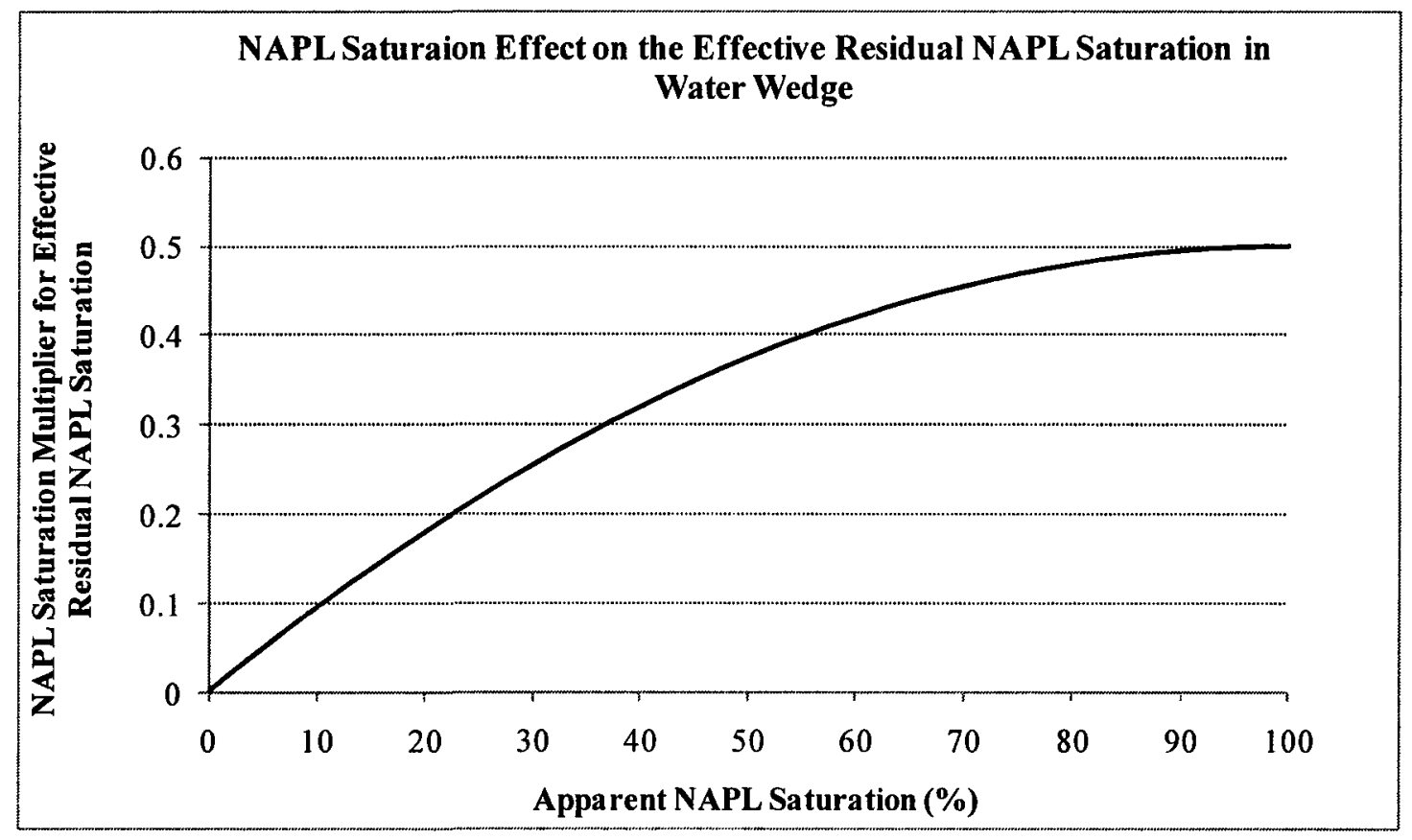

Figure 7.15: NAPL Saturation Effect on the Effective Residual NAPL Saturation in Water Wedges.

As mentioned earlier, the saturation of NAPL residing in water wedges depends on the combined effect of water and NAPL saturations. Therefore, checking the validity of the combined effect of water and NAPL saturations is more reasonable than checking 
each effect solely, which is not possible. The combined effect of water and NAPL saturations on the second component of the residual NAPL saturation is the product of the maximum effective residual NAPL saturation that could be present in water wedges by the first and the second multipliers, discussed earlier. In addition, a calibration term with a value of two was added to the first multiplier. The resulting relationship, which reflects the effect of water and NAPL saturation on the residual NAPL saturation enclosed in water wedges, is as follows:

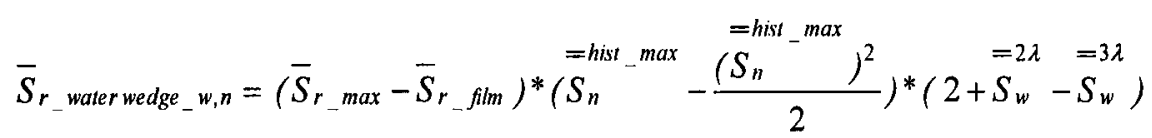

Where,

$\bar{S}_{r_{-} \text {waterwedge_w, } n}:$ effective residual NAPL saturation in water wedges due to water and NAPL saturation effect.

In order to check the validity of this component of the conceptual model, at imbibing heptane saturation of $10 \%$, the first component of the residual heptane (i.e., heptane films on water surfaces) was subtracted from the residual heptane saturations left in the porous medium after a volume of heptane, equivalent to $10 \%$ actual heptane saturation, imbibed into the porous medium and subsequently drained, at all water saturations. Then the remaining portions of the residual heptane saturations were plotted versus the corresponding water saturations. In addition, residual heptane saturations predicted according to Equation 7.4 were plotted in the same figure. Figure 7.16 and Figure 7.17 show the effective saturation of the heptane residing in water wedgesapparent water saturation relationships, at $10 \%$ actual heptane saturation, in the uniform sand and the well graded sand, respectively. As shown in the figures, the model 
predictions are slightly different than the experimental data but the trends are close which supports the conceptual model.

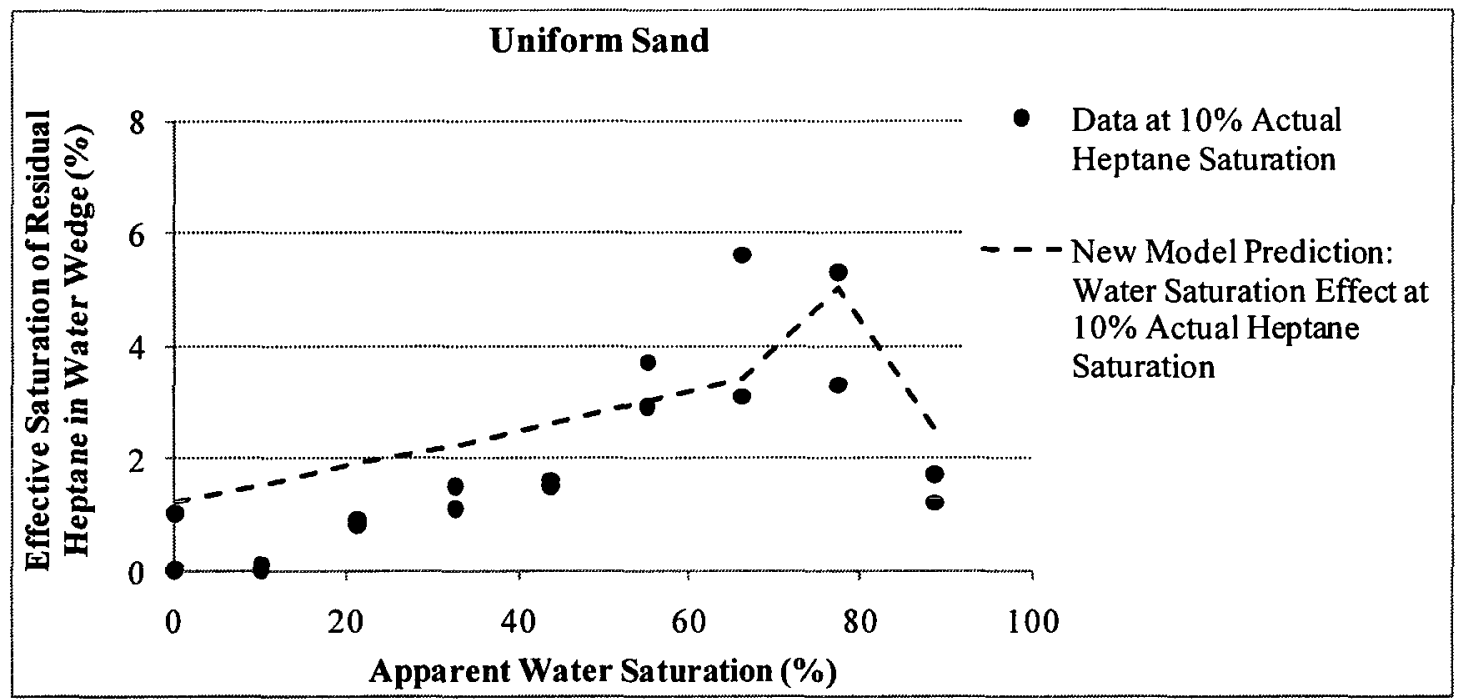

Figure 7.16: Effective Saturation of the Heptane Residing in Water WedgesApparent Water Saturation in Uniform Sand.

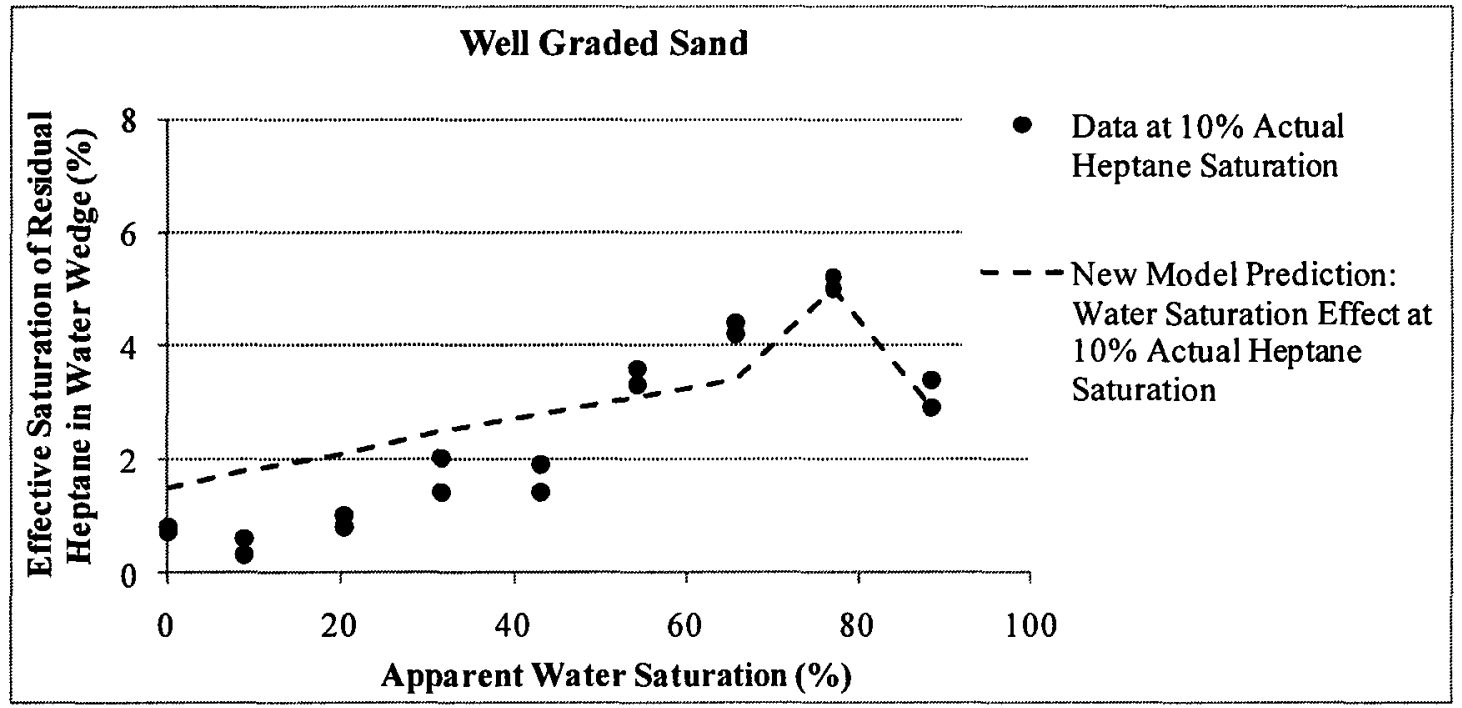

Figure 7.17: Effective Saturation of the Heptane Residing in Water WedgesApparent Water Saturation in Well Graded Sand.

Similarly, Figure 7.18 and Figure 7.19 show the effective saturation of the heptane residing in water wedges-apparent heptane saturation relationships, at $30 \%$ and 
$50 \%$ actual water saturations, in the uniform sand and the well graded sand, respectively. As shown in the figures, the model predictions are slightly different than the experimental data but the trends are close and this supports the conceptual model.

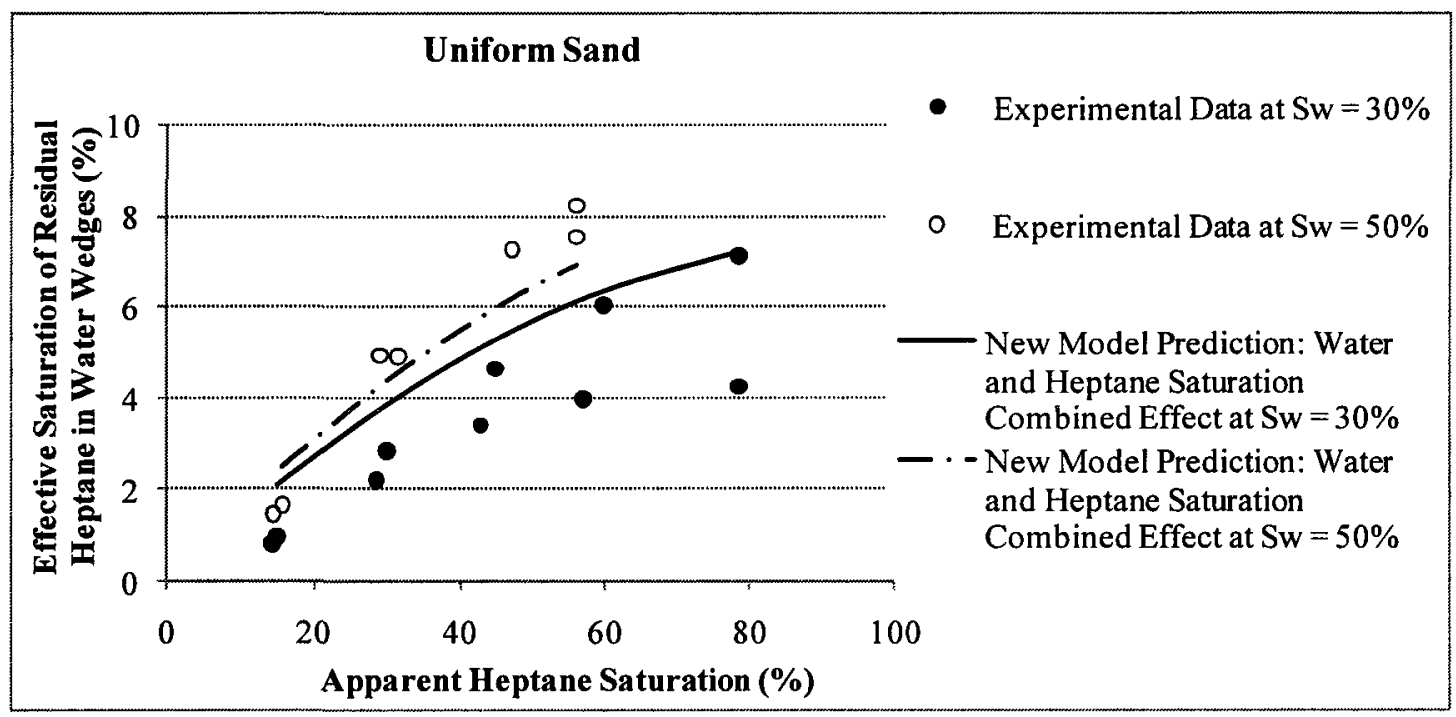

Figure 7.18: Effective Saturation of the Heptane Residing in Water WedgesApparent Heptane Saturation in Uniform Sand.

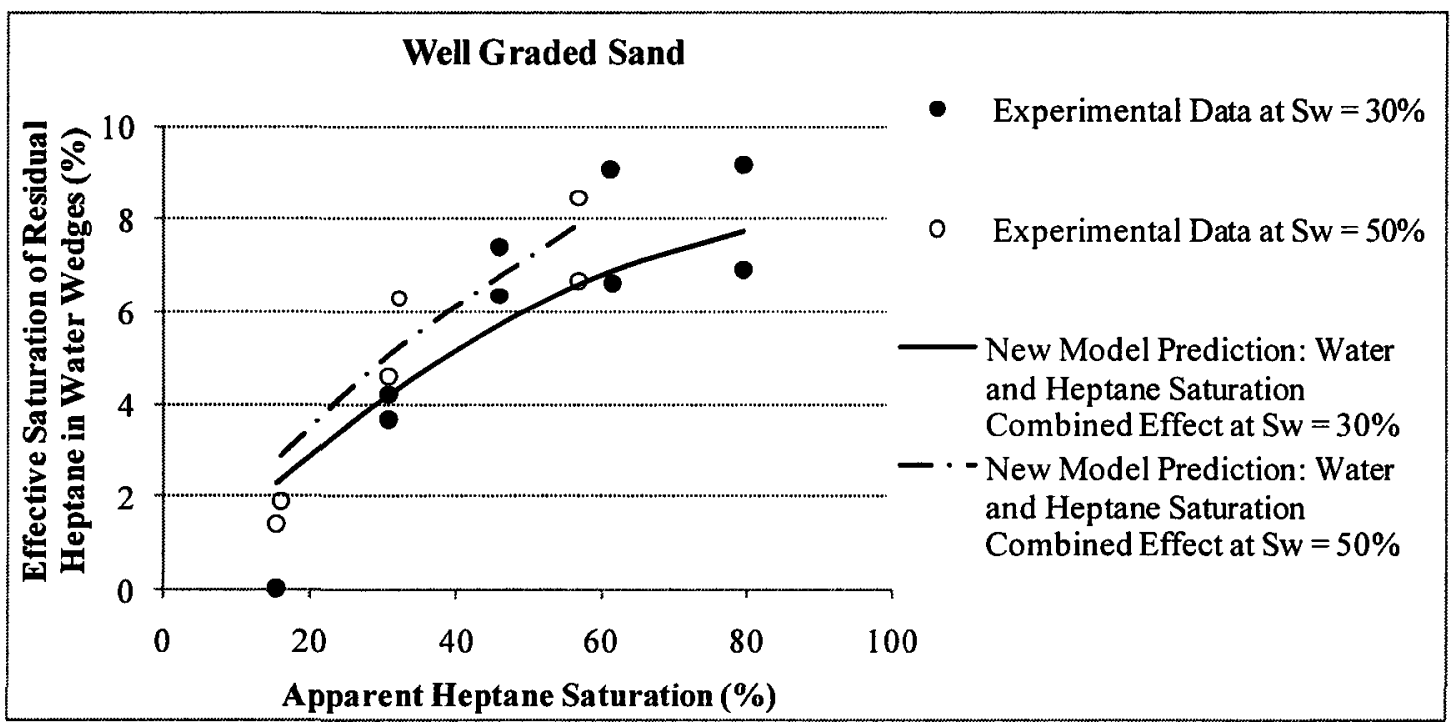

Figure 7.19: Effective Saturation of the Heptane Residing in Water WedgesApparent Heptane Saturation in Well Graded Sand. 
In summary, the new conceptual model, which predicts the effective residual NAPL saturation in a porous medium, includes two saturation components; NAPL film on water surfaces and NAPL residing in water wedges. The first component of the residual NAPL saturation depends only on water saturation as long as the imbibing NAPL volume is larger than the volume of the NAPL film. The second component of the residual NAPL depends on water and NAPL saturations. It is conceptualized that the NAPL film on water surfaces forms first and then, depending on the water and the imbibing NAPL saturations, a fraction of the imbibing NAPL resides in water wedges. Therefore, if the imbibing NAPL saturation is less than what is required to form the NAPL film on water surfaces, the residual NAPL saturation in the porous medium will be equivalent to the imbibing NAPL saturation. The new conceptual model is defined as follows:

$$
\begin{aligned}
& \bar{S}_{r}=\bar{S}_{n}^{=\text {hist }_{-} \text {max }} \\
& \text { if } \bar{S}_{n}{ }^{\text {hist }}{ }^{\max } \leq \bar{S}_{r_{-} \text {film }}
\end{aligned}
$$

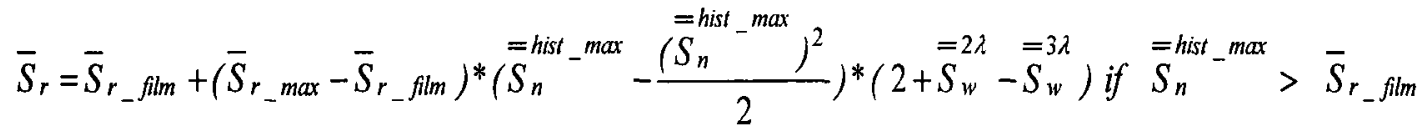

\subsubsection{Validation of Residual NAPL Saturation Conceptual Model}

The new conceptual model was used to predict the residual NAPL saturations in three experimental data sets; experimental data set (1), experimental data set (2), and Van Geel and Roy (2002) experimental data set. In addition, the model predictions were compared to the predictions of Van Geel and Roy (2002) linear and Land models, and Lenhard et al. (2004) model. 


\subsection{3.a. Experimental Data Set (1)}

The new conceptual model, the linear and Land models from Van Geel and Roy (2002), and the model developed by Lenhard et al. (2004) were used to predict the residual heptane saturations for this experimental data set. Both Van Geel and Roy (2002) and Lenhard et al. (2004) models assume that the effective residual NAPL saturation depends on three parameters; the initial apparent water saturation, the historic maximum apparent total liquid saturation, and the absolute maximum effective residual NAPL saturation. In the new model, it is assumed that the effective residual NAPL saturation depends on eight parameters; porous medium specific surface area, NAPL entry pressure head in NAPL/water two-phase system, porous medium grain size distribution (through fitting parameter $\lambda$ ), porous medium porosity, NAPL film thickness, apparent water saturation, historic maximum apparent NAPL saturation, and the absolute maximum effective residual NAPL saturation in the porous medium. The absolute maximum effective residual heptane saturation ranges from $8.7 \%$ to $15 \%$ with an average value of $12.1 \%$ in the uniform sand and it ranges from $8.6 \%$ to $17.5 \%$ with an average value of $12.7 \%$ in the well graded sand. The other input parameters were identified for each model prior to predicting the effective residual heptane saturations. Figure 7.20 through Figure 7.37 show the effective residual heptane saturation-apparent heptane saturation in uniform sand and well graded sand measured at actual water saturations corresponding to irreducible, $20 \%, 30 \%, 40 \%, 50 \%, 60 \%, 70 \%, 80 \%$, and $90 \%$.

As shown in Figure 7.20 through Figure 7.37, Van Geel and Roy (2002) and Lenhard et al. (2004) models could not predict the measured residual heptane saturation data in both porous media. There was one case where the Land model of Van Geel and 
Roy (2002), predicted the measured residual heptane saturation data in uniform sand at $30 \%$ actual water saturation as shown in Figure 7.24. In contrast, the new model predicted the measured residual heptane saturation data in most cases and provided a better fit than the models presented by Van Geel and Roy (2002) and Lenhard et al. (2004).

Van Geel and Roy (2002) linear model and Lenhard et al. (2004) model predictions are different than the measured data at the beginning, the trend, and the end. The beginning refers to the predicted or measured heptane that resided in the porous medium when a volume of heptane equivalent to $10 \%$ of the pore space imbibed into the porous medium and subsequently drained. The trend refers to the increase or decrease of residual heptane saturation when the heptane volume, which imbibed into the porous medium and subsequently drained, increased. The end refers to the predicted or measured heptane that resided in a porous medium when the imbibing heptane volume was large enough to attain $100 \%$ apparent total liquid saturation. Van Geel and Roy (2002) Land model predicted the trend but could not predict the measured data at the beginning and end.

One shortcoming of Van Geel and Roy (2002) and Lenhard (2004) models is that they assume the absolute maximum residual NAPL saturation occurs at the irreducible water saturation and that the apparent total liquid saturation, reached prior to NAPL drainage, is unity. This is not necessarily correct, since the absolute maximum residual heptane saturation occurs at $20 \%$ actual water saturation in the well graded sand and at $60 \%$ actual water saturation in the uniform sand. The models of Van Geel and Roy (2002) and Lenhard (2004), and the new model could not predict the effective residual 
heptane saturations at irreducible water saturation, as shown in Figure 7.20 and Figure 7.21. Another shortcoming of Van Geel and Roy (2002) and Lenhard et al. (2004) models is that they do not relate the residual NAPL saturation to soil properties (e.g., solids surface area and grain size distribution) or fluid properties (e.g., interfacial area, interfacial tension). Instead, they relate the residual NAPL saturation to the water saturation and the historic maximum apparent total liquid saturation.

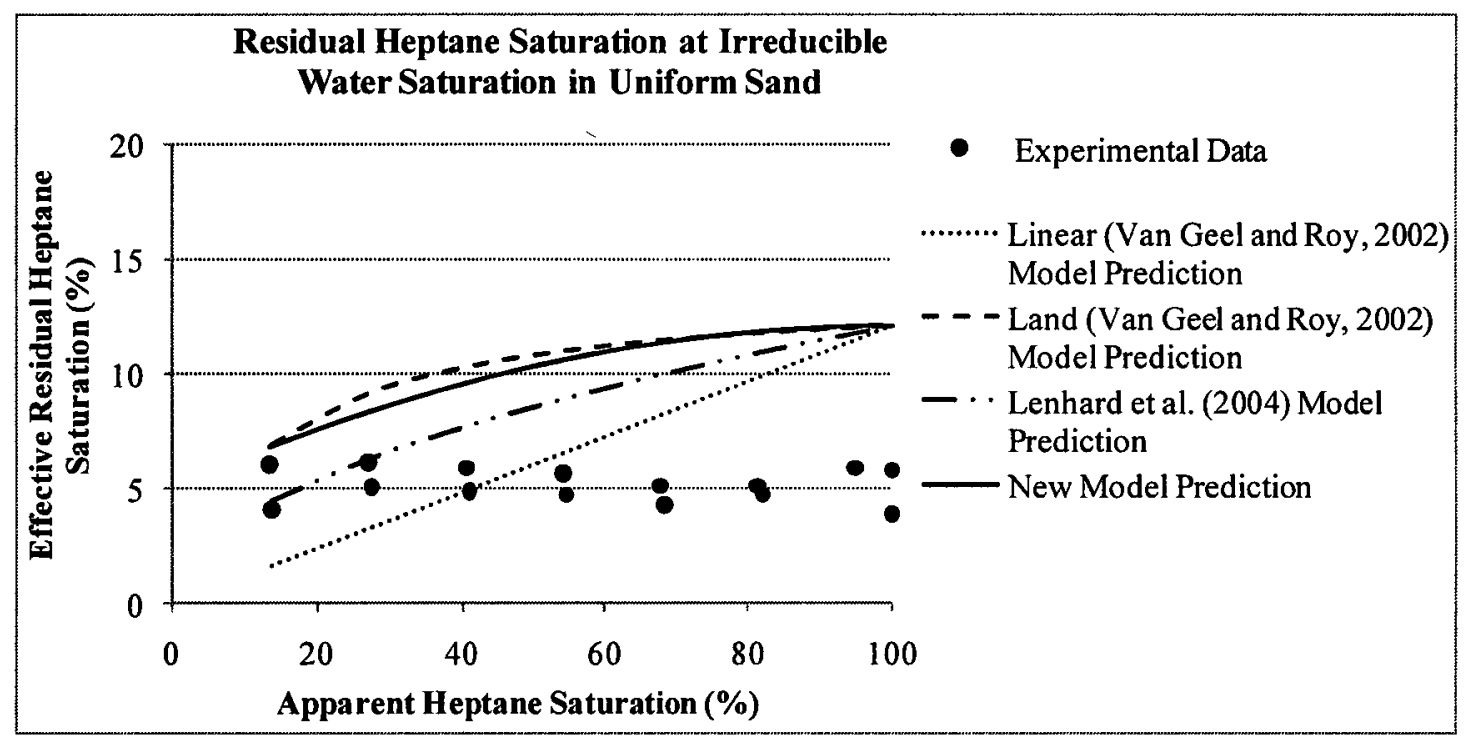

Figure 7.20: Effective Residual Heptane Saturation-Apparent Total Liquid Saturation at Irreducible Water Saturation in Uniform Sand. 


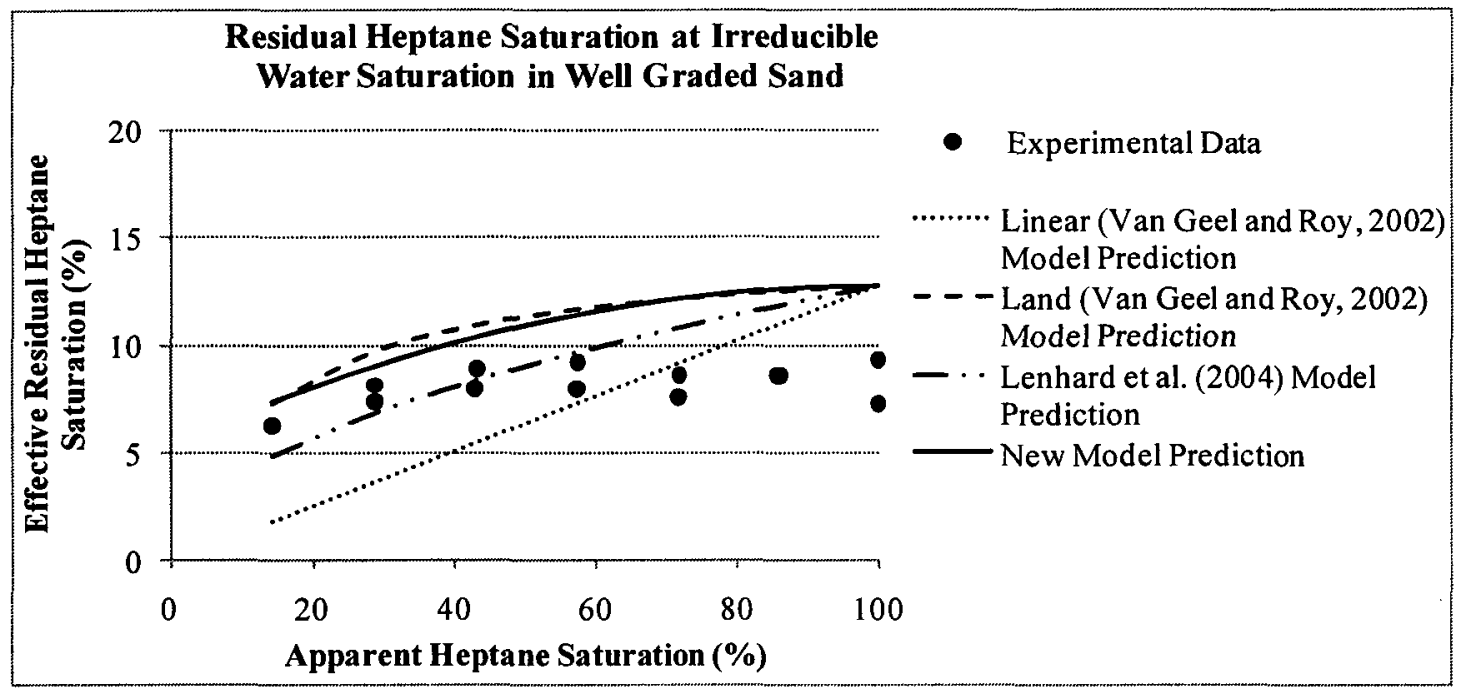

Figure 7.21: Effective Residual Heptane Saturation-Apparent Total Liquid Saturation at Irreducible Water Saturation in Well Graded Sand.

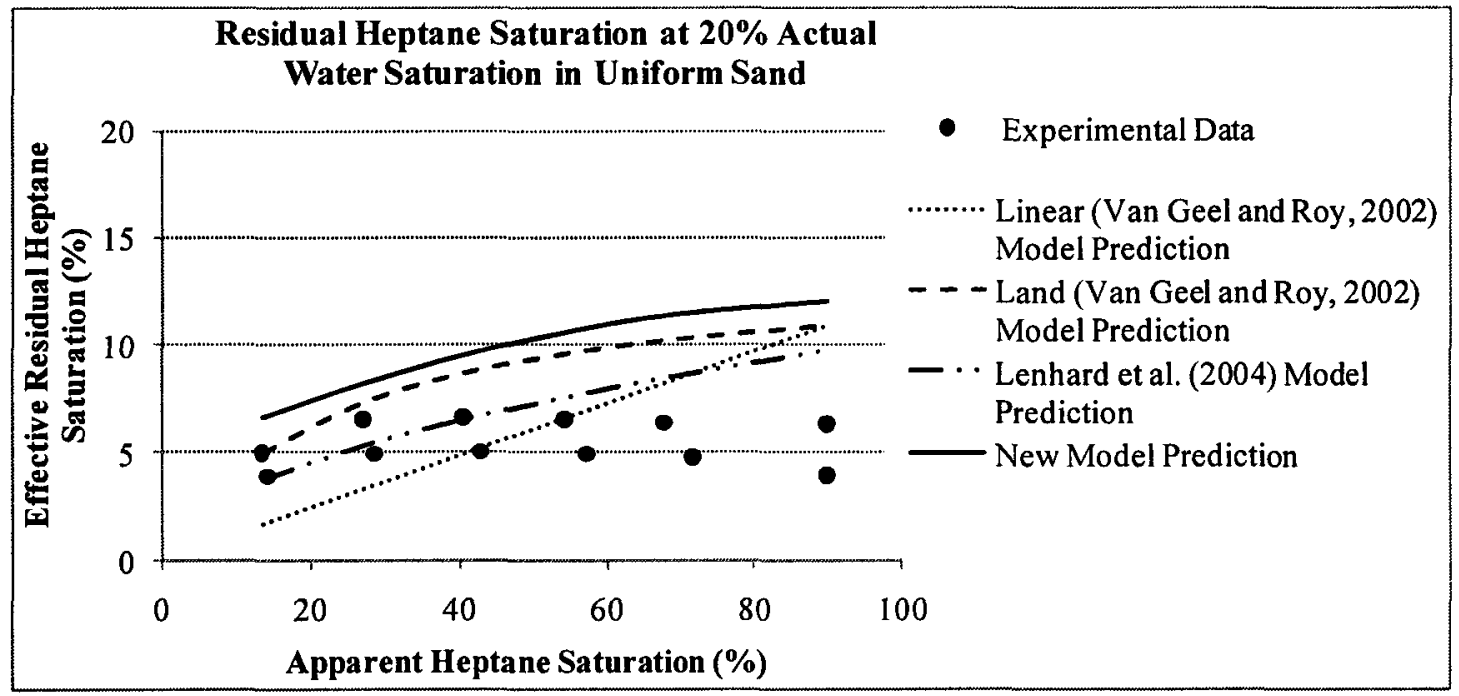

Figure 7.22: Effective Residual Heptane Saturation-Apparent Total Liquid Saturation at $20 \%$ Actual Water Saturation in Uniform Sand. 


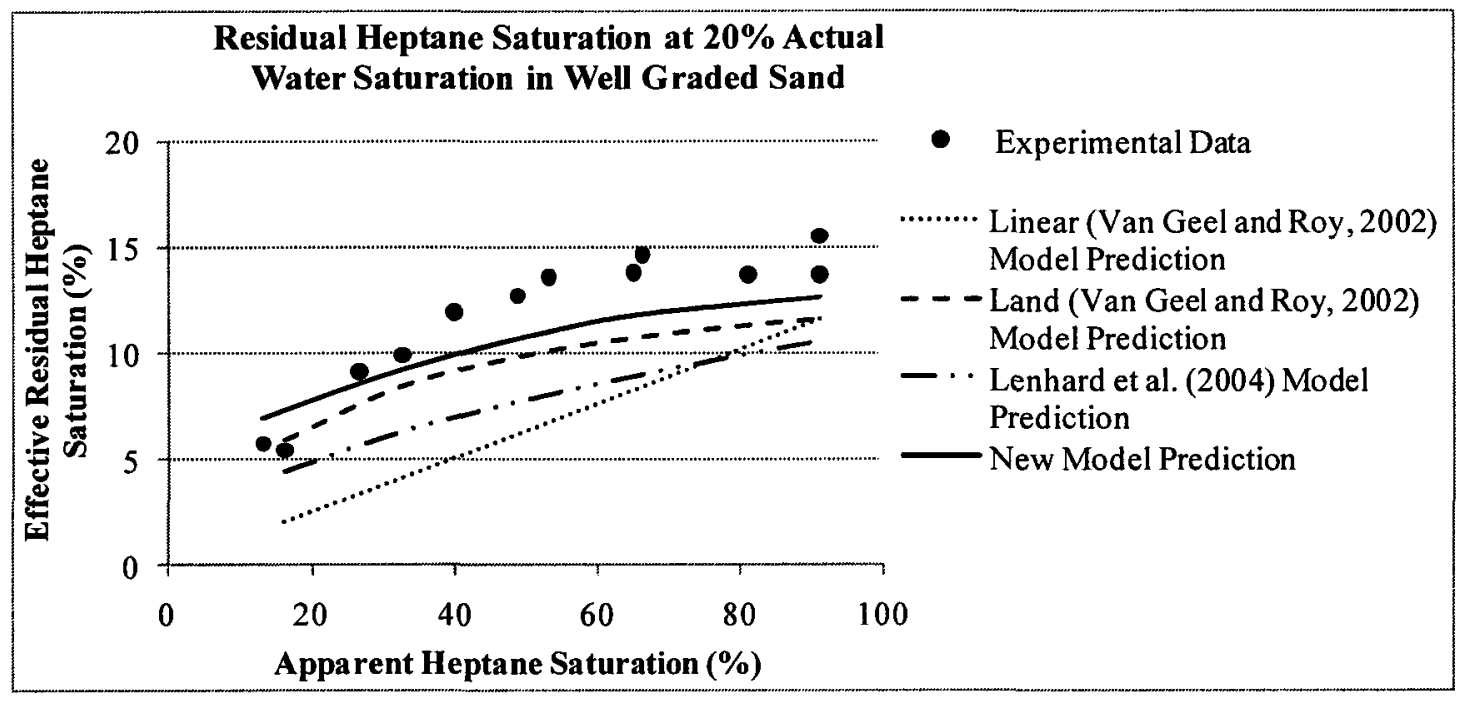

Figure 7.23: Effective Residual Heptane Saturation-Apparent Total Liquid Saturation at 20\% Actual Water Saturation in Well Graded Sand.

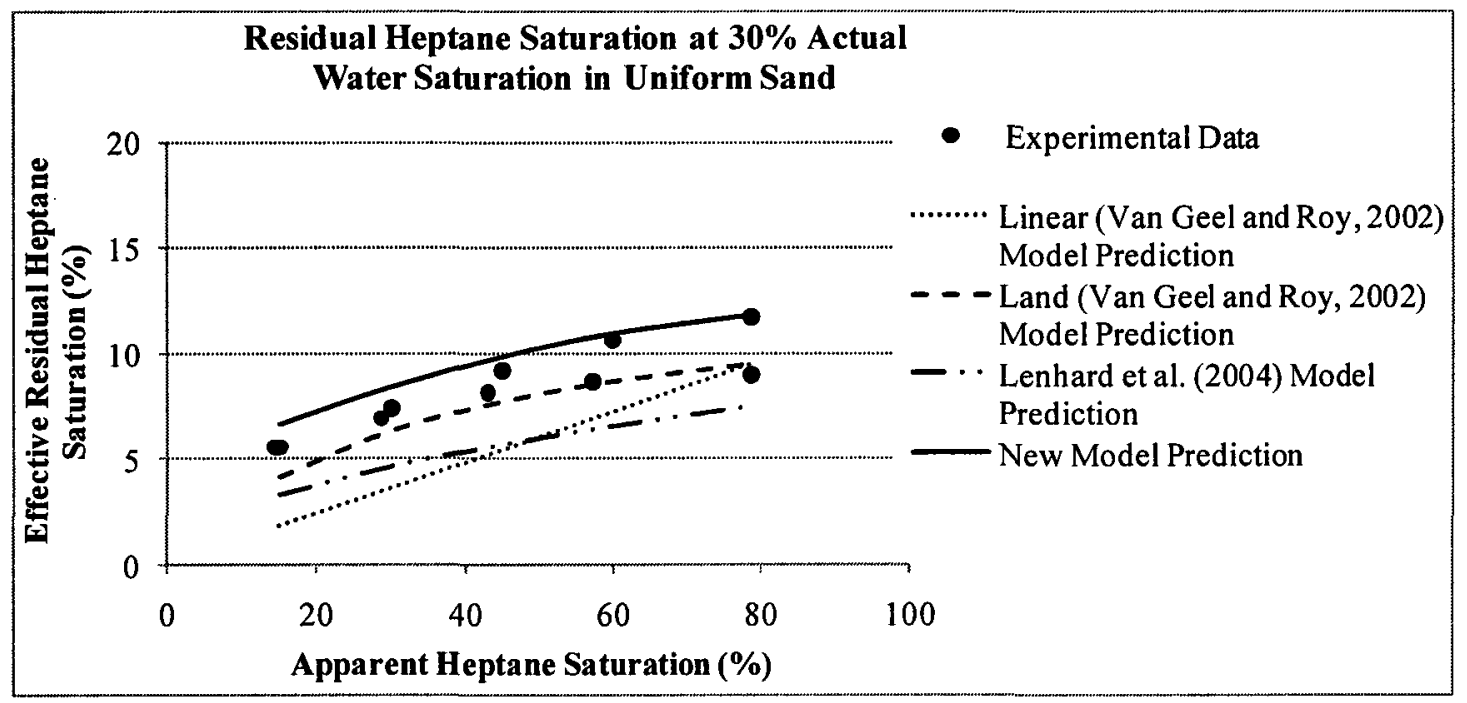

Figure 7.24: Effective Residual Heptane Saturation-Apparent Total Liquid Saturation at 30\% Actual Water Saturation in Uniform Sand. 


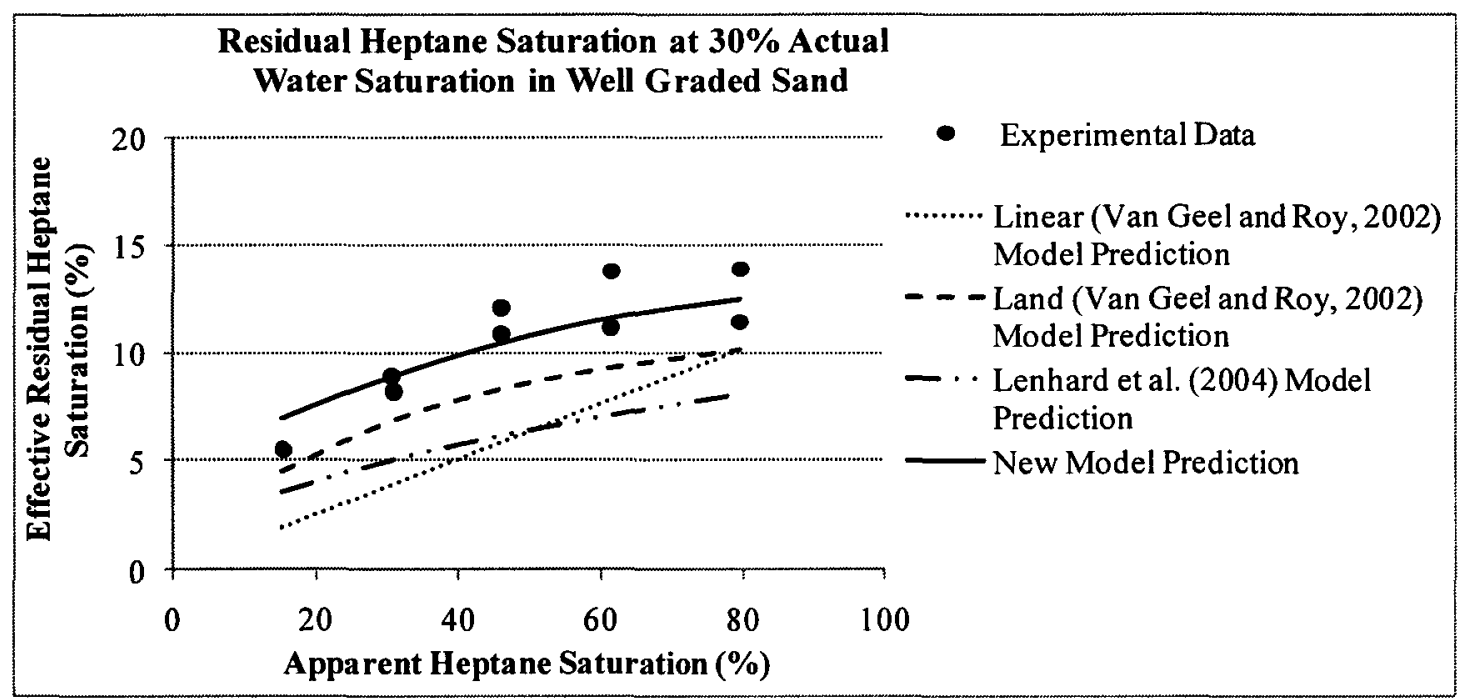

Figure 7.25: Effective Residual Heptane Saturation-Apparent Total Liquid Saturation at 30\% Actual Water Saturation in Well Graded Sand.

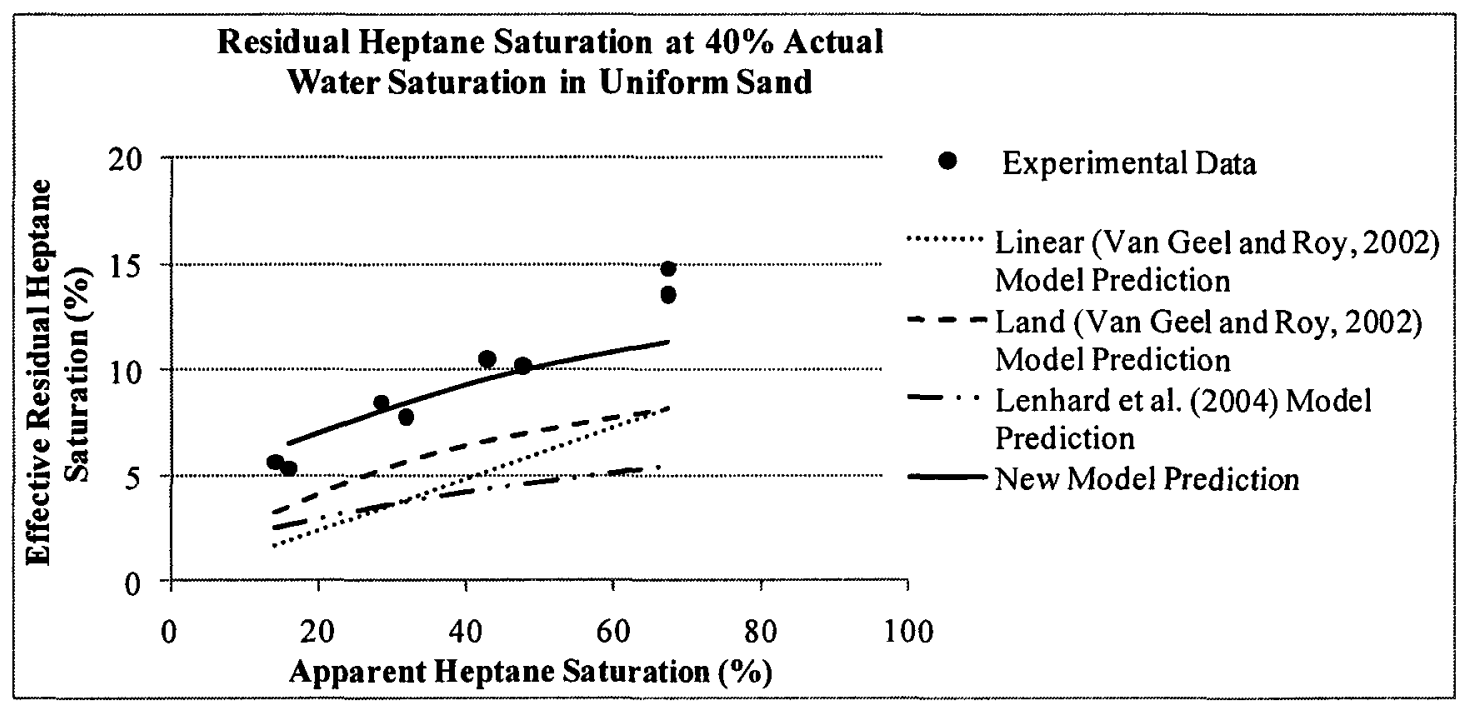

Figure 7.26: Effective Residual Heptane Saturation-Apparent Total Liquid Saturation at $\mathbf{4 0 \%}$ Actual Water Saturation in Uniform Sand. 


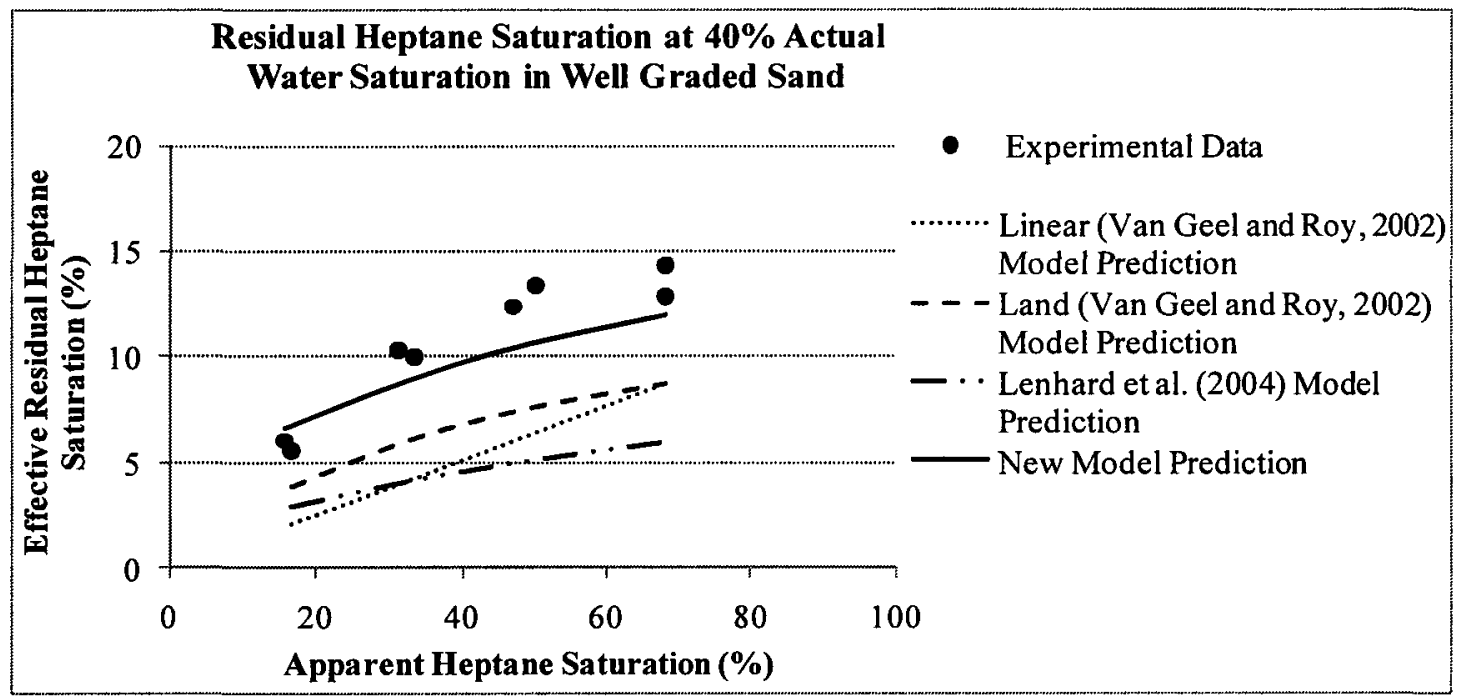

Figure 7.27: Effective Residual Heptane Saturation-Apparent Total Liquid Saturation at $\mathbf{4 0 \%}$ Actual Water Saturation in Well Graded Sand.

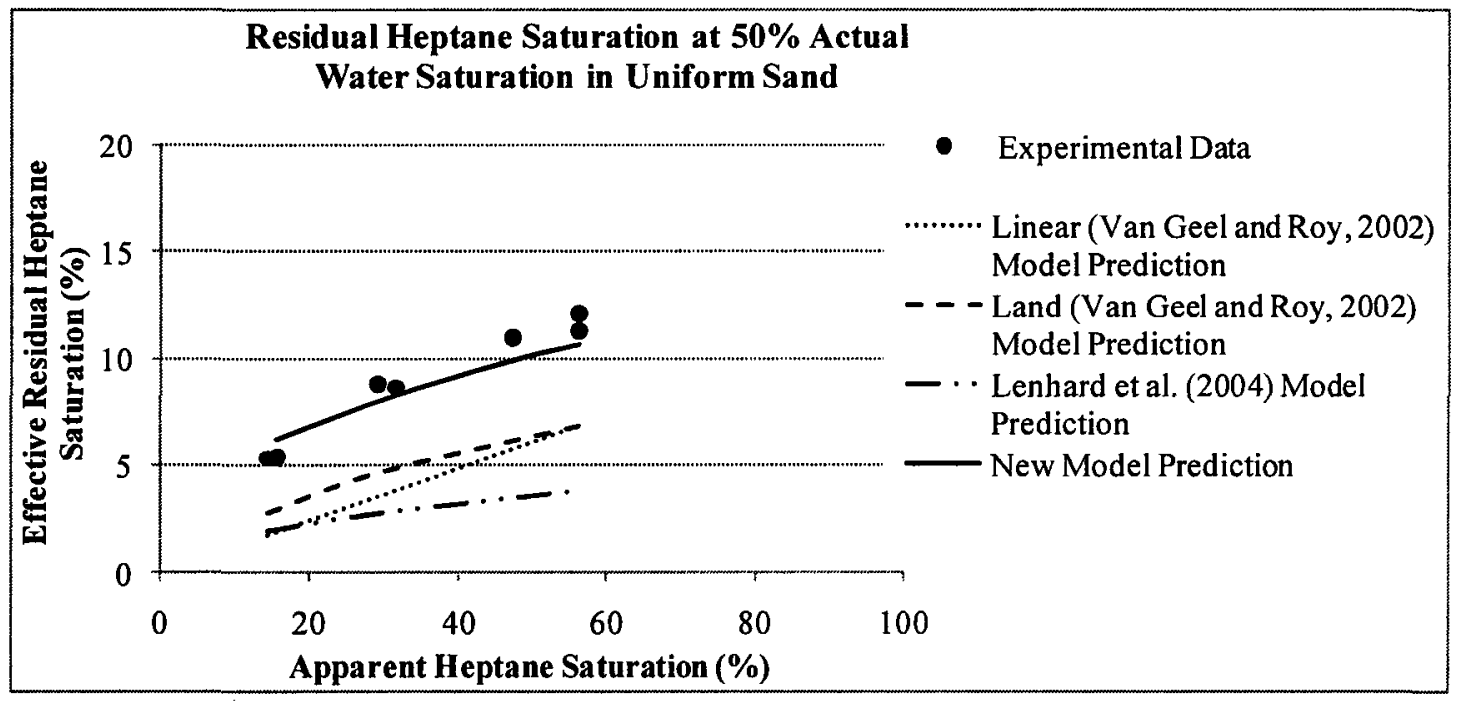

Figure 7.28: Effective Residual Heptane Saturation-Apparent Total Liquid Saturation at 50\% Actual Water Saturation in Uniform Sand. 


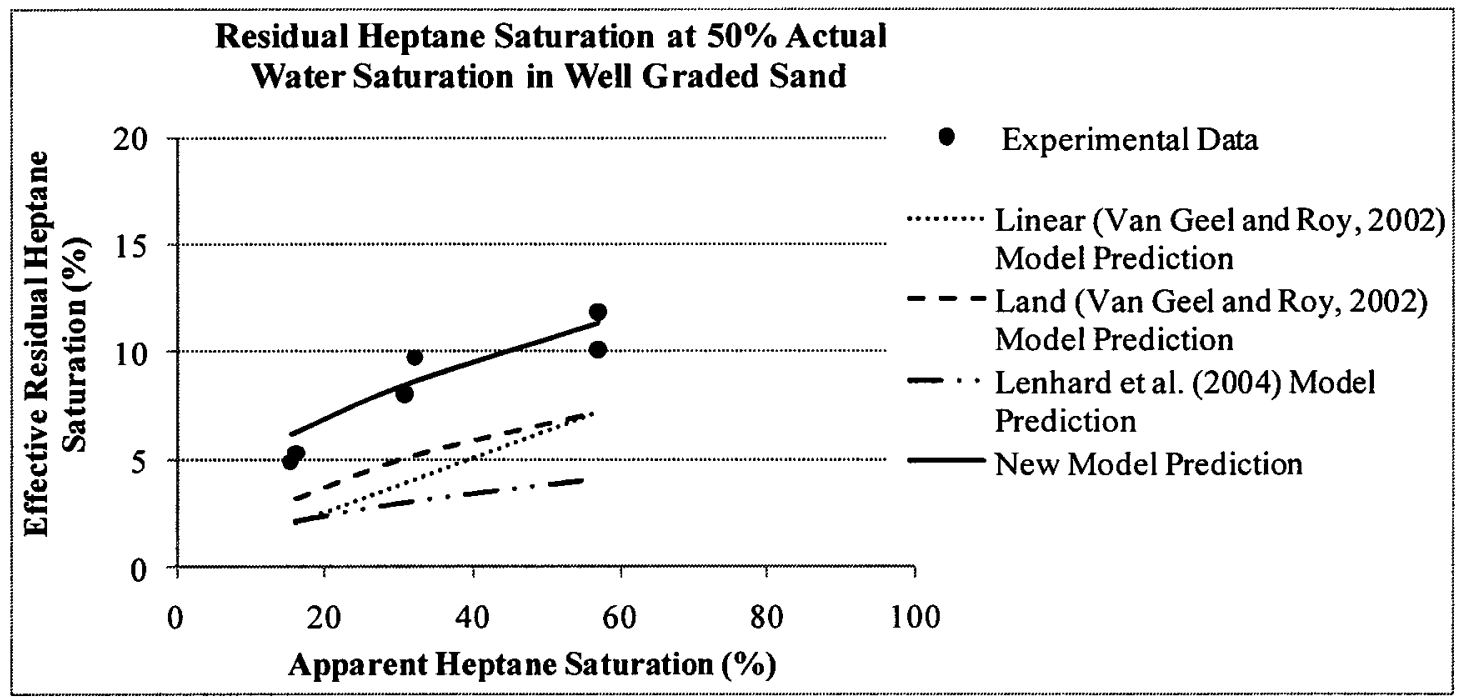

Figure 7.29: Effective Residual Heptane Saturation-Apparent Total Liquid Saturation at 50\% Actual Water Saturation in Well Graded Sand.

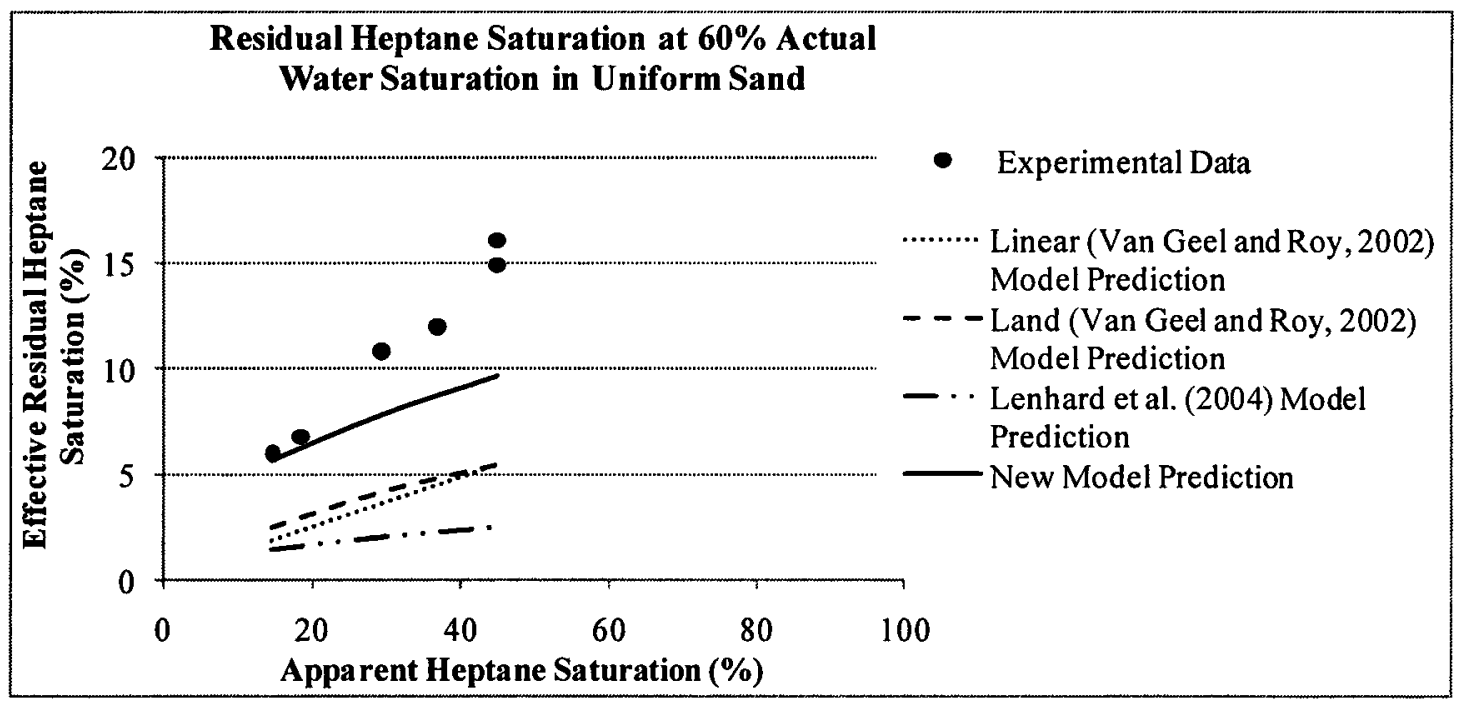

Figure 7.30: Effective Residual Heptane Saturation-Apparent Total Liquid Saturation at $\mathbf{6 0 \%}$ Actual Water Saturation in Uniform Sand. 


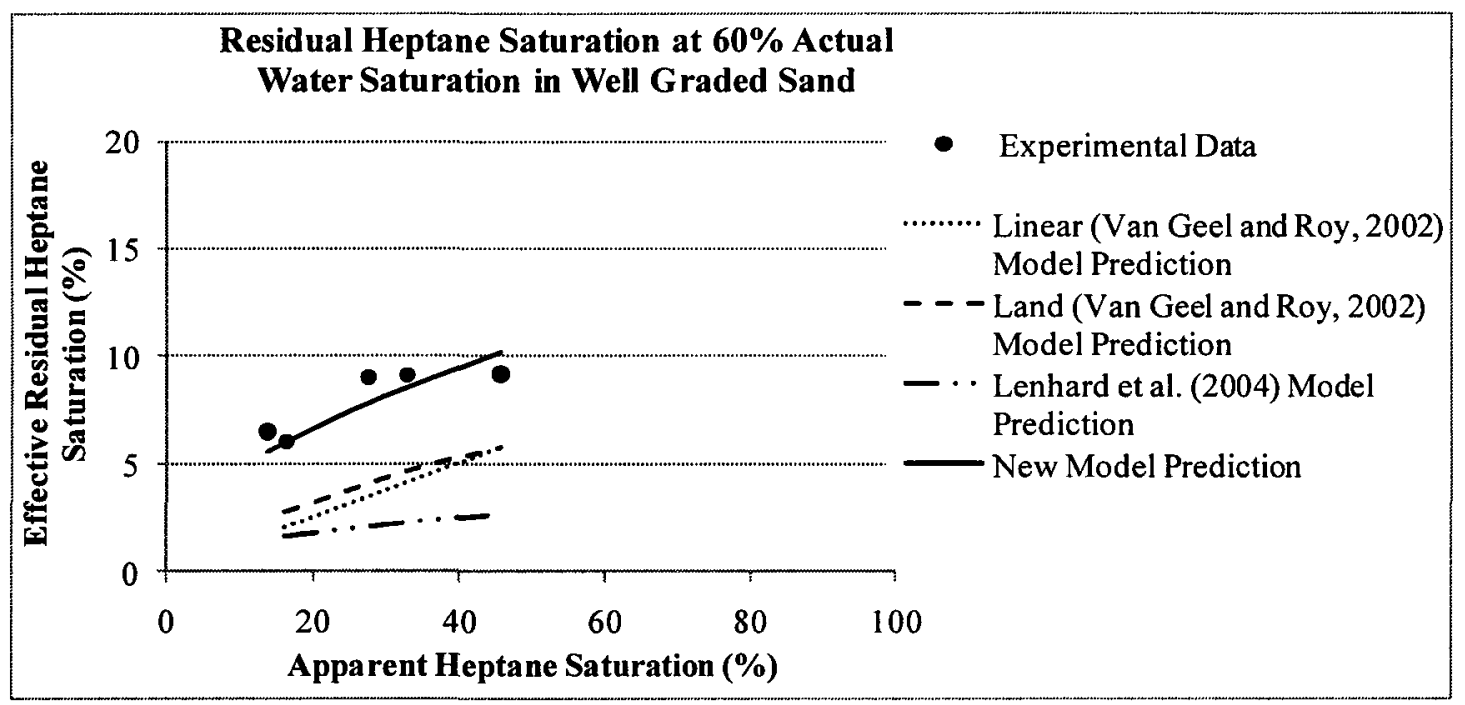

Figure 7.31: Effective Residual Heptane Saturation-Apparent Total Liquid Saturation at $60 \%$ Actual Water Saturation in Well Graded Sand.

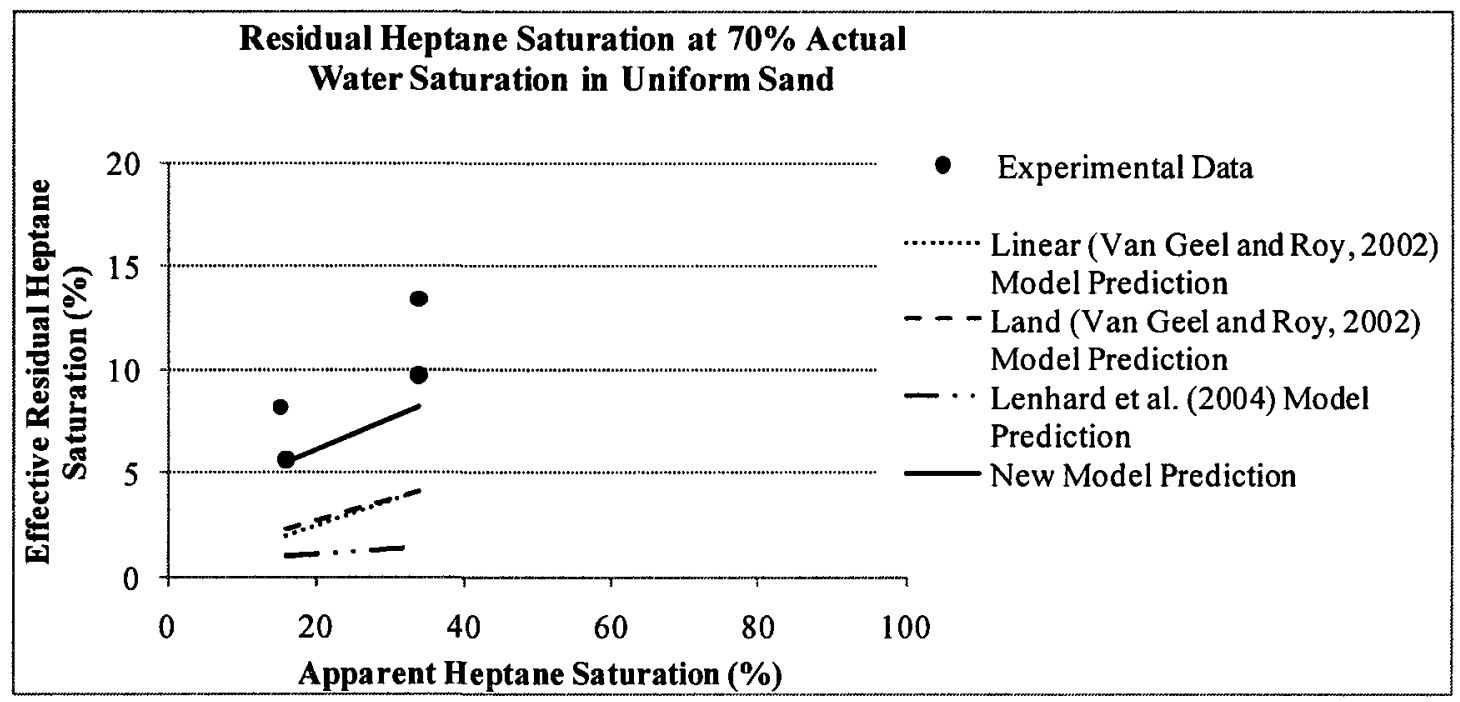

Figure 7.32: Effective Residual Heptane Saturation-Apparent Total Liquid Saturation at $\mathbf{7 0 \%}$ Actual Water Saturation in Uniform Sand. 


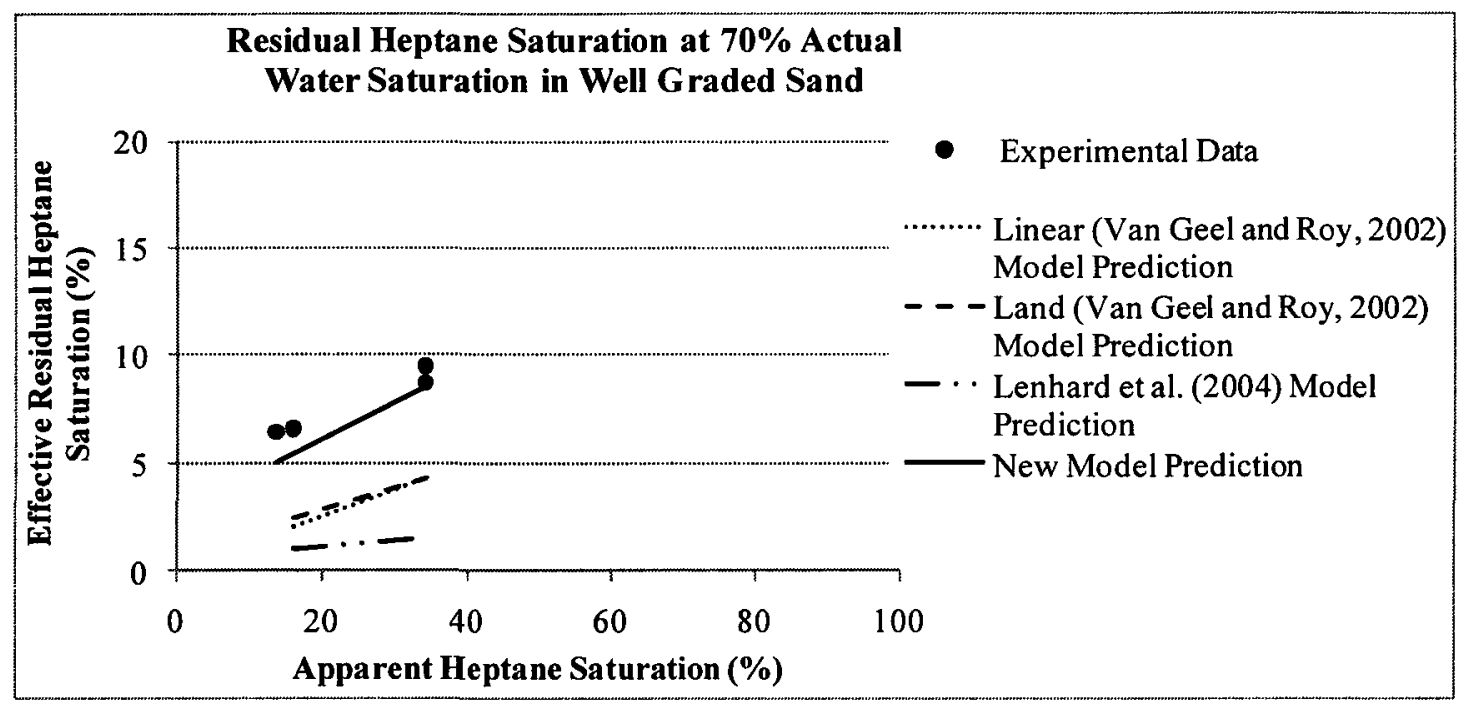

Figure 7.33: Effective Residual Heptane Saturation-Apparent Total Liquid Saturation at $\mathbf{7 0 \%}$ Actual Water Saturation in Well Graded Sand.

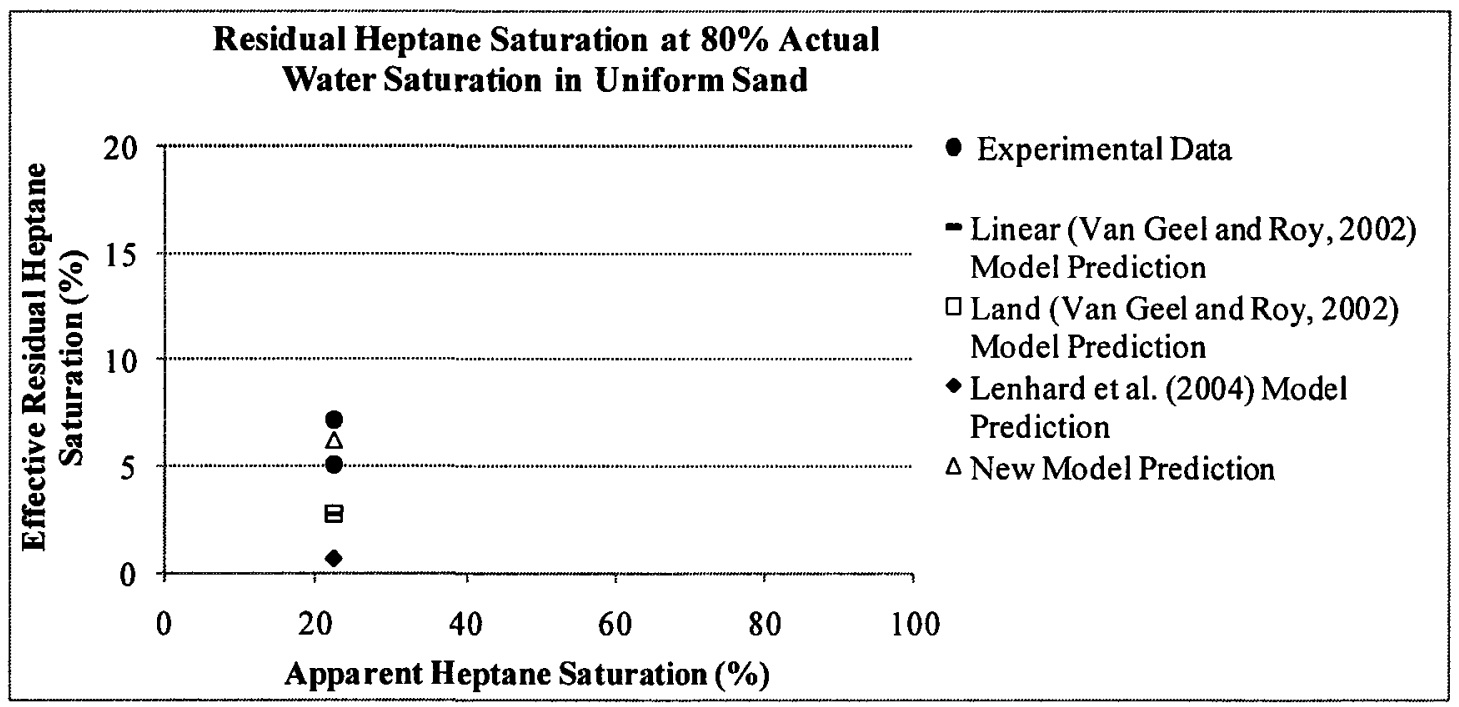

Figure 7.34: Effective Residual Heptane Saturation-Apparent Total Liquid Saturation at $80 \%$ Actual Water Saturation in Uniform Sand. 


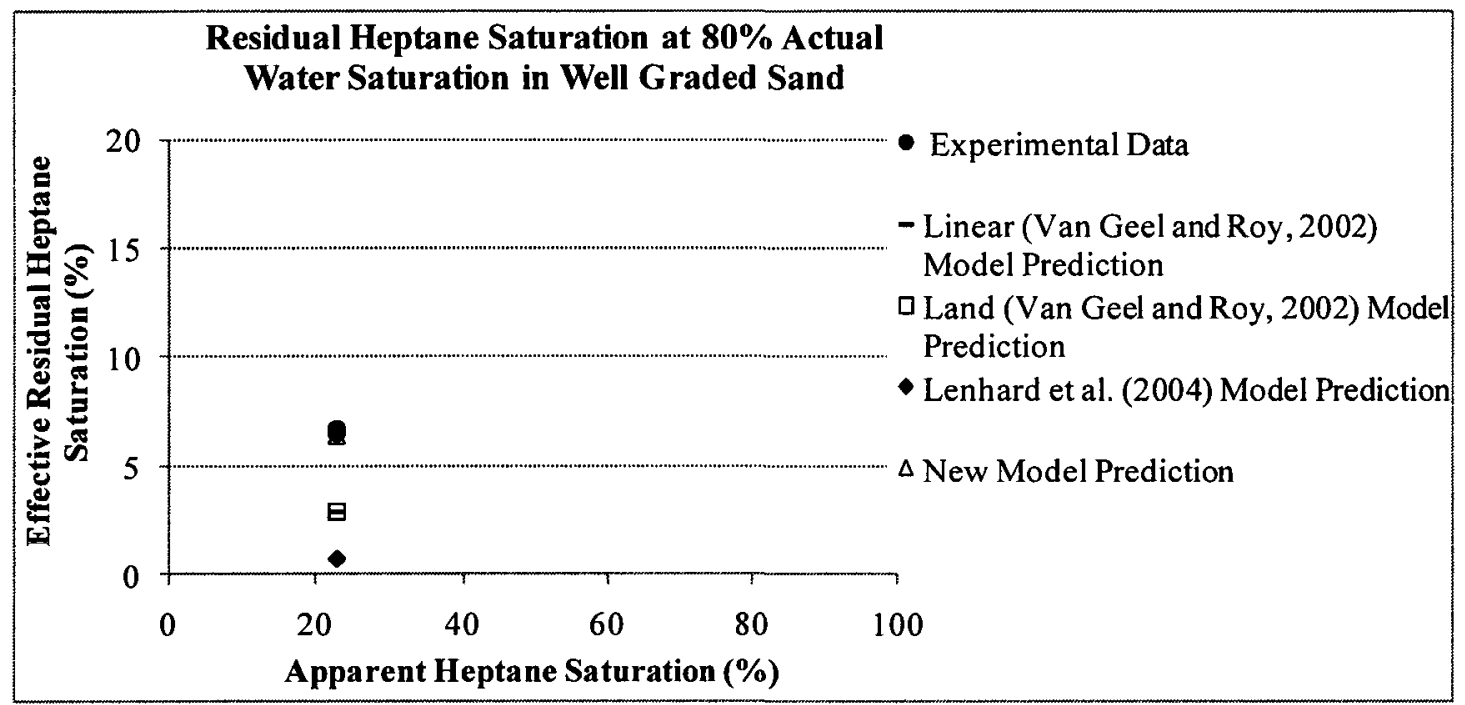

Figure 7.35: Effective Residual Heptane Saturation-Apparent Total Liquid Saturation at $\mathbf{8 0 \%}$ Actual Water Saturation in Well Graded Sand.

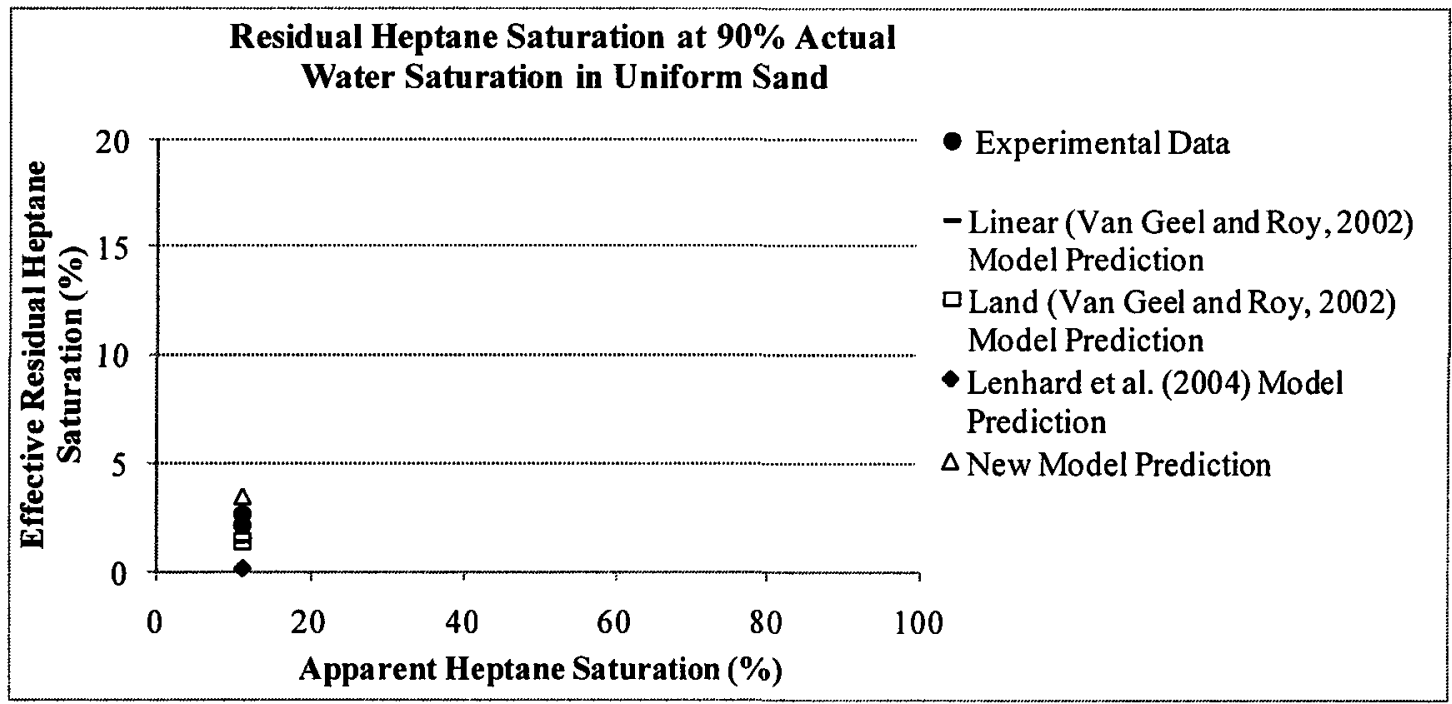

Figure 7.36: Effective Residual Heptane Saturation-Apparent Total Liquid Saturation at $\mathbf{9 0 \%}$ Actual Water Saturation in Uniform Sand. 


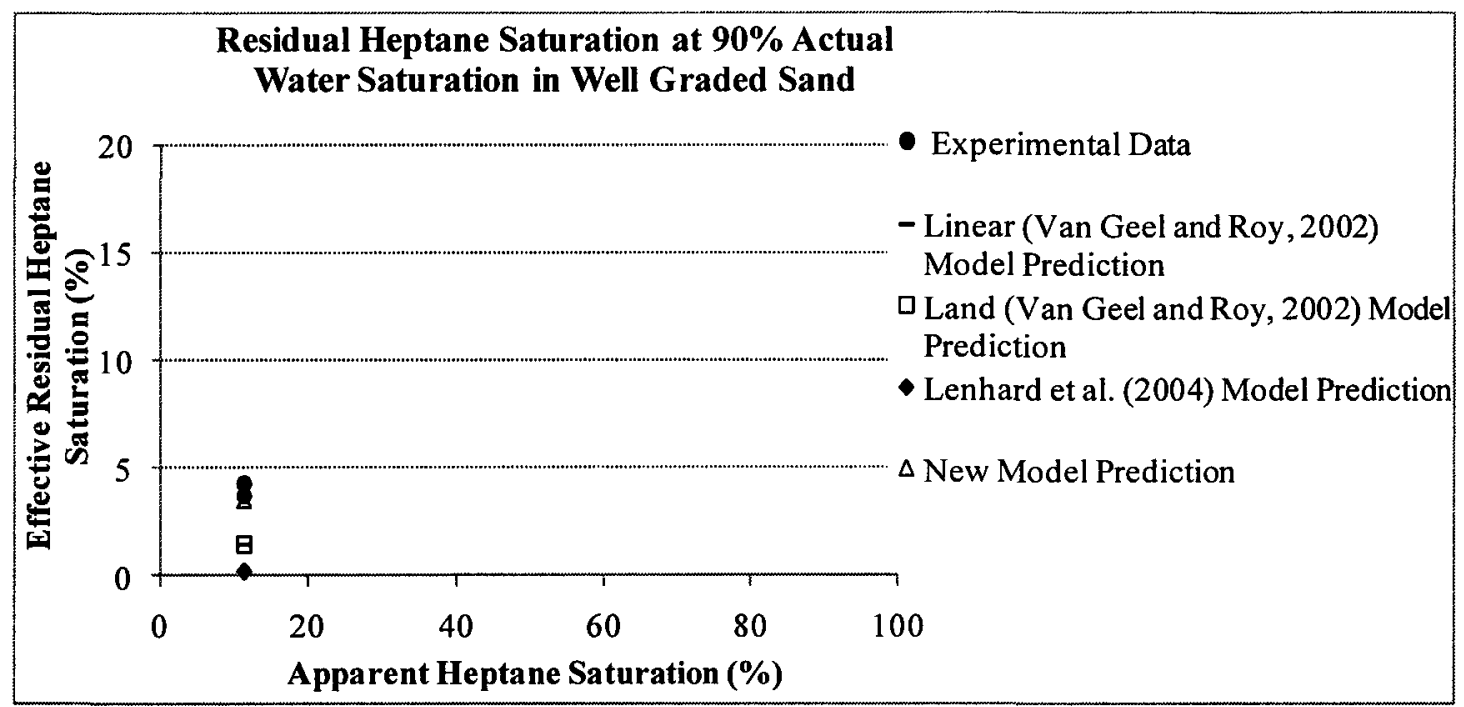

Figure 7.37: Effective Residual Heptane Saturation-Apparent Total Liquid Saturation at $\mathbf{9 0 \%}$ Actual Water Saturation in Well Graded Sand.

\subsection{3.b. Experimental Data Set (2)}

The new model, the linear and Land models developed by Van Geel and Roy (2002), and the model developed by Lenhard et al. (2004) were used to predict the residual heptane saturations for this experimental data set. The predictions of the models are shown in Table 7.2 for uniform sand and Table 7.3 for well graded sand. The new model predictions are closer to the effective residual heptane saturation data than Van Geel and Roy (2002) linear and Land models and Lenhard et al. (2004) model predictions. It is shown in the tables that the new model has the smallest sum of squared errors compared to the other models. 
Table 7.2: Measured Versus Predicted Effective Residual Heptane Saturations in Uniform Sand.

\begin{tabular}{|c|c|c|c|c|c|c|c|}
\hline \multirow{2}{*}{ Experiment } & \multirow{2}{*}{$\overline{\bar{S}}_{w}$} & \multirow{2}{*}{$\overline{\bar{S}}_{n}$} & \multirow{2}{*}{$\bar{S}_{r}$} & \multicolumn{2}{|c|}{ Van Geel and Roy (2002) } & \multirow{2}{*}{ Lenhard et al. (2004) } & \multirow{2}{*}{ New Model } \\
\hline & & & & Linear & Land & & \\
\hline \multirow{9}{*}{1} & 34.4 & 27.0 & 7.5 & 3.3 & 4.9 & 3.3 & 7.9 \\
\hline & 34.4 & 40.4 & 9.1 & 4.9 & 6.3 & 4.1 & 9.3 \\
\hline & 34.4 & 65.6 & 14.8 & 7.9 & 7.9 & 5.2 & 11.2 \\
\hline & 19.6 & 26.2 & 9.6 & 3.2 & 6.1 & 7.8 & 11.2 \\
\hline & 19.6 & 39.3 & 9.9 & 4.7 & 7.4 & 7.8 & 11.2 \\
\hline & 19.6 & 80.4 & 13.9 & 9.7 & 9.7 & 7.8 & 11.8 \\
\hline & 0.0 & 23.9 & $\overline{11.2}$ & 2.9 & 8.7 & 12.1 & 11.8 \\
\hline & 0.0 & 35.8 & 12.4 & 4.3 & 9.9 & 12.1 & 11.8 \\
\hline & 0.0 & 100.0 & 13.7 & 12.1 & 12.1 & 12.1 & 12.1 \\
\hline \multirow{9}{*}{2} & 33.6 & 27.1 & 9.1 & 3.3 & 5.0 & 3.4 & 7.9 \\
\hline & 33.6 & 40.6 & 11.1 & 4.9 & 6.4 & 4.2 & 9.3 \\
\hline & 33.6 & 66.4 & 15.1 & 8.0 & 8.0 & 5.3 & 11.3 \\
\hline & 18.5 & 26.6 & 11.6 & 3.2 & 6.2 & 8.0 & 11.3 \\
\hline & 18.5 & 39.8 & 11.1 & 4.8 & 7.6 & 8.0 & 11.3 \\
\hline & 18.5 & 81.5 & $\overline{14.6}$ & 9.9 & 9.9 & 8.0 & 11.9 \\
\hline & 0.0 & 22.7 & $\overline{11.5}$ & 2.8 & 8.6 & 12.1 & 11.9 \\
\hline & 0.0 & 34.0 & 10.3 & 4.1 & 9.8 & 12.1 & 11.9 \\
\hline & 0.0 & 100.0 & $\overline{12.0}$ & 12.1 & 12.1 & 12.1 & 12.1 \\
\hline \multirow{9}{*}{3} & 31.0 & 27.8 & 7.7 & 3.4 & 5.3 & 3.7 & 8.0 \\
\hline & 31.0 & 41.7 & 10.1 & 5.0 & 6.6 & 4.5 & 9.4 \\
\hline & 31.0 & 69.0 & 13.7 & 8.4 & 8.4 & 5.8 & 11.4 \\
\hline & 15.6 & 26.8 & 8.5 & 3.2 & 6.6 & 8.6 & 11.4 \\
\hline & 15.6 & 40.0 & 8.7 & 4.8 & 7.9 & 8.6 & 11.4 \\
\hline & 15.6 & 84.4 & 11.7 & 10.2 & 10.2 & 8.6 & 11.9 \\
\hline & 0.0 & 26.0 & 6.2 & 3.1 & 9.0 & $\overline{12.1}$ & 11.9 \\
\hline & 0.0 & 38.9 & 7.3 & 4.7 & 10.2 & 12.1 & 11.9 \\
\hline & 0.0 & 100.0 & 8.7 & 12.1 & 12.1 & 12.1 & 12.1 \\
\hline \multirow{9}{*}{4} & 37.3 & 25.4 & 8.9 & 3.1 & 4.6 & 3.0 & 7.6 \\
\hline & 37.3 & 38.1 & 10.9 & 4.6 & 5.9 & 3.7 & 9.0 \\
\hline & 37.3 & 62.7 & 12.9 & 7.6 & 7.6 & 4.8 & 11.1 \\
\hline & 20.2 & 26.8 & 10.3 & 3.2 & 6.1 & 7.7 & 11.1 \\
\hline & 20.2 & 40.2 & 12.1 & 4.9 & 7.5 & 7.7 & 11.1 \\
\hline & 20.2 & 79.8 & 13.3 & 9.7 & 9.7 & 7.7 & 11.8 \\
\hline & 0.0 & 26.2 & 11.3 & 3.2 & 9.0 & 12.1 & 11.8 \\
\hline & 0.0 & 39.4 & 12.1 & 4.8 & 10.2 & 12.1 & 11.8 \\
\hline & 0.0 & 100.0 & 13.1 & 12.1 & 12.1 & 12.1 & 12.1 \\
\hline \multirow{9}{*}{5} & 45.3 & 26.7 & 7.4 & 3.2 & 4.3 & 2.5 & 7.6 \\
\hline & 45.3 & 40.1 & 10.0 & 4.8 & 5.6 & 3.1 & 9.2 \\
\hline & 45.3 & 54.7 & 12.4 & 6.6 & 6.6 & 3.6 & 10.5 \\
\hline & 23.3 & 28.9 & 9.3 & 3.5 & 6.0 & 7.1 & 10.6 \\
\hline & 23.3 & 43.3 & 10.7 & 5.2 & 7.4 & 7.1 & 10.6 \\
\hline & 23.3 & 76.7 & 11.9 & 9.3 & 9.3 & 7.1 & 11.7 \\
\hline & 0.0 & 27.7 & 10.0 & 3.4 & 9.2 & 12.1 & 11.7 \\
\hline & 0.0 & 41.6 & 9.8 & 5.0 & 10.3 & 12.1 & 11.7 \\
\hline & 0.0 & 100.0 & 10.0 & 12.1 & 12.1 & 12.1 & 12.1 \\
\hline \multicolumn{4}{|c|}{ Sum of Squared Errors } & 1376.4 & 585.4 & 1030.7 & $\mathbf{1 7 2 . 5}$ \\
\hline
\end{tabular}


Table 7.3: Measured Versus Predicted Effective Residual Heptane Saturations in Well Graded Sand.

\begin{tabular}{|c|c|c|c|c|c|c|c|}
\hline \multirow[t]{2}{*}{ Experiment } & \multirow{2}{*}{$\overline{\bar{S}}_{w}$} & \multirow{2}{*}{$\overline{\bar{S}}_{n}$} & \multirow{2}{*}{$\bar{S}_{r}$} & \multicolumn{2}{|c|}{ Van Geel and Roy (2002) } & \multirow[t]{2}{*}{ Lenhard et al. (2004) } & \multirow[t]{2}{*}{ New Model } \\
\hline & & & & Linear & Land & & \\
\hline \multirow{9}{*}{1} & 33.8 & 25.9 & 7.7 & 3.3 & 5.1 & 3.5 & 8.0 \\
\hline & 33.8 & 38.9 & 9.9 & 4.9 & 6.5 & 4.3 & 9.6 \\
\hline & 33.8 & 66.2 & 12.8 & 8.4 & 8.4 & 5.6 & 11.9 \\
\hline & 19.2 & 25.7 & 11.7 & 3.3 & 6.3 & 8.3 & 11.8 \\
\hline & 19.2 & 38.6 & 14.0 & 4.9 & 7.8 & 8.3 & 11.8 \\
\hline & 19.2 & 80.8 & 14.5 & 10.3 & 10.3 & 8.3 & 12.5 \\
\hline & 0.0 & 24.3 & 10.8 & 3.1 & 9.1 & 12.7 & 12.4 \\
\hline & 0.0 & 36.4 & 11.2 & 4.6 & 10.4 & 12.7 & 12.4 \\
\hline & 0.0 & 100.0 & $\mathbf{1 3 . 5}$ & 12.7 & 12.7 & 12.7 & 12.7 \\
\hline \multirow{9}{*}{2} & 33.3 & 26.7 & 8.0 & 3.4 & 5.2 & 3.6 & 8.1 \\
\hline & 33.3 & 40.0 & 9.7 & 5.1 & 6.6 & 4.4 & 9.7 \\
\hline & 33.3 & 66.7 & 12.7 & 8.5 & 8.5 & 5.6 & 11.9 \\
\hline & 18.6 & 26.1 & 11.7 & 3.3 & 6.4 & 8.4 & 11.9 \\
\hline & 18.6 & 39.2 & 13.8 & 5.0 & 7.9 & 8.4 & 11.9 \\
\hline & 18.6 & 81.4 & 14.7 & 10.3 & 10.3 & 8.4 & 12.5 \\
\hline & 0.0 & 24.9 & 11.3 & 3.2 & 9.2 & 12.7 & 12.5 \\
\hline & 0.0 & 34.5 & 12.0 & 4.4 & 10.2 & 12.7 & 12.5 \\
\hline & 0.0 & 100.0 & 13.1 & 12.7 & 12.7 & 12.7 & 12.7 \\
\hline \multirow{9}{*}{3} & 40.8 & 32.3 & 6.7 & 4.1 & 5.4 & 3.3 & 8.6 \\
\hline & 40.8 & 48.5 & 7.5 & 6.2 & 6.8 & 4.0 & 10.5 \\
\hline & 40.8 & 59.2 & 8.0 & 7.5 & 7.5 & 4.5 & 11.4 \\
\hline & 24.7 & 32.3 & 8.9 & 4.1 & 6.6 & 7.2 & 11.4 \\
\hline & 24.7 & 48.5 & 10.0 & 6.2 & 8.0 & 7.2 & 11.4 \\
\hline & 24.7 & 75.3 & 10.4 & 9.6 & 9.6 & 7.2 & 12.3 \\
\hline & 0.0 & 31.1 & 6.8 & 3.9 & 9.9 & 12.7 & 12.2 \\
\hline & 0.0 & 46.6 & 7.6 & 5.9 & 11.1 & 12.7 & 12.2 \\
\hline & 0.0 & 100.0 & 10.8 & 12.7 & 12.7 & 12.7 & 12.7 \\
\hline \multirow{9}{*}{4} & 39.8 & 30.9 & 6.8 & 3.9 & 5.3 & 3.3 & 8.4 \\
\hline & 39.8 & 46.3 & 8.4 & 5.9 & 6.7 & 4.0 & 10.3 \\
\hline & 39.8 & 60.2 & 8.4 & 7.6 & 7.6 & 4.6 & 11.5 \\
\hline & 23.5 & 32.4 & 9.1 & 4.1 & 6.7 & 7.4 & 11.4 \\
\hline & 23.5 & 48.6 & 9.8 & 6.2 & 8.2 & 7.4 & 11.4 \\
\hline & 23.5 & 76.5 & 10.7 & 9.7 & 9.7 & 7.4 & 12.3 \\
\hline & 0.0 & 31.7 & 7.9 & 4.0 & 10.0 & 12.7 & 12.3 \\
\hline & 0.0 & 47.6 & 9.2 & 6.0 & 11.1 & 12.7 & 12.3 \\
\hline & 0.0 & 100.0 & 12.8 & 12.7 & 12.7 & 12.7 & 12.7 \\
\hline \multirow{9}{*}{5} & 38.5 & 26.9 & 9.2 & 3.4 & 4.9 & 3.2 & 8.0 \\
\hline & 38.5 & 40.3 & 11.2 & 5.1 & 6.3 & 3.9 & 9.7 \\
\hline & 38.5 & 61.5 & 12.1 & 7.8 & 7.8 & 4.8 & 11.6 \\
\hline & 21.6 & 28.1 & 12.8 & 3.6 & 6.4 & 7.8 & 11.6 \\
\hline & 21.6 & 42.0 & 14.3 & 5.3 & 7.8 & 7.8 & 11.6 \\
\hline & 21.6 & 78.4 & 15.3 & 10.0 & 10.0 & 7.8 & 12.4 \\
\hline & 0.0 & 26.8 & 12.4 & 3.4 & 9.4 & 12.7 & 12.4 \\
\hline & 0.0 & 40.1 & 13.0 & 5.1 & 10.7 & 12.7 & 12.4 \\
\hline & 0.0 & 100.0 & 15.9 & 12.7 & 12.7 & 12.7 & 12.7 \\
\hline \multicolumn{4}{|c|}{ Sum of Squared Errors } & 1268.5 & 512.1 & 869.6 & 204.6 \\
\hline
\end{tabular}




\subsection{3.c. Van Geel and Roy (2002) Experimental Data Set}

Van Geel and Roy (2002) conducted a series of residual heptane saturation experiments. In these experiments, residual heptane saturations were measured at different water and heptane saturations. This section aims to compare Van Geel and Roy (2002) linear and Land models and Lenhard et al (2004) model predictions to the new model predictions. The use of the new model implies knowledge of the porous medium specific surface area, heptane entry head and fitting parameter $\lambda$, and porous medium porosity. The porous medium specific surface area was assumed $1063.4 \mathrm{~cm}^{2} / \mathrm{cm}^{3}$ (i.e., equivalent to the specific surface area of the well graded sand). The heptane entry head and the fitting parameter, $\lambda$, were obtained by fitting the heptane /water capillary pressure head-actual water saturation data, presented in Van Geel and Roy (2002), to Brooks and Corey capillary pressure head-saturation relationship. The porous medium porosity is given in Van Geel and Roy (2002). The monolayer of water saturation was estimated as explained in Cary (1994). The dry end fitting parameter was calculated from Equation 7.1, as explained earlier in Section 7.2.2.a. These parameters are given in Table 7.4.

Table 7.4: Physical Properties of the Porous Medium.

\begin{tabular}{|l|c|}
\hline Specific Surface Area $\left(\mathrm{cm}^{2} / \mathrm{cm}^{3}\right)$ & 1063.4 \\
\hline Heptane Entry Head $\left(h_{b}\right)(\mathrm{cm})$ & 13.5 \\
\hline Porous Medium Porosity $(\eta)$ & 0.394 \\
\hline Fitting Parameter $(\lambda)$ & 1.1 \\
\hline Monolayer of Water Saturation $\left(S_{\text {monolayer }}\right)$ & $8.35 \times 10^{-5 *}$ \\
\hline Dry End Fitting Parameter $(I)$ & 128230.5 \\
\hline
\end{tabular}

*Determined as explained in Cary (1994) with an assumed depth of water equivalent to the thickness of a water monolayer.

Van Geel and Roy (2002) assumed a maximum effective residual heptane saturation in the porous medium equivalent to $20.1 \%$. In their experiments, the effective residual heptane saturation-apparent water saturation and the effective residual heptane 
saturation-apparent heptane saturation histories were tracked and the predictions of the models were obtained and compared, as shown in Table 7.5. The heptane film thickness, required for the new model was assumed $0.18 \mu \mathrm{m}$ (i.e., similar to the heptane film thickness in the uniform sand, as estimated in Section 7.2.2.a). Table 7.5 shows that, although the summation of the squared errors for the four models are comparable, Van Geel and Roy (2002) Land model has the lowest summation of the squared errors, which is close to the summation of the squared errors of the new model. The inability of the new model to predict these effective residual NAPL saturations may be due to an underestimation of the assumed porous medium specific surface area or the absolute maximum effective residual NAPL saturation. 
Table 7.5: Measured Versus Predicted Effective Residual Heptane Saturations for Van Geel and Roy (2002) Experimental Data Set.

\begin{tabular}{|c|c|c|c|c|c|c|}
\hline \multirow{2}{*}{$\overline{\bar{S}}_{w}$} & \multirow{2}{*}{$\overline{\bar{S}}_{n}$} & \multirow{2}{*}{$\bar{S}_{r}$} & \multicolumn{2}{|c|}{ Van Geel and Roy (2002) } & \multirow[t]{2}{*}{ Lenhard et al. (2004) } & \multirow[t]{2}{*}{ New Model } \\
\hline & & & Linear & Land & & \\
\hline 0.0 & 27.9 & 9.9 & 5.6 & 13.2 & 10.6 & 12.3 \\
\hline 0.0 & 53.9 & 13.7 & 10.8 & 17.2 & 14.8 & 16.9 \\
\hline 0.0 & 71.6 & 13.9 & 14.4 & 18.6 & 17.0 & 18.9 \\
\hline 0.0 & 100.0 & 14.3 & 20.1 & 20.1 & 20.1 & 20.1 \\
\hline 7.5 & 27.3 & 8.1 & 5.5 & 11.5 & 9.3 & 12.0 \\
\hline 3.9 & 50.1 & 11.8 & 10.1 & 15.9 & 13.4 & 16.3 \\
\hline 2.5 & 67.4 & 15.7 & 13.5 & 17.8 & 15.9 & 18.5 \\
\hline 1.4 & 98.6 & 18.0 & 19.8 & 19.8 & 19.5 & 20.1 \\
\hline 5.4 & 27.7 & 9.7 & 5.6 & 12.0 & 9.7 & 12.1 \\
\hline 2.2 & 66.3 & 12.7 & 13.3 & 17.8 & 15.8 & 18.4 \\
\hline 1.0 & 99.0 & 15.1 & 19.9 & 19.9 & 19.7 & 20.1 \\
\hline 1.9 & 26.6 & 9.7 & 5.4 & 12.5 & 10.1 & 12.0 \\
\hline 1.3 & 51.0 & 13.9 & 10.3 & 16.6 & 14.1 & 16.5 \\
\hline 0.0 & 69.5 & 18.6 & 14.0 & 18.5 & 16.8 & 18.7 \\
\hline 0.0 & 100.0 & 18.0 & 20.1 & 20.1 & 20.1 & 20.1 \\
\hline 11.2 & 25.2 & 9.5 & 5.1 & 10.3 & 8.4 & 11.4 \\
\hline 9.8 & 48.2 & 13.5 & 9.7 & 14.6 & 12.0 & 16.0 \\
\hline 9.2 & 65.2 & 14.1 & 13.1 & 16.5 & 14.1 & 18.3 \\
\hline 7.5 & 92.5 & 13.7 & 18.6 & 18.6 & 17.2 & 20.0 \\
\hline 10.7 & 27.0 & 7.9 & 5.4 & 10.8 & 8.8 & 11.8 \\
\hline 6.6 & 63.0 & 10.3 & 12.7 & 16.7 & 14.4 & 18.0 \\
\hline 5.1 & 94.9 & 12.5 & 19.1 & 19.1 & 18.1 & 20.1 \\
\hline 1.1 & 27.0 & 8.8 & 5.4 & 12.7 & 10.3 & 12.1 \\
\hline 0.0 & 48.7 & 12.9 & 9.8 & 16.6 & 14.0 & 16.1 \\
\hline 0.0 & 66.3 & 11.8 & 13.3 & 18.2 & 16.4 & 18.4 \\
\hline 0.0 & 100.0 & 14.4 & 20.1 & 20.1 & 20.1 & 20.1 \\
\hline 17.3 & 27.2 & 10.6 & 5.5 & 9.8 & 7.9 & 11.8 \\
\hline 12.4 & 67.3 & 13.6 & 13.5 & 16.1 & 13.5 & 18.5 \\
\hline 11.3 & 88.7 & 16.8 & 17.8 & 17.8 & 15.8 & 20.0 \\
\hline 29.8 & 29.9 & 11.2 & 6.0 & 8.9 & 6.5 & 12.2 \\
\hline 10.2 & 55.3 & 17.8 & 11.1 & 15.3 & 12.7 & 17.0 \\
\hline 7.3 & 92.7 & 19.8 & 18.6 & 18.6 & 17.3 & 20.0 \\
\hline 0.0 & 27.8 & 13.4 & 5.6 & 13.2 & 10.6 & 12.3 \\
\hline 0.0 & 72.9 & 20.5 & 14.7 & 18.7 & 17.2 & 19.0 \\
\hline 0.0 & 100.0 & 25.8 & 20.1 & 20.1 & 20.1 & 20.1 \\
\hline 11.1 & 30.7 & 9.8 & 6.2 & 11.5 & 9.3 & 12.6 \\
\hline 8.2 & 58.5 & 13.0 & 11.8 & 16.0 & 13.5 & 17.4 \\
\hline 5.9 & 76.7 & 14.2 & 15.4 & 17.9 & 16.1 & 19.3 \\
\hline 4.3 & 95.7 & 15.6 & 19.2 & 19.2 & 18.4 & 20.1 \\
\hline 36.4 & 29.0 & 11.6 & 5.8 & 8.1 & 5.5 & 12.0 \\
\hline 33.9 & 57.1 & 19.2 & 11.5 & 12.4 & 8.2 & 17.5 \\
\hline 32.6 & 67.4 & 22.7 & 13.6 & 13.6 & 9.1 & 18.8 \\
\hline 0.0 & 28.1 & 13.9 & 5.6 & 13.3 & 10.7 & 12.3 \\
\hline 0.0 & 73.9 & 13.6 & 14.8 & 18.8 & 17.3 & 19.1 \\
\hline 0.0 & 100.0 & 19.0 & 20.1 & 20.1 & 20.1 & 20.1 \\
\hline 13.4 & 31.8 & 9.7 & 6.4 & 11.4 & 9.1 & 12.9 \\
\hline 6.4 & 73.7 & 14.7 & 14.8 & 17.6 & 15.6 & 19.1 \\
\hline 4.8 & 95.2 & 22.1 & 19.1 & 19.1 & 18.2 & 20.1 \\
\hline 33.1 & 29.7 & 7.9 & 6.0 & 8.5 & 6.0 & 12.1 \\
\hline 31.6 & 68.4 & 17.8 & 13.8 & 13.8 & 9.4 & 18.9 \\
\hline \multicolumn{3}{|c|}{ quared } & 891.3 & 719.5 & 796.6 & 747.9 \\
\hline
\end{tabular}


In summary, the new model was able to closely predict the effective residual heptane saturation data for the two experimental data sets. The new model predictions were compared to Van Geel and Roy (2002) linear and Land models, and Lenhard et al. (2004) model predictions of the two experimental data sets and the experimental data set of Van Geel and Roy (2002) and showed that the new model better predicts the effective residual heptane saturations in a water-wet porous medium. The new model inputs include eight parameters porous medium specific surface area, NAPL entry pressure head in NAPL/water two-phase system, porous medium grain size distribution (through fitting parameter $\lambda$ ), porous medium porosity, NAPL film thickness, apparent water saturation, historic maximum apparent NAPL saturation, and the absolute maximum effective residual NAPL saturation in the porous medium. The inclusion of the porous medium and liquid specific parameters gives credit to the new model representing the effective residual NAPL saturations left in a water-wet porous medium.

\subsection{Residual NAPL Saturation in a Water-Wet Porous Medium with a NAPL-Wet Soil Lens of Different Grain Size Distribution}

As explained in Section 6.2 of Chapter 6, at the end of NAPL drainage pathway, the NAPL exists in the water-wet soil fraction at residual NAPL saturation. In contrast, the NAPL saturation in the NAPL-wet soil lens depends on whether the lens is isolated from or connected to the NAPL reservoir or/via other NAPL-wet soil lenses. Two conceptual models are described hereafter in Sections 7.3.1 and 7.3.2 for residual NAPL saturations in isolated and connected NAPL-wet soil lenses, of different grain size distribution than the water-wet soil fraction, respectively. These conceptual models are based on the conceptual models explained in Section 6.2 of Chapter 6 . 


\subsubsection{Conceptual Model for Residual NAPL Saturation in a Water-Wet Porous Medium with an Isolated NAPL-Wet Soil Lens}

\subsection{1.a. NAPL Imbibition Pathway}

To develop this conceptual model, first consider a porous medium that is an air/water two-phase system. As explained in Section 5.3.4 of Chapter 5, the water main drainage pathway starts at water-saturated water-wet soil fraction with partially-saturated NAPL-wet soil lens. If the water drains from the porous medium to a known water saturation, the water saturation in the water-wet soil fraction and in the NAPL-wet soil lens could be calculated as explained in Section 5.3.4 of Chapter 5. If a certain NAPL volume imbibes into the porous medium, the water saturations in the water-wet soil fraction and in the NAPL-wet soil lens can be calculated as explained in Section 5.4.4 of Chapter 5 and in Chapter 6. In contrast, the NAPL saturations in the NAPL-wet soil lens and in the water-wet soil fraction depend on the imbibing NAPL volume. If the imbibing NAPL volume is large, the NAPL imbibes into the NAPL-wet soil lens until the NAPL saturation in the NAPL-wet soil lens equals to the NAPL saturation lower limit (NSLL). The total liquid saturation in the water-wet soil fraction equals to the total liquid saturation lower limit (TLSLL), which is the summation of the water saturation and the residual NAPL saturation in the water-wet soil fraction. It is conceptualized that the residual NAPL exists in the water-wet soil fraction as NAPL film on water surfaces when the NAPL saturation in the NAPL-wet soil lens is less than NSLL.

As NAPL continues to imbibe into the porous medium, at saturations larger than the NSLL, NAPL distributes itself between the water-wet soil fraction and the NAPL-wet soil lens such that the capillary pressure is invariable in the REV. Similar to residual 
NAPL formation in a water-wet porous medium discussed in Section 7.2, residual NAPL in the water-wet soil fraction is composed of two components; NAPL film on water surfaces and NAPL residing in water wedges. The residual NAPL saturation in the waterwet soil fraction depends on the imbibing NAPL saturation into the water-wet soil fraction and the water saturation in the water-wet soil fraction.

If the imbibing NAPL volume is small, the NAPL imbibes into the NAPL-wet soil lens. It is also conceptualized that a small fraction of the NAPL imbibes into the water-wet soil fraction and forms NAPL film on water surfaces. A large NAPL volume is defined as the NAPL volume that is capable of attaining NAPL saturation in the NAPLwet soil lens at a value equal to or larger than the NSLL. The NAPL saturations in the water-wet soil fraction and in the NAPL-wet soil lens are defined as follows:

\section{Large NAPL Volume}

$$
\overline{\bar{S}}_{n_{N L}}=f\left(h_{a n}\right), h_{a n}=f\left(\overline{\bar{S}}_{t}\right), \overline{\bar{S}}_{n_{W W F}}=\overline{\bar{S}}_{t}-\overline{\bar{S}}_{T L L}+\bar{S}_{r} \text { if } \overline{\bar{S}}_{n_{N L}}>\overline{\bar{S}}_{n_{L L}}, \overline{\bar{S}}_{t}>\overline{\bar{S}}_{T L L}
$$

Where:

$\overline{\bar{S}}_{T L L}$ : apparent total liquid saturation lower limit.

\section{Small NAPL Volume}

$$
\begin{aligned}
& \overline{\bar{S}}_{n_{W W F}}=\bar{S}_{r_{-} f i l m} \\
& S_{n_{N L}}=\frac{V_{n}-\eta_{W W F} * V_{W W F} * S_{r_{-} f i m}}{\eta_{n w} * V_{N L}}
\end{aligned}
$$

Where:

$\overline{\bar{S}}_{n_{N L}}$ : apparent NAPL saturation in the NAPL-wet soil lens; 
$f\left(h_{a n}\right)$ : apparent NAPL saturation-air/NAPL capillary pressure head constitutive relationship in the NAPL-wet porous medium;

$f\left(\overline{\bar{S}}_{t}\right)$ : air/NAPL capillary pressure head-apparent total liquid saturation constitutive relationship in the water-wet porous medium;

$\overline{\bar{S}}_{n_{W W F}}:$ apparent NAPL saturation in the water-wet soil fraction; and

$V_{n}$ : imbibing NAPL volume.

\subsection{1.b. NAPL Drainage Pathway}

The NAPL drainage pathway starts where the NAPL imbibition pathway ends. If the imbibing NAPL volume is large, the NAPL drains from the porous medium until the NAPL saturation in the NAPL-wet soil lens equals to the NSLL. At this point, the NAPL exists in the water-wet soil fraction at residual NAPL saturation, that takes a form of NAPL film on water surfaces and NAPL residing in water wedges, and the NAPL saturation in the NAPL-wet soil lens equals to the NSLL. In contrast, if the imbibing NAPL volume is small, none of the NAPL drains from the porous medium. At this point, the NAPL exists in the water-wet soil fraction at residual NAPL saturation, that takes a form of NAPL film on water surfaces, and the NAPL saturation in the NAPL-wet soil lens remains as is, trapped in the NAPL-wet soil lens as the relative permeability of the NAPL in the surrounding soil is zero. The NAPL saturations in the water-wet soil fraction and in the NAPL-wet soil lens are defined as follows:

\section{Large NAPL Volume}

$$
\begin{aligned}
& \overline{\bar{S}}_{n_{N L}}=\overline{\bar{S}}_{n_{L L}} \\
& \overline{\bar{S}}_{n_{W W F}}=\bar{S}_{r}
\end{aligned}
$$




\section{Small NAPL Volume}

$$
\begin{aligned}
& \overline{\bar{S}}_{n_{W W F}}=\bar{S}_{r_{-} f i l m} \\
& S_{n_{N L}}=\frac{V_{n}-\eta_{W W F} * V_{W W F} * S_{r_{-} f l m}}{\eta_{m w} * V_{N L}}
\end{aligned}
$$

\subsubsection{Conceptual Model for Residual NAPL Saturation in a Water-Wet Porous Medium with a Connected NAPL-Wet Soil Lens}

\subsection{2.a. NAPL Imbibition Pathway}

The NAPL imbibition pathway for a connected NAPL-wet soil lens is similar to the NAPL imbibition pathway for an isolated NAPL-wet soil lens. Therefore, the same equations may be used to calculate liquid saturations in the water-wet soil fraction and in the NAPL-wet soil lens.

\subsection{2.b. NAPL Drainage Pathway}

The NAPL drainage pathway starts where the NAPL imbibition pathway ends. In contrast to the isolated NAPL-wet soil lens, the NAPL drainage pathways are similar for large and small imbibing NAPL volumes in the connected NAPL-wet soil lens. At the end of the NAPL drainage pathway, for a large imbibing NAPL volume, NAPL exists in the water-wet soil fraction at residual NAPL saturation that takes the form of NAPL film on water surfaces and NAPL residing in water wedges. For a small imbibing NAPL volume, NAPL exists in the water-wet soil fraction as NAPL film on water surfaces. In either case, NAPL exists in the NAPL-wet soil lens at irreducible NAPL saturation. As explained in Section 6.2 of Chapter 6, the residual NAPL saturation in the water-wet soil fraction is the same as the residual NAPL saturation in a water-wet porous medium. The 
irreducible NAPL saturation in the NAPL-wet soil lens is the same as the irreducible NAPL saturation in a NAPL-wet porous medium. The NAPL saturations in the water-wet soil fraction and in the NAPL-wet soil lens are defined as follows:

\section{Large NAPL Volume}

$$
\begin{aligned}
& S_{n_{N L}}=S_{i r_{N W}}^{a n} \\
& S_{n_{W W F}}=S_{r}
\end{aligned}
$$

\section{Small NAPL Volume}

$$
\begin{aligned}
& S_{n_{N L}}=S_{i r_{N W}}^{a n} \\
& S_{n_{W W F}}=S_{r_{-} f i l m}
\end{aligned}
$$

Where:

$$
\begin{aligned}
& S_{i r_{N W}}^{a n}: \text { irreducible NAPL saturation in air/NAPL two-phase system in the NAPL-wet } \\
& \text { porous medium. }
\end{aligned}
$$

\subsubsection{Residual NAPL Saturation in a Water-Wet Porous Medium with a NAPL- Wet Soil Lens of Similar Grain Size Distribution}

This section is a simplified scenario of Sections 7.3.1 and 7.3.2. In this section, the NSLL is equivalent to TLSLL. Therefore, the NAPL imbibition and drainage pathways and the equations developed to calculate NAPL saturations are similar to those presented in Sections 7.3.1 and 7.3.2. The equations developed in these two sections can be used to calculate the NAPL saturation in a water-wet soil fraction and in an isolated or a connected NAPL-wet soil lens. A simplified form of Equations 7.6 and 7.9 are Equations 7.17 and 7.18, respectively, which are given below. The conceptual model 
developed in Section 7.3.2, considering the grain size distribution of the connected NAPL-wet soil lens is similar to the grain size distribution of the water-wet soil fraction, is validated using the new conceptual model developed in Section 7.2 and the experimental data set (3).

$$
\begin{aligned}
& \overline{\bar{S}}_{n_{N L}}=\overline{\bar{S}}_{t}, \quad \overline{\bar{S}}_{n_{W W F}}=\overline{\bar{S}}_{t}-\overline{\bar{S}}_{T L L}+\bar{S}_{r} \text { if } \overline{\bar{S}}_{n_{N L}}=\overline{\bar{S}}_{t}>\overline{\bar{S}}_{T L L} \\
& \overline{\bar{S}}_{n_{N L}}=\overline{\bar{S}}_{T L L}
\end{aligned}
$$

\subsection{3.a. Conceptual Model Validation of the NAPL Drainage Pathway}

The experimental data set (3) includes residual heptane saturations measured in water-wet porous media with different size NAPL-wet soil lenses at different initial water and imbibing heptane saturations. The conceptual model described in Section 7.3.2 states that a NAPL saturation left in a water-wet soil fraction is equivalent to a residual NAPL saturation left in the water-wet porous medium at the same water and historic maximum imbibing NAPL saturation following NAPL imbibition and drainage. Moreover, a NAPL saturation left in a connected NAPL-wet soil lens should be equivalent to an irreducible NAPL saturation in the NAPL-wet porous medium following NAPL imbibition and drainage. In order to validate this conceptual model, residual heptane saturations in the water-wet soil fractions and irreducible heptane saturation in the NAPL-wet soil lenses were calculated from experimental data set (3). Provided that the irreducible heptane saturation in the NAPL-wet soil lens is assumed equivalent to the irreducible heptane saturation in a NAPL-wet porous medium, and depending on the NAPL-wet soil lens size, the residual heptane volumes left in the water-wet soil fractions were calculated from the experimental data. These residual heptane volumes were used to calculate the 
residual heptane saturations left in the water-wet soil fractions. The water saturations were identified in each water-wet soil fraction as well as the historic maximum heptane saturations imbibing into the water-wet soil fraction. The new conceptual model, described in Section 7.2, was then used to predict the residual heptane saturations in the water-wet soil fractions given the water and the historic maximum heptane saturations in the water-wet soil fractions.

The experimental data set (3) includes the residual heptane saturations in waterwet porous media with different size NAPL-wet soil lenses, corresponding to $90 \%$ waterwet-10\% NAPL-wet, 80\% water-wet-20\% NAPL-wet, 70\% water-wet-30\% NAPL-wet, and $60 \%$ water-wet-40\% NAPL-wet, in two sand types; uniform sand and well graded sand, at different initial water and imbibing heptane saturations. Table 7.6 through Table 7.13 present the measured residual heptane saturations (i.e., $\bar{S}_{r}$ ) within the water-wet soil fractions for the above porous media wettability configurations and compare these to the residual heptane saturations predicted by the new model. A brief review of the data contained in each table follows. The first column gives the apparent water saturation in the water-wet soil fraction $\left(\bar{S}_{w_{u v F}}\right)$. The second column is the volume of heptane allowed to imbibe into the porous medium prior to drainage. The third column is the heptane volume imbibing into the water-wet soil fraction $\left(V_{n_{\text {IVwF }}}\right)$ and is calculated as the total imbibing heptane volume less the imbibing heptane volume into the NAPL-wet soil lens. The latter is calculated as the volume of heptane in the NAPL-wet soil lens corresponding to an apparent heptane saturation equivalent to the apparent total liquid saturation in the water-wet soil fraction. The fourth column gives the apparent heptane saturation in the water-wet soil fraction $\left(\overline{\bar{S}}_{n_{W W F}}\right)$. If this value is greater than zero, it corresponds to a large 
imbibing heptane volume otherwise the imbibing heptane volume is small, as explained in Section 7.3.2.a and considering NSLL and TLSLL are equivalent. The fifth column gives the total residual heptane volume left in the porous medium and is denoted as $V_{r}$, which is the summation of the residual heptane volume in the water-wet soil fraction and the irreducible heptane volume in the NAPL-wet soil lens. The sixth column is the assumed irreducible heptane volume in the NAPL-wet soil lens $\left(V_{r_{N L}}\right)$. The latter is calculated based on the irreducible heptane saturation from an air/heptane $P_{c} \neg S$ experiment, and the NAPL-wet soil lens size and porosity. The seventh column is, therefore, the remaining residual heptane volume in the water-wet soil fraction $\left(V_{r_{w W F}}\right)$.

The eighth column gives the effective residual heptane saturation in the water-wet soil fraction $\left(\bar{S}_{r}\right)$, which is conceptualized to be equivalent to the effective residual heptane saturation in a water-wet porous medium. The ninth column gives the effective saturation of heptane film on water surfaces in the water-wet soil fraction $\left(\bar{S}_{r_{-}}\right.$film $)$, which is equivalent to that in a water-wet porous medium. In addition, the last column shows the new conceptual model prediction of the effective residual heptane saturation in the waterwet soil fraction. Table 7.6, Table 7.8, Table 7.10, and Table 7.12 show the data and the new model predictions for the uniform sand and Table 7.7, Table 7.9, Table 7.11, and Table 7.13 show the data and the new model predictions for the well graded sand. As shown in the tables, the new model predictions agree, in most cases, with the residual heptane saturations measured in the water-wet soil fractions and this validates the conceptual model presented in Section 7.3 for residual NAPL saturation in a water-wet porous medium with a connected NAPL-wet soil lens of similar grain size distribution. The disagreement between the new model predictions and the residual heptane saturation 
data may be attributed to underestimation or overestimation of the assumed irreducible heptane volumes in the NAPL-wet soil lenses or due to experimental errors.

Table 7.6:Measured Versus Predicted Effective Residual Heptane Saturations in 90\% Water-Wet-10\% NAPL-Wet Uniform Sand.

\begin{tabular}{|c|c|c|c|c|c|c|c|c|c|c|c|}
\hline \multicolumn{2}{|c|}{ Experiment } & $\overline{\bar{S}}_{w_{W W F}}$ & $V_{n}$ & $V_{n_{w w F}}$ & $\overline{\bar{S}}_{n_{W W F}}$ & $V_{r}$ & $V_{r_{N L}}$ & $V_{r_{w \gamma F}}$ & $\bar{S}_{r}$ & $\bar{S}_{r_{-} \text {film }}$ & $\begin{array}{l}\text { New } \\
\text { Model }\end{array}$ \\
\hline \multirow{18}{*}{\begin{tabular}{l} 
Z \\
\hdashline \\
0 \\
1 \\
3 \\
0 \\
0 \\
0
\end{tabular}} & \multirow{9}{*}{1} & 41.3 & 11.90 & 8.04 & 23.7 & 4.70 & 1.09 & 3.61 & 7.9 & 3.6 & 7.2 \\
\hline & & 41.3 & 17.80 & 11.83 & 34.9 & 6.20 & 1.09 & 5.11 & 11.2 & 3.6 & 8.6 \\
\hline & & 41.3 & 26.60 & 19.90 & 58.7 & 8.00 & 1.09 & 6.91 & $\overline{15.2}$ & 3.6 & 10.8 \\
\hline & & 21.7 & 11.90 & 7.74 & 20.8 & 6.30 & 1.09 & 5.21 & 11.4 & 4.4 & 10.8 \\
\hline & & 21.7 & 17.80 & 13.78 & 37.0 & 6.75 & 1.09 & 5.66 & 12.4 & 4.4 & 10.8 \\
\hline & & 21.7 & 34.85 & 29.17 & 78.3 & 7.55 & 1.09 & 6.46 & 14.2 & 4.4 & 11.8 \\
\hline & & 0.0 & 11.90 & 9.04 & 23.8 & 5.40 & 1.09 & 4.31 & 9.5 & 5.0 & 11.8 \\
\hline & & 0.0 & 17.80 & 15.67 & 41.3 & 5.25 & 1.09 & 4.16 & 9.1 & 5.0 & 11.8 \\
\hline & & 0.0 & 42.50 & 37.97 & 100.0 & 5.30 & 1.09 & 4.21 & 9.2 & 5.0 & 12.1 \\
\hline & \multirow{9}{*}{2} & 42.0 & 11.90 & 8.04 & 21.8 & 4.50 & 1.09 & 3.41 & 7.4 & 3.6 & 7.0 \\
\hline & & 42.0 & 17.80 & 12.71 & 34.5 & 5.70 & 1.09 & 4.61 & 10.0 & 3.6 & 8.6 \\
\hline & & 42.0 & 27.25 & 21.33 & 58.0 & 6.95 & 1.09 & 5.86 & 12.7 & 3.6 & 10.7 \\
\hline & & 22.7 & 11.90 & 8.53 & 22.8 & 4.85 & 1.09 & 3.76 & 8.1 & 4.4 & 10.8 \\
\hline & & 22.7 & 17.80 & 13.63 & 36.5 & 5.10 & 1.09 & 4.01 & 8.7 & 4.4 & 10.8 \\
\hline & & 22.7 & 34.70 & 28.87 & 77.3 & 6.15 & 1.09 & 5.06 & 11.0 & 4.4 & 11.7 \\
\hline & & 0.0 & 11.90 & 9.50 & 24.9 & 5.25 & 1.09 & 4.16 & 9.0 & 5.0 & 11.7 \\
\hline & & 0.0 & 17.80 & 15.76 & 41.3 & 4.85 & 1.09 & 3.76 & 8.1 & 5.0 & 11.7 \\
\hline & & 0.0 & 42.75 & 38.13 & 100.0 & 5.05 & 1.09 & 3.96 & 8.6 & 5.0 & 12.1 \\
\hline
\end{tabular}


Table 7.7: Measured Versus Predicted Effective Residual Heptane Saturations in 90\% Water-Wet-10\% NAPL-Wet Well Graded Sand.

\begin{tabular}{|c|c|c|c|c|c|c|c|c|c|c|c|}
\hline \multicolumn{2}{|c|}{ Experiment } & $\overline{\bar{S}}_{w_{W W F}}$ & $V_{n}$ & $V_{n_{w W F}}$ & $\overline{\bar{S}}_{n_{W W F}}$ & $V_{r}$ & $V_{r_{N L}}$ & $V_{r_{W W F}}$ & $\bar{S}_{r}$ & $\bar{S}_{r_{-} f i l m}$ & $\begin{array}{l}\text { New } \\
\text { Model }\end{array}$ \\
\hline \multirow{18}{*}{$\begin{array}{l}\mathbf{Z} \\
\dot{0} \\
1 \\
3 \\
3 \\
0 \\
0\end{array}$} & \multirow{9}{*}{1} & 41.1 & 10.60 & 7.16 & 21.1 & 4.00 & 0.87 & 3.13 & 7.7 & 3.4 & 7.0 \\
\hline & & 41.1 & 15.90 & 10.91 & 32.2 & 5.15 & 0.87 & 4.28 & 10.6 & 3.4 & 8.6 \\
\hline & & 41.1 & 25.85 & 19.98 & 58.9 & 6.35 & 0.87 & 5.48 & 13.6 & 3.4 & 11.4 \\
\hline & & 21.5 & 10.60 & 7.35 & 21.3 & 5.55 & 0.87 & 4.68 & 11.6 & 4.3 & 11.4 \\
\hline & & 21.5 & 15.90 & 12.40 & 36.0 & 5.95 & 0.87 & 5.08 & 12.6 & 4.3 & 11.4 \\
\hline & & 21.5 & 32.45 & 27.06 & 78.5 & 6.85 & 0.87 & 5.98 & 14.8 & 4.3 & 12.4 \\
\hline & & 0.0 & 10.60 & 8.00 & 24.6 & 5.40 & 0.87 & 4.53 & 11.2 & 5.3 & 12.4 \\
\hline & & 0.0 & 15.90 & 13.25 & 40.8 & 5.40 & 0.87 & 4.53 & 11.2 & 5.3 & 12.4 \\
\hline & & 0.0 & 37.25 & 32.47 & 100.0 & 5.75 & 0.87 & 4.88 & 12.1 & 5.3 & 12.7 \\
\hline & \multirow{9}{*}{2} & 41.5 & 10.60 & 7.16 & 20.8 & 3.80 & 0.87 & 2.93 & 7.2 & 3.4 & 7.0 \\
\hline & & 41.5 & 15.90 & 11.43 & 33.2 & 4.65 & 0.87 & 3.78 & 9.3 & 3.4 & 8.7 \\
\hline & & 41.5 & 25.45 & 20.11 & 58.5 & 5.35 & 0.87 & 4.48 & 11.0 & 3.4 & 11.4 \\
\hline & & 22.1 & 10.60 & 7.61 & 22.7 & 4.85 & 0.87 & 3.98 & 9.8 & 4.3 & 11.3 \\
\hline & & 22.1 & 15.90 & 12.33 & 36.7 & 5.45 & 0.87 & 4.58 & 11.2 & 4.3 & 11.3 \\
\hline & & 22.1 & 31.20 & 26.16 & 77.9 & 6.40 & 0.87 & 5.53 & 13.6 & 4.3 & 12.4 \\
\hline & & 0.0 & 10.60 & 8.61 & 26.8 & 5.30 & 0.87 & 4.43 & 10.9 & 5.3 & 12.3 \\
\hline & & 0.0 & 15.90 & 13.27 & 41.3 & 5.55 & 0.87 & 4.68 & 11.5 & 5.3 & 12.3 \\
\hline & & 0.0 & 36.85 & 32.15 & 100.0 & 5.95 & 0.87 & 5.08 & 12.5 & 5.3 & 12.7 \\
\hline
\end{tabular}

Table 7.8: Measured Versus Predicted Effective Residual Heptane Saturations in 80\% Water-Wet-20\% NAPL-Wet Uniform Sand.

\begin{tabular}{|c|c|c|c|c|c|c|c|c|c|c|c|}
\hline \multicolumn{2}{|c|}{ Experiment } & $\overline{\bar{S}}_{w_{W W F}}$ & $V_{n}$ & $V_{n_{W W F}}$ & $\overline{\bar{S}}_{n_{W W F}}$ & $V_{r}$ & $V_{r_{N L}}$ & $V_{r_{w V F}}$ & $\bar{S}_{r}$ & $\bar{S}_{r_{-} \text {film }}$ & $\begin{array}{l}\text { New } \\
\text { Model }\end{array}$ \\
\hline \multirow{18}{*}{ 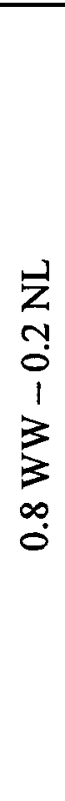 } & \multirow{9}{*}{1} & 50.1 & 11.90 & 4.17 & 12.1 & 5.90 & 2.18 & 3.72 & 8.9 & 3.3 & 5.3 \\
\hline & & 50.1 & 17.80 & 7.09 & 20.7 & 7.70 & 2.18 & 5.52 & 13.2 & 3.3 & 6.6 \\
\hline & & 50.1 & 29.90 & 17.13 & 49.9 & 8.10 & 2.18 & 5.92 & 14.2 & 3.3 & 10.1 \\
\hline & & 28.8 & 11.90 & 5.92 & 16.1 & 6.20 & 2.18 & 4.02 & 9.6 & 4.2 & 10.2 \\
\hline & & 28.8 & 17.80 & 9.49 & 25.9 & 6.90 & 2.18 & 4.72 & 11.3 & 4.2 & 10.2 \\
\hline & & 28.8 & 38.55 & 26.15 & 71.2 & 7.65 & 2.18 & 5.47 & 13.1 & 4.2 & 11.5 \\
\hline & & 0.0 & 11.90 & 6.90 & 17.8 & 6.80 & 2.18 & 4.62 & 11.1 & 5.1 & 11.5 \\
\hline & & 0.0 & 17.80 & 11.45 & 29.5 & 6.45 & 2.18 & 4.27 & 10.2 & 5.1 & 11.5 \\
\hline & & 0.0 & 52.15 & 38.86 & 100.0 & 6.95 & 2.18 & 4.77 & $\overline{11.4}$ & 5.1 & 12.1 \\
\hline & \multirow{9}{*}{2} & 49.1 & 11.90 & 4.17 & 13.0 & 5.20 & 2.18 & 3.02 & 7.4 & 3.3 & 5.5 \\
\hline & & 49.1 & 17.80 & 6.95 & 21.7 & 6.15 & 2.18 & 3.97 & 9.7 & 3.3 & 6.8 \\
\hline & & 49.1 & 29.25 & 16.30 & 50.9 & 7.90 & 2.18 & 5.72 & 14.0 & 3.3 & 10.2 \\
\hline & & 27.4 & 11.90 & 5.72 & 17.6 & 6.00 & 2.18 & 3.82 & 9.3 & 4.2 & 10.2 \\
\hline & & 27.4 & 17.80 & 9.69 & 29.7 & 6.50 & 2.18 & 4.32 & $\overline{10.6}$ & 4.2 & 10.2 \\
\hline & & 27.4 & 35.15 & 23.67 & 72.6 & 7.10 & 2.18 & 4.92 & 12.0 & 4.2 & 11.6 \\
\hline & & 0.0 & 11.90 & 6.19 & 17.8 & 5.20 & 2.18 & 3.02 & 7.4 & 5.0 & 11.6 \\
\hline & & 0.0 & 17.80 & 9.69 & 27.9 & 4.95 & 2.18 & 2.77 & 6.8 & 5.0 & 11.6 \\
\hline & & 0.0 & 45.15 & 34.80 & 100.0 & 5.15 & 2.18 & 2.97 & 7.3 & 5.0 & 12.1 \\
\hline
\end{tabular}


Table 7.9: Measured Versus Predicted Effective Residual Heptane Saturations in 80\% Water-Wet-20\% NAPL-Wet Well Graded Sand.

\begin{tabular}{|c|c|c|c|c|c|c|c|c|c|c|c|}
\hline \multicolumn{2}{|c|}{ Experiment } & $\overline{\bar{S}}_{w_{W W F}}$ & $V_{n}$ & $V_{n_{w w F}}$ & $\overline{\bar{S}}_{n_{W W F}}$ & $V_{r}$ & $V_{r_{N L}}$ & $V_{r_{W W F F}}$ & $\bar{S}_{r}$ & $\bar{S}_{r_{-} \text {film }}$ & $\begin{array}{l}\text { New } \\
\text { Model }\end{array}$ \\
\hline \multirow{18}{*}{ 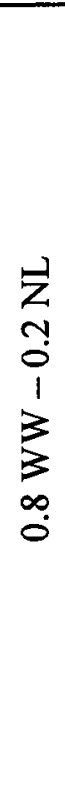 } & \multirow{9}{*}{1} & 48.2 & 10.60 & 3.71 & 17.6 & 4.20 & 1.75 & 2.45 & 6.9 & 3.0 & 6.3 \\
\hline & & 48.2 & 15.90 & 6.89 & 32.7 & 5.15 & 1.75 & 3.40 & 9.5 & 3.0 & 8.6 \\
\hline & & 48.2 & 20.80 & 10.92 & 51.8 & 5.75 & 1.75 & 4.00 & 11.2 & 3.0 & 10.8 \\
\hline & & 26.1 & 10.60 & 4.70 & 18.7 & 5.25 & 1.75 & 3.50 & 9.8 & 4.1 & 10.8 \\
\hline & & 26.1 & 15.90 & 8.84 & 35.2 & 6.55 & 1.75 & 4.80 & $\overline{13.4}$ & 4.1 & 10.8 \\
\hline & & 26.1 & 27.70 & 18.54 & 73.9 & 7.05 & 1.75 & 5.30 & 14.8 & 4.1 & 12.2 \\
\hline & & 0.0 & 10.60 & 5.99 & 19.5 & 6.00 & 1.75 & 4.25 & 11.9 & 5.3 & 12.2 \\
\hline & & $\overline{0.0}$ & 15.90 & 10.74 & 35.0 & 6.05 & 1.75 & 4.30 & 12.0 & 5.3 & 12.2 \\
\hline & & 0.0 & 41.00 & 30.71 & 100.0 & 6.90 & 1.75 & 5.15 & 14.4 & 5.3 & 12.7 \\
\hline & \multirow{9}{*}{2} & 47.8 & 10.60 & 3.71 & 12.4 & 4.40 & 1.75 & 2.65 & 7.5 & 3.0 & 5.3 \\
\hline & & 47.8 & 15.90 & 7.09 & 23.7 & 5.20 & 1.75 & 3.45 & 9.7 & 3.0 & 7.2 \\
\hline & & 47.8 & 27.40 & 15.61 & 52.2 & 6.05 & 1.75 & 4.30 & 12.1 & 3.0 & 10.9 \\
\hline & & 25.5 & 10.60 & 4.64 & 15.2 & 5.90 & 1.75 & 4.15 & 11.7 & 4.1 & 10.8 \\
\hline & & 25.5 & 15.90 & 8.97 & 29.5 & 6.35 & 1.75 & 4.60 & 13.0 & 4.1 & 10.8 \\
\hline & & 25.5 & 32.90 & 22.66 & 74.5 & 6.80 & 1.75 & 5.05 & 14.3 & 4.1 & 12.3 \\
\hline & & 0.0 & 10.60 & 5.88 & 20.2 & 5.70 & 1.75 & 3.95 & 11.2 & 5.2 & 12.2 \\
\hline & & 0.0 & 15.90 & 10.74 & 36.8 & 5.80 & 1.75 & 4.05 & 11.4 & 5.2 & 12.2 \\
\hline & & 0.0 & 38.90 & 29.16 & 100.0 & 6.00 & 1.75 & 4.25 & 12.0 & 5.2 & 12.7 \\
\hline
\end{tabular}

Table 7.10: Measured Versus Predicted Effective Residual Heptane Saturations in 70\% Water-Wet-30\% NAPL-Wet Uniform Sand.

\begin{tabular}{|c|c|c|c|c|c|c|c|c|c|c|c|}
\hline \multicolumn{2}{|c|}{ Experiment } & $\overline{\bar{S}}_{w_{W W F}}$ & $V_{n}$ & $V_{n_{w W F}}$ & $\overline{\bar{S}}_{n_{W W F}}$ & $V_{r}$ & $V_{r_{N L}}$ & $V_{r_{w W F}}$ & $\bar{S}_{r}$ & $\bar{S}_{r_{-} \text {film }}$ & $\begin{array}{l}\text { New } \\
\text { Model }\end{array}$ \\
\hline \multirow{18}{*}{$\begin{array}{l}1 \\
z \\
m \\
0 \\
1 \\
3 \\
\vdots \\
0\end{array}$} & \multirow{9}{*}{1} & 57.6 & 11.90 & 0.30 & 1.0 & 4.50 & 3.27 & 1.23 & 3.5 & 2.8 & 3.0 \\
\hline & & 57.6 & 17.80 & 3.04 & 10.2 & 5.35 & 3.27 & 2.08 & 5.9 & 2.8 & 4.6 \\
\hline & & 57.6 & 31.50 & 12.71 & 42.4 & 7.40 & 3.27 & 4.13 & 11.8 & 2.8 & 9.3 \\
\hline & & 32.2 & 11.90 & 4.13 & 14.1 & 5.85 & 3.27 & 2.58 & 7.4 & 4.0 & 9.5 \\
\hline & & 32.2 & 17.80 & 5.80 & 19.8 & 5.65 & 3.27 & 2.38 & 6.8 & 4.0 & 9.5 \\
\hline & & 32.2 & 38.05 & 19.85 & 67.8 & 6.90 & 3.27 & 3.63 & 10.4 & 4.0 & 11.3 \\
\hline & & 0.0 & 11.90 & 5.17 & 17.2 & 5.35 & 3.27 & 2.08 & 5.9 & 5.0 & 11.4 \\
\hline & & 0.0 & 17.80 & 9.18 & 30.6 & 5.05 & 3.27 & 1.78 & 5.1 & 5.0 & 11.4 \\
\hline & & 0.0 & 47.75 & 29.99 & 100.0 & 6.15 & 3.27 & 2.88 & 8.2 & 5.0 & 12.1 \\
\hline & \multirow{9}{*}{2} & 57.8 & 11.90 & 0.30 & 1.1 & 4.60 & 3.27 & 1.33 & 3.8 & 2.8 & 3.0 \\
\hline & & 57.8 & 17.80 & 2.69 & 9.8 & 5.45 & 3.27 & 2.18 & 6.2 & 2.8 & 4.6 \\
\hline & & 57.8 & 30.70 & 11.62 & 42.2 & 7.45 & 3.27 & 4.18 & 11.9 & 2.8 & 9.3 \\
\hline & & 32.6 & 11.90 & 4.18 & 14.8 & 5.75 & 3.27 & 2.48 & 7.0 & 4.0 & 9.4 \\
\hline & & 32.6 & 17.80 & 5.56 & 19.6 & 5.95 & 3.27 & 2.68 & 7.6 & 4.0 & 9.4 \\
\hline & & 32.6 & 37.05 & 19.10 & 67.4 & 7.45 & 3.27 & 4.18 & 11.9 & 4.0 & 11.3 \\
\hline & & 0.0 & 11.90 & 5.39 & 17.9 & 5.65 & 3.27 & 2.38 & 6.8 & 5.0 & 11.3 \\
\hline & & 0.0 & 17.80 & 10.00 & 33.2 & 5.15 & 3.27 & 1.88 & 5.3 & 5.0 & 11.3 \\
\hline & & 0.0 & 47.00 & 30.15 & 100.0 & 5.85 & 3.27 & 2.58 & 7.3 & 5.0 & 12.1 \\
\hline
\end{tabular}


Table 7.11: Measured Versus Predicted Effective Residual Heptane Saturations in 70\% Water-Wet-30\% NAPL-Wet Well Graded Sand.

\begin{tabular}{|c|c|c|c|c|c|c|c|c|c|c|c|}
\hline \multicolumn{2}{|c|}{ Experiment } & $\overline{\bar{S}}_{w_{W W F}}$ & $V_{n}$ & $V_{n_{w W F}}$ & $\overline{\bar{S}}_{n_{W W F}}$ & $V_{r}$ & $V_{r_{N L}}$ & $V_{r_{\text {HWF }}}$ & $\bar{S}_{r}$ & $\bar{S}_{r_{-} f i l m}$ & $\begin{array}{l}\text { New } \\
\text { Model }\end{array}$ \\
\hline \multirow{18}{*}{$\begin{array}{l}\vec{z} \\
m \\
0 \\
1 \\
3 \\
3 \\
\vdots \\
0\end{array}$} & \multirow{9}{*}{1} & 57.1 & 10.60 & 0.27 & 1.3 & 3.50 & 2.62 & 0.88 & 2.9 & 2.5 & 2.7 \\
\hline & & 57.1 & 15.90 & 2.41 & 11.8 & 4.75 & 2.62 & 2.13 & 6.9 & 2.5 & 4.9 \\
\hline & & 57.1 & 24.50 & 8.81 & 42.9 & 6.10 & 2.62 & 3.48 & 11.3 & 2.5 & 9.8 \\
\hline & & 31.5 & 10.60 & 3.48 & 15.3 & 5.30 & 2.62 & 2.68 & 8.7 & 3.8 & 9.9 \\
\hline & & 31.5 & 15.90 & 5.32 & 23.4 & 5.85 & 2.62 & 3.23 & $\mathbf{1 0 . 5}$ & 3.8 & 9.9 \\
\hline & & 31.5 & 30.30 & 15.57 & 68.5 & 6.80 & 2.62 & 4.18 & 13.6 & 3.8 & 12.0 \\
\hline & & 0.0 & 10.60 & 4.80 & 20.5 & 5.30 & 2.62 & 2.68 & 8.7 & 5.2 & 12.0 \\
\hline & & 0.0 & 15.90 & 8.23 & 35.2 & 5.10 & 2.62 & 2.48 & 8.1 & 5.2 & 12.0 \\
\hline & & 0.0 & 37.40 & 23.36 & 100.0 & 6.15 & 2.62 & 3.53 & 11.5 & 5.2 & 12.7 \\
\hline & \multirow{9}{*}{2} & 58.1 & 10.60 & 0.27 & 1.4 & 3.90 & 2.62 & 1.28 & 4.1 & 2.5 & 2.8 \\
\hline & & 58.1 & 15.90 & 1.90 & 9.9 & 4.20 & 2.62 & 1.58 & 5.0 & 2.5 & 4.5 \\
\hline & & 58.1 & 25.20 & 8.05 & 41.9 & 6.10 & 2.62 & 3.48 & 11.0 & 2.5 & 9.7 \\
\hline & & 33.0 & 10.60 & 3.48 & 14.8 & 5.45 & 2.62 & 2.83 & 9.0 & 3.8 & 9.8 \\
\hline & & 33.0 & 15.90 & 5.09 & 21.6 & 6.10 & 2.62 & 3.48 & 11.0 & 3.8 & 9.8 \\
\hline & & 33.0 & 31.05 & 15.82 & 67.0 & 6.60 & 2.62 & 3.98 & 12.6 & 3.8 & 11.9 \\
\hline & & 0.0 & 10.60 & 4.77 & 20.8 & 5.65 & 2.62 & 3.03 & 9.6 & 5.3 & 11.9 \\
\hline & & 0.0 & 15.90 & 8.20 & 35.7 & 5.45 & 2.62 & 2.83 & 9.0 & 5.3 & 11.9 \\
\hline & & 0.0 & 37.10 & 22.98 & 100.0 & 6.70 & 2.62 & 4.08 & 13.0 & 5.3 & 12.7 \\
\hline
\end{tabular}

Table 7.12: Measured Versus Predicted Effective Residual Heptane Saturations in $60 \%$ Water-Wet-40\% NAPL-Wet Uniform Sand.

\begin{tabular}{|c|c|c|c|c|c|c|c|c|c|c|c|}
\hline \multicolumn{2}{|c|}{ Experiment } & $\overline{\bar{S}}_{w_{W w F}}$ & $V_{n}$ & $V_{n_{W W F}}$ & $\overline{\bar{S}}_{n_{W W W F}}$ & $V_{r}$ & $V_{r_{N L}}$ & $V_{r_{\text {wWF }}}$ & $\bar{S}_{r}$ & $\bar{S}_{r_{-} f i l m}$ & $\begin{array}{l}\text { New } \\
\text { Model }\end{array}$ \\
\hline \multirow{18}{*}{$\begin{array}{l}z \\
\dot{z} \\
0 \\
1 \\
z \\
0 \\
0 \\
0\end{array}$} & \multirow{9}{*}{1} & 69.8 & 11.90 & 0.00 & 0.0 & 4.45 & 4.35 & 0.10 & 0.3 & 2.0 & 2.0 \\
\hline & & 69.8 & 17.80 & 0.10 & 0.8 & 5.35 & 4.35 & 1.00 & 3.4 & 2.0 & 2.2 \\
\hline & & 69.8 & 27.50 & 3.59 & 30.2 & 6.55 & 4.35 & 2.20 & 7.4 & 2.0 & 7.5 \\
\hline & & 39.6 & 11.90 & 2.20 & 11.3 & 5.30 & 4.35 & 0.95 & 3.2 & 3.6 & 8.0 \\
\hline & & 39.6 & 17.80 & 1.49 & 7.6 & 5.45 & 4.35 & 1.10 & 3.7 & 3.6 & 8.0 \\
\hline & & 39.6 & 34.80 & 11.78 & 60.4 & 7.75 & 4.35 & 3.40 & 11.5 & 3.6 & 10.9 \\
\hline & & 0.0 & 11.90 & 3.43 & 15.5 & 6.40 & 4.35 & 2.05 & 6.9 & 4.9 & 11.0 \\
\hline & & 0.0 & 17.80 & 6.43 & 29.0 & 6.35 & 4.35 & 2.00 & 6.8 & 4.9 & 11.0 \\
\hline & & 0.0 & 44.00 & 22.13 & 100.0 & 7.35 & 4.35 & 3.00 & 10.1 & 4.9 & 12.1 \\
\hline & \multirow{9}{*}{2} & 69.5 & 11.90 & 0.00 & 0.0 & 5.40 & 4.35 & 1.05 & 3.6 & 2.0 & 2.0 \\
\hline & & 69.5 & 17.80 & 1.05 & 8.0 & 5.70 & 4.35 & 1.35 & 4.6 & 2.0 & 3.7 \\
\hline & & 69.5 & 27.95 & 4.00 & 30.5 & 7.75 & 4.35 & 3.40 & 11.6 & 2.0 & 7.6 \\
\hline & & 39.0 & 11.90 & 3.40 & 17.1 & 5.85 & 4.35 & 1.50 & 5.1 & 3.6 & 8.1 \\
\hline & & 39.0 & 17.80 & 1.53 & 7.7 & 5.85 & 4.35 & 1.50 & 5.1 & 3.6 & 8.1 \\
\hline & & 39.0 & 35.45 & 12.12 & 61.0 & 8.20 & 4.35 & 3.85 & 13.2 & 3.6 & 10.9 \\
\hline & & 0.0 & 11.90 & 3.85 & 16.8 & 6.45 & 4.35 & 2.10 & 7.2 & 4.9 & 11.0 \\
\hline & & 0.0 & 17.80 & 7.05 & 30.7 & 5.95 & 4.35 & 1.60 & 5.5 & 4.9 & 11.0 \\
\hline & & 0.0 & 44.30 & 22.96 & 100.0 & 6.10 & 4.35 & 1.75 & 6.0 & 4.9 & 12.1 \\
\hline
\end{tabular}


Table 7.13: Measured Versus Predicted Effective Residual Heptane Saturations in 60\% Water-Wet-40\% NAPL-Wet Well Graded Sand.

\begin{tabular}{|c|c|c|c|c|c|c|c|c|c|c|c|}
\hline \multicolumn{2}{|c|}{ Experiment } & $\overline{\bar{S}}_{w_{W w F}}$ & $V_{n}$ & $V_{n_{W W F}}$ & $\overline{\bar{S}}_{n_{W W F}}$ & $V_{r}$ & $V_{r_{N L}}$ & $V_{r_{t r W F}}$ & $\bar{S}_{r}$ & $\bar{S}_{r_{-} \text {film }}$ & $\begin{array}{l}\text { New } \\
\text { Model }\end{array}$ \\
\hline \multirow{18}{*}{$\begin{array}{l}\vec{Z} \\
\dot{J} \\
0 \\
1 \\
3 \\
3 \\
0 \\
0\end{array}$} & \multirow{9}{*}{1} & 69.8 & 10.60 & 0.00 & 0.0 & 4.00 & 3.49 & 0.51 & 1.9 & 1.7 & 1.7 \\
\hline & & 69.8 & 15.90 & 0.51 & 2.9 & 4.35 & 3.49 & 0.86 & 3.3 & 1.7 & 2.4 \\
\hline & & 69.8 & 26.60 & 5.34 & 30.2 & 5.40 & 3.49 & 1.91 & 7.3 & 1.7 & 7.8 \\
\hline & & 39.7 & 10.60 & 1.91 & 9.6 & 4.95 & 3.49 & 1.46 & 5.6 & 3.3 & 8.3 \\
\hline & & 39.7 & 15.90 & 2.50 & 12.6 & 5.10 & 3.49 & 1.61 & 6.1 & 3.3 & 8.3 \\
\hline & & 39.7 & 32.00 & 12.01 & 60.3 & 5.55 & 3.49 & 2.06 & 7.9 & 3.3 & 11.5 \\
\hline & & 0.0 & $10: 60$ & 3.06 & 17.0 & 4.85 & 3.49 & 1.36 & 5.2 & 5.2 & 11.5 \\
\hline & & 0.0 & 15.90 & 6.05 & 33.6 & 5.10 & 3.49 & 1.61 & 6.1 & 5.2 & 11.5 \\
\hline & & 0.0 & 35.35 & 18.00 & 100.0 & 6.15 & 3.49 & 2.66 & 10.1 & 5.2 & 12.7 \\
\hline & \multirow{9}{*}{2} & 69.5 & 10.60 & 0.00 & 0.0 & 4.80 & 3.49 & 1.31 & 5.0 & 1.7 & 1.7 \\
\hline & & 69.5 & 15.90 & 1.31 & 8.7 & 5.65 & 3.49 & 2.16 & 8.3 & 1.7 & 3.7 \\
\hline & & 69.5 & 25.60 & 4.60 & 30.5 & 5.60 & 3.49 & 2.11 & 8.1 & 1.7 & 7.8 \\
\hline & & 39.2 & 10.60 & 2.11 & 11.6 & 5.10 & 3.49 & 1.61 & 6.2 & 3.3 & 8.3 \\
\hline & & 39.2 & 15.90 & 1.72 & 9.5 & 5.20 & 3.49 & 1.71 & 6.6 & 3.3 & 8.3 \\
\hline & & 39.2 & 31.35 & 11.03 & 60.8 & 6.55 & 3.49 & 3.06 & 11.8 & 3.3 & 11.5 \\
\hline & & 0.0 & 10.60 & 3.20 & 18.4 & 5.00 & 3.49 & 1.51 & 5.8 & 5.1 & 11.5 \\
\hline & & 0.0 & 15.90 & 5.76 & 33.1 & 5.25 & 3.49 & 1.76 & 6.8 & 5.1 & 11.5 \\
\hline & & 0.0 & 35.10 & 17.38 & 100.0 & 6.00 & 3.49 & 2.51 & 9.6 & 5.1 & 12.7 \\
\hline
\end{tabular}

\subsection{Summary}

This chapter focused on the development and validation of a new conceptual model for residual NAPL saturation in a water-wet porous medium. The new model assumes that the effective residual NAPL saturation depends on eight parameters; porous medium specific surface area, NAPL entry pressure head in NAPL/water two-phase system, porous medium grain size distribution (through the fitting parameter $\lambda$ ), porous medium porosity, NAPL film thickness, apparent water saturation, historic maximum apparent NAPL saturation, and the absolute maximum effective residual NAPL saturation in the porous medium. Model predications were compared to the effective residual heptane saturations measured under a large range of experimental conditions. In 
most cases, the model was capable of predicting the measured effective residual heptane saturations. Then, two conceptual models were developed to describe the residual NAPL saturations in a water-wet porous medium with an isolated or a connected NAPL-wet soil lens, respectively. The conceptual model that described the residual NAPL saturation in a water-wet porous medium with a connected NAPL-wet soil lens, of similar grain size distribution, was then validated. In most cases, the model predictions agreed with the measured effective residual heptane saturations. 


\section{Chapter 8. Summary, Contributions, and Recommendations}

\subsection{Summary}

This research focused on the effects of porous medium wettability on $P_{c}-S$ relationships in two and three-phase systems using a spreading LNAPL. The porous medium wettability refers to a water-wet porous medium and a water-wet porous medium with NAPL-wet soil lenses. Using the knowledge of $P_{c}-S$ relationships in a water-wet porous medium and in a NAPL-wet porous medium, conceptual models were developed for $P_{c}-S$ relationships in air/NAPL, air/water, and NAPL/water two-phase systems in a water-wet porous medium with a NAPL-wet soil lens. Conceptual models were also developed for a three-phase system in a water-wet porous medium with a NAPL-wet soil lens. The research also proposed new conceptual models for residual NAPL saturation in a water-wet porous medium and in a water-wet porous medium with NAPL-wet soil lenses. In this research, the following tasks were undertaken:

- Initially a comprehensive literature review was completed to investigate porous medium and immiscible fluid phase characteristics, and interactions between the immiscible fluid phases and porous media through capillarity. Then the mass balance equations, constitutive relations, and deficiencies in the current multiphase flow treatment were presented. A review of the work pertaining to incorporation of wettability and residual NAPL saturation into multiphase flow equations, which is the research focus, was completed.

- A detailed description of the experimental work was presented. Two sands with different grain size distributions, uniform and well graded, were exposed to 
different saturation pathways in Tempe cells and Buchner funnels under different wettability conditions. In addition, a porous medium with a grain size distribution similar to the uniform sand was generated from glass beads and exposed to different saturation pathways in a glass box apparatus to visually view the NAPL distribution at selected saturation values under the different wettability conditions. The saturation pathways include $P_{c} \rightarrow S$ pathways in two and three-phase systems and residual NAPL saturation experiments. The NAPL used was heptane and it corresponds to a spreading LNAPL.

- During the course of the initial experiments, a study was performed on the effectiveness of the traditional procedure that has been widely published in the literature to render hydrophilic silica sands and porous ceramics hydrophobic. This study showed that the traditional procedure results in excess unbound OTS on the treated surfaces. Experimental results indicated that treatment with a 5\% (by volume) OTS solution in ethanol followed by rinsing in ethanol resulted in hydrophobic porous media, but also resulted in excess OTS (i.e., present but not bonded to silica sands or porous ceramics), which dissolved in most solvents used in multiphase flow experiments. Excess OTS was found to dissolve in heptane, decane, soltrol 100 , soltrol 220 , toluene, and TCE. It was shown that OTSimpacted solvents rendered both water-coated and dry water-wet silica sands hydrophobic. An enhanced washing technique for NAPL-wet silica sands and porous ceramics was presented. This enhanced washing technique results in a hydrophobic porous medium that is free of excess OTS. 
- A comprehensive analysis of $P_{c}-S$ data in air/heptane, air/water, and heptane/water two-phase systems under different soil wettability conditions, corresponding to water-wet porous medium with different size NAPL-wet soil lenses was presented. The water-wet porous medium with a NAPL-wet soil lens consists of two parts; a water-wet soil fraction and a NAPL-wet soil lens. The approach taken in this research was to consider the water-wet porous medium with the NAPL-wet soil lens as the field scale REV and to derive constitutive relationships for this REV scale based on our knowledge of the constitutive relationships of each soil fraction (water-wet and NAPL-wet). Based on the REV scale two-phase data and supported by pore scale images, conceptual models to describe the $P_{c} \neg S$ relationship for each fluid pair for an REV consisting of a water-wet porous medium with a NAPL-wet soil lens were formulated. Then, the developed conceptual models were validated using the $P_{c}-S$ data.

- Conceptual models were developed in a three-phase system for air/heptane capillary pressure-total liquid saturation and heptane/water capillary pressurewater saturation relationships in a water-wet porous medium with a NAPL-wet soil lens. Two scenarios corresponding to isolated and connected NAPL-wet soil lenses were considered. The conceptual model for air/heptane capillary pressuretotal liquid saturation relationship was validated for a connected NAPL-wet soil lens and constant water saturation. In addition, Leverett assumption in this porous medium was checked.

- A comprehensive analysis for residual heptane saturation data and development of a new conceptual model for residual NAPL saturation in a water-wet porous 
medium were presented. This conceptual model assumes that the residual NAPL saturation depends on eight parameters; porous medium specific surface area, NAPL entry pressure head in NAPL/water two-phase system, porous medium grain size distribution (through the fitting parameter $\lambda$ ), porous medium porosity, NAPL film thickness, apparent water saturation, historic maximum apparent NAPL saturation, and the absolute maximum effective residual NAPL saturation in the porous medium. The new model was validated using the effective residual heptane saturations measured under a large range of experimental conditions. Comparison of the new model predictions to the predictions of Van Geel and Roy (2002) linear and Land models and Lenhard et al. (2004) model, using the effective residual heptane saturations measured in this research and in Van Geel and Roy (2002), was in the favour of the new model. Two conceptual models were developed to describe the residual NAPL saturations in a water-wet porous medium with an isolated or a connected NAPL-wet soil lens, respectively. The conceptual model that described the residual NAPL saturation in a water-wet porous medium with a connected NAPL-wet soil lens was then validated. In most cases, the model predictions agreed with the measured effective residual heptane saturations.

\subsection{Contributions}

This research addressed the effectiveness of the traditional soil treatment procedure. It also addressed the $P_{c}-S$ relationships in a water-wet porous medium with a NAPL-wet soil lens. In addition, it addressed the residual NAPL saturation in a water-wet 
porous medium and in a water-wet porous medium with a NAPL-wet soil lens. The main contributions of this research can be summarized as follows:

- An enhanced washing technique for hydrophobic silica sands and porous ceramics was developed. The traditional procedure that has been widely published in the literature to render hydropholic silica sands and porous ceramics hydrophobic results in excess unbound OTS on treated surfaces. This unbound OTS migrates through the NAPL from the NAPL-wet sands or porous ceramics to the adjacent water-wet sands or porous ceramics rendering them hydrophobic. In the enhanced washing technique, hydrophilic silica sand was rendered hydrophobic according to the traditional treatment procedure. The silica sands were then placed in hexane in a soxhlet extractor for 24 hours. The hexane was replaced with fresh hexane after 12 hours. After drying the treated sand sample, it should be washed in the soxhlet extractor with the NAPL type that will be used in the multiphase flow experiments for 12 hours. The only restriction on using this procedure is that it cannot be used for solvents with boiling points higher than $100^{\circ} \mathrm{C}$ as the OTS bond to the soil grains may be broken at these temperatures. The proposed enhanced washing technique results in hydrophobic silica sands and porous ceramics free of excess unbound OTS.

- A large set of $P_{c} \rightarrow S$ data were measured in two and three-phase systems in waterwet porous media and in water-wet porous media with NAPL-wet soil lenses. In addition, a large number of residual heptane saturation experiments, corresponding to a large range of experimental conditions, were conducted in 
water-wet porous media and in water-wet porous media with NAPL-wet soil lenses.

- Conceptual models for $P_{c}-S$ relationships in two and three-phase systems in a water-wet porous medium with NAPL-wet soil lenses were developed. The conceptual models were developed in two-phase systems for air/NAPL, air/water, and NAPL/water. Conceptual models were also developed for air/NAPL capillary pressure-total liquid saturation and NAPL/water capillary pressure-water saturation relationships in three-phase systems.

- A conceptual model for residual NAPL saturation in a water-wet porous medium was developed. This conceptual model provides a physical description of the distribution of residual NAPL at the pore scale and relates the residual NAPL saturation to saturation histories and porous medium properties through the inclusion of eight parameters within the model; porous medium specific surface area, NAPL entry pressure head in NAPL/water two-phase system, porous medium grain size distribution (through the fitting parameter $\lambda$ ), porous medium porosity, NAPL film thickness, apparent water saturation, historic maximum apparent NAPL saturation, and the absolute maximum effective residual NAPL saturation in the porous medium. Moreover, conceptual models were developed for residual NAPL saturation in water-wet porous media with isolated or connected NAPL-wet soil lenses. 


\subsection{Recommendations}

Based on the findings of this research, the following are recommended for future research:

- Development of a general soil treatment technique that is capable of rendering water-wet solids NAPL-wet, regardless of the NAPL used in flow or relative permeability- $P_{c} \rightarrow S$ experiments;

- Development of conceptual models for $k_{r}-S$ relationship in a water-wet porous medium with isolated or connected NAPL-wet soil lenses;

- Further validation of the developed constitutive relationships in laboratory NAPL spill experiments both in one and two dimensional flow experiments for isolated and connected NAPL-wet soil lenses;

- Testing the effect of the Brooks and Corey parameter (i.e., $\lambda$ ) on the residual NAPL saturation-water saturation relationship. This requires testing soils with various grain size distributions ranging from uniform to well graded with different median grain sizes (i.e., $50^{\text {th }}$ percentile);

- Validation of the new conceptual model for residual NAPL saturation in soils with various grain size distributions using different NAPLs; and

- Investigation of the implications of the NAPL-wet soil lenses on the effectiveness of the remedial alternatives. In addition, development of a technique for remediating contaminated sites with NAPL present in NAPL-wet soil lenses. 


\section{REFERENCES}

Adamson, A.W. 1997. Physical Chemistry of Surfaces $\left(6^{\text {th }}\right.$ ed. $)$. John Wiley \& Sons, Inc., New York, USA.

Al-Raoush R.I., and Wilson, C.S. 2005. A Pore Scale Investigation of a Multiphase Porous Media System. Journal of Contaminant Hydrology. Vol. 77, pp. 67- 89.

Anderson, W. 1987. Wettability Literature Survey. Part 4. Effects of Wettability on Capillary Pressure. Journal of Petroleum Technology. Vol 39. pp. 1283-1300.

Anderson, R., Larson, G., and Smith, C., 1991. Silicon Compounds: Register and Review 5th. Huls America Inc, Piscataway, NJ.

Aveyard, R., Binks, B.P., Clark, S, and Mead, J. 1986. Interfacial Tension Minima in OilWater-Surfactant Systems; Behaviour of Alkane-Aqueous $\mathrm{NaCl}$ Systems Containing Aerosol OT. Journal of Chemical Society, Faraday Transactions. 1, 82, $125-142$.

Bear, J., 1988. Dynamics of Fluids in Porous Media. Dover, New York

Bradford, S.A. and Leij, F.J, 1995a. Wettability Effects on Scaling Two- and Three-Fluid Capillary Pressure-Saturation Relations. Environmental Science and Technology Journal. Vol. 29, pp. $1446-1455$.

Bradford, S.A. and Leij, F. J., 1995b. Fractional Wettability Effects on Two- and ThreeFluid Capillary Pressure-Saturation Relations. Journal of Contaminant Hydrology. Vol. 20, pp. 89-105.

Bradford, S.A. and F. Leij. 1996. Predicting Two- and Three- Fluid Capillary PressureSaturation Relationships of Porous Media with Fractional Wettability. Water Resources Research. Vol 32(2). pp. 251-259. 
Bradford, S., Abriola L., and Rathfelder K. 1998. Flow and Entrapment of Dense Nonaqueous Phase Liquids in Physically and Chemically Heterogeneous Aquifer Formations. Advances in Water Resources. Vol. 22. (2), pp. 117-132.

Bradford, S., Vendlinski, R., and Abriola, L. 1999. The Entrapment and Long-term Dissolution of Tetachloroethylene in Fractional Wettability Porous Media. Water Resources Research. Vol. 35 (10). pp. 2955-2964.

Brooks, R.H. and Corey, A.T., 1966. Properties of Porous Media Affecting Fluid Flow. Journal of Irrigation and Drainage Division, Proc. ASCE, Vol. 92, IR 2, pp. 6188.

Brunauer, S., Emmett, P. H., and Teller, E., 1938. Adsorption of Gases in Multi Molecular Layers. Journal of the American Chemical Society, Vol. 60, pp. 309-319. Cary, J.W., 1994. Estimating the Surface Area of Fluid Phase Interfaces in Porous Media. Journal of Contaminant Hydrology, 15, 243- 248.

Cha, K. and Kim, D., 2001. Investigation of the Tribological Behavior of Octadecyltrichlorosilane Deposited on Silicon. Wear. Vol. 251, 1169-1176.

Collins, R. E., 1961. Flow of Fluids through Porous Materials. Reinhold Publishing Corporation, New York.

Corey, A.T., 1977. Mechanics of Heterogeneous Fluids in Porous Media. Water Resources, Colorado, USA.

Corey, A.T., 1994. Mechanics of Immiscible Fluids in Porous Media. Water Resources, Colorado, USA.

Craig, F. F. 1971. The Reservoir Engineering Aspects of Water Flooding. Monograph Series, Vol. 3, Society of Petroleum Engineers, Richardson. TX (cited in O'Carroll et al. 2004). 
CRC Handbook of Chemistry and Physics, 68th Edition, CRC Press, Boca Raton, Fl, 1987. (cited in Selker et al. 1999)

Dane, J.H., Oostrom, M., and Missildine, B.C., 1992. An Improved Method for the Determination of Capillary Pressure - Saturation Curves Involving TCE, Water and Air. Journal of Contaminant Hydrology, Volume 11, pp. $69-81$.

Dullien, F. A. L., 1991. Porous Media. Academic Press Limited, London, U.K.

Dwarakanath, D., Jackson, R.E., and Pope, G.A., 2002. Influence of Wettability on the Recovery of NAPLs from Alluvium. Environ. Sci. Technol. 36, pp. 227-231.

Dyal, R S. and Hendricks, S. B., 1950. Total Surface of Clays in Polar Liquids as a Characteristic Index. Soil Science, Vol. 69, pp.421-432.

Faust, Charles R. (GeoTrans Inc, Herndon, VA, USA) 1985. Transport of Immiscible Fluids Within and Below the Unsaturated Zone. Water Resour. Res. v 21, n 4, 587 596.

Gardner, R., 1955. Relation of Temperature to Moisture Tension of Soil. Soil Science, Vol. 79, pp. $257-265$.

Helmig, R., 1997. Multiphase Flow and Transport Processes in the Subsurface. Springer Verlag Berlin Heidelberg

Hillel, D. 1980. Fundamentals of Soil Physics. Academic Press, San Diego, CA.

Hofstee, C., Dane, J.H., and Hill, W.E., 1997. Three-Fluid Retention in Porous Media Involving Water, PCE, and air. J. Contam. Hydrol. 25, 235 - 247.

Hofstee, C., Oostrom, M., Dane, J.H., and Walker, R.C., 1998. Infiltration and Redistribution of Perchloroethylene in Partially Saturated, Stratified Porous Media. Journal of Contaminant Hydrology, Vol. 34, pp. 293 - 313. 
Kalaydjian, F. and Tixier, M., 1991. Effects of the Spreading Coefficients on Gasroil Capillary Pressure Curves in Presence of Connate Water. SCA conference paper, Paper number 9106.

Kluth, G.J., Sung, M.M., and Maboudian, R., 1997. Thermal behaviour of alkylsiloxane self-assembled monolayers on the oxidized $\operatorname{Si}(100)$ surface. Langmuir 17 (2), $3775-3780$.

Kuppusamy, T., Sheng, J., Parker, J.C., and Lenhard, R.L., 1987. Finite Element Analysis of Multiphase Immiscible Flow through Soils. Water Resources Research, Volume 23. Issue 4, pp. $625-631$.

Land, C.S., 1968. Calculation of Imbibition Relative Permeability for Two- and ThreePhase Flow from Rock Properties .Soc. Pet. Eng. J., 149-156 (June).

Lenhard, R.J. and Parker, J.C., 1987a. Measurement and Prediction of Saturation Pressure Relationships in Three - Phase Porous Media System. Journal of Contaminant Hydrology, 1 (1987) 407-424.

Lenhard, R.J. and Parker, J.C., 1987b. A model for Hysteretic Constitutive Relations Governing Multiphase Flow: 2. Permeability-saturation relations. Water Resources Research. 23, $2197-2206$.

Lenhard, R.J. and Parker, J.C., 1988. Experimental Validation of the Theory of Extending Two-Phase Saturation-Pressure Relations to Three-Phase Systems for Monotonic Drainage Paths. Water Resources Research. 24, 373 - 380.

Lenhard, R.J., 1992. Measurement and Modelling of Three-Phase Saturation- Pressure Hysteresis. J. Contam. Hydrol. 9, 243 - 269. 
Lenhard, R.J., Oostrom, M., and Dane, J.H., 2004. A Constitutive Model for Air-NAPLWater Flow in the Vadose Zone Accounting for Residual NAPL in Strongly Water Wet Porous Media. Journal of Contaminant Hydrology 71:261-282.

Leverett, M.C., 1941. Capillary Behaviour in Porous Solids. Petrol. Trans., AIME, Volume 142, pp. $152-169$.

Leverett, M.C. and Lewis, W. B. 1941. Steady Flow of Gas-Oil-Water Mixtures through Unconsolidated Sands. Petroleum Technology, Issue 1206, pp. $107-116$.

Leverett, M.C., Lewis, W.B., and True, M.E., 1942. Dimensional-Model Studies of OilField Behavior. Pet. Technol., Tech. Paper 1413, January, pp. 175-193.

Lowry, M. and Miller C., 1995. Pore-scale Modeling of Non-wetting Phase Residual in Porous Media. Water Resources Research, Vol. 31, No. 3, pp. 455-473.

Luque de Castro, M.D. and Garcia-Ayuso, L.E. 1998. Soxhlet Extraction of Solid Materials: an outdated technique with a promising innovative future. Analytica Chimica Acta. 369, 1-10.

Maoz, R. and Sagiv, J., 1984. On the Formation and Structure of Self-assembling Monolayers. I. A Comparative ATR-Wettability Study of Langmuir-Blodgett and Adsorbed Films on Flat Substrates and Glass Microbeads. Journal of Colloid Interface Science. Vol. 100, pp. 465-496.

Mayer, A.S. and Miller, C.T., 1992. The Influence of Porous Medium Characteristics and Measurement Scale on Pore-scale Distributions of Residual Nonaqueous-Phase Liquids. Journal of Contaminant Hydrology, Vo. 11, pp. $189-213$.

McWhorter, D.B. and Kueper, B.H., 1996. Mechanics and Mathematics of the Movement of Dense, Non-Aqueous Phase Liquids (DNAPLs) in Porous 
Media. Chapter 3, Dense Chlorinated Solvents and Other DNAPLs in Groundwater, Edited by J.F. Pankow and J.A. Cherry, Waterloo Educational Services.

Meinardus, H.W., Dwarakanath, V., Ewing, J., Hirasaki, G.J., Jackson, R.E., Jin, M., Ginn, J.S., Londergan, J.T., Miller, C.A., and Pope, G.A., 2002. Performance Assessment of NAPL Remediation in Heterogeneous Alluvium. Journal of Contaminant Hydrology 54, pp. 173- 193.

Mercer, J.W. and Cohen, R.M., 1990. A Review of Immiscible Fluids in the Subsurface: Properties, Models, Characterization and Remediation. Journal of Contaminant Hydrology 6, $107-163$.

Miller, C.T., Christakos, G., Imhoff, P.T., McBride, J.F., and Pedit, J.A., 1998. Multiphase Flow and Transport Modelling in Heterogeneous Porous Media: Challenges and Approaches. Advances in Water Resources Journal, Vol. 21, No. 2, pp. $77-120$.

O'Carroll D., S. Bradford, and L. Abriola. 2004. Infiltration of PCE in a System Containing Wettability Variations. Journal of Contaminant Hydrology. Vol. 73, pp. 39-63.

O'Caroll, D., Abriola, L., Polityka, T., Bradford, S., and Demond, A. 2005. Prediction of Two-Phase Capillary Pressure-Saturation Relationships in Fractional Wettability Systems. Journal of Contaminant Hydrology, Vol. 77, pp. 247-270.

Parker, J.C., Lenhard, R.J., and Kuppusamy, T., 1987. A Parametric Model for Constitutive Properties Governing Multiphase Fluid Flow in Porous Media. Water Resources Research, 23: 618-624. 
Parker, J.C. and Lenhard, R.J., 1987. A Model for Hysteretic Constitutive Relations Governing Multiphase Flow: 1. Saturation - Pressure Relations. Water Resour. Res. $23,2187-2196$.

Pennell, K., Pope G., and Abriola, L. 1996. Influence of Viscous and Buoyancy Forces on the Mobilization of Residual tetracHloroethylene during Surfactant Flushing. Environmental Science and Technology. Vol. 30 (4). pp. 1328-1335. (cited in O'Carroll et al. 2004)

Powers, S. and Tamblin, M. 1995. Wettability of Porous meDia after Exposure to Synthetic Gasolines. Journal of Contaminant Hydrology. Vol 19 (2). pp. 105-125.

Reeves, P.C. and Celia, M.A., 1996. A Functional relAtionship between Capillary Pressure, Saturation, and Interfacial Area as Revealed by a Pore-Scale Network Model. Water Resources Research, Vol. 32, No. 8, pp. 2345 - 2358.

Rose, C.W., 1966. Agricultural Physics. Pergamon, Oxford, 226 pp.

Schreiber, F. 2000. Structure and Growth of Self-assembling Monolayers. Progress in Surface Science. Vol. 65, pp. $151-257$.

Schumacher, B.A., Shines, K.C., Burton, J.V., and Papp, M.L., 1990. Comparison of Three Methods for Soil Homogenization. Soil Science Society of America Journal, Volume (54), pp. $1187-1190$.

Schwille, F., 1988. Dense Chlorinated Solvents in Porous and Fractured Media: Model Experiments. ed. Chelsea, MI, Lewis Publishers, Inc.

Selker, J.S., Keller, C.K., and McCord. J.T., 1999. Vadose Zone Processes CRC Press LLC, New York, USA. 
Silberzan, P., Leger, L., Ausserre, D., and Benattar, J.J. 1991. Silanation of Silica Surfaces. A New Method of Constructing Pure or Mixed Monolayers. Langmuir. Vol. 7, pp. 1647-1651.

Taber, J.J., 1968, Dynamic and Static Forces Required to Remove a Discontinuous Oil Phase from Porous Media Containing Both Oil and Water. American Institute of Mining, Metallurgical, and Petroleum Engineers.

Thomson, N.R., Graham, D.N., and Farquhar G.J. 1992. One-Dimensional Immiscible Displacement Experiments. Journal of Contaminant Hydrology 10, 197-223.

Van Geel, P.G. and Sykes, J.F., 1994. Laboratory and model simulations of a LNAPL spill in a variably-saturated sand,1. Laboratory Experiment and Image Analysis Techniques. Journal of Contaminant Hydrology, Volume 17, pp. $1-25$.

Van Geel, P.J. and Roy, S.D., 2002. A Proposed Model to Include a Residual NAPL Saturation in Hysteretic Capillary Pressure-Saturation Relationship. J. Contam. Hydrol. 58, 79- 110 .

Van Genuchten, M.Th., 1980. A Closed-Form Equation for Predicting the Hydraulic Conductivity of Unsaturated Soils. Soil Sci. Soc. Am. J. 44, $892-898$.

Van Genuchten, M.Th. and Nielsen, D.R., 1985. On Describing and Predicting the Hydraulic Properties of Unsaturated Soils. Ann. Geophys. 3 (5), 615-628.

Wardlaw, N.C., 1982. The Effects of Geometry, Wettability, Viscosity and Interfacial Tension on Trapping in Single Pore-Throat Pairs. Journal of Canadian Petroleum Technology 21, no. 1:21-27. 
White, M.D., Oostrom, M., and Lenhard, R.J. 2004. A Practical Model for Mobile, Residual, and Entrapped NAPL in Water-Wet Porous Media. Ground Water 42:734-46.

Wilson, J.L. and Conrad, S.H. 1984. Is Physical Displacement of Residual Hydrocarbons a Realistic Possibility in Aquifer Restoration? Proceedings of the NWWA/API Conference on Petroleum Hydrocarbons and Organic Chemicals in Ground Water Prevention, Detection and Restoration, Houston, Texas, USA.

Wipfler, E.L. and Van der Zee, S.E.A.T.M., 2001. A Set of Constitutive Relationships Accounting for Residual NAPL in the Unsaturated Zone. J. Contam. Hydrol. 50, 53 -77 .

Yoshida, W, Castro, R. P., Jou, J. D., and Cohen, Y. 2001. Multilayer Alkoxysilane Silylation of Oxide Surfaces. Langmuir. Vol. 17, pp. 5882-5888.

Zalidis, G.C., Wallace, R. B., and Voice, T.C., 1998. Influence of Initial Water Saturation on the Residual Saturation of an Organic Liquid in the Vadose Zone. Journal of Water Resources Management. 12, 81-93. 